\title{
Cobalt (II)-Catalyzed Stereoselective Olefin Isomerization: Facile Access to Acyclic Trisubstituted Alkenes
}

Sheng Zhang, Deepika Bedi, Lu Cheng, Daniel K. Unruh, Guigen Li and Michael Findlater*

Department of Chemistry \& Biochemistry, Texas Tech University, Lubbock TX, 79409, USA.

\section{Supporting Information}

Table of Contents

Part I Experimental Section

1. General information

2. General procedure for the synthesis of substrates

3. Deuterium labeling substrate synthesis

4. General procedure for the synthesis of ligands

5. Optimization of reaction conditions

6. General procedures for alkene isomerization

7. Procedure for gram scale alkene isomerization

8. Synthesis of deuterium labeled product

9. Procedure for catalyst-recycling experiment

10. Procedure for control experimens

11. Single crystal structures of the product and precatalyst

12. Photophysical properties of the selected products

13. Experimental data for alkene isomerization products

Part II NMR Spectra 


\section{General Information}

${ }^{1} \mathrm{H}$ NMR, ${ }^{2} \mathrm{H}$ NMR, ${ }^{13} \mathrm{C}$ NMR, ${ }^{31} \mathrm{P}$ NMR and ${ }^{19} \mathrm{~F}$ NMR were recorded on a Jeol $400 \mathrm{MHz}$ spectrometer ( ${ }^{1} \mathrm{H}$ NMR: $400 \mathrm{MHz},{ }^{2} \mathrm{H}$ NMR: $61 \mathrm{MHz},{ }^{13} \mathrm{C}$ NMR: $100 \mathrm{MHz},{ }^{31} \mathrm{P}$ NMR: $162 \mathrm{MHz},{ }^{19} \mathrm{~F}$ NMR: $376 \mathrm{MHz}$ ). The chemical shifts ( $\delta$ ) and coupling constants (J) were expressed in ppm and $\mathrm{Hz}$ respectively. ${ }^{1} \mathrm{H}$ NMR spectra were referenced to the solvent residual peak $\left(\mathrm{CDCl}_{3}, \delta 7.26 \mathrm{ppm}\right)$ and ${ }^{13} \mathrm{C}\{1 \mathrm{H}\}$ NMR spectra were referenced to the solvent residual peak $\left(\mathrm{CDCl}_{3}, \delta 77.0 \mathrm{ppm}\right)$. A single value was given from the center of the multiplets. IR was recorded on Nicolet iS5 FTIR spectrometer. High Resolution mass spectra were obtained using Thermo Scientific Exactive mass spectrometer, Santa Clara, CA with HESI electrospray with $50 \%$ acetonitrile water containing either $0.1 \%$ Formic acid at infusion rate of $5 \mu \mathrm{L} / \mathrm{min}$. GC-MS data were acquired using Thermo Scientific ${ }^{\mathrm{TM}} \mathrm{ISQ}^{\mathrm{TM}}$ Single Quadrupole system. Photoluminescence property of the solid samples were acquired using FLS980 series of fluorescence spectrometers. All solvents were purified and dried according to the standard procedures unless otherwise noted. Commercially substrates were purchased and used directly.

\section{General procedure for the synthesis of substrates (1e as an example)}

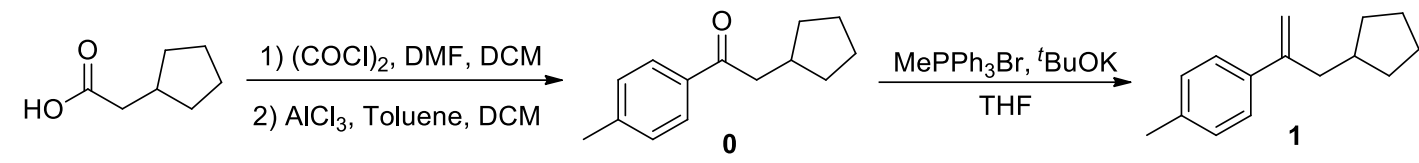

Step 1: To a solution of 2-cyclopentylacetic acid (2.56 g, $20.0 \mathrm{mmol}$ ) in $40 \mathrm{~mL} \mathrm{CH}_{2} \mathrm{Cl}_{2}$ at $0{ }^{\circ} \mathrm{C}$, was dropwise added oxalyl chloride $(2.03 \mathrm{~mL}, 24.0 \mathrm{mmol}, 1.2$ equiv.). Then a catalytic amount of anhydrous DMF (150 $\mu \mathrm{L}$ ) was carefully added and the resulting mixture was allowed to warm to room temperature, stirring for $2 \mathrm{~h}$. The reaction mixture was concentrated in vacuo and the acyl chloride was used immediately without further purification.

Step 2: To a mixed solution of DCM and toluene $(30 \mathrm{~mL}, 2 / 1)$ containing the above acyl chloride was added anhydrous $\mathrm{AlCl}_{3}\left(2.93 \mathrm{~g}, 22 \mathrm{mmol}, 1.1\right.$ equiv.) in portions at $0{ }^{\circ} \mathrm{C}$. The resulting mixture was stirred at the same temperature for $30 \mathrm{~min}$. The reaction mixture was poured into chilled $\mathrm{HCl}$ solution $(2 \mathrm{M}, 100 \mathrm{~mL})$ and extracted with ethyl acetate three times $(3 \times 50 \mathrm{~mL})$. The combined organic phase was washed with sat. $\mathrm{NaCl}(200 \mathrm{~mL})$. It was then dried over $\mathrm{Na}_{2} \mathrm{SO}_{4}$ and evaporated in vacuo. The product 2-cyclopentyl-1-( $p$-tolyl)ethanone $(3.1 \mathrm{~g}, 77 \%$ yield) was isolated by flash column chromatography (hexanes/EtOAc 50/1 followed by hexanes/EtOAc 20/1).

Step 3: To a suspension of ${ }^{\mathrm{t}} \mathrm{BuOK}$ ( $1.35 \mathrm{~g}, 12 \mathrm{mmol}, 1.2$ equiv.) in anhydrous THF (20 mL) was added $\mathrm{MePPh}_{3} \mathrm{Br}$ ( $4.28 \mathrm{~g}, 12 \mathrm{mmol}, 1.2$ equiv.) under argon atmosphere. The suspension was stirred at room temperature for $1 \mathrm{~h}$, and then 2-cyclopentyl-1-( $p$-tolyl)ethanone $(2.02 \mathrm{~g}, 10 \mathrm{mmol})$ was added. The resulting mixture was stirred at same temperature for $1 \mathrm{~h}$ (In the cases of 10-1ah and 1ao-1aq, higher temperature $50{ }^{\circ} \mathrm{C}$ is required). Then the mixture was filtered through a short pad of silica gel, which was subsequently washed with hexanes $(200 \mathrm{~mL})$. After evaporation of the organic solvent, the residue was purified by silica gel column chromatography (hexanes followed by hexanes/EtOAc 100/1) to provide $1.91 \mathrm{~g}$ (95\% yield) of $1 \mathrm{e}$ as a colorless oil. 
<smiles>C=C(CC)c1ccccc1</smiles>

But-1-en-2-ylbenzene (1a): The title substrate was synthesized from propiophenone $(10 \mathrm{mmol})$ according to the above procedure Step 3. Colorless oil; $1.28 \mathrm{~g}, 97 \%$ yield; ${ }^{1} \mathrm{H}$ NMR (400 MHz, $\left.\mathrm{CDCl}_{3}\right)$ : $7.46(\mathrm{~m}, 2 \mathrm{H}), 7.37(\mathrm{~m}, 2 \mathrm{H}), 7.30(\mathrm{~m}, 1 \mathrm{H}), 5.32(\mathrm{~m}, 1 \mathrm{H}), 5.10(\mathrm{q}, J=1.6 \mathrm{~Hz}, 1 \mathrm{H}), 2.56(\mathrm{q}, J=$ $7.2 \mathrm{~Hz}, 2 \mathrm{H}), 1.15(\mathrm{t}, J=7.4 \mathrm{~Hz}, 3 \mathrm{H})$; These data are in accordance with the literature. ${ }^{1}$<smiles>C=C(CCC)c1ccccc1</smiles>

Pent-1-en-2-ylbenzene (1b): The title substrate was synthesized from 1-phenylbutan-1-one (10 mmol) according to the above procedure Step 3. Colorless oil; $1.31 \mathrm{~g}, 90 \%$ yield; ${ }^{1} \mathrm{H}$ NMR (400 $\left.\mathrm{MHz}, \mathrm{CDCl}_{3}\right): 7.44(\mathrm{~m}, 2 \mathrm{H}), 7.36(\mathrm{~m}, 2 \mathrm{H}), 7.29(\mathrm{~m}, 1 \mathrm{H}), 5.31(\mathrm{~d}, J=1.6 \mathrm{~Hz}, 1 \mathrm{H}), 5.09(\mathrm{q}, J=1.6 \mathrm{~Hz}$, $1 \mathrm{H}), 2.52(\mathrm{t}, J=8.0 \mathrm{~Hz}, 2 \mathrm{H}), 1.52(\mathrm{~m}, 2 \mathrm{H}), 0.96(\mathrm{t}, J=6.0 \mathrm{~Hz}, 3 \mathrm{H})$; These data are in accordance with the literature. ${ }^{1}$<smiles>C=C(CCCC)c1ccccc1</smiles>

Hex-1-en-2-ylbenzene (1c): The title substrate was synthesized from 1-phenylpentan-1-one (10 mmol) according to the above procedure Step 3. Colorless oil; $1.42 \mathrm{~g}, 89 \%$ yield; ${ }^{1} \mathrm{H}$ NMR (400 $\left.\mathrm{MHz}, \mathrm{CDCl}_{3}\right): 7.45(\mathrm{~m}, 2 \mathrm{H}), 7.36(\mathrm{~m}, 2 \mathrm{H}), 7.29(\mathrm{~m}, 1 \mathrm{H}), 5.30(\mathrm{~m}, 1 \mathrm{H}), 5 . .09(\mathrm{q}, J=1.6 \mathrm{~Hz}, 1 \mathrm{H}), 2.54$ $(\mathrm{t}, J=7.6 \mathrm{~Hz}, 2 \mathrm{H}), 1.43(\mathrm{~m}, 4 \mathrm{H}), 0.94(\mathrm{t}, J=7.2 \mathrm{~Hz}, 3 \mathrm{H})$; These data are in accordance with the literature. $^{2}$<smiles>C=C(CCc1ccccc1)c1ccccc1</smiles>

But-3-ene-1,3-diyldibenzene (1d): The title substrate was synthesized from 1,3-diphenylpropan-1-one (5 mmol) according to the above procedure Step 3. Colorless oil; $1.0 \mathrm{~g}$, 96\% yield; $\left.{ }^{1} \mathrm{H} \mathrm{NMR} \mathrm{(400} \mathrm{MHz,} \mathrm{CDCl}_{3}\right): 7.51(\mathrm{~m}, 2 \mathrm{H}), 7.42(\mathrm{~m}, 2 \mathrm{H}), 7.35(\mathrm{~m}, 3 \mathrm{H}), 7.26(\mathrm{~m}, 3 \mathrm{H}), 5.37$ $(\mathrm{s}, 1 \mathrm{H}), 5.14(\mathrm{~s}, 1 \mathrm{H}), 2.87(\mathrm{~m}, 4 \mathrm{H})$. These data are in accordance with the literature. ${ }^{3}$<smiles>Cc1ccc(C(=O)CC2CCCC2)cc1</smiles>

2-Cyclopentyl-1-(p-tolyl)ethanone (0e): The title substrate was synthesized from 2-cyclopentylacetic acid (20 mmol) according to the above procedure Step 1-2. Colorless oil; $3.1 \mathrm{~g}$, $77 \%$ yield; ${ }^{1} \mathrm{H}$ NMR (400 MHz, CDCl $): 7.86$ (d, $J=8.0 \mathrm{~Hz}, 2 \mathrm{H}$ ), 7.24 (d, $J=8.4 \mathrm{~Hz}, 2 \mathrm{H}$ ), 2.95 (d, $J=$ $7.2 \mathrm{~Hz}, 2 \mathrm{H}), 2.40(\mathrm{~s}, 3 \mathrm{H}), 2.36(\mathrm{~m}, 1 \mathrm{H}), 1.86(\mathrm{~m}, 2 \mathrm{H}), 1.59(\mathrm{~m}, 4 \mathrm{H}), 1.18(\mathrm{~m}, 2 \mathrm{H})$. These data are in accordance with the literature. ${ }^{4}$ 
<smiles>C=C(CC1CCCC1)c1ccc(C)cc1</smiles>

1-(3-Cyclopentylprop-1-en-2-yl)-4-methylbenzene (1e): The title substrate was synthesized from 2-cyclopentyl-1-( $p$-tolyl)ethanone $(10 \mathrm{mmol})$ according to the above procedure Step 3. Colorless oil; $1.91 \mathrm{~g}$, 95\% yield; ${ }^{1} \mathrm{H}$ NMR (400 MHz, $\left.\mathrm{CDCl}_{3}\right): 7.35(\mathrm{~d}, J=8.4 \mathrm{~Hz}, 2 \mathrm{H}), 7.18(\mathrm{~d}, J=8.4 \mathrm{~Hz}, 2 \mathrm{H}$ ), $5.25(\mathrm{~d}, J=1.6 \mathrm{~Hz}, 1 \mathrm{H}), 5.04(\mathrm{~d}, J=1.2 \mathrm{~Hz}, 1 \mathrm{H}), 2.53(\mathrm{~d}, J=7.6 \mathrm{~Hz}, 2 \mathrm{H}), 2.39(\mathrm{~s}, 3 \mathrm{H}), 1.96(\mathrm{~m}, 1 \mathrm{H})$, $1.67(\mathrm{~m}, 4 \mathrm{H}), 1.51(\mathrm{~m}, 2 \mathrm{H}), 1.20(\mathrm{~m}, 2 \mathrm{H}) ;{ }^{13} \mathrm{C} \mathrm{NMR}\left(100 \mathrm{MHz}, \mathrm{CDCl}_{3}\right): 148.2,138.6,136.9,128.9$, 126.1, 112.0, 42.0, 38.2, 32.4, 25.0, 21.1; IR (neat): 2946, 2864, 1624, 1512, 1449, 889, 823; GC-MS: $t_{R}=9.51 \mathrm{~min}, \mathrm{~m} / \mathrm{z}=200.2$.<smiles>Cc1ccc(C(=O)CC2CC3CCC2C3)cc1</smiles>

2-(Bicyclo[2.2.1]heptan-2-yl)-1-(p-tolyl)ethanone (0f): The title substrate was synthesized from 2-norbornaneacetic acid (20 mmol) according to the above procedure Step 1-2. White solid, m.p. 66-67 ${ }^{\circ} \mathrm{C} ; 3.74 \mathrm{~g}, 82 \%$ yield; ${ }^{1} \mathrm{H} \mathrm{NMR}\left(400 \mathrm{MHz}, \mathrm{CDCl}_{3}\right): 7.85(\mathrm{~d}, J=8.4 \mathrm{~Hz}, 2 \mathrm{H}), 7.24(\mathrm{~d}, J=8.0 \mathrm{~Hz}$, 2H), $2.93(\mathrm{dd}, J=7.6 \mathrm{~Hz}, J=16.0 \mathrm{~Hz}, 1 \mathrm{H}$ ), $2.75(\mathrm{dd}, J=7.6 \mathrm{~Hz}, J=16.0 \mathrm{~Hz}, 1 \mathrm{H}$ ), $2.40(\mathrm{~s}, 3 \mathrm{H}), 2.22$ (br, 1H), $2.04(\mathrm{~m}, 2 \mathrm{H}), 1.57(\mathrm{~m}, 1 \mathrm{H}), 1.48(\mathrm{~m}, 2 \mathrm{H}), 1.37(\mathrm{~m}, 1 \mathrm{H}), 1.23(\mathrm{~m}, 1 \mathrm{H}), 1.12(\mathrm{~m}, 3 \mathrm{H}) ;{ }^{13} \mathrm{C}$ NMR $\left(100 \mathrm{MHz}, \mathrm{CDCl}_{3}\right): 199.7,143.5,134.8,129.2,128.2,45.6,41.2,38.2,38.0,36.7,35.4,29.9$, 28.6, 21.6; IR (neat): 2929, 2863, 1672, 1599, 1224, 1180, 799; GC-MS: $t_{R}=10.84 \mathrm{~min}, \mathrm{~m} / \mathrm{z}$ $=228.2$.<smiles>C=C(CC1CC2CCC1C2)c1ccc(C)cc1</smiles>

2-(2-(p-Tolyl)allyl)bicyclo[2.2.1]heptanes (1f): The title substrate was synthesized from 2-(bicyclo[2.2.1]heptan-2-yl)-1-(p-tolyl)ethanone (10 mmol) according to the above procedure Step 3. Colorless oil; $2.10 \mathrm{~g}, 93 \%$ yield; ${ }^{1} \mathrm{H}$ NMR $\left(400 \mathrm{MHz}, \mathrm{CDCl}_{3}\right): 7.31$ (d, $\left.J=8.0 \mathrm{~Hz}, 2 \mathrm{H}\right), 7.14$ (d, $J=8.0 \mathrm{~Hz}, 2 \mathrm{H}), 5.24(\mathrm{~d}, J=1.6 \mathrm{~Hz}, 1 \mathrm{H}), 4.98(\mathrm{~m}, 1 \mathrm{H}), 2.49(\mathrm{dd}, J=7.6 \mathrm{~Hz}, J=14.4 \mathrm{~Hz}, 1 \mathrm{H}), 2.36(\mathrm{~s}$, $3 \mathrm{H}), 2.26(\mathrm{dd}, J=7.6 \mathrm{~Hz}, J=14.4 \mathrm{~Hz}, 1 \mathrm{H}), 2.20(\mathrm{br}, 1 \mathrm{H}), 2.02(\mathrm{br}, 1 \mathrm{H}), 1.55(\mathrm{~m}, 1 \mathrm{H}), 1.46(\mathrm{~m}, 2 \mathrm{H})$, $1.35(\mathrm{~m}, 2 \mathrm{H}), 1.09(\mathrm{~m}, 4 \mathrm{H}) ;{ }^{13} \mathrm{C} \mathrm{NMR}\left(100 \mathrm{MHz}, \mathrm{CDCl}_{3}\right): 147.3,138.8,136.9,128.9,126.1,112.3$, 42.4, 40.8, 39.9, 37.7, 36.7, 35.3, 29.9, 28.8, 21.1; IR (neat): 2945, 2866, 1512, 1455, 889, 822; GC-MS: $t_{R}=10.2 \mathrm{~min}, \mathrm{~m} / \mathrm{z}=226.2$.<smiles>Cc1ccc(C(=O)CC[C@H](C)[C@H]2CC[C@H]3[C@H]4CC[C@H]5CCCC[C@]5(C)[C@H]4CC[C@@]32C)cc1</smiles>

$(R)-4-((5 S, 8 R, 9 S, 10 S, 13 R, 14 S, 17 R)-10,13-D i m e t h y l h e x a d e c a h y d r o-1 H$-cyclopenta[ $a]$ phenanthren17-yl)-1-( $p$-tolyl)pentan-1-one (0g): The title substrate was synthesized from $5 \beta$-Cholanic acid $(1 \mathrm{~g}$, $2.78 \mathrm{mmol}$ ) according to the above procedure Step 1-2. White solid, m.p. $143-144^{\circ} \mathrm{C} ; 0.96 \mathrm{~g}, 80 \%$ 
yield; ${ }^{1} \mathrm{H}$ NMR (400 MHz, CDCl 3 ): $7.86(\mathrm{~d}, J=8.4 \mathrm{~Hz}, 2 \mathrm{H}), 7.25(\mathrm{~d}, J=8.0 \mathrm{~Hz}, 2 \mathrm{H}), 2.97(\mathrm{~m}, 1 \mathrm{H}), 2.85$ $(\mathrm{m}, 1 \mathrm{H}), 2.41(\mathrm{~s}, 3 \mathrm{H}), 1.97(\mathrm{~m}, 1 \mathrm{H}), 1.87(\mathrm{~m}, 3 \mathrm{H}), 1.73(\mathrm{~m}, 3 \mathrm{H}), 1.56(\mathrm{~m}, 2 \mathrm{H}), 1.37(\mathrm{~m}, 6 \mathrm{H}), 1.22(\mathrm{~m}$, $10 \mathrm{H}), 1.06(\mathrm{~m}, 2 \mathrm{H}), 0.98(\mathrm{~d}, J=6.4 \mathrm{~Hz}, 3 \mathrm{H}), 0.91(\mathrm{~s}, 3 \mathrm{H}), 0.86(\mathrm{dd}, J=4.0 \mathrm{~Hz}, J=12.8 \mathrm{~Hz}, 1 \mathrm{H}), 0.65$ (s, 3H); ${ }^{13} \mathrm{C}$ NMR (100 MHz, CDCl $): 200.8,143.5,134.6,129.2,128.2,56.6,56.1,43.7,42.8,40.5$, 40.3, 37.6, 35.9, 35.6, 35.4, 35.3, 30.6, 28.3, 27.5, 27.2, 27.0, 26.6, 24.27, 24.25, 21.6, 21.3, 20.8, 18.6, 12.1; IR (neat): 2923, 2855, 1674, 1606, 1446, 1179, 815, 755; HRMS: calcd. for $\mathrm{C}_{31} \mathrm{H}_{46} \mathrm{O}$ $[\mathrm{M}+\mathrm{H}]^{+}$435.3621, found: 435.3626 .

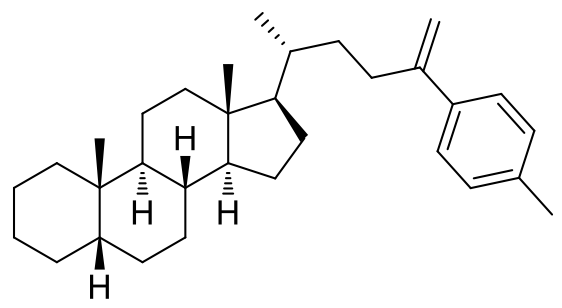

$(5 S, 8 R, 9 S, 10 S, 13 R, 14 S, 17 R)-10,13-D i m e t h y l-17-((R)-5$-( $p$-tolyl)hex-5-en-2-yl)hexadecahydro-1H-cy clopenta[a]phenanthrene $(\mathbf{1} \mathbf{g})$ : The title substrate was synthesized from the corresponding ketone (435 mg, $1 \mathrm{mmol}$ ) according to the above procedure Step 3. White solid, m.p. $97-99{ }^{\circ} \mathrm{C}$; $416 \mathrm{mg}, 96 \%$ yield; ${ }^{1} \mathrm{H}$ NMR $\left(400 \mathrm{MHz}, \mathrm{CDCl}_{3}\right): 7.31(\mathrm{~d}, J=8.4 \mathrm{~Hz}, 2 \mathrm{H}), 7.14(\mathrm{~d}, J=8.0 \mathrm{~Hz}, 2 \mathrm{H}), 5.22$ $(\mathrm{d}, J=1.6 \mathrm{~Hz}, 1 \mathrm{H}), 5.00(\mathrm{q}, J=1.2 \mathrm{~Hz}, 1 \mathrm{H}), 2.55(\mathrm{~m}, 1 \mathrm{H}), 2.34(\mathrm{~m}, 4 \mathrm{H}), 1.97(\mathrm{~m}, 1 \mathrm{H}), 1.85(\mathrm{~m}, 1 \mathrm{H})$, $1.74(\mathrm{~m}, 3 \mathrm{H}), 1.53(\mathrm{~m}, 2 \mathrm{H}), 1.39(\mathrm{~m}, 6 \mathrm{H}), 1.20(\mathrm{~m}, 11 \mathrm{H}), 1.05(\mathrm{~m}, 2 \mathrm{H}), 0.97(\mathrm{~d}, J=6.4 \mathrm{~Hz}, 3 \mathrm{H}), 0.92$ (s, 3H), $0.89(\mathrm{~m}, 2 \mathrm{H}), 0.64(\mathrm{~s}, 3 \mathrm{H}) ;{ }^{13} \mathrm{C}$ NMR $\left(100 \mathrm{MHz}, \mathrm{CDCl}_{3}\right): 149.1,138.5,136.9,128.9,125.9$, 111.0, 56.6, 56.0, 43.71, 42.72, 40.5, 40.3, 37.6, 35.9, 35.7, 35.3, 34.8, 31.9, 28.2, 27.5, 27.2, 27.0, 26.6, 24.3, 24.2, 21.3, 21.1, 20.8, 18.6, 12.0; IR (neat): 2917, 2860, 1678, 1449, 1380, 887,820; HRMS: calcd. for $\mathrm{C}_{32} \mathrm{H}_{48}[\mathrm{M}]^{+} 432.3751$, found: 432.3755 .<smiles>C=C(CC)c1ccc(C)cc1</smiles>

1-(But-1-en-2-yl)-4-methylbenzene (1h): The title substrate was synthesized from 1-( $p$-tolyl)propan-1-one $(10 \mathrm{mmol})$ according to the above procedure Step 3. Colorless oil; $1.36 \mathrm{~g}$, 93\% yield; ${ }^{1} \mathrm{H} \mathrm{NMR}\left(400 \mathrm{MHz}, \mathrm{CDCl}_{3}\right): 7.35(\mathrm{~d}, J=8.0 \mathrm{~Hz}, 2 \mathrm{H}), 7.17(\mathrm{~d}, J=8.0 \mathrm{~Hz}, 2 \mathrm{H}), 5.28(\mathrm{~m}, 1 \mathrm{H})$, $5.04(\mathrm{q}, J=1.2 \mathrm{~Hz}, 1 \mathrm{H}), 2.53(\mathrm{q}, J=7.6 \mathrm{~Hz}, 2 \mathrm{H}), 2.37(\mathrm{~s}, 3 \mathrm{H}), 1.13(\mathrm{t}, J=7.6 \mathrm{~Hz}, 3 \mathrm{H})$; These data are in accordance with the literature. ${ }^{5}$<smiles>C=C(CC)c1ccc(OC)cc1</smiles>

1-(But-1-en-2-yl)-4-methoxybenzene (1i): The title substrate was synthesized from 1-(4-methoxyphenyl)propan-1-one $(10 \mathrm{mmol})$ according to the above procedure Step 3. Colorless oil; $1.53 \mathrm{~g}$, $94 \%$ yield; ${ }^{1} \mathrm{H} \mathrm{NMR}\left(400 \mathrm{MHz}, \mathrm{CDCl}_{3}\right): 7.38$ (d, $\left.J=8.8 \mathrm{~Hz}, 2 \mathrm{H}\right), 6.88(\mathrm{~d}, J=8.8 \mathrm{~Hz}, 2 \mathrm{H}$ ), $5.23(\mathrm{~m}, 1 \mathrm{H}), 4.99(\mathrm{q}, J=1.2 \mathrm{~Hz}, 1 \mathrm{H}), 3.83(\mathrm{~s}, 3 \mathrm{H}), 2.51(\mathrm{q}, J=7.6 \mathrm{~Hz}, 2 \mathrm{H}), 1.12(\mathrm{t}, J=7.6 \mathrm{~Hz}, 3 \mathrm{H})$; These data are in accordance with the literature. ${ }^{5}$ 
(1)

1-(But-1-en-2-yl)-4-fluorobenzene (1j): The title substrate was synthesized from 1-(4-fluorophenyl)propan-1-one $(10 \mathrm{mmol})$ according to the above procedure Step 3. Colorless oil; $1.41 \mathrm{~g}, 94 \%$ yield; ${ }^{1} \mathrm{H}$ NMR $\left(400 \mathrm{MHz}, \mathrm{CDCl}_{3}\right): 7.39$ (dd, $\left.J=5.6 \mathrm{~Hz}, J=8.8 \mathrm{~Hz}, 2 \mathrm{H}\right), 7.02(\mathrm{t}, J=8.8 \mathrm{~Hz}$, $2 \mathrm{H}), 5.23(\mathrm{~m}, 1 \mathrm{H}), 5.05(\mathrm{~m}, 1 \mathrm{H}), 2.50(\mathrm{~m}, 2 \mathrm{H}), 1.11(\mathrm{t}, J=7.6 \mathrm{~Hz}, 3 \mathrm{H}) ;{ }^{19} \mathrm{~F} \mathrm{NMR}\left(376 \mathrm{MHz}, \mathrm{CDCl}_{3}\right)$ : -116.2; These data are in accordance with the literature. ${ }^{6}$<smiles>C=C(CC)c1cccc(F)c1F</smiles>

1-(But-1-en-2-yl)-2,3-difluorobenzene (1k): The title substrate was synthesized from 1-(2,3-difluorophenyl)propan-1-one $(1 \mathrm{~g}, 5.9 \mathrm{mmol})$ according to the above procedure Step 3. olorless oil; $0.92 \mathrm{~g}, 93 \%$ yield; ${ }^{1} \mathrm{H}$ NMR (400 MHz, $\left.\mathrm{CDCl}_{3}\right): 7.04(\mathrm{~m}, 3 \mathrm{H}), 5.26$ (q, J=1.2 Hz, 1H), $5.17(\mathrm{~s}, 1 \mathrm{H}), 2.48$ (q, $J=7.2 \mathrm{~Hz}, 2 \mathrm{H}), 1.06(\mathrm{t}, J=7.4 \mathrm{~Hz}, 3 \mathrm{H}) ;{ }^{13} \mathrm{C} \mathrm{NMR}\left(100 \mathrm{MHz}, \mathrm{CDCl}_{3}\right): 150.8$ (dd, $\left.J_{F-C}=246.1 \mathrm{~Hz}, J_{F-C}=13.5 \mathrm{~Hz}\right), 147.9\left(\mathrm{dd}, J_{F-C}=247.3 \mathrm{~Hz}, J_{F-C}=12.7 \mathrm{~Hz}\right), 145.4\left(\mathrm{q}, J_{F-C}=1.2 \mathrm{~Hz}\right)$, $132.5\left(\mathrm{~d}, J_{F-C}=11.2 \mathrm{~Hz}\right), 124.5\left(\mathrm{t}, J_{F-C}=3.2 \mathrm{~Hz}\right), 123.6\left(\mathrm{dd}, J_{F-C}=7.1 \mathrm{~Hz}, J_{F-C}=4.7 \mathrm{~Hz}\right), 115.7\left(\mathrm{~d}, J_{F-C}=\right.$ $17.3 \mathrm{~Hz}), 115.3\left(\mathrm{~d}, J_{F-C}=2.8 \mathrm{~Hz}\right), 29.2\left(\mathrm{~d}, J_{F-C}=2.9 \mathrm{~Hz}\right), 12.6 ;{ }^{19} \mathrm{~F} N M R\left(376 \mathrm{MHz}, \mathrm{CDCl}_{3}\right):-139.2$, -142.0; IR (neat): 2970, 1622, 1585, 1473, 1267, 954, 906, 786, 732; GC-MS: $t_{R}=6.75 \mathrm{~min}, \mathrm{~m} / \mathrm{z}$ $=168.1$.<smiles>C=C(CC)c1ccc(Cl)cc1</smiles>

1-(But-1-en-2-yl)-4-chlorobenzene (11): The title substrate was synthesized from 1-(4-chlorophenyl)propan-1-one $(10 \mathrm{mmol})$ according to the above procedure Step 3. Colorless

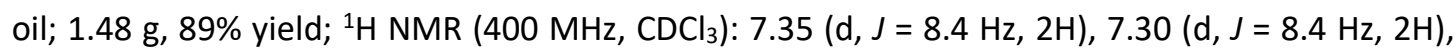
$5.27(\mathrm{~m}, 1 \mathrm{H}), 5.08(\mathrm{q}, J=1.2 \mathrm{~Hz}, 1 \mathrm{H}), 2.49(\mathrm{~m}, 2 \mathrm{H}), 1.10(\mathrm{t}, J=7.4 \mathrm{~Hz}, 3 \mathrm{H})$; These data are in accordance with the literature. ${ }^{5}$<smiles>C=C(CC)c1cccc(Cl)c1</smiles>

1-(But-1-en-2-yl)-3-chlorobenzene (1m): The title substrate was synthesized from 1-(3-chlorophenyl)propan-1-one $(10 \mathrm{mmol})$ according to the above procedure Step 3. Colorless oil; $1.52 \mathrm{~g}, 91 \%$ yield; ${ }^{1} \mathrm{H}$ NMR (400 MHz, $\left.\mathrm{CDCl}_{3}\right): 7.42(\mathrm{~m}, 1 \mathrm{H}), 7.30(\mathrm{~m}, 1 \mathrm{H}), 7.26(\mathrm{~m}, 2 \mathrm{H}), 5.30(\mathrm{~s}$, $1 \mathrm{H}), 5.12(\mathrm{~m}, 1 \mathrm{H}), 2.50(\mathrm{~m}, 2 \mathrm{H}), 1.12(\mathrm{t}, J=7.4 \mathrm{~Hz}, 3 \mathrm{H})$; These data are in accordance with the literature. $^{5}$ 
(1)

1-Bromo-4-(but-1-en-2-yl)benzene (1n): The title substrate was synthesized from 1-(4-bromophenyl)propan-1-one $(10 \mathrm{mmol})$ according to the above procedure Step 3. Colorless oil; $2.02 \mathrm{~g}$, 96\% yield; ${ }^{1} \mathrm{H}$ NMR (400 MHz, $\left.\mathrm{CDCl}_{3}\right): 7.45(\mathrm{~d}, J=8.8 \mathrm{~Hz}, 2 \mathrm{H}), 7.28(\mathrm{~d}, J=8.4 \mathrm{~Hz}, 2 \mathrm{H}$ ), $5.27(\mathrm{~m}, 1 \mathrm{H}), 5.08(\mathrm{q}, J=1.6 \mathrm{~Hz}, 1 \mathrm{H}), 2.48(\mathrm{~m}, 2 \mathrm{H}), 1.10(\mathrm{t}, J=7.4 \mathrm{~Hz}, 3 \mathrm{H})$; These data are in accordance with the literature. ${ }^{5}$<smiles>C=C(Cc1ccccc1)c1ccccc1</smiles>

Prop-2-ene-1,2-diyldibenzene (10): The title substrate was synthesized from 1,2-diphenylethanone (10 mmol) according to the above procedure Step 3. Colorless oil; $1.69 \mathrm{~g}$, 87\% yield; ${ }^{1} \mathrm{H} \mathrm{NMR}\left(400 \mathrm{MHz}, \mathrm{CDCl}_{3}\right): 7.47(\mathrm{~m}, 2 \mathrm{H}), 7.26(\mathrm{~m}, 8 \mathrm{H}), 5.53(\mathrm{~m}, 1 \mathrm{H}), 5.05(\mathrm{~m}, 1 \mathrm{H}), 3.87$ $(\mathrm{s}, 2 \mathrm{H})$; These data are in accordance with the literature. ${ }^{7}$<smiles>Cc1ccc(C(=O)Cc2ccccc2)cc1</smiles>

2-Phenyl-1-( $p$-tolyl)ethanone $(\mathbf{O} p)$ : The title substrate was synthesized from 2-phenylacetyl chloride $(20 \mathrm{mmol})$ according to the above procedure Step 2. White solid; $2.90 \mathrm{~g}$, $69 \%$ yield; ${ }^{1} \mathrm{H}$ NMR (400 MHz, CDCl $)$ : $7.92(\mathrm{~d}, J=8.4 \mathrm{~Hz}, 2 \mathrm{H}), 7.33(\mathrm{~m}, 2 \mathrm{H}), 7.26(\mathrm{~m}, 5 \mathrm{H}), 4.26(\mathrm{~s}, 2 \mathrm{H}), 2.41(\mathrm{~s}$, $3 \mathrm{H})$; These data are in accordance with the literature. ${ }^{8}$<smiles>C=C(Cc1ccccc1)c1ccc(C)cc1</smiles>

1-Methyl-4-(3-phenylprop-1-en-2-yl)benzene (1p): The title substrate was synthesized from 2-phenyl-1-( $p$-tolyl)ethanone $(10 \mathrm{mmol})$ according to the above procedure Step 3. Colorless oil; $1.90 \mathrm{~g}, 91 \%$ yield; ${ }^{1} \mathrm{H}$ NMR (400 MHz, $\left.\mathrm{CDCl}_{3}\right): 7.39(\mathrm{~d}, J=8.0 \mathrm{~Hz}, 2 \mathrm{H}), 7.30(\mathrm{~m}, 4 \mathrm{H}), 7.22(\mathrm{~m}, 1 \mathrm{H})$, $7.14(\mathrm{~d}, J=8.0 \mathrm{~Hz}, 2 \mathrm{H}), 5.53(\mathrm{~d}, J=1.6 \mathrm{~Hz}, 1 \mathrm{H}), 5.03(\mathrm{q}, J=1.2 \mathrm{~Hz}, 1 \mathrm{H}), 3.87(\mathrm{~s}, 2 \mathrm{H}), 2.36(\mathrm{~s}, 3 \mathrm{H})$; These data are in accordance with the literature. ${ }^{9}$<smiles>CC(C)(C)c1ccc(C(=O)Cc2ccccc2)cc1</smiles>

1-(4-(tert-Butyl)phenyl)-2-phenylethanone (0q): The title substrate was synthesized from 2-phenylacetyl chloride $(20 \mathrm{mmol})$ and tert-butylbenzene $(10 \mathrm{~mL})$ according to the above procedure Step 2. White solid; $3.68 \mathrm{~g}, 73 \%$ yield; ${ }^{1} \mathrm{H} \mathrm{NMR} \mathrm{(400} \mathrm{MHz,} \mathrm{CDCl}_{3}$ ): 7.96 (d, $J=8.8 \mathrm{~Hz}$, $2 \mathrm{H}), 7.47(\mathrm{~d}, J=8.8 \mathrm{~Hz}, 2 \mathrm{H}), 7.29(\mathrm{~m}, 5 \mathrm{H}), 4.27(\mathrm{~s}, 2 \mathrm{H}), 1.34(\mathrm{~s}, 9 \mathrm{H})$. These data are in accordance with the literature. ${ }^{10}$ 
<smiles>C=C(Cc1ccccc1)c1ccc(C(C)(C)C)cc1</smiles>

1-(tert-Butyl)-4-(3-phenylprop-1-en-2-yl)benzene (1q): The title substrate was synthesized from 1-(4-(tert-Butyl)phenyl)-2-phenylethanone $(10 \mathrm{mmol})$ according to the above procedure Step 3. Colorless oil; $2.15 \mathrm{~g}, 86 \%$ yield; ${ }^{1} \mathrm{H}$ NMR $\left(400 \mathrm{MHz}, \mathrm{CDCl}_{3}\right): 7.40(\mathrm{~d}, J=8.4 \mathrm{~Hz}, 2 \mathrm{H}), 7.32(\mathrm{~d}, J=8.4$ $\mathrm{Hz}, 2 \mathrm{H}), 7.26(\mathrm{~m}, 4 \mathrm{H}), 7.19(\mathrm{~m}, 1 \mathrm{H}), 5.52(\mathrm{~m}, 1 \mathrm{H}), 4.97(\mathrm{~m}, 1 \mathrm{H}), 3.83(\mathrm{~s}, 2 \mathrm{H}), 1.30(\mathrm{~s}, 9 \mathrm{H})$; These data are in accordance with the literature. ${ }^{11}$<smiles>O=C(Cc1ccccc1)c1ccc(-c2ccccc2)cc1</smiles>

1-([1,1'-Biphenyl]-4-yl)-2-phenylethanone (Or): The title substrate was synthesized from 2-phenylacetyl chloride $(20 \mathrm{mmol})$ and 1,1'-biphenyl $(9.2 \mathrm{~g}, 60 \mathrm{mmol})$ according to the above procedure Step 2. White solid; $4.25 \mathrm{~g}, 78 \%$ yield; ${ }^{1} \mathrm{H} \mathrm{NMR}\left(400 \mathrm{MHz}, \mathrm{CDCl}_{3}\right): 8.11(\mathrm{~d}, \mathrm{~J}=8.8 \mathrm{~Hz}$, $2 \mathrm{H}), 7.69(\mathrm{~d}, J=8.4 \mathrm{~Hz}, 2 \mathrm{H}), 7.63(\mathrm{~m}, 2 \mathrm{H}), 7.48(\mathrm{~m}, 2 \mathrm{H}), 7.42(\mathrm{~m}, 1 \mathrm{H}), 7.32(\mathrm{~m}, 5 \mathrm{H}), 4.33(\mathrm{~s}, 2 \mathrm{H})$; These data are in accordance with the literature. ${ }^{12}$<smiles>C=C(Cc1ccccc1)c1ccc(-c2ccccc2)cc1</smiles>

4-(3-Phenylprop-1-en-2-yl)-1,1'-biphenyl (1r): The title substrate was synthesized from 1-([1,1'-biphenyl]-4-yl)-2-phenylethanone $(10 \mathrm{mmol})$ according to the above procedure Step 3. White solid, m.p. $85-86{ }^{\circ} \mathrm{C} ; 2.62 \mathrm{~g}, 97 \%$ yield; ${ }^{1} \mathrm{H}$ NMR $\left(400 \mathrm{MHz}, \mathrm{CDCl}_{3}\right): 7.60(\mathrm{~m}, 2 \mathrm{H}), 7.55(\mathrm{~m}$, 4H), $7.44(\mathrm{~m}, 2 \mathrm{H}), 7.32(\mathrm{~m}, 5 \mathrm{H}), 7.22(\mathrm{~m}, 1 \mathrm{H}), 5.60(\mathrm{~s}, 1 \mathrm{H}), 5.08(\mathrm{~s}, 1 \mathrm{H}), 3.90(\mathrm{~s}, 2 \mathrm{H}) ;{ }^{13} \mathrm{C}$ NMR $(100$ $\left.\mathrm{MHz}, \mathrm{CDCl}_{3}\right): 146.2,140.6,140.2,139.6,139.5,128.9,128.7,128.4,127.2,126.9,126.5,126.1$, 114.6, 41.5. IR (neat): $2911,2845,1621,1514,1400,892,824,734,690 ;$ GC-MS: $t_{R}=12.54 \mathrm{~min}$, $\mathrm{m} / \mathrm{z}=270.2$.<smiles>COc1ccc(C(=O)Cc2ccccc2)cc1</smiles>

1-(4-Methoxyphenyl)-2-phenylethanone (0s): The title substrate was synthesized from 2-phenylacetyl chloride $(20 \mathrm{mmol})$ and anisole $(10 \mathrm{~mL})$ according to the above procedure Step 2. White solid; $3.40 \mathrm{~g}, 75 \%$ yield; ${ }^{1} \mathrm{H}$ NMR $\left(400 \mathrm{MHz}, \mathrm{CDCl}_{3}\right): 8.00(\mathrm{~d}, J=8.8 \mathrm{~Hz}, 2 \mathrm{H}), 7.28(\mathrm{~m}, 5 \mathrm{H})$, $6.93(\mathrm{~d}, J=8.8 \mathrm{~Hz}, 2 \mathrm{H}), 4.24(\mathrm{~s}, 2 \mathrm{H}), 3.86(\mathrm{~s}, 3 \mathrm{H})$. These data are in accordance with the literature..$^{13}$<smiles>C=C(Cc1ccccc1)c1ccc(OC)cc1</smiles>

1-Methoxy-4-(3-phenylprop-1-en-2-yl)benzene (1s): The title substrate was synthesized from 
1-(4-methoxyphenyl)-2-phenylethanone $(10 \mathrm{mmol})$ according to the above procedure Step 3. Colorless oil; 2.06 g, $92 \%$ yield; ${ }^{1} \mathrm{H}$ NMR $\left(400 \mathrm{MHz}, \mathrm{CDCl}_{3}\right): 7.40(\mathrm{~d}, J=8.8 \mathrm{~Hz}, 2 \mathrm{H}), 7.24(\mathrm{~m}, 5 \mathrm{H})$, $6.84(\mathrm{~d}, J=8.8 \mathrm{~Hz}, 2 \mathrm{H}), 5.45(\mathrm{~m}, 1 \mathrm{H}), 4.97(\mathrm{q}, J=1.2 \mathrm{~Hz}, 1 \mathrm{H}), 3.84(\mathrm{~s}, 2 \mathrm{H}), 3.80(\mathrm{~s}, 3 \mathrm{H})$. These data are in accordance with the literature. ${ }^{14}$<smiles>O=C(Cc1ccccc1)c1ccc(F)cc1</smiles>

1-(4-Fluorophenyl)-2-phenylethanone (0t): The title substrate was synthesized from 2-phenylacetyl chloride $(20 \mathrm{mmol})$ and fluorobenzene $(10 \mathrm{~mL})$ according to the above procedure Step 2. White solid; $3.47 \mathrm{~g}, 81 \%$ yield; ${ }^{1} \mathrm{H}$ NMR $\left(400 \mathrm{MHz}, \mathrm{CDCl}_{3}\right): 8.05$ (dd, $J=5.2 \mathrm{~Hz}, J=8.8 \mathrm{~Hz}$, $2 \mathrm{H}), 7.35(\mathrm{~m}, 2 \mathrm{H}), 7.27(\mathrm{~m}, 3 \mathrm{H}), 7.13(\mathrm{t}, J=8.8 \mathrm{~Hz}, 2 \mathrm{H}), 4.27(\mathrm{~s}, 2 \mathrm{H}) .{ }^{19} \mathrm{~F} \mathrm{NMR}\left(376 \mathrm{MHz}, \mathrm{CDCl}_{3}\right)$ : -105.0; These data are in accordance with the literature. ${ }^{15}$<smiles>C=C(Cc1ccccc1)c1ccc(F)cc1</smiles>

1-Fluoro-4-(3-phenylprop-1-en-2-yl)benzene (1t): The title substrate was synthesized from 1-(4-fluorophenyl)-2-phenylethanone $(10 \mathrm{mmol})$ according to the above procedure Step 3. Colorless oil; $1.97 \mathrm{~g}$, $93 \%$ yield; ${ }^{1} \mathrm{H}$ NMR $\left(400 \mathrm{MHz}, \mathrm{CDCl}_{3}\right): 7.41$ (dd, $J=5.6 \mathrm{~Hz}, J=8.8 \mathrm{~Hz}, 2 \mathrm{H}$ ), $7.30(\mathrm{~m}, 2 \mathrm{H}), 7.22(\mathrm{~m}, 3 \mathrm{H}), 6.99(\mathrm{t}, J=8.4 \mathrm{~Hz}, 2 \mathrm{H}), 5.46(\mathrm{~s}, 1 \mathrm{H}), 5.06(\mathrm{~s}, 1 \mathrm{H}), 3.84(\mathrm{~s}, 2 \mathrm{H}) ;{ }^{19} \mathrm{~F} \mathrm{NMR}$ $\left(376 \mathrm{MHz}, \mathrm{CDCl}_{3}\right):-115.5$; These data are in accordance with the literature. ${ }^{16}$<smiles>C=C(Cc1ccccc1)c1ccc(Cl)cc1</smiles>

1-Chloro-4-(3-phenylprop-1-en-2-yl)benzene (1u): The title substrate was synthesized from 1-(4-chlorophenyl)-2-phenylethanone $(10 \mathrm{mmol})$ according to the above procedure Step 3. Colorless oil; $2.01 \mathrm{~g}, 88 \%$ yield; ${ }^{1} \mathrm{H}$ NMR $\left(400 \mathrm{MHz}, \mathrm{CDCl}_{3}\right): 7.36(\mathrm{~d}, J=8.8 \mathrm{~Hz}, 2 \mathrm{H}), 7.25(\mathrm{~m}, 7 \mathrm{H})$, $5.49(\mathrm{~m}, 1 \mathrm{H}), 5.07(\mathrm{q}, J=1.2 \mathrm{~Hz}, 1 \mathrm{H}), 3.82(\mathrm{~s}, 2 \mathrm{H})$. These data are in accordance with the literature. ${ }^{16}$<smiles>C=C(Cc1ccccc1)c1ccc2ccccc2c1</smiles>

2-(3-Phenylprop-1-en-2-yl)naphthalene (1v): The title substrate was synthesized from 1-(naphthalen-2-yl)-2-phenylethanone $(10 \mathrm{mmol})$ according to the above procedure Step 3. Colorless oil; $2.12 \mathrm{~g}, 87 \%$ yield; ${ }^{1} \mathrm{H}$ NMR $\left(400 \mathrm{MHz}, \mathrm{CDCl}_{3}\right): 7.89(\mathrm{~s}, 1 \mathrm{H}), 7.81(\mathrm{~m}, 3 \mathrm{H}), 7.66$ (dd, $J=$ $1.6 \mathrm{~Hz}, J=8.4 \mathrm{~Hz}, 1 \mathrm{H}), 7.48(\mathrm{~m}, 2 \mathrm{H}), 7.30(\mathrm{~m}, 4 \mathrm{H}), 7.21(\mathrm{~m}, 1 \mathrm{H}), 5.68(\mathrm{~s}, 1 \mathrm{H}), 5.17(\mathrm{~s}, 1 \mathrm{H}), 4.00(\mathrm{~s}$, $2 \mathrm{H}) ;$ These data are in accordance with the literature. ${ }^{16}$<smiles>Cc1ccc(CC(=O)c2ccc(C)cc2)cc1</smiles> 
1,2-Di- $p$-tolylethanone $(\mathbf{0 w})$ : The title substrate was synthesized from 2-( $p$-tolyl)acetic acid (20 $\mathrm{mmol}$ ) according to the above procedure Step1-Step 2. White solid; $3.10 \mathrm{~g}, 69 \%$ yield; ${ }^{1} \mathrm{H}$ NMR (400 MHz, CDCl $): 7.92$ (d, J = 8.4 Hz, 2H), 7.25 (d, J = 8.4 Hz, 2H), 7.16 (d, J=8.4 Hz, 2H), 7.13 (d, J $=8.4 \mathrm{~Hz}, 2 \mathrm{H}), 4.22(\mathrm{~s}, 2 \mathrm{H}), 2.40(\mathrm{~s}, 3 \mathrm{H}), 2.32(\mathrm{~s}, 3 \mathrm{H})$; These data are in accordance with the literature. ${ }^{17}$<smiles>C=C(Cc1ccc(C)cc1)c1ccc(C)cc1</smiles>

4,4'-(Prop-2-ene-1,2-diyl)bis(methylbenzene) (1w): The title substrate was synthesized from 1,2-di- $p$-tolylethanone $(10 \mathrm{mmol})$ according to the above procedure Step3. White solid, m.p. 35-36 ${ }^{\circ} \mathrm{C}$; $2.04 \mathrm{~g}$, 92\% yield; ${ }^{1} \mathrm{H}$ NMR $\left(400 \mathrm{MHz}, \mathrm{CDCl}_{3}\right): 7.39(\mathrm{~m}, 2 \mathrm{H}), 7.15(\mathrm{~m}, 6 \mathrm{H}), 5.51(\mathrm{t}, \mathrm{J}=1.2$ $\left.\mathrm{Hz}, 1 \mathrm{H}), 5.03(\mathrm{t}, J=1.2 \mathrm{~Hz}, 1 \mathrm{H}), 3.84(\mathrm{~s}, 3 \mathrm{H}), 2.36(\mathrm{~s}, 3 \mathrm{H}), 2.35(\mathrm{~s}, 3 \mathrm{H}) ;{ }^{13} \mathrm{C} \mathrm{NMR} \mathrm{(100} \mathrm{MHz}, \mathrm{CDCl}_{3}\right)$ : $146.8,137.9,137.1,136.5,135.4,129.0,128.9,128.7,126.0,113.6,41.1,21.05,20.99$. IR (neat): $3022,2915,1625,1513,1428,897,820,788 ;$ GC-MS: $t_{R}=10.36 \mathrm{~min}, \mathrm{~m} / \mathrm{z}=222.2$.<smiles>Cc1ccc(C(=O)Cc2ccccc2C)cc1</smiles>

2-(o-Tolyl)-1-( $p$-tolyl)ethanone (0x): The title substrate was synthesized from 2-(o-tolyl)acetic acid (20 mmol) according to the above procedure Step1-Step 2. White solid; $3.65 \mathrm{~g}, 81 \%$ yield; ${ }^{1} \mathrm{H}$ NMR (400 MHz, CDCl $\left.)_{3}\right): 7.94$ (d, J = 8.4 Hz, 2H), 7.28 (d, J = 8.0 Hz, 2H), $7.17(\mathrm{~m}, 4 \mathrm{H}), 4.29(\mathrm{~s}, 2 \mathrm{H})$, $2.43(\mathrm{~s}, 3 \mathrm{H}), 2.27(\mathrm{~s}, 3 \mathrm{H})$; These data are in accordance with the literature. ${ }^{18}$<smiles>C=C(Cc1ccccc1C)c1ccc(C)cc1</smiles>

1-Methyl-2-(2-(p-tolyl)allyl)benzene (1x): The title substrate was synthesized from 2-(o-tolyl)-1-( $p$-tolyl)ethanone $(10 \mathrm{mmol})$ according to the above procedure Step3. White solid; $2.10 \mathrm{~g}, 95 \%$ yield; ${ }^{1} \mathrm{H} \mathrm{NMR}\left(400 \mathrm{MHz}, \mathrm{CDCl}_{3}\right): 7.40$ (d, $\left.J=8.4 \mathrm{~Hz}, 2 \mathrm{H}\right), 7.18(\mathrm{~m}, 6 \mathrm{H}), 5.44$ (q, J $=1.2$ $\mathrm{Hz}, 1 \mathrm{H}), 4.71(\mathrm{q}, J=1.2 \mathrm{~Hz}, 1 \mathrm{H}), 3.78(\mathrm{~s}, 2 \mathrm{H}), 2.37(\mathrm{~s}, 3 \mathrm{H}), 2.32(\mathrm{~s}, 3 \mathrm{H})$; These data are in accordance with the literature. ${ }^{9}$<smiles>Cc1ccc(C(=O)Cc2cccc(C)c2)cc1</smiles>

2-(m-Tolyl)-1-( $p$-tolyl)ethanone (0y): The title substrate was synthesized from 2-( $m$-tolyl)acetic acid (20 mmol) according to the above procedure Step1-Step 2. White solid, m.p. 65-66 ${ }^{\circ} \mathrm{C} ; 2.82 \mathrm{~g}$, $63 \%$ yield; ${ }^{1} \mathrm{H} N M R\left(400 \mathrm{MHz}, \mathrm{CDCl}_{3}\right): 7.93(\mathrm{~d}, J=8.0 \mathrm{~Hz}, 2 \mathrm{H}), 7.26(\mathrm{~d}, J=8.0 \mathrm{~Hz}, 2 \mathrm{H}), 7.22(\mathrm{t}, J=$ $7.4 \mathrm{~Hz}, 1 \mathrm{H}), 7.08(\mathrm{~m}, 3 \mathrm{H}), 4.23(\mathrm{~s}, 2 \mathrm{H}), 2.41(\mathrm{~s}, 3 \mathrm{H}), 2.33(\mathrm{~s}, 3 \mathrm{H}) ;{ }^{13} \mathrm{C} \mathrm{NMR}\left(100 \mathrm{MHz}, \mathrm{CDCl}_{3}\right): 197.4$, 143.9, 138.2, 134.6, 134.1, 130.1, 129.3, 128.7, 128.5, 127.6, 126.4, 45.3, 21.6, 21.4. IR (neat): $2899,1677,1603,1323,1175,804,762 ;$ GC-MS: $t_{R}=10.86 \mathrm{~min}, \mathrm{~m} / \mathrm{z}=224.2$. 
<smiles>C=C(Cc1cccc(C)c1)c1ccc(C)cc1</smiles>

1-Methyl-3-(2-(p-tolyl)allyl)benzene (1y): The title substrate was synthesized from 2-( $m$-tolyl)-1-( $p$-tolyl)ethanone $(10 \mathrm{mmol})$ according to the above procedure Step3. Colorless oil; $2.04 \mathrm{~g}, 92 \%$ yield; ${ }^{1} \mathrm{H}$ NMR $\left(400 \mathrm{MHz}, \mathrm{CDCl}_{3}\right): 7.37(\mathrm{~m}, 2 \mathrm{H}), 7.11(\mathrm{~m}, 6 \mathrm{H}), 5.50(\mathrm{t}, J=1.4 \mathrm{~Hz}, 1 \mathrm{H})$, $5.00(\mathrm{t}, J=1.4 \mathrm{~Hz}, 1 \mathrm{H}), 3.81(\mathrm{~s}, 2 \mathrm{H}), 2.34(\mathrm{~s}, 6 \mathrm{H})$; These data are in accordance with the literature. ${ }^{9}$<smiles>Cc1ccc(C(=O)Cc2ccc(-c3ccccc3)cc2)cc1</smiles>

2-([1,1'-Biphenyl]-4-yl)-1-( $p$-tolyl)ethanone (0z): The title substrate was synthesized from 2-([1,1'-biphenyl]-4-yl)acetic acid (20 mmol) according to the above procedure Step1-Step 2. White solid, m.p. $158-159^{\circ} \mathrm{C} ; 5.00 \mathrm{~g}, 87 \%$ yield; ${ }^{1} \mathrm{H}$ NMR $\left(400 \mathrm{MHz}, \mathrm{CDCl}_{3}\right): 7.95$ (d, J=8.4 Hz, 2H), $7.57(\mathrm{~m}, 4 \mathrm{H}), 7.43(\mathrm{~m}, 2 \mathrm{H}), 7.35(\mathrm{~d}, J=10.0 \mathrm{~Hz}, 3 \mathrm{H}), 7.28(\mathrm{~m}, 2 \mathrm{H}), 4.31(\mathrm{~s}, 2 \mathrm{H}), 2.42(\mathrm{~s}, 3 \mathrm{H}) ;{ }^{13} \mathrm{C}$ NMR $\left(100 \mathrm{MHz}, \mathrm{CDCl}_{3}\right): 197.2,144.1,140.8,139.7,134.1,133.8,129.9,129.3,128.8,128.7,127.4$, $127.2,127.0,45.0,21.6$. IR (neat): $1685,1603,1485,1406,999,810,748,685$; GC-MS: $t_{R}=14.4$ $\min , \mathrm{m} / \mathrm{z}=286.2$.<smiles>C=C(Cc1ccc(-c2ccccc2)cc1)c1ccc(C)cc1</smiles>

4-(2-(p-Tolyl)allyl)-1,1'-biphenyl (1z): The title substrate was synthesized from 2-([1,1'-biphenyl]-4-yl)-1-( $p$-tolyl)ethanone $(10 \mathrm{mmol})$ according to the above procedure Step3. White solid, m.p. $115-116{ }^{\circ} \mathrm{C} ; 2.75 \mathrm{~g}, 97 \%$ yield; ${ }^{1} \mathrm{H}$ NMR $\left(400 \mathrm{MHz}, \mathrm{CDCl}_{3}\right): 7.60(\mathrm{~m}, 2 \mathrm{H}), 7.53(\mathrm{~d}, J$ $=8.4 \mathrm{~Hz}, 2 \mathrm{H}), 7.43(\mathrm{~m}, 4 \mathrm{H}), 7.34(\mathrm{~m}, 3 \mathrm{H}), 7.14(\mathrm{~d}, J=8.4 \mathrm{~Hz}, 2 \mathrm{H}), 5.54(\mathrm{~s}, 1 \mathrm{H}), 5.07(\mathrm{~s}, 1 \mathrm{H}), 3.90(\mathrm{~s}$, 2H), 2.35 (s, 3H); ${ }^{13} \mathrm{C}$ NMR (100 MHz, $\left.\mathrm{CDCl}_{3}\right): 146.5,141.0,138.9,138.8,137.7,137.2,129.3$, 129.0, 128.7, 127.04, 127.00, 126.9, 126.0, 113.9, 41.2, 21.1; IR (neat): 2933, 2860, 1513, 1450, $1381,887,821,743$; GC-MS: $t_{R}=13.11 \mathrm{~min}, \mathrm{~m} / \mathrm{z}=284.2$.<smiles>COc1ccc(CC(=O)c2ccc(C)cc2)cc1</smiles>

2-(4-Methoxyphenyl)-1-(p-tolyl)ethanone (0aa): The title substrate was synthesized from 2-(4-methoxyphenyl)acetic acid (20 mmol) according to the above procedure Step1-Step 2. White solid; $3.07 \mathrm{~g}, 64 \%$ yield; ${ }^{1} \mathrm{H}$ NMR $\left(400 \mathrm{MHz}, \mathrm{CDCl}_{3}\right): 7.91(\mathrm{~d}, J=8.0 \mathrm{~Hz}, 2 \mathrm{H}), 7.25(\mathrm{~d}, J=8.0 \mathrm{~Hz}, 2 \mathrm{H})$, $7.18(\mathrm{~d}, J=11.2 \mathrm{~Hz}, 2 \mathrm{H}), 6.86(\mathrm{~d}, J=8.8 \mathrm{~Hz}, 2 \mathrm{H}), 4.20(\mathrm{~s}, 2 \mathrm{H}), 3.78(\mathrm{~s}, 3 \mathrm{H}), 2.40(\mathrm{~s}, 3 \mathrm{H})$; These data are in accordance with the literature. ${ }^{17}$<smiles>C=C(Cc1ccc(OC)cc1)c1ccc(C)cc1</smiles>

1-Methoxy-4-(2-(p-tolyl)allyl)benzene (1aa): The title substrate was synthesized from 
2-(4-methoxyphenyl)-1-(p-tolyl)ethanone $(10 \mathrm{mmol})$ according to the above procedure Step3. Colorless oil; $2.24 \mathrm{~g}$, 94\% yield; ${ }^{1} \mathrm{H}$ NMR $\left(400 \mathrm{MHz}, \mathrm{CDCl}_{3}\right): 7.36$ (d, $\left.J=8.4 \mathrm{~Hz}, 2 \mathrm{H}\right), 7.16$ (d, $J=8.8$ $\mathrm{Hz}, 2 \mathrm{H}), 7.12(\mathrm{~d}, J=8.0 \mathrm{~Hz}, 2 \mathrm{H}), 6.83(\mathrm{~d}, J=8.8 \mathrm{~Hz}, 2 \mathrm{H}), 5.47(\mathrm{~m}, 1 \mathrm{H}), 4.99(\mathrm{~m}, 1 \mathrm{H}), 3.79(\mathrm{~s}, 5 \mathrm{H})$, $2.34(\mathrm{~s}, 3 \mathrm{H})$. These data are in accordance with the literature. ${ }^{19}$<smiles>Cc1ccc(C(=O)Cc2ccc(F)cc2)cc1</smiles>

2-(4-Fluorophenyl)-1-( $p$-tolyl)ethanone (0ab): The title substrate was synthesized from 2-(4-fluorophenyl)acetic acid $(20 \mathrm{mmol}$ ) according to the above procedure Step1-Step 2. White solid; $2.70 \mathrm{~g}, 59 \%$ yield; ${ }^{1} \mathrm{H}$ NMR $\left(400 \mathrm{MHz}, \mathrm{CDCl}_{3}\right): 7.91(\mathrm{~d}, J=8.0 \mathrm{~Hz}, 2 \mathrm{H}), 7.27(\mathrm{~d}, J=8.0 \mathrm{~Hz}, 2 \mathrm{H}$ ), $7.22(\mathrm{~m}, 2 \mathrm{H}), 7.01(\mathrm{~m}, 2 \mathrm{H}), 4.24(\mathrm{~s}, 2 \mathrm{H}), 2.41(\mathrm{~s}, 3 \mathrm{H}) .{ }^{19} \mathrm{~F}$ NMR $\left(376 \mathrm{MHz}, \mathrm{CDCl}_{3}\right)$ : -116.3; These data are in accordance with the literature. ${ }^{17}$<smiles>C=C(Cc1ccc(F)cc1)c1ccc(C)cc1</smiles>

1-Fluoro-4-(2-( $p$-tolyl)allyl)benzene (1ab): The title substrate was synthesized from 2-(4-fluorophenyl)-1-( $p$-tolyl)ethanone $(10 \mathrm{mmol})$ according to the above procedure Step3. White solid, m.p. $38-39{ }^{\circ} \mathrm{C} ; 2.07 \mathrm{~g}$, $92 \%$ yield; ${ }^{1} \mathrm{H}$ NMR $\left(400 \mathrm{MHz}, \mathrm{CDCl}_{3}\right)$ : 7.34 (m, 2H), $7.20(\mathrm{~m}, 2 \mathrm{H}), 7.12$ $(\mathrm{m}, 2 \mathrm{H}), 6.98(\mathrm{~m}, 2 \mathrm{H}), 5.49(\mathrm{t}, J=1.2 \mathrm{~Hz}, 1 \mathrm{H}), 5.00(\mathrm{t}, J=1.2 \mathrm{~Hz}, 1 \mathrm{H}), 3.82(\mathrm{~s}, 2 \mathrm{H}), 2.34(\mathrm{~s}, 3 \mathrm{H}) ;{ }^{13} \mathrm{C}$ NMR $\left(100 \mathrm{MHz}, \mathrm{CDCl}_{3}\right): 161.4\left(\mathrm{~d}, J_{F-C}=242.4 \mathrm{~Hz}\right), 146.6,137.5,137.3,135.2\left(\mathrm{~d}, J_{F-C}=3.2 \mathrm{~Hz}\right)$, $130.2\left(\mathrm{~d}, \mathrm{~J}_{F-C}=7.7 \mathrm{~Hz}\right), 129.0,125.9,115.1\left(\mathrm{~d}, \mathrm{~J}_{F-C}=21 \mathrm{~Hz}\right), 113.8,40.8,21.1 ;{ }^{19} \mathrm{~F} \mathrm{NMR}(376 \mathrm{MHz}$, $\mathrm{CDCl}_{3}$ ): -118.0 ; IR (neat): 2980, 2920, 1624, 1599, 1506, 1218, 893, 822, 776, 509; GC-MS: $t_{R}=$ $9.98 \mathrm{~min}, \mathrm{~m} / \mathrm{z}=226.2$.<smiles>Cc1ccc(C(=O)Cc2ccc(Cl)cc2)cc1</smiles>

2-(4-Chlorophenyl)-1-(p-tolyl)ethanone (0ac): The title substrate was synthesized from 2-(4-chlorophenyl)acetic acid $(20 \mathrm{mmol})$ according to the above procedure Step1-Step 2. White solid; $4.47 \mathrm{~g}, 91 \%$ yield; ${ }^{1} \mathrm{H}$ NMR $\left(400 \mathrm{MHz}, \mathrm{CDCl}_{3}\right): 7.90(\mathrm{~d}, J=8.0 \mathrm{~Hz}, 2 \mathrm{H}), 7.28(\mathrm{~m}, 4 \mathrm{H}), 7.19(\mathrm{~d}, J$ $=8.8 \mathrm{~Hz}, 2 \mathrm{H}), 4.23(\mathrm{~s}, 2 \mathrm{H}), 2.41(\mathrm{~s}, 3 \mathrm{H})$. These data are in accordance with the literature. ${ }^{17}$<smiles>C=C(Cc1ccc(Cl)cc1)c1ccc(C)cc1</smiles>

1-Chloro-4-(2-( $p$-tolyl)allyl)benzene (1ac): The title substrate was synthesized from 2-(4-chlorophenyl)-1-( $p$-tolyl)ethanone $(10 \mathrm{mmol})$ according to the above procedure Step3. White solid, $72-73{ }^{\circ} \mathrm{C} ; 2.22 \mathrm{~g}$, 91\% yield; ${ }^{1} \mathrm{H}$ NMR $\left(400 \mathrm{MHz}, \mathrm{CDCl}_{3}\right): 7.32(\mathrm{~m}, 2 \mathrm{H}), 7.24(\mathrm{~m}, 2 \mathrm{H})$, $7.16(\mathrm{~m}, 2 \mathrm{H}), 7.11(\mathrm{~m}, 2 \mathrm{H}), 5.49(\mathrm{~s}, 1 \mathrm{H}), 5.00(\mathrm{t}, J=1.2 \mathrm{~Hz}, 1 \mathrm{H}), 3.80(\mathrm{~s}, 2 \mathrm{H}), 2.33(\mathrm{~s}, 3 \mathrm{H}) ;{ }^{13} \mathrm{C}$ NMR (100 MHz, $\mathrm{CDCl}_{3}$ ): 146.2, 138.1, 137.5, 137.4, 131.8, 130.2, 129.0, 128.4, 125.9, 114.0, 41.0, 21.1; IR (neat): 2980, 2918, 1622, 1488, 1404, 1086, 896, 825, 798; GC-MS: $t_{R}=10.72 \mathrm{~min}, \mathrm{~m} / \mathrm{z}=242.1$. 
<smiles>Cc1ccc(C(=O)Cc2cccc3ccccc23)cc1</smiles>

2-(Naphthalen-1-yl)-1-(p-tolyl)ethanone (0ad): The title substrate was synthesized from 2-(naphthalen-1-yl)acetic acid $(20 \mathrm{mmol}$ ) according to the above procedure Step1-Step 2. Light yellow solid; $3.05 \mathrm{~g}, 59 \%$ yield; ${ }^{1} \mathrm{H}$ NMR $\left(400 \mathrm{MHz}, \mathrm{CDCl}_{3}\right): 7.99(\mathrm{~d}, J=8.4 \mathrm{~Hz}, 2 \mathrm{H}), 7.88(\mathrm{~m}, 2 \mathrm{H})$, $7.80(\mathrm{~d}, J=8.0 \mathrm{~Hz}, 1 \mathrm{H}), 7.49(\mathrm{~m}, 2 \mathrm{H}), 7.43(\mathrm{t}, J=7.6 \mathrm{~Hz}, 1 \mathrm{H}), 7.36(\mathrm{~d}, J=6.8 \mathrm{~Hz}, 1 \mathrm{H}), 7.28(\mathrm{~d}, J=8.0$ $\mathrm{Hz}, 2 \mathrm{H}), 4.72(\mathrm{~s}, 2 \mathrm{H}), 2.43(\mathrm{~s}, 3 \mathrm{H})$. These data are in accordance with the literature. ${ }^{20}$<smiles>C=C(Cc1cccc2ccccc12)c1ccc(C)cc1</smiles>

1-(2-(p-tolyl)allyl)naphthalene (1ad): The title substrate was synthesized from 2-(naphthalen-1-yl)-1-( $p$-tolyl)ethanone $(10 \mathrm{mmol})$ according to the above procedure Step3. Colorless oil; $2.26 \mathrm{~g}$, $88 \%$ yield; ${ }^{1} \mathrm{H}$ NMR $\left(400 \mathrm{MHz}, \mathrm{CDCl}_{3}\right): 8.03(\mathrm{~m}, 1 \mathrm{H}), 7.90(\mathrm{~m}, 1 \mathrm{H}), 7.78(\mathrm{~m}$, $1 \mathrm{H}), 7.46(\mathrm{~m}, 6 \mathrm{H}), 7.19(\mathrm{~d}, \mathrm{~J}=8.0 \mathrm{~Hz}, 2 \mathrm{H}), 5.53(\mathrm{~m}, 1 \mathrm{H}), 4.77(\mathrm{~m}, 1 \mathrm{H}), 4.27(\mathrm{~s}, 2 \mathrm{H}), 2.39(\mathrm{~s}, 3 \mathrm{H})$. These data are in accordance with the literature. ${ }^{9}$<smiles>Cc1ccc(C(=O)Cc2ccc3ccccc3c2)cc1</smiles>

2-(Naphthalen-2-yl)-1-(p-tolyl)ethanone (0ae): The title substrate was synthesized from 2-(naphthalen-2-yl)acetic acid $(20 \mathrm{mmol}$ ) according to the above procedure Step1-Step 2. Light yellow solid; $4.46 \mathrm{~g}, 86 \%$ yield; ${ }^{1} \mathrm{H}$ NMR $\left(400 \mathrm{MHz}, \mathrm{CDCl}_{3}\right): 7.96(\mathrm{~d}, J=8.0 \mathrm{~Hz}, 2 \mathrm{H}), 7.80(\mathrm{~m}, 3 \mathrm{H})$, $7.73(\mathrm{~s}, 1 \mathrm{H}), 7.44(\mathrm{~m}, 3 \mathrm{H}), 7.26(\mathrm{~d}, J=8.4 \mathrm{~Hz}, 2 \mathrm{H}), 4.43(\mathrm{~s}, 2 \mathrm{H}), 2.40(\mathrm{~s}, 3 \mathrm{H})$. These data are in accordance with the literature..$^{21}$<smiles>C=C(Cc1ccc2ccccc2c1)c1ccc(C)cc1</smiles>

2-(2-(p-Tolyl)allyl)naphthalene (1ae): The title substrate was synthesized from 2-(naphthalen-2-yl)-1-( $p$-tolyl)ethanone $(10 \mathrm{mmol})$ according to the above procedure Step3. White solid, m.p. $76-77^{\circ} \mathrm{C} ; 2.40 \mathrm{~g}, 93 \%$ yield; ${ }^{1} \mathrm{H}$ NMR $\left(400 \mathrm{MHz}, \mathrm{CDCl}_{3}\right): 7.80(\mathrm{~m}, 3 \mathrm{H}), 7.70(\mathrm{~s}, 1 \mathrm{H})$, $7.45(\mathrm{~m}, 2 \mathrm{H}), 7.40(\mathrm{~m}, 3 \mathrm{H}), 7.11(\mathrm{~d}, J=8.0 \mathrm{~Hz}, 2 \mathrm{H}), 5.55(\mathrm{~d}, J=0.8 \mathrm{~Hz}, 1 \mathrm{H}), 5.06(\mathrm{q}, J=1.2 \mathrm{~Hz}, 1 \mathrm{H})$, $4.01(\mathrm{~s}, 2 \mathrm{H}), 2.33(\mathrm{~s}, 3 \mathrm{H}) ;{ }^{13} \mathrm{C} \mathrm{NMR}\left(100 \mathrm{MHz}, \mathrm{CDCl}_{3}\right): 146.5,137.8,137.22,137.20,133.6,132.1$, 129.0, 127.8, 127.6, 127.53, 127.52, 127.2, 126.0, 125.8, 125.2, 114.0, 41.8, 21.1. IR (neat): 2917, $2860,1513,903,888,820,735 ;$ GC-MS: $t_{R}=12.02 \mathrm{~min}, \mathrm{~m} / \mathrm{z}=258.2$. 
<smiles>C=C(Cc1ccsc1)c1ccccc1</smiles>

3-(2-Phenylallyl)thiophene (1af): The title substrate was synthesized from 1-phenyl-2-(thiophen-3-yl)ethan-1-one $(10 \mathrm{mmol})$ according to the above procedure Step3. Light yellow oil, $1.6 \mathrm{~g}, 80 \%$ yield; ${ }^{1} \mathrm{H}$ NMR $\left(400 \mathrm{MHz}, \mathrm{CDCl}_{3}\right)$ : $7.49(\mathrm{~m}, 2 \mathrm{H}), 7.31(\mathrm{~m}, 4 \mathrm{H}), 6.99(\mathrm{~m}, 2 \mathrm{H})$, $5.52(\mathrm{~s}, 1 \mathrm{H}), 5.12(\mathrm{~s}, 1 \mathrm{H}), 3.88(\mathrm{~s}, 2 \mathrm{H})$; These data are in accordance with the literature. ${ }^{22}$<smiles>C=C(Cc1ccc(N(C)C)cc1)c1ccccc1</smiles>

$\mathrm{N}, \mathrm{N}$-Dimethyl-4-(2-phenylallyl)aniline (1ag): The title substrate was synthesized from 2-(4-(dimethylamino)phenyl)-1-phenylethan-1-one $(10 \mathrm{mmol})$ according to the above procedure Step3. White solid, $1.73 \mathrm{~g}, 73 \%$ yield; ${ }^{1} \mathrm{H}$ NMR $\left(400 \mathrm{MHz}, \mathrm{CDCl}_{3}\right): 7.45(\mathrm{~d}, J=7.2 \mathrm{~Hz}, 2 \mathrm{H}), 7.27(\mathrm{~m}$, $3 \mathrm{H}), 7.10(\mathrm{~d}, J=8.4 \mathrm{~Hz}, 2 \mathrm{H}), 6.68(\mathrm{~d}, J=8.8 \mathrm{~Hz}, 2 \mathrm{H}), 5.46(\mathrm{~s}, 1 \mathrm{H}), 5.01(\mathrm{~s}, 1 \mathrm{H}), 3.75(\mathrm{~s}, 2 \mathrm{H}), 2.91(\mathrm{~s}$, $6 \mathrm{H})$; These data are in accordance with the literature. ${ }^{23}$<smiles>C=C(Cc1ccc(C(=O)OC)cc1)c1ccccc1</smiles>

Methyl 4-(2-phenylallyl)benzoate (1ah): The title substrate was synthesized from methyl 4-(2-oxo-2-phenylethyl)benzoate ( $5 \mathrm{mmol}$ ) according to the above procedure Step3. Colorless oil; $0.8 \mathrm{~g}, 63 \%$ yield; ${ }^{1} \mathrm{H}$ NMR $\left(400 \mathrm{MHz}, \mathrm{CDCl}_{3}\right): 7.93(\mathrm{~d}, J=7.6 \mathrm{~Hz}, 2 \mathrm{H}), 7.39(\mathrm{~d}, J=7.2 \mathrm{~Hz}, 2 \mathrm{H}$ ), 7.27 $(\mathrm{m}, 5 \mathrm{H}), 5.52(\mathrm{~s}, 1 \mathrm{H}), 5.04(\mathrm{t}, J=1.2 \mathrm{~Hz}, 2 \mathrm{H}), 3.87(\mathrm{~m}, 5 \mathrm{H})$; These data are in accordance with the literature. ${ }^{24}$<smiles>C=C1CCC(c2ccccc2)CC1</smiles>

(4-Methylenecyclohexyl)benzene (1ai): The title substrate was synthesized from 4-phenylcyclohexanone $(10 \mathrm{mmol})$ according to the above procedure Step3. Colorless oil, $1.68 \mathrm{~g}$, $98 \%$ yield; $\left.{ }^{1} \mathrm{H} \mathrm{NMR} \mathrm{(400} \mathrm{MHz,} \mathrm{CDCl}_{3}\right): 7.30(\mathrm{~m}, 2 \mathrm{H}), 7.20(\mathrm{~m}, 3 \mathrm{H}), 4.69(\mathrm{q}, \mathrm{J}=1.2 \mathrm{~Hz}, 2 \mathrm{H}), 2.68(\mathrm{~m}$, $1 \mathrm{H}), 2.44(\mathrm{~m}, 2 \mathrm{H}), 2.20(\mathrm{~m}, 2 \mathrm{H}), 2.00(\mathrm{~m}, 2 \mathrm{H}), 1.56(\mathrm{~m}, 2 \mathrm{H})$; These data are in accordance with the literature..$^{25}$<smiles>C=C1CCCc2ccccc21</smiles>

1-Methylene-1,2,3,4-tetrahydronaphthalene (1aj): The title substrate was synthesized from 3,4-dihydronaphthalen-1(2H)-one (10 mmol) according to the above procedure Step3. Colorless oil; $1.38 \mathrm{~g}, 96 \%$ yield; ${ }^{1} \mathrm{H} \mathrm{NMR}\left(400 \mathrm{MHz}, \mathrm{CDCl}_{3}\right): 7.67(\mathrm{~m}, 1 \mathrm{H}), 7.18(\mathrm{~m}, 2 \mathrm{H}), 7.12(\mathrm{~m}, 1 \mathrm{H}), 5.50(\mathrm{q}$, $J=0.8 \mathrm{~Hz}, 1 \mathrm{H}), 4.97(\mathrm{q}, J=1.2 \mathrm{~Hz}, 1 \mathrm{H}), 2.87(\mathrm{t}, J=6.4 \mathrm{~Hz}, 2 \mathrm{H}), 2.57(\mathrm{~m}, 2 \mathrm{H}), 1.90(\mathrm{~m}, 2 \mathrm{H})$. These 
data are in accordance with the literature. ${ }^{26}$<smiles>C=C1CCc2ccccc2C1</smiles>

2-Methylene-1,2,3,4-tetrahydronaphthalene (1ak): The title substrate was synthesized from 3,4-dihydronaphthalen-1(2H)-one $(10 \mathrm{mmol})$ with a prolonged reaction time $24 \mathrm{~h}$ according to the above procedure Step3. Colorless oil; $1.26 \mathrm{~g}, 88 \%$ yield; ${ }^{1} \mathrm{H}$ NMR $\left(400 \mathrm{MHz}, \mathrm{CDCl}_{3}\right): 7.16(\mathrm{~m}, 4 \mathrm{H})$, $4.91(\mathrm{~m}, 2 \mathrm{H}), 3.58(\mathrm{~s}, 2 \mathrm{H}), 2.91(\mathrm{t}, J=6.4 \mathrm{~Hz}, 2 \mathrm{H}), 2.52(\mathrm{t}, J=6.4 \mathrm{~Hz}, 2 \mathrm{H})$. These data are in accordance with the literature. ${ }^{27}$<smiles>Cc1ccc(C(=O)CCCCCCCCC(=O)c2ccc(C)cc2)cc1</smiles>

1,10-Di-p-tolyldecane-1,10-dione (0al): The title substrate was synthesized from sebacoyl chloride (10 mmol), $\mathrm{AlCl}_{3}(2.93 \mathrm{~g}, 22 \mathrm{mmol}, 2.2$ equiv.) and toluene $(10 \mathrm{~mL})$ according to the above procedure Step 2. white solid, m.p. $100-101{ }^{\circ} \mathrm{C} ; 2.10 \mathrm{~g}, 60 \%$ yield; ${ }^{1} \mathrm{H}$ NMR $(400 \mathrm{MHz}$, $\left.\mathrm{CDCl}_{3}\right): 7.86(\mathrm{~d}, J=8.0 \mathrm{~Hz}, 4 \mathrm{H}), 7.25(\mathrm{~d}, J=8.4 \mathrm{~Hz}, 4 \mathrm{H}), 2.93(\mathrm{t}, J=7.4 \mathrm{~Hz}, 4 \mathrm{H}), 2.40(\mathrm{~s}, 6 \mathrm{H}), 1.72(\mathrm{~m}$ 4H), $1.38(\mathrm{~m}, 8 \mathrm{H}) ;{ }^{13} \mathrm{C} \mathrm{NMR}\left(100 \mathrm{MHz}, \mathrm{CDCl}_{3}\right): 200.2,143.5,134.5,129.2,128.1,38.4,29.3,24.4$, 21.6; IR (neat): 2923, 2908, 1681, 1603, 1405, 1288, 1228, 1179, 974, 814; HRMS: calcd. for $\mathrm{C}_{24} \mathrm{H}_{30} \mathrm{O}_{2}[\mathrm{M}]^{+} 350.2240$, found: 350.2230 .<smiles>C=C(CCCCCCCCC(=C)c1ccc(C)cc1)c1ccc(C)cc1</smiles>

4,4'-(Dodeca-1,11-diene-2,11-diyl)bis(methylbenzene) (1al): The title substrate was synthesized from 1,10-di-p-tolyldecane-1,10-dione ( $2 \mathrm{~g}, 5.7 \mathrm{mmol}$ ), ${ }^{t} \mathrm{BuOK}$ (1.54 g, $13.7 \mathrm{mmol}, 2.4$ equiv.) and $\mathrm{MePPh}_{3} \mathrm{Br}$ (4.89 g, $13.7 \mathrm{mmol}, 2.4$ equiv.) according to the above procedure Step3. White solid, m.p. $34-36{ }^{\circ} \mathrm{C} ; 1.82 \mathrm{~g}, 92 \%$ yield; ${ }^{1} \mathrm{H}$ NMR $\left(400 \mathrm{MHz}, \mathrm{CDCl}_{3}\right): 7.31$ (d, J = 8.4 Hz, $\left.4 \mathrm{H}\right), 7.14$ (d, $J=8.0 \mathrm{~Hz}, 4 \mathrm{H}), 5.24(\mathrm{~d}, J=1.6 \mathrm{~Hz}, 2 \mathrm{H}), 5.01(\mathrm{q}, J=1.6 \mathrm{~Hz}, 2 \mathrm{H}), 2.48(\mathrm{t}, J=7.6 \mathrm{~Hz}, 4 \mathrm{H}), 1.43(\mathrm{~m}, 4 \mathrm{H})$, $1.30(\mathrm{~m}, 8 \mathrm{H}) ;{ }^{13} \mathrm{C} \mathrm{NMR}\left(100 \mathrm{MHz}, \mathrm{CDCl}_{3}\right): 148.5,138.5,136.9,128.9,125.9,111.2,35.3,29.4,29.3$ 28.2, 21.1; IR (neat): 2980, 2922, 2852, 1623, 1513, 1457, 890, 823; GC-MS: $t_{R}=14.7 \mathrm{~min}, \mathrm{~m} / \mathrm{z}$ $=346.3$.<smiles>Cc1ccc(C(=O)CCCCC(=O)c2ccc(C)cc2)cc1</smiles>

1,6-Di-p-tolylhexane-1,6-dione (0am): The title substrate was synthesized from adipic acid (20 mmol) according to the above procedure Step1-Step 2. White solid, m.p. $143-144{ }^{\circ} \mathrm{C} ; 4.5 \mathrm{~g}, 77 \%$ yield; ${ }^{1} \mathrm{H} \mathrm{NMR}\left(400 \mathrm{MHz}, \mathrm{CDCl}_{3}\right): 7.86(\mathrm{~d}, J=8.0 \mathrm{~Hz}, 4 \mathrm{H}), 7.25(\mathrm{~d}, J=8.0 \mathrm{~Hz}, 4 \mathrm{H}), 3.01(\mathrm{t}, J=6.8 \mathrm{~Hz}$, 4H), 2.40 (s, 6H), $1.82(\mathrm{~m}, 4 \mathrm{H}) ;{ }^{13} \mathrm{C} \mathrm{NMR}\left(100 \mathrm{MHz}, \mathrm{CDCl}_{3}\right)$ : 199.7, 143.7, 134.4, 129.2, 128.1, 38.3, 24.0, 21.6; IR (neat): $1672,1603,1404,1368,1258,1177,963,803,734$; GC-MS: $t_{R}=14.21 \mathrm{~min}$, $\mathrm{m} / \mathrm{z}=294.2$. 
<smiles>C=C(CCCCC(=C)c1ccc(C)cc1)c1ccc(C)cc1</smiles>

4,4'-(Octa-1,7-diene-2,7-diyl)bis(methylbenzene) (1am): The title substrate was synthesized from 1,6-Di-p-tolylhexane-1,6-dione (1.47 g, $5.0 \mathrm{mmol}),{ }^{t} \mathrm{BuOK}$ (1.34 g, $12 \mathrm{mmol}, 2.4$ equiv.) and $\mathrm{MePPh}_{3} \mathrm{Br}(4.28 \mathrm{~g}, 12 \mathrm{mmol}, 2.4$ equiv.) according to the above procedure Step3. White solid, m.p. 49-50 ${ }^{\circ} \mathrm{C} ; 1.40$ g, 97\% yield; ${ }^{1} \mathrm{H}$ NMR (400 MHz, CDCl 3 ): 7.30 (d, $\left.J=8.0 \mathrm{~Hz}, 4 \mathrm{H}\right), 7.14$ (d, J=8.0 Hz, $4 \mathrm{H}), 5.23(\mathrm{~d}, J=1.2 \mathrm{~Hz}, 2 \mathrm{H}), 5.00(\mathrm{q}, J=1.2 \mathrm{~Hz}, 2 \mathrm{H}), 2.49(\mathrm{t}, J=6.4 \mathrm{~Hz}, 4 \mathrm{H}), 2.36(\mathrm{~s}, 6 \mathrm{H}), 1.50(\mathrm{~m}$, $4 \mathrm{H}) ;{ }^{13} \mathrm{C} \mathrm{NMR}\left(100 \mathrm{MHz}, \mathrm{CDCl}_{3}\right): 148.3,138.3,136.9,128.9,125.9,111.4,35.1,27.9,21.1 ; \mathrm{IR}$ (neat): 2919, 2852, 1619, 1512, 1126, 1014, 886, 821; GC-MS: $t_{R}=12.05 \mathrm{~min}, \mathrm{~m} / \mathrm{z}=290.2$.<smiles>Cc1ccc(C(=O)CCCC(=O)c2ccc(C)cc2)cc1</smiles>

1,5-Di-p-tolylpentane-1,5-dione (0an): The title substrate was synthesized from glutaroyl dichloride (10 mmol), $\mathrm{AlCl}_{3}(2.93 \mathrm{~g}, 22 \mathrm{mmol}, 2.2$ equiv.) and toluene $(10 \mathrm{~mL})$ according to the above procedure Step1-Step 2. White solid, m.p. $110-111^{\circ} \mathrm{C} ; 1.68 \mathrm{~g}, 60 \%$ yield; ${ }^{1} \mathrm{H}$ NMR (400 $\mathrm{MHz}, \mathrm{CDCl}_{3}$ ): $7.88(\mathrm{~d}, J=8.4 \mathrm{~Hz}, 4 \mathrm{H}), 7.25(\mathrm{~d}, J=8.4 \mathrm{~Hz}, 4 \mathrm{H}), 3.08(\mathrm{t}, J=7.0 \mathrm{~Hz}, 4 \mathrm{H}), 2.40(\mathrm{~s}, 6 \mathrm{H})$, 2.18 (m, 2H); ${ }^{13} \mathrm{C} N M R\left(100 \mathrm{MHz}, \mathrm{CDCl}_{3}\right): 199.5,143.8,134.3,129.2,128.1,37.5,21.6,18.9$; IR (neat): $1672,1602,1409,1278,1177,977,823,748 ;$ GC-MS: $t_{R}=13.32 \mathrm{~min}, \mathrm{~m} / \mathrm{z}=280.2$.<smiles>C=C(CCCC(=C)c1ccc(C)cc1)c1ccc(C)cc1</smiles>

4,4'-(Hepta-1,6-diene-2,6-diyl)bis(methylbenzene) (1an): The title substrate was synthesized from

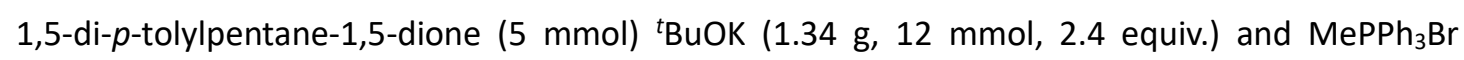
( $4.28 \mathrm{~g}, 12 \mathrm{mmol}, 2.4$ equiv.) according to the above procedure Step3. Colorless oil; $1.35 \mathrm{~g}, 98 \%$ yield; ${ }^{1} \mathrm{H}$ NMR (400 MHz, CDCl$)$ : $7.28(\mathrm{~m}, 4 \mathrm{H}), 7.13(\mathrm{~m}, 4 \mathrm{H}), 5.27(\mathrm{~m}, 1 \mathrm{H}), 5.02(\mathrm{~m}, 1 \mathrm{H}), 2.54(\mathrm{t}, \mathrm{J}=$ $7.4 \mathrm{~Hz}, 4 \mathrm{H}), 2.36(\mathrm{~s}, 6 \mathrm{H}), 1.63(\mathrm{~m}, 2 \mathrm{H}) ;{ }^{13} \mathrm{C} \mathrm{NMR}\left(100 \mathrm{MHz}, \mathrm{CDCl}_{3}\right): 148.0,138.2,137.0,128.9$, $125.9,111.6,34.8,26.6,21.1$; IR (neat): $2920,1623,1512,1456,888,822,733$; GC-MS: $t_{R}=11.65$ $\min , \mathrm{m} / \mathrm{z}=276.2$.

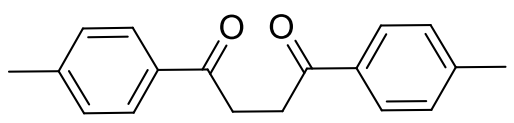

1,4-Di-p-tolylbutane-1,4-dione (0ao): The title substrate was synthesized from succinyl dichloride (10 mmol) according to the above procedure Step 2. White solid; $1.94 \mathrm{~g}, 73 \%$ yield; ${ }^{1} \mathrm{H}$ NMR (400 $\mathrm{MHz}, \mathrm{CDCl}_{3}$ ): $7.94(\mathrm{~d}, J=8.4 \mathrm{~Hz}, 4 \mathrm{H}), 7.27(\mathrm{~d}, J=8.0 \mathrm{~Hz}, 4 \mathrm{H}), 3.43(\mathrm{~s}, 4 \mathrm{H}), 2.42(\mathrm{~s}, 6 \mathrm{H})$. These data are in accordance with the literature. ${ }^{28}$

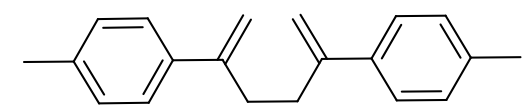

4,4'-(Hexa-1,5-diene-2,5-diyl)bis(methylbenzene) (1ao): The title substrate was synthesized from 1,4-di-p-tolylbutane-1,4-dione $(5 \mathrm{mmol})$ according to the above procedure Step3. Colorless oil; $1.02 \mathrm{~g}, 68 \%$ yield; ${ }^{1} \mathrm{H} \mathrm{NMR}\left(400 \mathrm{MHz}, \mathrm{CDCl}_{3}\right): 7.30(\mathrm{~d}, J=8.0 \mathrm{~Hz}, 4 \mathrm{H}), 7.14(\mathrm{~d}, J=8.4 \mathrm{~Hz}, 4 \mathrm{H}$ ), 5.26 $(\mathrm{d}, J=1.6 \mathrm{~Hz}, 2 \mathrm{H}), 5.01(\mathrm{~s}, 2 \mathrm{H}), 2.63(\mathrm{~s}, 4 \mathrm{H}), 2.36(\mathrm{~s}, 6 \mathrm{H})$. These data are in accordance with the 
literature. ${ }^{29}$<smiles>Cc1ccc(C(=O)Cc2cccc(CC(=O)c3ccc(C)cc3)c2)cc1</smiles>

2,2'-(1,3-Phenylene)bis(1-( $p$-tolyl)ethanone) (0ap): The title substrate was synthesized from 2,2'-(1,3-phenylene)diacetic acid $(10 \mathrm{mmol})$ according to the above procedure Step1-Step 2. White solid, m.p. $62-63{ }^{\circ} \mathrm{C} ; 3.15 \mathrm{~g}$, 92\% yield; ${ }^{1} \mathrm{H}$ NMR $\left(400 \mathrm{MHz}, \mathrm{CDCl}_{3}\right): 7.90$ (d, J=8.4 Hz, 4H), $7.28(\mathrm{~d}, J=7.6 \mathrm{~Hz}, 1 \mathrm{H}), 7.24(\mathrm{~d}, J=8.8 \mathrm{~Hz}, 4 \mathrm{H}), 7.19(\mathrm{~s}, 1 \mathrm{H}), 7.15(\mathrm{~d}, J=7.6 \mathrm{~Hz}, 2 \mathrm{H}), 4.23(\mathrm{~s}, 4 \mathrm{H})$, 2.40 (s, 6H); $\left.{ }^{13} \mathrm{C} \mathrm{NMR} \mathrm{(100} \mathrm{MHz,} \mathrm{CDCl}_{3}\right): 197.1,143.9,135.0,134.0,130.5,129.2,128.8,128.7$, 127.9, 45.2, 21.6; IR (neat): 2980, 2902, 1678, 1604, 1324, 1219, 1204, 1179, 1003, 817, 801, 749; HRMS: calcd. for $\mathrm{C}_{24} \mathrm{H}_{22} \mathrm{O}_{2}[\mathrm{M}+\mathrm{H}]^{+} 343.1693$, found: 343.1703 .<smiles>C=C(Cc1cccc(CC(=C)c2ccc(C)cc2)c1)c1ccc(C)cc1</smiles>

1,3-Bis(2-(p-tolyl)allyl)benzene (0ap): The title substrate was synthesized from 2,2'-(1,3-phenylene)bis(1-( $p$-tolyl)ethanone) $(5 \mathrm{mmol})$ according to the above procedure Step3. Colorless oil; $1.39 \mathrm{~g}$, 82\% yield; ${ }^{1} \mathrm{H}$ NMR $\left(400 \mathrm{MHz}, \mathrm{CDCl}_{3}\right): 7.32(\mathrm{~d}, J=8.0 \mathrm{~Hz}, 4 \mathrm{H}), 7.18(\mathrm{~m}, 1 \mathrm{H})$, $7.09(\mathrm{~m}, 7 \mathrm{H}), 5.46(\mathrm{~s}, 2 \mathrm{H}), 4.94(\mathrm{~m}, 2 \mathrm{H}), 3.79(\mathrm{~s}, 4 \mathrm{H}), 2.34(\mathrm{~s}, 6 \mathrm{H}) ;{ }^{13} \mathrm{C} \mathrm{NMR}\left(100 \mathrm{MHz}, \mathrm{CDCl}_{3}\right)$ : 146.7, 139.5, 137.9, 137.0, 129.7, 128.9, 128.3, 126.6, 126.0, 113.6, 41.5, 21.1.; IR (neat): 2980, $2916,1623,1513,1436,892,822,734,703$; GC-MS: $t_{R}=14.4 \mathrm{~min}, \mathrm{~m} / \mathrm{z}=338.3$.

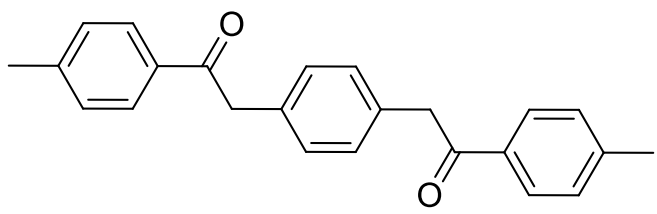

2,2'-(1,4-Phenylene)bis(1-( $p$-tolyl)ethanone) (0aq): The title substrate was synthesized from 2,2'-(1,4-phenylene)diacetic acid $(20 \mathrm{mmol})$ according to the above procedure Step1-Step 2. White solid, m.p. $210-213^{\circ} \mathrm{C} ; 5.7 \mathrm{~g}, 83 \%$ yield; ${ }^{1} \mathrm{H} \mathrm{NMR}\left(400 \mathrm{MHz}, \mathrm{CDCl}_{3}\right): 7.90(\mathrm{~d}, J=8.0 \mathrm{~Hz}, 4 \mathrm{H})$, $7.24(\mathrm{~d}, J=8.0 \mathrm{~Hz}, 4 \mathrm{H}), 7.22(\mathrm{~s}, 4 \mathrm{H}), 4.22(\mathrm{~s}, 4 \mathrm{H}), 2.40(\mathrm{~s}, 6 \mathrm{H}) ;{ }^{13} \mathrm{C} \mathrm{NMR}\left(100 \mathrm{MHz}, \mathrm{CDCl}_{3}\right): 197.2$, 144.0, 134.1, 133.3, 129.7, 129.3, 128.7, 45.0, 21.6; IR (neat): 1685, 1599, 1402, 1330, 1196, 1001, 811; HRMS: calcd. for $\mathrm{C}_{24} \mathrm{H}_{22} \mathrm{O}_{2}[\mathrm{M}+\mathrm{H}]^{+} 343.1693$, found: 343.1704 .

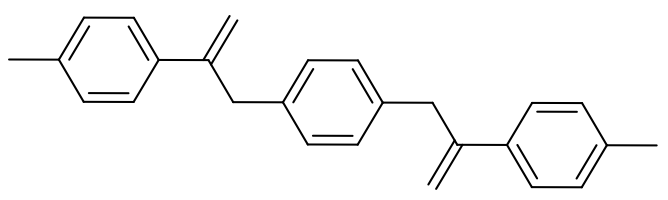

1,4-Bis(2-(p-tolyl)allyl)benzene (1aq): The title substrate was synthesized from 2,2'-(1,4-phenylene)bis(1-( $p$-tolyl)ethanone) $(5 \mathrm{mmol})$ according to the above procedure Step3. White solid, m.p. $114-115^{\circ} \mathrm{C} ; 1.45 \mathrm{~g}, 86 \%$ yield; ${ }^{1} \mathrm{H}$ NMR $\left(400 \mathrm{MHz}, \mathrm{CDCl}_{3}\right): 7.34(\mathrm{~d}, J=8.0 \mathrm{~Hz}, 4 \mathrm{H})$, $7.13(\mathrm{~s}, 4 \mathrm{H}), 7.10(\mathrm{~s}, 4 \mathrm{H}), 5.46(\mathrm{~d}, J=1.2 \mathrm{~Hz}, 2 \mathrm{H}), 4.95(\mathrm{q}, J=1.2 \mathrm{~Hz}, 2 \mathrm{H}), 3.78(\mathrm{~s}, 4 \mathrm{H}), 2.33(\mathrm{~s}, 6 \mathrm{H})$; $\left.{ }^{13} \mathrm{C} \mathrm{NMR} \mathrm{(100} \mathrm{MHz,} \mathrm{CDCl}\right): 146.7,137.9,137.3,137.1,128.9,128.8,125.9,113.6,41.1,21.1 ;$ IR (neat): $2980,2907,2683,1512,1435,891,822,736,567$; GC-MS: $t_{R}=15.04 \mathrm{~min}, \mathrm{~m} / \mathrm{z}=338.2$. 
<smiles>Cc1ccc(C(=O)CCC(C)C(=O)c2ccc(C)cc2)cc1</smiles>

2-Methyl-1,5-di-p-tolylpentane-1,5-dione (0ar): The title substrate was synthesized from 2-methylpentanedioic acid $(10 \mathrm{mmol})$ according to the above procedure Step1-Step 2. White solid; $2.1 \mathrm{~g}, 72 \%$ yield; ${ }^{1} \mathrm{H}$ NMR ( $\left.400 \mathrm{MHz}, \mathrm{CDCl}_{3}\right): 7.89(\mathrm{~d}, J=8.0 \mathrm{~Hz}, 2 \mathrm{H}), 7.83(\mathrm{~d}, J=8.4 \mathrm{~Hz}, 2 \mathrm{H}$ ), $7.26(\mathrm{~d}, J=8.0 \mathrm{~Hz}, 2 \mathrm{H}), 7.22(\mathrm{~d}, J=8.4 \mathrm{~Hz}, 2 \mathrm{H}), 3.63(\mathrm{~m}, 1 \mathrm{H}), 3.06(\mathrm{~m}, 1 \mathrm{H}), 2.86(\mathrm{~m}, 1 \mathrm{H}), 2.40(\mathrm{~s}$, $3 \mathrm{H}), 2.39(\mathrm{~s}, 3 \mathrm{H}), 2.25(\mathrm{~m}, 1 \mathrm{H}), 1.90(\mathrm{~m}, 1 \mathrm{H}), 1.23(\mathrm{~d}, J=7.2 \mathrm{~Hz}, 3 \mathrm{H})$. These data are in accordance with the literature. ${ }^{30}$<smiles>C=C(CCC(C)C(=C)c1ccc(C)cc1)c1ccc(C)cc1</smiles>

4,4'-(3-Methylhepta-1,6-diene-2,6-diyl)bis(methylbenzene) (1ar): The title substrate was synthesized from 2-methyl-1,5-di-p-tolylpentane-1,5-dione $(5 \mathrm{mmol})$ according to the above procedure Step3. Colorless oil; $1.0 \mathrm{~g}, 69 \%$ yield; ${ }^{1} \mathrm{H} \mathrm{NMR}\left(400 \mathrm{MHz}, \mathrm{CDCl}_{3}\right): 7.29(\mathrm{~d}, J=8.0 \mathrm{~Hz}, 2 \mathrm{H})$ $7.25(\mathrm{~d}, J=8.0 \mathrm{~Hz}, 2 \mathrm{H}), 7.14(\mathrm{~d}, J=8.0 \mathrm{~Hz}, 4 \mathrm{H}), 5.25(\mathrm{~m}, 2 \mathrm{H}), 5.03(\mathrm{~m}, 2 \mathrm{H}), 2.75(\mathrm{~m}, 1 \mathrm{H}), 2.55(\mathrm{t}, J=$ $8.0 \mathrm{~Hz}, 2 \mathrm{H}), 2.39(\mathrm{~s}, 6 \mathrm{H}), 1.73(\mathrm{~m}, 1 \mathrm{H}), 1.54(\mathrm{~m}, 1 \mathrm{H}), 1.18(\mathrm{~d}, J=6.8 \mathrm{~Hz}, 3 \mathrm{H}) ;{ }^{13} \mathrm{C}$ NMR $(100 \mathrm{MHz}$, $\left.\mathrm{CDCl}_{3}\right): 154.1,148.3,139.9,138.2,136.9,136.7,128.9,128.8,126.5,125.9,111.4,110.5,37.4$, 34.6, 32.9, 21.1, 20.2. IR (neat): $2960,2921,1623,1511,1456,889,822,733$; GC-MS: $t_{R}=11.68$ $\min , \mathrm{m} / \mathrm{z}=290.2$.<smiles>C=CC(C)c1ccccc1</smiles>

But-3-en-2-ylbenzene (1as): The title substrate was synthesized from 2-phenylpropanal (10 mmol) according to the above procedure Step3. Colorless oil; $1.2 \mathrm{~g}$, $91 \%$ yield; ${ }^{1} \mathrm{H}$ NMR (400 MHz, $\left.\mathrm{CDCl}_{3}\right)$ : $7.31(\mathrm{~m}, 2 \mathrm{H}), 7.23(\mathrm{~m}, 3 \mathrm{H}), 6.01(\mathrm{~m}, 1 \mathrm{H}), 5.05(\mathrm{~m}, 2 \mathrm{H}), 3.47(\mathrm{~m}, 1 \mathrm{H}), 1.37(\mathrm{~d}, J=7.2 \mathrm{~Hz}, 3 \mathrm{H})$; These data are in accordance with the literature. ${ }^{31}$<smiles>C=C(CC)C1CCCCC1</smiles>

But-1-en-2-ylcyclohexane (1at): The title substrate was synthesized from 1-cyclohexylpropan-1-one (10 mmol) according to the above procedure Step3. Colorless oil; $1.3 \mathrm{~g}$, 95\% yield; ${ }^{1} \mathrm{H}$ NMR (400 MHz, $\left.\mathrm{CDCl}_{3}\right): 4.69(\mathrm{~m}, 1 \mathrm{H}), 4.67(\mathrm{~m}, 1 \mathrm{H}), 2.04(\mathrm{~m}, 2 \mathrm{H}), 1.77(\mathrm{~m}, 6 \mathrm{H}), 1.20$ $(\mathrm{m}, 5 \mathrm{H}), 1.02(\mathrm{t}, J=7.4 \mathrm{~Hz}, 3 \mathrm{H})$; These data are in accordance with the literature. ${ }^{32}$ 


\section{Deuterium labeling substrate synthesis}

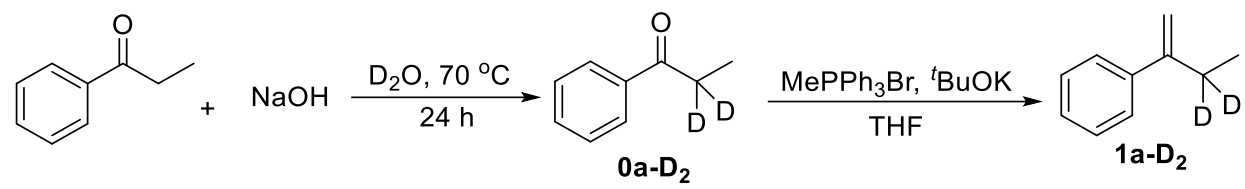

A mixture of propiophenone (1.6 mL,12 mmol), $\mathrm{NaOH}(0.04 \mathrm{~g}, 1 \mathrm{mmol})$ and $\mathrm{D}_{2} \mathrm{O}(10 \mathrm{~mL})$ was stirred at $70{ }^{\circ} \mathrm{C}$ for $24 \mathrm{~h}$ under argon. The reaction mixture was extracted with ethyl ether ( $3 * 30$ $\mathrm{mL}$ ). The combined organic phase was dried with anhydrous $\mathrm{MgSO}_{4}$. The crude product was purified by silica gel column chromatography (hexane/EtOAc $=30 / 1$ ) affording product $\mathbf{0 a}-\mathbf{D}_{\mathbf{2}}(1.4$ g, $87 \%$ yield).

The wittig reaction was performed as the procedure step 3 in substrate synthesis (with shorter reaction time $20 \mathrm{~min})$. $\mathbf{0} \mathbf{a}-\mathbf{D}_{\mathbf{2}}(\mathbf{1 0} \mathrm{mmol})$ readily converted to $1.26 \mathrm{~g}$ product $\mathbf{1} \mathbf{a}-\mathbf{D}_{\mathbf{2}}(\mathbf{9 4} \%$ yield).<smiles>[2H]C([2H])(C)C(=O)c1ccccc1</smiles>

0a-D $\mathrm{D}_{2}$ : Colorless oil; $1.4 \mathrm{~g}, 87 \%$ yield; ${ }^{1} \mathrm{H}$ NMR $\left(400 \mathrm{MHz}, \mathrm{CDCl}_{3}\right): 7.97$ (d, $\left.J=7.2 \mathrm{~Hz}, 2 \mathrm{H}\right), 7.55(\mathrm{t}, J=$ $7.4 \mathrm{~Hz}, 1 \mathrm{H}), 7.46(\mathrm{t}, J=7.6 \mathrm{~Hz}, 2 \mathrm{H}), 2.98(\mathrm{~m}, 0.2 \mathrm{H}), 1.21(\mathrm{~s}, 3 \mathrm{H}) ;{ }^{2} \mathrm{H}$ NMR $\left(61.4 \mathrm{MHz}, \mathrm{Et}_{2} \mathrm{O}\right): 3.53$ (q, $J=1.02 \mathrm{~Hz}) ;{ }^{13} \mathrm{C}$ NMR $\left(100 \mathrm{MHz}, \mathrm{CDCl}_{3}\right): 200.97,136.88,132.88,128.54,127.98,31.12(\mathrm{~m}), 8.12$; IR (neat): $3060,2975,1680,1448,1272,938,758$. These data are in accordance with the literature. ${ }^{33}$<smiles>[2H]C([2H])(C)C(=C)c1ccccc1</smiles>

1a-D $\mathrm{D}_{2}$ : Colorless oil; $1.26 \mathrm{~g}$, $94 \%$ yield; ${ }^{1} \mathrm{H}$ NMR $\left(400 \mathrm{MHz}, \mathrm{CDCl}_{3}\right): 7.44(\mathrm{~m}, 2 \mathrm{H}), 7.34(\mathrm{~m}, 2 \mathrm{H}), 7.28$ $(\mathrm{m}, 1 \mathrm{H}), 5.30(\mathrm{~s}, 1 \mathrm{H}), 5.08(\mathrm{~s}, 1 \mathrm{H}), 2.52(\mathrm{~m}, 0.2 \mathrm{H}), 1.11(\mathrm{~s}, 3 \mathrm{H}) ;{ }^{2} \mathrm{H}$ NMR $\left(61.4 \mathrm{MHz}, \mathrm{Et}_{2} \mathrm{O}\right): 2.42(\mathrm{q}, J$ $=0.6 \mathrm{~Hz}) ;{ }^{13} \mathrm{C} \mathrm{NMR}\left(100 \mathrm{MHz}, \mathrm{CDCl}_{3}\right): 149.9,141.4,128.2,127.2,126.0,110.9,27.5(\mathrm{~m}), 12.7 ; \mathrm{IR}$ (neat): 3080, 2964, 1623, 1493, 1023, 885, 775, 694; GC-MS: $t_{R}=6.79 \mathrm{~min}, \mathrm{~m} / \mathrm{z}=134.2$. 
4. General procedure for the synthesis of ligands (L1 as an example)

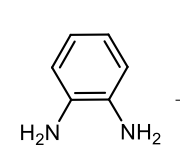

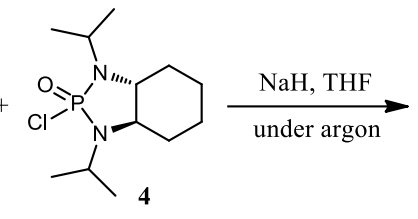
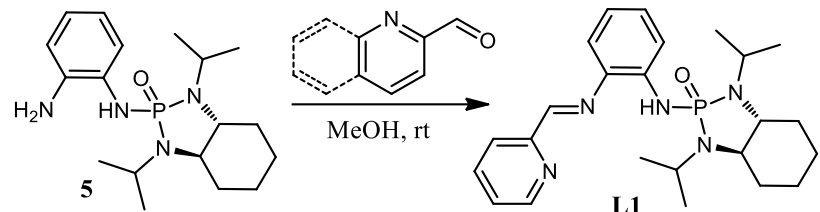

To a $250 \mathrm{~mL}$ schlenk flask was added a solution of o-Phenylenediamine (3) $(30 \mathrm{mmol}, 3.24 \mathrm{~g})$ in anhydrous THF $(80 \mathrm{~mL})$ and the solution was cooled to $0{ }^{\circ} \mathrm{C}$ under argon atmosphere. To the above solution, $\mathrm{NaH}$ ( $60 \%$ in mineral oil) $(30 \mathrm{mmol}, 1.2 \mathrm{~g}$ ) was added portionwise under argon atmosphere. The resulting solution was warmed to $40{ }^{\circ} \mathrm{C}$ and stirred at $40{ }^{\circ} \mathrm{C}$ for 1 hour, then was cooled to $0{ }^{\circ} \mathrm{C}$. To the solution was added a solution of $4^{34}(20 \mathrm{mmol}, 5.57 \mathrm{~g})$ in $20 \mathrm{~mL}$ anhydrous THF. The resulting solution was warmed to $40{ }^{\circ} \mathrm{C}$ and stirred at $40{ }^{\circ} \mathrm{C}$ overnight. To quench the reaction, $50 \mathrm{~mL}$ saturated $\mathrm{NH}_{4} \mathrm{Cl}$ aqueous solution was slowly added to the reaction mixture. The resulting suspension was extracted with diethyl ether for three times $(3 \times 100 \mathrm{~mL})$, and the combined organic phase was dried over anhydrous $\mathrm{MgSO}_{4}$. After filtering $\mathrm{MgSO}_{4}$, the organic solvent was evaporated and the product was purified by silica gel column chromatography $\left(\mathrm{CH}_{2} \mathrm{Cl}_{2} / \mathrm{EtOAc} 2 / 1\right.$ followed by EtOAc/MeOH $\left.20 / 1\right)$ to provide $3.7 \mathrm{~g}$ (53\% yield) of 5 as a white solid.

To a solution of 5 (350 mg, $1 \mathrm{mmol})$ in methanol $(5 \mathrm{~mL}$ ) was added picolinaldehyde (190 $\mu \mathrm{L}, 2$ $\mathrm{mmol})$. The solution was stirred overnight at room temperature then the solvent was evaporated. The residue was purified by silica gel column chromatography (EtOAc/Hexane/Et ${ }_{3} \mathrm{~N} \mathrm{50/50/1}$ followed by EtOAc/MeOH/Et ${ }_{3} \mathrm{~N} \mathrm{100/5/1)} \mathrm{to} \mathrm{give} \mathrm{the} \mathrm{corresponding} \mathrm{L1} \mathrm{(431} \mathrm{mg,} \mathrm{98 \%} \mathrm{yield).}$

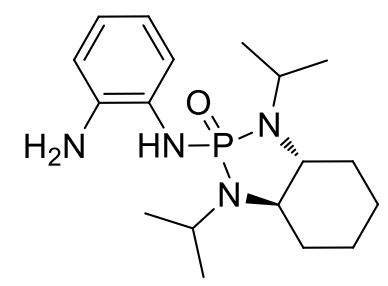

(3aR,7aR)-2-((2-Aminophenyl)amino)-1,3-diisopropyloctahydro-1H-benzo[d][1,3,2]diazaphosphol e 2-oxide (5): white solid, m.p.: $216-218{ }^{\circ} \mathrm{C} ;{ }^{1} \mathrm{H}$ NMR $\left(400 \mathrm{MHz}, \mathrm{CDCl}_{3}\right): 7.50(\mathrm{~d}, J=8.0 \mathrm{~Hz}, 1 \mathrm{H})$, $6.77(\mathrm{~m}, 1 \mathrm{H}), 6.61(\mathrm{~m}, 2 \mathrm{H}), 6.42(\mathrm{~d}, J=6.0 \mathrm{~Hz}, 1 \mathrm{H}), 4.45(\mathrm{~s}, 2 \mathrm{H}), 3.51(\mathrm{~m}, 2 \mathrm{H}), 3.04(\mathrm{~m}, 2 \mathrm{H}), 2.07$ $(\mathrm{m}, 2 \mathrm{H}), 1.78(\mathrm{~m}, 2 \mathrm{H}), 1.40(\mathrm{~d}, J=6.8 \mathrm{~Hz}, 3 \mathrm{H}), 1.32(\mathrm{~m}, 4 \mathrm{H}), 1.21(\mathrm{~d}, J=6.4 \mathrm{~Hz}, 3 \mathrm{H}), 1.07(\mathrm{~d}, J=6.8$ $\mathrm{Hz}, 3 \mathrm{H}), 0.94\left(\mathrm{~d}, J=6.4 \mathrm{~Hz}, 3 \mathrm{H}\right.$ ); ${ }^{31} \mathrm{P}$ NMR $\left(162 \mathrm{MHz}, \mathrm{CDCl}_{3}\right): 18.8 ;{ }^{13} \mathrm{C} \mathrm{NMR}\left(100 \mathrm{MHz}, \mathrm{CDCl}_{3}\right)$ : $138.8\left(\mathrm{~d}, J_{P-C}=8.2 \mathrm{~Hz}\right), 128.7,122.3,120.1\left(\mathrm{~d}, J_{P-C}=2.0 \mathrm{~Hz}\right), 117.6,116.1,60.7\left(\mathrm{~d}, J_{P-C}=13.8 \mathrm{~Hz}\right)$, $59.6\left(d, J_{P-C}=10.6 \mathrm{~Hz}\right), 44.6\left(\mathrm{~d}, J_{P_{-} C}=2.5 \mathrm{~Hz}\right), 43.9\left(\mathrm{~d}, J_{P-C}=4.9 \mathrm{~Hz}\right), 30.6\left(\mathrm{~d}, J_{P_{-} C}=8.2 \mathrm{~Hz}\right), 29.7(\mathrm{~d}$, $\left.J_{P-C}=11.0 \mathrm{~Hz}\right), 24.4,24.2,22.6\left(\mathrm{~d}, J_{P-C}=2.6 \mathrm{~Hz}\right), 21.1\left(\mathrm{~d}, J_{P-C}=3.2 \mathrm{~Hz}\right), 19.8\left(\mathrm{~d}, J_{P-C}=1.2 \mathrm{~Hz}\right), 19.7(\mathrm{~d}$ $J_{P-C}=2.7 \mathrm{~Hz}$ ); IR (neat): 3231, 2925, 2852, 1653, 1504, 1281, 1202, 1178, 1018, 946, 751; HRMS: calcd. for $\mathrm{C}_{18} \mathrm{H}_{31} \mathrm{~N}_{4} \mathrm{OP}[\mathrm{M}+\mathrm{H}]^{+}$351.2308, found: 351.2307 . 
<smiles>Nc1ccccc1NP1(=O)N(Cc2ccccc2)[C@@H]2CCCC[C@H]2N1Cc1ccccc1</smiles>

(3aR,7aR)-2-((2-Aminophenyl)amino)-1,3-dibenzyloctahydro-1H-benzo[d][1,3,2]diazaphosphole 2-oxide (6): white solid, m.p.: 190-192 ${ }^{\circ} \mathrm{C}$; ${ }^{1} \mathrm{H}$ NMR $\left(400 \mathrm{MHz}, \mathrm{CDCl}_{3}\right): 7.33(\mathrm{~m}, 4 \mathrm{H}), 7.19(\mathrm{~m}, 7 \mathrm{H})$, $6.91(\mathrm{t}, J=7.6 \mathrm{~Hz}, 1 \mathrm{H}), 6.68(\mathrm{~m}, 2 \mathrm{H}), 6.02(\mathrm{~d}, J=7.2 \mathrm{~Hz}, 1 \mathrm{H}), 4.40(\mathrm{~m}, 2 \mathrm{H}), 4.06(\mathrm{~m}, 3 \mathrm{H}), 3.60(\mathrm{dd}, J$ $=16.4 \mathrm{~Hz}, J=8.8 \mathrm{~Hz}, 1 \mathrm{H}), 2.93(\mathrm{~m}, 2 \mathrm{H}), 1.74(\mathrm{~d}, J=9.6 \mathrm{~Hz}, 1 \mathrm{H}), 1.56(\mathrm{~m}, 3 \mathrm{H}), 1.12(\mathrm{~m}, 3 \mathrm{H}), 0.94(\mathrm{~m}$, 1H); $\left.{ }^{31} \mathrm{P} \mathrm{NMR} \mathrm{(162} \mathrm{MHz,} \mathrm{CDCl} 3\right): 24.3 ;{ }^{13} \mathrm{C} \mathrm{NMR}\left(100 \mathrm{MHz}, \mathrm{CDCl}_{3}\right): 140.4\left(\mathrm{~d}, \mathrm{JP}_{\mathrm{P}-\mathrm{C}}=4.3 \mathrm{~Hz}\right), 140.1$ (d, $\left.J_{P-C}=7.1 \mathrm{~Hz}\right), 138.9\left(\mathrm{~d}, J_{P-C}=4.9 \mathrm{~Hz}\right), 128.3,128.1,128.0,127.3,126.9,126.8,126.6,123.9,122.4$ $\left(d, J_{P-C}=2.1 \mathrm{~Hz}\right), 118.1,116.3,65.0\left(d, J_{P-C}=10.4 \mathrm{~Hz}\right), 63.0\left(\mathrm{~d}, J_{P-C}=10.0 \mathrm{~Hz}\right), 47.3\left(\mathrm{~d}, J_{P-C}=3.0 \mathrm{~Hz}\right)$, $46.4\left(d, J_{P-C}=4.9 \mathrm{~Hz}\right), 30.2\left(\mathrm{~d}, J_{P-C}=7.8 \mathrm{~Hz}\right), 29.3\left(\mathrm{~d}, J_{P-C}=10.4 \mathrm{~Hz}\right), 24.2,24.1 ; \mathrm{IR}$ (neat): 3413, $3261,2949,1589,1504,1440,1279,1201,1179,940,782$; HRMS: calcd. for $\mathrm{C}_{26} \mathrm{H}_{31} \mathrm{~N}_{4} \mathrm{OP}[\mathrm{M}+\mathrm{H}]^{+}$ 447.2308, found: 447.2302 .

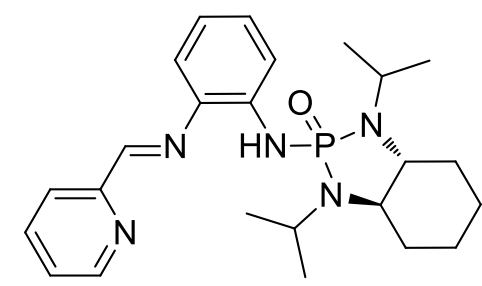

(3aR,7aR)-1,3-Diisopropyl-2-((2-((E)-(pyridin-2-ylmethylene)amino)phenyl)amino)octahydro- $1 \mathrm{H}$-b enzo[d][1,3,2]diazaphosphole 2-oxide (L1): yellow solid, m.p.: $137-139{ }^{\circ} \mathrm{C} ;{ }^{1} \mathrm{H}$ NMR $(400 \mathrm{MHz}$, $\left.\mathrm{CDCl}_{3}\right): 8.70(\mathrm{~s}, 1 \mathrm{H}), 8.68(\mathrm{~m}, 1 \mathrm{H}), 8.27(\mathrm{~d}, J=7.6 \mathrm{~Hz}, 1 \mathrm{H}), 7.81(\mathrm{~m}, 1 \mathrm{H}), 7.52(\mathrm{dd}, J=8.0 \mathrm{~Hz}, J=0.8$ $\mathrm{Hz}, 1 \mathrm{H}), 7.36(\mathrm{~m}, 1 \mathrm{H}), 7.20(\mathrm{~m}, 2 \mathrm{H}), 6.90(\mathrm{~m}, 1 \mathrm{H}), 6.28(\mathrm{~d}, J=6.8 \mathrm{~Hz}, 1 \mathrm{H}), 3.48(\mathrm{~m}, 2 \mathrm{H}), 3.15(\mathrm{~m}$, $2 \mathrm{H}), 2.12(\mathrm{~m}, 2 \mathrm{H}), 1.83(\mathrm{~m}, 2 \mathrm{H}), 1.39(\mathrm{~m}, 4 \mathrm{H}), 1.34(\mathrm{~d}, J=6.8 \mathrm{~Hz}, 3 \mathrm{H}), 1.20(\mathrm{~d}, J=6.8 \mathrm{~Hz}, 3 \mathrm{H}), 1.17$ $(\mathrm{d}, J=6.8 \mathrm{~Hz}, 3 \mathrm{H}), 0.99$ (d, J = 6.4 Hz, 3H); ${ }^{31} \mathrm{P} \mathrm{NMR}\left(162 \mathrm{MHz}, \mathrm{CDCl}_{3}\right): 16.8 ;{ }^{13} \mathrm{C} \mathrm{NMR}(100 \mathrm{MHz}$, $\left.\mathrm{CDCl}_{3}\right): 158.6,154.6,149.7,138.2,137.4\left(\mathrm{~d}, \mathrm{~J}_{\mathrm{P}-\mathrm{C}}=8.9 \mathrm{~Hz}\right), 136.8,128.3,125.3,121.9,120.7,117.1$ $\left(d, J_{P-C}=1.6 \mathrm{~Hz}\right), 117.0,60.8\left(d, J_{P-C}=10.7 \mathrm{~Hz}\right), 59.7\left(d, J_{P-C}=10.6 \mathrm{~Hz}\right), 44.9\left(d, J_{P-C}=2.4 \mathrm{~Hz}\right), 44.2$ $\left(d, J_{P-C}=4.8 \mathrm{~Hz}\right), 30.5\left(\mathrm{~d}, J_{P-C}=8.2 \mathrm{~Hz}\right), 30.0\left(\mathrm{~d}, J_{P-C}=11.3 \mathrm{~Hz}\right), 24.5\left(\mathrm{~d}, J_{\mathrm{P}-\mathrm{C}}=1.0 \mathrm{~Hz}\right), 24.4\left(\mathrm{~d}, J_{P-C}=\right.$ $1.4 \mathrm{~Hz}), 22.5\left(\mathrm{~d}, J_{\mathrm{P}-\mathrm{C}}=2.8 \mathrm{~Hz}\right), 21.6\left(\mathrm{~d}, J_{\mathrm{P}-\mathrm{C}}=4.7 \mathrm{~Hz}\right), 20.3\left(\mathrm{~d}, J_{\mathrm{P}-\mathrm{C}}=2.4 \mathrm{~Hz}\right), 20.1\left(\mathrm{~d}, J_{\mathrm{P}-\mathrm{C}}=0.8 \mathrm{~Hz}\right) ; \mathrm{IR}$ (neat): 3361, 2970, 2934, 2865, 1585, 1488, 1385, 1201, 1180, 1015, 990, 977, 747; HRMS: calcd. for $\mathrm{C}_{24} \mathrm{H}_{34} \mathrm{~N}_{5} \mathrm{OP}[\mathrm{M}+\mathrm{H}]^{+} 440.2574$, found: 440.2563 .

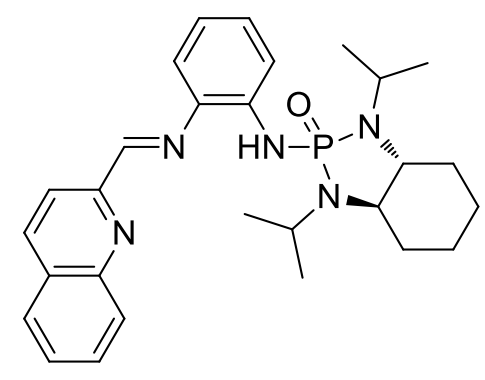


(3aR,7aR)-1,3-diisopropyl-2-((2-((E)-(quinolin-2-ylmethylene)amino)phenyl)amino)octahydro-1Hbenzo[d][1,3,2] diazaphosphole 2-oxide (L2): yellow solid; ${ }^{1} \mathrm{H}$ NMR $\left(400 \mathrm{MHz}, \mathrm{CDCl}_{3}\right): 8.89(\mathrm{~s}, 1 \mathrm{H})$, $8.40(\mathrm{~d}, J=8.8 \mathrm{~Hz}, 1 \mathrm{H}), 8.25(\mathrm{~d}, J=8.4 \mathrm{~Hz}, 1 \mathrm{H}), 8.15(\mathrm{~d}, J=8.0 \mathrm{~Hz}, 1 \mathrm{H}), 7.87(\mathrm{~d}, J=8.0 \mathrm{~Hz}, 1 \mathrm{H}$ ), $7.76(\mathrm{~m}, 1 \mathrm{H}), 7.59(\mathrm{~m}, 2 \mathrm{H}), 7.32(\mathrm{~m}, 1 \mathrm{H}), 7.20(\mathrm{~m}, 1 \mathrm{H}), 6.92(\mathrm{~m}, 1 \mathrm{H}), 6.40(\mathrm{~d}, J=7.2 \mathrm{~Hz}, 1 \mathrm{H}), 3.50$ $(\mathrm{m}, 2 \mathrm{H}), 3.16(\mathrm{~m}, 2 \mathrm{H}), 2.12(\mathrm{~m}, 2 \mathrm{H}), 1.83(\mathrm{~m}, 2 \mathrm{H}), 1.37(\mathrm{~m}, 7 \mathrm{H}), 1.22(\mathrm{~d}, J=6.4 \mathrm{~Hz}, 3 \mathrm{H}), 1.18(\mathrm{~d}, J=$ $6.8 \mathrm{~Hz}, 3 \mathrm{H}), 1.00$ (d, $J=6.8 \mathrm{~Hz}, 3 \mathrm{H}$ ); ${ }^{31} \mathrm{P} \mathrm{NMR}\left(162 \mathrm{MHz}, \mathrm{CDCl}_{3}\right): 16.9 ;{ }^{13} \mathrm{C} \mathrm{NMR}\left(100 \mathrm{MHz}, \mathrm{CDCl}_{3}\right)$ : $158.3,154.6,147.9,138.3\left(d, J_{P-C}=0.9 \mathrm{~Hz}\right), 137.0\left(d, J_{P-C}=8.7 \mathrm{~Hz}\right), 136.7,129.9,129.5,128.9$, 128.5, 127.8, 127.7, 120.6, 118.8, $117.1\left(\mathrm{~d}, J_{P-C}=1.5 \mathrm{~Hz}\right), 116.9,60.7\left(\mathrm{~d}, J_{P-C}=10.8 \mathrm{~Hz}\right), 59.6\left(\mathrm{~d}, J_{P-C}\right.$ $=10.7 \mathrm{~Hz}), 44.8\left(\mathrm{~d}, J_{P-C}=2.3 \mathrm{~Hz}\right), 44.0\left(\mathrm{~d}, J_{P-C}=4.7 \mathrm{~Hz}\right), 30.4\left(\mathrm{~d}, J_{P-C}=8.2 \mathrm{~Hz}\right), 29.8\left(\mathrm{~d}, J_{P-C}=11.3 \mathrm{~Hz}\right)$, $24.4\left(\mathrm{~d}, J_{P-C}=0.7 \mathrm{~Hz}\right), 24.3\left(\mathrm{~d}, J_{P-C}=1.4 \mathrm{~Hz}\right), 22.4\left(\mathrm{~d}, J_{P-C}=2.7 \mathrm{~Hz}\right), 21.4\left(\mathrm{~d}, J_{P-C}=4.6 \mathrm{~Hz}\right), 20.2\left(\mathrm{~d}, J_{P-C}\right.$ $=1.3 \mathrm{~Hz}$ ), 20.0; HRMS: calcd. for $\mathrm{C}_{28} \mathrm{H}_{36} \mathrm{~N}_{5} \mathrm{OP}[\mathrm{M}]^{+} 489.2651$, found: 489.2648 .<smiles>O=P1(Nc2ccccc2/N=C/c2ccccn2)N(Cc2ccccc2)[C@@H]2CCCC[C@H]2N1Cc1ccccc1</smiles>

(3aR,7aR)-1,3-Dibenzyl-2-((2-((E)-(pyridin-2-ylmethylene)amino)phenyl)amino)octahydro-1H-ben zo[d][1,3,2]diazaphosphole 2-oxide (L3): yellow solid, m.p.: 129-131 ${ }^{\circ} \mathrm{C} ;{ }^{1} \mathrm{H} \mathrm{NMR}\left(400 \mathrm{MHz}, \mathrm{CDCl}_{3}\right)$ : $8.69(\mathrm{~d}, J=4.4 \mathrm{~Hz}, 1 \mathrm{H}), 8.66(\mathrm{~s}, 1 \mathrm{H}), 8.23(\mathrm{~d}, J=8.0 \mathrm{~Hz}, 1 \mathrm{H}), 7.79(\mathrm{t}, J=7.8 \mathrm{~Hz}, 1 \mathrm{H}), 7.38(\mathrm{~m}, 4 \mathrm{H})$, $7.31(\mathrm{~m}, 2 \mathrm{H}), 7.22(\mathrm{~m}, 4 \mathrm{H}), 7.14(\mathrm{~m}, 4 \mathrm{H}), 6.97(\mathrm{t}, J=7.8 \mathrm{~Hz}, 1 \mathrm{H}), 6.39(\mathrm{~d}, J=6.8 \mathrm{~Hz}, 1 \mathrm{H}), 4.40(\mathrm{~m}$, $2 \mathrm{H}), 3.99(\mathrm{dd}, J=15.2 \mathrm{~Hz}, J=8.8 \mathrm{~Hz}, 1 \mathrm{H}), 3.83(\mathrm{dd}, J=16.4 \mathrm{~Hz}, J=9.2 \mathrm{~Hz}, 1 \mathrm{H}), 3.15(\mathrm{~m}, 2 \mathrm{H}), 1.74$ $(\mathrm{m}, 4 \mathrm{H}), 1.19(\mathrm{~m}, 3 \mathrm{H}), 1.10(\mathrm{~m}, 1 \mathrm{H}) ;{ }^{31} \mathrm{P} \mathrm{NMR}\left(162 \mathrm{MHz}, \mathrm{CDCl}_{3}\right): 21.7 ;{ }^{13} \mathrm{C} \mathrm{NMR}\left(100 \mathrm{MHz}, \mathrm{CDCl}_{3}\right)$ : 158.6, 154.3, 149.5, $139.5\left(\mathrm{~d}, J_{P-C}=3.8 \mathrm{~Hz}\right), 138.7\left(\mathrm{~d}, J_{P-C}=5.9 \mathrm{~Hz}\right), 137.3\left(\mathrm{~d}, J_{P-C}=8.9 \mathrm{~Hz}\right), 137.0(\mathrm{~d}$, $\left.J_{P-C}=0.7 \mathrm{~Hz}\right), 136.6,128.3,128.12,128.09,128.0,127.5,126.8\left(d, J_{P-C}=4.6 \mathrm{~Hz}\right), 125.2,121.9$, 121.1, 117.0, $116.6\left(d, J_{P-C}=1.7 \mathrm{~Hz}\right), 64.7\left(d, J_{P-C}=10.8 \mathrm{~Hz}\right), 63.5\left(\mathrm{~d}, J_{P-C}=10.0 \mathrm{~Hz}\right), 47.4\left(\mathrm{~d}, J_{P-C}=\right.$ $2.3 \mathrm{~Hz}), 46.5\left(d, J_{P-C}=5.1 \mathrm{~Hz}\right), 30.1\left(d, J_{P-C}=7.7 \mathrm{~Hz}\right), 29.5\left(d, J_{P-C}=11.0 \mathrm{~Hz}\right), 24.2,24.1$; IR (neat): 2937, 1587, 1494, 1322, 1211, 1173, 930, 739; HRMS: calcd. for $\mathrm{C}_{32} \mathrm{H}_{34} \mathrm{~N}_{5} \mathrm{OP}[\mathrm{M}+\mathrm{H}]^{+} 536.2574$, found: 536.2565 . 


\section{Optimization of reaction conditions}

Table S1. Cobalt catalyzed stereoselective olefin isomerization ${ }^{a}$

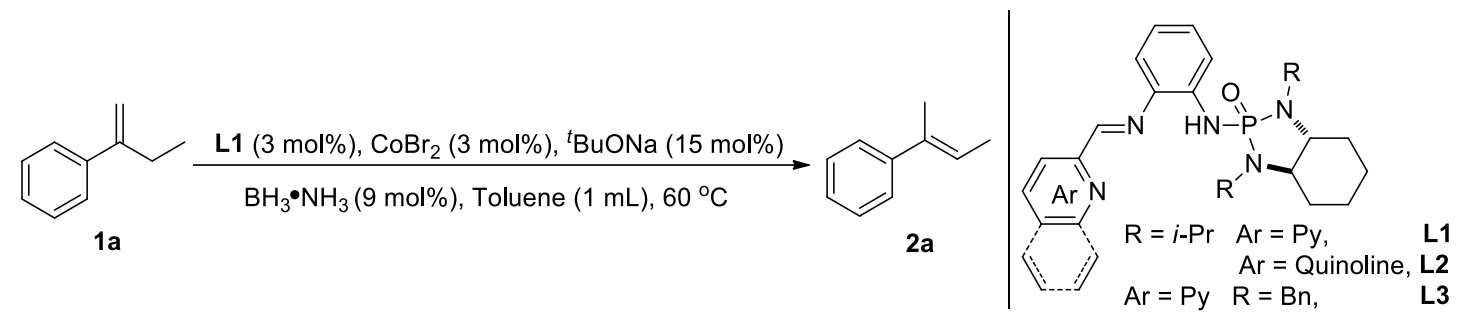

\begin{tabular}{|c|c|c|c|}
\hline Entry & Changes from standard conditions & Yield $(\%)^{b}$ & $E / Z^{c}$ \\
\hline 1 & None & 95 & $104 / 1$ \\
\hline 2 & Removal of $\mathrm{CoBr}_{2}$ & trace & n.d. \\
\hline 3 & Removal of ${ }^{t} \mathrm{BuONa}$ & trace & n.d. \\
\hline 4 & Removal of $\mathrm{BH}_{3} \cdot \mathrm{NH}_{3}$ & trace & n.d. \\
\hline 5 & Removal of $\mathbf{L} \mathbf{1}$ & 12 & $5 / 1$ \\
\hline 6 & Only using ${ }^{t} \mathrm{BuONa}$ as catalyst & trace & n.d. \\
\hline 7 & $\mathbf{L} \mathbf{2}$ was used instead of $\mathbf{L} \mathbf{1}$ & 96 & $11 / 1$ \\
\hline 8 & $\mathbf{L} \mathbf{3}$ was used instead of $\mathbf{L} \mathbf{1}$ & 96 & $38 / 1$ \\
\hline 9 & $\mathrm{PhSiH}_{3}$ was used instead of $\mathrm{BH}_{3} \cdot \mathrm{NH}_{3}$ & 62 & $8 / 1$ \\
\hline 10 & THF was used instead of toluene & 87 & $11 / 1$ \\
\hline 11 & ${ }^{t} \mathrm{BuOK}$ was used instead of ${ }^{t} \mathrm{BuONa}$ & 68 & $7 / 1$ \\
\hline
\end{tabular}

${ }^{a}$ The reaction of $1 \mathrm{a}(0.5 \mathrm{mmol})$ was performed in the presence of $\mathrm{CoBr}_{2}(0.015 \mathrm{mmol}, 3 \mathrm{~mol} \%), \mathrm{L}$ (0.015 mmol, $3 \mathrm{~mol} \%), t$ BuONa (0.075 mmol, $15 \mathrm{~mol} \%)$ and $\mathrm{BH}_{3} \cdot \mathrm{NH}_{3}(0.045 \mathrm{mmol}, 9 \mathrm{~mol} \%)$ in

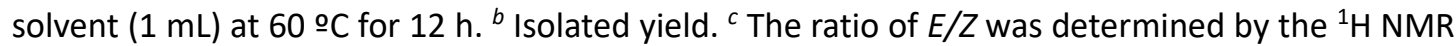
of crude product.

Entry 1

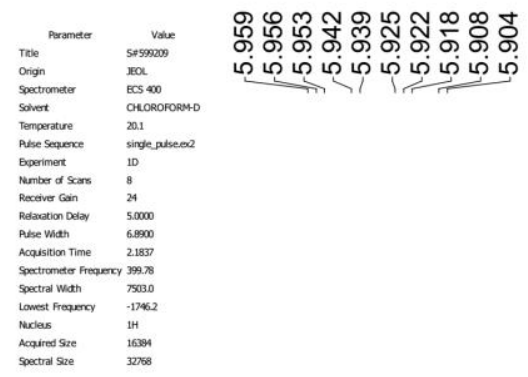

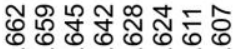

ம

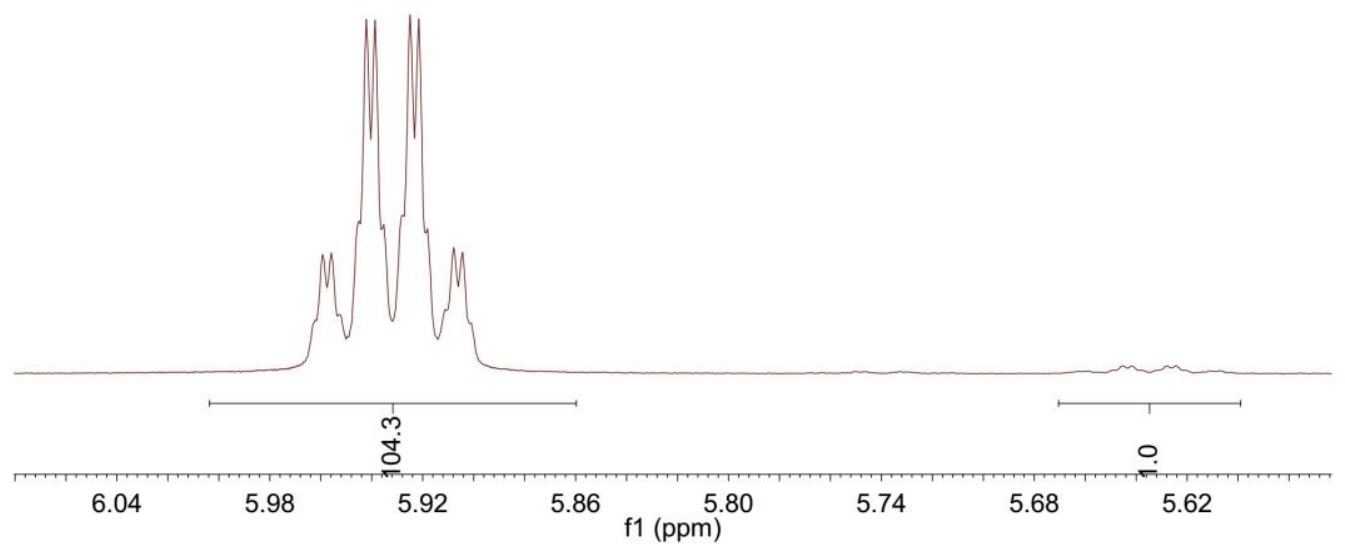


Entry 5
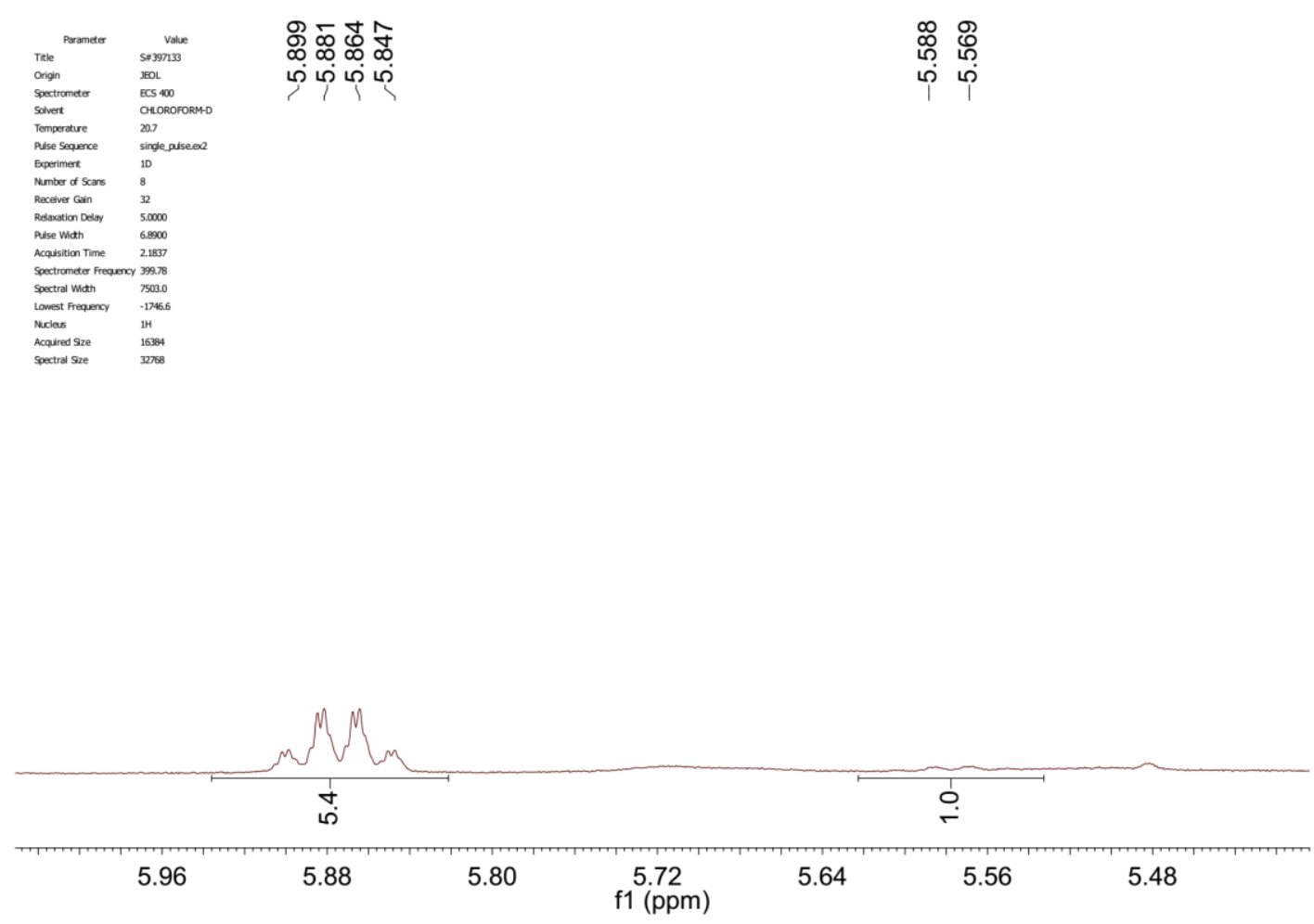

Entry 7

\begin{tabular}{|c|c|}
\hline Prameter & Value \\
\hline Inte & sestoro \\
\hline Origin & \\
\hline sectromear & $\operatorname{cec} 440$ \\
\hline solvert & CHOROFORM- \\
\hline $\begin{array}{l}\text { Temperature } \\
\text { Puise secquente }\end{array}$ & 20.0 \\
\hline $\begin{array}{l}\text { Pistese seanence } \\
\text { Experimert }\end{array}$ & $\begin{array}{l}\text { singe passeos } 2 \\
10\end{array}$ \\
\hline Nember of scars & 10 \\
\hline Recetiver Gain & 28 \\
\hline Relaxation Delay & 50000 \\
\hline Pulse Wath & 64450 \\
\hline Acquistion Time & 2.1897 \\
\hline Spectrometer Frequenc & 39978 \\
\hline Spectral Woth & 75030 \\
\hline Lowst Frequeny & -17466 \\
\hline Nacleus & \\
\hline caured Sze & 16384 \\
\hline
\end{tabular}

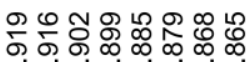

ما

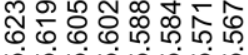

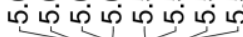

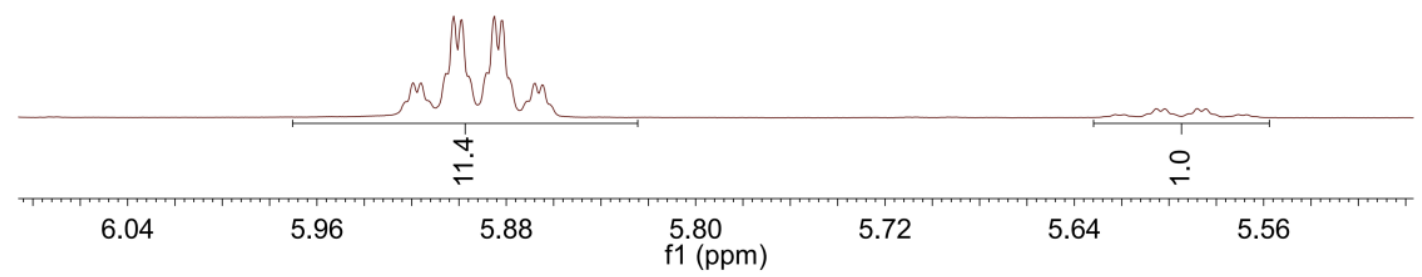


Entry 8

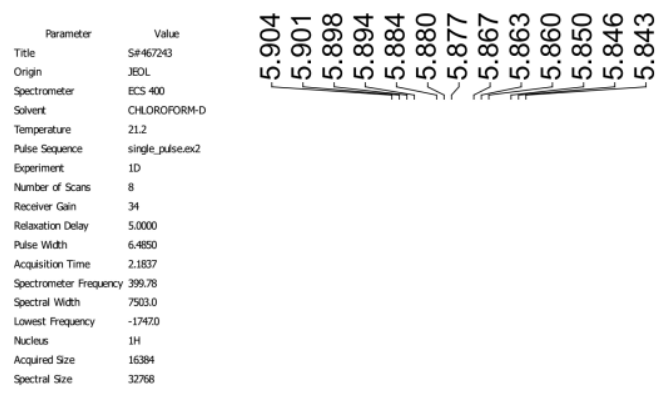

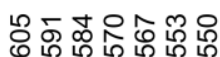

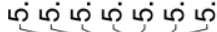

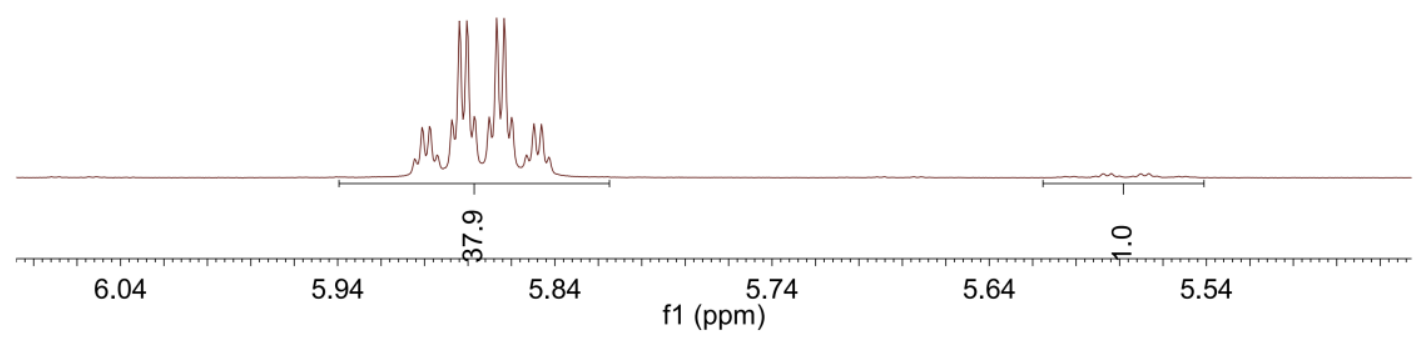

Entry 9

\begin{tabular}{|c|c|}
\hline & \\
\hline & Ses55r94 \\
\hline $\begin{array}{l}\text { Onigin } \\
\text { spectrome }\end{array}$ & 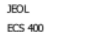 \\
\hline $\begin{array}{l}\text { soctrom } \\
\text { solvert }\end{array}$ & $\begin{array}{l}\text { ECE } 400 \\
\text { CHOROFORM-D }\end{array}$ \\
\hline Temperature & 203 \\
\hline Puise sequence & singe paseor2 2 \\
\hline Experimert & 10 \\
\hline Number $d \mathrm{~s}$ sars & 8 \\
\hline Recever Gion & \\
\hline Redaxation Detion & 50000 \\
\hline Pulse Wath & 6.8000 \\
\hline Acaustion Time & 21837 \\
\hline spectrometer Freagen & \\
\hline spectral With & 75030 \\
\hline Lowest frequency & . \\
\hline Narien & 14 \\
\hline Acaired Sze & ${ }_{16384}$ \\
\hline ctra Sze & 32738 \\
\hline
\end{tabular}

둥

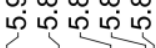

등 矛品吕

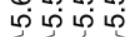

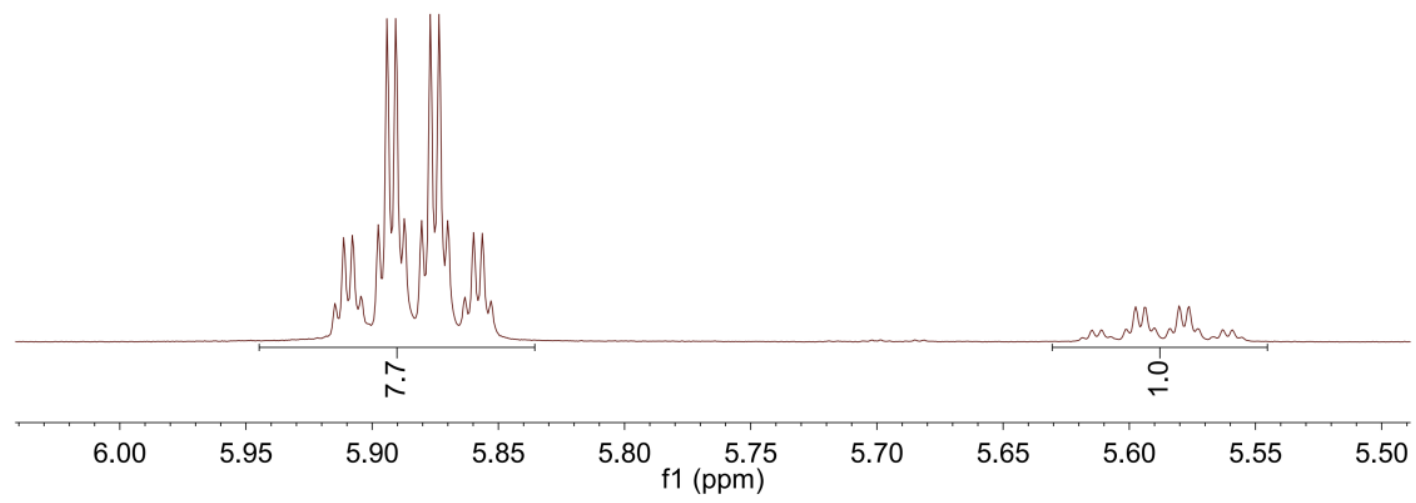


Entry 10

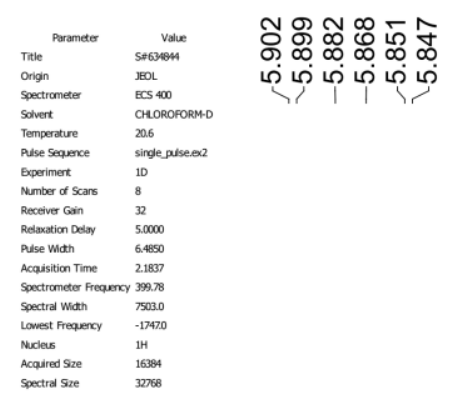

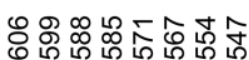

ما

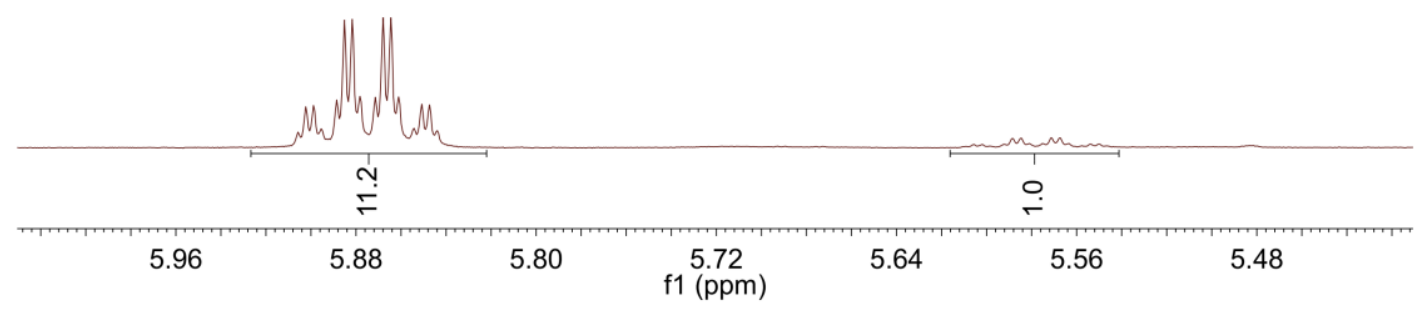

Entry 11

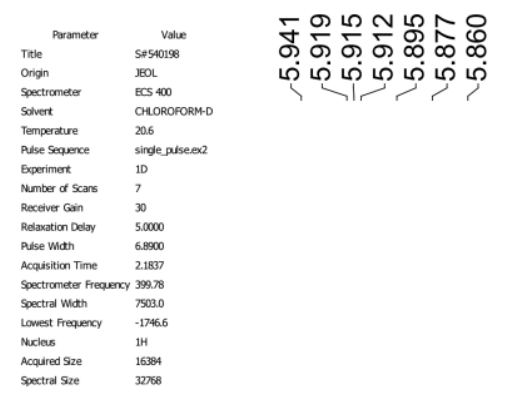

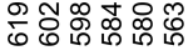

"م

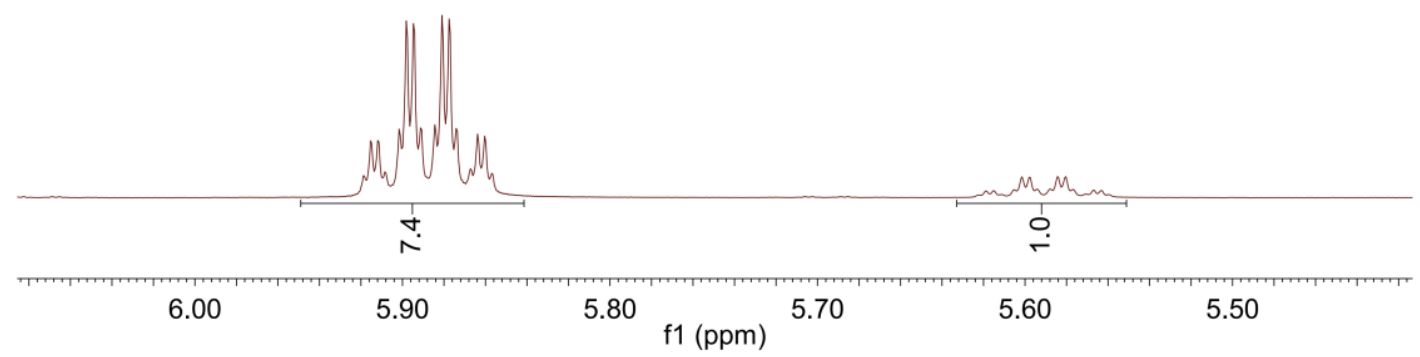


Table S2: Performance of representative cobalt catalysis systems in olefin isomerization

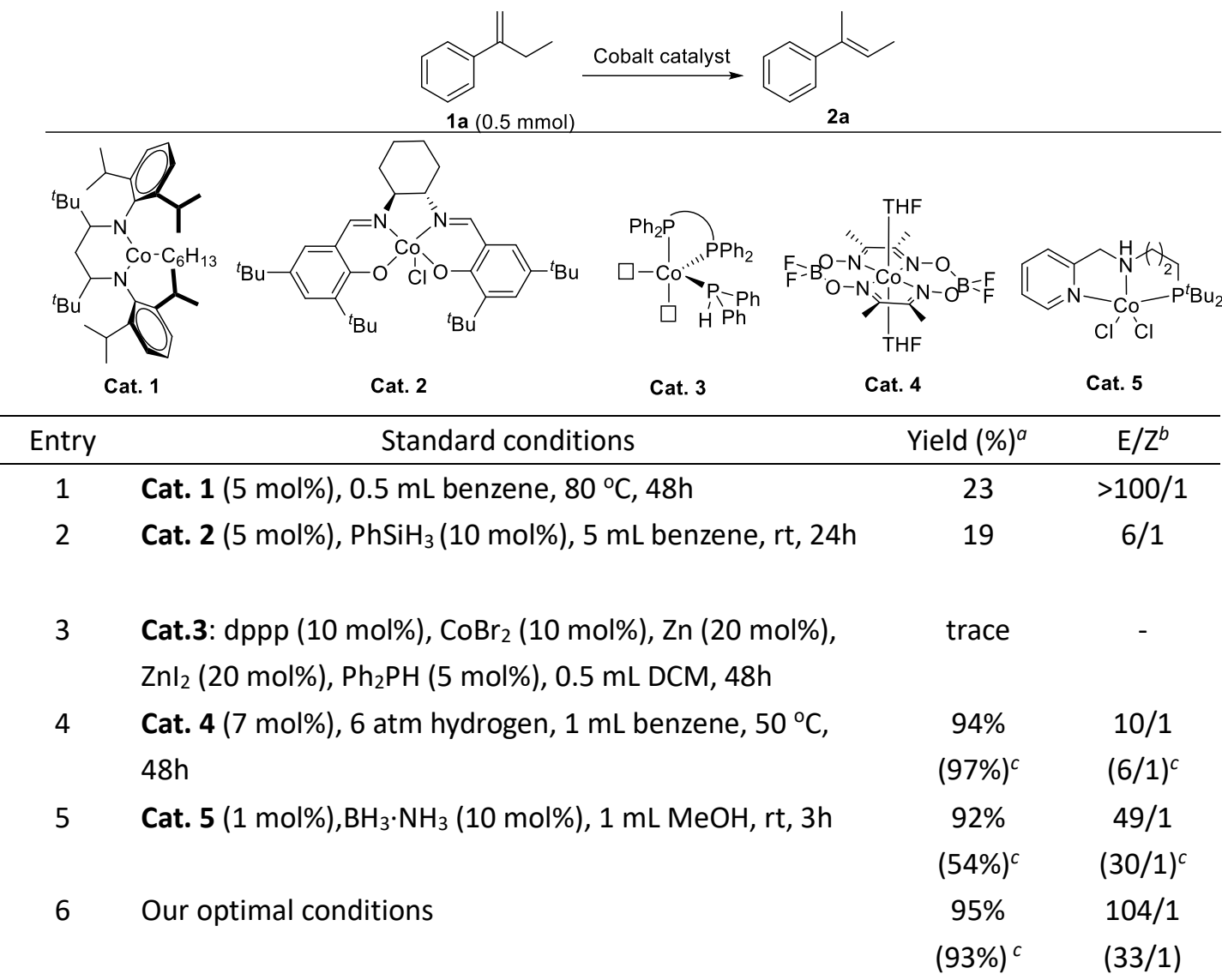

${ }^{a}$ Isolated yield. ${ }^{b}$ The ratio of $E / Z$ was determined by the ${ }^{1} \mathrm{H}$ NMR of crude product. ${ }^{c}$ The data in parentheses was obtained using $\alpha$-benzylstyrene as a substrate.

Entry 1
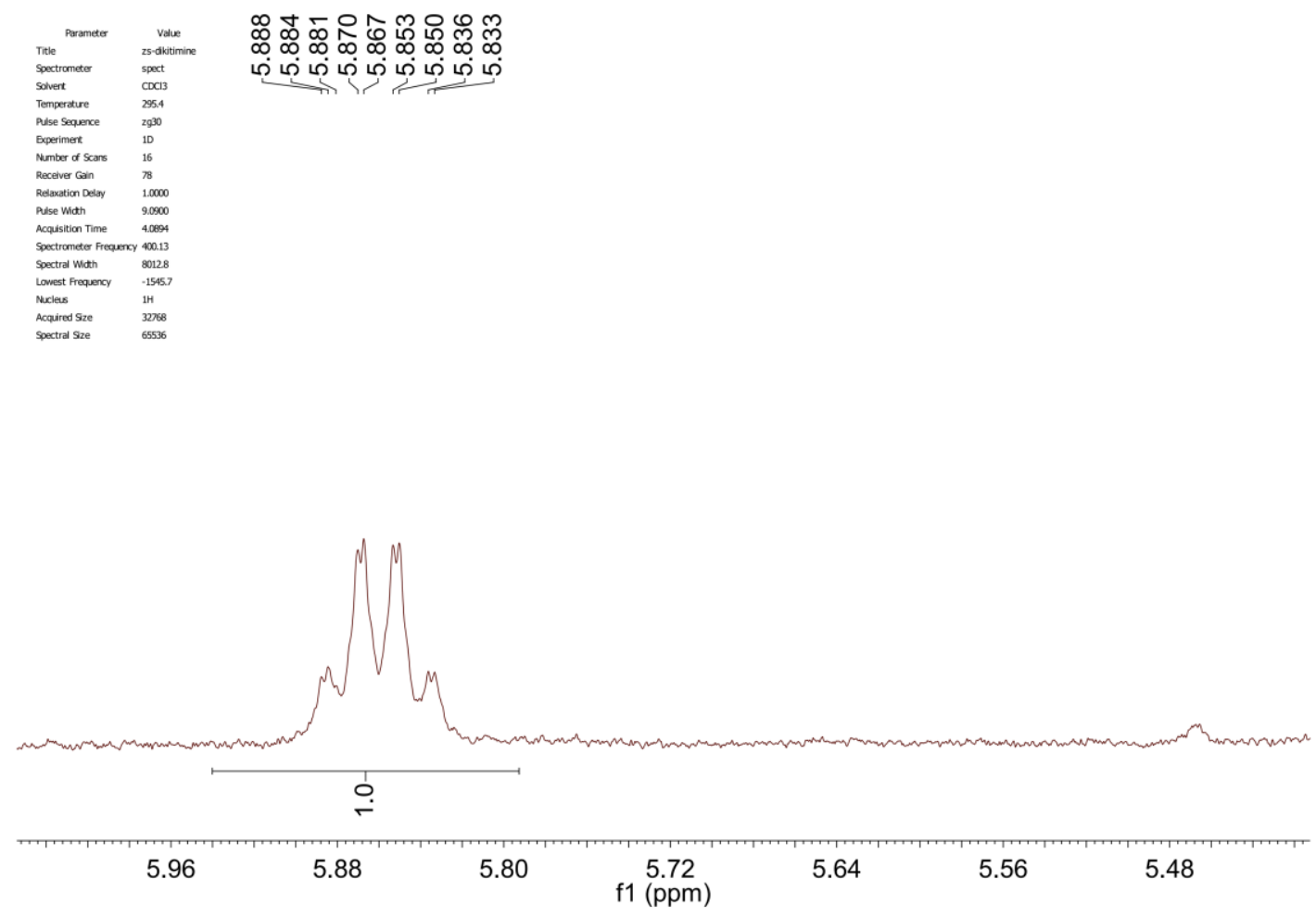
Entry 2

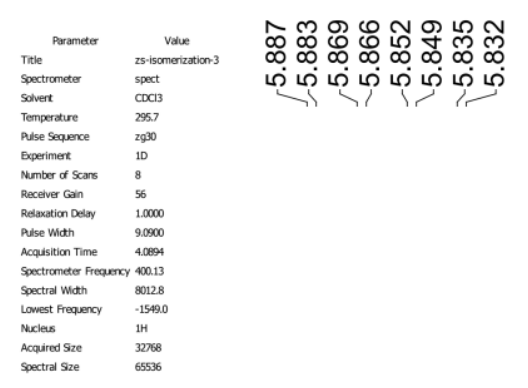

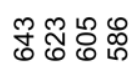

ம் மீ

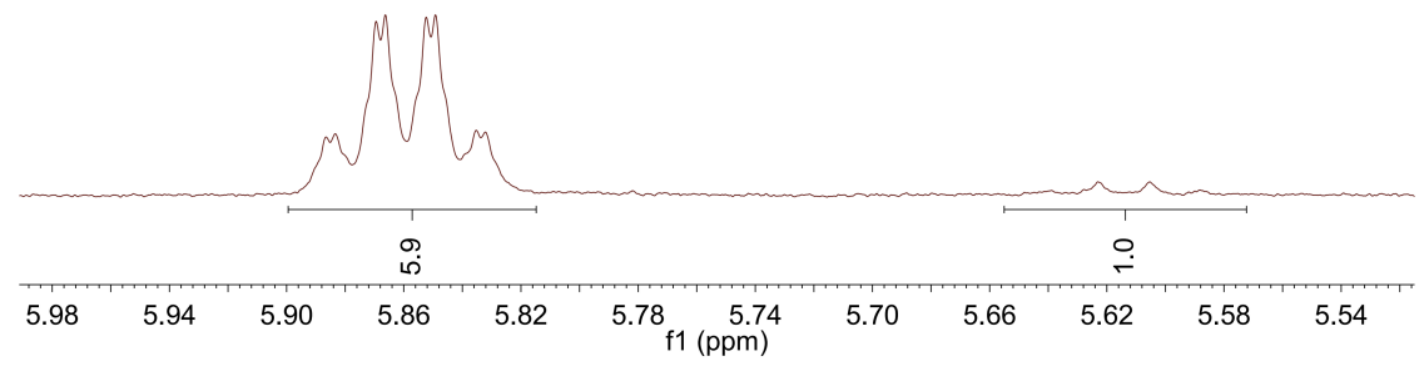

Entry 4

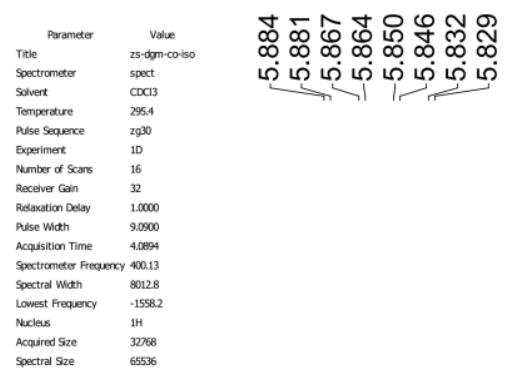

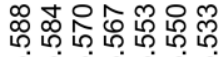

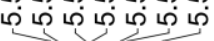

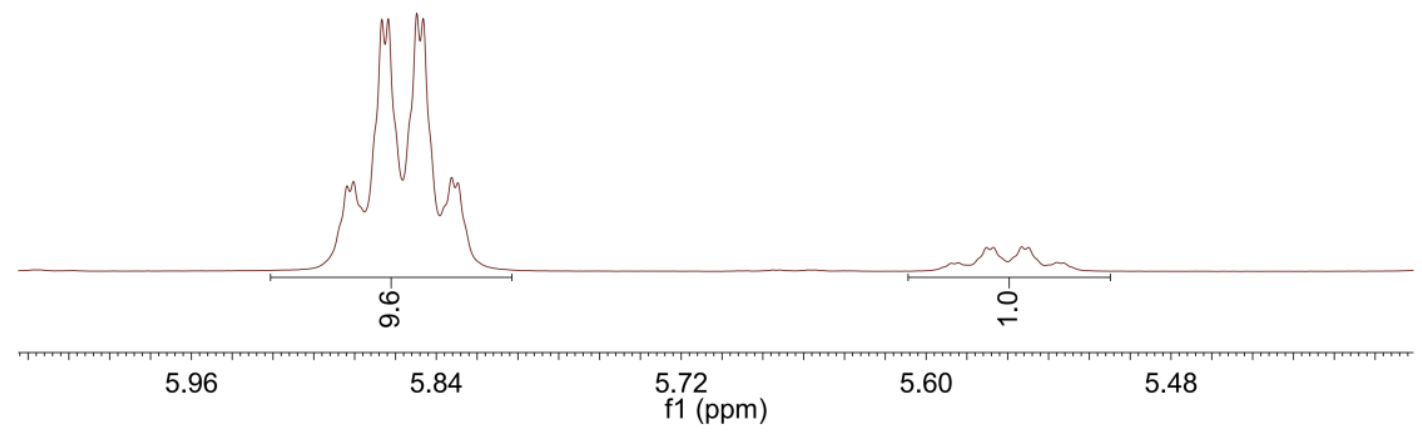


Entry 4 parenthesis

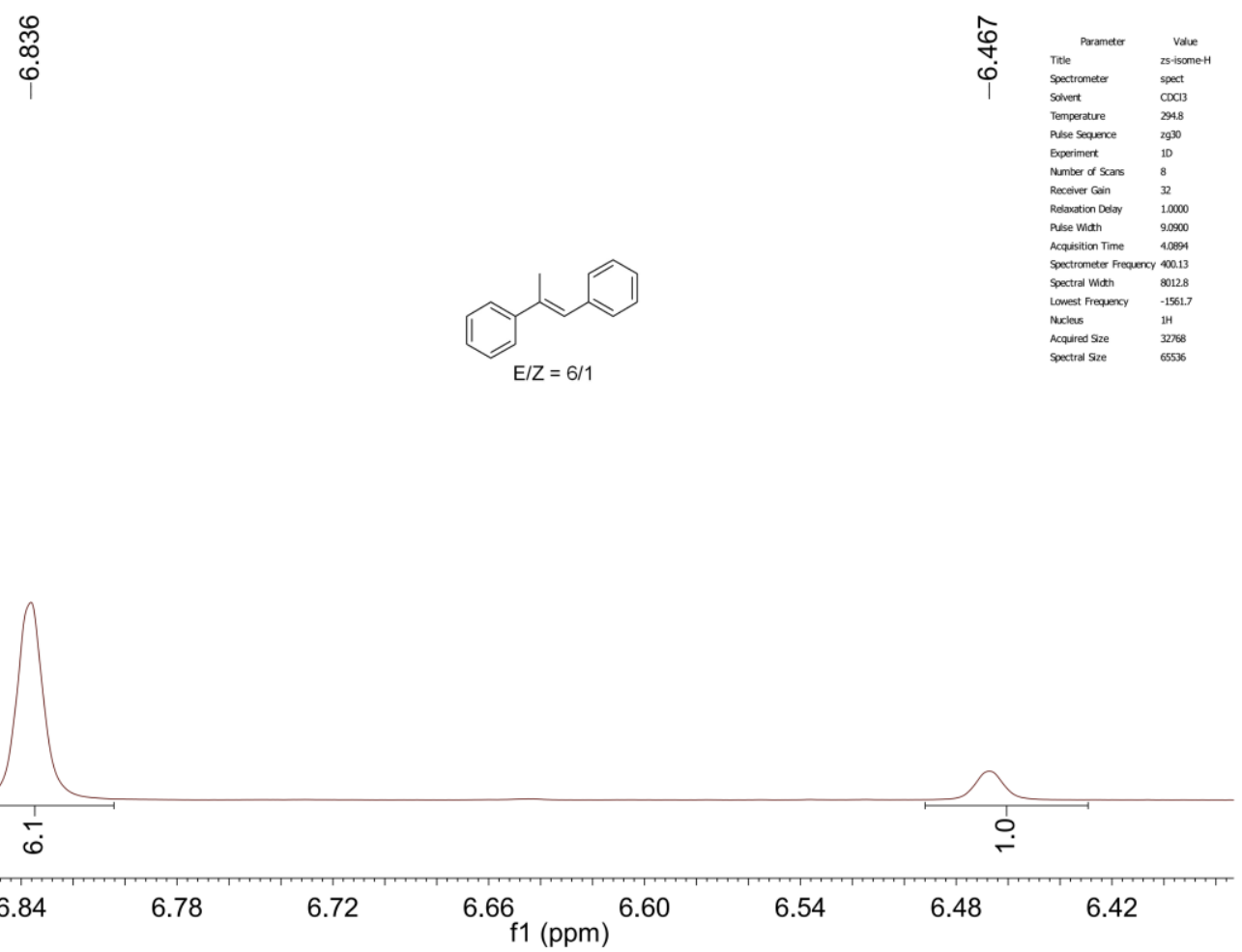

Entry 5

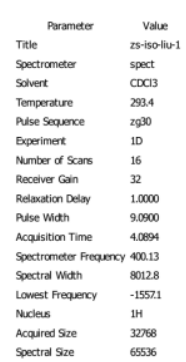

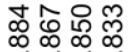

ம் ம் ம்

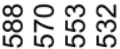

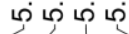

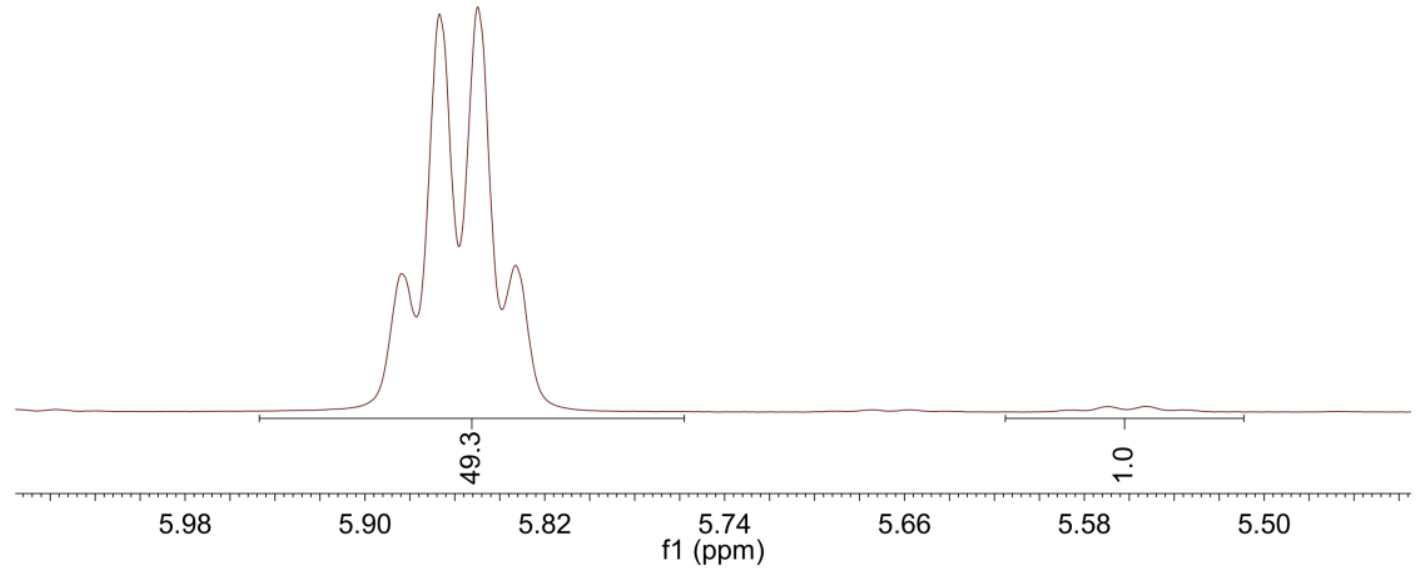




\section{Entry 5 parenthesis}

$\hat{\infty}$
0
0

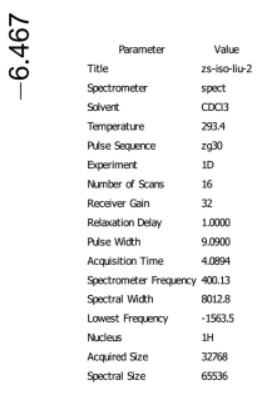

$E / Z=30 / 1$

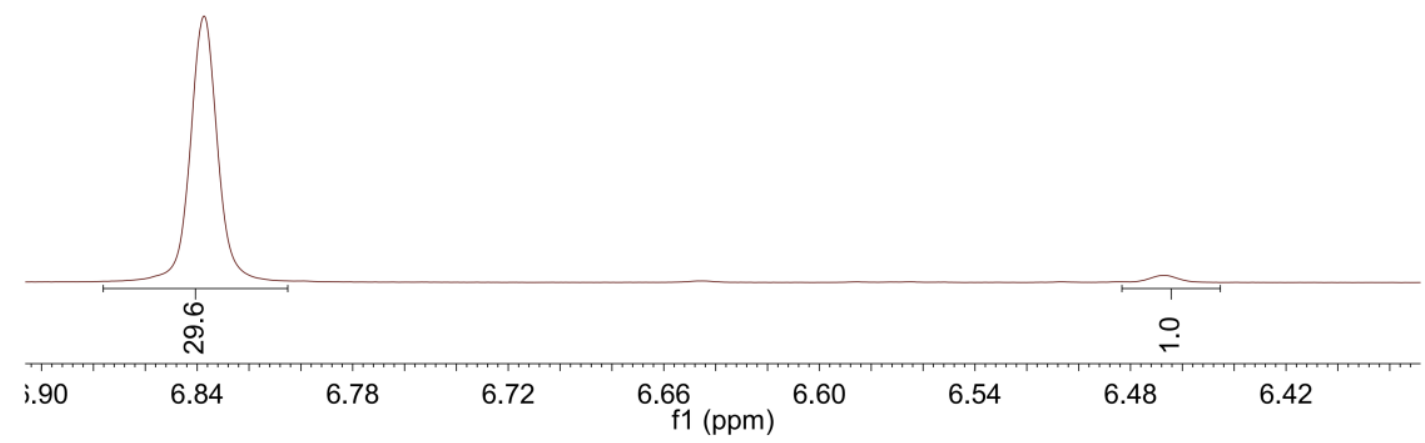

Entry 6 see Entry 1 of Table S1

Entry 6 parenthesis

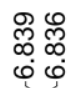
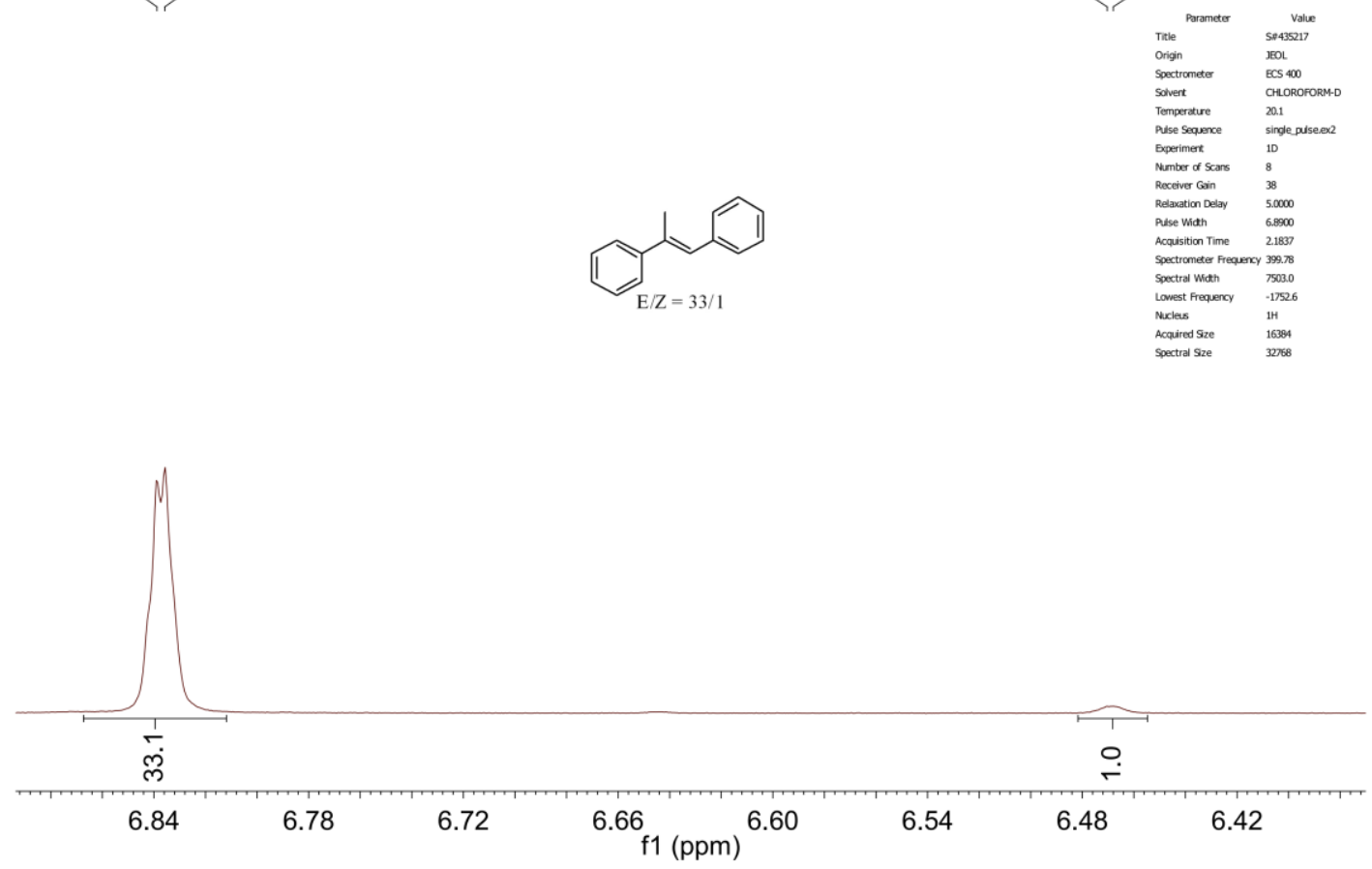


\section{Entry 1 reaction conditions ${ }^{35}$}

Inside an argon-atmosphere glovebox, to a borosilicate glass vial was added $\alpha$-ethylstyrene 1a (75 $\mu \mathrm{L}, 0.50 \mathrm{mmol})$, cat. $1(16.15 \mathrm{mg}, 0.025 \mathrm{mmol})^{36}$ and $\mathrm{C}_{6} \mathrm{H}_{6}(0.5 \mathrm{~mL})$. The reaction mixture was removed from the glovebox and stirred at $80{ }^{\circ} \mathrm{C}$ under an argon atmosphere for 48 hours. the crude product can be obtained through a short pipette column with hexane as eluent. It can be used for $E / Z$ selectivity determination. The crude product was further purified by silica gel column chromatography (pentane used as eluent) affording $15 \mathrm{mg}$.

\section{Entry 2 reaction conditions ${ }^{37}$}

Inside an argon-atmosphere glovebox, cat. 2 (16 mg, $0.025 \mathrm{mmol})^{38}$ was then added via syringe as a solution in benzene $(2.5 \mathrm{~mL})$ to a borosilicate glass vial and allowed to concentrate to dryness under a flow of argon. Benzene $(5 \mathrm{~mL})$ and $\alpha$-ethylstyrene 1a $(75 \mu \mathrm{L}, 0.50 \mathrm{mmol})$ was added was then added, which was subsequently added via a micro-syringe. The resulting homogeneous solution turned a dark black-green color. Reductant $\mathrm{PhSiH}_{3}(0.05 \mathrm{mmol}, 6.2 \mu \mathrm{L})$ was then added directly via a micro-syringe, which resulted in a distinct color change to a shade of orange, within minutes. The reaction mixture was removed from the glovebox and stirred at room temperature under an argon atmosphere for 24 hours. The crude product can be obtained through a short pipette column with hexane as eluent. It can be used for $E / Z$ selectivity determination. The crude product was further purified by silica gel column chromatography (pentane used as eluent) affording $13 \mathrm{mg}$.

\section{Entry 3 reaction conditions ${ }^{39}$}

Inside an argon-atmosphere glovebox, anhydrous zinc iodide (31.9 $\mathrm{mg}, 0.1 \mathrm{mmol})$, zinc powder (6.5 mg, $0.1 \mathrm{mmol})$ and the respective cobalt complex $\mathrm{CoBr}_{2}(\mathrm{dppp})(31.5 \mathrm{mg}, 0.05 \mathrm{mmol}$ ) were suspended in $0.5 \mathrm{~mL}$ anhydrous dichloromethane in a borosilicate glass vial. After stirring for 10 min of $\mathrm{Ph}_{2} \mathrm{PH}(0.025 \mathrm{mmol}, 4.4 \mu \mathrm{L})$ were added and after additional stirring for $10 \mathrm{~min} 0.50 \mathrm{mmol}$ of $\alpha$-ethylstyrene 1a $(75 \mu \mathrm{L}, 0.50 \mathrm{mmol})$ were added. The reaction mixture was removed from the glovebox and stirred at room temperature under an argon atmosphere for 48 hours. The crude product can be obtained through a short pipette column with hexane as eluent. It can be used for $E / Z$ selectivity determination.

\section{Entry 4 reaction conditions ${ }^{40}$}

A solution of cat. $4(20 \mathrm{mg}, 0.035 \mathrm{mmol})^{41}$ and benzene $(1 \mathrm{~mL})$ were prepared in a Fischer-Porter reactor, and $\alpha$-ethylstyrene $1 \mathrm{a}(75 \mu \mathrm{L}, 0.50 \mathrm{mmol})$ was added. The vessel was sealed and then pressurized to $6 \mathrm{~atm}$, and then placed in an oil bath at $50{ }^{\circ} \mathrm{C}$. After stirring for $48 \mathrm{~h}$, the reaction was allowed to return to room temperature, andthe $\mathrm{H}_{2}$ pressure was vented. The crude product can be obtained through a short pipette column with hexane as eluent. It can be used for $E / Z$ selectivity determination. The crude product was further purified by silica gel column chromatography (pentane used as eluent) affording $62 \mathrm{mg}$.

\section{Entry 5 reaction conditions ${ }^{42}$}

Inside an argon-atmosphere glovebox, cat.5 $(0.005 \mathrm{mmol}, 2.1 \mathrm{mg})$, ammonia borane ( $0.05 \mathrm{mmol}$, $1.5 \mathrm{mg}), \alpha$-ethylstyrene $1 \mathrm{a}(75 \mu \mathrm{L}, 0.50 \mathrm{mmol})$ and methanol $(1 \mathrm{~mL})$ were added sequentially to a borosilicate glass vial. The reaction was stirred for 3 hours at room temperature. The resulting 
solution was concentrated in vacuum and the residue was purified by silica gel column chromatography eluted with ethyl acetate/petroleum ether to give the isomerization product. The crude product can be obtained through a short pipette column with hexane as eluent. It can be used for $E / Z$ selectivity determination. The crude product was further purified by silica gel column chromatography (pentane used as eluent) affording $61 \mathrm{mg}$.

\section{Entry 6 reaction conditions}

Inside an argon-atmosphere glovebox, a borosilicate glass vial was charged with $\mathrm{CoBr}_{2}$ (3.3 mg, $0.015 \mathrm{mmol}$ ) and $\mathbf{L} \mathbf{1}(6.6 \mathrm{mg}, 0.015 \mathrm{mmol}),{ }^{t} \mathrm{BuONa}(7.2 \mathrm{mg}, 0.15 \mathrm{mmol})$, with anhydrous toluene $(1 \mathrm{~mL})$ as solvent. The resulting mixture was stirred at room temperature for 3 hours to form a precatalyst. Subsequently, $\mathrm{BH}_{3} \cdot \mathrm{NH}_{3}(1.4 \mathrm{mg}, 0.045 \mathrm{mmol})$ was added and the mixture was stirred for $5 \mathrm{~min}$ before adding $\alpha$-ethylstyrene $1 \mathrm{a}(75 \mu \mathrm{L}, 0.5 \mathrm{mmol})$. The reaction mixture was removed from the glovebox and stirred at $60{ }^{\circ} \mathrm{C}$ under an argon atmosphere for 12 hours. The crude product can be obtained through a short pipette column with hexane as eluent. It can be used for $E / Z$ selectivity determination. The crude product was further purified by silica gel column chromatography (pentane used as eluent) affording $63 \mathrm{mg}$ (95\% yield, $E / Z=104 / 1)$ product $2 \mathrm{a}$ as colorless oil. 


\section{General procedures for alkene isomerization}

Condition I (use for $2 \mathrm{a}-2 \mathrm{c}, \mathbf{2 h}, \mathbf{2 j}, \mathbf{2 m}$ )

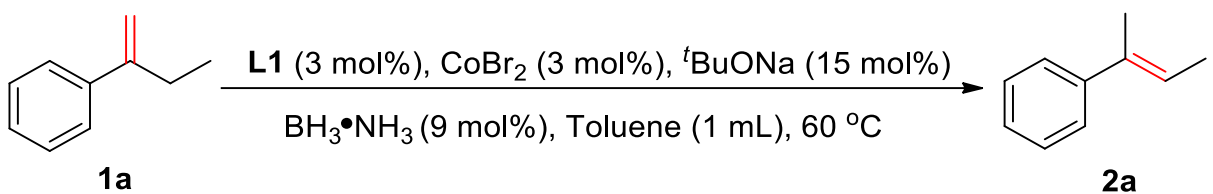

Inside an argon-atmosphere glovebox, a borosilicate glass vial was charged with $\mathrm{CoBr}_{2}$ (6.5 $\mathrm{mg}, 0.03 \mathrm{mmol}$ ) and $\mathbf{L} 1(13.2 \mathrm{mg}, 0.03 \mathrm{mmol}),{ }^{t} \mathrm{BuONa}(14.4 \mathrm{mg}, 0.15 \mathrm{mmol})$, with anhydrous toluene $(1 \mathrm{~mL})$ as solvent. The resulting mixture was stirred at room temperature for 3 hours to form a precatalyst. Subsequently, $\mathrm{BH}_{3} \cdot \mathrm{NH}_{3}(2.7 \mathrm{mg}, 0.09 \mathrm{mmol})$ was added and the mixture was stirred for $5 \mathrm{~min}$ before adding $\alpha$-ethylstyrene 1a $(149 \mu \mathrm{L}, 1 \mathrm{mmol})$. The reaction mixture was removed from the glovebox and stirred at $60^{\circ} \mathrm{C}$ under an argon atmosphere for 12 hours. The crude product can be obtained through a short pipette column with hexane as eluent. It can be used for $E / Z$ selectivity determination. The crude product was further purified by silica gel column chromatography (pentane used as eluent) affording $126 \mathrm{mg}$ (95\% yield, $E / Z=107 / 1)$ product $\mathbf{2 a}$ as colorless oil.

Condition II (use for 2d-2f, 2i, 2k, 2l, 2n-2ac, 2aj-2ao, 2ar)

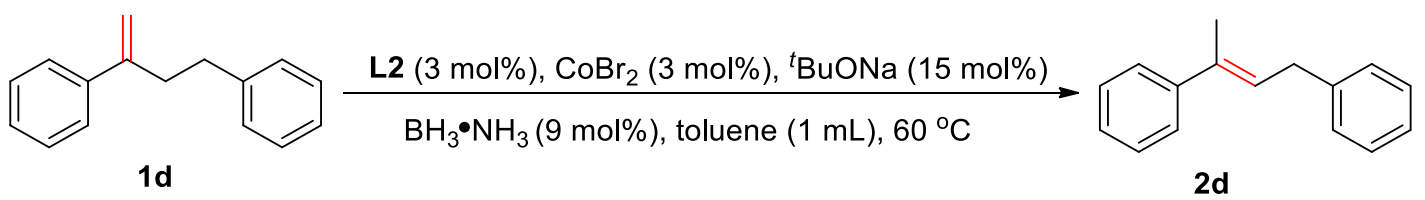

Inside an argon-atmosphere glovebox, a borosilicate glass vial was charged with $\mathrm{CoBr}_{2}$ (6.5 $\mathrm{mg}, 0.03 \mathrm{mmol}$ ) and $\mathbf{L} 2(14.7 \mathrm{mg}, 0.03 \mathrm{mmol}),{ }^{t} \mathrm{BuONa}(14.4 \mathrm{mg}, 0.15 \mathrm{mmol})$, with anhydrous toluene $(1 \mathrm{~mL})$ as solvent. The resulting mixture was stirred at room temperature for 3 hours to form a precatalyst. Subsequently, $\mathrm{BH}_{3} \cdot \mathrm{NH}_{3}(2.7 \mathrm{mg}, 0.09 \mathrm{mmol})$ was added and the mixture was stirred for $5 \mathrm{~min}$ before adding but-3-ene-1,3-diyldibenzene $1 \mathrm{~d}$ (208 $\mathrm{mg}, 1 \mathrm{mmol})$. The reaction mixture was removed from the glovebox and stirred at $60{ }^{\circ} \mathrm{C}$ under an argon atmosphere for 12 hours. The crude product can be obtained through a short pipette column with hexane as eluent. It can be used for $E / Z$ selectivity determination. The crude product was further purified by silica gel column chromatography (pentane as eluent) affording $169 \mathrm{mg}$ ( $81 \%$ yield, $E / Z=35 / 1$ ) product $\mathbf{2 d}$ as colorless oil.

Condition III (use for 2g, 2af-2ah, 2ap, 2aq)

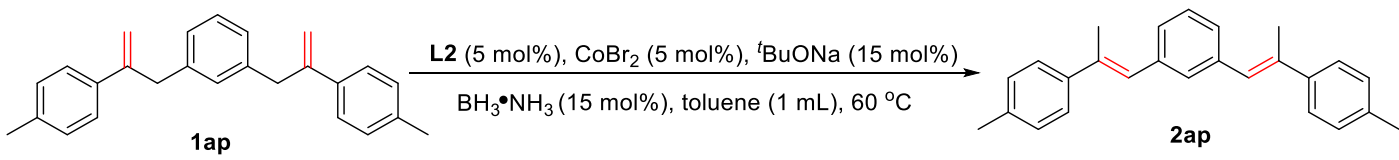

Inside an argon-atmosphere glovebox, a borosilicate glass vial was charged with $\mathrm{CoBr}_{2}$ (10.9 $\mathrm{mg}, 0.05 \mathrm{mmol}$ ) and $\mathbf{L} 2(24.5 \mathrm{mg}, 0.05 \mathrm{mmol}),{ }^{t} \mathrm{BuONa}(14.4 \mathrm{mg}, 0.15 \mathrm{mmol})$, with anhydrous toluene $(1 \mathrm{~mL})$ as solvent. The resulting mixture was stirred at room temperature for 3 hours to form a precatalyst. Subsequently, $\mathrm{BH}_{3} \cdot \mathrm{NH}_{3}(4.5 \mathrm{mg}, 0.15 \mathrm{mmol})$ was added and the mixture was stirred for $5 \mathrm{~min}$ before adding 1,3-bis(2-( $p$-tolyl)allyl)benzene 1ap $(208 \mathrm{mg}, 1 \mathrm{mmol})$. The reaction mixture was removed from the glovebox and stirred at $60^{\circ} \mathrm{C}$ under an argon atmosphere for 12 hours. The crude product can be obtained through a short pipette column with DCM as 
eluent. It can be used for $E / Z$ selectivity determination. The crude product was further purified by silica gel column chromatography (pentane followed by ether/pentane $=1 / 200$ ) affording $274 \mathrm{mg}$ ( $81 \%$ yield, $E / Z=52 / 1$ ) product 2 ap as white solid. (In case of $\mathbf{2 g}$, the reaction was performed with $0.5 \mathrm{mmol}$ scale.)

Condition IV (use for 2ai)

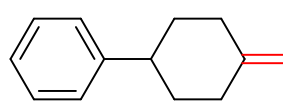

1ai

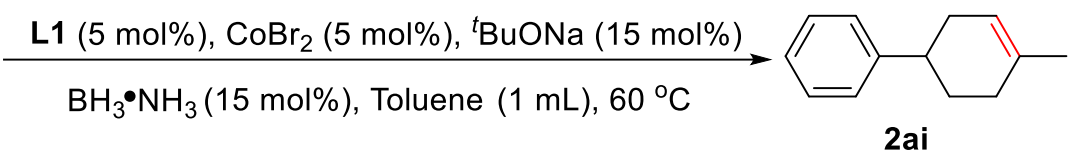

2ai

Inside an argon-atmosphere glovebox, a borosilicate glass vial was charged with $\mathrm{CoBr}_{2}$ (10.9 $\mathrm{mg}, 0.05 \mathrm{mmol}$ ) and $\mathbf{L} 1(22.0 \mathrm{mg}, 0.05 \mathrm{mmol})$, ${ }^{\mathrm{t}} \mathrm{BuONa}(14.4 \mathrm{mg}, 0.15 \mathrm{mmol})$, with anhydrous toluene $(1 \mathrm{~mL})$ as solvent. The resulting mixture was stirred at room temperature for 3 hours to form a precatalyst. Subsequently, $\mathrm{BH}_{3} \cdot \mathrm{NH}_{3}(4.5 \mathrm{mg}, 0.15 \mathrm{mmol})$ was added and the mixture was stirred for $5 \mathrm{~min}$ before adding (4-methylenecyclohexyl)benzene 1ai (172 mg, $1 \mathrm{mmol})$. The reaction mixture was removed from the glovebox and stirred at $60^{\circ} \mathrm{C}$ under an argon atmosphere for 12 hours. The solvent was removed in vacuum. The resulting residue was purified by silica gel column chromatography (pentane used as eluent) affording $155 \mathrm{mg}$ (90\% yield) product 2ai as colorless oil.

\section{Procedure for gram scale alkene isomerization}

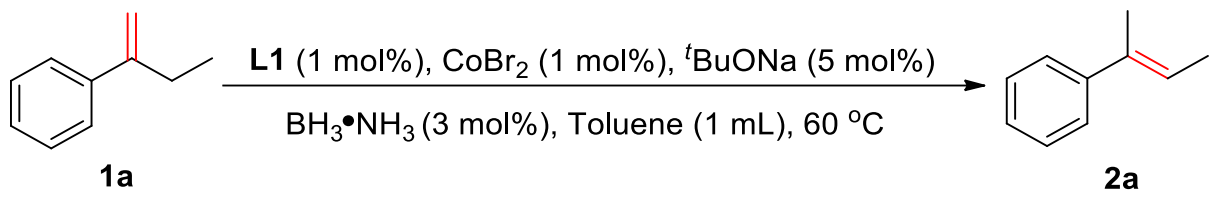

Inside an argon-atmosphere glovebox, a borosilicate glass vial was charged with $\mathrm{CoBr}_{2}(21.8$ $\mathrm{mg}, 0.1 \mathrm{mmol}$ ) and $\mathbf{L} \mathbf{1}(44.0 \mathrm{mg}, 0.1 \mathrm{mmol}),{ }^{t} \mathrm{BuONa}(48.0 \mathrm{mg}, 0.5 \mathrm{mmol})$, with anhydrous toluene $(1 \mathrm{~mL})$ as solvent. The resulting mixture was stirred at room temperature for 3 hours to form a precatalyst. Subsequently, $\mathrm{BH}_{3} \cdot \mathrm{NH}_{3}(9.0 \mathrm{mg}, 0.3 \mathrm{mmol})$ was added and the mixture was stirred for $5 \mathrm{~min}$ before adding $\alpha$-ethylstyrene $1 \mathrm{a}(1.49 \mathrm{~mL}, 10 \mathrm{mmol})$. The reaction mixture was removed from the glovebox and stirred at $60 \stackrel{\circ}{\circ}$ under an argon atmosphere for 12 hours. The crude product can be obtained through a short plug of silicon gel using hexane as eluent. It can be used for $E / Z$ selectivity determination. The crude product was further purified by silica gel column chromatography (pentane used as eluent) affording $1.24 \mathrm{~g}(94 \%$ yield, $E / Z=64 / 1$ ) product $2 \mathrm{a}$ as colorless oil. 


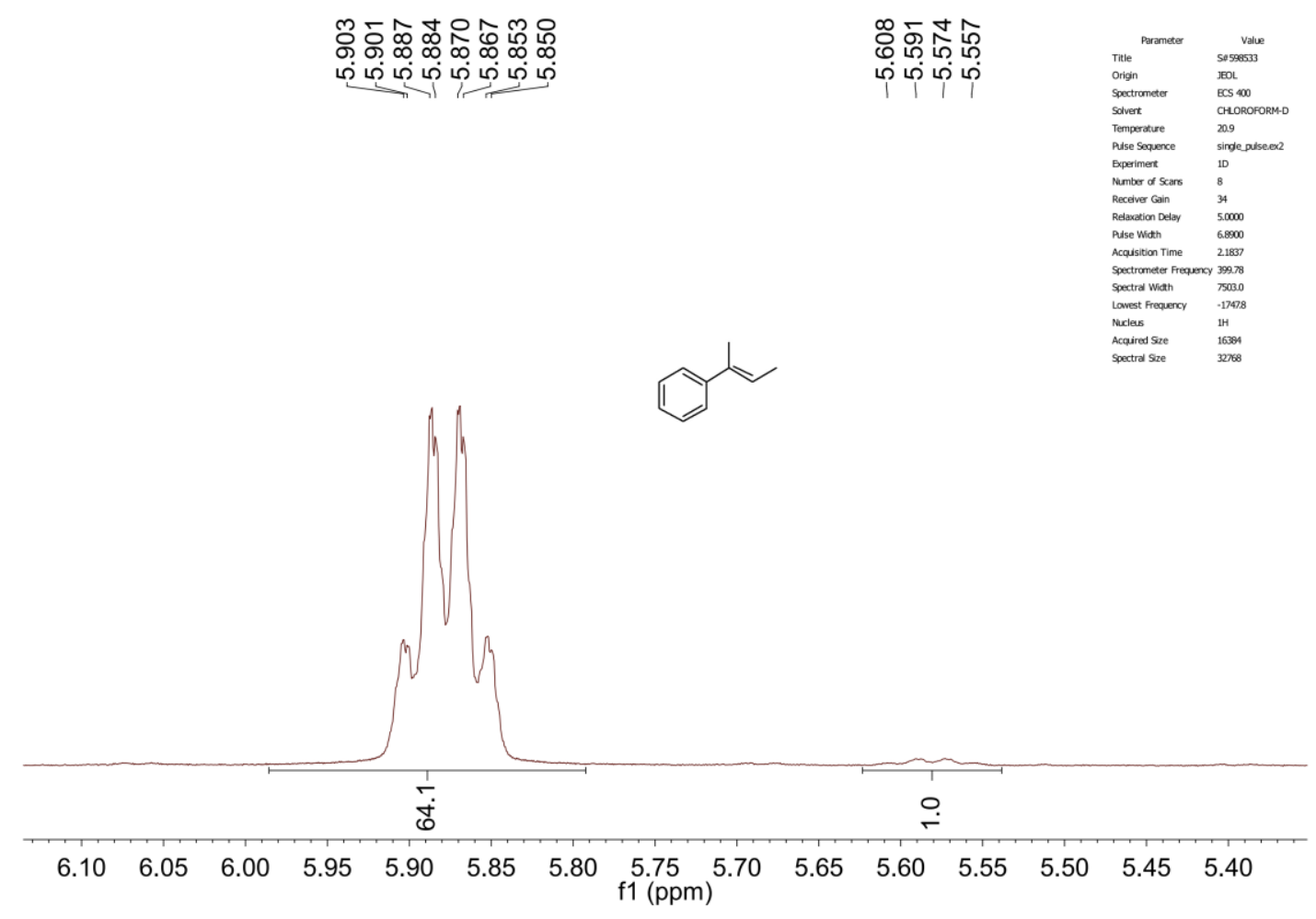

\section{Synthesis of deuterium labeled product}

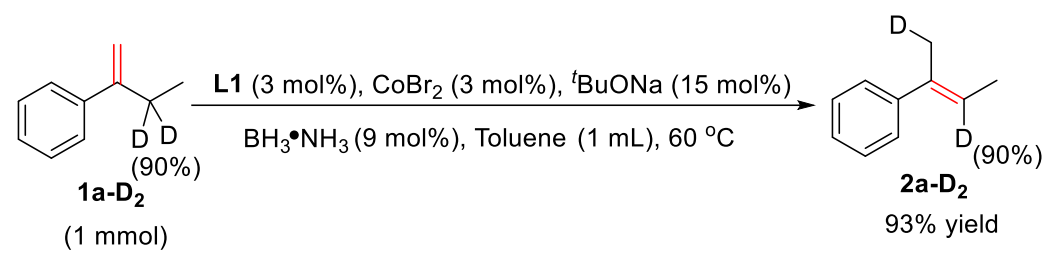

This reaction was carried out following the procedure Condition I using $\mathbf{1} \mathbf{a}-\mathbf{D}_{\mathbf{2}}$ as reactant. The resulting deuterium product (125 $\mathrm{mg}$ ) was obtained with $93 \%$ yield and $90 \%$ deuterium content.

(E)-(but-2-en-2-yl-1,3- $d_{2}$ ) benzene (2a- $\left.\mathbf{D}_{2}\right)$ : $125 \mathrm{mg}, 93 \%$ yield; colorless oil; ${ }^{1} \mathrm{H}$ NMR (400 MHz, $\left.\mathrm{CDCl}_{3}\right)$ : $7.39(\mathrm{~m}, 2 \mathrm{H}), 7.32(\mathrm{~m}, 2 \mathrm{H}), 7.23(\mathrm{~m}, 1 \mathrm{H}), 5.89(\mathrm{~m}, 0.1 \mathrm{H}), 2.04(\mathrm{~m}, 2 \mathrm{H}), 1.81(\mathrm{~s}, 3 \mathrm{H}) ;{ }^{2} \mathrm{H}$ $\operatorname{NMR}\left(61.4 \mathrm{MHz}, \mathrm{Et}_{2} \mathrm{O}\right): 6.45(\mathrm{q}, J=1.06 \mathrm{~Hz}), 2.60(\mathrm{~d}, J=2.39 \mathrm{~Hz}) ;{ }^{13} \mathrm{C} \mathrm{NMR}\left(100 \mathrm{MHz}, \mathrm{CDCl}_{3}\right)$ : 144.0, $135.4(\mathrm{t}, J=5.7 \mathrm{~Hz}), 128.1,126.4,125.5,122.5,15.4(\mathrm{~d}, J=3.8 \mathrm{~Hz}), 14.3(\mathrm{~d}, J=12.6 \mathrm{~Hz}) ; \mathrm{IR}$ (neat): 2922, 2853, 1686, 1442, 1264, 1025, 738, 763, 695; GC-MS: $\mathrm{t}_{\mathrm{R}}=8.71 \mathrm{~min}, \mathrm{~m} / \mathrm{z}=134.2$. 


\section{Procedure for catalyst-recycling experiment}

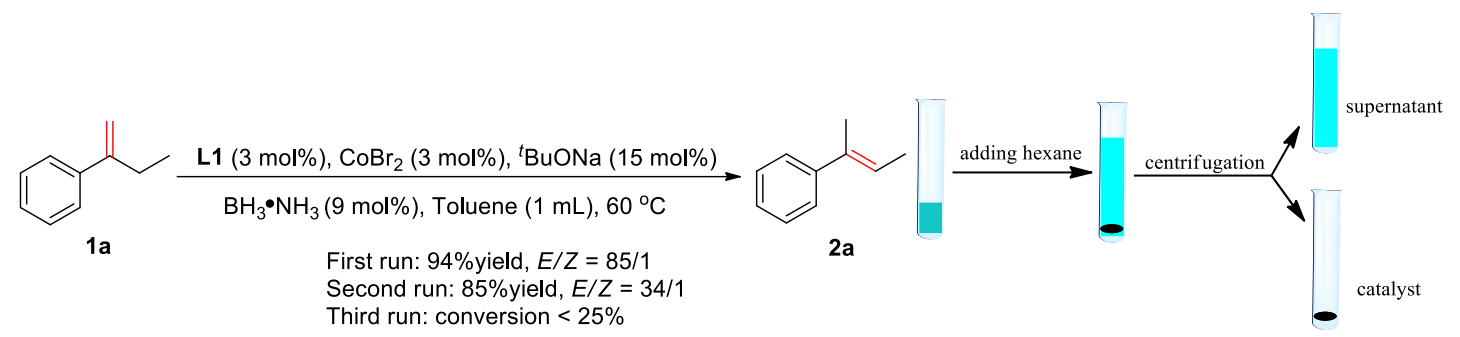

Inside an argon-atmosphere glovebox, a borosilicate glass vial was charged with $\mathrm{CoBr}_{2}$ (13.1 $\mathrm{mg}, 0.06 \mathrm{mmol}$ ) and $\mathbf{L} \mathbf{1}(26.4 \mathrm{mg}, 0.06 \mathrm{mmol}),{ }^{\mathrm{t}} \mathrm{BuONa}(28.8 \mathrm{mg}, 0.3 \mathrm{mmol})$, with anhydrous toluene $(1 \mathrm{~mL})$ as solvent. The resulting mixture was stirred at room temperature for 3 hours to form a precatalyst. Subsequently, $\mathrm{BH}_{3} \cdot \mathrm{NH}_{3}(5.4 \mathrm{mg}, 0.18 \mathrm{mmol})$ was added and the mixture was stirred for $5 \mathrm{~min}$ before adding $\alpha$-ethylstyrene 1a $(298 \mu \mathrm{L}, 2 \mathrm{mmol})$. The reaction mixture was removed from the glovebox and stirred at $60^{\circ} \mathrm{C}$ under an argon atmosphere for 12 hours. After the reaction cooled to room temperature, hexane $(30 \mathrm{~mL})$ was added and the catalyst precipitated from the mixture. The precipitate was removed by centrifugation at about $5000 \mathrm{rpm}$ for $5 \mathrm{~min}$, and washed with hexane twice $(15 \times 2 \mathrm{~mL})$. The combined supernatant was evaporated in vacuum and the residue was purified by silica gel column chromatography (pentane as eluent) affording product $2 \mathrm{a}$ ( $248 \mathrm{mg}, 94 \%$ yield, $E / Z=85 / 1$ ). The precipitate was transferred to a vial and dried in vacuum, which can be used as catalyst in second run. To maintain the activity of recycled catalyst, an extra $\mathrm{NaO}^{t} \mathrm{Bu}(23 \mathrm{mg}, 0.24 \mathrm{mmol})$ and $\mathrm{BH}_{3} \cdot \mathrm{NH}_{3}(5.4 \mathrm{mg}, 0.18 \mathrm{mmol})$ has to be added in the beginning of every run. The recycling experiment was performed in three times and the yields are the average value.

First run

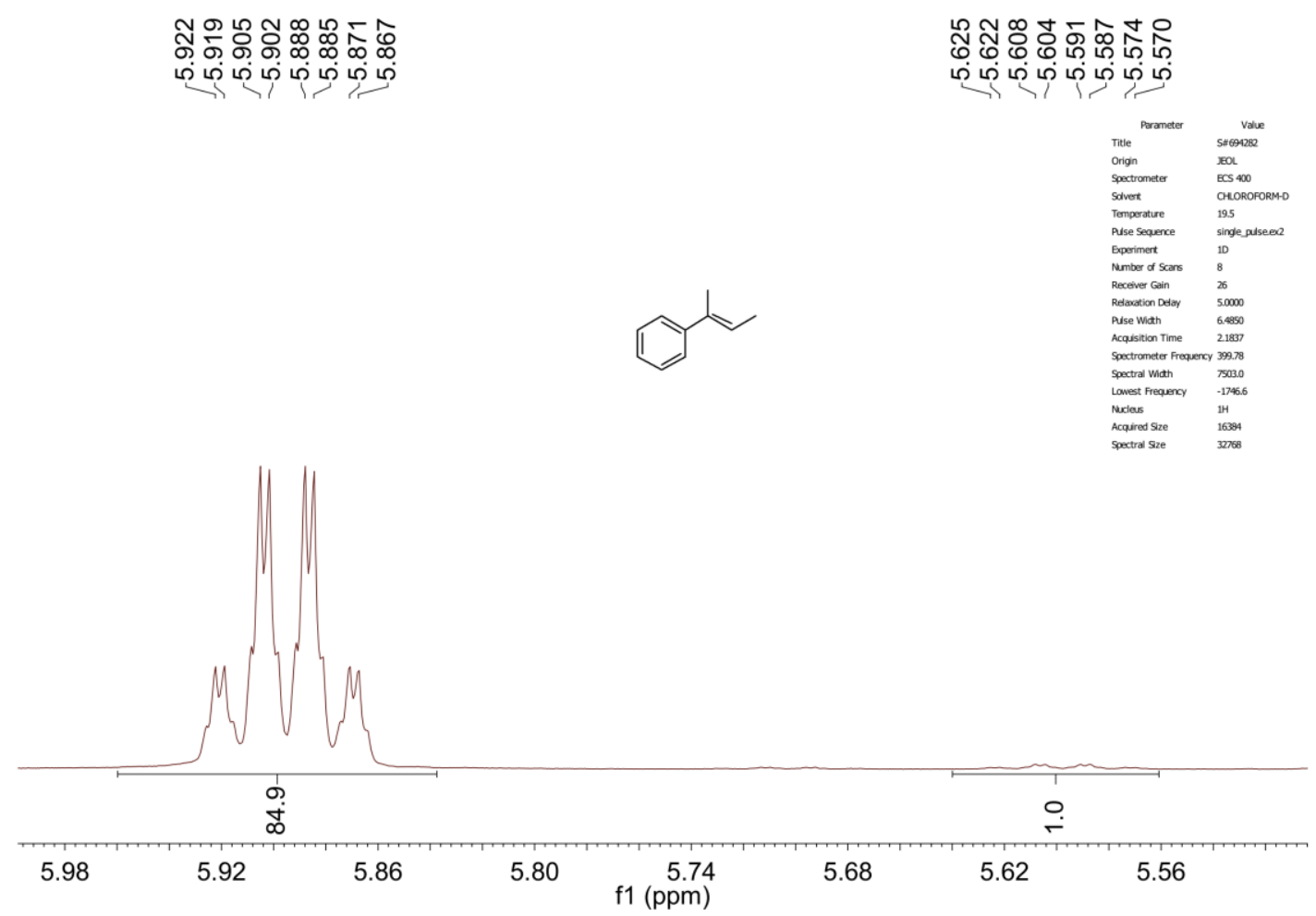


Second run

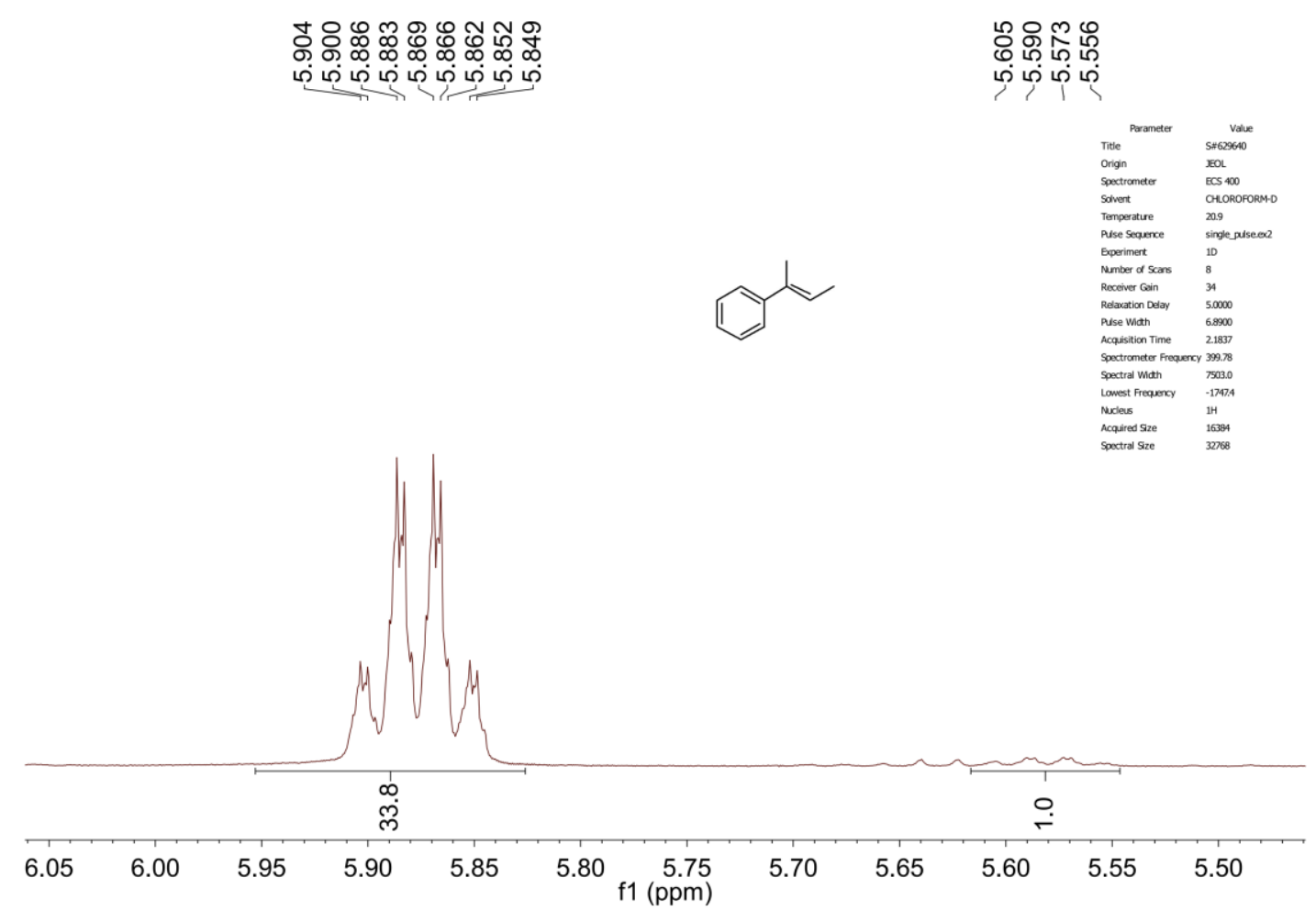




\section{Procedure for control experimens}

10.1 Crossover experiment

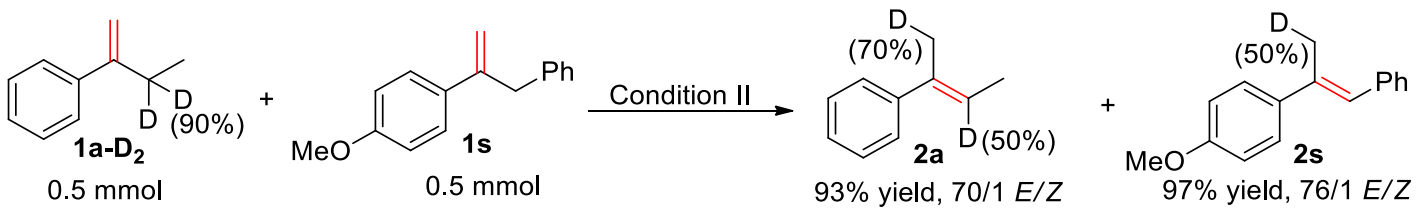

The crossover experiment was performed with the procedure Condition II using $0.5 \mathrm{mmol}$ $1 \mathrm{a}-\mathbf{D}_{\mathbf{2}}$ and $0.5 \mathrm{mmol} \mathbf{1 s}$ as substrate. The product $2 \mathrm{a}$ and $\mathbf{2 s}$ was obtained with $93 \%$ and $97 \%$ yields respectively. The spectra of products $2 \mathrm{a}$ and $\mathbf{2 s}$ were shown as below. $2 a^{1} \mathrm{H} N M R$

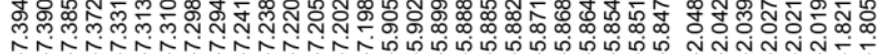
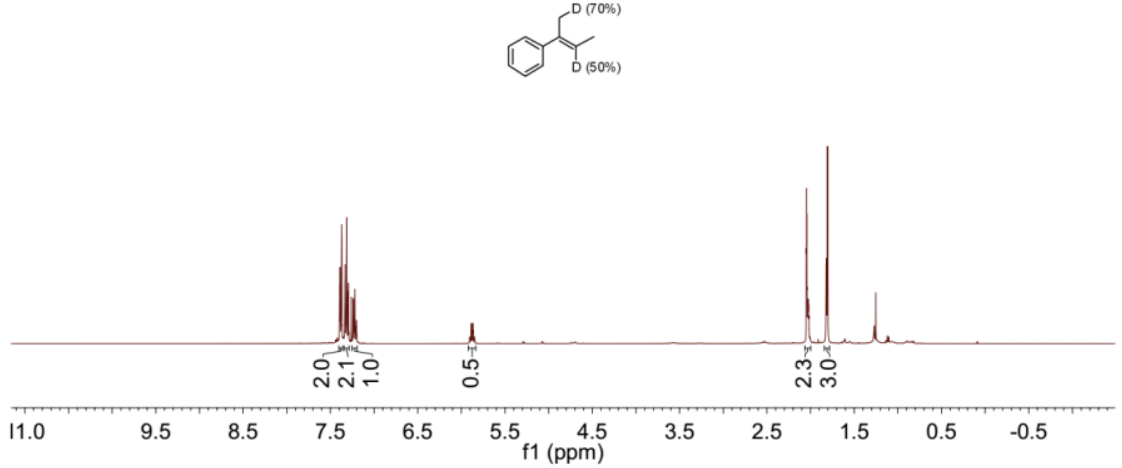

2a $E / Z$

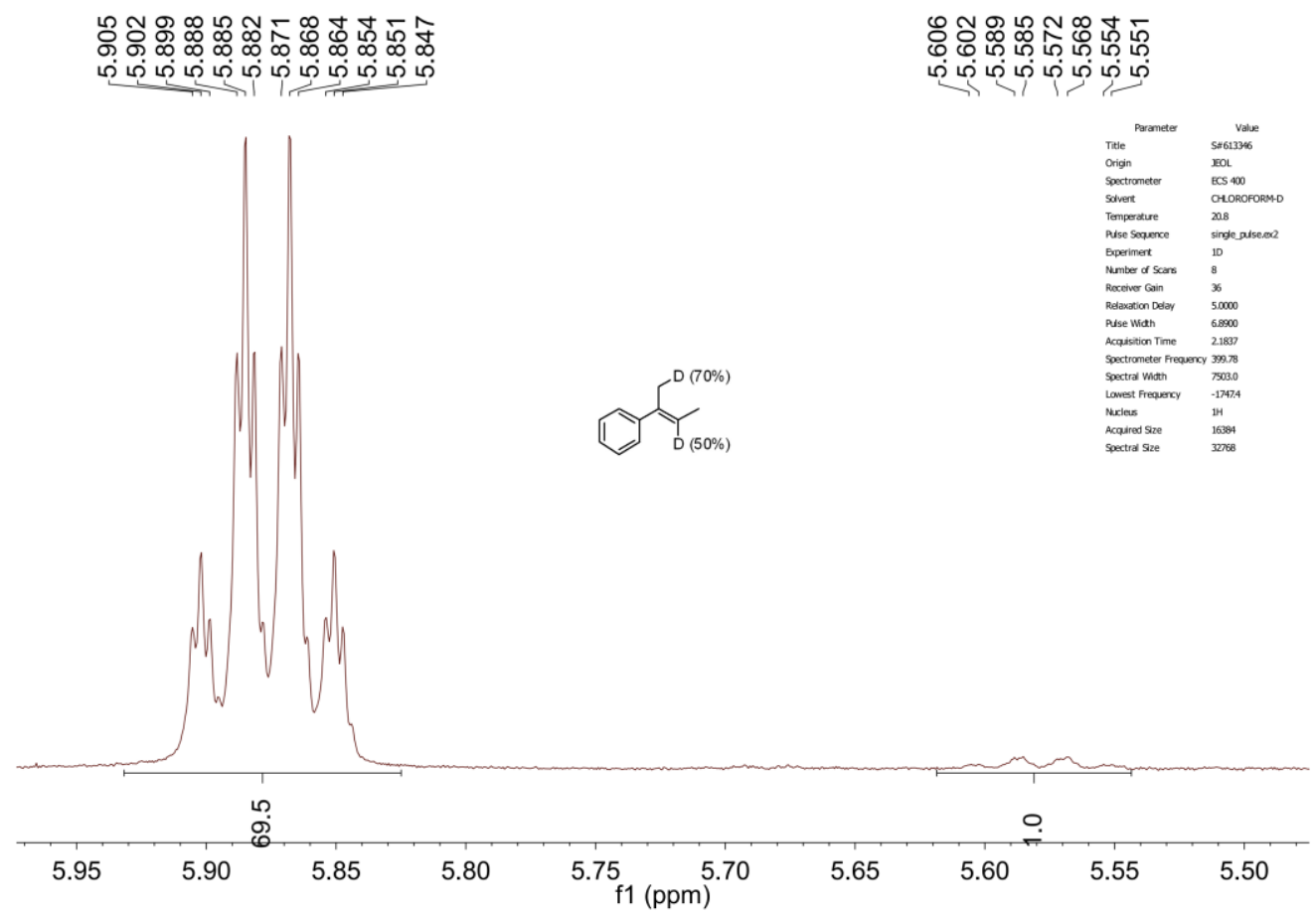


2s ${ }^{1} \mathrm{H} N M R$
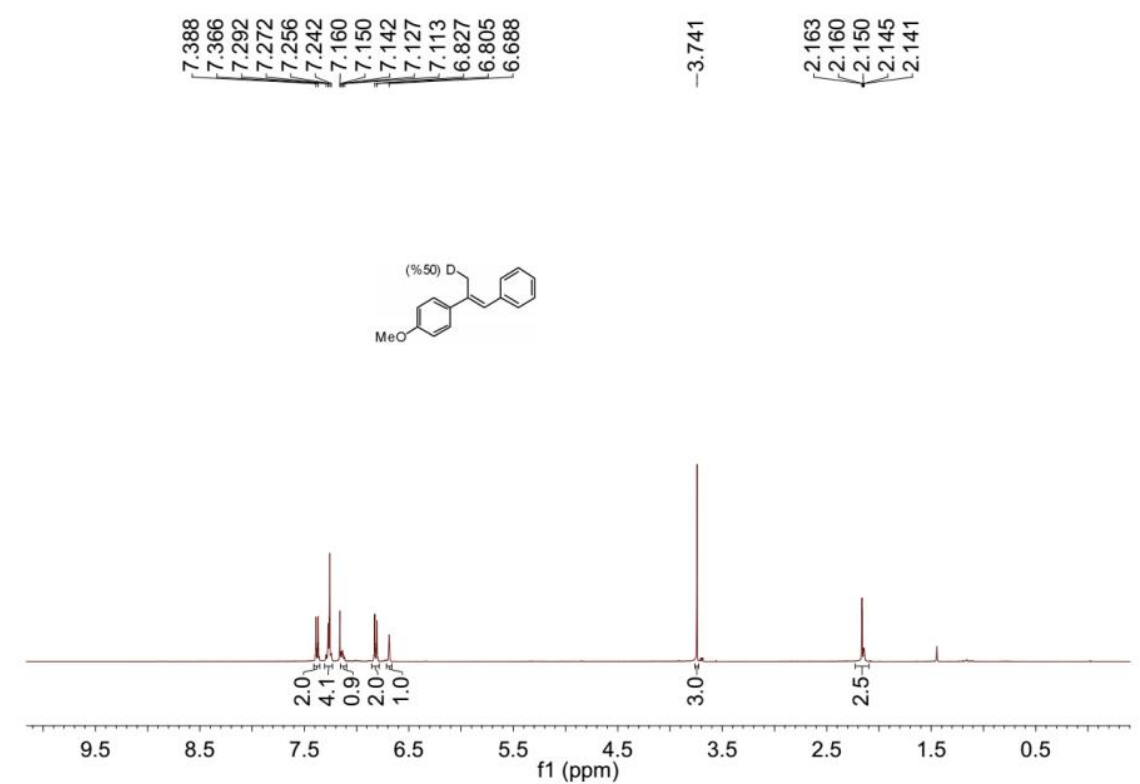

2s $E / Z$

$\infty$
0
0
0
0

必㧀

ن
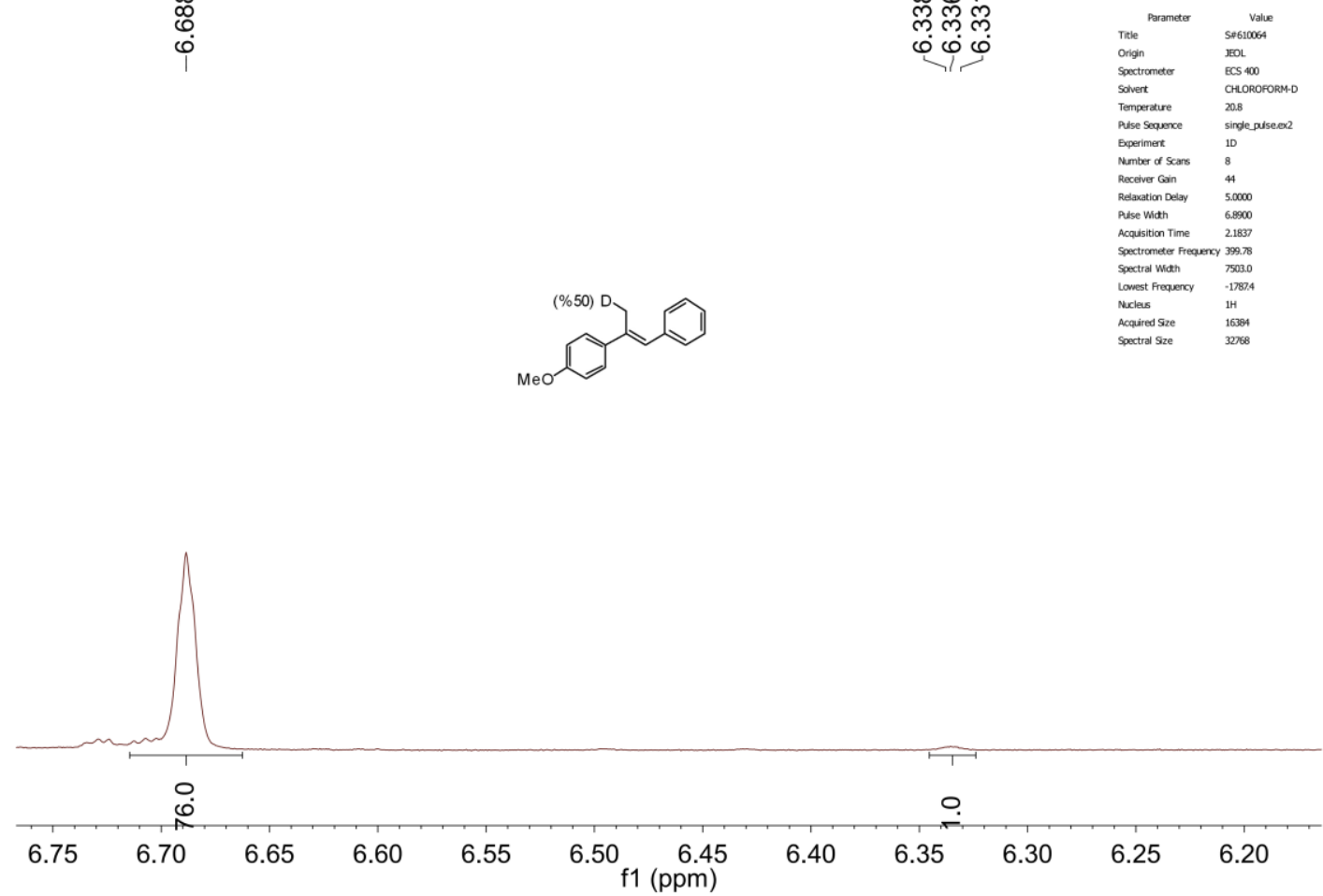
10.2 Radical trapping experiment

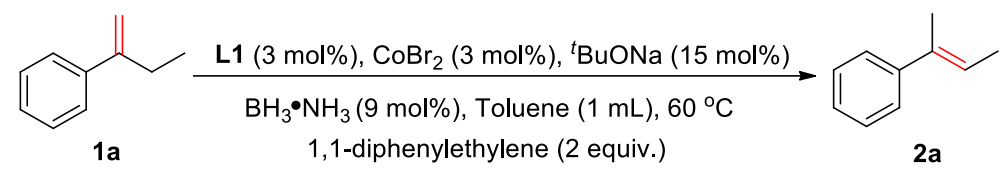

Inside an argon-atmosphere glovebox, a borosilicate glass vial was charged with $\mathrm{CoBr}_{2}$ (6.5 $\mathrm{mg}, 0.03 \mathrm{mmol}$ ) and $\mathbf{L} 1(13.2 \mathrm{mg}, 0.03 \mathrm{mmol}),{ }^{t} \mathrm{BuONa}(14.4 \mathrm{mg}, 0.15 \mathrm{mmol})$, with anhydrous toluene $(1 \mathrm{~mL})$ as solvent. The resulting mixture was stirred at room temperature for 3 hours to form a precatalyst. Subsequently, $\mathrm{BH}_{3} \cdot \mathrm{NH}_{3}(2.7 \mathrm{mg}, 0.09 \mathrm{mmol})$ was added and the mixture was stirred for 5 min before adding $\alpha$-ethylstyrene 1a (149 $\mu \mathrm{L}, 1 \mathrm{mmol}$ ) and 1,1-diphenylethylene (353 $\mu \mathrm{L}, 2 \mathrm{mmol})$. The reaction mixture was removed from the glovebox and stirred at $60^{\circ} \mathrm{C}$ under an argon atmosphere for 12 hours. The crude product can be obtained through a short pipette column with hexane as eluent. It can be used for $E / Z$ selectivity determination. The crude product was further purified by silica gel column chromatography (pentane used as eluent) affording 123 $\mathrm{mg}(93 \%$ yield, $E / Z=65 / 1)$ product $2 \mathrm{a}$ as colorless oil.

2a $E / Z$

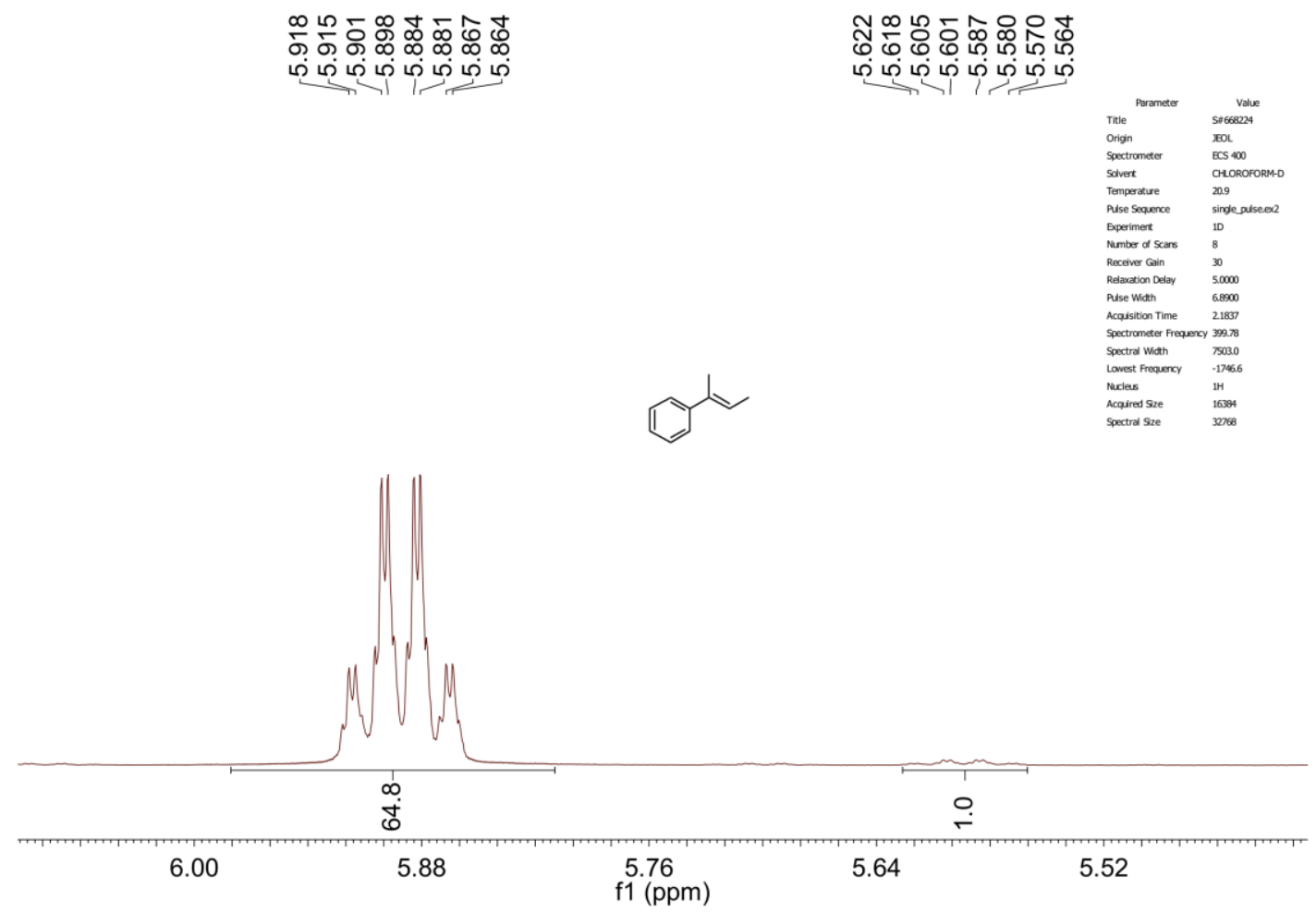

10.3 KIE study

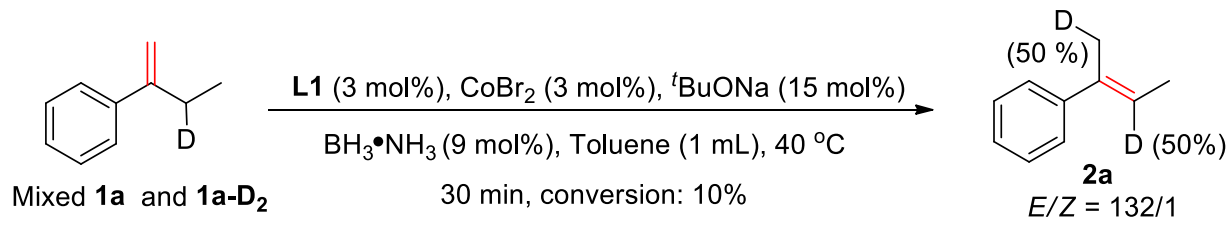

Inside an argon-atmosphere glovebox, a borosilicate glass vial was charged with $\mathrm{CoBr}_{2}$ (6.5 $\mathrm{mg}, 0.03 \mathrm{mmol}$ ) and $\mathbf{L} 1(13.2 \mathrm{mg}, 0.03 \mathrm{mmol}),{ }^{\mathrm{t}} \mathrm{BuONa}(14.4 \mathrm{mg}, 0.15 \mathrm{mmol})$, with anhydrous 
toluene $(1 \mathrm{~mL})$ as solvent. The resulting mixture was stirred at room temperature for 3 hours to form a precatalyst. Subsequently, $\mathrm{BH}_{3} \cdot \mathrm{NH}_{3}(2.7 \mathrm{mg}, 0.09 \mathrm{mmol})$ was added and the mixture was stirred for $5 \mathrm{~min}$ before adding $1 \mathrm{a}-\mathrm{D}_{\mathbf{2}}(83 \mu \mathrm{L}, 0.56 \mathrm{mmol}$ ) and $1 \mathrm{a}(66 \mu \mathrm{L}, 0.44 \mathrm{mmol})$ (to form 1a- $\left.\mathbf{D}_{1}\right)$. The reaction mixture was removed from the glovebox and stirred at $40{ }^{\circ} \mathrm{C}$ under an argon atmosphere for $30 \mathrm{~min}$. The crude product can be obtained through a short pipette column with hexane as eluent. It can be used for $E / Z$ selectivity determination. The crude product was further purified by silica gel column chromatography (pentane used as eluent) affording $12 \mathrm{mg}$ ( $9 \%$ yield, $E / Z=132 / 1)$ product $2 \mathrm{a}$ as colorless oil.

\section{2a ${ }^{1} \mathrm{H} N M R$}

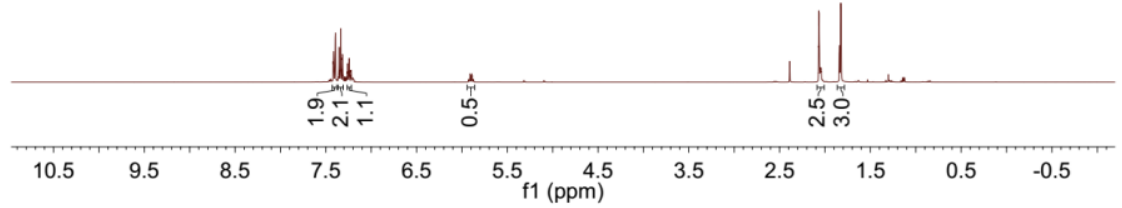

2a $E / Z$

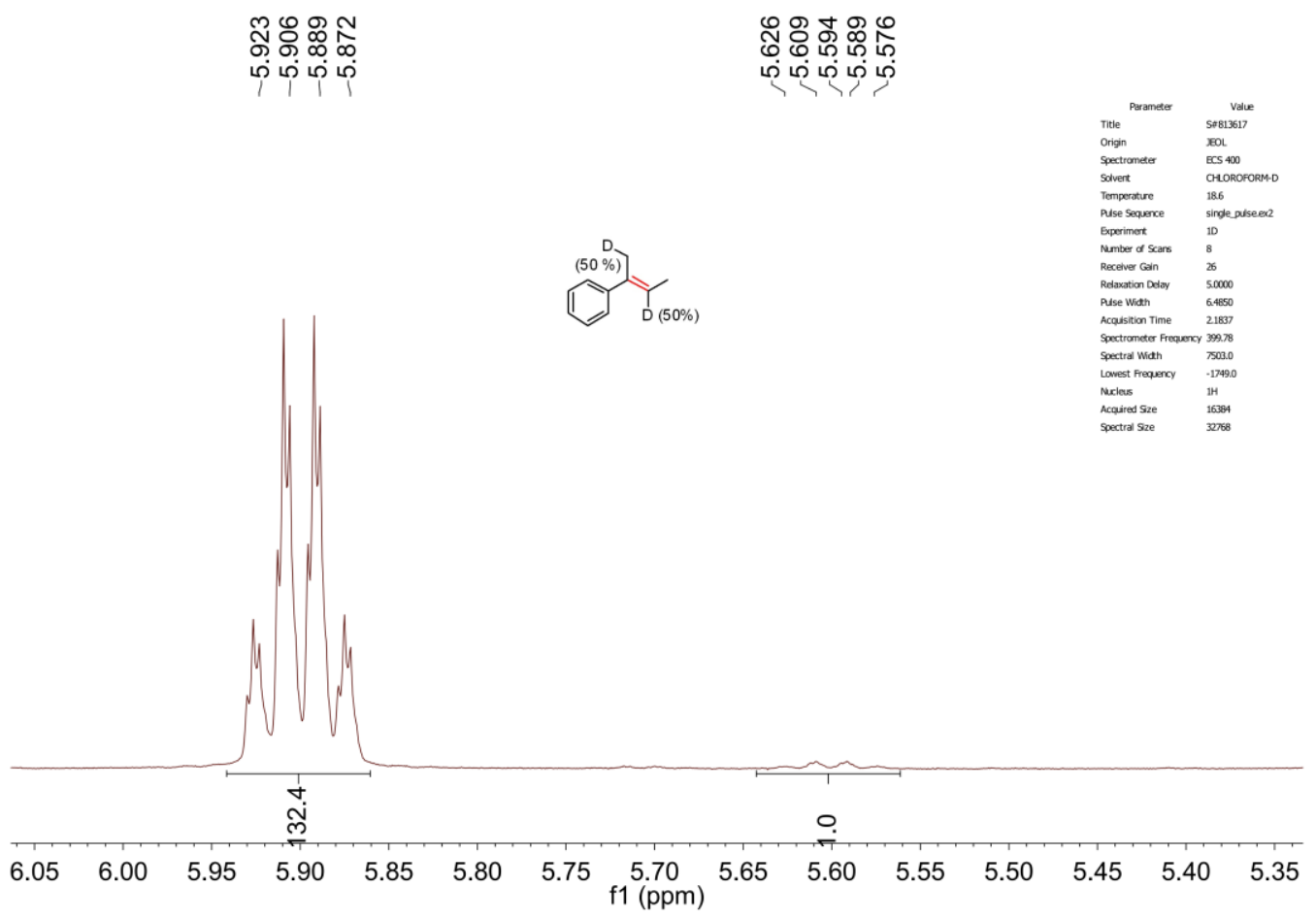


10.4 Steroselectivity origin study

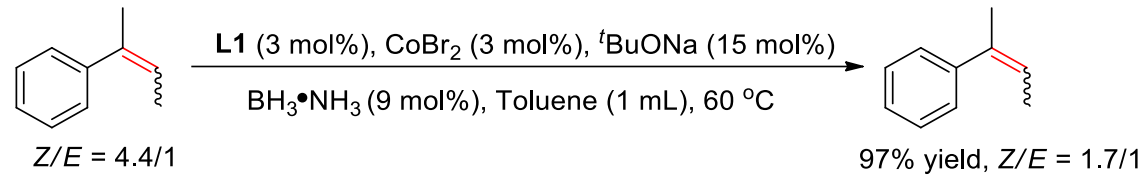

This reaction was carried out following the procedure Condition I. The spectra $(E / Z)$ of reactant and product were shown as below.

reactant $E / Z$

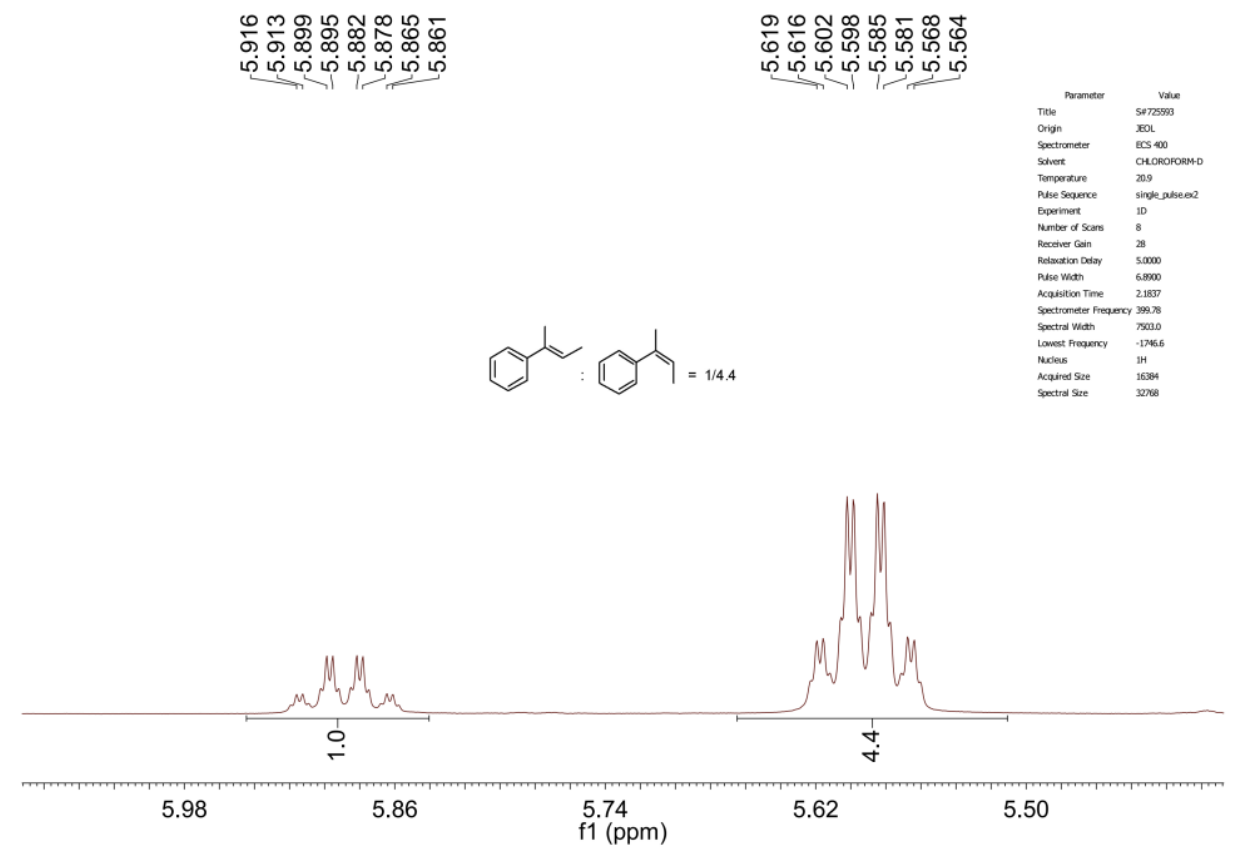

product $E / Z$

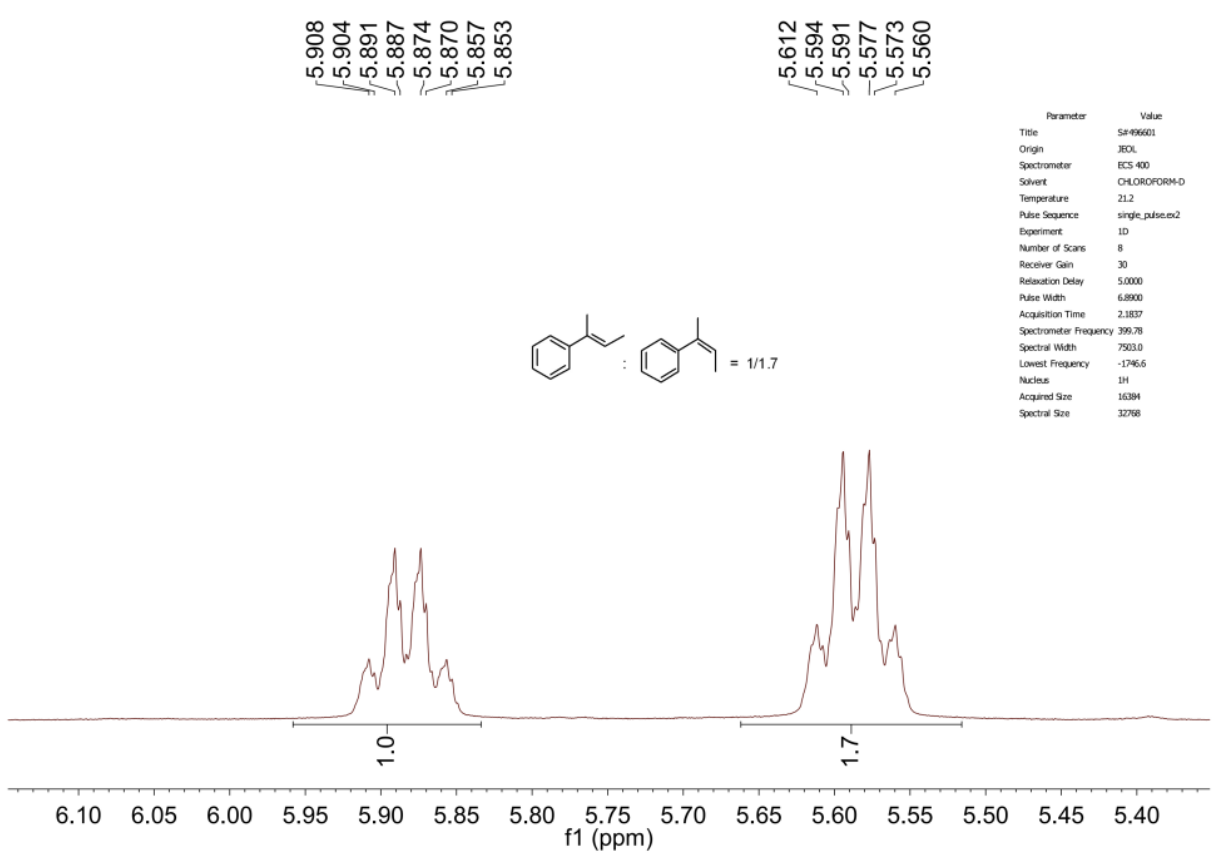




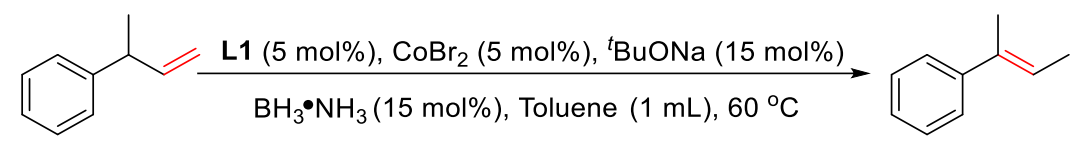

$97 \%$ yield, $E / Z=20 / 1$

This reaction was carried out following the procedure Condition IV. The spectra (E/Z) of product were shown as below.
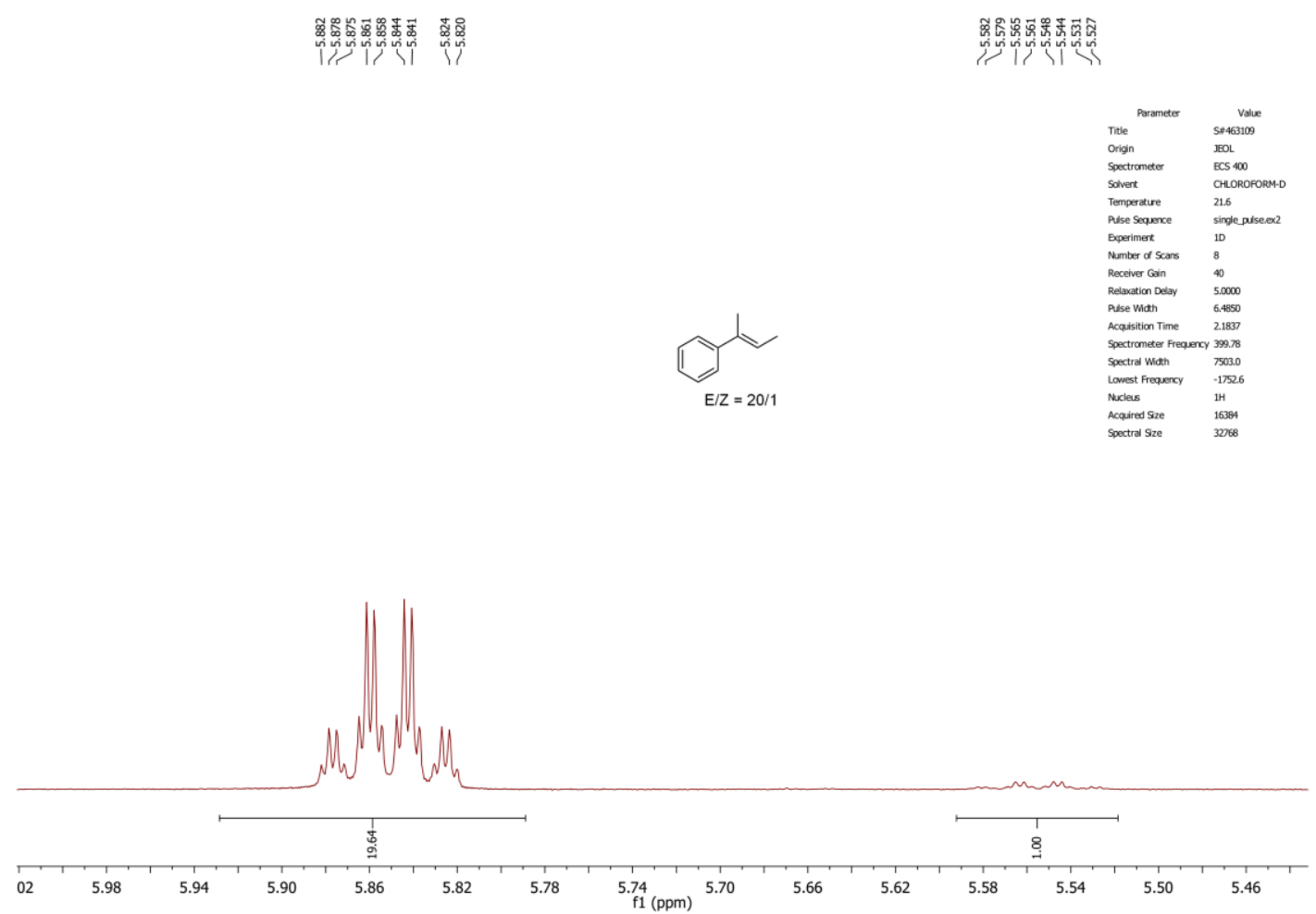
$10.5 \pi-\pi$ Stacking effect study

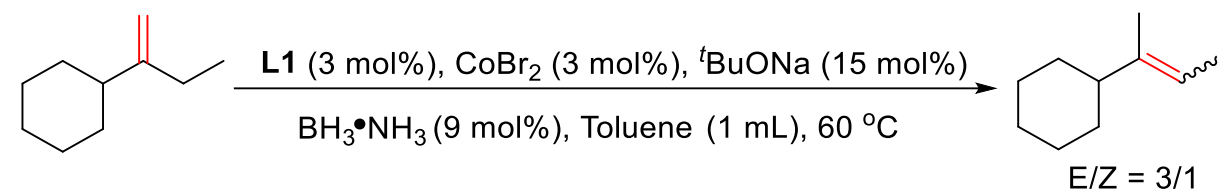

This reaction was carried out following the procedure Condition I. The spectra $(E / Z)$ of crude mixture of reaction were shown as below.
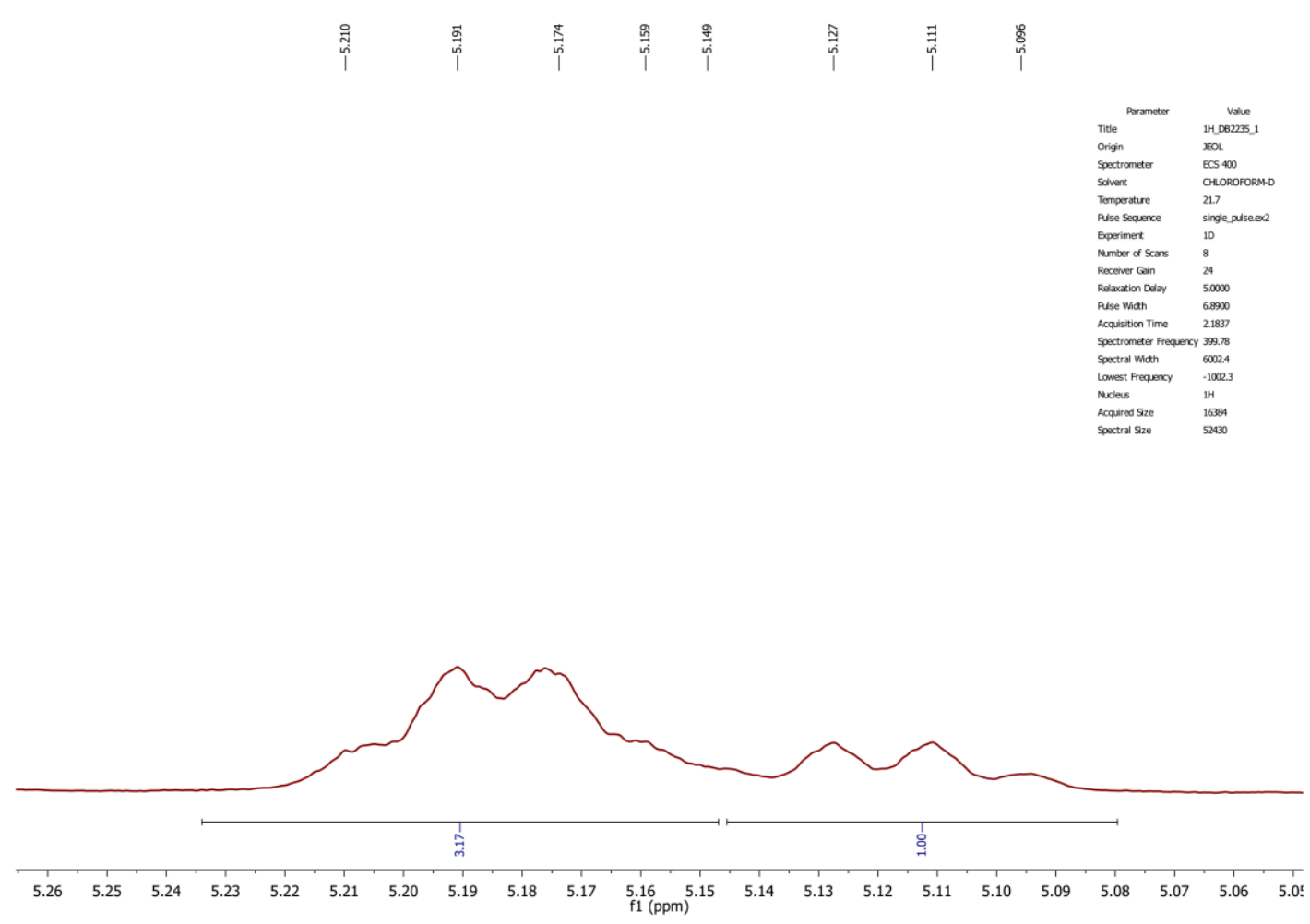


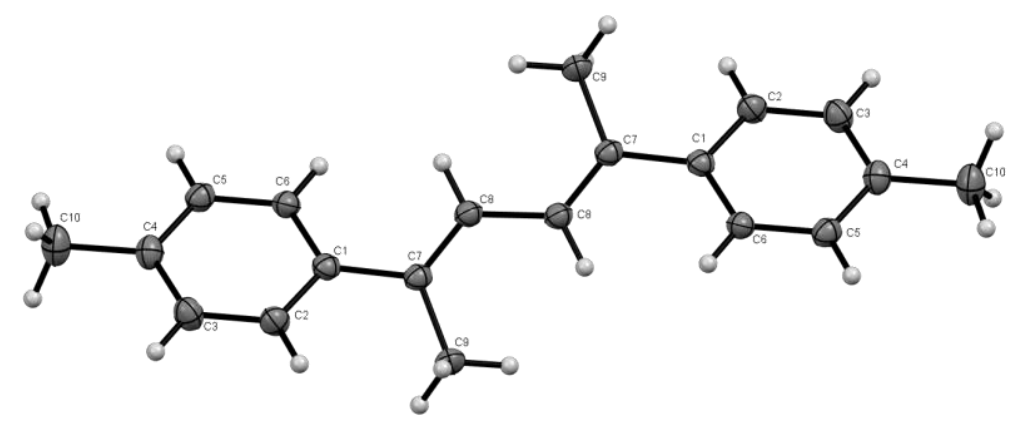

Figure S1. The thermal ellipsoids (2ao) are represented at 50\% probability. Carbon and hydrogen atoms are represented by gray and white ellipsoids, respectively. (The crystal was obtained from the solution of $2 \mathrm{ao}$ in dichloromethane)

Table S3. Crystal data and structure refinement for 2 ao.

\begin{tabular}{|c|c|}
\hline Identification code & fin19_14 \\
\hline Crystal color & colorless \\
\hline Crystal habit & irregular \\
\hline Empirical formula & $\mathrm{C}_{20} \mathrm{H}_{22}$ \\
\hline Formula weight & 262.37 \\
\hline Temperature & $100(2) \mathrm{K}$ \\
\hline Wavelength & $1.54178 \AA$ \\
\hline Crystal system & Monoclinic \\
\hline Space group & $P 2_{1} / c$ \\
\hline \multirow[t]{3}{*}{ Unit cell dimensions } & $a=13.3703(2) \AA \quad$ alpha $=90^{\circ}$. \\
\hline & $\mathrm{b}=6.66930(10) \AA \quad$ beta $=103.9530(10)^{\circ}$ \\
\hline & $\mathrm{c}=8.81190(10) \AA \quad$ gamma $=90^{\circ}$. \\
\hline Volume & $762.577(19) \AA^{3}$ \\
\hline z & 2 \\
\hline Calculated density & $1.143 \mathrm{Mg} / \mathrm{m}^{3}$ \\
\hline Absorption coefficient & $0.475 \mathrm{~mm}^{-1}$ \\
\hline Crystal size & $0.265 \times 0.155 \times 0.067 \mathrm{~mm}$ \\
\hline Theta range for data collection & 3.406 to $77.357^{\circ}$ \\
\hline Limiting indices & $-16<=\mathrm{h}<=16,-8<=\mathrm{k}<=8,-11<=\mathrm{k}<=10$ \\
\hline Reflections collected / unique & $13895 / 1600[R($ int $)=0.0357]$ \\
\hline Completeness to theta $=67.679^{\circ}$ & $100.0 \%$ \\
\hline Refinement method & Full-matrix least-squares on F2 \\
\hline Data / restraints / parameters & $1600 / 0 / 93$ \\
\hline Goodness-of-fit on $\mathrm{F}^{2}$ & 1.047 \\
\hline Final $R$ indices $[\mid>2 \operatorname{sigma}(I)]$ & $R 1=0.0430, w R 2=0.1230$ \\
\hline $\mathrm{R}$ indices (all data) & $R 1=0.0455, w R 2=0.1259$ \\
\hline Largest diff. peak and hole & .260 and -0.187 e. $\AA^{-3}$ \\
\hline
\end{tabular}


Precatalyst single crystal preparation:

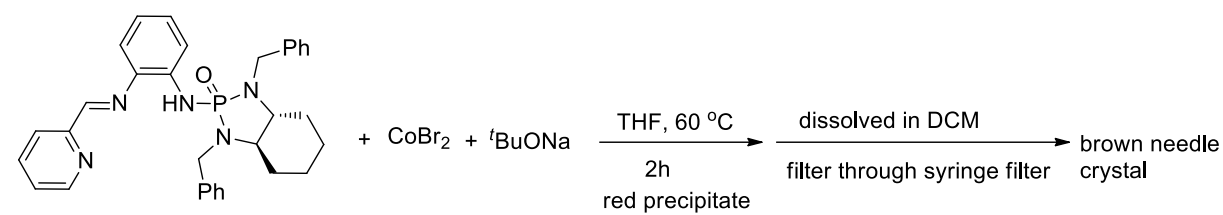

Inside an argon-atmosphere glovebox, a borosilicate glass vial was charged with $\mathrm{CoBr}_{2}$ (43.7 $\mathrm{mg}, 0.2 \mathrm{mmol}$ ) and $\mathbf{L} 3(107.1 \mathrm{mg}, 0.2 \mathrm{mmol}),{ }^{t} \mathrm{BuONa}(19.2 \mathrm{mg}, 0.2 \mathrm{mmol})$, with anhydrous THF $(1 \mathrm{~mL})$ as solvent. The reaction mixture was removed from the glovebox and stirred at $60^{\circ} \mathrm{C}$ under an argon atmosphere for 2 hours. The desired complex precipitated from the solution and it was obtained by filtration in the glovebox. The desired complex was dissolved in the DCM and filtered through a syringe filter to remove the sodium bromide. The complex DCM solution was covered with toluene. After two or three days, the complex crystals can be been collected from the solution.

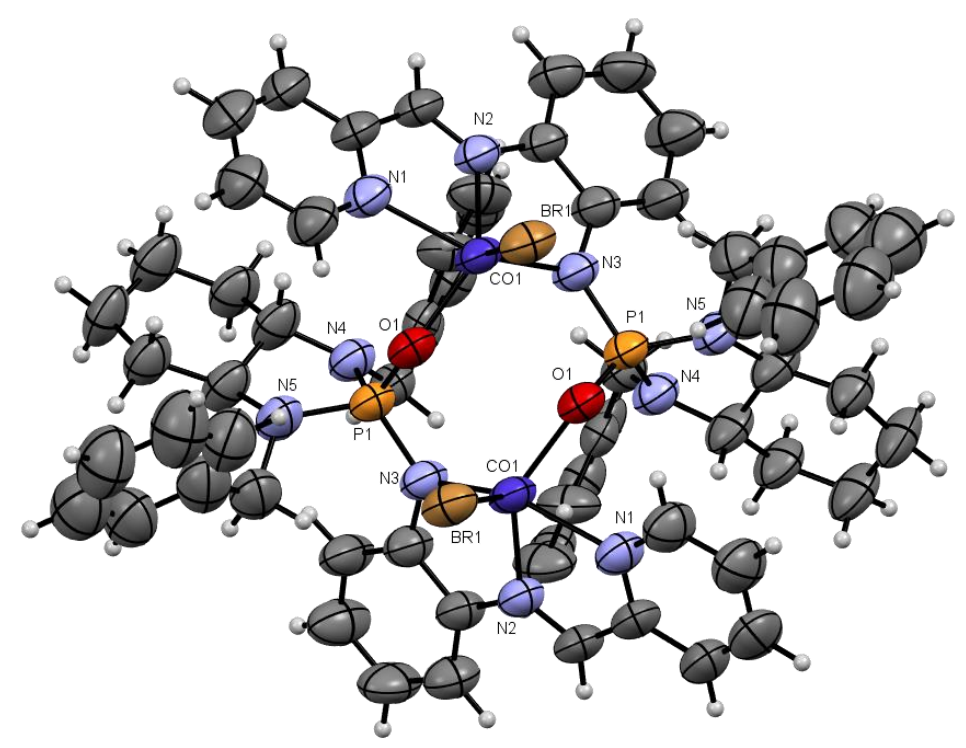

Figure S2. The thermal ellipsoids are represented at 50\% probability. Carbon, hydrogen, nitrogen, oxygen, phosphorus, bromine and cobalt atoms are represented by gray, white, light blue, red, light orange, orange and purple ellipsoids, respectively. The B sites of disordered atoms were omitted for clarity. 
Table S4. Crystal data and structure refinement for precatalyst.

\begin{tabular}{|c|c|}
\hline Identification code & fin19_21 \\
\hline Crystal color & red \\
\hline Crystal habit & needle \\
\hline Empirical formula & $\mathrm{C}_{64} \mathrm{H}_{66} \mathrm{Br}_{2} \mathrm{Co}_{2} \mathrm{~N}_{10} \mathrm{O}_{2} \mathrm{P}_{2}$ \\
\hline Formula weight & 1346.88 \\
\hline Temperature & $100(2) \mathrm{K}$ \\
\hline Wavelength & $1.54178 \AA$ \\
\hline Crystal system & Monoclinic \\
\hline Space group & $\mathrm{C} 2 / \mathrm{c}$ \\
\hline Unit cell dimensions & $\mathrm{a}=21.1593(7) \AA \quad$ alpha $=90^{\circ}$. \\
\hline & $\begin{array}{cc}b=21.8994(6) \AA & \text { beta }=112.926(3)^{\circ} . \\
c=20.9154(5) \AA & \text { gamma }=90^{\circ} .\end{array}$ \\
\hline Volume & 8926.1(5) $\AA^{3}$ \\
\hline Z & 4 \\
\hline Calculated density & $1.002 \mathrm{Mg} / \mathrm{m}^{3}$ \\
\hline bsorption coefficient & $4.584 \mathrm{~mm}^{-1}$ \\
\hline$F(000)$ & 2760 \\
\hline Crystal size & $0.469 \times 0.066 \times 0.058 \mathrm{~mm}$ \\
\hline range for data collection & 3.035 to $77.231^{\circ}$ \\
\hline Limiting indices & $-26<=\mathrm{h}<=26,-27<=\mathrm{k}<=27,-26<=\mathrm{k}<=19$ \\
\hline ctions collected / unique & $57024 / 9235[R($ int $)=0.0650]$ \\
\hline leteness to theta $=67.679^{\circ}$ & $99.8 \%$ \\
\hline Refinement method & Full-matrix least-squares on $\mathrm{F}^{2}$ \\
\hline / restraints / parameters & 9235 / 893 / 507 \\
\hline Goodness-of-fit on $\mathrm{F}^{2}$ & 1.093 \\
\hline al R indices [I>2sigma(I)] & $\mathrm{R} 1=0.0928, w R 2=0.2811$ \\
\hline $\mathrm{R}$ indices (all data) & $R 1=0.1068, w R 2=0.2950$ \\
\hline Extinction coefficient & $0.00091(10)$ \\
\hline gest diff. peak and hole & 0.992 and -0.428 e. $\AA^{-3}$ \\
\hline
\end{tabular}



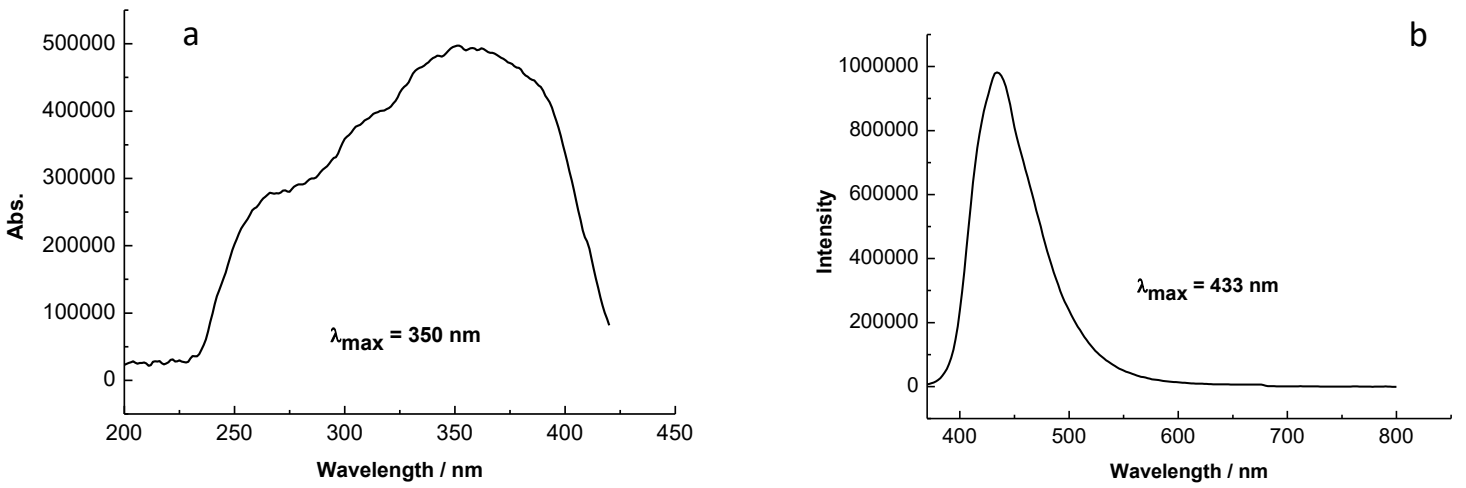

Figure S3. a) Photoluminescence absorption spectrum of $\mathbf{2 z}$ in solid powders b) Photoluminescence emission spectrum of $\mathbf{2 z}$ in solid powders upon excitation at $350 \mathrm{~nm}$

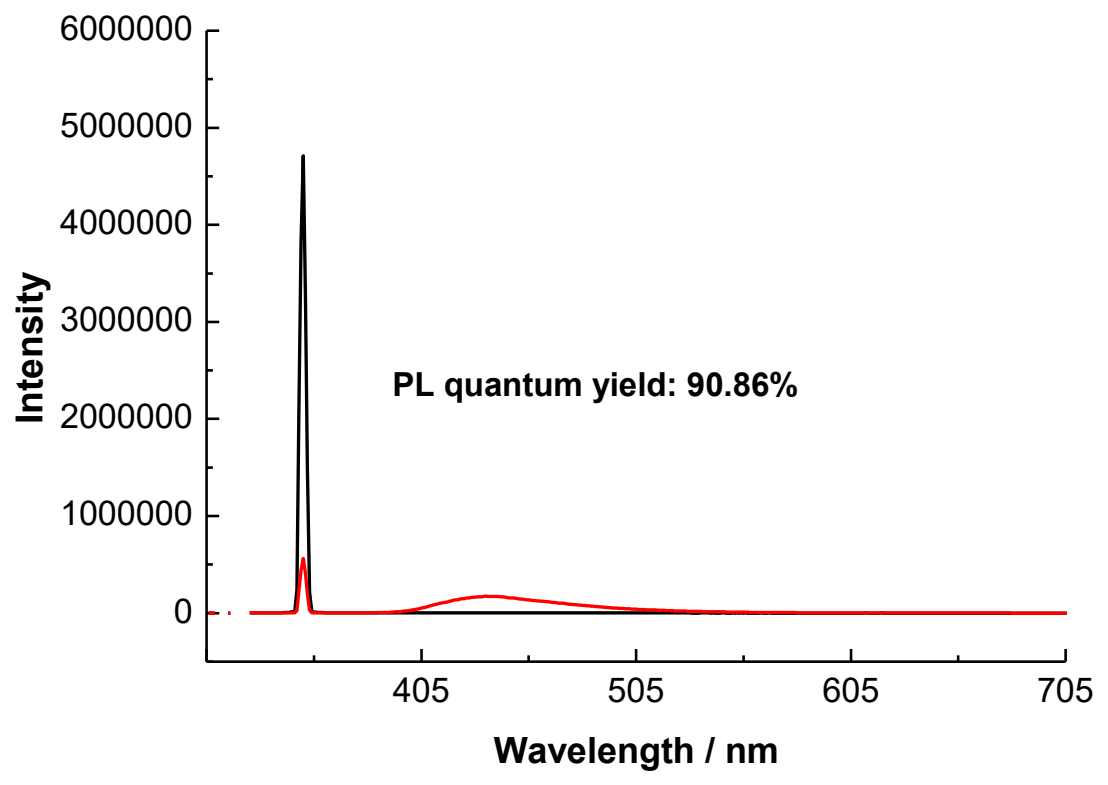

Figure S4. a) Photoluminescence quantum yield of $\mathbf{2 z}$ in solid powders upon excitation at $350 \mathrm{~nm}$ 

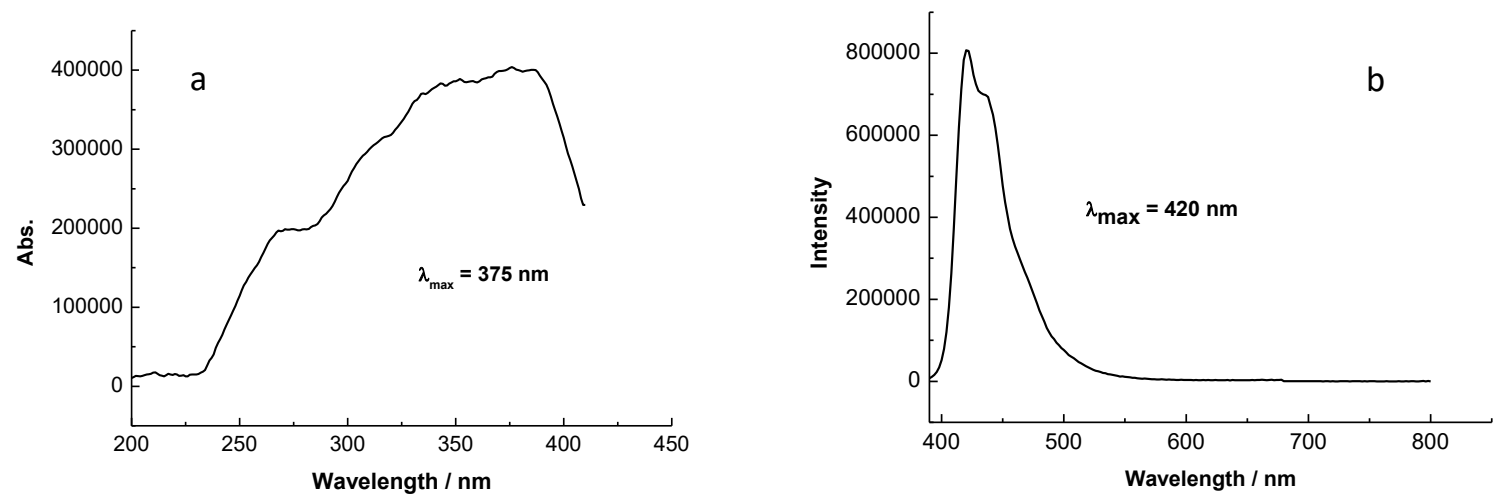

Figure S5. a) Photoluminescence absorption spectrum of 2 ao in solid powders b) Photoluminescence emission spectrum of 2 ao in solid powders upon excitation at $375 \mathrm{~nm}$

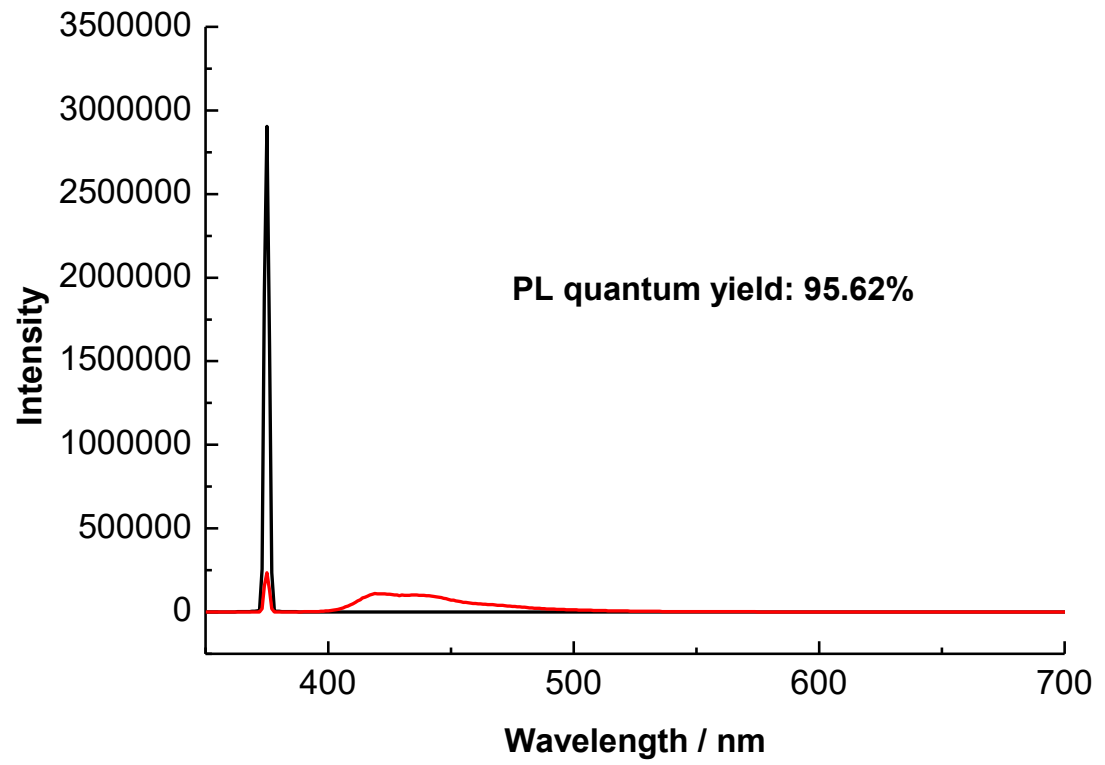

Figure S6. a) Photoluminescence quantum yield of 2 ao in solid powders upon excitation at 375 $\mathrm{nm}$ 

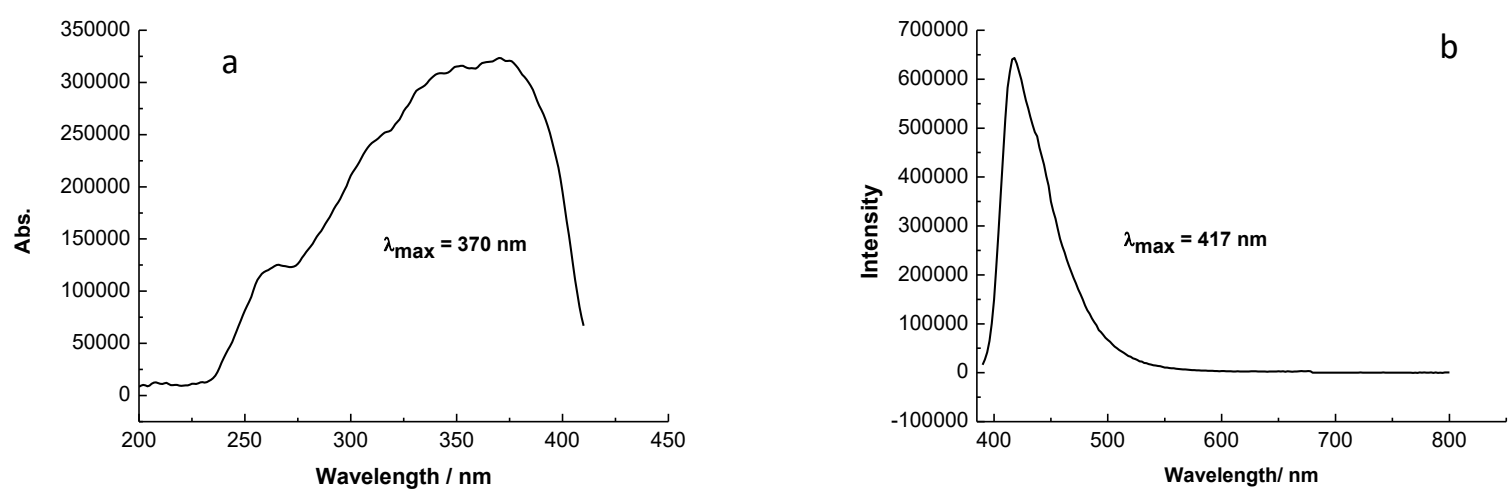

Figure S7. a) Photoluminescence absorption spectrum of 2 aq in solid powders b) Photoluminescence emission spectrum of 2 aq in solid powders upon excitation at $370 \mathrm{~nm}$

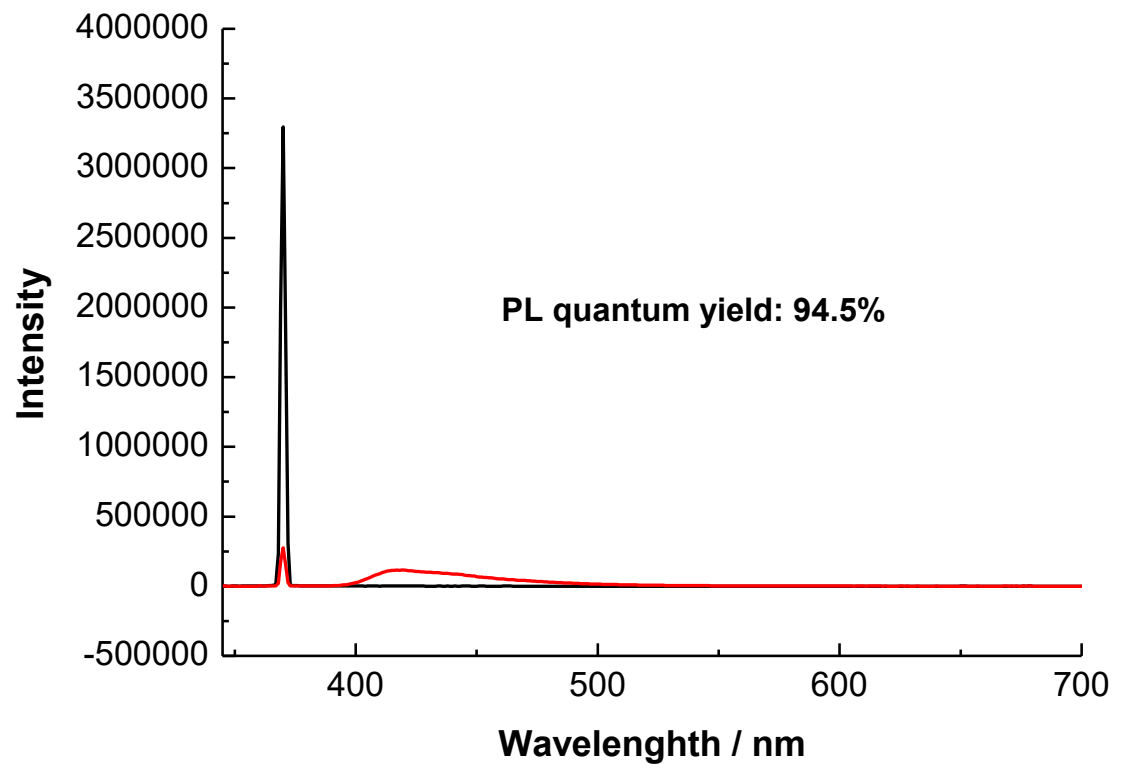

Figure 58. a) Photoluminescence quantum yield of 2 aq in solid powders upon excitation at 370 $\mathrm{nm}$ 


\section{Experimental data of alkene isomerization products}<smiles>C/C=C(\C)c1ccccc1</smiles>

(E)-But-2-en-2-ylbenzene (2a): Colorless oil; $126 \mathrm{mg}, 95 \%$ yield; $E / Z=107 / 1 ;{ }^{1} \mathrm{H}$ NMR (400 MHz, $\left.\mathrm{CDCl}_{3}\right)$ : $7.41(\mathrm{~m}, 2 \mathrm{H}), 7.34(\mathrm{~m}, 2 \mathrm{H}), 7.25(\mathrm{~m}, 1 \mathrm{H}), 5.90(\mathrm{~m}, 1 \mathrm{H}), 2.07(\mathrm{~m}, 3 \mathrm{H}), 1.84(\mathrm{~m}, 3 \mathrm{H}) ;{ }^{13} \mathrm{C} N M R$ $\left(100 \mathrm{MHz}, \mathrm{CDCl}_{3}\right): 144.0,135.5,128.1,126.4,125.5,122.4,15.5,14.3$. These data are in accordance with the literature. ${ }^{43}$<smiles>CC/C=C(\C)c1ccccc1</smiles>

(E)-Pent-2-en-2-ylbenzene(2b): Colorless oil; $117 \mathrm{mg}, 80 \%$ yield; $E / Z=28 / 1 ;{ }^{1} \mathrm{H}$ NMR (400 MHz, $\left.\mathrm{CDCl}_{3}\right)$ : $7.41(\mathrm{~m}, 2 \mathrm{H}), 7.33(\mathrm{~m}, 2 \mathrm{H}), 7.23(\mathrm{~m}, 1 \mathrm{H}), 5.80(\mathrm{~m}, 1 \mathrm{H}), 2.24(\mathrm{~m}, 2 \mathrm{H}), 2.06(\mathrm{~m}, 3 \mathrm{H}), 1.09(\mathrm{t}, \mathrm{J}$ $=7.4 \mathrm{~Hz}, 3 \mathrm{H}) ;{ }^{13} \mathrm{C} \mathrm{NMR}\left(100 \mathrm{MHz}, \mathrm{CDCl}_{3}\right): 143.9,134.0,130.3,128.1,126.4,125.6,22.0,15.6$, 14.1. These data are in accordance with the literature. ${ }^{44}$<smiles>CCC/C=C(\C)c1ccccc1</smiles>

(E)-Hex-2-en-2-ylbenzene (2c): Colorless oil; $134 \mathrm{mg}, 84 \%$ yield; $E / Z=38 / 1 ;{ }^{1} \mathrm{H} \mathrm{NMR}(400 \mathrm{MHz}$, $\left.\mathrm{CDCl}_{3}\right)$ : $7.42(\mathrm{~m}, 2 \mathrm{H}), 7.34(\mathrm{~m}, 2 \mathrm{H}), 7.24(\mathrm{~m}, 1 \mathrm{H}), 5.82(\mathrm{~m}, 1 \mathrm{H}), 2.21(\mathrm{q}, J=7.3 \mathrm{~Hz}, 2 \mathrm{H}), 2.07(\mathrm{~s}, 3 \mathrm{H})$, $1.52(\mathrm{~m}, 2 \mathrm{H}), 1.00(\mathrm{t}, J=7.4 \mathrm{~Hz}, 3 \mathrm{H}) ;{ }^{13} \mathrm{C}$ NMR $\left(100 \mathrm{MHz}, \mathrm{CDCl}_{3}\right): 144.0,134.6,128.6,128.1,126.4$, $125.6,30.8,22.8,15.8,13.9$. These data are in accordance with the literature. ${ }^{45}$<smiles>C/C(=C\Cc1ccccc1)c1ccccc1</smiles>

(E)-But-2-ene-1,3-diyldibenzene (2d): Colorless oil; $169 \mathrm{mg}, 81 \%$ yield; $E / Z=35 / 1 ;{ }^{1} \mathrm{H}$ NMR (400 $\left.\mathrm{MHz}, \mathrm{CDCl}_{3}\right): 7.49(\mathrm{~m}, 2 \mathrm{H}), 7.38(\mathrm{~m}, 4 \mathrm{H}), 7.29(\mathrm{~m}, 4 \mathrm{H}), 6.06(\mathrm{~m}, 1 \mathrm{H}), 3.65(\mathrm{~d}, J=7.2 \mathrm{~Hz}, 2 \mathrm{H}), 2.22$ (m, 3H); ${ }^{13} \mathrm{C}$ NMR $\left(100 \mathrm{MHz}, \mathrm{CDCl}_{3}\right): 143.6,141.0,135.6,128.45,128.40,128.2,126.71,126.69$, $125.9,125.7,34.9,15.9$. These data are in accordance with the literature. ${ }^{46}$<smiles>CC(=CC1CCCC1)c1ccc(C)cc1</smiles>

(E)-1-(1-cyclopentylprop-1-en-2-yl)-4-methylbenzene (2e): Colorless oil; $170 \mathrm{mg}, 85 \%$ yield; $E / Z=$ 130/1; ${ }^{1} \mathrm{H} \mathrm{NMR} \mathrm{(400} \mathrm{MHz,} \mathrm{CDCl} 3$ ): 7.32 (d, $J=8.4 \mathrm{~Hz}, 2 \mathrm{H}$ ), 7.14 (d, $J=8.4 \mathrm{~Hz}, 2 \mathrm{H}$ ), 5.72 (dd, $J=8.8$ $\mathrm{Hz}, J=1.2 \mathrm{~Hz}, 1 \mathrm{H}), 2.82(\mathrm{~m}, 1 \mathrm{H}), 2.36(\mathrm{~s}, 3 \mathrm{H}), 2.07(\mathrm{~d}, J=1.2 \mathrm{~Hz}, 3 \mathrm{H}), 1.90(\mathrm{~m}, 2 \mathrm{H}), 1.69(\mathrm{~m}, 4 \mathrm{H})$, $1.34(\mathrm{~m}, 2 \mathrm{H}) ;{ }^{13} \mathrm{C} \mathrm{NMR}\left(100 \mathrm{MHz}, \mathrm{CDCl}_{3}\right): 141.1,135.9,133.4,133.0,128.8,125.4,39.7,33.6,25.4$, 21.0, 15.9 ; IR (neat): 2949, 2865, 1684, 1513, 1449, 1266, 811; GC-MS: $t_{R}=9.76 \mathrm{~min}, \mathrm{~m} / \mathrm{z}=200.2$.<smiles>CC(=CC1CC2CCC1C2)c1ccc(C)cc1</smiles>

2-((E)-2-(p-Tolyl)prop-1-en-1-yl)bicyclo[2.2.1] heptanes (2f): Colorless oil; $199 \mathrm{mg}, 88 \%$ yield, $E / Z=$ 15/1; ${ }^{1} \mathrm{H} \mathrm{NMR}\left(400 \mathrm{MHz}, \mathrm{CDCl}_{3}\right): 7.28(\mathrm{~d}, J=8.4 \mathrm{~Hz}, 2 \mathrm{H}), 7.11(\mathrm{~d}, J=8.0 \mathrm{~Hz}, 2 \mathrm{H}), 5.66(\mathrm{~m}, 1 \mathrm{H}), 2.41$ $(\mathrm{m}, 1 \mathrm{H}), 2.34(\mathrm{~s}, 3 \mathrm{H}), 2.27(\mathrm{br}, 1 \mathrm{H}), 2.07(\mathrm{br}, 1 \mathrm{H}), 2.02(\mathrm{~d}, J=1.2 \mathrm{~Hz}, 3 \mathrm{H}), 1.65(\mathrm{~m}, 1 \mathrm{H}), 1.53(\mathrm{~m}, 2 \mathrm{H})$, 
$1.45(\mathrm{~m}, 1 \mathrm{H}), 1.28(\mathrm{~m}, 2 \mathrm{H}), 1.19(\mathrm{~m}, 2 \mathrm{H}) ;{ }^{13} \mathrm{C} \mathrm{NMR}\left(100 \mathrm{MHz}, \mathrm{CDCl}_{3}\right): 141.1,136.0,134.8,132.3$, 128.8, 125.4, 43.1, 41.3, 39.5, 36.5, 36.1, 29.7, 29.0, 21.0, 16.1; IR (neat): 2946, 2866, 1684, 1513, $1455,1266,1064,809 ;$ GC-MS: $t_{R}=9,94 \mathrm{~min}, \mathrm{~m} / \mathrm{z}=226.2$.

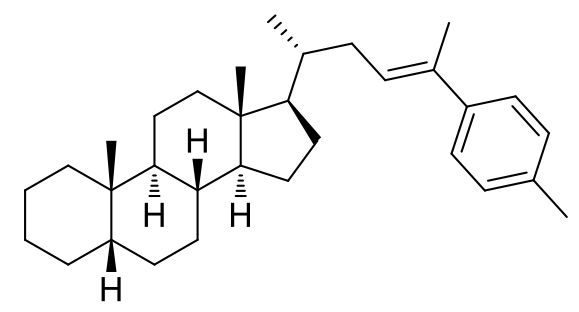

$(5 S, 8 R, 9 S, 10 S, 13 R, 14 S, 17 R)-10,13-D i m e t h y l-17-((R, E)$-5-( $p$-tolyl)hex-4-en-2-yl)hexadecahydro-1Hcyclopenta[a]phenanthrene (2g): Colorless oil; $193 \mathrm{mg}, 89 \%$ yield (0.5 mmol scale); $E / Z=24 / 1 ;{ }^{1} \mathrm{H}$ NMR (400 MHz, CDCl $): 7.30(\mathrm{~d}, J=8.0 \mathrm{~Hz}, 2 \mathrm{H}), 7.13(\mathrm{~d}, J=8.0 \mathrm{~Hz}, 2 \mathrm{H}), 5.78(\mathrm{~m}, 1 \mathrm{H}), 2.35(\mathrm{~s}, 3 \mathrm{H})$, $2.02(\mathrm{~s}, 3 \mathrm{H}), 1.92(\mathrm{~m}, 2 \mathrm{H}), 1.76(\mathrm{~m}, 3 \mathrm{H}), 1.58(\mathrm{~m}, 2 \mathrm{H}), 1.40(\mathrm{~m}, 7 \mathrm{H}), 1.24(\mathrm{~m}, 9 \mathrm{H}), 1.09(\mathrm{~m}, 3 \mathrm{H})$, $0.97(\mathrm{~d}, J=6.8 \mathrm{~Hz}, 3 \mathrm{H}), 0.93(\mathrm{~s}, 3 \mathrm{H}), 0.89(\mathrm{~m}, 2 \mathrm{H}), 0.68(\mathrm{~s}, 3 \mathrm{H}) ;{ }^{13} \mathrm{C} \mathrm{NMR}\left(100 \mathrm{MHz}, \mathrm{CDCl}_{3}\right): 141.5$, 136.1, 135.0, 128.9, 126.8, 125.6, 56.7, 56.5, 43.8, 42.9, 40.6, 40.3, 37.7, 37.0, 36.0, 35.5, 35.4, 28.6, 27.6, 27.4, 27.1, 26.7, 24.45, 24.40, 21.5, 21.1, 20.9, 19.1, 16.1, 12.2. IR (neat): 2921, 2858, 1448, 1375, 810, 520; HRMS: calcd. for $\mathrm{C}_{32} \mathrm{H}_{48}[\mathrm{M}]^{+} 432.3751$, found: 432.3747 .<smiles>CC=C(C)c1ccc(C)cc1</smiles>

(E)-1-(But-2-en-2-yl)-4-methylbenzene (2h): Colorless oil; $130 \mathrm{mg}, 89 \%$ yield; $E / Z=25 / 1 ;{ }^{1} \mathrm{H}$ NMR (400 MHz, $\mathrm{CDCl}_{3}$ ): 7.30 (d, J = 8.4 Hz, 2H), $7.15(\mathrm{~d}, J=8.4 \mathrm{~Hz}, 2 \mathrm{H}), 5.87(\mathrm{~m}, 1 \mathrm{H}), 2.37(\mathrm{~s}, 3 \mathrm{H}), 2.05$ $(\mathrm{m}, 3 \mathrm{H}), 1.82(\mathrm{~d}, J=6.8 \mathrm{~Hz}, 3 \mathrm{H}) ;{ }^{13} \mathrm{C} \mathrm{NMR}\left(100 \mathrm{MHz}, \mathrm{CDCl}_{3}\right): 141.1,136.0,135.2,128.8,125.4$, $121.6,21.0,15.4,14.3$; IR (neat): 2918, 2859, 1511, 1448, 1378, 801, 780; GC-MS: $t_{R}=7.82 \mathrm{~min}$, $\mathrm{m} / \mathrm{z}=146.1$.<smiles>C/C=C(\C)c1ccc(OC)cc1</smiles>

(E)-1-(but-2-en-2-yl)-4-methoxybenzene (2i): Colorless oil; $141 \mathrm{mg}, 87 \%$ yield; $E / Z=20 / 1 ;{ }^{1} \mathrm{H}$ NMR (400 MHz, CDCl $)$ : 7.33 (d, $J=8.8 \mathrm{~Hz}, 2 \mathrm{H}), 6.87(\mathrm{~d}, J=8.8 \mathrm{~Hz}, 2 \mathrm{H}), 5.81(\mathrm{~m}, 1 \mathrm{H}), 3.82(\mathrm{~s}, 3 \mathrm{H}), 2.03$ $(\mathrm{t}, \mathrm{J}=1.2 \mathrm{~Hz}, 3 \mathrm{H}), 1.81(\mathrm{~m}, 3 \mathrm{H}) ;{ }^{13} \mathrm{C}$ NMR $\left(100 \mathrm{MHz}, \mathrm{CDCl}_{3}\right): 158.3,136.6,134.8,126.5,120.8$, $113.5,55.2,15.5,14.2$. These data are in accordance with the literature. ${ }^{47}$<smiles>CC=C(C)c1ccc(F)cc1</smiles>

(E)-1-(but-2-en-2-yl)-4-fluorobenzene (2j): Colorless oil; $125 \mathrm{mg}, 83 \%$ yield; $E / Z=21 / 1 ;{ }^{1} \mathrm{H} N M R$ $\left(400 \mathrm{MHz}, \mathrm{CDCl}_{3}\right): 7.32$ (dd, $\left.J=5.6 \mathrm{~Hz}, J=8.8 \mathrm{~Hz}, 2 \mathrm{H}\right), 7.99(\mathrm{t}, J=8.8 \mathrm{~Hz}, 2 \mathrm{H}), 5.80(\mathrm{~m}, 1 \mathrm{H}), 2.01(\mathrm{t}$, $J=1.2 \mathrm{~Hz}, 3 \mathrm{H}), 1.79(\mathrm{~m}, 3 \mathrm{H}) ;{ }^{13} \mathrm{C} \mathrm{NMR}\left(100 \mathrm{MHz}, \mathrm{CDCl}_{3}\right): 161.7\left(\mathrm{~d}, \mathrm{~J}_{F-C}=243.5 \mathrm{~Hz}\right), 140.1\left(\mathrm{~d}, J_{F-C}=\right.$ $3.3 \mathrm{~Hz}$ ), 134.5, $126.9\left(\mathrm{~d}, J_{F-C}=7.8 \mathrm{~Hz}\right), 122.3\left(\mathrm{~d}, J_{F-C}=1.3 \mathrm{~Hz}\right), 114.8\left(\mathrm{~d}, J_{F-C}=21.0 \mathrm{~Hz}\right), 15.6,14.3$; ${ }^{19} \mathrm{~F}$ NMR (376 MHz, CDCl$)_{3}$ : -117.6; IR (neat): 2970, 2918, 1602, 1507, 1222, 1159, 813, 554; GC-MS: $t_{R}=7.24 \mathrm{~min}, \mathrm{~m} / \mathrm{z}=150.1$. 
<smiles>C/C=C(\C)c1cccc(F)c1F</smiles>

(E)-1-(but-2-en-2-yl)-2,3-difluorobenzene (2k): Colorless oil; $143 \mathrm{mg}, 85 \%$ yield; $E / Z=76 / 1 ;{ }^{1} \mathrm{H}$ NMR (400 MHz, CDCl $): 6.98(\mathrm{~m}, 3 \mathrm{H}), 5.71(\mathrm{~m}, 1 \mathrm{H}), 2.00(\mathrm{q}, J=1.2 \mathrm{~Hz}, 3 \mathrm{H}), 1.79(\mathrm{~m}, 3 \mathrm{H}) ;{ }^{13} \mathrm{C} \mathrm{NMR}$ $\left(100 \mathrm{MHz}, \mathrm{CDCl}_{3}\right): 150.8\left(\mathrm{dd}, \mathrm{J}_{F-C}=245.6 \mathrm{~Hz}, \mathrm{~J}_{F-C}=13.7 \mathrm{~Hz}\right), 147.9\left(\mathrm{dd}, \mathrm{J}_{F-C}=246.6 \mathrm{~Hz}, \mathrm{~J}_{F-C}=12.6 \mathrm{~Hz}\right.$ ), $135.0\left(\mathrm{~d}, J_{F-C}=10.9 \mathrm{~Hz}\right), 131.0\left(\mathrm{~d}, J_{F-C}=3.6 \mathrm{~Hz}\right), 126.8\left(\mathrm{~d}, J_{F-C}=2.4 \mathrm{~Hz}\right), 124.2\left(\mathrm{t}, J_{F-C}=3.3 \mathrm{~Hz}\right), 123.5$ $\left(\mathrm{dd}, J_{F-C}=4.7 \mathrm{~Hz}, J_{F-C}=7.2 \mathrm{~Hz}\right), 115.0\left(\mathrm{~d}, J_{F-C}=17.3 \mathrm{~Hz}\right), 16.5\left(\mathrm{~d}, J_{F-C}=3.4 \mathrm{~Hz}\right), 14.0 ;{ }^{19} \mathrm{~F}$ NMR $(376$ $\mathrm{MHz}, \mathrm{CDCl}_{3}$ ): -139.1, -141.9; IR (neat): 2979, 1622, 1585, 1473, 1259, 1216, 778, 723; GC-MS: $t_{R}=$ $7.11 \mathrm{~min}, \mathrm{~m} / \mathrm{z}=169.1$.<smiles>CC=C(C)c1ccc(Cl)cc1</smiles>

(E)-1-(but-2-en-2-yl)-4-chlorobenzene (2I): Colorless oil; $155 \mathrm{mg}, 93 \%$ yield; $E / Z=18 / 1 ;{ }^{1} \mathrm{H}$ NMR $\left(400 \mathrm{MHz}, \mathrm{CDCl}_{3}\right): 7.31(\mathrm{~d}, J=8.8 \mathrm{~Hz}, 2 \mathrm{H}), 7.27(\mathrm{~d}, J=8.8 \mathrm{~Hz}, 2 \mathrm{H}), 5.87(\mathrm{~m}, 1 \mathrm{H}), 2.02(\mathrm{t}, J=1.2 \mathrm{~Hz}$, $3 \mathrm{H}), 1.81(\mathrm{~m}, 3 \mathrm{H}) ;{ }^{13} \mathrm{C} \mathrm{NMR}\left(100 \mathrm{MHz}, \mathrm{CDCl}_{3}\right): 142.4,134.4,132.0,128.2,126.8,123.0,15.4,14.3$; These data are in accordance with the literature. ${ }^{48}$<smiles>CC=C(C)c1cccc(Cl)c1</smiles>

(E)-1-(But-2-en-2-yl)-4-chlorobenzene (2m): Colorless oil; $160 \mathrm{mg}$, 96\% yield; $E / Z=79 / 1 ;{ }^{1} \mathrm{H}$ NMR (400 MHz, $\left.\mathrm{CDCl}_{3}\right)$ : $7.36(\mathrm{~m}, 1 \mathrm{H}), 7.22(\mathrm{~m}, 3 \mathrm{H}), 5.90(\mathrm{~m}, 1 \mathrm{H}), 2.02(\mathrm{~m}, 3 \mathrm{H}), 1.82(\mathrm{dd}, J=6.8 \mathrm{~Hz}, J=$ $0.8 \mathrm{~Hz}, 3 \mathrm{H}) ;{ }^{13} \mathrm{C}$ NMR $\left(100 \mathrm{MHz}, \mathrm{CDCl}_{3}\right): 145.8,134.4,134.0,129.3,126.3,125.7,123.7,123.6$, $15.3,14.3$; IR (neat): 2980, 2917, 1691, 1591, 1563, 1421, 1249, 1081, 776, 689; GC-MS: $t_{R}=7.9$ $\min , \mathrm{m} / \mathrm{z}=166.1$.<smiles>CC=C(C)c1ccc(Br)cc1</smiles>

(E)-1-Bromo-4-(but-2-en-2-yl)benzene (2n): Colorless oil; $203 \mathrm{mg}$, 96\% yield, $E / Z=14 / 1 ;{ }^{1} \mathrm{H}$ NMR $\left(400 \mathrm{MHz}, \mathrm{CDCl}_{3}\right): 7.42(\mathrm{~d}, J=8.8 \mathrm{~Hz}, 2 \mathrm{H}), 7.23(\mathrm{~d}, J=8.8 \mathrm{~Hz}, 2 \mathrm{H}), 5.86(\mathrm{~m}, 1 \mathrm{H}), 2.00(\mathrm{t}, J=1.2 \mathrm{~Hz}$, 3H), 1.79 (m, 3H); $\left.{ }^{13} \mathrm{C} \mathrm{NMR} \mathrm{(100} \mathrm{MHz,} \mathrm{CDCl}\right)$ : 142.8, 134.5, 131.1, 127.1, 123.1, 120.1, 15.3, 14.4; IR (neat): 2980, 2916, 1686, 1587, 1487, 1395, 1263, 1076, 1007, 806; GC-MS: $t_{R}=8.71 \mathrm{~min}, \mathrm{~m} / \mathrm{z}$ $=210.0$.<smiles>C/C(=C\c1ccccc1)c1ccccc1</smiles>

(E)-Prop-1-ene-1,2-diyldibenzene (2o): White solid; $188 \mathrm{mg}, 97 \%$ yield; $E / Z=61 / 1 ;{ }^{1} \mathrm{H}$ NMR (400 
$\left.\mathrm{MHz}, \mathrm{CDCl}_{3}\right): 7.53(\mathrm{~m}, 2 \mathrm{H}), 7.38(\mathrm{~m}, 6 \mathrm{H}), 7.27(\mathrm{~m}, 2 \mathrm{H}), 6.85(\mathrm{q}, J=1.2 \mathrm{~Hz}, 1 \mathrm{H}), 2.29(\mathrm{~d}, J=1.2 \mathrm{~Hz}$, $3 \mathrm{H}) ;{ }^{13} \mathrm{C}$ NMR $\left(100 \mathrm{MHz}, \mathrm{CDCl}_{3}\right): 143.9,138.3,137.4,129.1,128.3,128.2,127.7,127.2,126.4$, 126.0, 17.5. These data are in accordance with the literature. ${ }^{49}$<smiles>C/C(=C\c1ccccc1)c1ccc(C)cc1</smiles>

(E)-1-Methyl-4-(1-phenylprop-1-en-2-yl)benzene (2p): White solid; $200 \mathrm{mg}$, 96\% yield; $E / Z=72 / 1$; ${ }^{1} \mathrm{H}$ NMR $\left(400 \mathrm{MHz}, \mathrm{CDCl}_{3}\right): 7.45(\mathrm{~d}, J=8.4 \mathrm{~Hz}, 2 \mathrm{H}), 7.39(\mathrm{~m}, 4 \mathrm{H}), 7.26(\mathrm{~m}, 1 \mathrm{H}), 7.20(\mathrm{~d}, J=8.0 \mathrm{~Hz}$, $2 \mathrm{H}), 6.84(\mathrm{q}, J=1.2 \mathrm{~Hz}, 1 \mathrm{H}), 2.39(\mathrm{~s}, 3 \mathrm{H}), 2.29(\mathrm{~d}, J=1.2 \mathrm{~Hz}, 3 \mathrm{H}) ;{ }^{13} \mathrm{C} \mathrm{NMR}\left(100 \mathrm{MHz}, \mathrm{CDCl}_{3}\right)$ : $141.0,138.5,137.2,136.9,129.1,129.0,128.1,126.9,126.3,125.8,21.1,17.4$; These data are in accordance with the literature. ${ }^{49}$<smiles>CC(=Cc1ccccc1)c1ccc(C(C)(C)C)cc1</smiles>

(E)-1-(tert-butyl)-4-(1-phenylprop-1-en-2-yl)benzene (2q): White solid, m.p. 88-90 ${ }^{\circ} \mathrm{C} ; 215 \mathrm{mg}, 86 \%$ yield; $E / Z=78 / 1 ;{ }^{1} \mathrm{H} \mathrm{NMR}\left(400 \mathrm{MHz}, \mathrm{CDCl}_{3}\right): 7.52(\mathrm{~d}, J=8.4 \mathrm{~Hz}, 2 \mathrm{H}), 7.44(\mathrm{~d}, J=8.0 \mathrm{~Hz}, 2 \mathrm{H}), 7.40$ $(\mathrm{m}, 4 \mathrm{H}), 7.27(\mathrm{~m}, 1 \mathrm{H}), 6.88(\mathrm{~s}, 1 \mathrm{H}), 2.32(\mathrm{~s}, 3 \mathrm{H}), 1.38(\mathrm{~s}, 9 \mathrm{H}) ;{ }^{13} \mathrm{C} \mathrm{NMR}\left(100 \mathrm{MHz}, \mathrm{CDCl}_{3}\right): 150.2$, 140.9, 138.5, 137.1, 129.1, 128.1, 127.1, 126.3, 125.6, 125.2, 34.5, 31.3, 17.4; IR (neat): 2948, $2899,2863,1507,1362,1269,1113,840,819,746,700 ;$ GC-MS: $t_{R}=11.20 \mathrm{~min}, \mathrm{~m} / \mathrm{z}=250.2$.<smiles>CC(=Cc1ccccc1)c1ccc(-c2ccccc2)cc1</smiles>

(E)-4-(1-Phenylprop-1-en-2-yl)-1,1'-biphenyl (2r): White solid; $246 \mathrm{mg}, 91 \%$ yield; $E / Z=49 / 1 ;{ }^{1} \mathrm{H}$ NMR (400 MHz, CDCl $\left.)_{3}\right): 7.66(\mathrm{~m}, 6 \mathrm{H}), 7.49(\mathrm{~m}, 2 \mathrm{H}), 7.40(\mathrm{~m}, 5 \mathrm{H}), 7.30(\mathrm{~m}, 1 \mathrm{H}), 6.96(\mathrm{q}, J=1.2 \mathrm{~Hz}$, $1 \mathrm{H}), 2.36(\mathrm{~d}, J=1.6 \mathrm{~Hz}, 3 \mathrm{H}) ;{ }^{13} \mathrm{C} \mathrm{NMR}\left(100 \mathrm{MHz}, \mathrm{CDCl}_{3}\right): 142.8,140.7,139.9,138.3,136.8,129.2$, $128.8,128.2,127.7,127.2,126.99,126.96,126.5,126.4,17.4$. These data are in accordance with the literature. ${ }^{50}$<smiles>COc1ccc(/C(C)=C/c2ccccc2)cc1</smiles>

(E)-1-Methoxy-4-(1-phenylprop-1-en-2-yl)benzene (2s): White solid; $218 \mathrm{mg}, 97 \%$ yield; $E / Z=$ 53/1; ${ }^{1} \mathrm{H} \mathrm{NMR}\left(400 \mathrm{MHz}, \mathrm{CDCl}_{3}\right): 7.50(\mathrm{~d}, J=8.8 \mathrm{~Hz}, 2 \mathrm{H}), 7.39(\mathrm{~m}, 4 \mathrm{H}), 7.25(\mathrm{~m}, 1 \mathrm{H}), 6.94(\mathrm{~d}, J=$ $8.8 \mathrm{~Hz}, 2 \mathrm{H}), 6.81(\mathrm{~s}, 1 \mathrm{H}), 3.85(\mathrm{~s}, 3 \mathrm{H}), 2.28(\mathrm{~d}, J=1.2 \mathrm{~Hz}, 3 \mathrm{H}) ;{ }^{13} \mathrm{C} \mathrm{NMR}\left(100 \mathrm{MHz}, \mathrm{CDCl}_{3}\right): 158.9$, $138.5,136.7,136.3,129.1,128.1,127.0,126.23,126.19,113.6,55.3,17.4$. These data are in accordance with the literature..$^{49}$ 
<smiles>C/C(=C\c1ccccc1)c1ccc(F)cc1</smiles>

(E)-1-Fluoro-4-(1-phenylprop-1-en-2-yl)benzene (2t): White solid; $191 \mathrm{mg}, 90 \%$ yield, $E / Z=48 / 1$; ${ }^{1} \mathrm{H} \mathrm{NMR}\left(400 \mathrm{MHz}, \mathrm{CDCl}_{3}\right): 7.50$ (dd, $\left.J=8.8 \mathrm{~Hz}, J=5.2 \mathrm{~Hz}, 2 \mathrm{H}\right), 7.38(\mathrm{~m}, 4 \mathrm{H}), 7.27(\mathrm{~m}, 1 \mathrm{H}), 7.07(\mathrm{t}, J$ $=8.8 \mathrm{~Hz}, 2 \mathrm{H}), 6.80(\mathrm{~s}, 1 \mathrm{H}), 2.27(\mathrm{~d}, J=1.6 \mathrm{~Hz}, 3 \mathrm{H}) ;{ }^{13} \mathrm{C} \mathrm{NMR}\left(100 \mathrm{MHz}, \mathrm{CDCl}_{3}\right): 162.1\left(\mathrm{~d}, \mathrm{~J}_{F-C}=244.8\right.$ $\mathrm{Hz}), 139.9\left(\mathrm{~d}, J_{F-C}=3.4 \mathrm{~Hz}\right), 138.1,136.4,129.1,128.2,127.6\left(\mathrm{~d}, J_{F-C}=1.3 \mathrm{~Hz}\right), 127.5\left(\mathrm{~d}, J_{F-C}=8.0\right.$ $\mathrm{Hz}), 126.5,115.1\left(\mathrm{~d}, \mathrm{~J}_{\mathrm{F}-\mathrm{C}}=21.1 \mathrm{~Hz}\right), 17.6 .{ }^{19} \mathrm{~F} \mathrm{NMR}\left(376 \mathrm{MHz}, \mathrm{CDCl}_{3}\right):-115.6$; These data are in accordance with the literature. ${ }^{51}$<smiles>CC(=Cc1ccccc1)c1ccc(Cl)cc1</smiles>

(E)-1-Chloro-4-(1-phenylprop-1-en-2-yl)benzene (2u): White solid; $215 \mathrm{mg}$, 94\% yield; $E / Z=21 / 1$; ${ }^{1} \mathrm{H} \mathrm{NMR}\left(400 \mathrm{MHz}, \mathrm{CDCl}_{3}\right): 7.46(\mathrm{~d}, J=8.4 \mathrm{~Hz}, 2 \mathrm{H}), 7.37(\mathrm{~m}, 6 \mathrm{H}), 7.27(\mathrm{~m}, 1 \mathrm{H}), 6.83(\mathrm{~s}, 1 \mathrm{H}), 2.26(\mathrm{~d}$, $J=1.2 \mathrm{~Hz}, 3 \mathrm{H}) ;{ }^{13} \mathrm{C} \mathrm{NMR}\left(100 \mathrm{MHz}, \mathrm{CDCl}_{3}\right): 142.3,138.0,136.2,132.9,129.1,128.4,128.2,128.1$, $127.3,126.7,17.4$; These data are in accordance with the literature. ${ }^{52}$<smiles>CC(=Cc1ccccc1)c1ccc2ccccc2c1</smiles>

(E)-2-(1-phenylprop-1-en-2-yl)naphthalene (2v): White solid; $239 \mathrm{mg}, 98 \%$ yield; $E / Z=107 / 1 ;{ }^{1} \mathrm{H}$ NMR (400 MHz, CDCl 3 ): $7.97(\mathrm{~s}, 1 \mathrm{H}), 7.88(\mathrm{~m}, 3 \mathrm{H}), 7.75(\mathrm{~m}, 1 \mathrm{H}), 7.47(\mathrm{~m}, 6 \mathrm{H}), 7.29(\mathrm{~m}, 1 \mathrm{H}), 7.05(\mathrm{~s}$, 1H), 2.43 (s, 3H); $\left.{ }^{13} \mathrm{C} \mathrm{NMR} \mathrm{(100} \mathrm{MHz,} \mathrm{CDCl}_{3}\right): 141.1,138.3,137.2,133.4,132.7,129.2,128.22$, $128.19,128.1,127.8,127.5,126.5,126.2,125.8,124.7,124.4,17.5$. These data are in accordance with the literature. ${ }^{49}$<smiles>CC(=Cc1ccc(C)cc1)c1ccc(C)cc1</smiles>

(E)-4,4'-(Prop-1-ene-1,2-diyl)bis(methylbenzene) (2w): White solid; $180 \mathrm{mg}, 81 \%$ yield; $E / Z=36 / 1$; ${ }^{1} \mathrm{H} \mathrm{NMR}\left(400 \mathrm{MHz}, \mathrm{CDCl}_{3}\right): 7.45(\mathrm{~m}, 2 \mathrm{H}), 7.29(\mathrm{~m}, 2 \mathrm{H}), 7.20(\mathrm{~d}, J=8.8 \mathrm{~Hz}, 4 \mathrm{H}), 6.82(\mathrm{~s}, 1 \mathrm{H}), 2.39(\mathrm{~d}$, 6H), 2.29 (m, 3H); $\left.{ }^{13} \mathrm{C} \mathrm{NMR} \mathrm{(100} \mathrm{MHz,} \mathrm{CDCl} 3\right): 141.2,136.8,136.5,136.0,135.6,129.04,129.97$, $128.8,126.8,125.8,21.2,21.1,17.4$; These data are in accordance with the literature..$^{53}$<smiles>C/C(=C\c1ccccc1C)c1ccc(C)cc1</smiles>

(E)-1-Methyl-2-(2-(p-tolyl)prop-1-en-1-yl)benzene (2x): Colorless oil; $202 \mathrm{mg}, 91 \%$ yield; $E / Z=$ 111/1; $\left.{ }^{1} \mathrm{H} \mathrm{NMR} \mathrm{(400} \mathrm{MHz,} \mathrm{CDCl} 3\right): 7.53(\mathrm{~d}, J=8.0 \mathrm{~Hz}, 2 \mathrm{H}), 7.29(\mathrm{~m}, 6 \mathrm{H}), 6.90(\mathrm{~s}, 1 \mathrm{H}), 2.45(\mathrm{~s}, 3 \mathrm{H})$, $2.37(\mathrm{~s}, 3 \mathrm{H}), 2.18(\mathrm{~d}, J=1.6 \mathrm{~Hz}, 3 \mathrm{H}) ;{ }^{13} \mathrm{C} \mathrm{NMR}\left(100 \mathrm{MHz}, \mathrm{CDCl}_{3}\right): 140.5,137.7,136.93,136.92$, 136.7, 129.8, 129.3, 129.0, 126.7, 125.9, 125.8, 125.3, 21.1, 20.0, 17.1; IR (neat): 3018, 2980, $2917,1512,1447,1377,1067,809,745,729 ;$ GC-MS: $t_{R}=10.54 \mathrm{~min}, \mathrm{~m} / \mathrm{z}=222.2$. 
<smiles>CC(=Cc1cccc(C)c1)c1ccc(C)cc1</smiles>

(E)-1-Methyl-3-(2-(p-tolyl)prop-1-en-1-yl)benzene (2y): Colorless oil; $211 \mathrm{mg}, 95 \%$ yield; $E / Z=$ 112/1; ${ }^{1} \mathrm{H}$ NMR (400 MHz, $\left.\mathrm{CDCl}_{3}\right): 7.44(\mathrm{~d}, J=8.0 \mathrm{~Hz}, 2 \mathrm{H}), 7.28(\mathrm{t}, J=7.8 \mathrm{~Hz}, 1 \mathrm{H}), 7.19(\mathrm{~m}, 4 \mathrm{H})$, $7.07(\mathrm{~d}, J=7.6 \mathrm{~Hz}, 1 \mathrm{H}), 6.82(\mathrm{~s}, 1 \mathrm{H}), 2.394(\mathrm{~s}, 3 \mathrm{H}), 2.386(\mathrm{~s}, 3 \mathrm{H}), 2.29(\mathrm{~d}, J=1.2 \mathrm{~Hz}, 3 \mathrm{H}) ;{ }^{13} \mathrm{C} \mathrm{NMR}$ (100 MHz, $\mathrm{CDCl}_{3}$ ): 141.1, 138.4, 137.6, 137.0, 136.8, 129.9, 129.0, 128.0, 127.1, 127.0, 126.2, $125.8,21.5,21.1,17.4$; IR (neat): 2980, 2917, 1600, 1512, 1447, 909, 812, 787; GC-MS: $t_{R}=10.76$ $\min , \mathrm{m} / \mathrm{z}=222.2$.<smiles>C/C(=C\c1ccc(-c2ccccc2)cc1)c1ccc(C)cc1</smiles>

(E)-4-(2-(p-Tolyl)prop-1-en-1-yl)-1,1'-biphenyl (2z): White solid, m.p. 144-145 ${ }^{\circ} \mathrm{C} ; 259 \mathrm{mg}, 91 \%$ yield; $E / Z=90 / 1 ;{ }^{1} \mathrm{H} \mathrm{NMR}\left(400 \mathrm{MHz}, \mathrm{CDCl}_{3}\right): 7.64(\mathrm{~m}, 4 \mathrm{H}), 7.47(\mathrm{~m}, 6 \mathrm{H}), 7.37(\mathrm{~m}, 1 \mathrm{H}), 7.11(\mathrm{~d}, J=$ $8.0 \mathrm{~Hz}, 2 \mathrm{H}), 6.87(\mathrm{~s}, 1 \mathrm{H}), 2.40(\mathrm{~s}, 3 \mathrm{H}), 2.34(\mathrm{~d}, J=1.2 \mathrm{~Hz}, 3 \mathrm{H}) ;{ }^{13} \mathrm{C} \mathrm{NMR}\left(100 \mathrm{MHz}, \mathrm{CDCl}_{3}\right): 141.0$, 140.8, 139.0, 137.48, 137.46, 137.0, 129.6, 129.0, 128.8, 127.2, 127.0, 126.8, 126.5, 125.8, 21.1, 17.6; IR (neat): 3026, 2917, 1596, 1505, 1448, 1221, 1221, 878, 829; GC-MS: $t_{R}=14.2 \mathrm{~min}, \mathrm{~m} / \mathrm{z}=$ 284.2.<smiles>COc1ccc(/C=C(\C)c2ccc(C)cc2)cc1</smiles>

(E)-1-Methoxy-4-(2-(p-tolyl)prop-1-en-1-yl)benzene (2aa): White solid; $212 \mathrm{mg} ; 89 \%$ yield; $E / Z=$ $71 / 1 ;{ }^{1} \mathrm{H} \mathrm{NMR}\left(400 \mathrm{MHz}, \mathrm{CDCl}_{3}\right): 7.42(\mathrm{~d}, J=8.0 \mathrm{~Hz}, 2 \mathrm{H}), 7.31(\mathrm{~d}, J=8.8 \mathrm{~Hz}, 2 \mathrm{H}), 7.18(\mathrm{~d}, J=8.0 \mathrm{~Hz}$, $2 \mathrm{H}), 6.92(\mathrm{~d}, J=8.8 \mathrm{~Hz}, 2 \mathrm{H}), 6.76(\mathrm{~s}, 1 \mathrm{H}), 3.84(\mathrm{~s}, 3 \mathrm{H}), 2.37(\mathrm{~s}, 3 \mathrm{H}), 2.26(\mathrm{~m}, 3 \mathrm{H}) ;{ }^{13} \mathrm{C} \mathrm{NMR}(100$ $\mathrm{MHz}, \mathrm{CDCl}_{3}$ ): 158.0, 141.2, 136.7, 135.7, 131.0, 130.3, 129.0, 126.5, 125.8, 113.5, 55.2, 21.1, 17.4 . These data are in accordance with the literature. ${ }^{54}$<smiles>CC(=Cc1ccc(F)cc1)c1ccc(C)cc1</smiles>

(E)-1-Fluoro-4-(2-( $p$-tolyl)prop-1-en-1-yl)benzene (2ab): White solid, m.p. 177-178 ${ }^{\circ} \mathrm{C} ; 204 \mathrm{mg}, 90 \%$ yield; $E / Z=54 / 1 ;{ }^{1} \mathrm{H} \mathrm{NMR}\left(400 \mathrm{MHz}, \mathrm{CDCl}_{3}\right): 7.42(\mathrm{~d}, J=8.0 \mathrm{~Hz}, 2 \mathrm{H}), 7.31(\mathrm{~m}, 2 \mathrm{H}), 7.19(\mathrm{~m}, 2 \mathrm{H})$, $7.06(\mathrm{t}, J=8.8 \mathrm{~Hz}, 2 \mathrm{H}), 6.77(\mathrm{~s}, 1 \mathrm{H}), 2.38(\mathrm{~s}, 3 \mathrm{H}), 2.24(\mathrm{~s}, 3 \mathrm{H}) ;{ }^{13} \mathrm{C} \mathrm{NMR}\left(100 \mathrm{MHz}, \mathrm{CDCl}_{3}\right): 161.3(\mathrm{~d}$, $\left.J_{F-C}=244.6 \mathrm{~Hz}\right), 140.8,137.2\left(\mathrm{~d}, J_{F-C}=1.4 \mathrm{~Hz}\right), 137.0,134.4\left(\mathrm{~d}, J_{F-C}=3.3 \mathrm{~Hz}\right), 130.7,130.6,129.0$, 125.8, $115.0\left(\mathrm{~d}, J_{F-C}=21.2 \mathrm{~Hz}\right), 21.1,17.3 ;{ }^{19} \mathrm{~F} \mathrm{NMR}\left(376 \mathrm{MHz}, \mathrm{CDCl}_{3}\right):-116.4$; IR (neat): 3026, $2917,1596,1503,1221,878,828,810,521$; GC-MS: $t_{R}=10.40 \mathrm{~min}, \mathrm{~m} / \mathrm{z}=226.1$. 
<smiles>CC(=Cc1ccc(Cl)cc1)c1ccc(C)cc1</smiles>

(E)-1-chloro-4-(2-( $p$-tolyl)prop-1-en-1-yl)benzene (2ac): White solid; $235 \mathrm{mg}, 97 \%$ yield; $E / Z=$ 58/1; ${ }^{1} \mathrm{H} \mathrm{NMR}\left(400 \mathrm{MHz}, \mathrm{CDCl}_{3}\right): 7.43(\mathrm{~d}, J=8.0 \mathrm{~Hz}, 2 \mathrm{H}), 7.35$ (d, $\left.J=8.4 \mathrm{~Hz}, 2 \mathrm{H}\right), 7.29$ (d, J=8.4 Hz, $2 \mathrm{H}), 7.20(\mathrm{~d}, J=8.0 \mathrm{~Hz}, 2 \mathrm{H}), 6.76(\mathrm{~s}, 1 \mathrm{H}), 2.39(\mathrm{~s}, 3 \mathrm{H}), 2.26(\mathrm{~m}, 3 \mathrm{H}) ;{ }^{13} \mathrm{C} \mathrm{NMR}\left(100 \mathrm{MHz}, \mathrm{CDCl}_{3}\right)$ : $140.7,138.0,137.2,136.9,132.0,130.4,129.0,128.3,125.8,125.7,21.1,17.4$. These data are in accordance with the literature..$^{55}$<smiles>C/C(=C\c1cccc2ccccc12)c1ccc(C)cc1</smiles>

(E)-1-(2-( $p$-Tolyl)prop-1-en-1-yl)naphthalene (2ad): Colorless oil; $243 \mathrm{mg} ; 94 \%$ yield; $E / Z=48 / 1$; ${ }^{1} \mathrm{H} \mathrm{NMR}\left(400 \mathrm{MHz}, \mathrm{CDCl}_{3}\right): 8.10(\mathrm{~m}, 1 \mathrm{H}), 7.94(\mathrm{~m}, 1 \mathrm{H}), 7.85(\mathrm{~d}, J=8.0 \mathrm{~Hz}, 1 \mathrm{H}), 7.62(\mathrm{~d}, J=8.0 \mathrm{~Hz}$, 2H), $7.55(\mathrm{~m}, 3 \mathrm{H}), 7.49(\mathrm{~d}, J=7.2 \mathrm{~Hz}, 1 \mathrm{H}), 7.34(\mathrm{~s}, 1 \mathrm{H}), 7.30(\mathrm{~d}, J=8.0 \mathrm{~Hz}, 2 \mathrm{H}), 2.47(\mathrm{~s}, 3 \mathrm{H}), 2.19$ (m, 3H); ${ }^{13} \mathrm{C} \mathrm{NMR}\left(100 \mathrm{MHz}, \mathrm{CDCl}_{3}\right): 140.3,138.6,137.1,135.8,134.2,132.2,129.1,128.4,127.1$, 126.7, 125.82, 125.79, 125.7, 125.3, 125.2, 124.7, 21.1, 17.4; IR (neat): 2980, 2915, 1508, 1442, $1391,1067,810,779,758 ;$ GC-MS: $t_{R}=12.27 \mathrm{~min}, \mathrm{~m} / \mathrm{z}=258.2$.<smiles>C/C(=C\c1ccc2ccccc2c1)c1ccc(C)cc1</smiles>

(E)-2-(2-(p-Tolyl)prop-1-en-1-yl)naphthalene (2ae): White solid, m.p. 105-106 ${ }^{\circ} \mathrm{C} ; 225 \mathrm{mg}, 87 \%$ yield; $E / Z=87 / 1$; ${ }^{1} \mathrm{H} \mathrm{NMR}\left(400 \mathrm{MHz}, \mathrm{CDCl}_{3}\right): 7.84(\mathrm{~m}, 4 \mathrm{H}), 7.49(\mathrm{~m}, 5 \mathrm{H}), 7.23(\mathrm{~d}, J=8.4 \mathrm{~Hz}, 2 \mathrm{H})$, $6.99(\mathrm{~s}, 1 \mathrm{H}), 2.41(\mathrm{~s}, 3 \mathrm{H}), 2.37(\mathrm{~d}, J=1.2 \mathrm{~Hz}, 3 \mathrm{H}) ;{ }^{13} \mathrm{C} \mathrm{NMR}\left(100 \mathrm{MHz}, \mathrm{CDCl}_{3}\right): 141.0,137.7,137.0$, 136.0, 133.3, 132.0, 129.0, 127.9, 127.69, 127.67, 127.6, 127.5, 126.9, 126.0, 125.9, 125.6, 21.10, 17.58; IR (neat): 2912, 1510, 1376, 947, 904, 856, 826, 814, 739; GC-MS: $t_{R}=12.78 \mathrm{~min}, \mathrm{~m} / \mathrm{z}=$ 258.2 .<smiles>CC(=Cc1ccsc1)c1ccccc1</smiles>

(E)-3-(2-phenylprop-1-en-1-yl)thiophene (2af): White solid, m.p. 58-60; $190 \mathrm{mg}, 95 \%$ yield, $E / Z=$ 33/1; ${ }^{1} \mathrm{H} \mathrm{NMR}\left(400 \mathrm{MHz}, \mathrm{CDCl}_{3}\right): 7.52(\mathrm{~m}, 2 \mathrm{H}), 7.36(\mathrm{~m}, 3 \mathrm{H}), 7.29(\mathrm{~m}, 2 \mathrm{H}), 7.21(\mathrm{~d}, \mathrm{~J}=4.8 \mathrm{~Hz}, 1 \mathrm{H})$, $6.79(\mathrm{~s}, 1 \mathrm{H}), 2.36(\mathrm{~s}, 3 \mathrm{H}) ;{ }^{13} \mathrm{C} \mathrm{NMR}\left(100 \mathrm{MHz}, \mathrm{CDCl}_{3}\right): 144.1,139.3,136.8,129.0,128.3,127.0$, 126.0, 124.9, 122.8, 122.0, 18.08; IR (neat): ; GC-MS: $t_{R}=9.67 \mathrm{~min}, \mathrm{~m} / \mathrm{z}=200.1$. 
<smiles>C/C(=C\c1ccc(N(C)C)cc1)c1ccccc1</smiles>

(E)-N,N-dimethyl-4-(2-phenylprop-1-en-1-yl)aniline (2ag): White solid, m.p. $88-90{ }^{\circ} \mathrm{C} ; 180 \mathrm{mg}, 76 \%$ yield; $E / Z=22 / 1 ;{ }^{1} \mathrm{H} \mathrm{NMR}\left(400 \mathrm{MHz}, \mathrm{CDCl}_{3}\right): 7.51(\mathrm{~m}, 2 \mathrm{H}), 7.33(\mathrm{~m}, 4 \mathrm{H}), 7.24(\mathrm{~m}, 1 \mathrm{H}), 6.75(\mathrm{~m}, 3 \mathrm{H})$, $2.98(\mathrm{~d}, J=1.6 \mathrm{~Hz}, 6 \mathrm{H}), 2.31(\mathrm{~s}, 3 \mathrm{H}) ;{ }^{13} \mathrm{C} \mathrm{NMR}\left(100 \mathrm{MHz}, \mathrm{CDCl}_{3}\right): 149.1,144.6,134.1,130.2,128.2$, $127.8,126.7,126.6,125.9,112.1,40.5,17.5 ;$ IR (neat): ; GC-MS: $t_{R}=11.71 \mathrm{~min}, \mathrm{~m} / \mathrm{z}=237.2$.<smiles>COC(=O)c1ccc(/C=C(\C)c2ccccc2)cc1</smiles>

Methyl (E)-4-(2-phenylprop-1-en-1-yl)benzoate (2ah): crude product, $47 \%$ conversion; $E / Z=8 / 1$; ${ }^{1} \mathrm{H} \mathrm{NMR}\left(400 \mathrm{MHz}, \mathrm{CDCl}_{3}\right): 8.05(\mathrm{~d}, J=8.4 \mathrm{~Hz}, 2 \mathrm{H}), 7.53(\mathrm{~d}, J=7.2 \mathrm{~Hz}, 2 \mathrm{H}), 7.40(\mathrm{~m}, 5 \mathrm{H}), 6.85(\mathrm{~s}$, $1 \mathrm{H}), 3.93(\mathrm{~s}, 3 \mathrm{H}), 2.31(\mathrm{~s}, 3 \mathrm{H})$; These data are in accordance with the literature. ${ }^{56}$<smiles>CC1=CCC(c2ccccc2)CC1</smiles>

4-Methyl-1,2,3,6-tetrahydro-1,1'-biphenyl (2ai): Colorless oil; $155 \mathrm{mg} ; 90 \%$ yield; ${ }^{1} \mathrm{H}$ NMR (400 $\left.\mathrm{MHz}, \mathrm{CDCl}_{3}\right)$ : $7.38(\mathrm{~m}, 2 \mathrm{H}), 7.28(\mathrm{~m}, 3 \mathrm{H}), 5.57(\mathrm{~m}, 1 \mathrm{H}), 2.82(\mathrm{~m}, 1 \mathrm{H}), 2.27(\mathrm{~m}, 3 \mathrm{H}), 2.06(\mathrm{~m}, 2 \mathrm{H})$, $1.86(\mathrm{~m}, 1 \mathrm{H}), 1.79(\mathrm{~s}, 3 \mathrm{H}) ;{ }^{13} \mathrm{C} \mathrm{NMR}\left(100 \mathrm{MHz}, \mathrm{CDCl}_{3}\right): 147.3,133.9,128.3,126.9,125.9,120.8$, $40.0,33.6,30.7,30.1,23.5$. These data are in accordance with the literature. ${ }^{57}$<smiles>CC1=CCCc2ccccc21</smiles>

4-Methyl-1,2-dihydronaphthalene (2aj): Colorless oil; $137 \mathrm{mg}, 95 \%$ yield; ${ }^{1} \mathrm{H}$ NMR $(400 \mathrm{MHz}$, $\left.\mathrm{CDCl}_{3}\right): 7.20(\mathrm{~m}, 4 \mathrm{H}), 5.88(\mathrm{~m}, 1 \mathrm{H}), 2.78(\mathrm{t}, J=8.2 \mathrm{~Hz}, 2 \mathrm{H}), 2.27(\mathrm{~m}, 2 \mathrm{H}), 2.08(\mathrm{q}, J=1.6 \mathrm{~Hz}, 3 \mathrm{H}) ;{ }^{13} \mathrm{C}$ NMR (100 MHz, CDCl $)$ : 136.3, 135.8, 132.1, 127.3, 126.6, 126.3, 125.4, 122.7, 28.3, 23.2, 19.3. These data are in accordance with the literature..$^{58}$<smiles>CC1=Cc2ccccc2CC1</smiles>

3-Methyl-1,2-dihydronaphthalene (2ak): Colorless oil; $123 \mathrm{mg}$; 85\% yield; regioselectivity = 29/1; ${ }^{1} \mathrm{H} \mathrm{NMR}\left(400 \mathrm{MHz}, \mathrm{CDCl}_{3}\right): 7.13(\mathrm{~m}, 3 \mathrm{H}), 6.98(\mathrm{~d}, J=7.2 \mathrm{~Hz}, 1 \mathrm{H}), 6.24(\mathrm{q}, J=1.5 \mathrm{~Hz}, 1 \mathrm{H}), 2.84(\mathrm{t}, J=$ $8.2 \mathrm{~Hz}, 2 \mathrm{H}), 2.26(\mathrm{~m}, 2 \mathrm{H}), 1.93(\mathrm{~m}, 3 \mathrm{H}) ;{ }^{13} \mathrm{C} N M R\left(100 \mathrm{MHz}, \mathrm{CDCl}_{3}\right): 138.2,135.0,134.0,127.1$, $126.3,125.9,125.0,122.7,28.8,28.0,23.5$. These data are in accordance with the literature. ${ }^{59}$<smiles>CC(=CCCCCCCC=C(C)c1ccc(C)cc1)c1ccc(C)cc1</smiles>

4,4'-((2E,10E)-Dodeca-2,10-diene-2,11-diyl)bis(methylbenzene) (2al): Colorless oil; 308 mg, 89\% 
yield; $E / Z=65 / 1 ;{ }^{1} \mathrm{H} \mathrm{NMR}\left(400 \mathrm{MHz}, \mathrm{CDCl}_{3}\right): 7.29(\mathrm{~d}, J=8.4 \mathrm{~Hz}, 4 \mathrm{H}), 7.12(\mathrm{~d}, J=8.0 \mathrm{~Hz}, 4 \mathrm{H}), 5.76$ $(\mathrm{m}, 2 \mathrm{H}), 2.34(\mathrm{~s}, 6 \mathrm{H}), 2.19$ (q, $J=7.2 \mathrm{~Hz}, 4 \mathrm{H}), 2.02(\mathrm{~m}, 6 \mathrm{H}), 1.43(\mathrm{~m}, 8 \mathrm{H}) ;{ }^{13} \mathrm{C} \mathrm{NMR}(100 \mathrm{MHz}$, $\mathrm{CDCl}_{3}$ ): 141.1, 136.0, 134.2, 128.8, 127.9, 125.4, 29.6, 29.3, 28.7, 21.0, 15.8; IR (neat): 2923, 2855, $1682,1606,1514,1456,1267,1071,813$; GC-MS: $t_{R}=15.13 \mathrm{~min}, \mathrm{~m} / \mathrm{z}=346.4$.<smiles>CC(=CCCC=C(C)c1ccc(C)cc1)c1ccc(C)cc1</smiles>

4,4'-((2E,6E)-Octa-2,6-diene-2,7-diyl)bis(methylbenzene) (2am): White solid, m.p. 60-61 ${ }^{\circ} \mathrm{C} ; 270$ mg, $93 \%$ yield; $E / Z=34 / 1 ;{ }^{1} \mathrm{H}$ NMR $\left(400 \mathrm{MHz}, \mathrm{CDCl}_{3}\right): 7.28$ (d, $\left.J=8.0 \mathrm{~Hz}, 4 \mathrm{H}\right), 7.12$ (d, $J=8.0 \mathrm{~Hz}$, $4 \mathrm{H}), 5.80(\mathrm{~m}, 2 \mathrm{H}), 2.34(\mathrm{~m}, 10 \mathrm{H}), 2.04(\mathrm{~d}, J=1.2 \mathrm{~Hz}, 6 \mathrm{H}) ;{ }^{13} \mathrm{C} \mathrm{NMR}\left(100 \mathrm{MHz}, \mathrm{CDCl}_{3}\right): 141.0,136.1$, 134.9, 128.8, 127.0, 125.5, 28.8, 21.0, 15.9; IR (neat): 2914, 2854, 1510, 1373, 1056, 808, 546, 490; GC-MS: $t_{R}=12.73 \mathrm{~min}, \mathrm{~m} / \mathrm{z}=290.2$.<smiles>CC(=CCC=C(C)c1ccc(C)cc1)c1ccc(C)cc1</smiles>

4,4'-((2E,5E)-Hepta-2,5-diene-2,6-diyl)bis(methylbenzene) (2an): Colorless oil; $243 \mathrm{mg}, 88 \%$ yield; $E / Z=26 / 1 ;{ }^{1} \mathrm{H} \mathrm{NMR}\left(400 \mathrm{MHz}, \mathrm{CDCl}_{3}\right): 7.33(\mathrm{~d}, J=8.4 \mathrm{~Hz}, 4 \mathrm{H}), 7.15(\mathrm{~d}, J=8.0 \mathrm{~Hz}, 4 \mathrm{H}), 5.84(\mathrm{~m}, 1 \mathrm{H})$, $3.12(\mathrm{t}, J=7.2 \mathrm{~Hz}, 2 \mathrm{H}), 2.37(\mathrm{~s}, 6 \mathrm{H}), 2.12(\mathrm{~s}, 6 \mathrm{H}) ;{ }^{13} \mathrm{C} \mathrm{NMR}\left(100 \mathrm{MHz}, \mathrm{CDCl}_{3}\right)$ : 140.9, 136.2, 134.9, 128.8, 125.53, 125.48, 28.5, 21.0, 15.9; IR (neat): 2980, 2918, 1683, 1511, 1447,1376, 1061, 809; GC-MS: $t_{R}=12.32 \mathrm{~min}, \mathrm{~m} / \mathrm{z}=276.3$.<smiles>CC(=CC=C(C)c1ccc(C)cc1)c1ccc(C)cc1</smiles>

4,4'-((2E,4E)-Hexa-2,4-diene-2,5-diyl)bis(methylbenzene) (2ao): White solid, m.p. 142-144 ${ }^{\circ} \mathrm{C} ; 252$ mg, 96\% yield; $\left.E / Z=52 / 1 ;{ }^{1} \mathrm{H} \mathrm{NMR} \mathrm{(400} \mathrm{MHz,} \mathrm{CDCl}_{3}\right): 7.41$ (d, $J=8.4 \mathrm{~Hz}, 4 \mathrm{H}$ ), 7.17 (d, $J=8.0 \mathrm{~Hz}$, 4H), 6.81 (s, 2H), 2.37 (s, 6H), $\left.2.24(\mathrm{~s}, 6 \mathrm{H}) ;{ }^{13} \mathrm{C} \mathrm{NMR} \mathrm{(100} \mathrm{MHz,} \mathrm{CDCl} 3\right): 140.9,136.7,136.3,129.0$, 125.6, 123.2, 21.1, 16.1; IR (neat): 2916, 1596, 1504, 1447, 1222, 878, 809, 780; GC-MS: $t_{R}=12.3$ $\min , \mathrm{m} / \mathrm{z}=262.2$.<smiles>C/C(=C\c1cccc(/C=C(\C)c2ccc(C)cc2)c1)c1ccc(C)cc1</smiles>

1,3-Bis((E)-2-(p-tolyl)prop-1-en-1-yl)benzene (2ap): White solid, m.p. $76-78^{\circ} \mathrm{C} ; 274 \mathrm{mg}, 81 \%$ yield; $E / Z=52 / 1 ;{ }^{1} \mathrm{H} \mathrm{NMR}\left(400 \mathrm{MHz}, \mathrm{CDCl}_{3}\right): 7.45(\mathrm{~d}, J=8.0 \mathrm{~Hz}, 4 \mathrm{H}), 7.38(\mathrm{~m}, 2 \mathrm{H}), 7.25$ (dd, $J=7.6 \mathrm{~Hz}, J$ $=1.6 \mathrm{~Hz}, 2 \mathrm{H}), 7.20(\mathrm{~d}, J=8.0 \mathrm{~Hz}, 4 \mathrm{H}), 6.85(\mathrm{~s}, 6 \mathrm{H}), 2.39(\mathrm{~s}, 6 \mathrm{H}), 2.31(\mathrm{~d}, J=1.2 \mathrm{~Hz}, 6 \mathrm{H}) ;{ }^{13} \mathrm{C} \mathrm{NMR}$ (100 MHz, $\mathrm{CDCl}_{3}$ ): 141.0, 138.3, 137.3, 136.9, 129.9, 129.0, 127.9, 127.2, 126.9, 125.8, 21.1, 17.5; IR (neat): $3026,2980,2917,1596,1504,1222,878,828,810,780 ;$ GC-MS: $t_{R}=15.6 \mathrm{~min}, \mathrm{~m} / \mathrm{z}=$ 338.3 . 


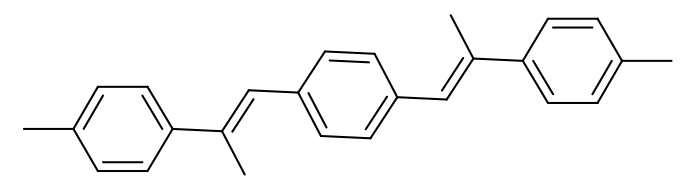

1,4-Bis((E)-2-(p-tolyl)prop-1-en-1-yl)benzene (2aq): White solid, m.p. 177-178 ${ }^{\circ} \mathrm{C} ; 294 \mathrm{mg}, 87 \%$ yield; $E / Z=16 / 1 ;{ }^{1} \mathrm{H} \mathrm{NMR}\left(400 \mathrm{MHz}, \mathrm{CDCl}_{3}\right.$ ): 7.45 (d, $J=8.0 \mathrm{~Hz}, 4 \mathrm{H}$ ), 7.39 (s, 4H), 7.20 (d, $J=8.0$ $\mathrm{Hz}, 4 \mathrm{H}), 6.84(\mathrm{q}, J=1.2 \mathrm{~Hz}, 2 \mathrm{H}), 2.39(\mathrm{~s}, 6 \mathrm{H}), 2.33(\mathrm{~d}, J=1.2 \mathrm{~Hz}, 6 \mathrm{H}) ;{ }^{13} \mathrm{C} \mathrm{NMR}\left(100 \mathrm{MHz}, \mathrm{CDCl}_{3}\right)$ : 141.1, 137.2, 136.9, 136.5, 129.0, 128.9, 126.8, 125.8, 21.1, 17.6; IR (neat): 3017, 2915, 2857, 1501, 1456, 1114, 885, 847, 802, 549; HRMS: calcd. for $\mathrm{C}_{26} \mathrm{H}_{26}[\mathrm{M}+\mathrm{H}]^{+} 339.2107$, found: 339.2098 .<smiles>C=C(c1ccc(C)cc1)C(C)C/C=C(\C)c1ccc(C)cc1</smiles>

(E)-4,4'-(3-Methylhepta-1,5-diene-2,6-diyl)bis(methylbenzene) (2ar): Colorless oil; $258 \mathrm{mg}, 89 \%$ yield; $E / Z=52 / 1 ;{ }^{1} \mathrm{H}$ NMR $\left(400 \mathrm{MHz}, \mathrm{CDCl}_{3}\right): 7.32(\mathrm{~d}, J=8.0 \mathrm{~Hz}, 2 \mathrm{H}), 7.29$ (d, $J=8.4 \mathrm{~Hz}, 2 \mathrm{H}$ ), 7.19 $(\mathrm{d}, J=8.0 \mathrm{~Hz}, 2 \mathrm{H}), 7.16(\mathrm{~d}, J=8.0 \mathrm{~Hz}, 2 \mathrm{H}), 5.80(\mathrm{~m}, 1 \mathrm{H}), 5.26(\mathrm{~s}, 1 \mathrm{H}), 5.10(\mathrm{~s}, 1 \mathrm{H}), 2.90(\mathrm{~m}, 1 \mathrm{H})$, $2.45(\mathrm{~m}, 1 \mathrm{H}), 2.40(\mathrm{~s}, 3 \mathrm{H}), 2.38(\mathrm{~s}, 3 \mathrm{H}), 2.26(\mathrm{~m}, 1 \mathrm{H}), 2.02(\mathrm{~s}, 3 \mathrm{H}), 1.22(\mathrm{~d}, J=6.8 \mathrm{~Hz}, 2 \mathrm{H}) ;{ }^{13} \mathrm{C} N M R$ (100 MHz, $\left.\mathrm{CDCl}_{3}\right): 154.0,141.2,139.9,136.8,136.1,135.2,128.9,128.8,126.6,126.1,125.5$, 110.7, 38.2, 35.0, 21.1, 21.0, 19.5, 16.0; IR (neat): 2962, 2920, 1683, 1511, 1456, 1375, 1018, 894, 813; GC-MS: $t_{R}=11.97 \mathrm{~min}, \mathrm{~m} / \mathrm{z}=290.3$. 


\section{References:}

1. Molloy, J. J.; Metternich, J. B.; Daniliuc, C. G.; Watson, A. J. B.; Gilmour, R. Angew. Chem. Int. Ed. 2018, 57, 3168.

2. Abramovitch, A.; Varghese, J. P.; Marek, I. Org. Lett.2004, 6, 621.

3. Chen, Z.-W.; Dong, V. M. Nat. Commun.2017, 8, article number: 784.

4. Zhu, X.-T.; Ye, C.-Q.; Li, Y.-J.; Bao, H.-L. Chem. Eur. J. 2017, 23, 10254.

5. McIntyre, S.; Hörmann, E.; Menges, F.; Smidt, S. P.; Pfaltz, A. Adv. Synth. Catal. 2005, 347, 282.

6. Berthiol, F.; Doucet, H.; Santelli, M. Eur. J. Org. Chem. 2003, 1091.

7. Standley, E. A.; Jamison, T. F. J. Am. Chem. Soc.2013,135, 1585.

8. Miao, T,; Wang, G.-W. Chem. Commun. 2011, 47, 9501.

9. Wu, Q.; Wang, L.-L.; Jin, R.-Z.; Kang, C.-Q.; Bian, Z.; Du, Z.-J.; Ma, X.-Y.; Guo, H.-Q.; Gao, L.-X. Eur. J. Org. Chem. 2016, 32, 5415.

10. Arisawa, M.; Kuwajima, M.; Toriyama, F.; Li, G.-Z.; Yamaguchi, M. Org. Lett. 2012, 14, 3804.

11. Qin, L.; Ren, X.-F.; Lu, Y.-P.; Li, Y.-X.; Zhou, J.-R. Angew. Chem. Int. Ed. 2012, 51, 5915.

12. Wu, J.-C.; Gong, L.-B.; Xia, Y.-Z.; Song, R.-J.; Xie, Y.-X.; Li, J.-H. Angew. Chem. Int. Ed. 2012, 51, 9909.

13. Landers, B.; Berini, C.; Wang, C.; Navarro, O. J. Org. Chem. 2011, 76, 1390.

14. Zheng, C.-W.; Stahl, S. S. Chem. Commun. 2015, 51, 12771.

15. Vaitla, J.; Bayer, A.; Hopmann, K. H. Angew. Chem. Int. Ed. 2017, 56, 4277.

16. Zhou, Z.-B.; Hou, Z.-L.; Yang, F.; Yao, B. Tetrahedron 2018, 74, 7228.

17. Liu, J.; Zhou, X.-Y.; Rao, H.-H.; Xiao, F.-H.; Li, C.-J.; Deng, G.-J. Chem. Eur. J. 2011, 17, 7996.

18. Ayitou, A. J.-L.; Flynn, K.; Jockusch, S.; Khan, S. I.; Garcia-Garibay, M. A. J. Am. Chem. Soc.2016, 138, 2644.

19. Luo, H.; Hu, G.; Li, P.-F. J. Org. Chem. 2019, 84, 10569.

20. Takise, R.; Muto, K.; Yamaguchi, J.; Itami, K. Angew. Chem. Int. Ed. 2014, 53, 6791.

21. Kangani, C. O.; Day, B. W. Org. Lett. 2008, 10, 2645.

22. Dong, C.; Zhang, L.; Xue, X.; Li, H.; Yu, Z.; Tang, W.; Xu, L. RSC Adv. 2014, 4, 11152.

23. Dichirarante, V.; Fagnoni, M.; Albini, A. J. Org. Chem. 2010, 75, 1271.

24. Cui, X.; Wang, S.; Zhang, Y.; Deng, W.; Qian, Q.; Gong, H. Org. Biomol. Chem. 2013, 11, 3094.

25. Soulard, V.; Villa, G.; Vollmar, D. P.; Renaud, P. J. Am. Chem. Soc. 2018, 140, 155.

26. Phan, D. H. T.; Kou, K. G. M.; Dong, V. M. J. Am. Chem. Soc. 2010, 132, 16354.

27. Yan, T.-H.; Tsai, C.-C.; Chien, C.-T.; Cho, C.-C.; Huang, P.-C. Org. Lett. 2014, 6, 4961.

28. Hua, G.-X.; Henry, J. B.; Li, Y.; Mount, A. R.; Slawin, A. M. Z.; Woollins, J. D. Org. Biomol. Chem. 2010, 8, 1655.

29. Pratsch, G.; Overman, L. E. J. Org. Chem. 2015, 80, 11388.

30. Tutkowski, B. M.; Grigalunas, M.; Wiest, O.; Helquist, P. Tetrahedron Lett. 2015, 56, 3468.

31. López-Pérez, A.; Adrio, J.; Carretero, J. C. Org. Lett. 2009, 11, 5514.

32. Cahiez, G.; Gager, O.; Habiak, V. Synthesis 2018, 16, 2636.

33. Sabot, C.; Kumar, K. A.; Antheaume, C.; Mioskowski, C. J. Org. Chem. 2007, 72, 5001.

34. Kattuboina, A.; Kaur, P.; Ai, T.; Li, G.-G. Chem. Biol. Drug. Des. 2008, 71, 216.

35. Chen, C.; Dugan, T. R.; Brennessel, W. W.; Weix, D. J.; Holland, P. L. J. Am. Chem. Soc. 2014, $136,945$. 
36. For synthesis procedure, see: (a) Budzelaar, P. H. M.; van Oort, A. B.; Orpen, A. G. Eur. J. Inorg. Chem. 1998, 1485; (b) Holland, P. L.; Cundari, T. R.; Parez, L. L.; Eckert, N. A.; Lachicotte, R. J. J. Am. Chem. Soc. 2002, 124, 14416.

37. Crossley, S. W. M.; Barabé, F.; Shenvi, R. J. Am. Chem. Soc. 2014, 136, 16788.

38. For synthesis procedure, see: Ford, D. D.; Nielsen, L. P. C.; Zuend, S. J.; Musgrave, C. B.; Jacobsen, E. N. J. Am. Chem. Soc. 2013, 135, 15595.

39. Schmidt, A.; Nödling, A. R.; Hilt, G. Angew. Chem. Int. Ed. 2015, 54, 801.

40. Li, G.; Kuo, J. L.; Han, A.; Abuyuan, J. M.; Young, L. C.; Norton, J. R.; Palmer, J. H. J. Am. Chem. Soc. 2016, 138, 7698 .

41. For synthesis procedure, see: (a) Li, G.; Han, A.; Pulling, M. E.; Estes, D. P.; Norton, J. R. J. Am. Chem. Soc. 2012, 134, 14662; (b) Li, G.; Estes, D. P.; Norton, J. R.; Ruccolo, S.; Sattler, A.; Sattler, W. Inorg. Chem. 2014, 53, 10743.

42. Liu, X.; Zhang, W.; Wang, Y.; Zhang, Z.-X.; Jiao, L.; Liu, Q. J. Am. Chem. Soc. 2018, 140, 6873.

43. Huang, R.-Z.; Lau, K. K.; Li, Z.-F.; Liu, T.-L.; Zhao, Y. J. Am. Chem. Soc. 2018, 140, 14647.

44. Wei, W.; Dai, X.-J.; Wang, H.-N.; Li, C.-C.; Yang, X.-B.; Li, C.-J. Chem. Sci. 2017, 8, 8193.

45. Murai, M.; Nishimura, K.; Takai, K. Chem. Commun. 2019, 55, 2769.

46. Wang, Y.-J.; Shao, Z.-H.; Zhang, K.; Liu, Q. Angew. Chem. Int. Ed. 2018, 57, 15143.

47. Pieterse, T.; Visser, M.; Marais, C.; Bezuidenhoudt, B. C. B. Synlett 2016, 27, 1541.

48. Lim, H. J.; Smith, C. R.; RajanBabu, T. V. J. Org. Chem. 2009, 74, 4565.

49. Yang, Z.-Z.; Chen, X.-Y.; Kong, W.; Xia, S.-Y.; Zheng, R.-W.; Luo, F.; Zhu, G.-G. Org. Biomol. Chem. 2013, 11, 2175.

50. O’Neill, J.; Yoo, K. S.; Jung, K. W. Tetrahedron Lett. 2008, 49, 7307.

51. Kim, H. R.; Yun, J. Chem. Commun. 2011, 47, 2943.

52. Lasch, R.; Fehler, S. K.; Heinrich, M. R. Org. Lett. 2016, 18, 1586.

53. Knappke, C. E. I.; Neudörfl, J. M.; von Wangelin, A. J. Org. Biomol. Chem. 2010, 8, 1695.

54. Yang, F.-L.; Ma, X.-T.; Tian, S.-K. Chem. Eur. J. 2012, 18, 1582.

55. Scholz, M.; Ulbrich, H. K.; Soehnlein, O.; Lindbom, L.; Mattern, A.; Dannhardt, G. Bioorg. Med. Chem. 2009, 17, 558.

56. Das, A.; Chaudhuri, R.; Liu, R.-S. Chem. Commun. 2009, 4046.

57. Liu, X.-F.; Zhang, W.; Wang, Y.-J.; Zhang, Z.-X.; Jiao, L.; Liu, Q. J. Am. Chem. Soc. 2018, 140, 6873.

58. Meng, Q.-Y.; Schirmer, T. E.; Katou, K., König, B. Angew. Chem. Int. Ed. 2019, 58, 5723.

59. Scheiper, B.; Bonnekessel, M.; Krause, H.; Fürster, A. J. Org. Chem. 2004, 69, 3943. 


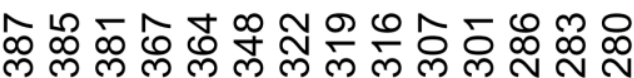
NNNNNNNNNN
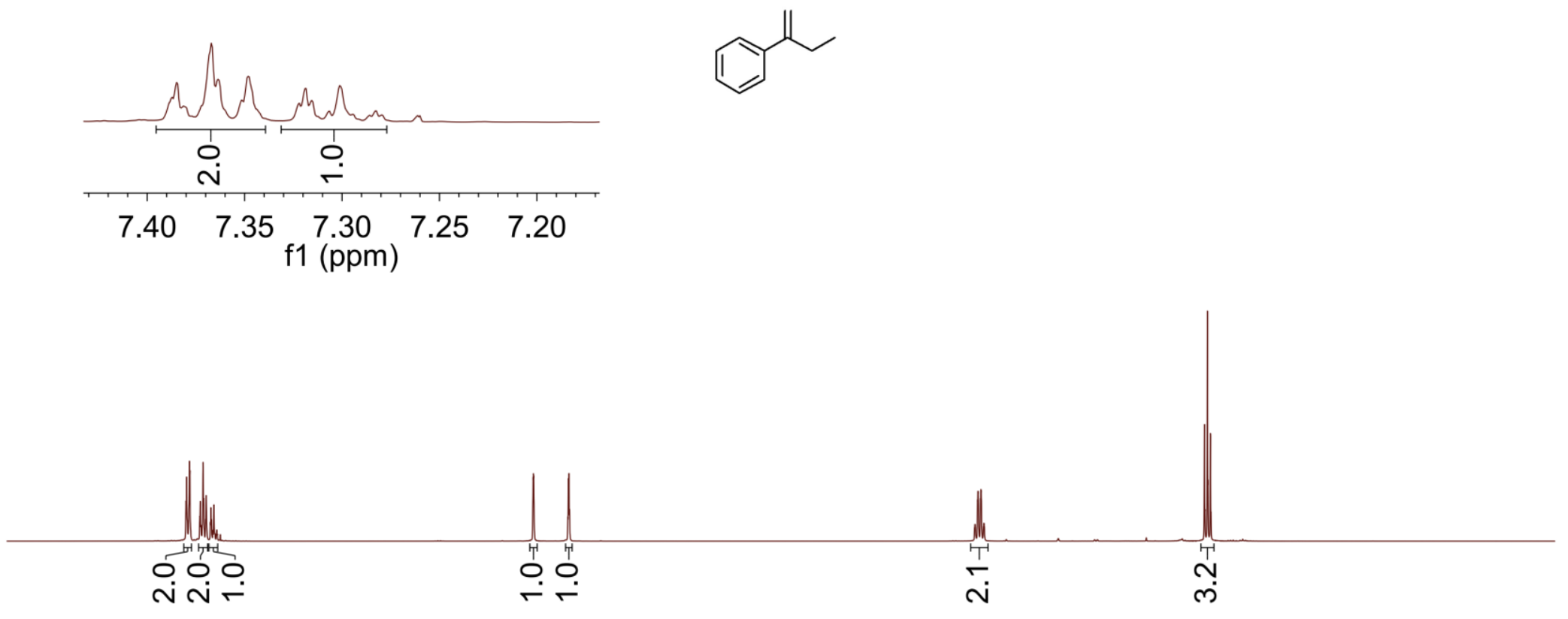

$\begin{array}{lrllllllll}\begin{array}{l}4.0 \\ \text { f1 }(\mathrm{ppm})\end{array} & 3.5 & 3.0 & 2.5 & 2.0 & 1.5 & 1.0 & 0.5 & 0.0 & -0.5\end{array}$


1b ${ }^{1} \mathrm{H}$ NMR

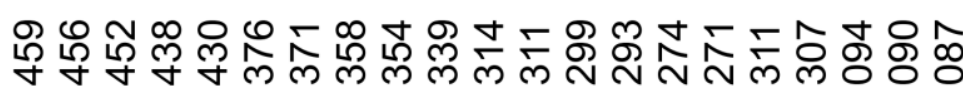

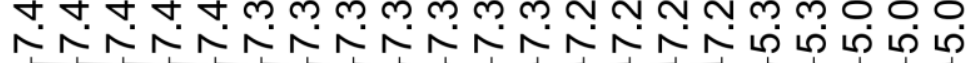

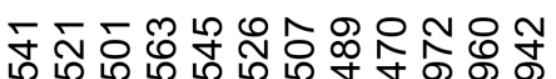

-

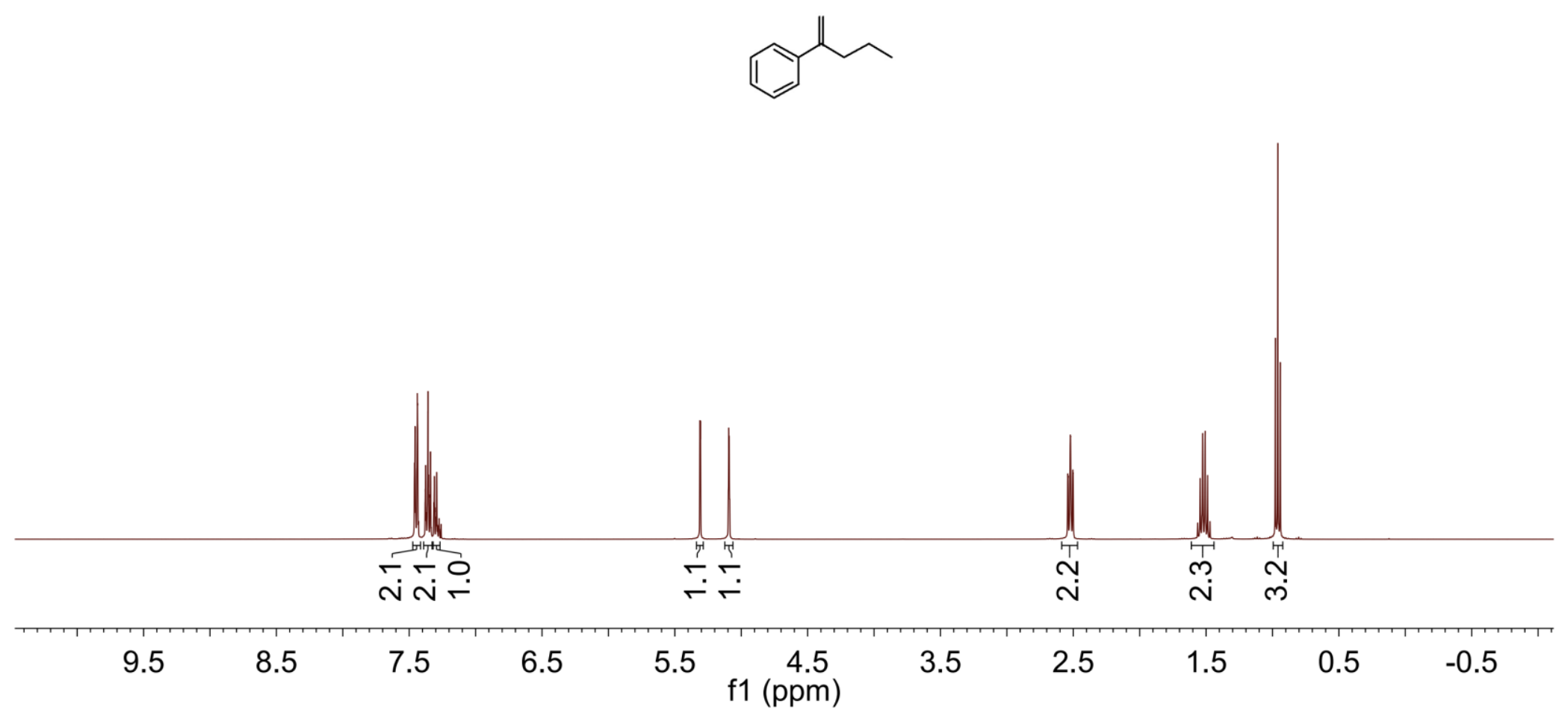




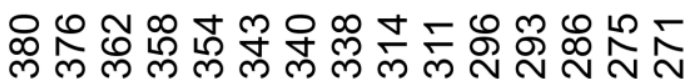

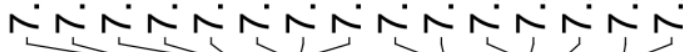
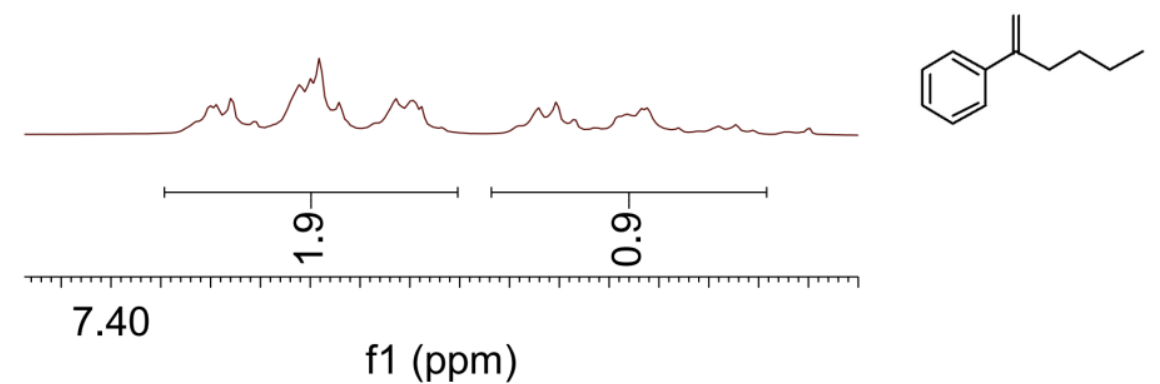

$$
f 1(\mathrm{ppm})
$$

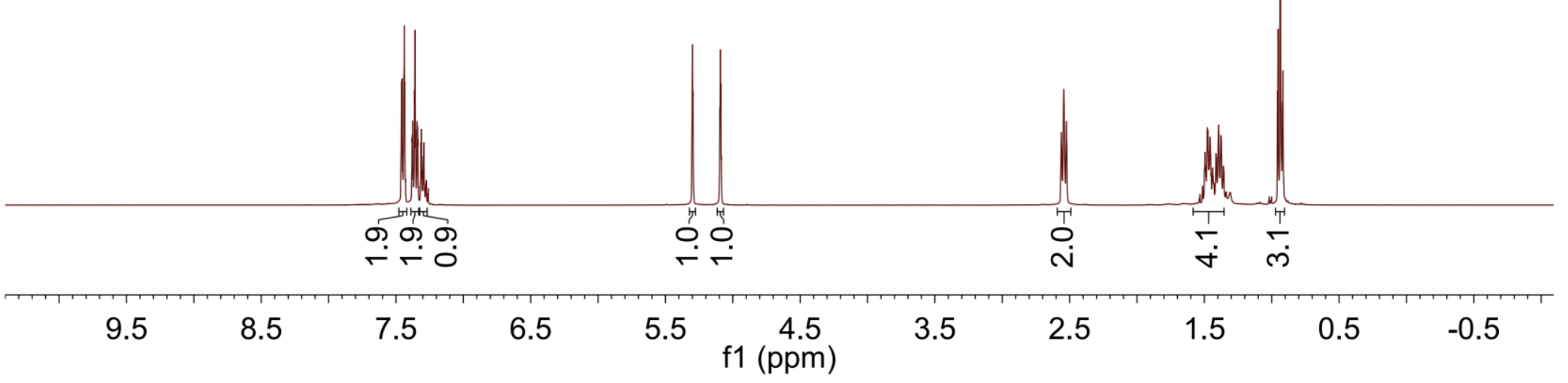




\begin{tabular}{|c|c|c|}
\hline 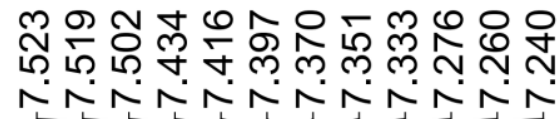 & 纺 & 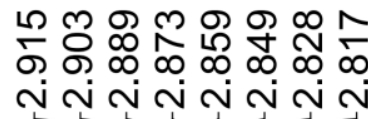 \\
\hline
\end{tabular}

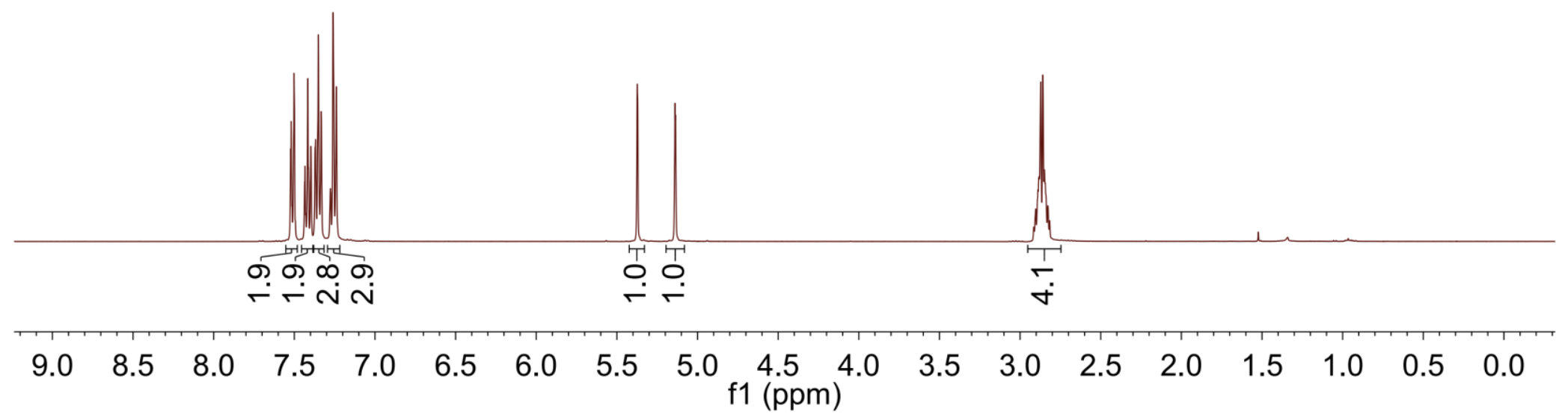




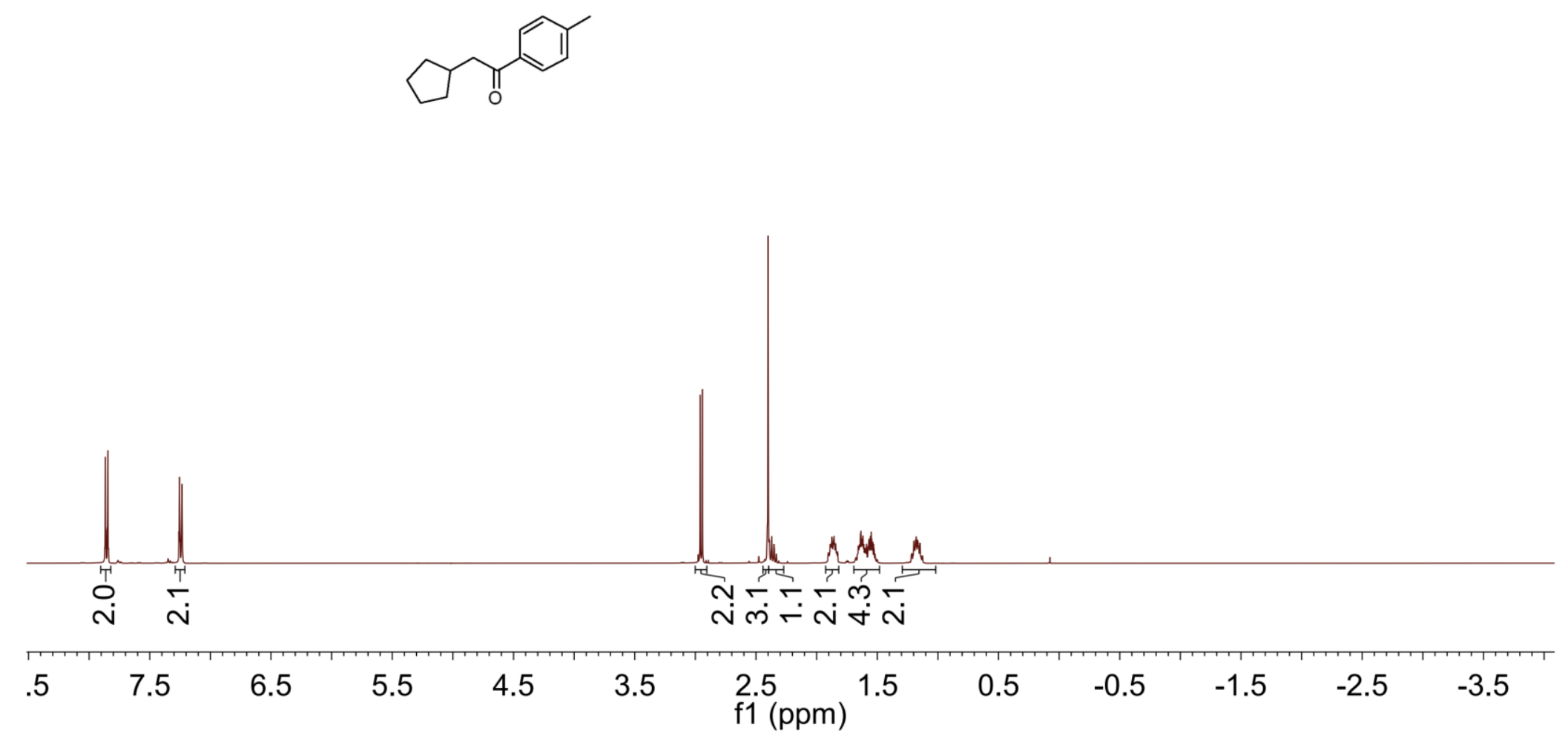




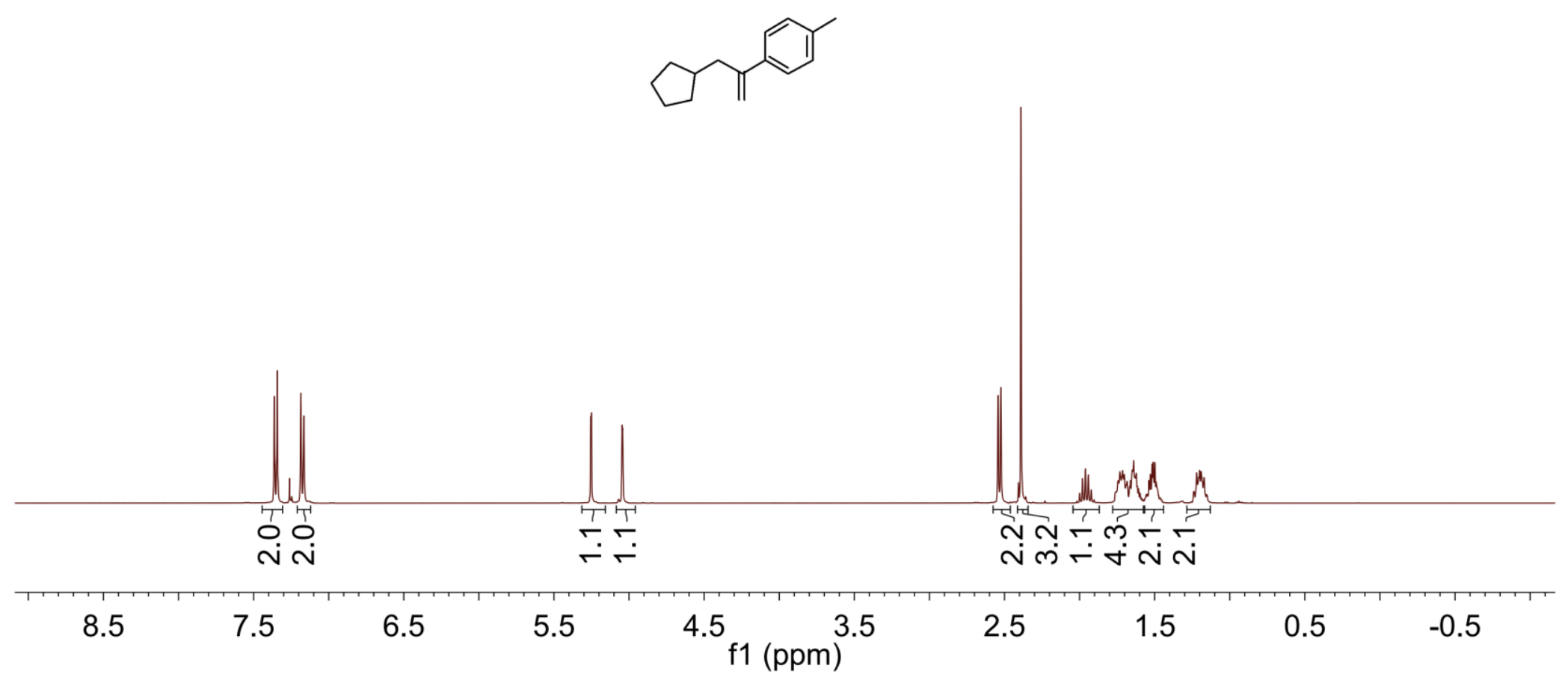




\begin{tabular}{|c|c|c|}
\hline 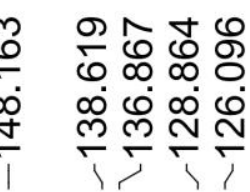 & 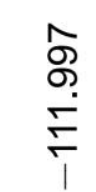 & 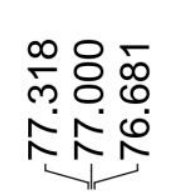 \\
\hline
\end{tabular}
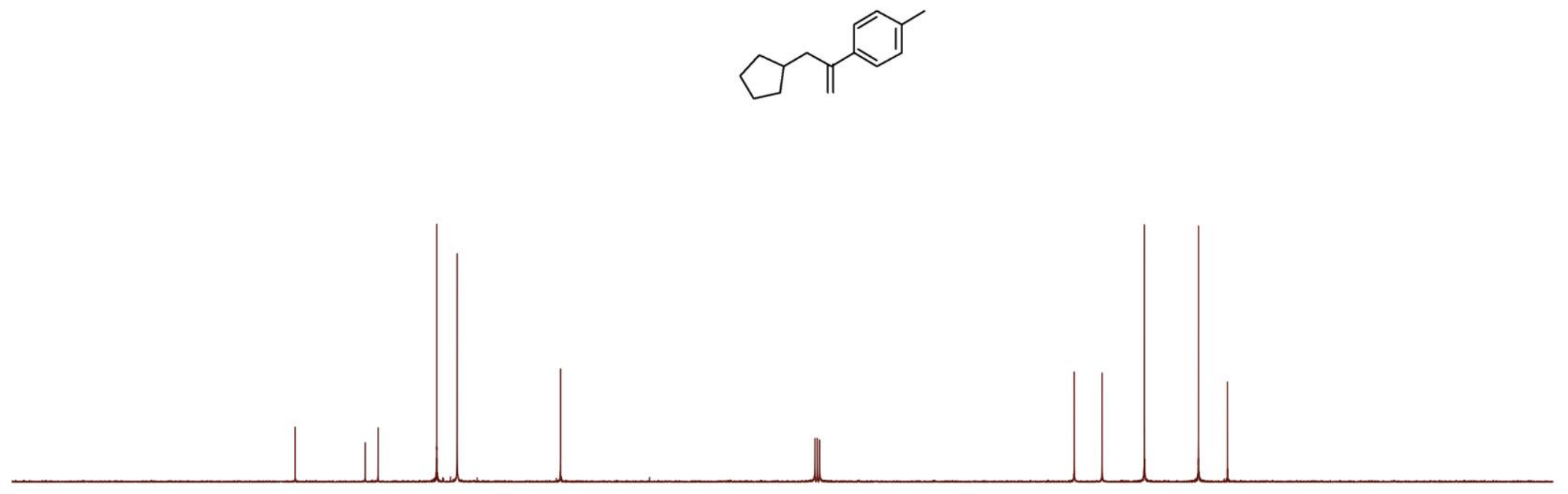


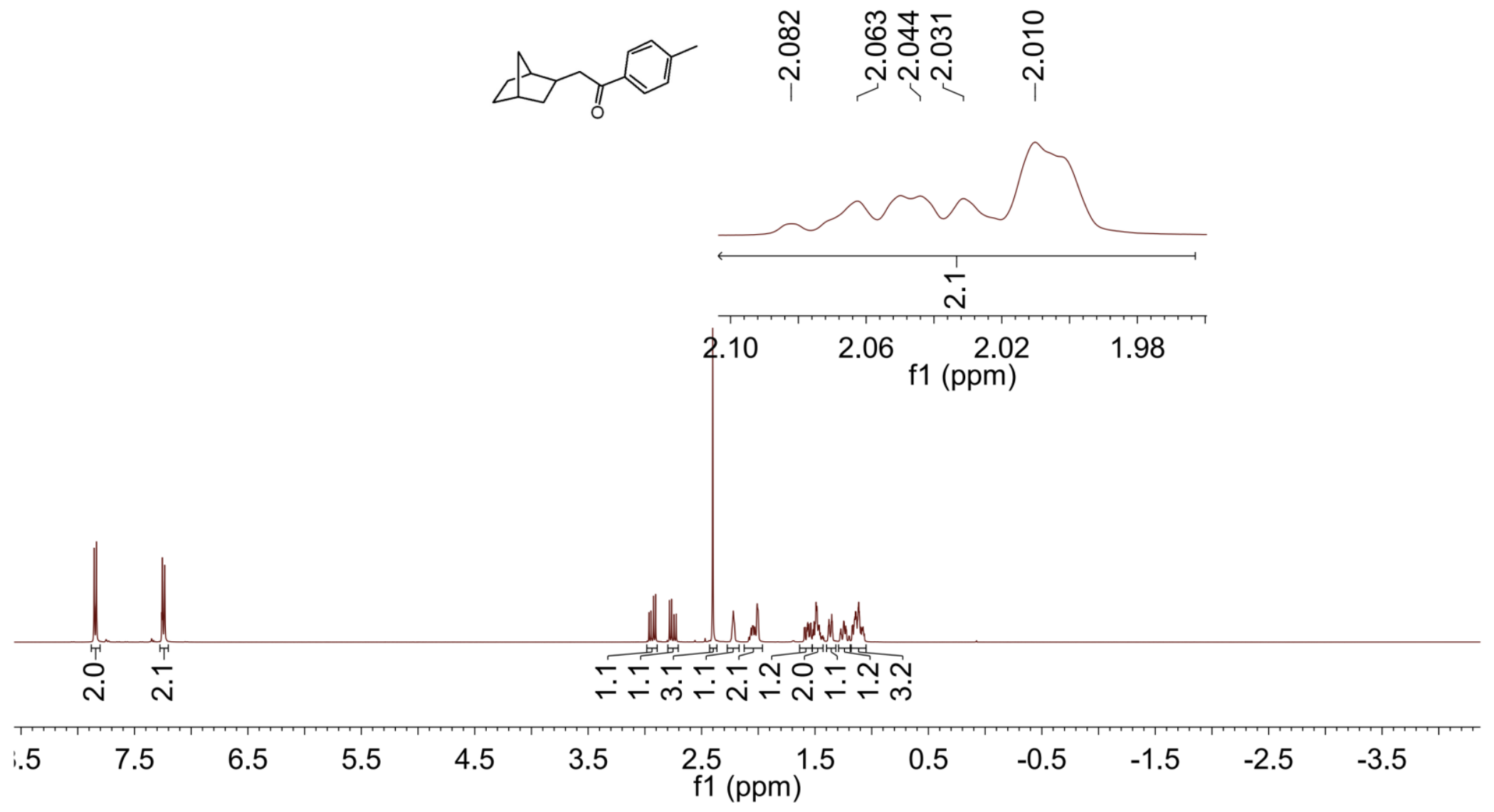




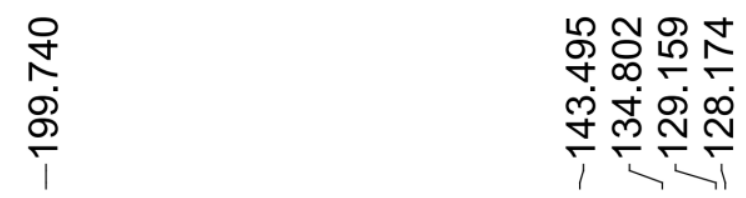

กิ

ヘヘN

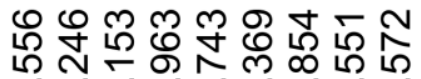

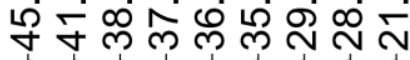
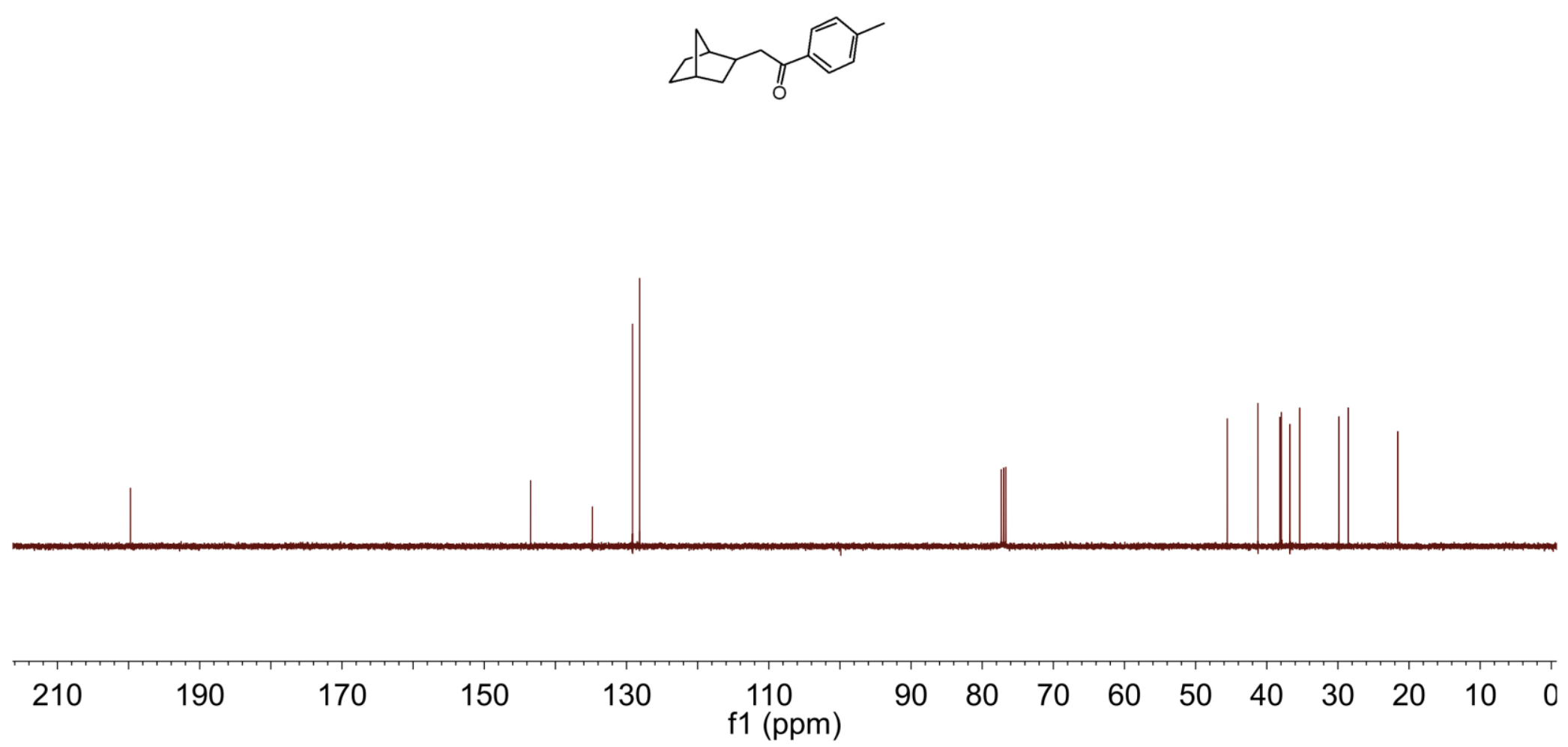


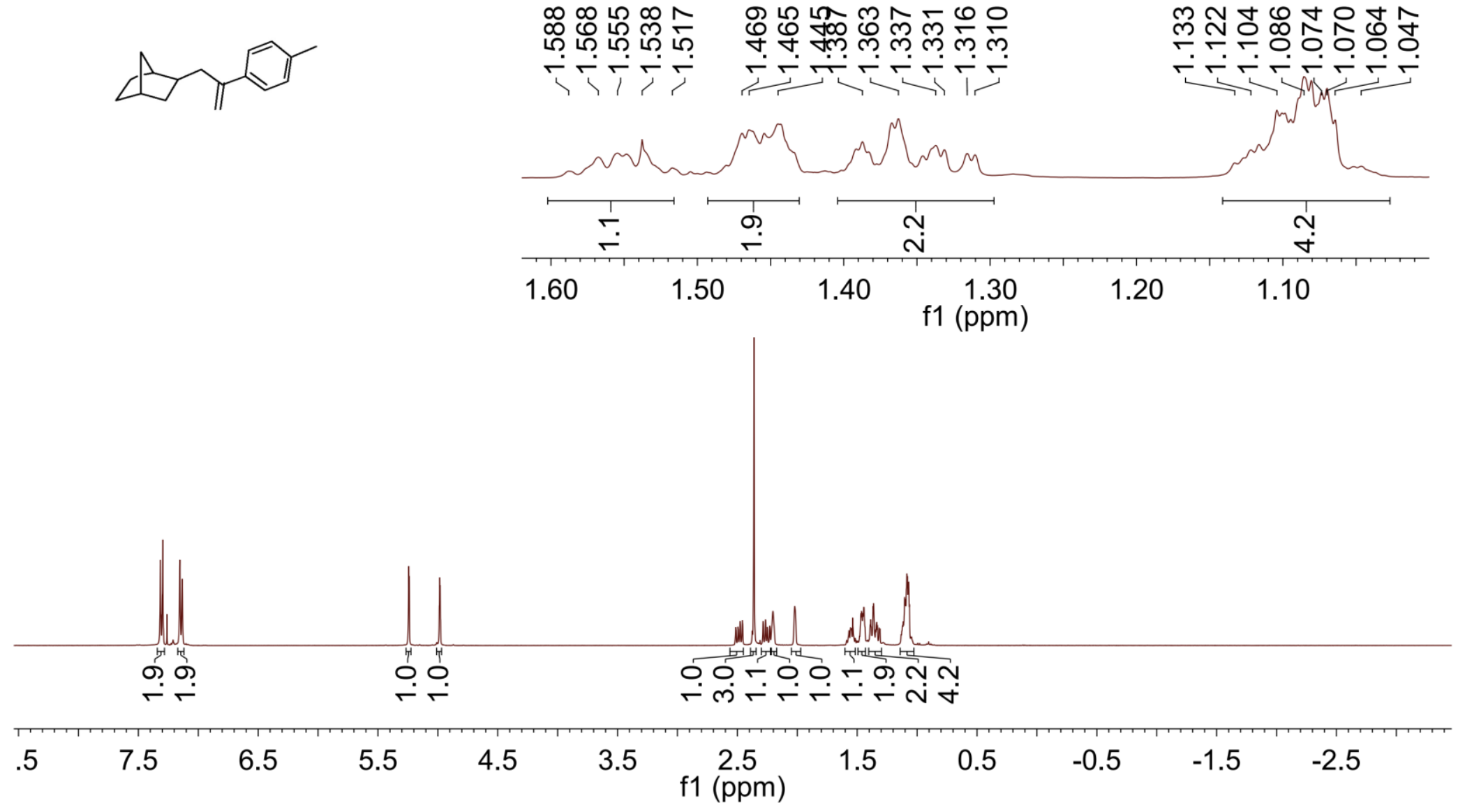




\begin{tabular}{|c|c|c|c|}
\hline 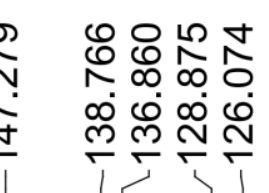 & 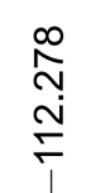 & 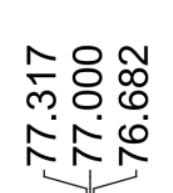 & 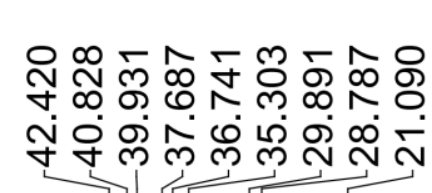 \\
\hline
\end{tabular}

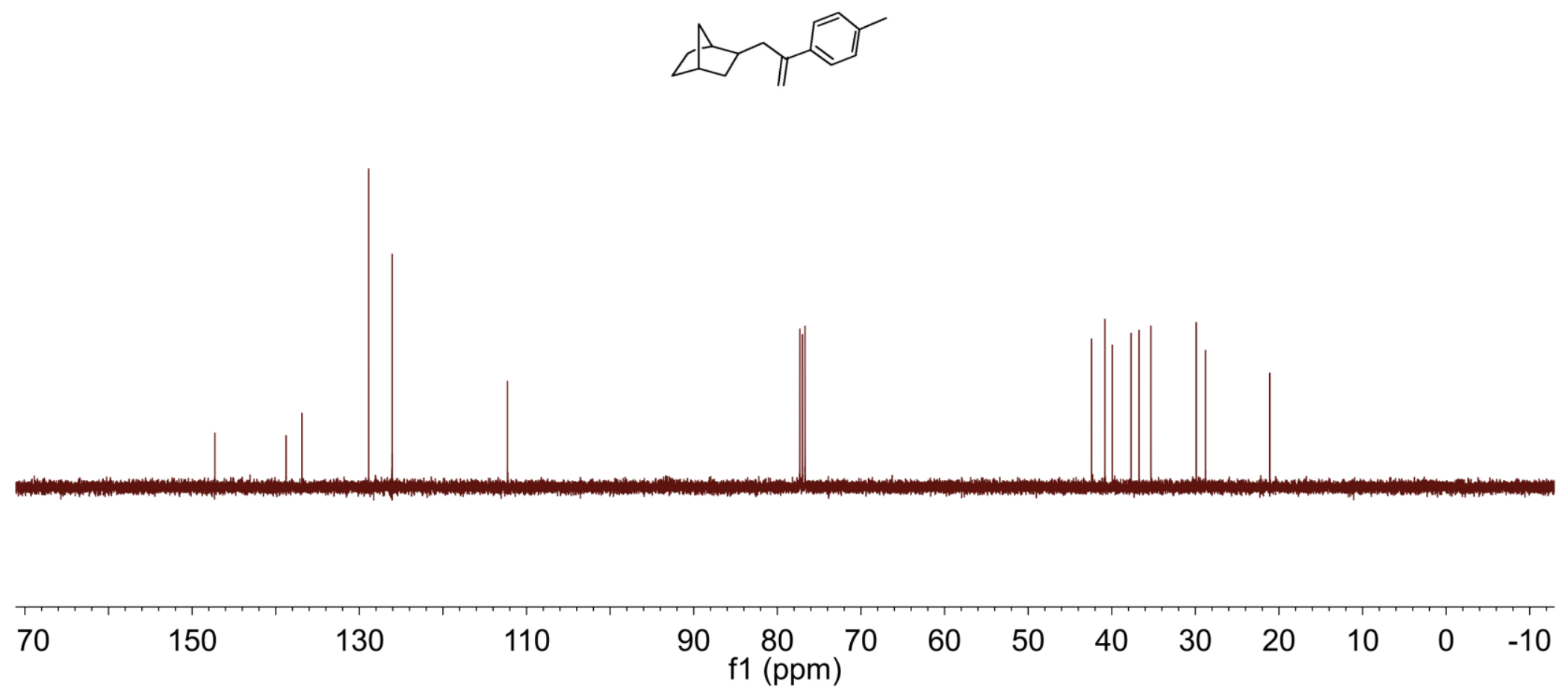




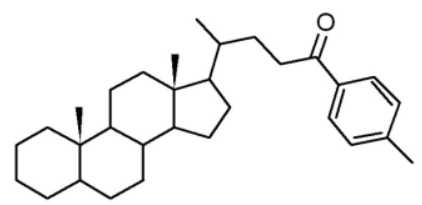

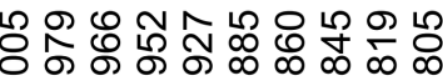

min่

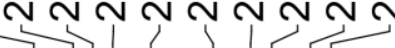
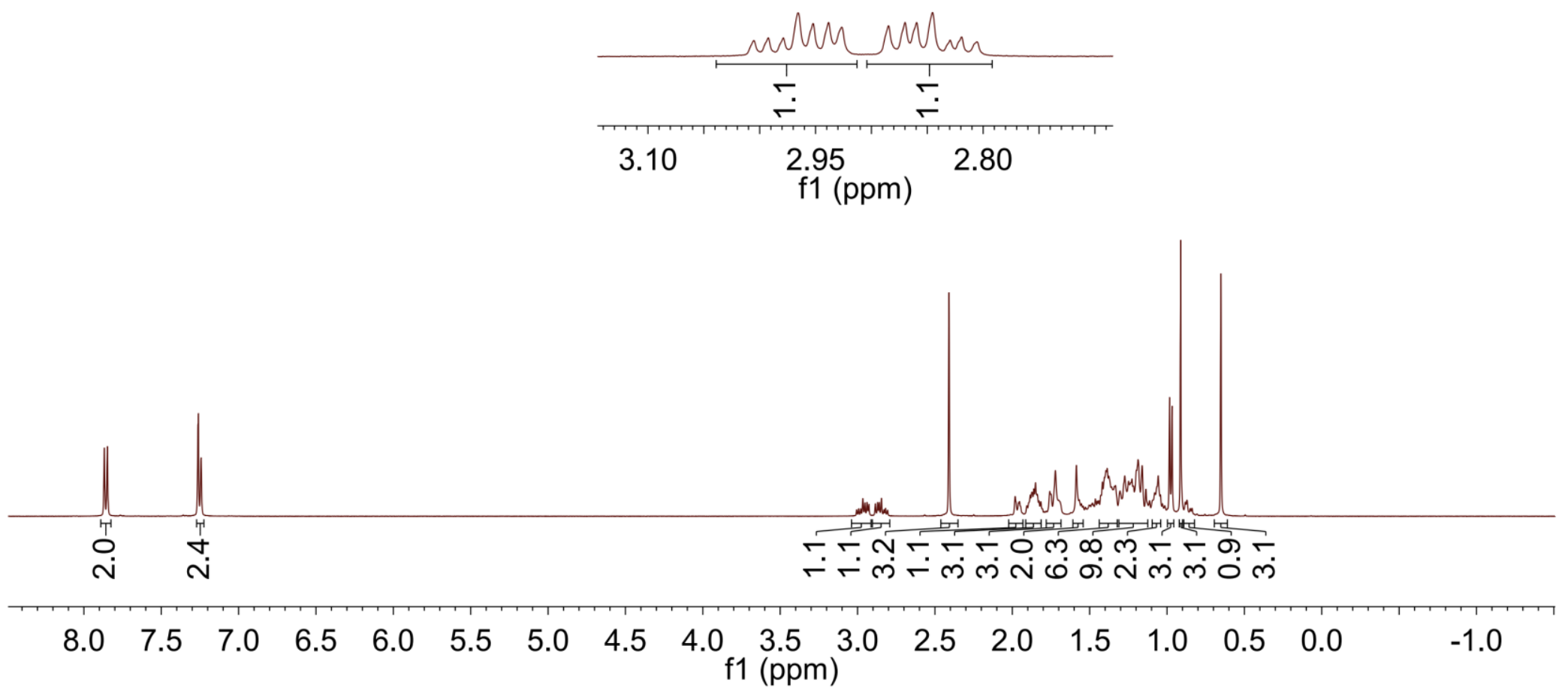


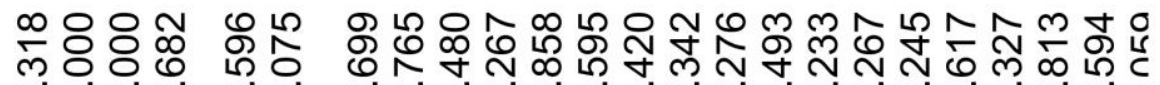

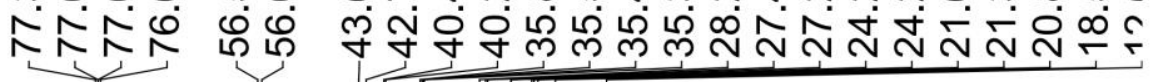

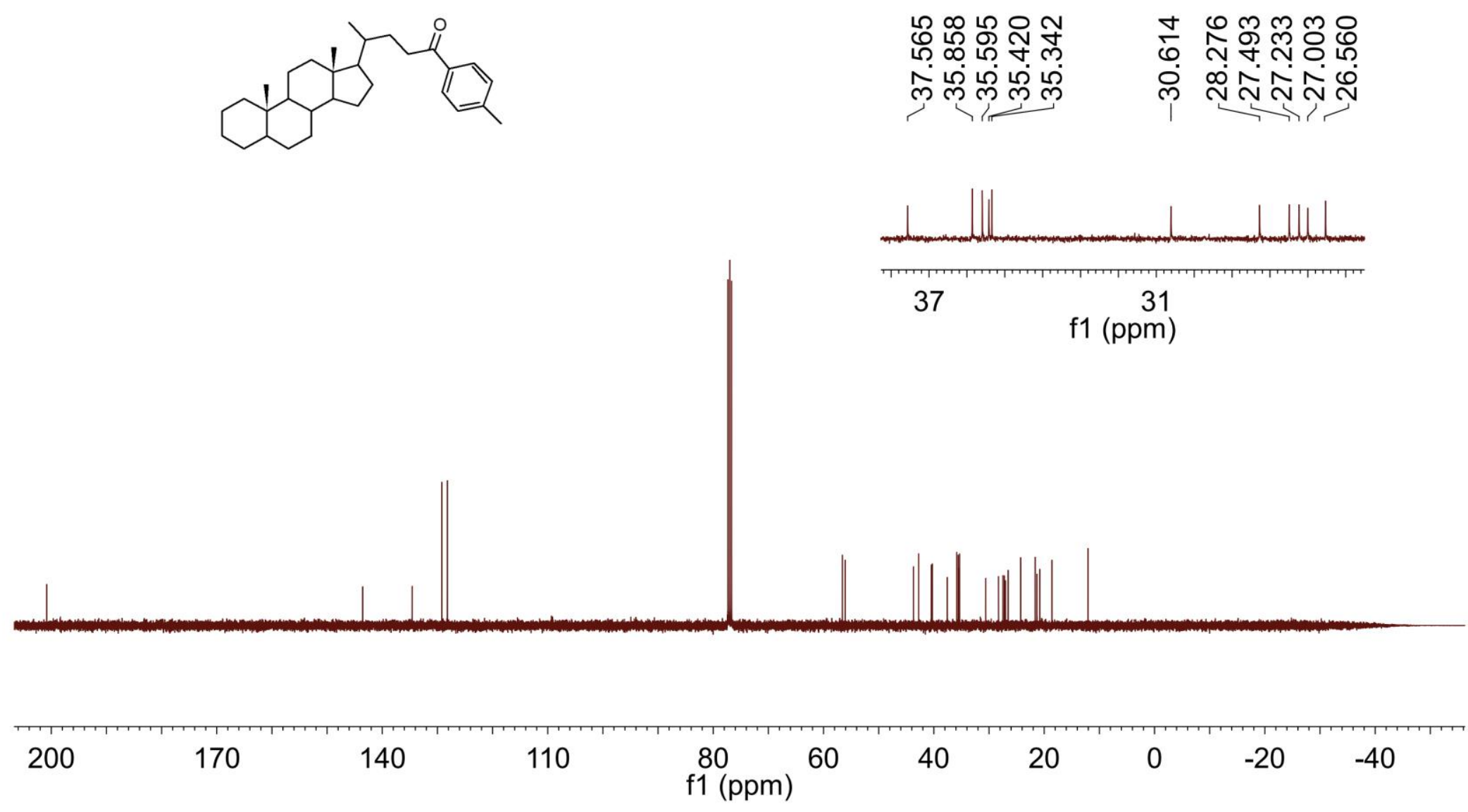




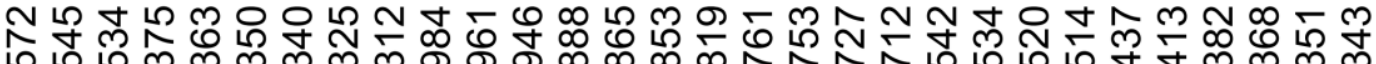

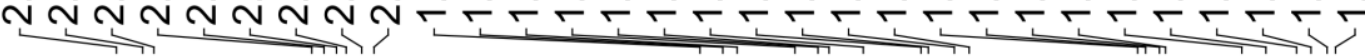

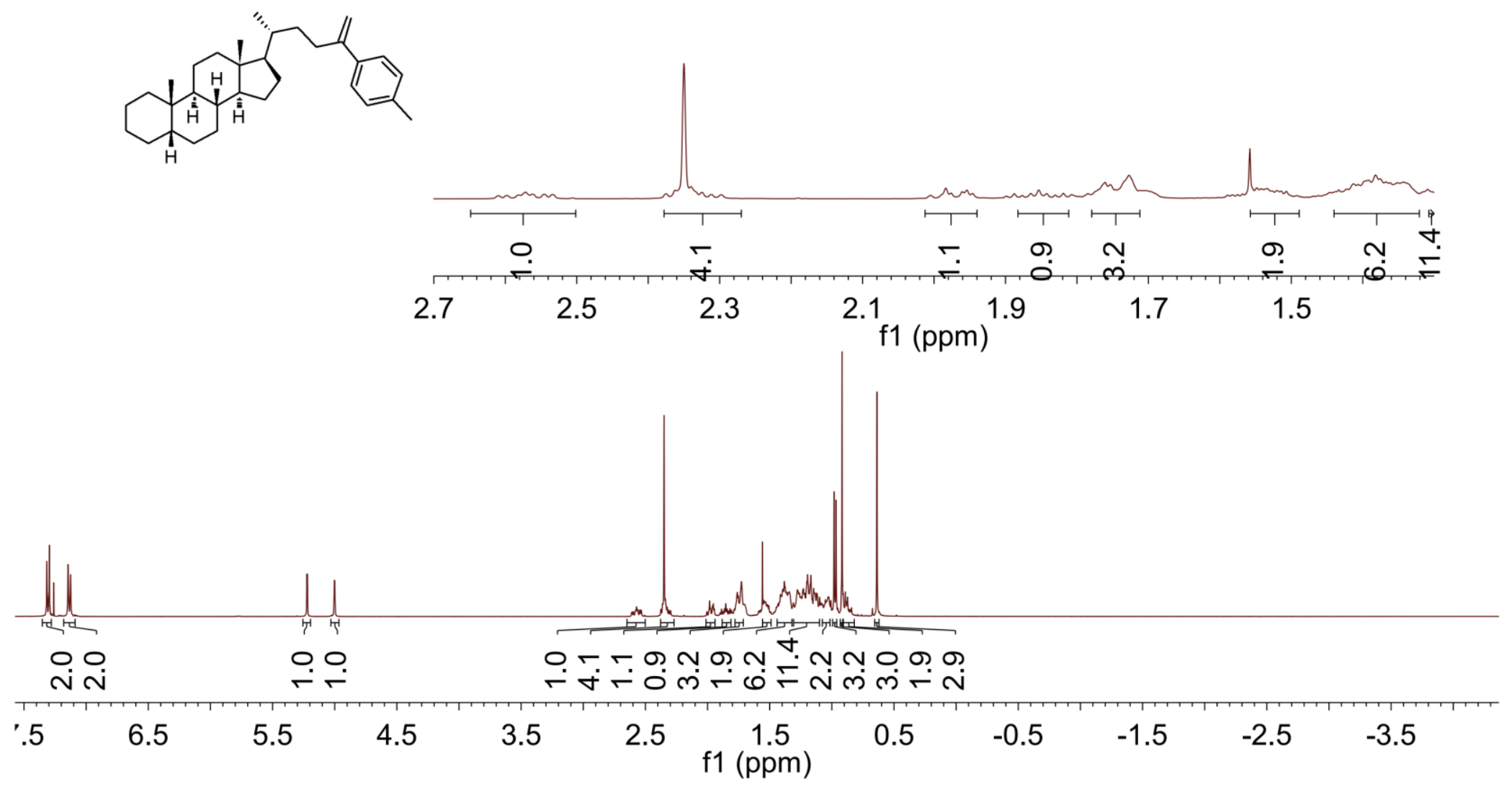



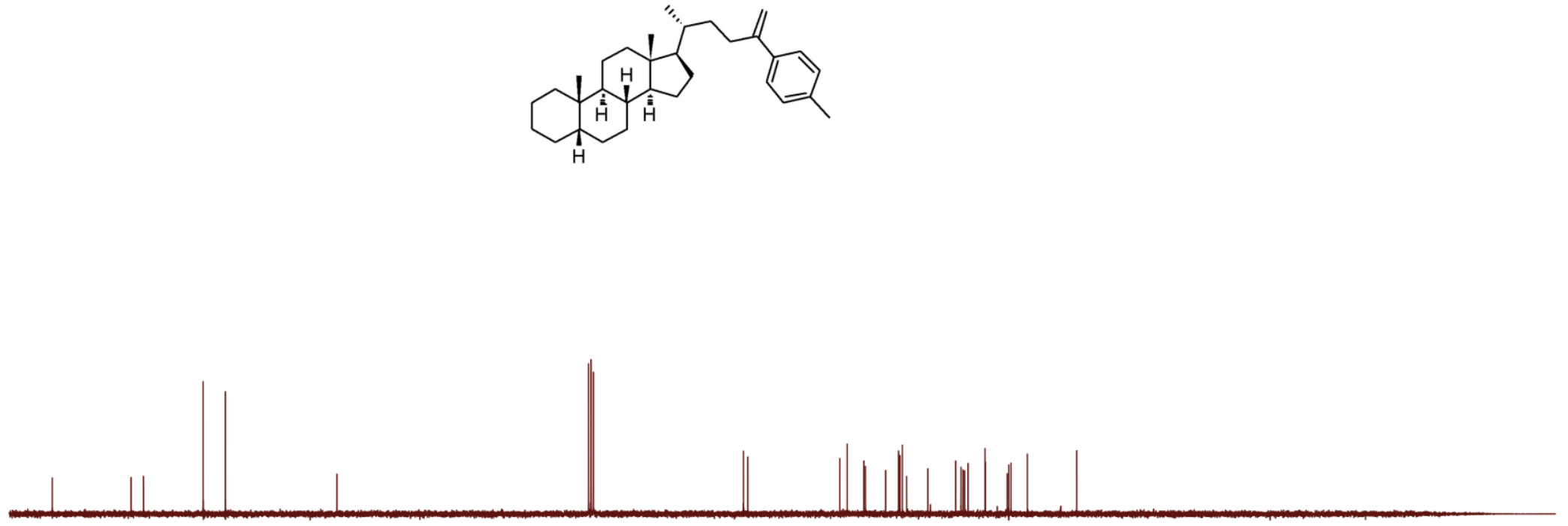

\begin{tabular}{lllllllllllllll}
\hline 150 & 130 & 110 & 90 & 80 & 70 & $\underset{\mathrm{f} 1(\mathrm{ppm})}{60}$ & 40 & 30 & 20 & 10 & 0 & -20 & -40
\end{tabular}


1h ${ }^{1}$ H NMR

\begin{tabular}{|c|c|c|}
\hline 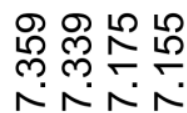 & 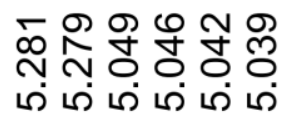 & 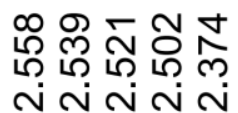 \\
\hline
\end{tabular}

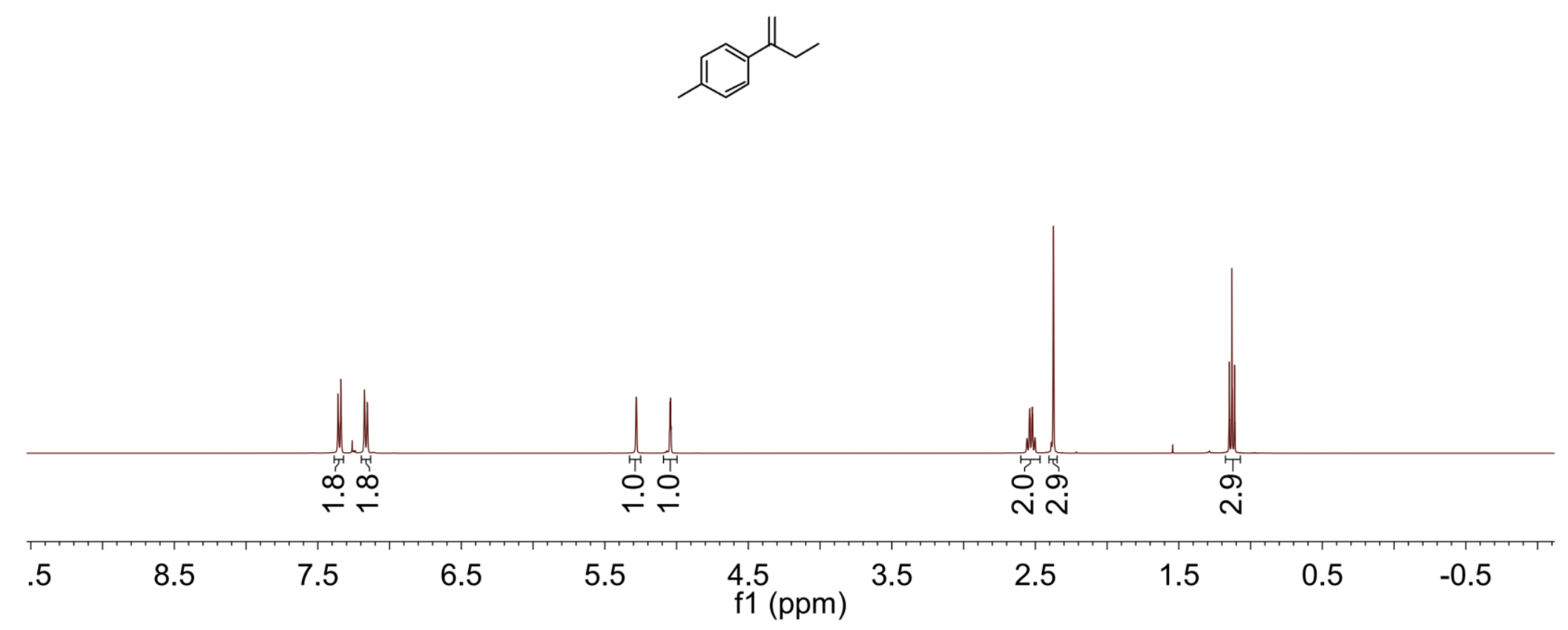




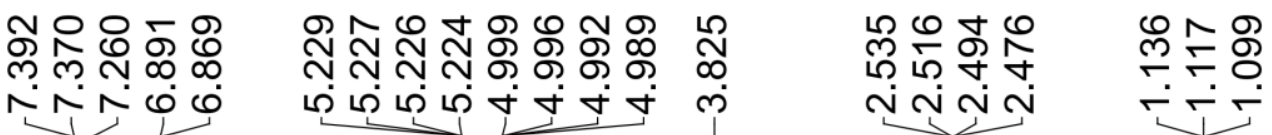

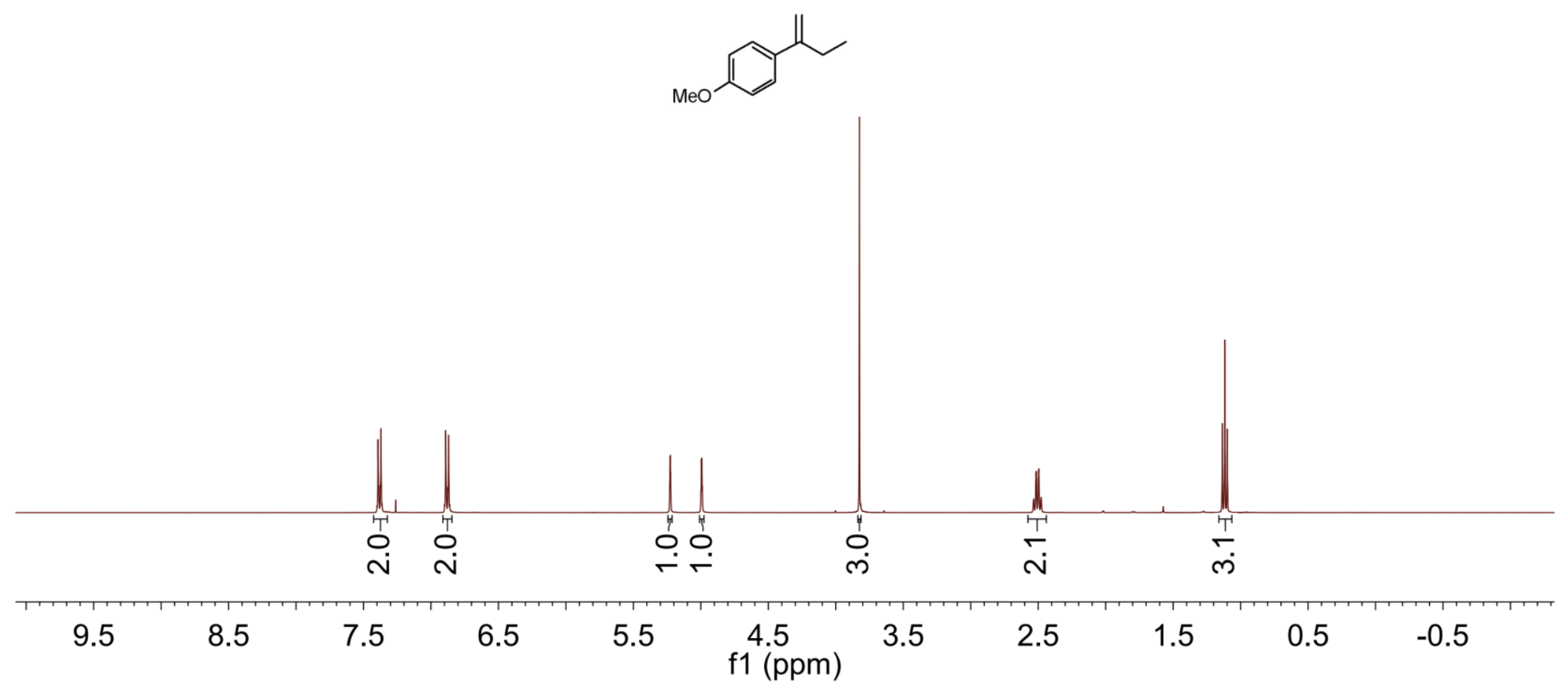


${ }_{1 j}{ }^{1} H_{N M R}$

\begin{tabular}{|c|c|c|}
\hline 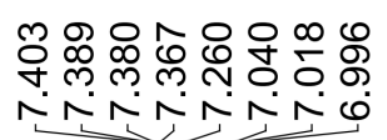 & 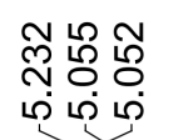 & 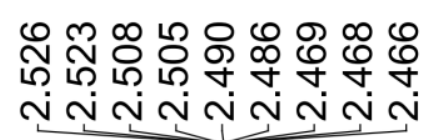 \\
\hline
\end{tabular}

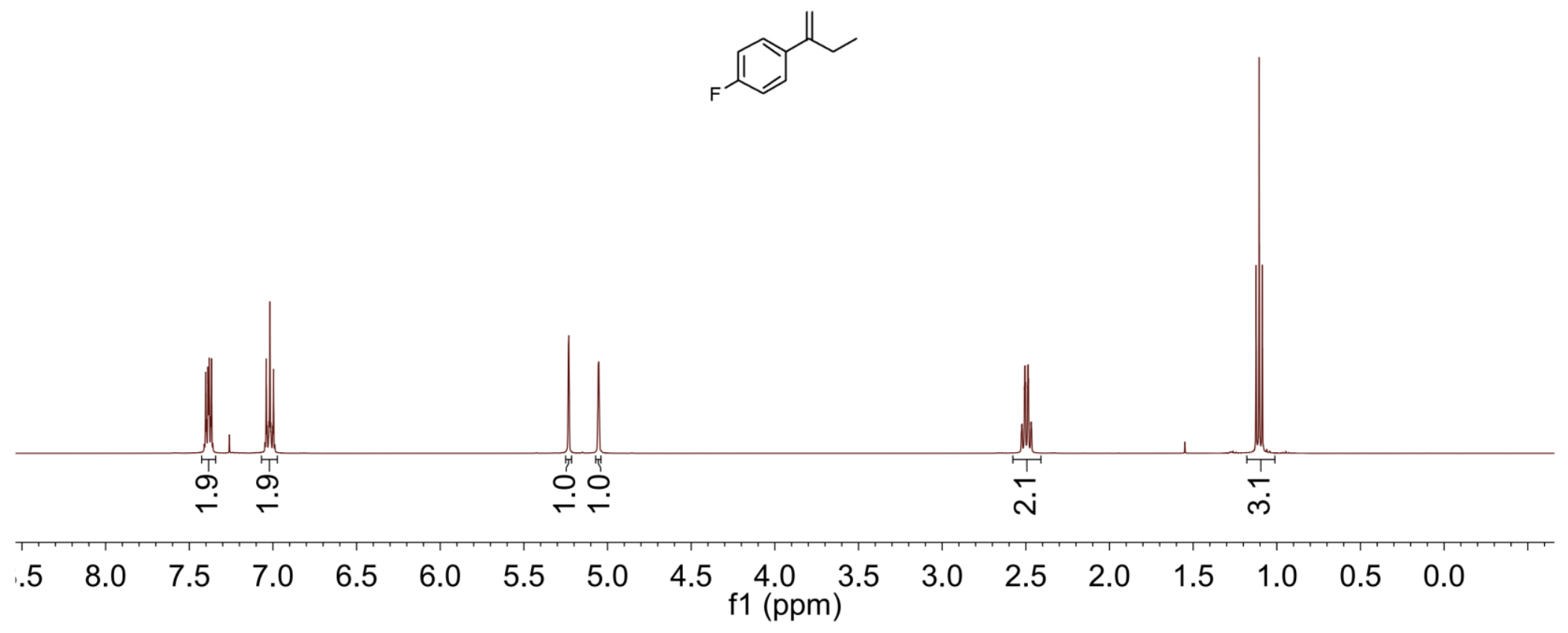


1j ${ }^{19}$ F NMR
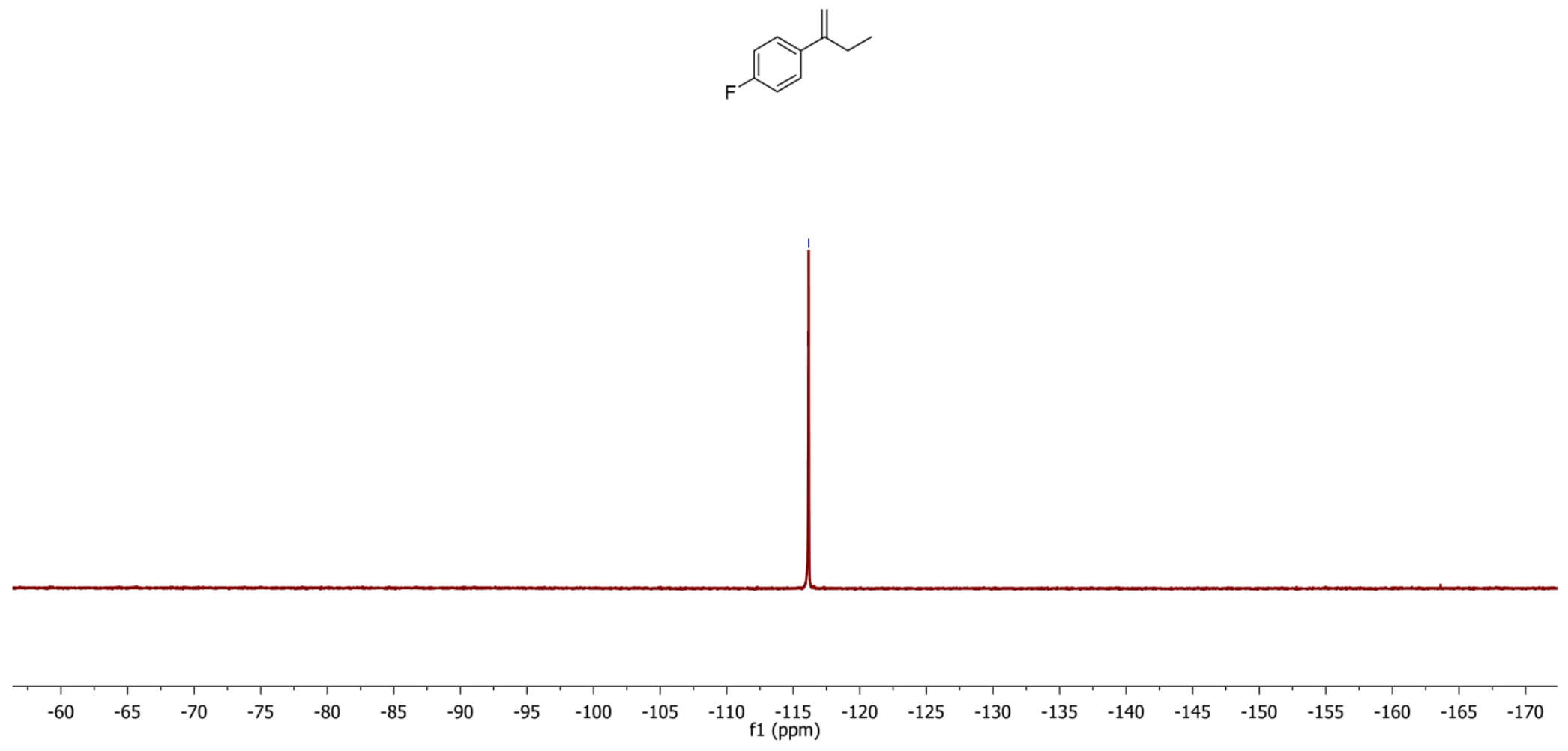
$\mathbf{1 k}{ }^{1} \mathrm{H} N M R$

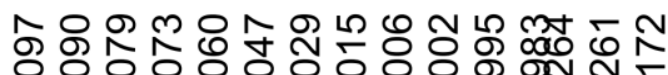 \\ ب.}

N N

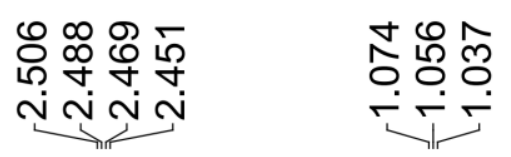
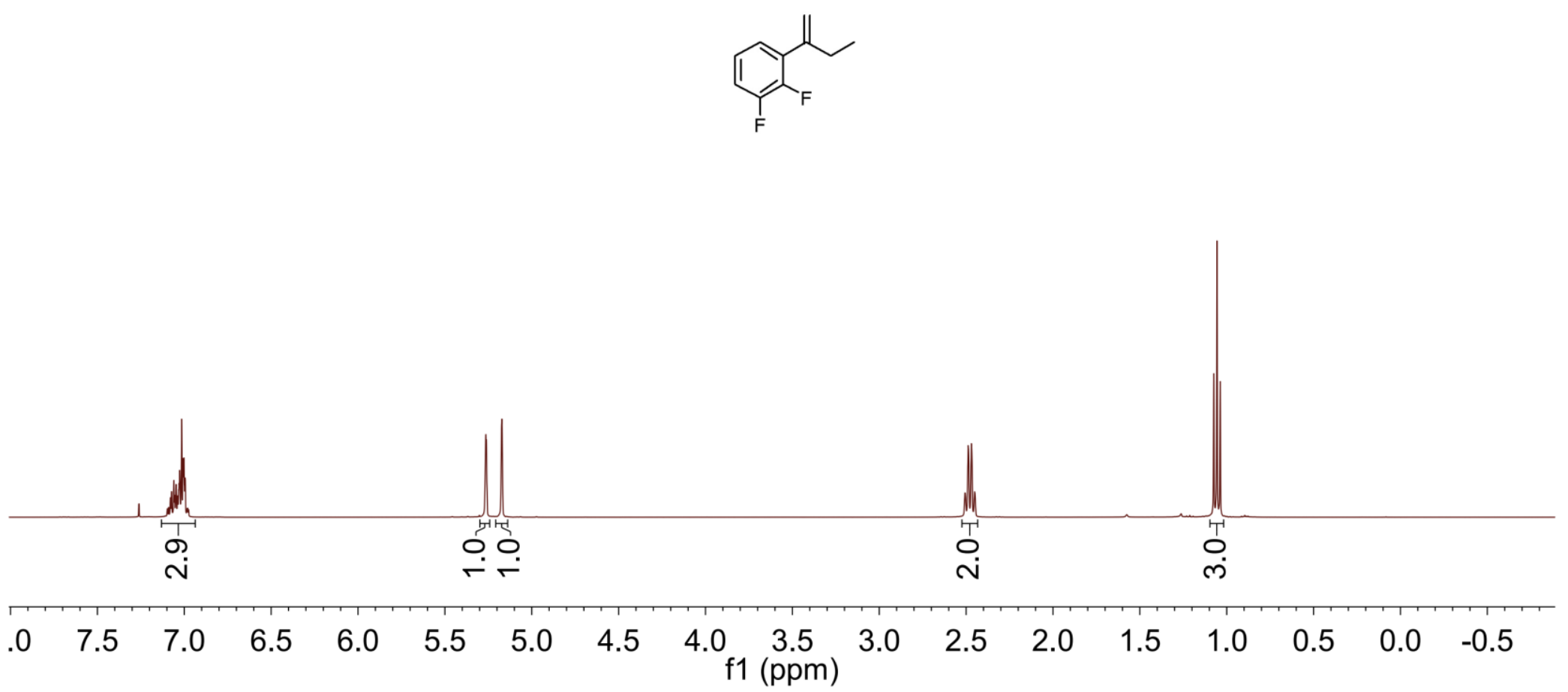


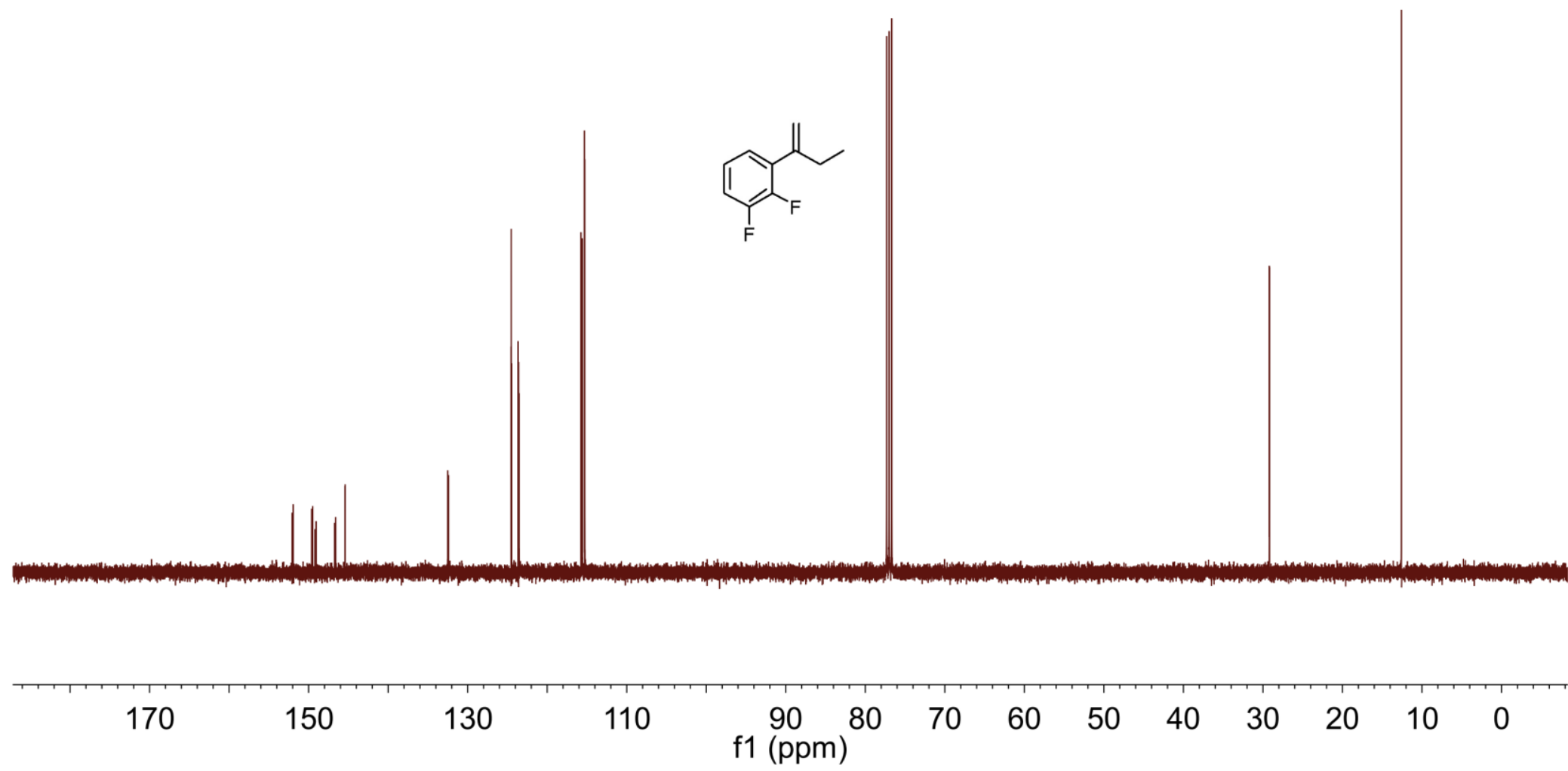



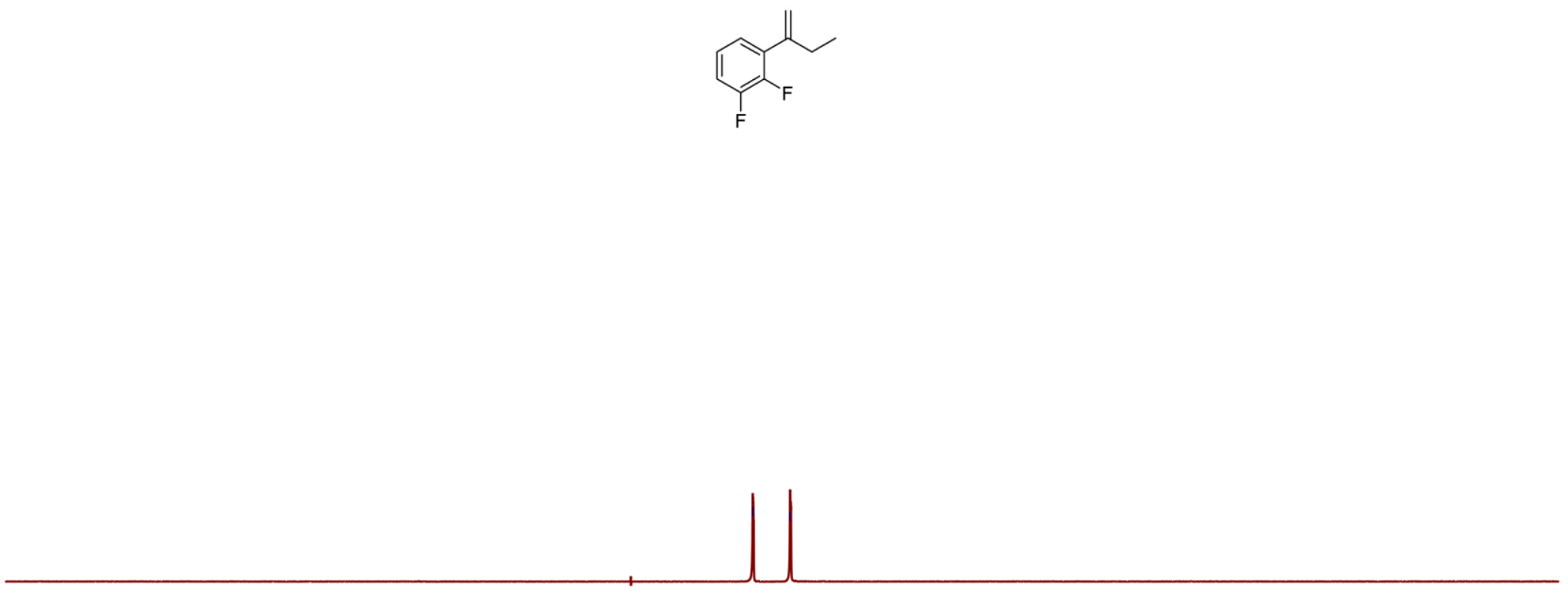


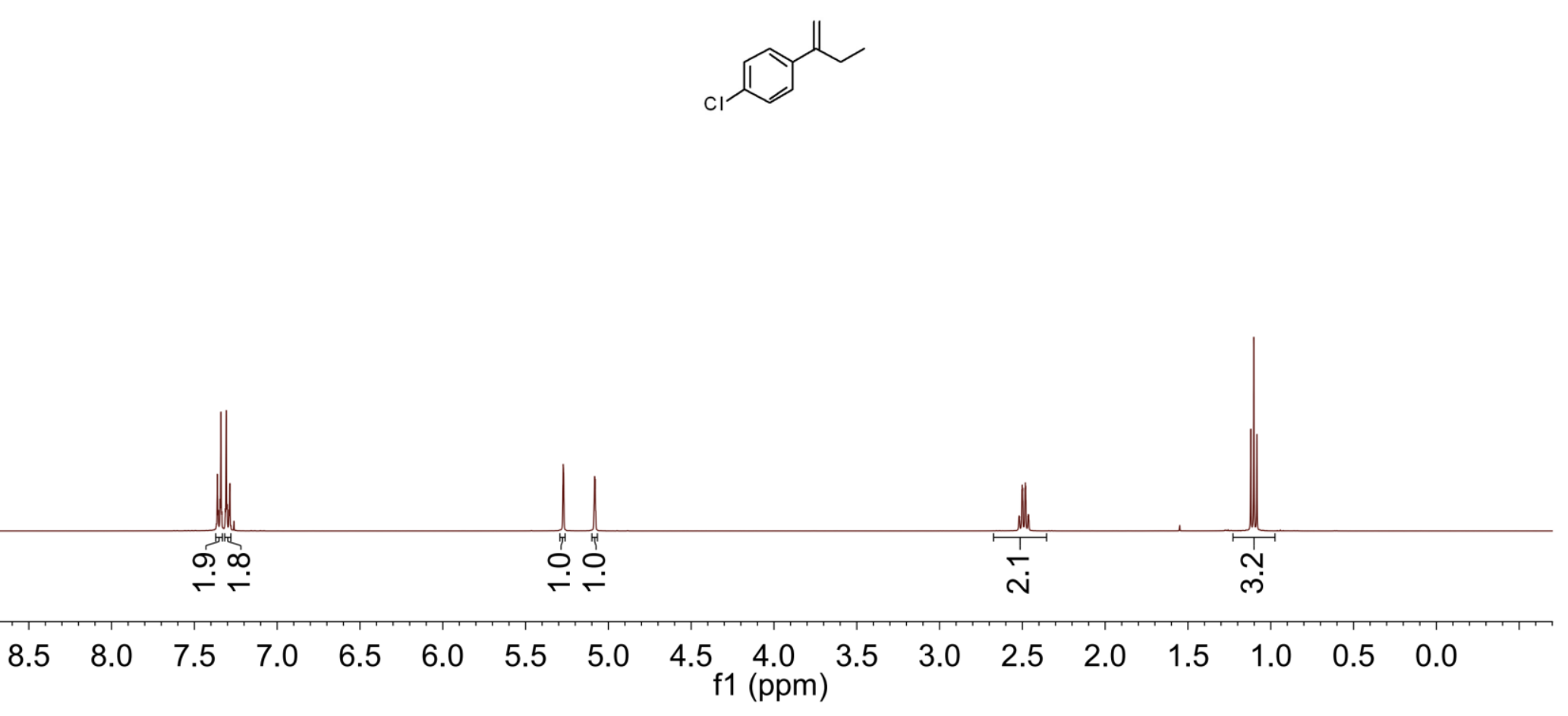




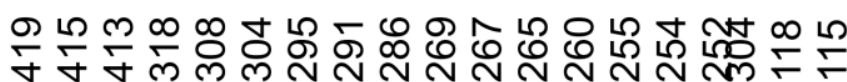

寸 寸 オ m

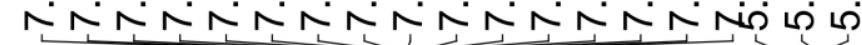

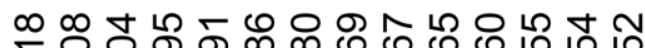

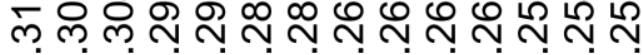

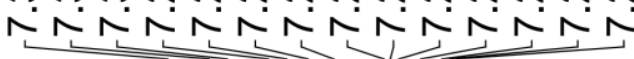
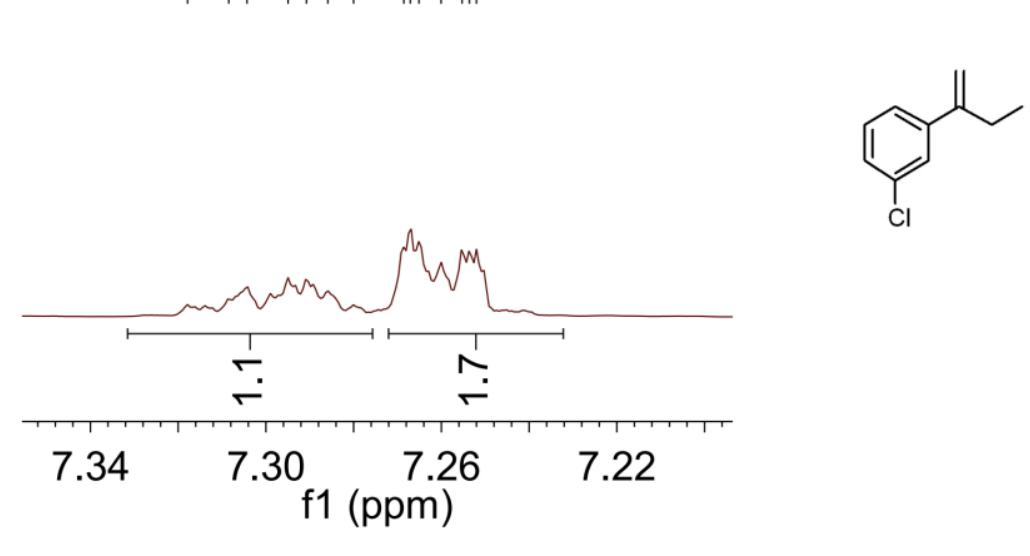

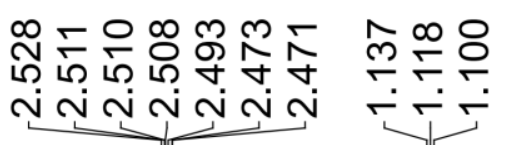

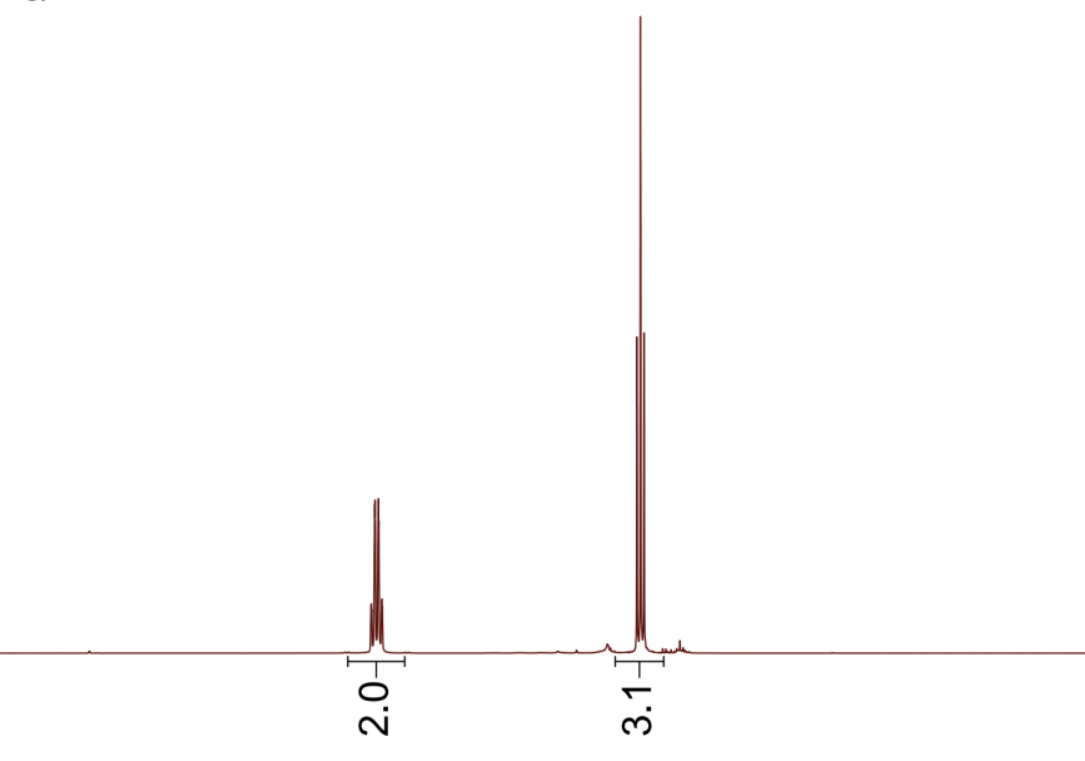

\begin{tabular}{|c|c|c|c|c|c|c|c|c|c|}
\hline 1.5 & 8.5 & 7.5 & 6.5 & 5.5 & $\begin{array}{c}4.5 \\
\mathrm{f} 1\end{array}$ & 3.5 & 2.5 & 1.5 & 0.5 \\
\hline
\end{tabular}




\begin{tabular}{|c|c|c|}
\hline 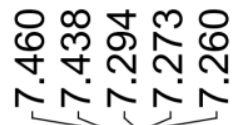 & 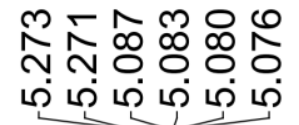 & 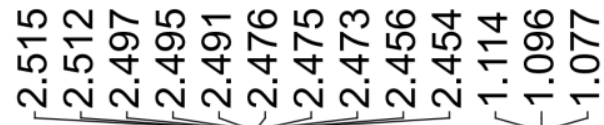 \\
\hline
\end{tabular}

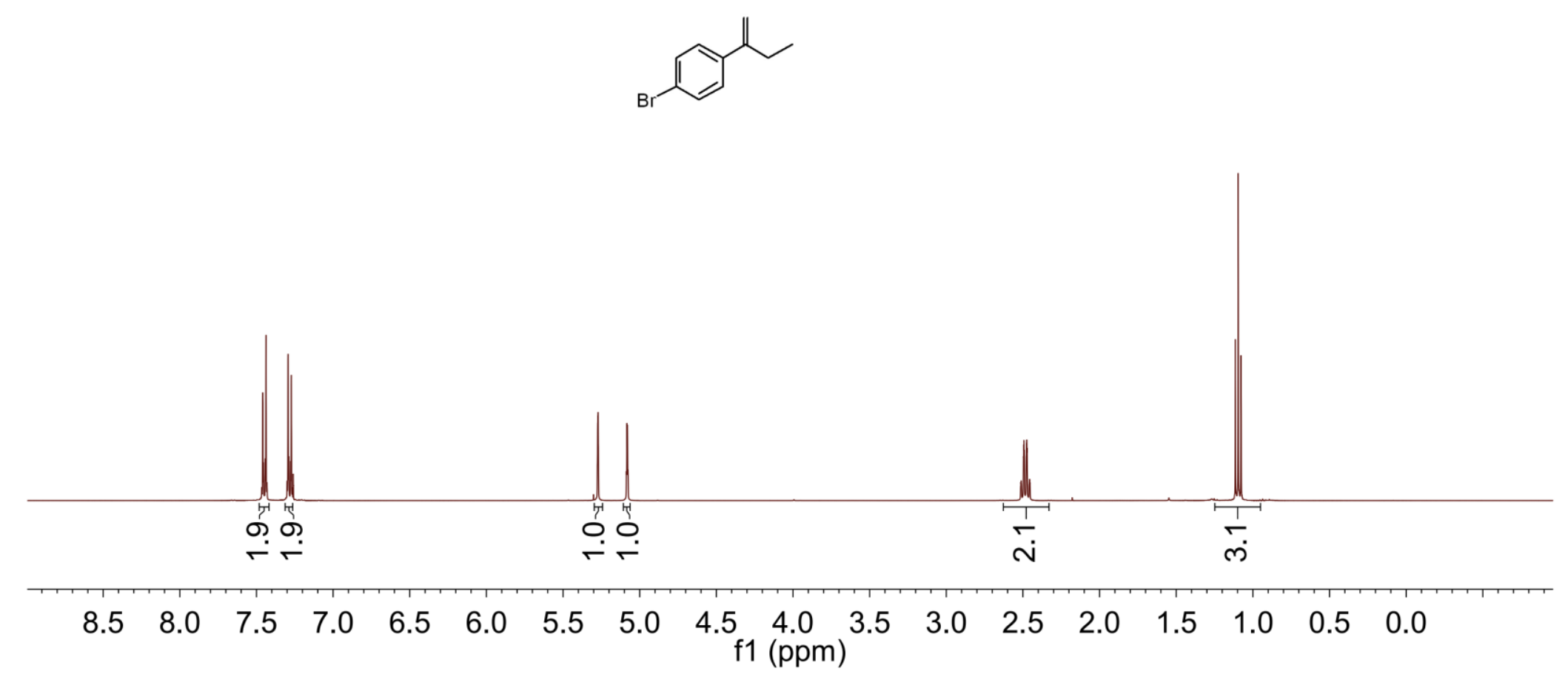




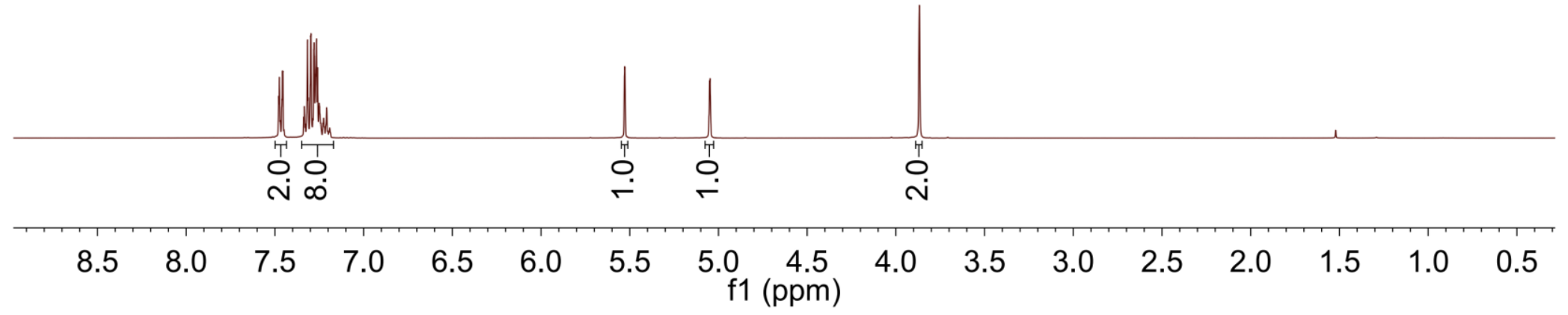


Op ${ }^{1} \mathrm{H}$ NMR

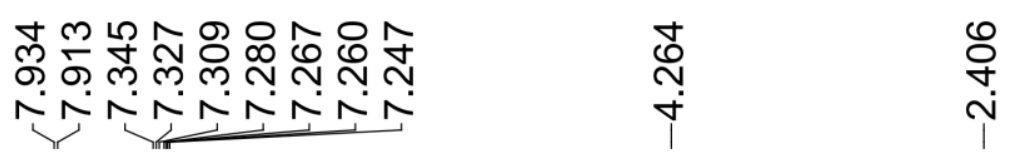
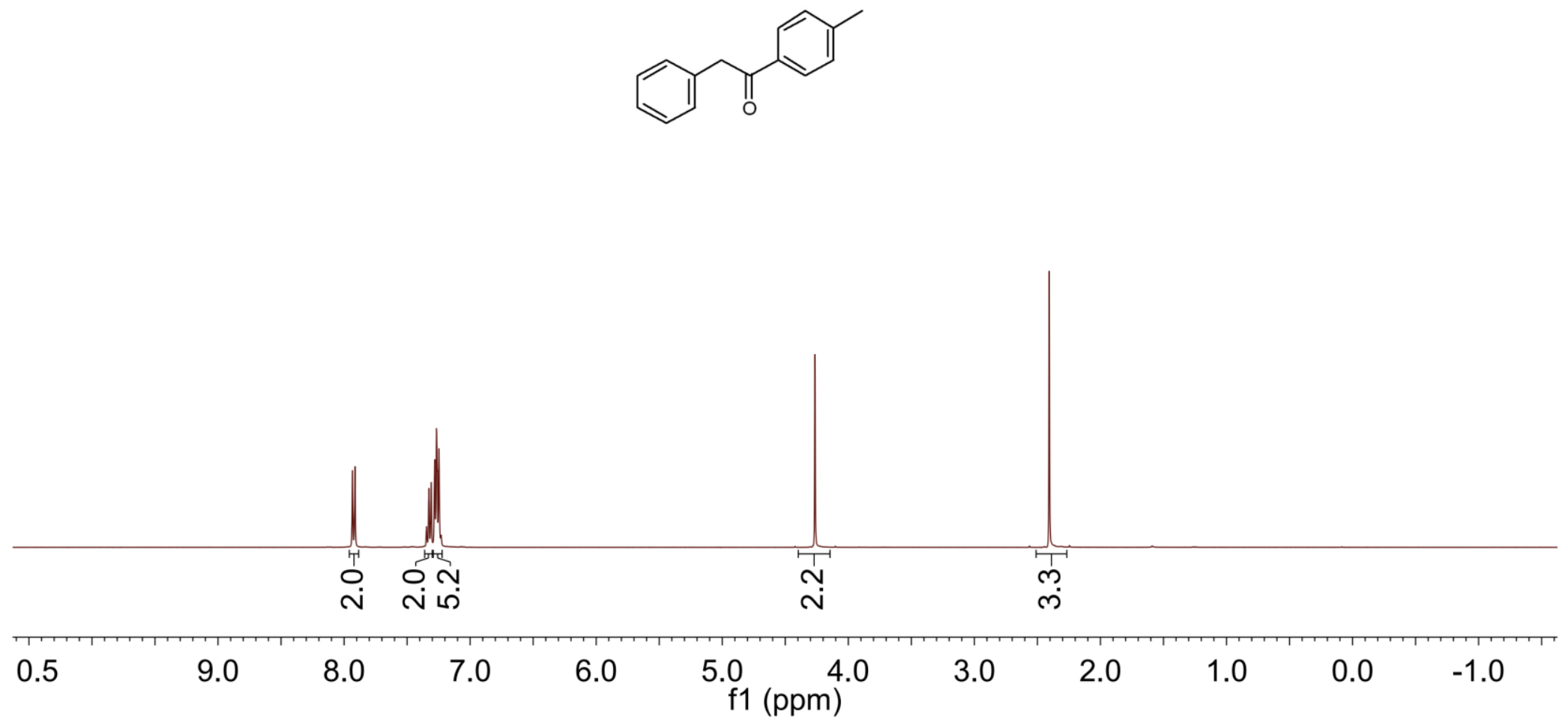


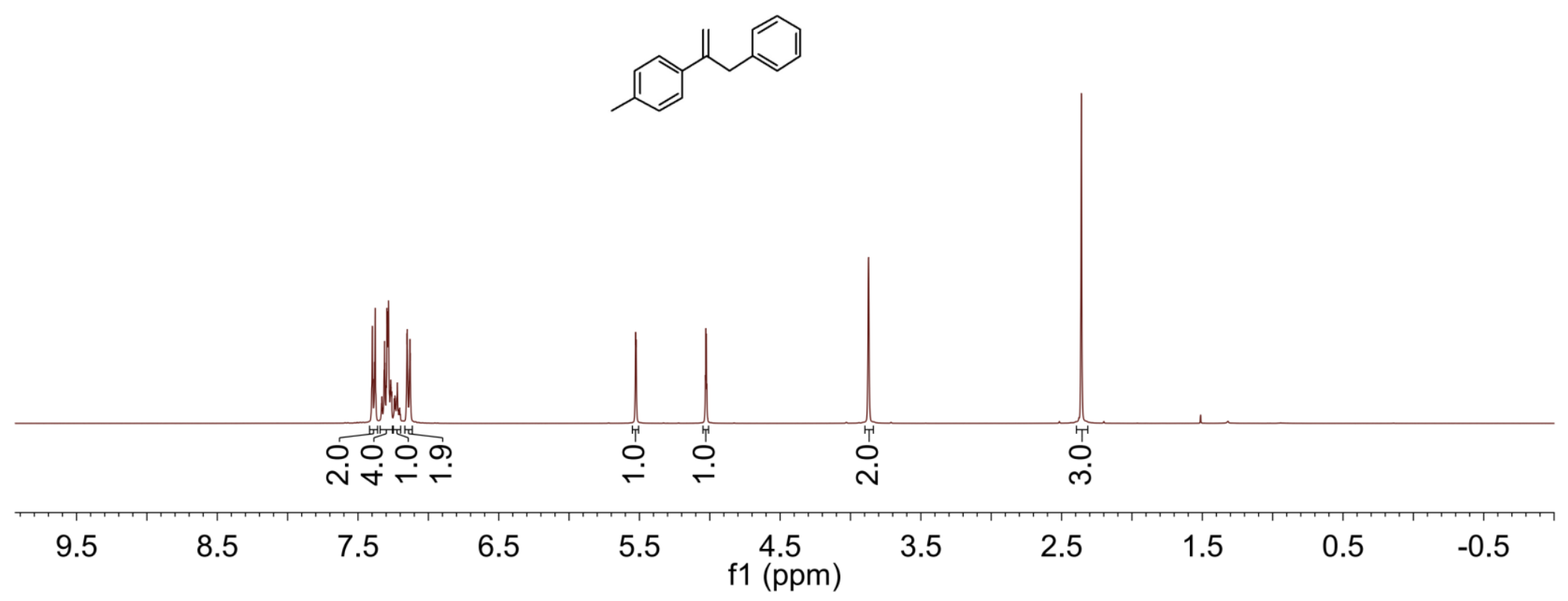



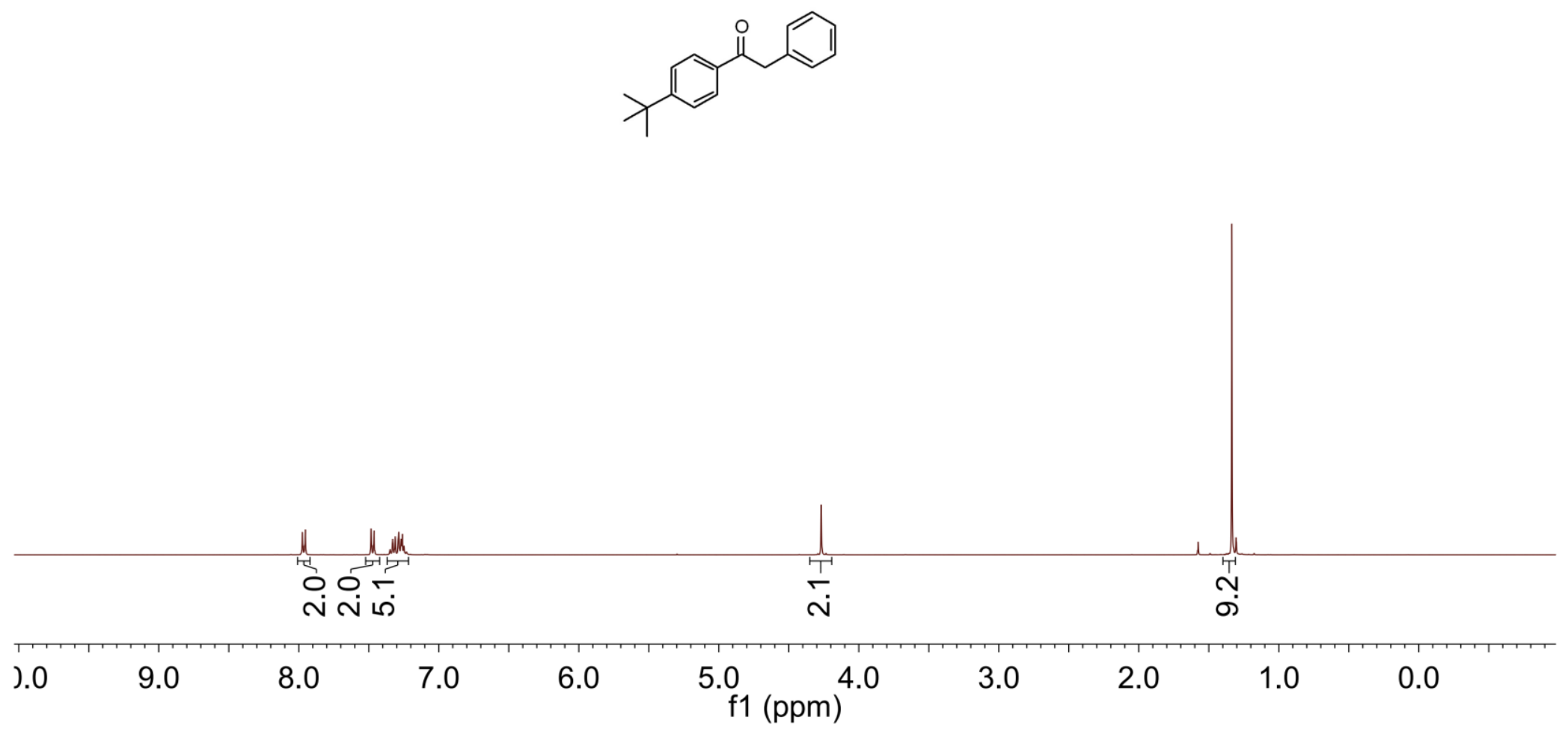


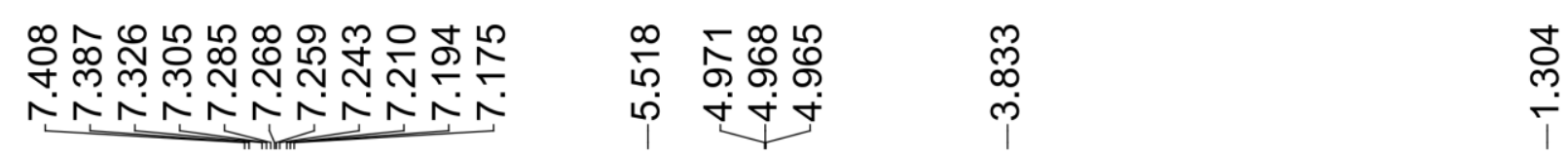
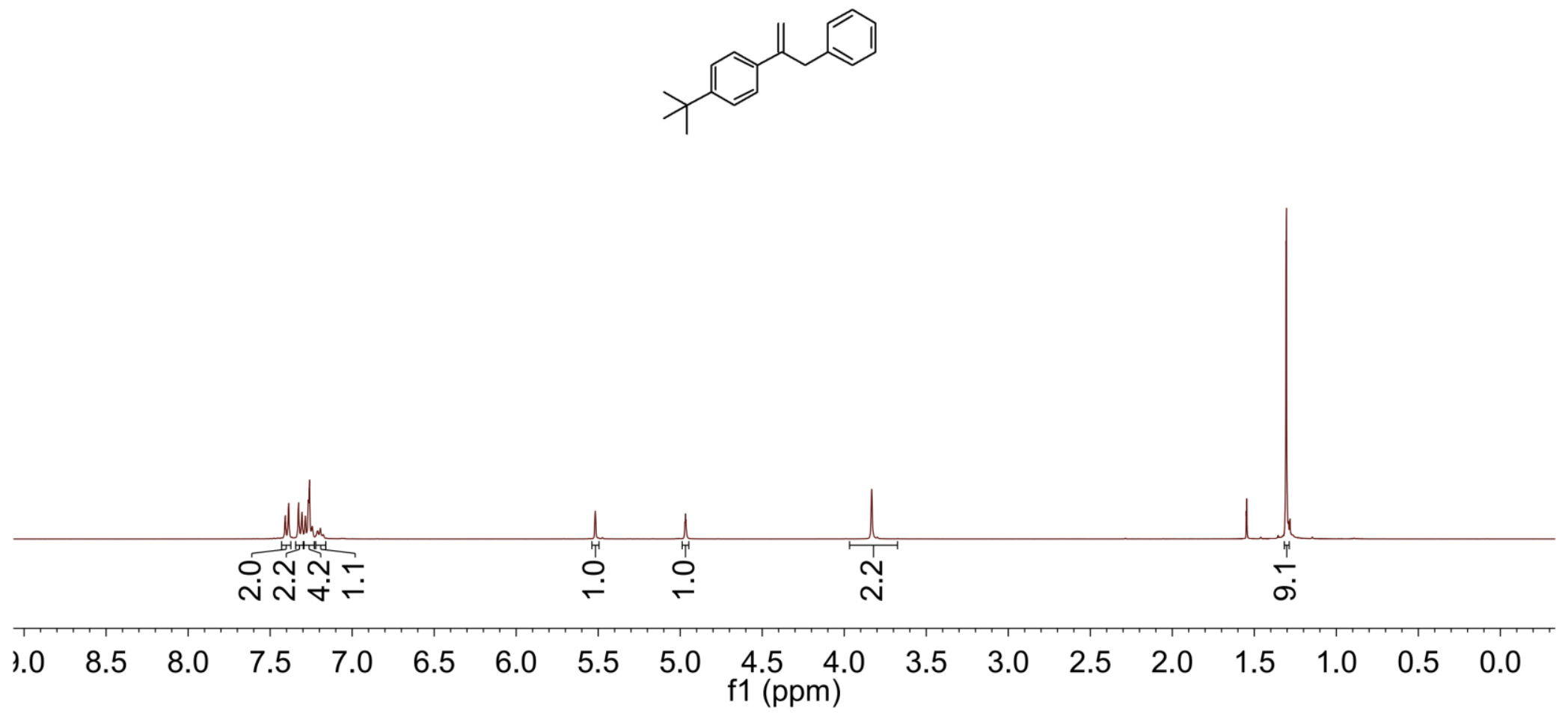


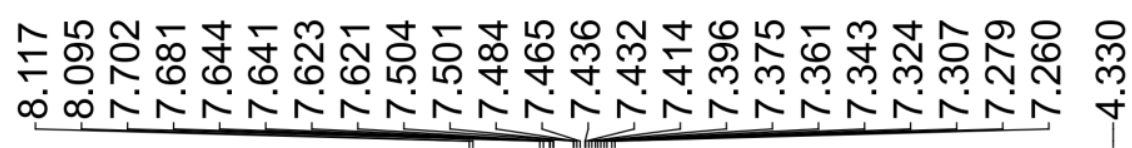

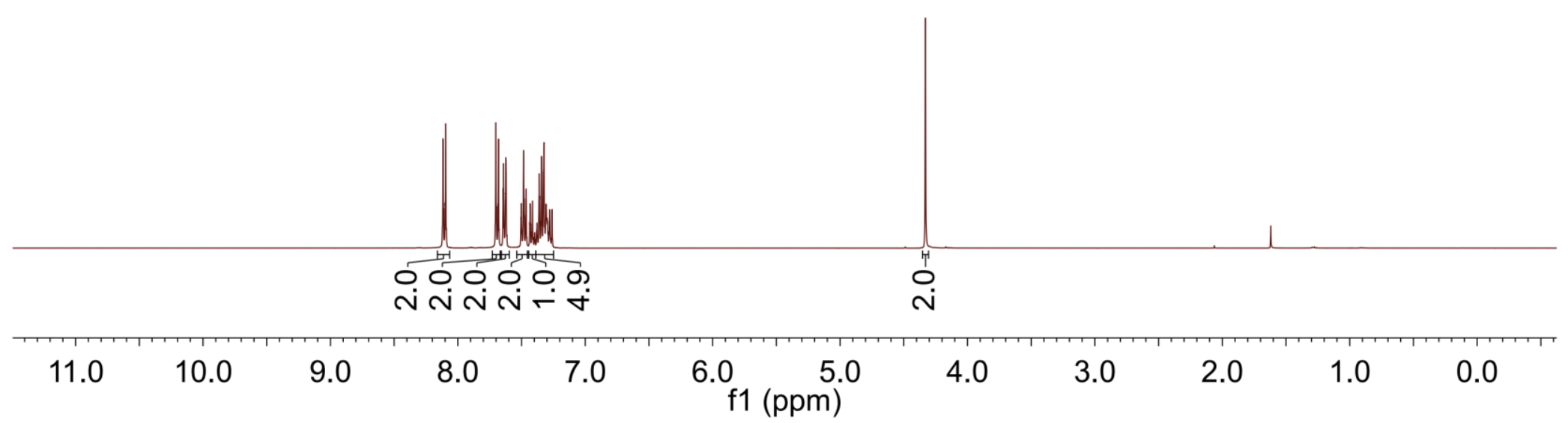




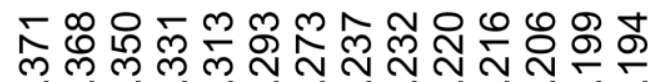

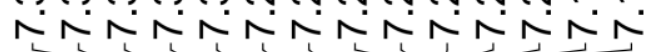

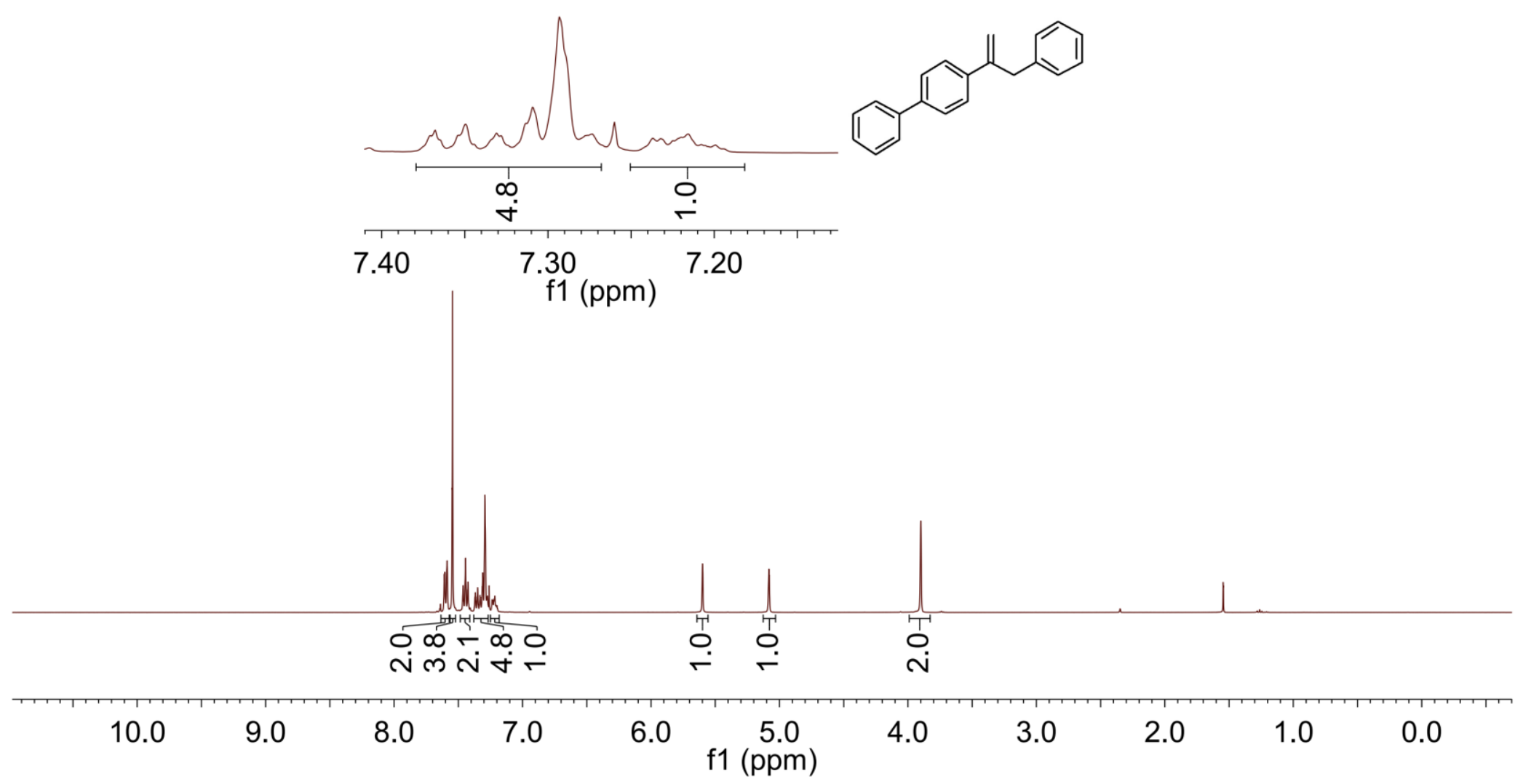




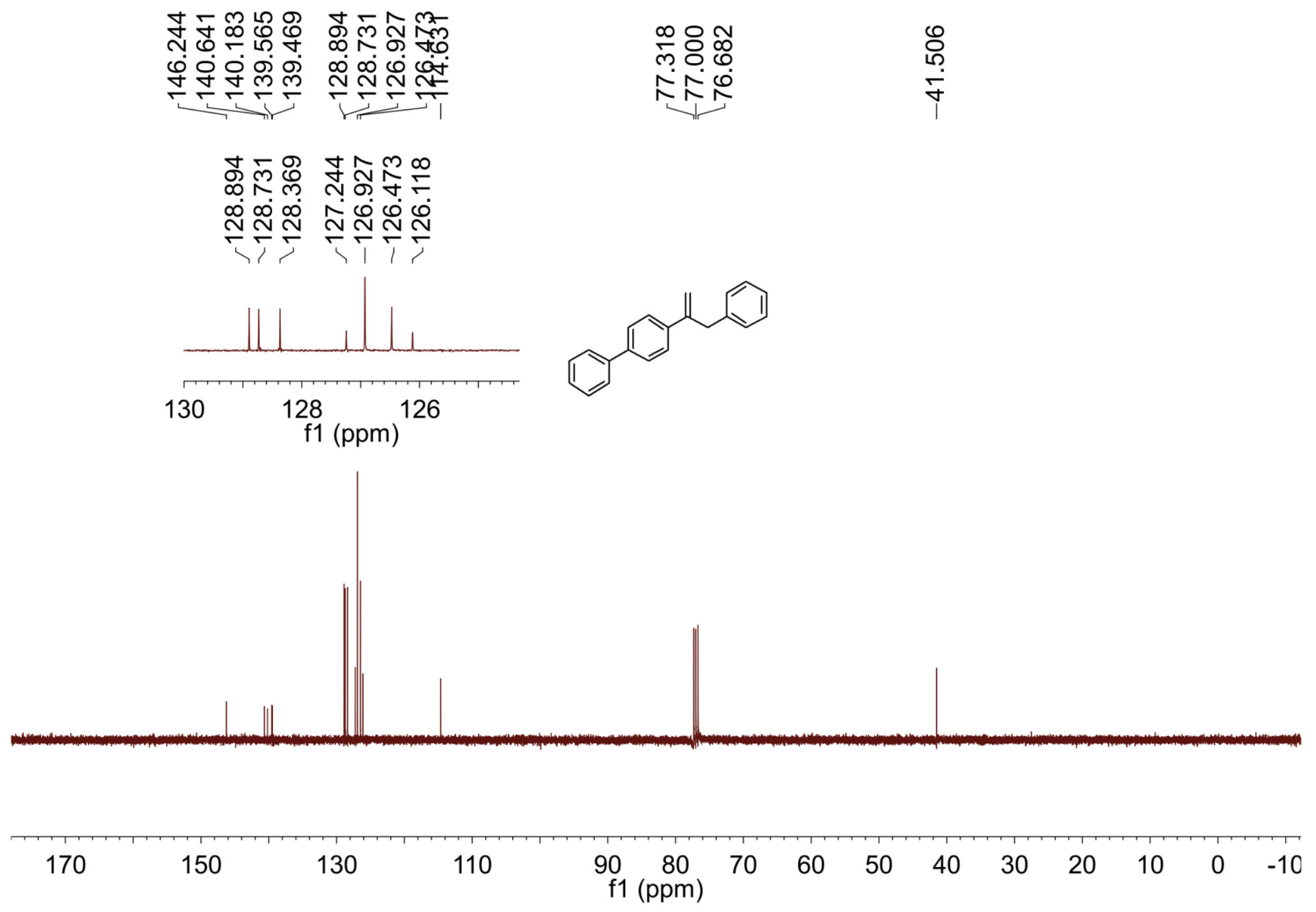



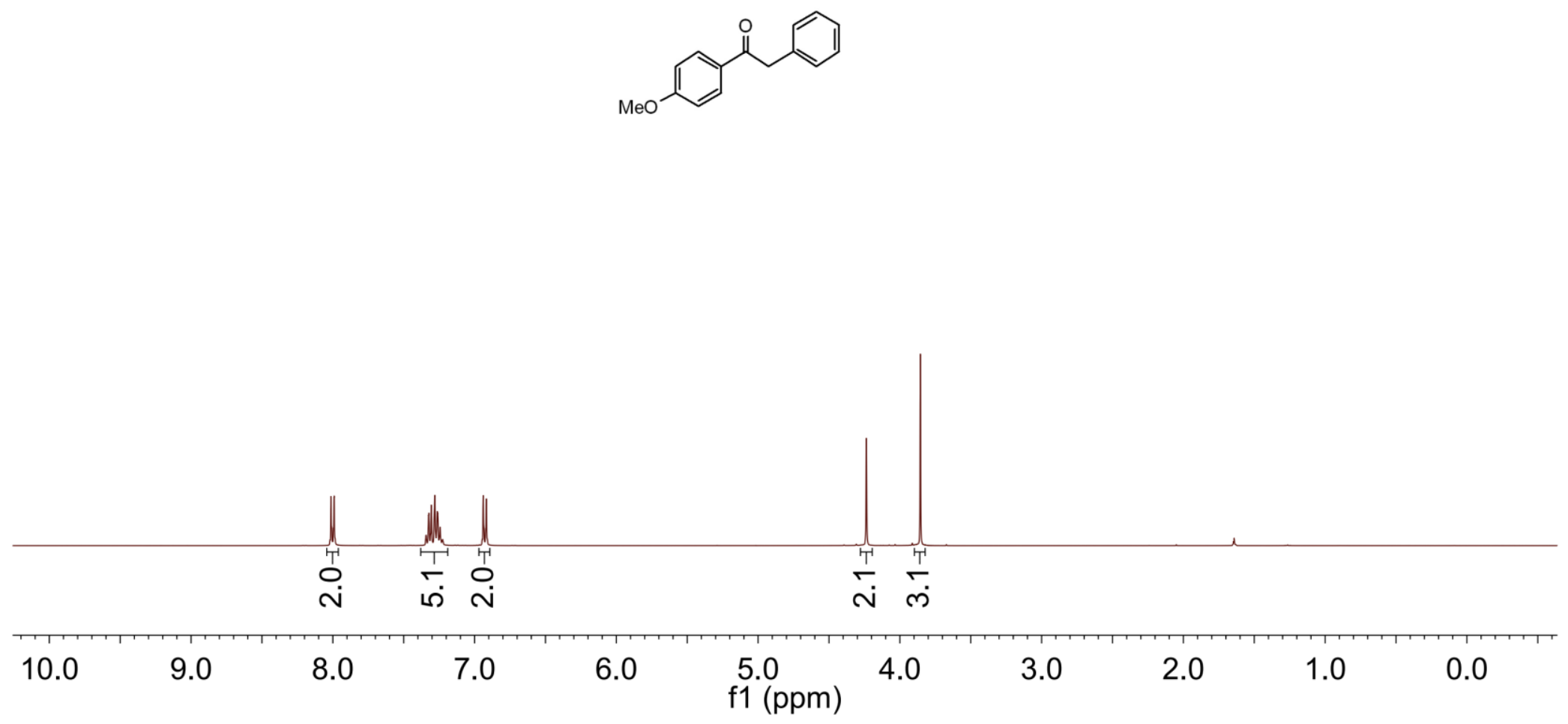


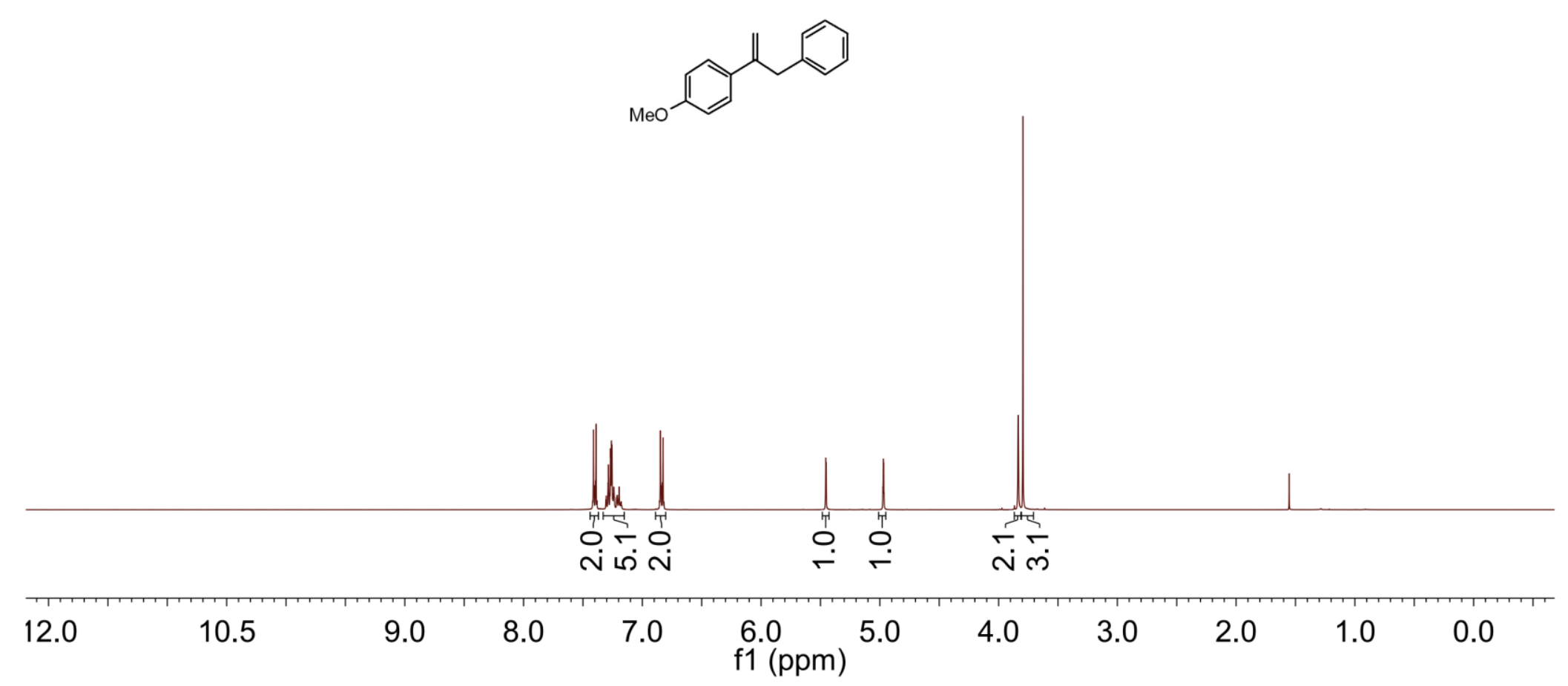



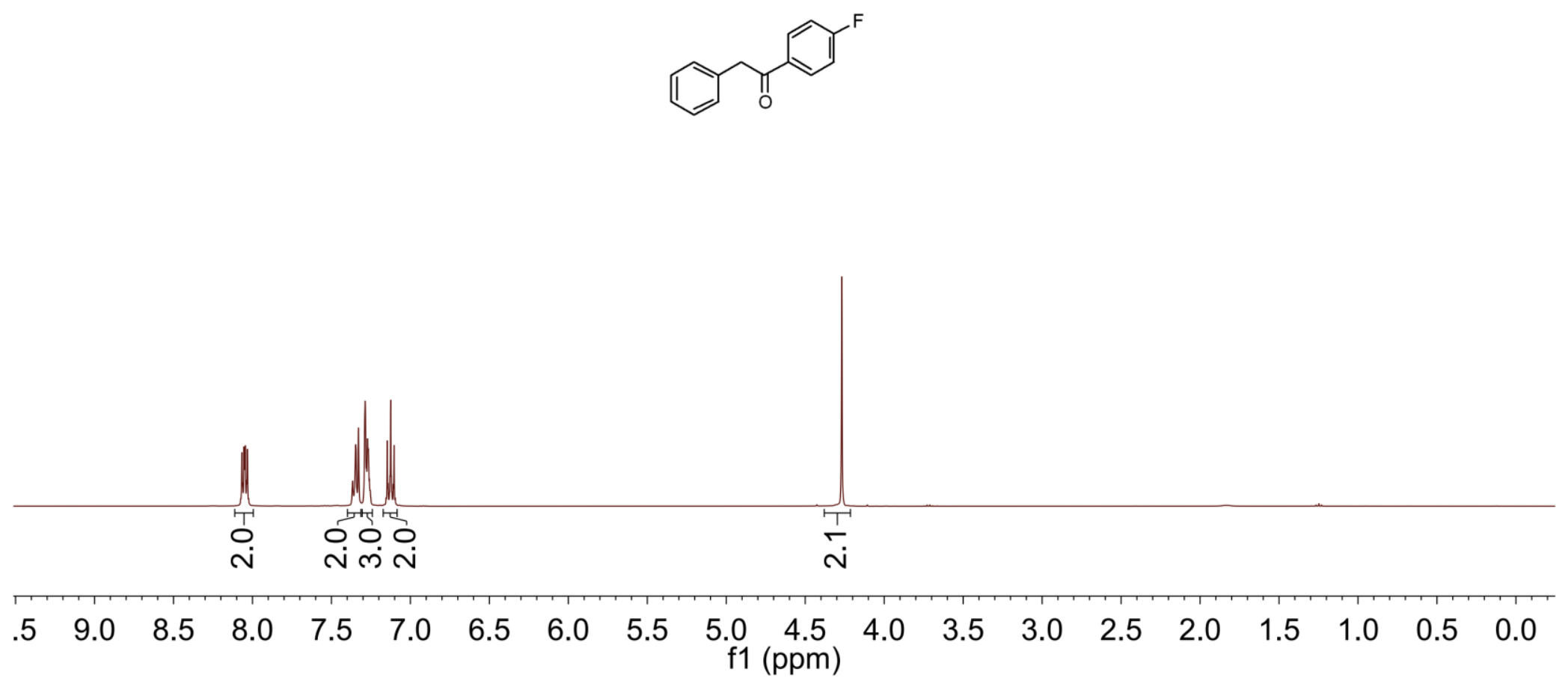
Ot ${ }^{19} \mathrm{~F}$ NMR

i̊
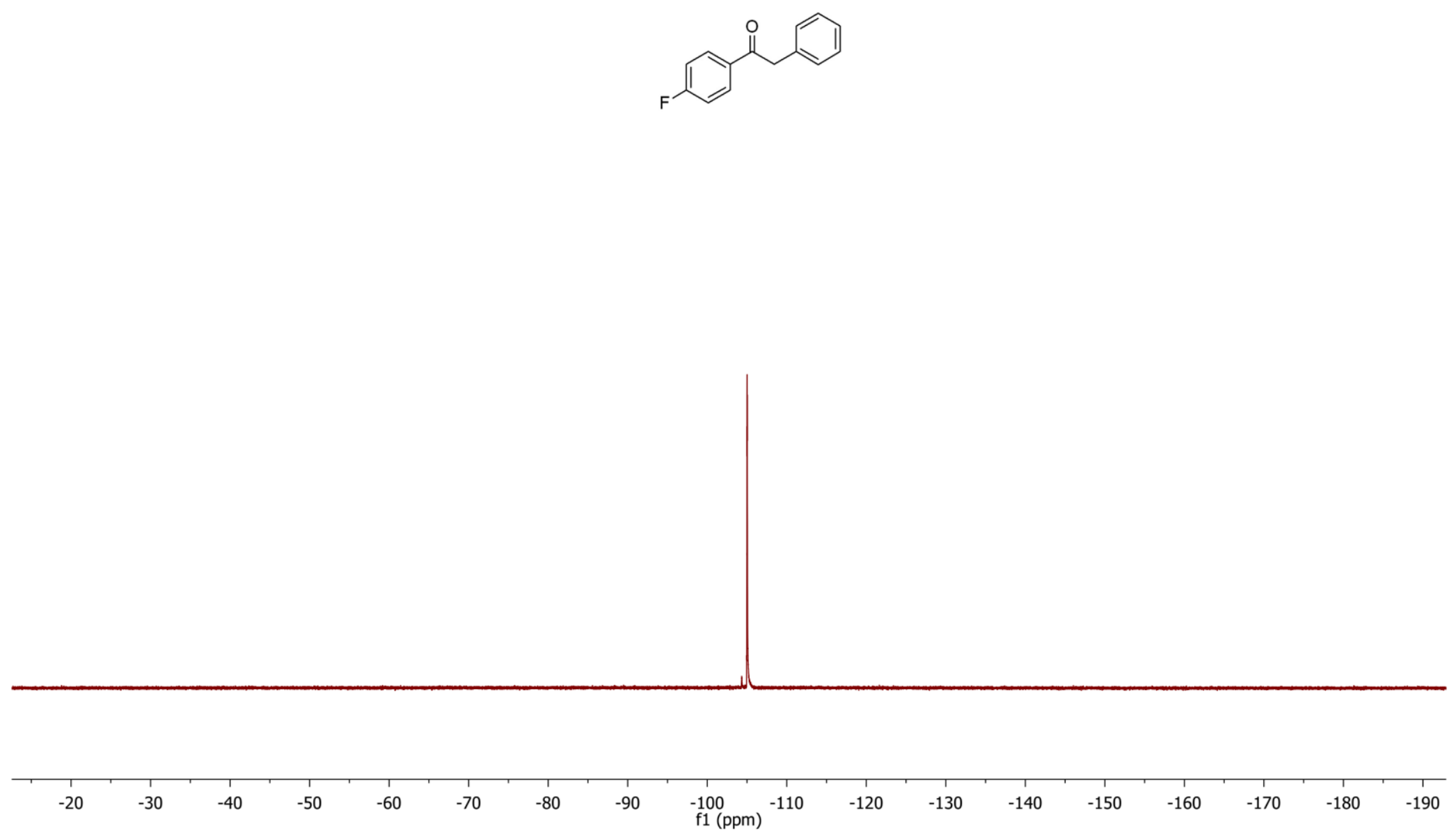
1t ${ }^{1} \mathrm{H}$ NMR

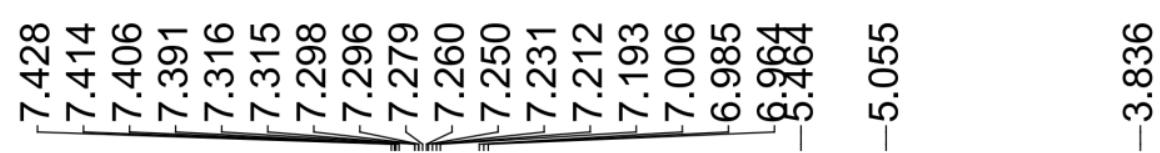

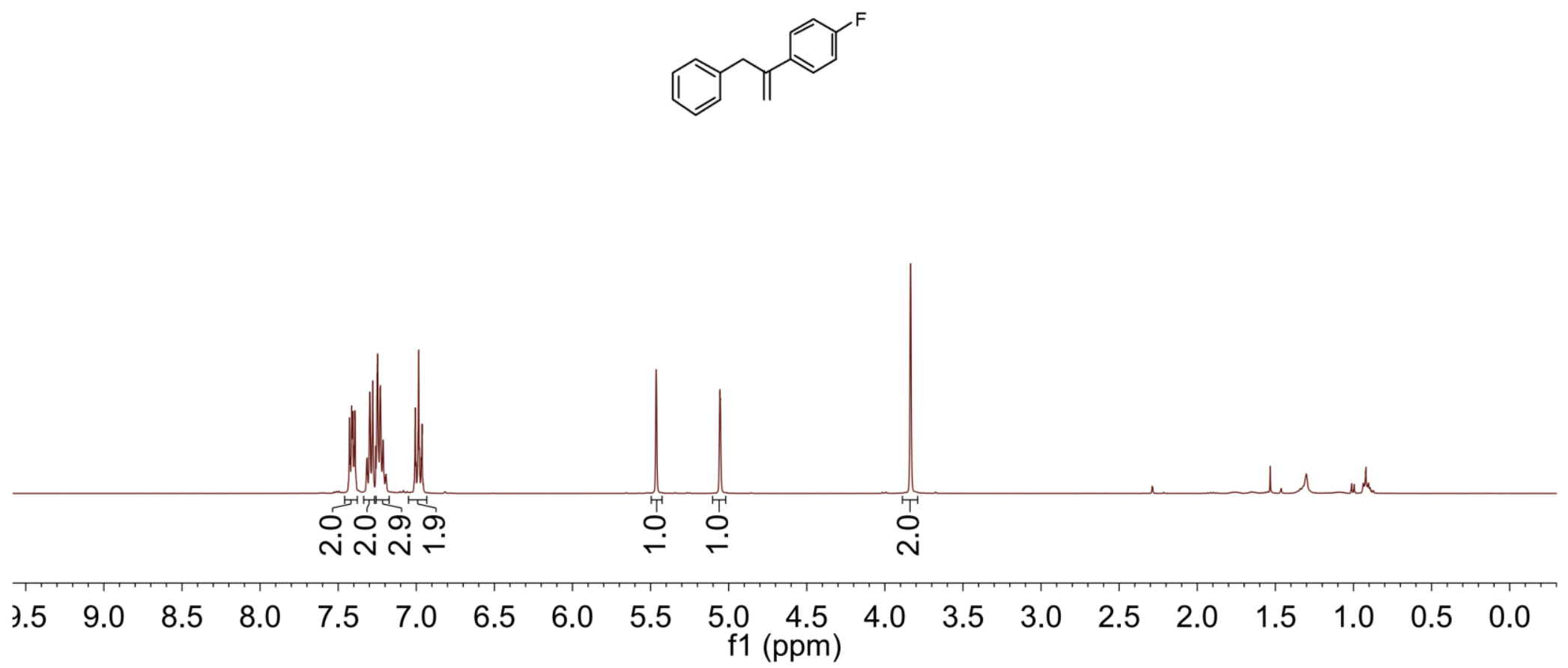


1t ${ }^{19}$ F NMR
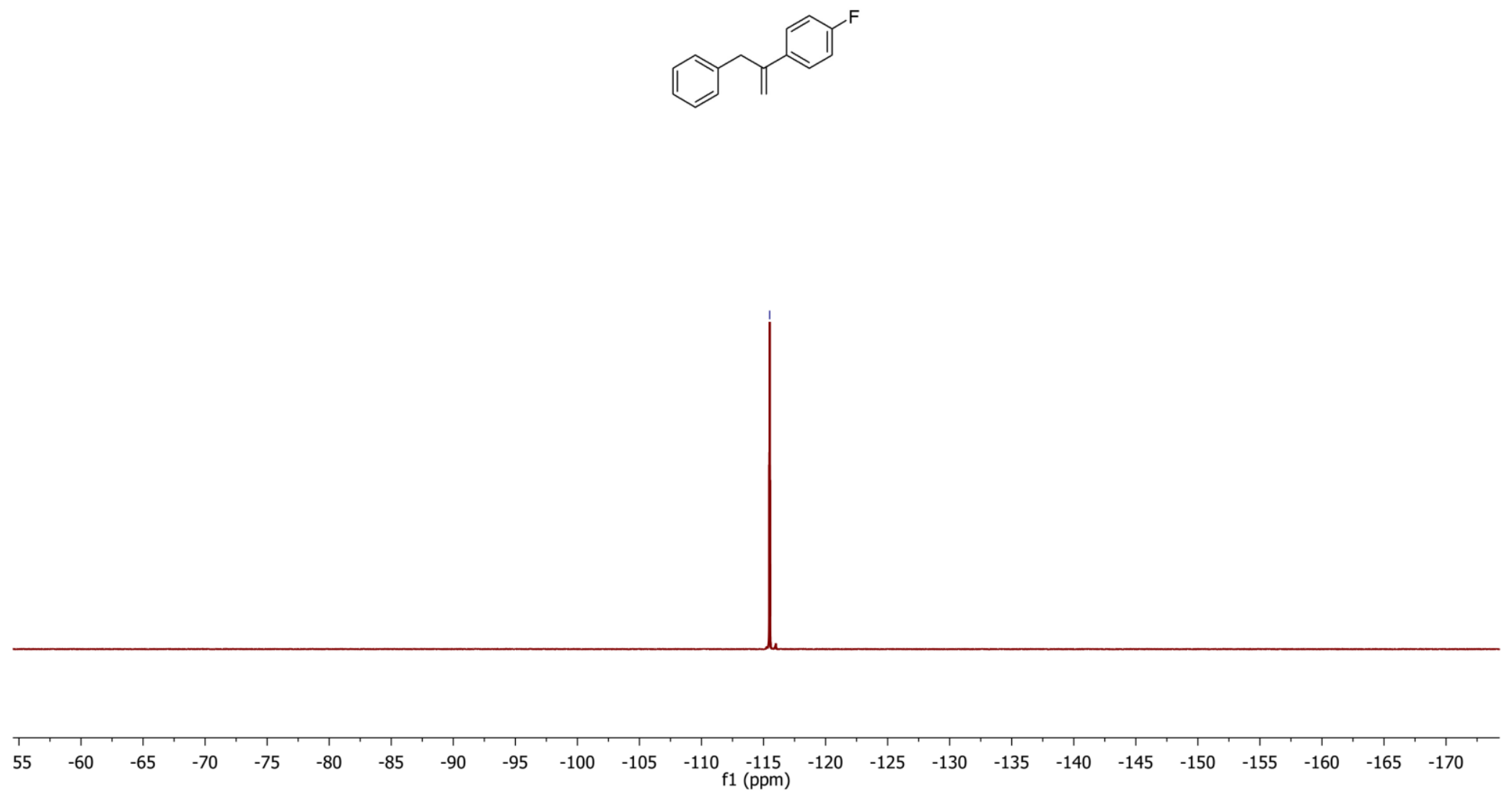
1u ${ }^{1} \mathrm{H}$ NMR

\begin{tabular}{|c|c|c|}
\hline 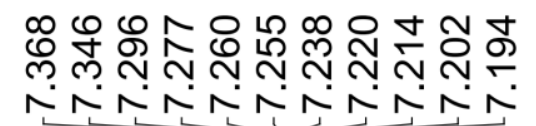 & 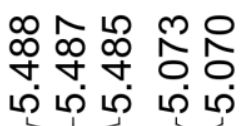 & $\frac{N}{\infty}$ \\
\hline
\end{tabular}
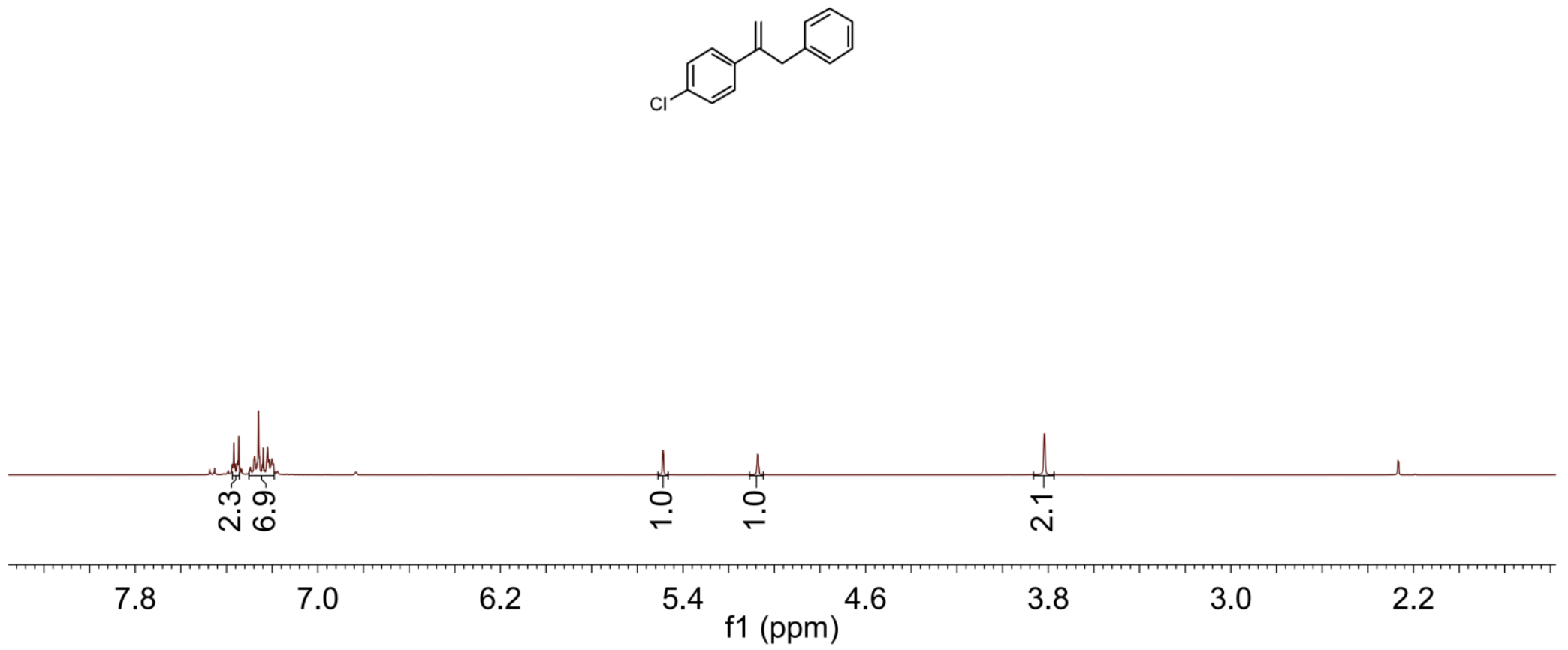


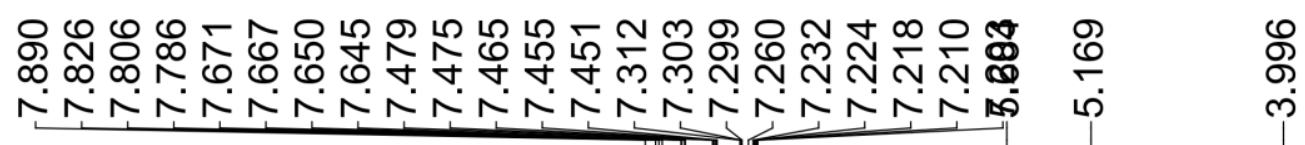

స๐ํㅇำ

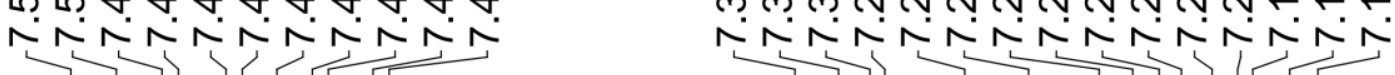
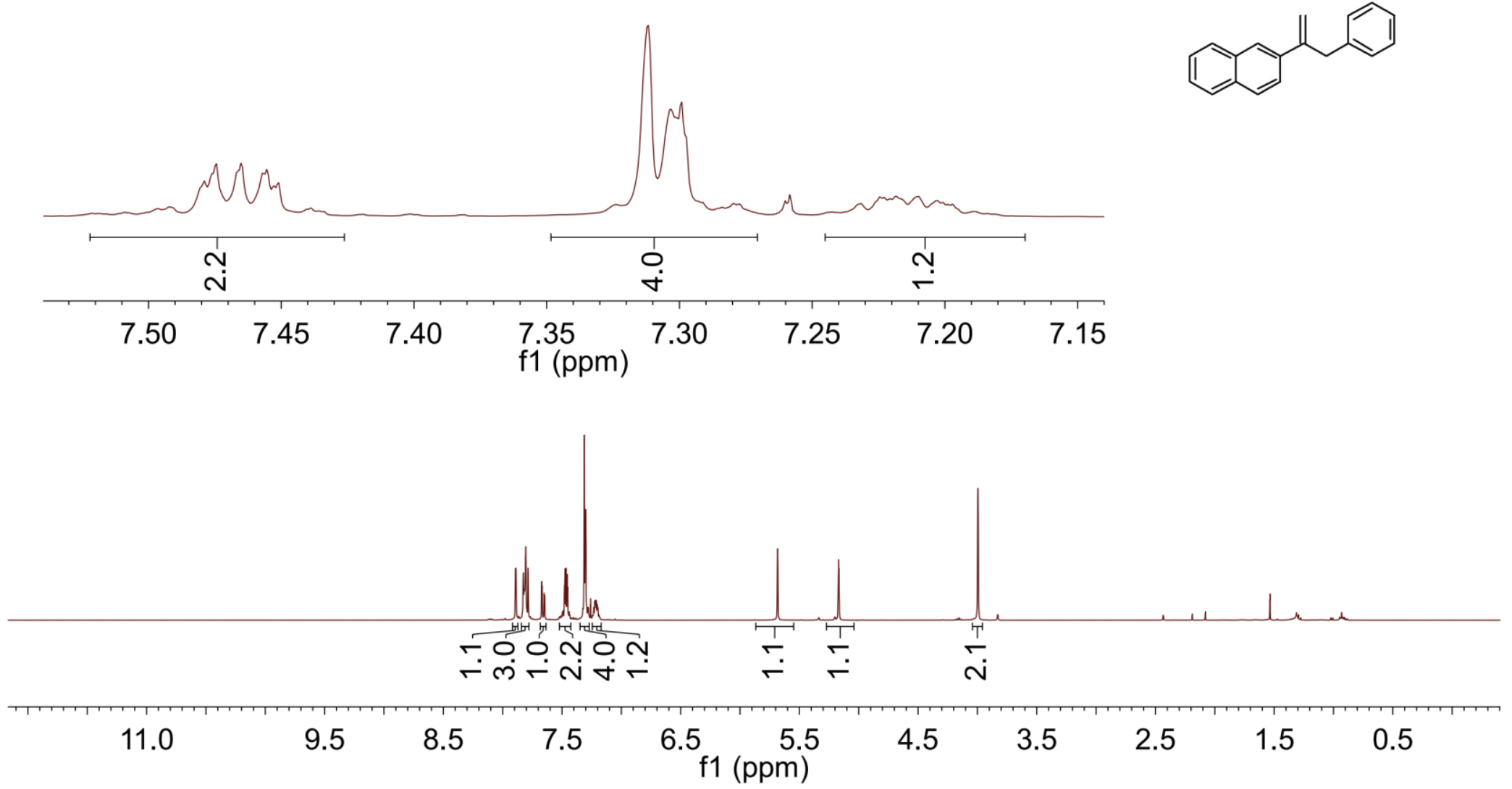


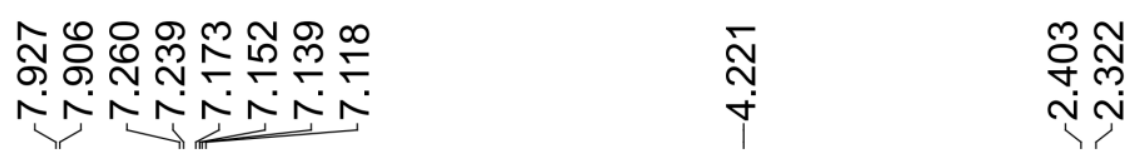

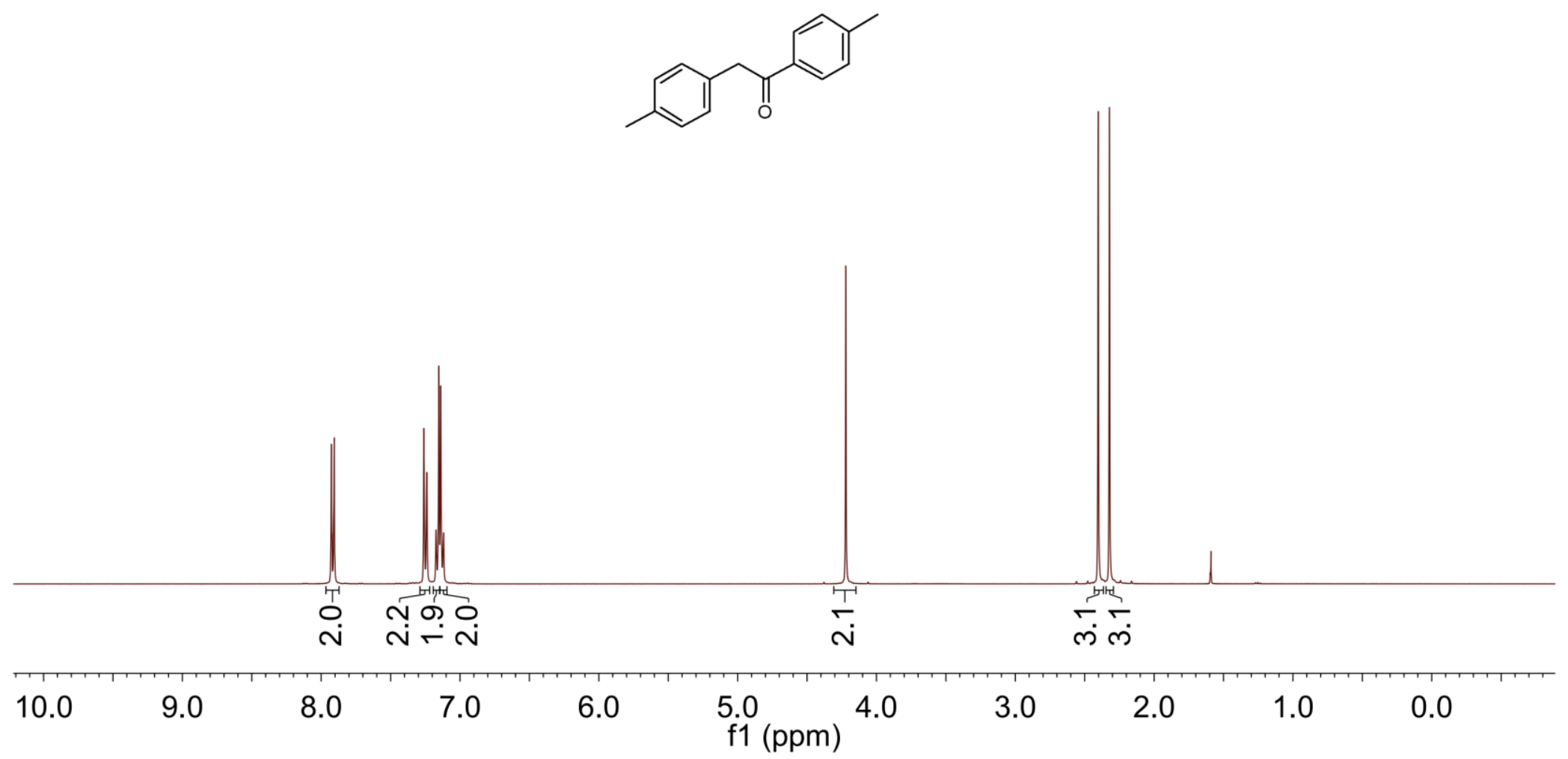




\begin{tabular}{|c|c|c|}
\hline 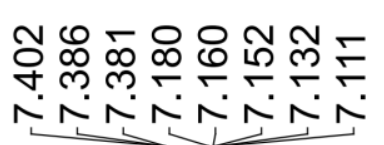 & 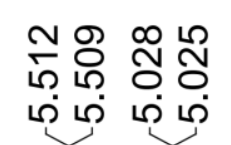 & $\begin{array}{l}\prod_{\infty} \\
\infty \\
m\end{array}$ \\
\hline
\end{tabular}

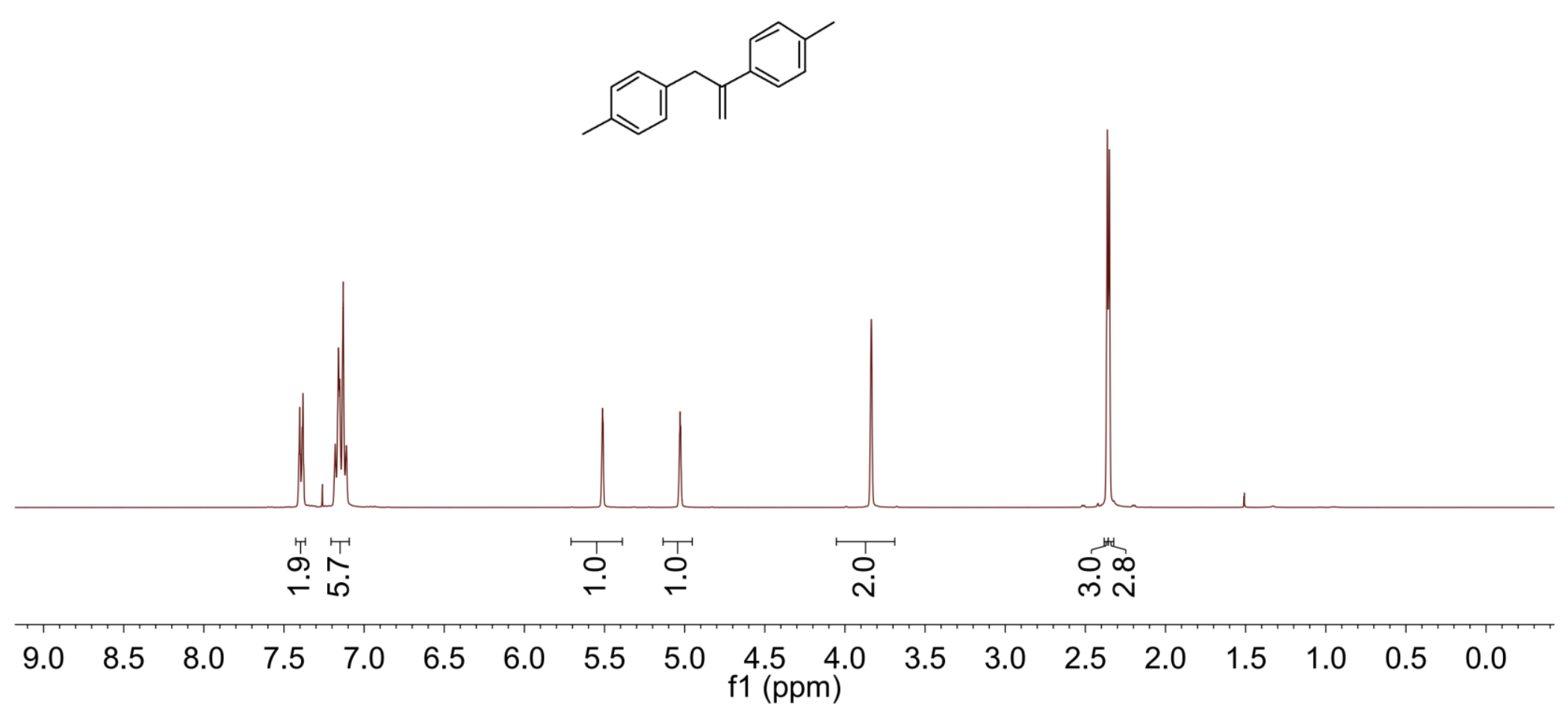



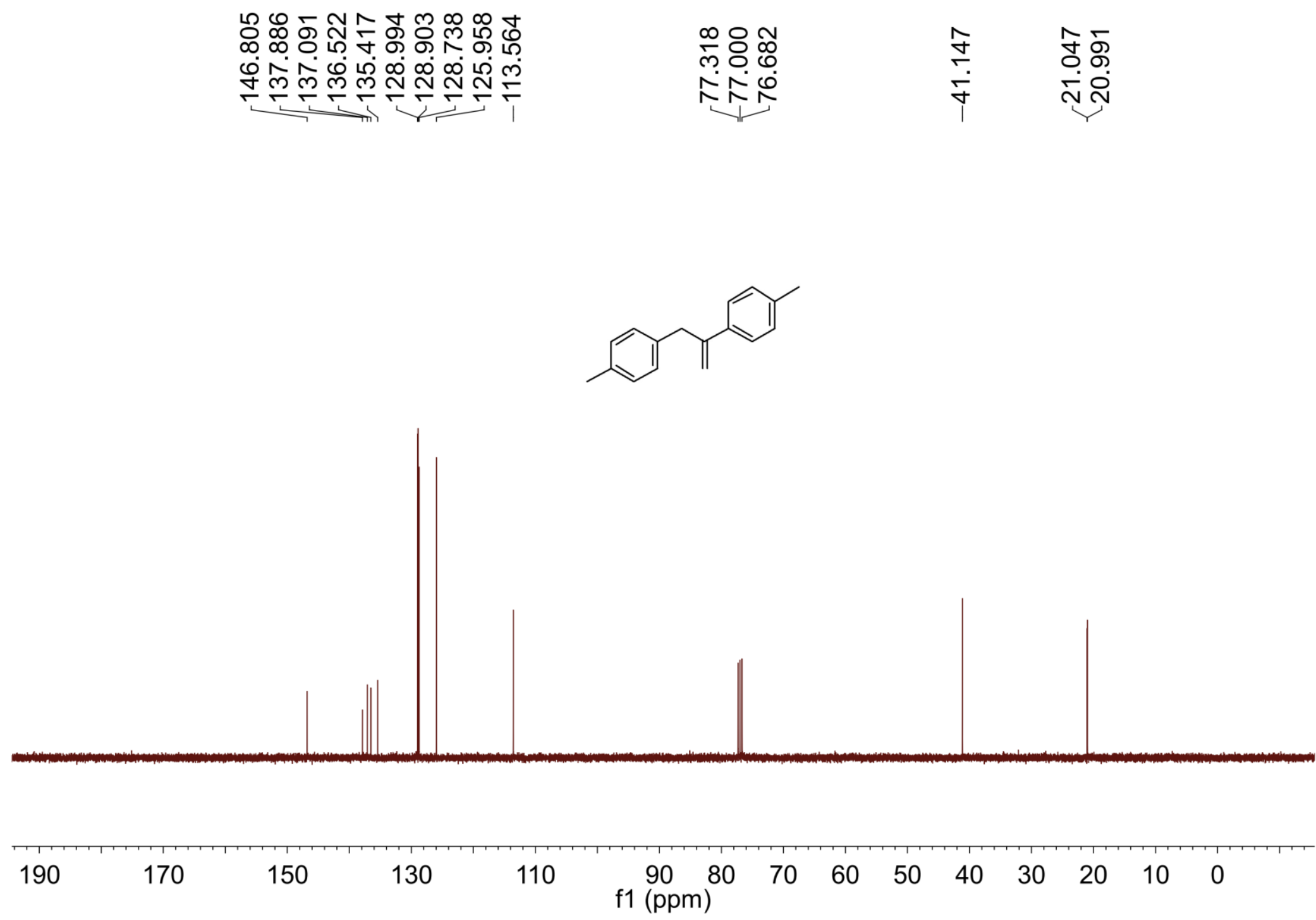

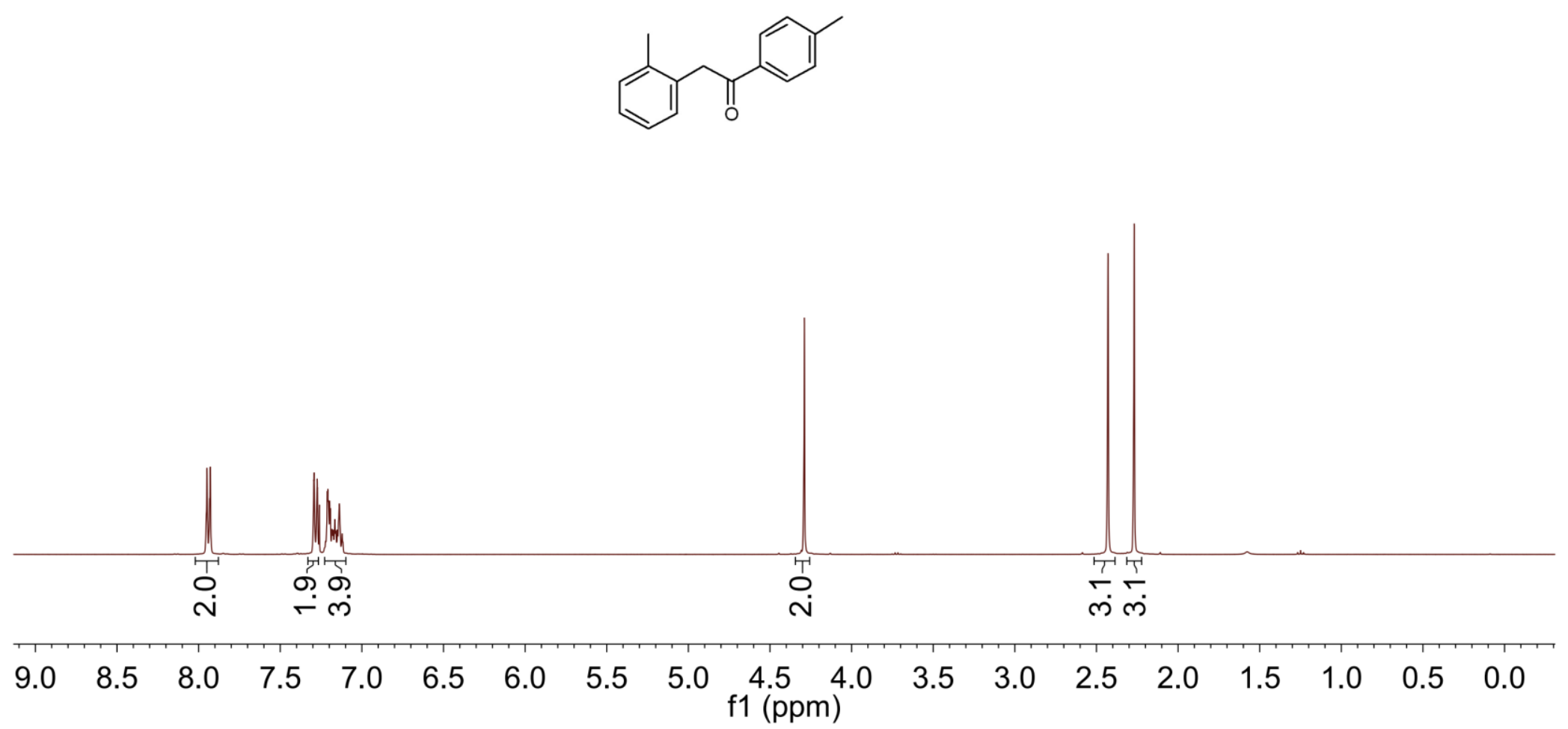


\begin{tabular}{|c|c|c|c|}
\hline 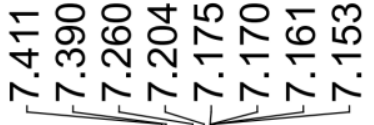 & 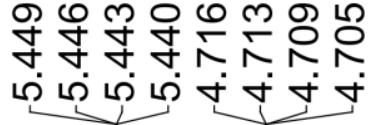 & $\stackrel{n}{n}$ & 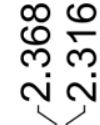 \\
\hline
\end{tabular}

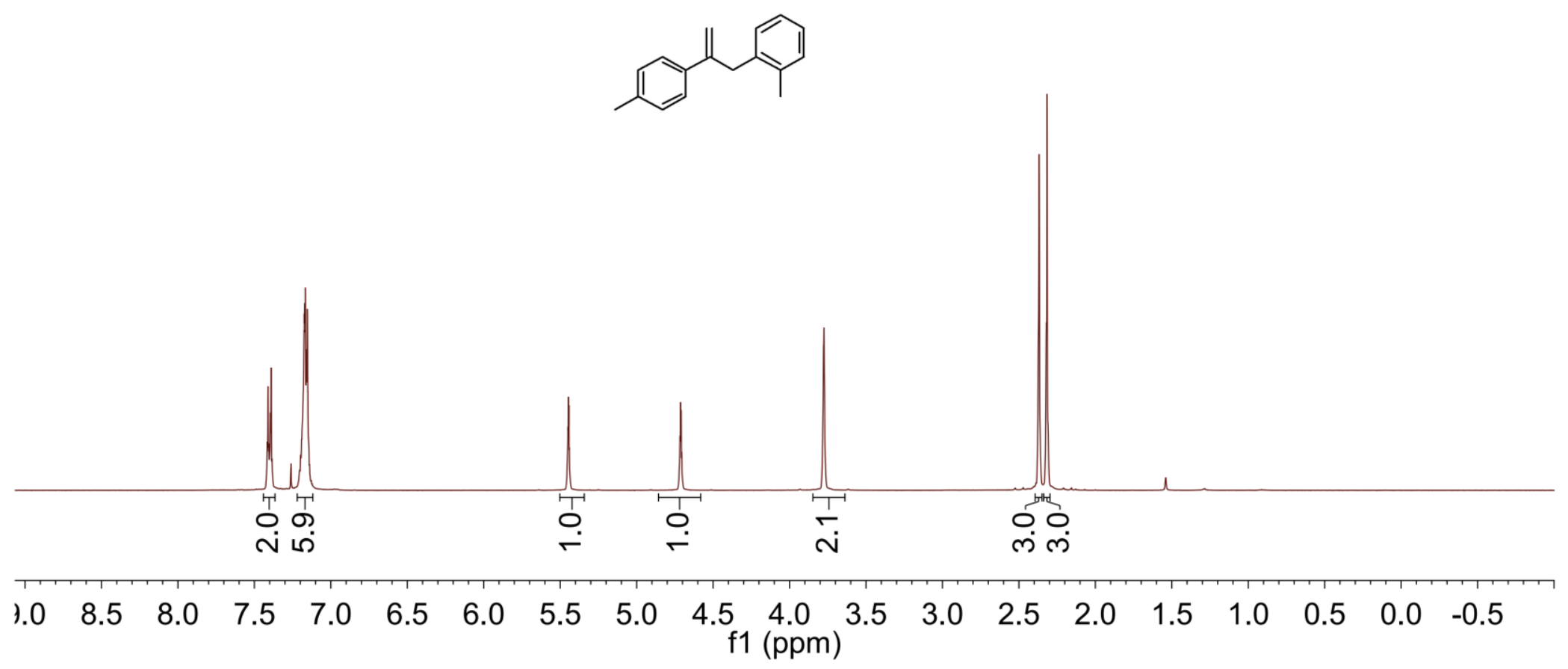


Oy ${ }^{1} \mathrm{H}$ NMR

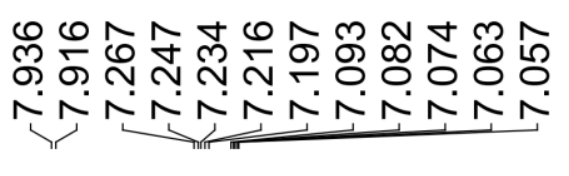

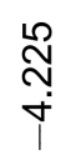

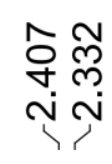
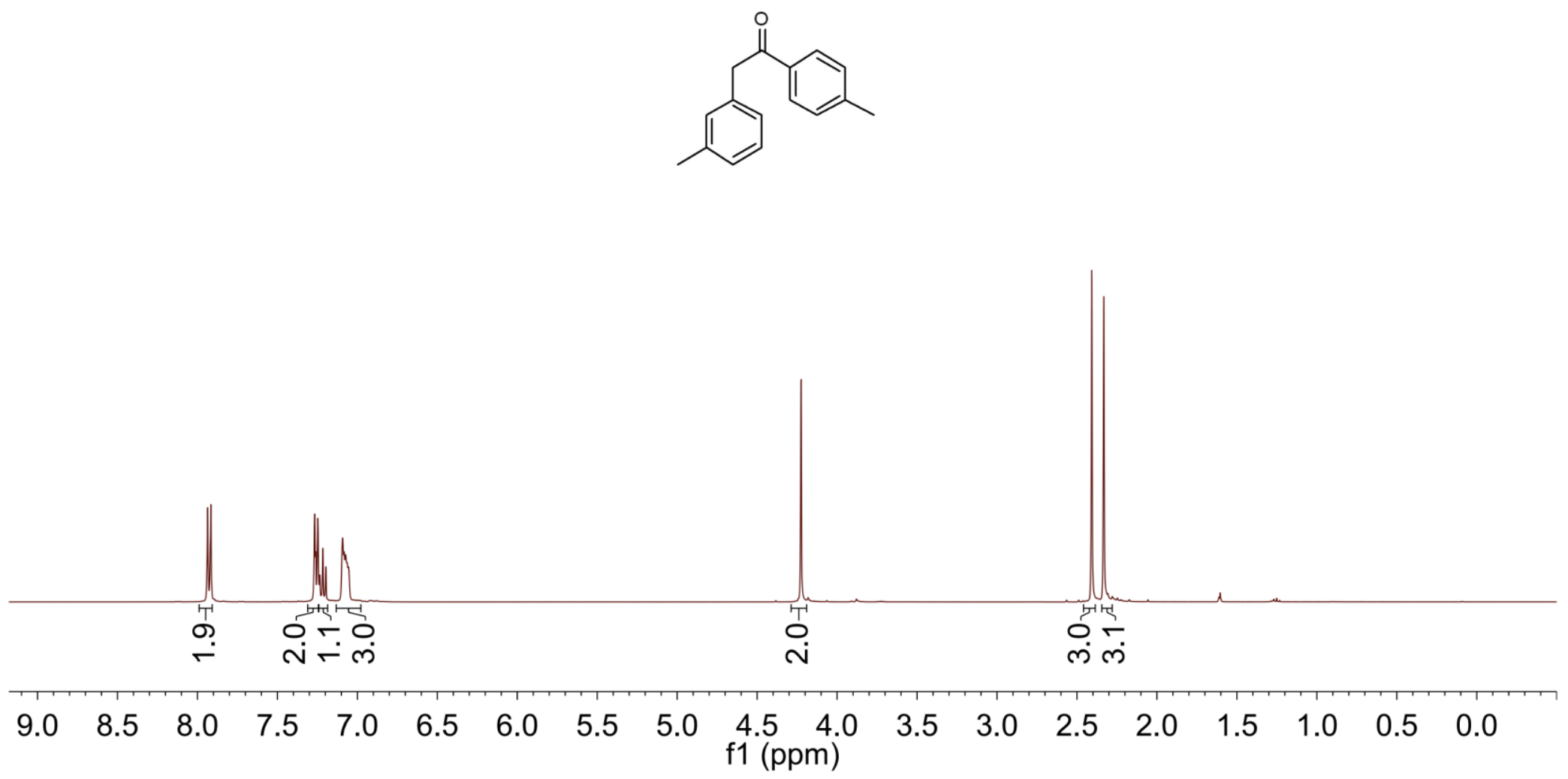

S109 
Oy ${ }^{13}$ C NMR

ڤิ

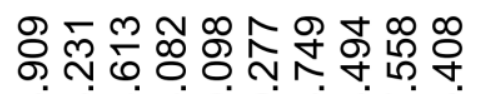

ฺ่

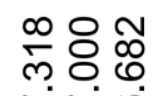

令会

m

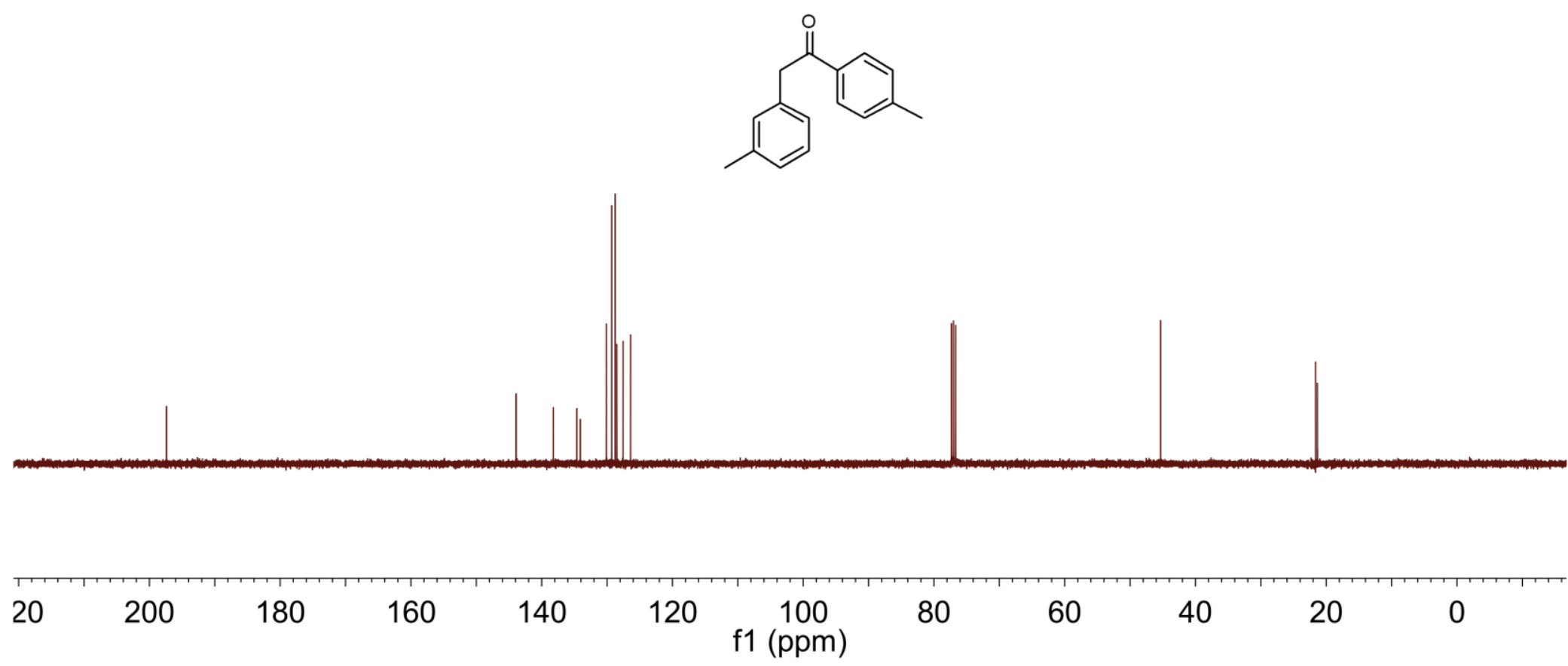


1y ${ }^{1}$ H NMR

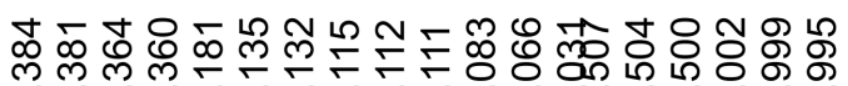

$\underset{\infty}{\infty}$

$\stackrel{\text { กิ }}{\text { ก }}$

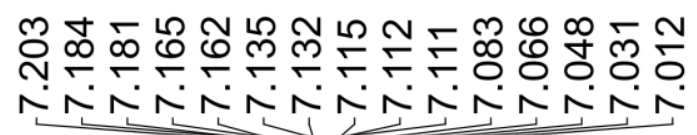
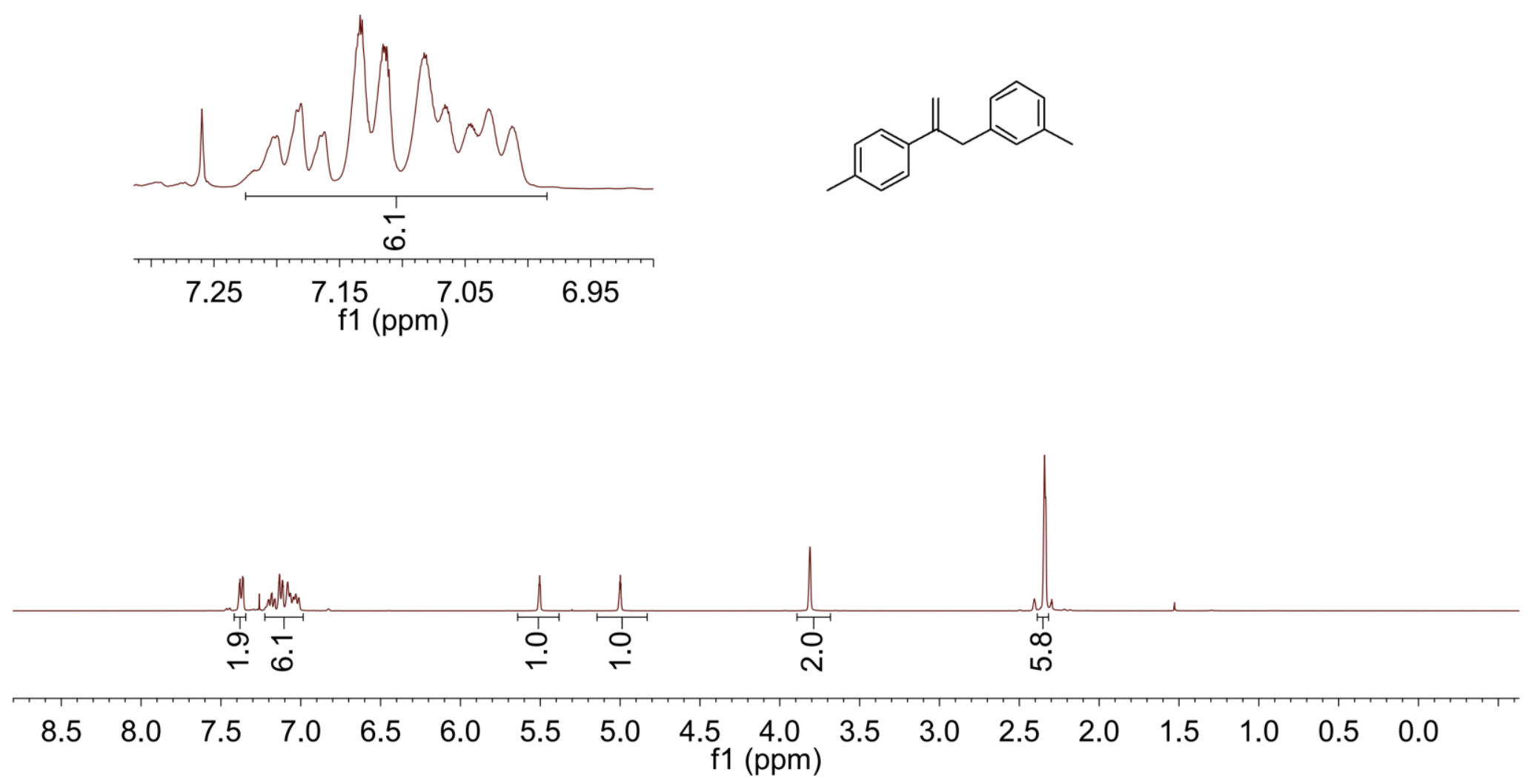

S111 


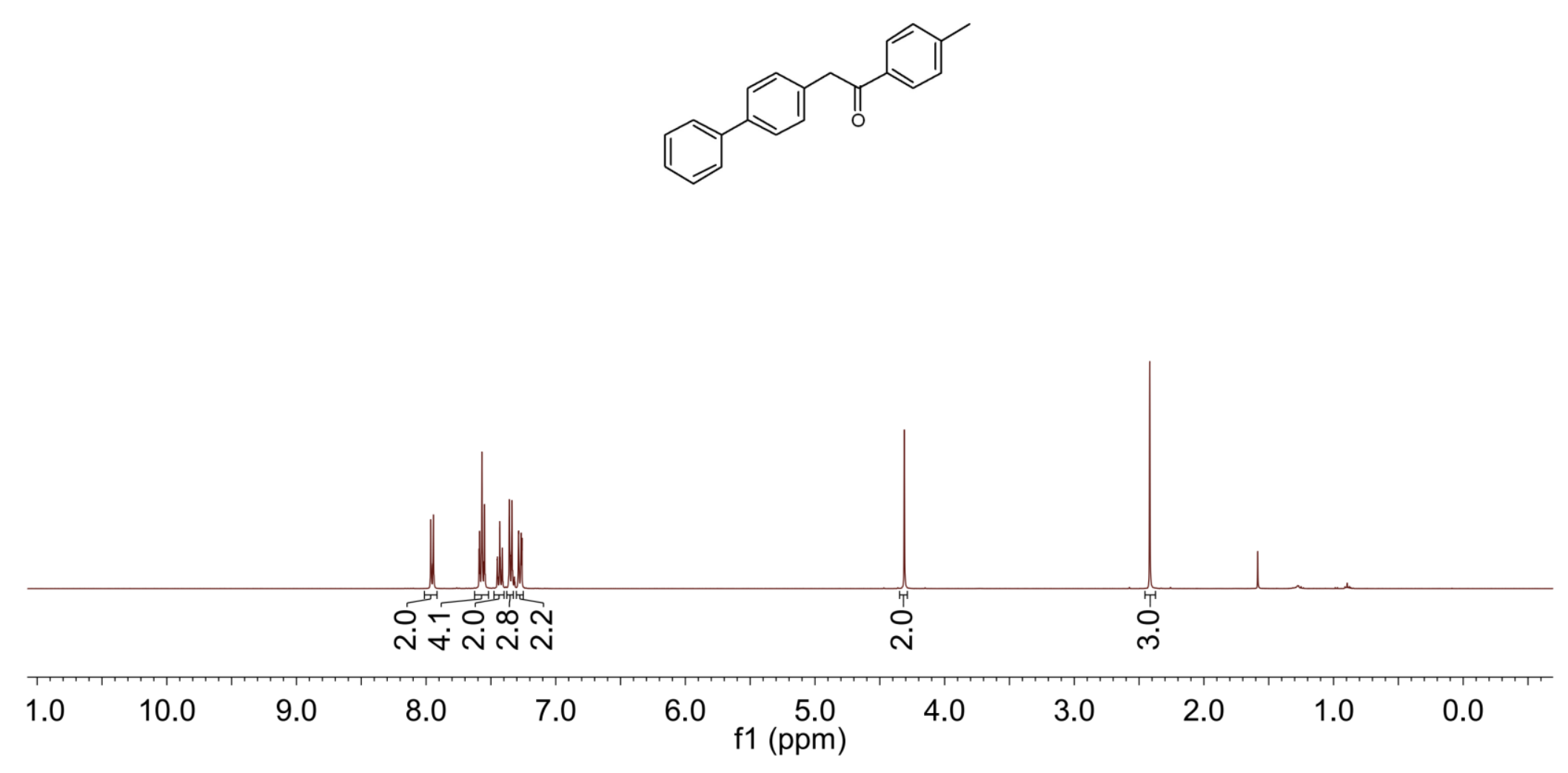



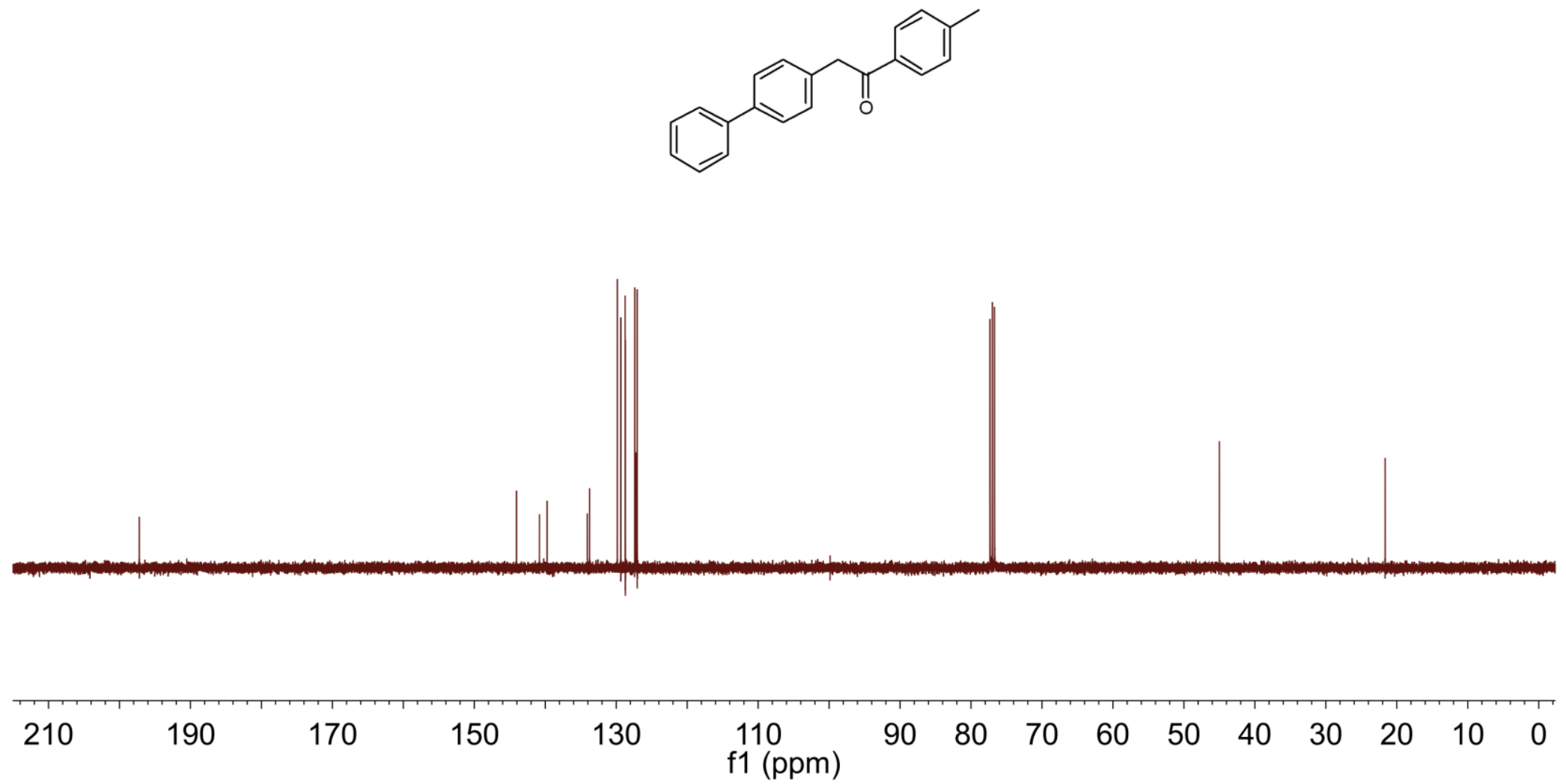


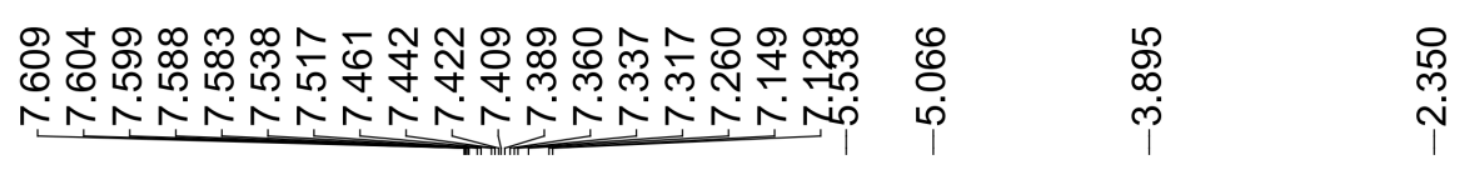

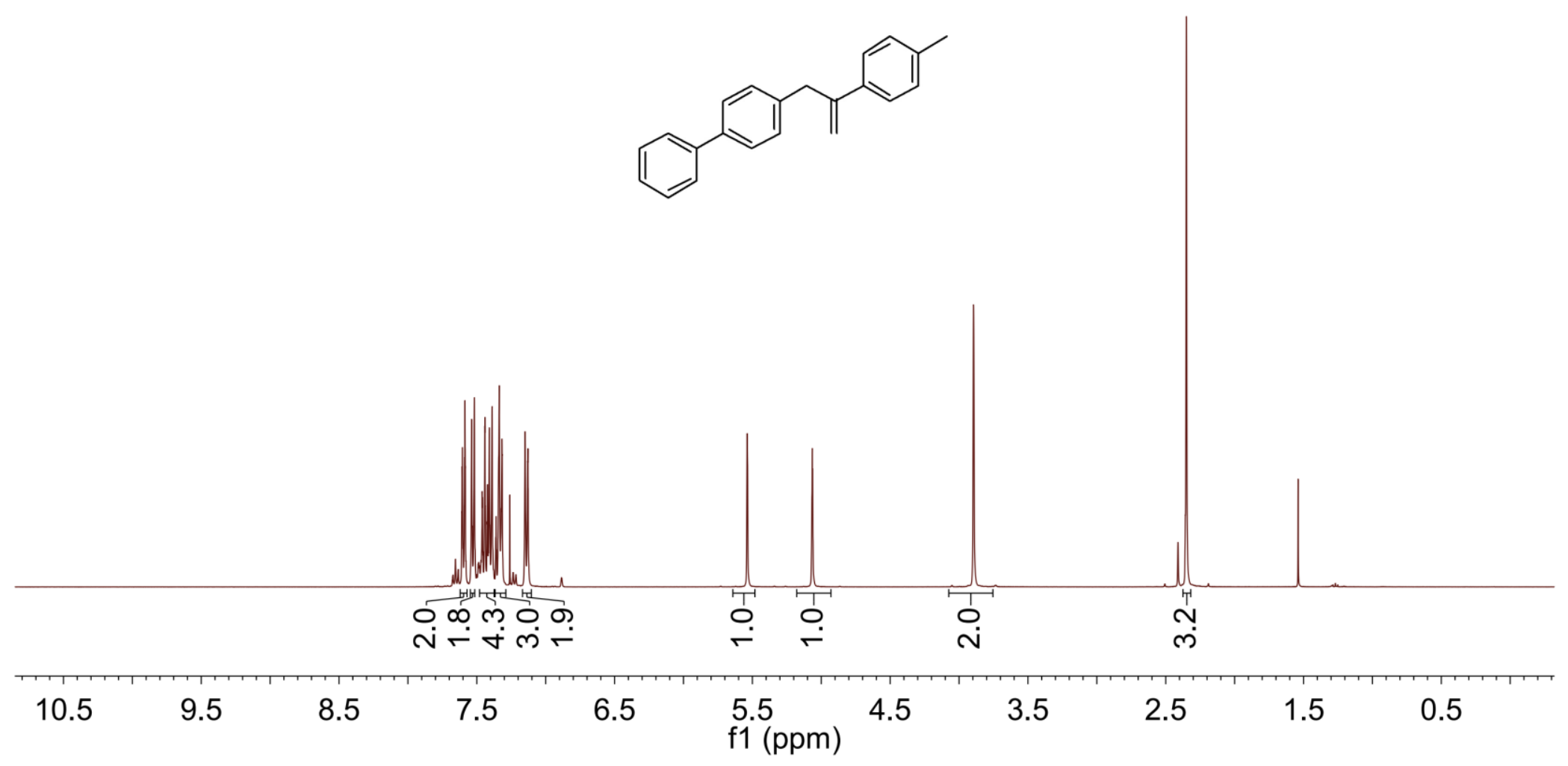




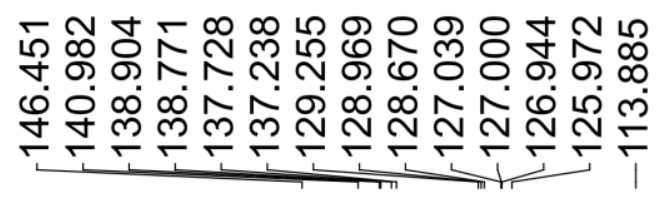

๓ 융

Nㅗ

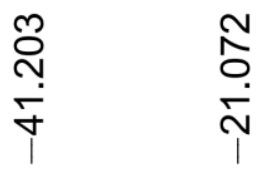
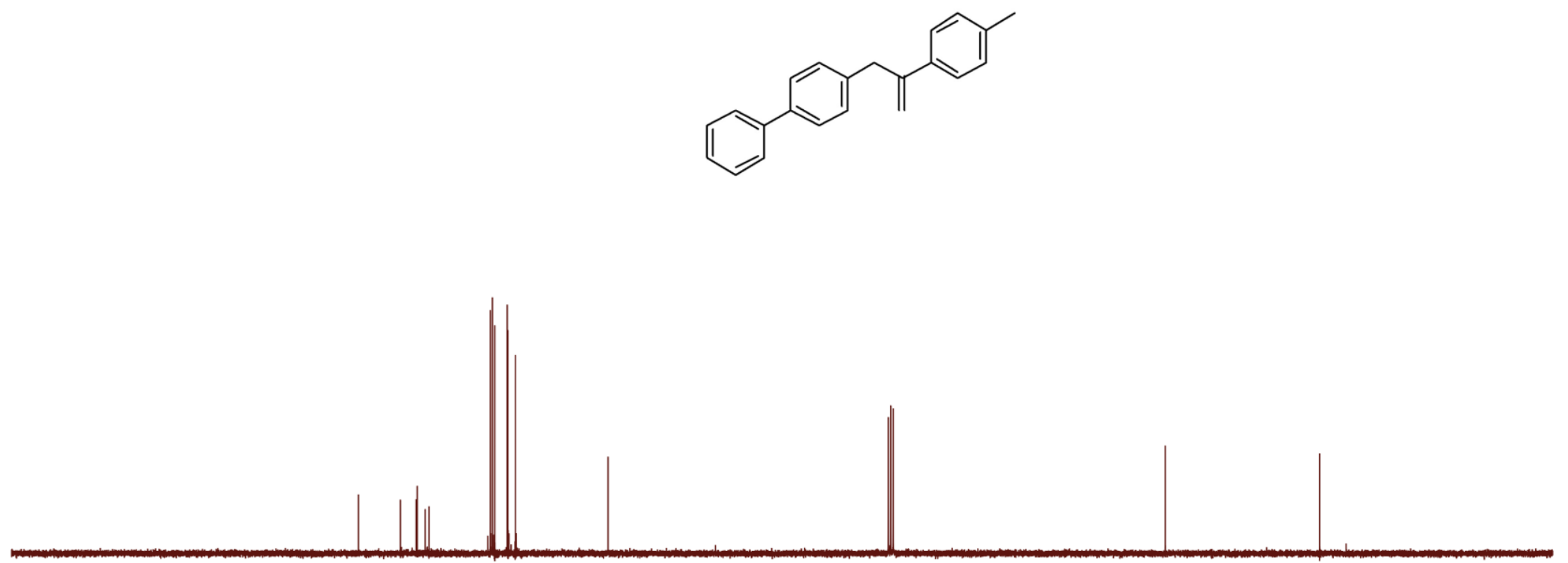

90

170

150

130

110

90

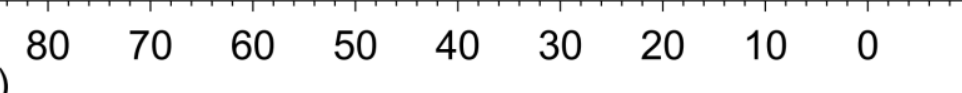




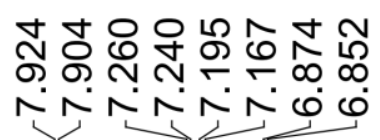

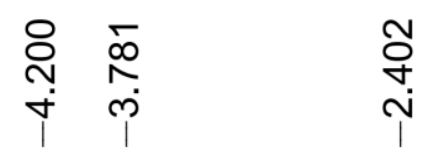
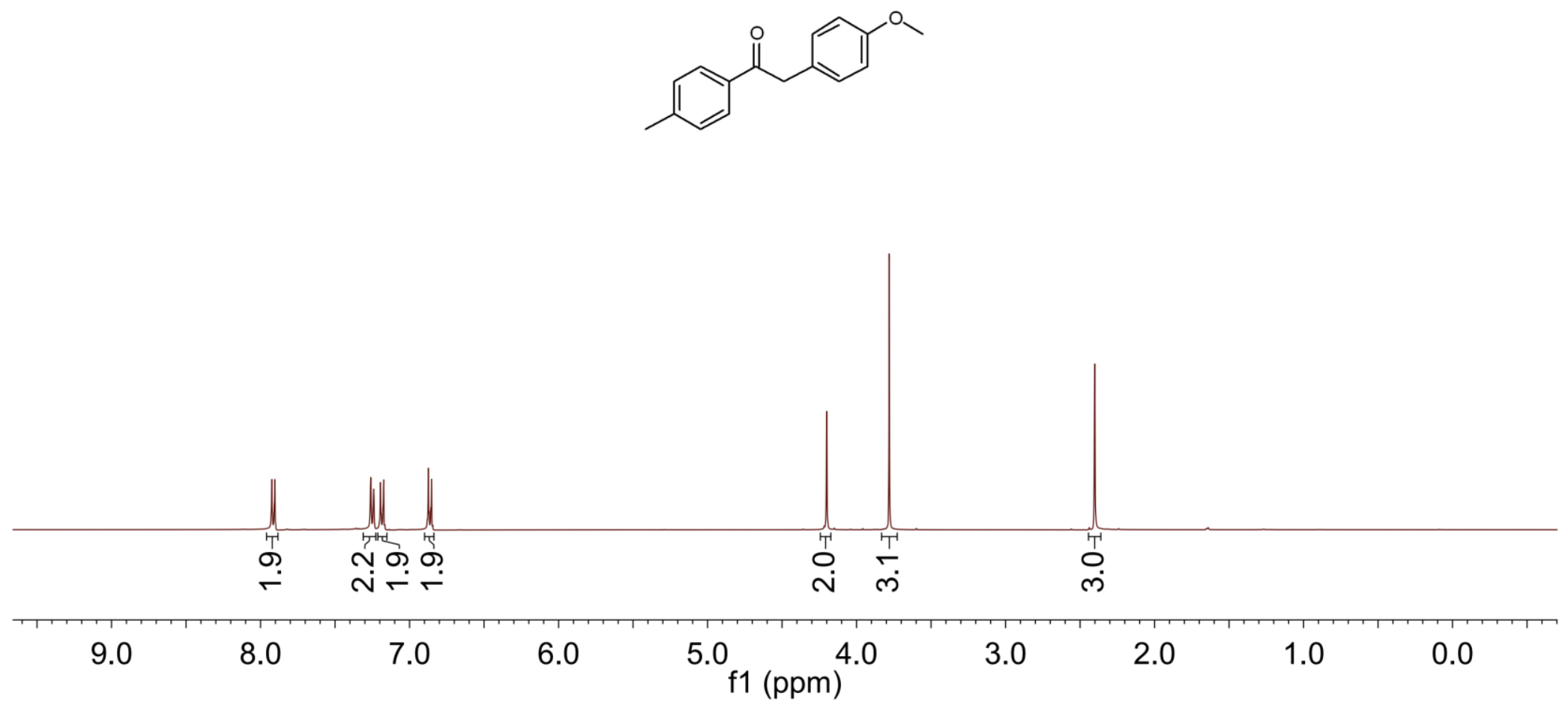


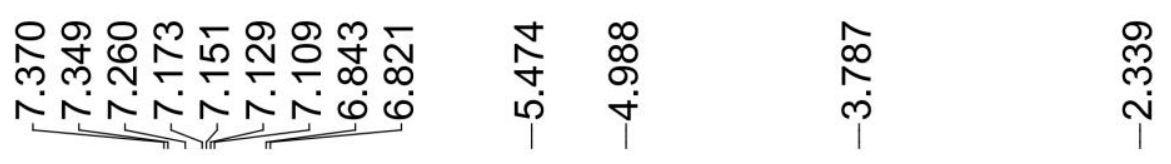

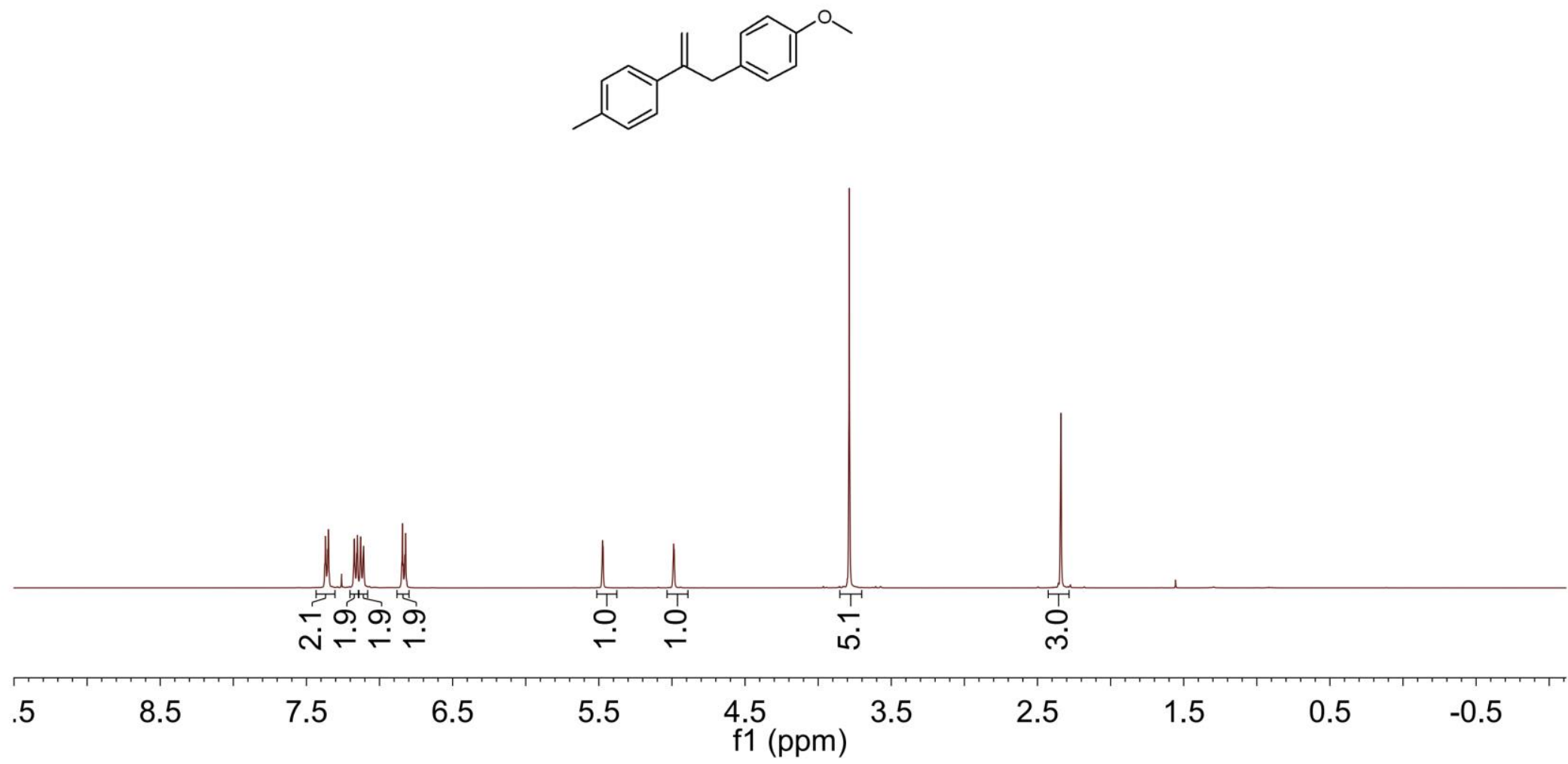




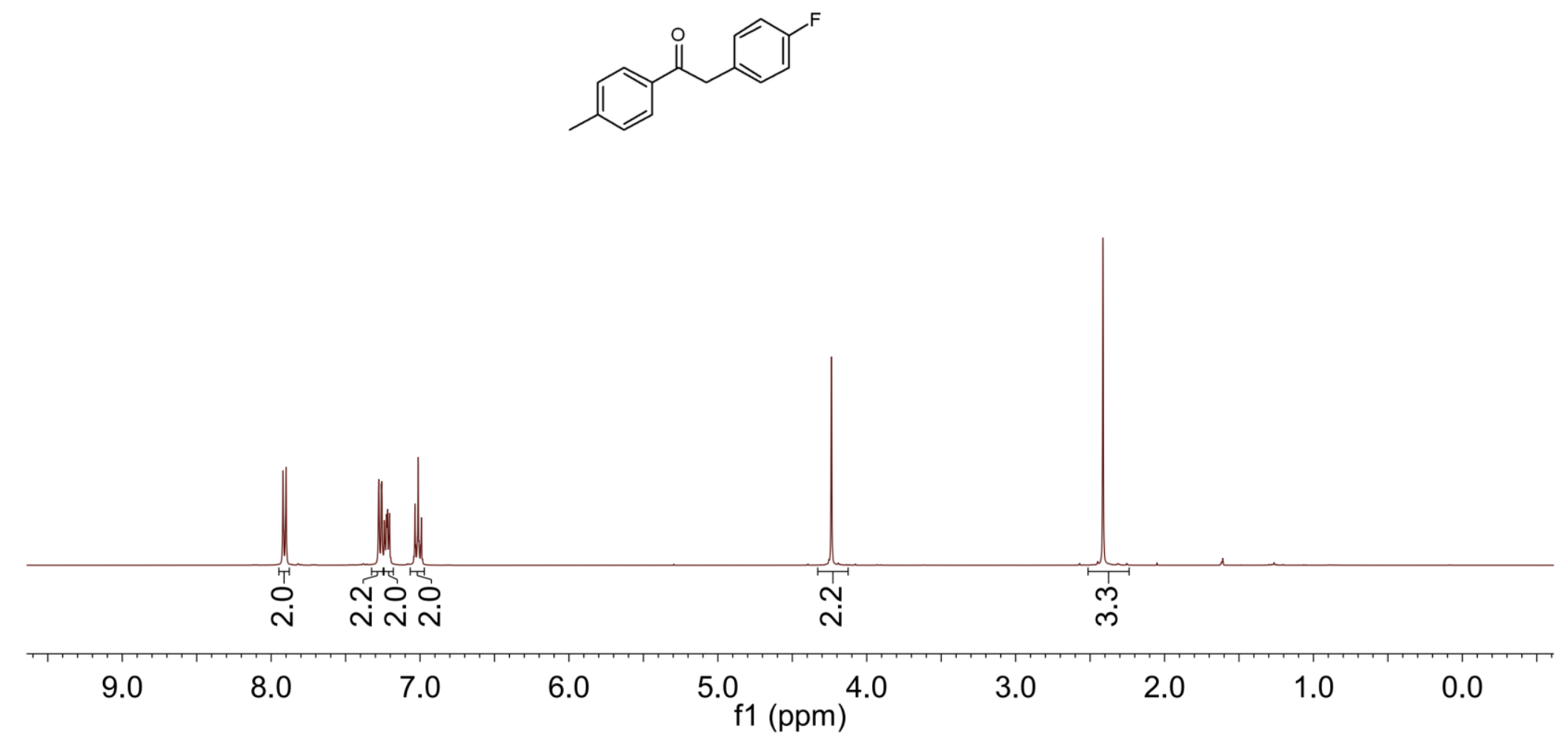



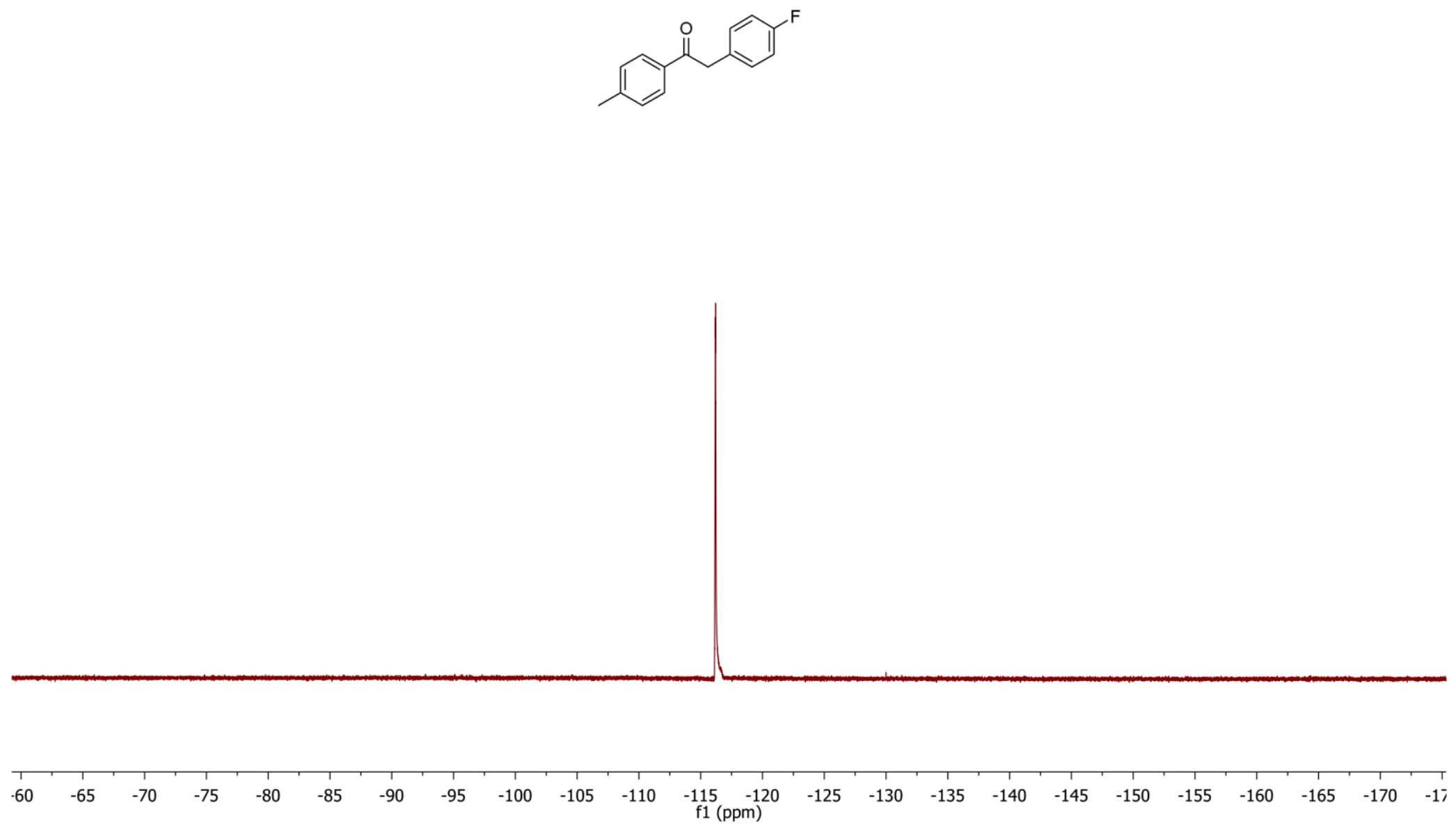


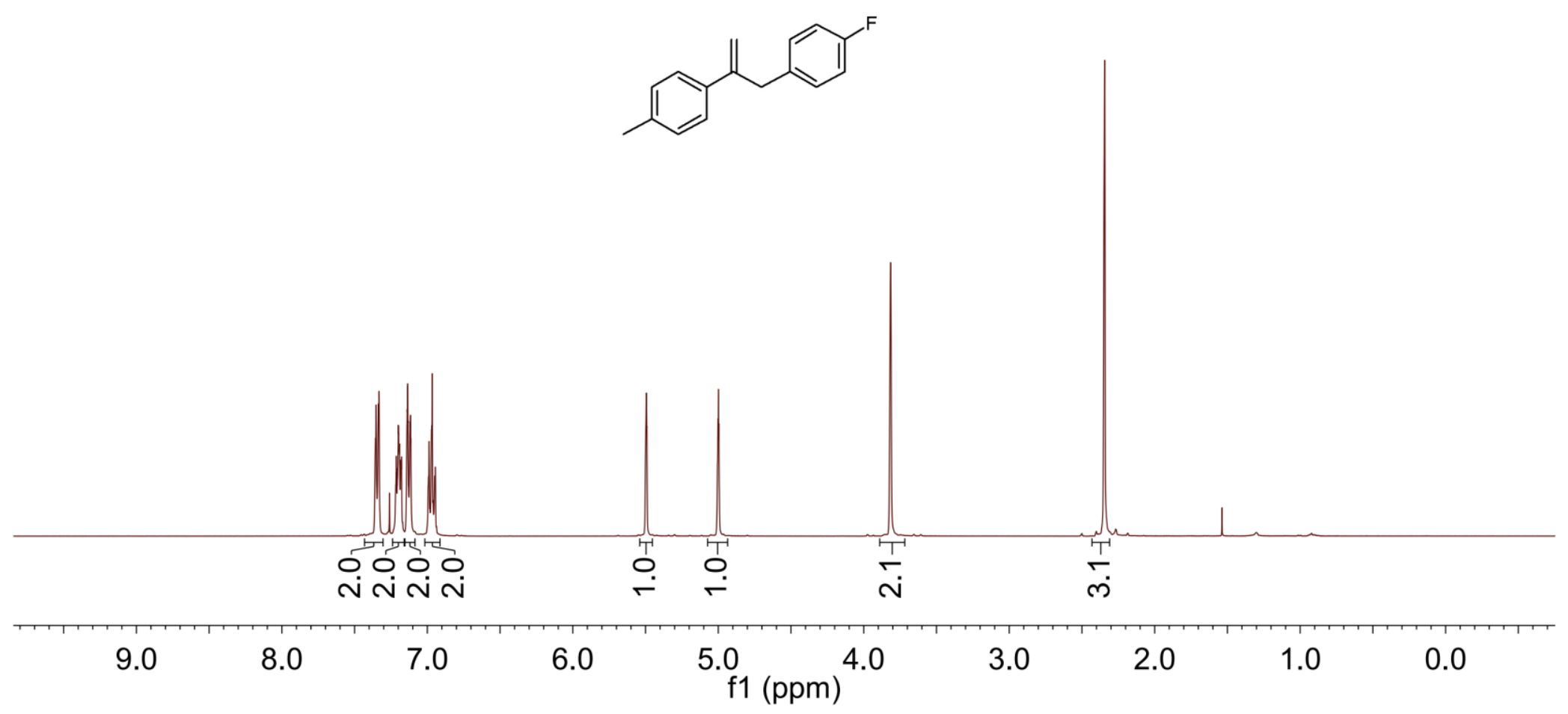



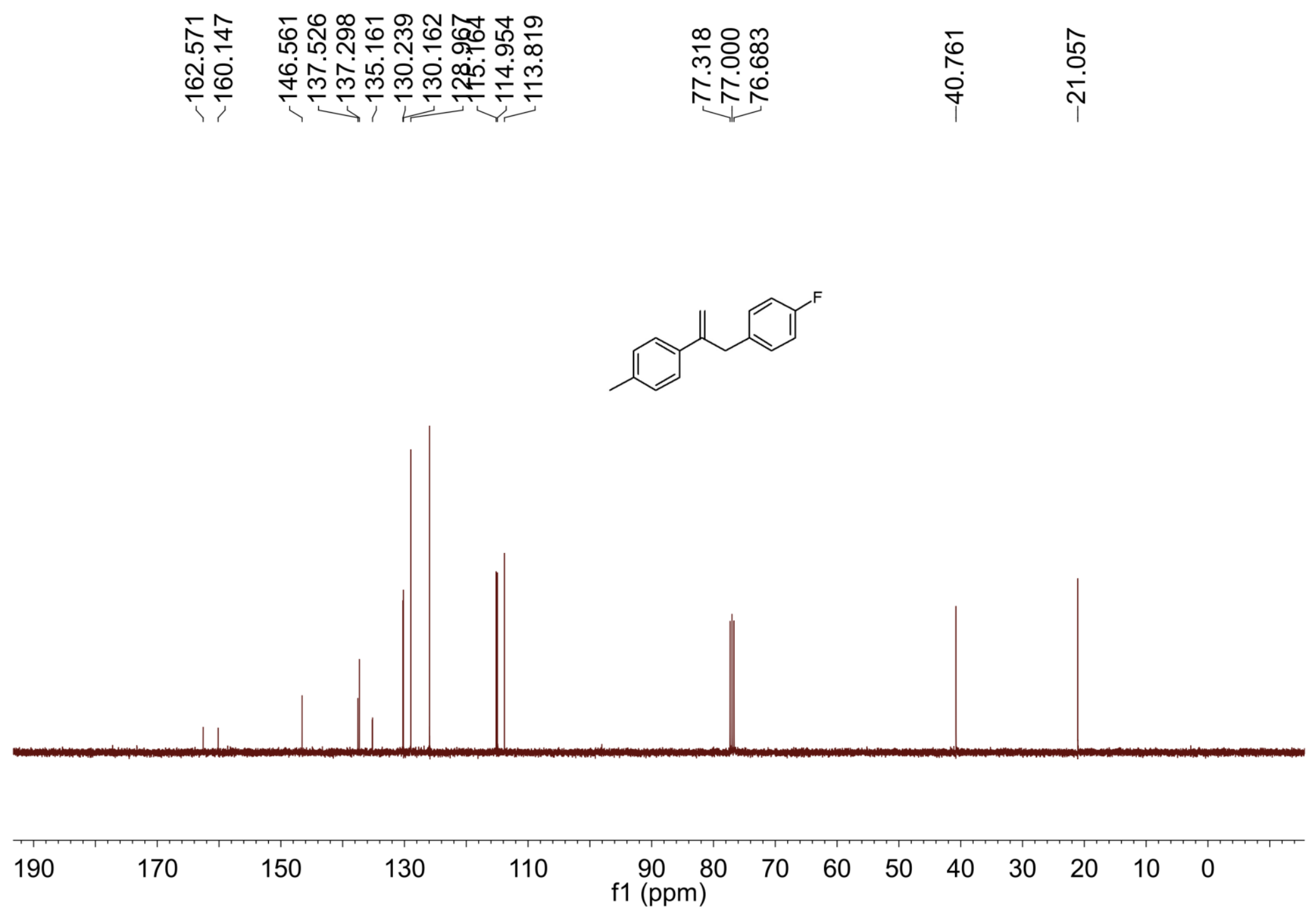
$1 a b{ }^{19} \mathrm{~F}$ NMR

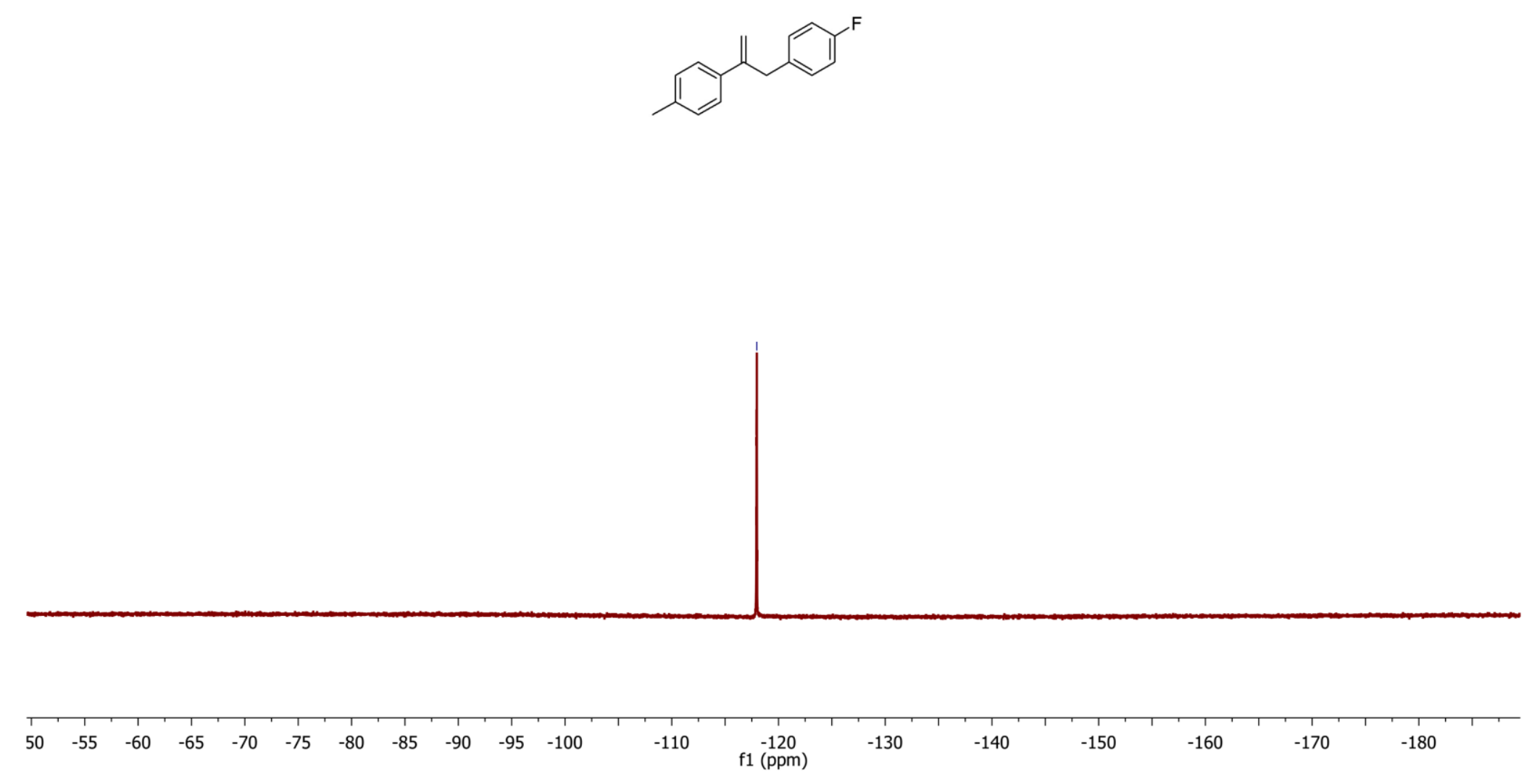


Oac ${ }^{1} \mathrm{H}$ NMR

تِ

$\underset{\text { ஸ̃ }}{\text { † }}$

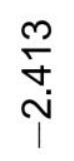
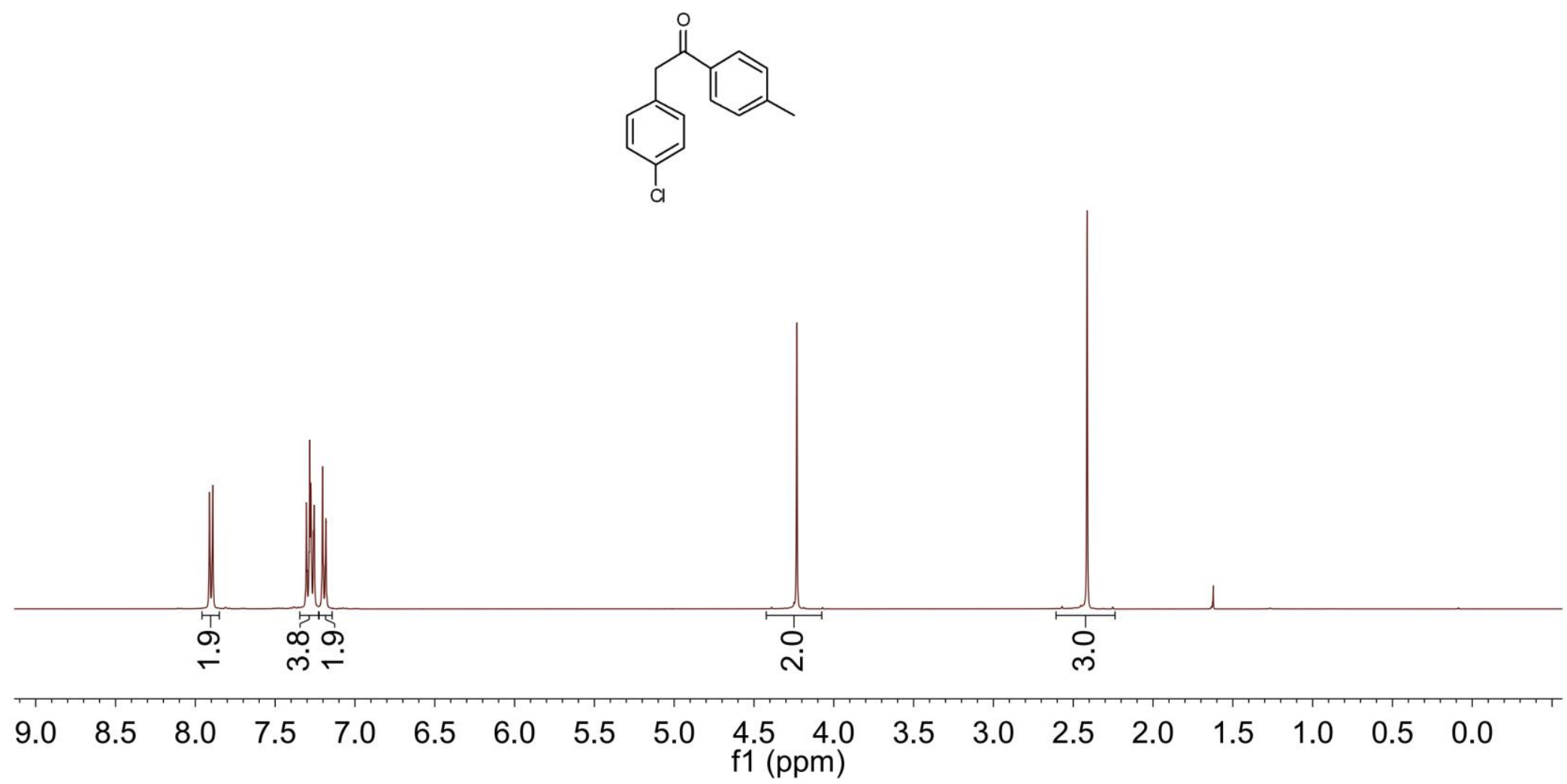

S123 


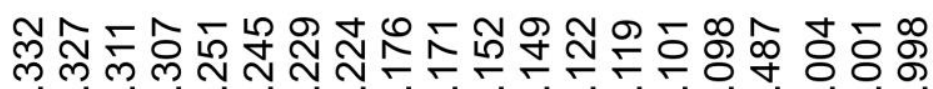

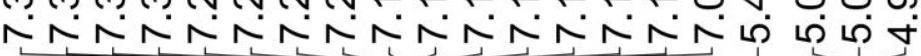

$\stackrel{8}{\infty}$

$\bar{m}$

Ni
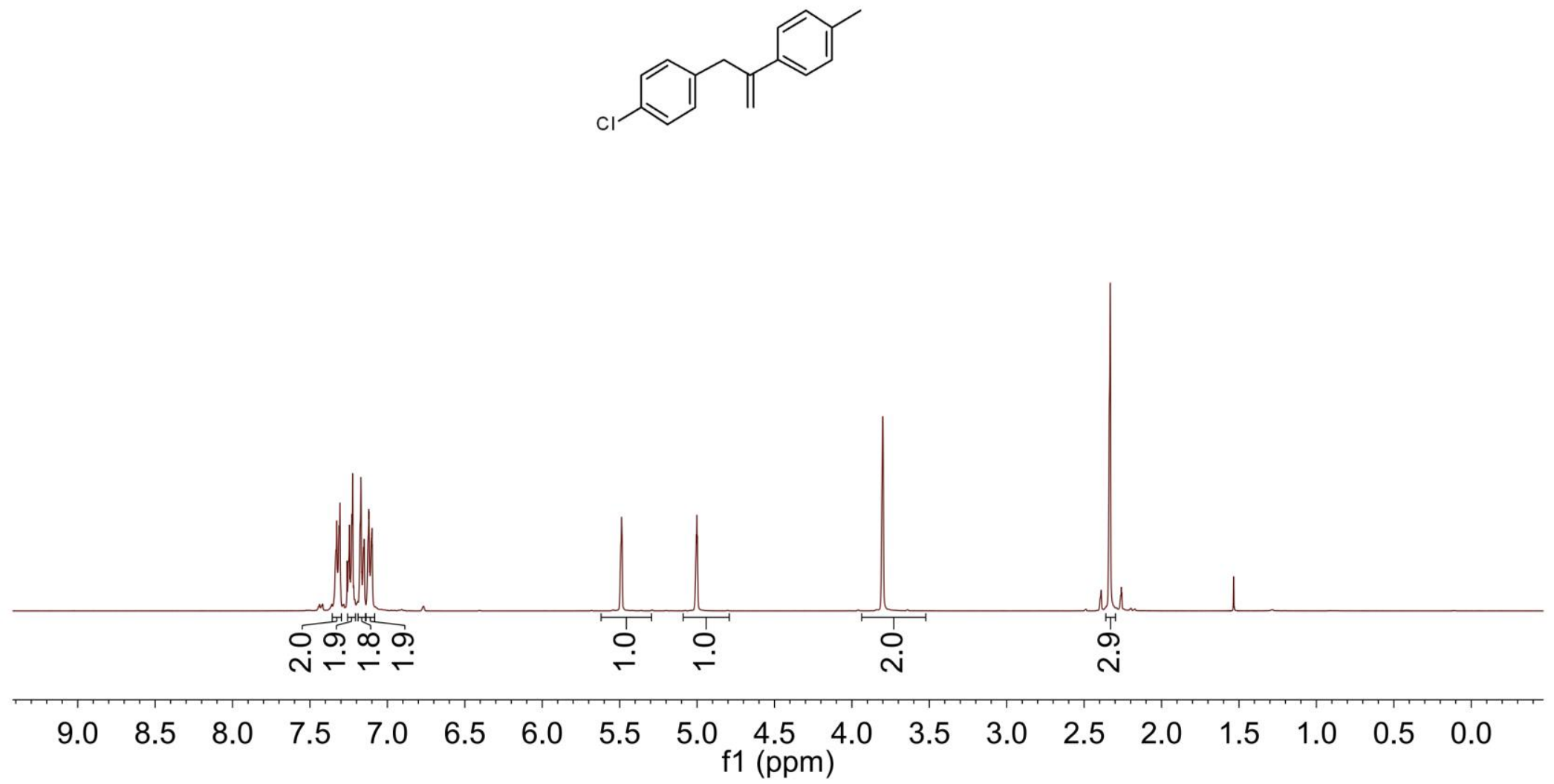


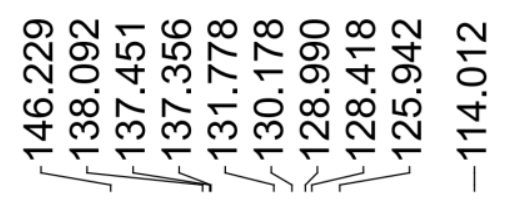

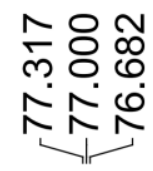

$\stackrel{8}{\mathscr{S}}$
$\stackrel{9}{+}$

$\stackrel{\hat{\sim}}{\stackrel{i}{i}}$
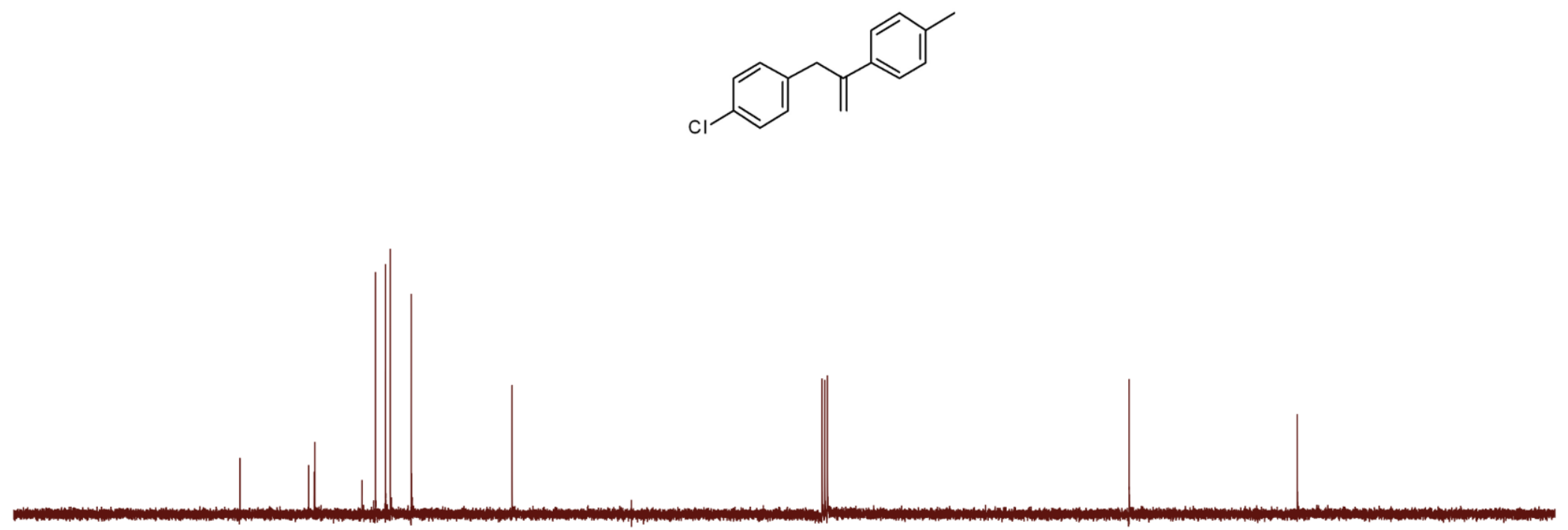

$\begin{array}{lllllllllllllllll}170 & 160 & 150 & 140 & 130 & 120 & 110 & 100 & 90 \underset{\mathrm{f} 1(\mathrm{ppm})}{80} & 70 & 60 & 50 & 40 & 30 & 20 & 10 & 0\end{array}$ 
$\operatorname{Oad}^{1} \mathrm{H}$ NMR

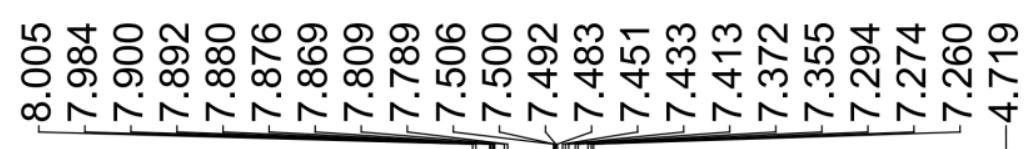

$\stackrel{\text { ্ָ }}{\text { i }}$

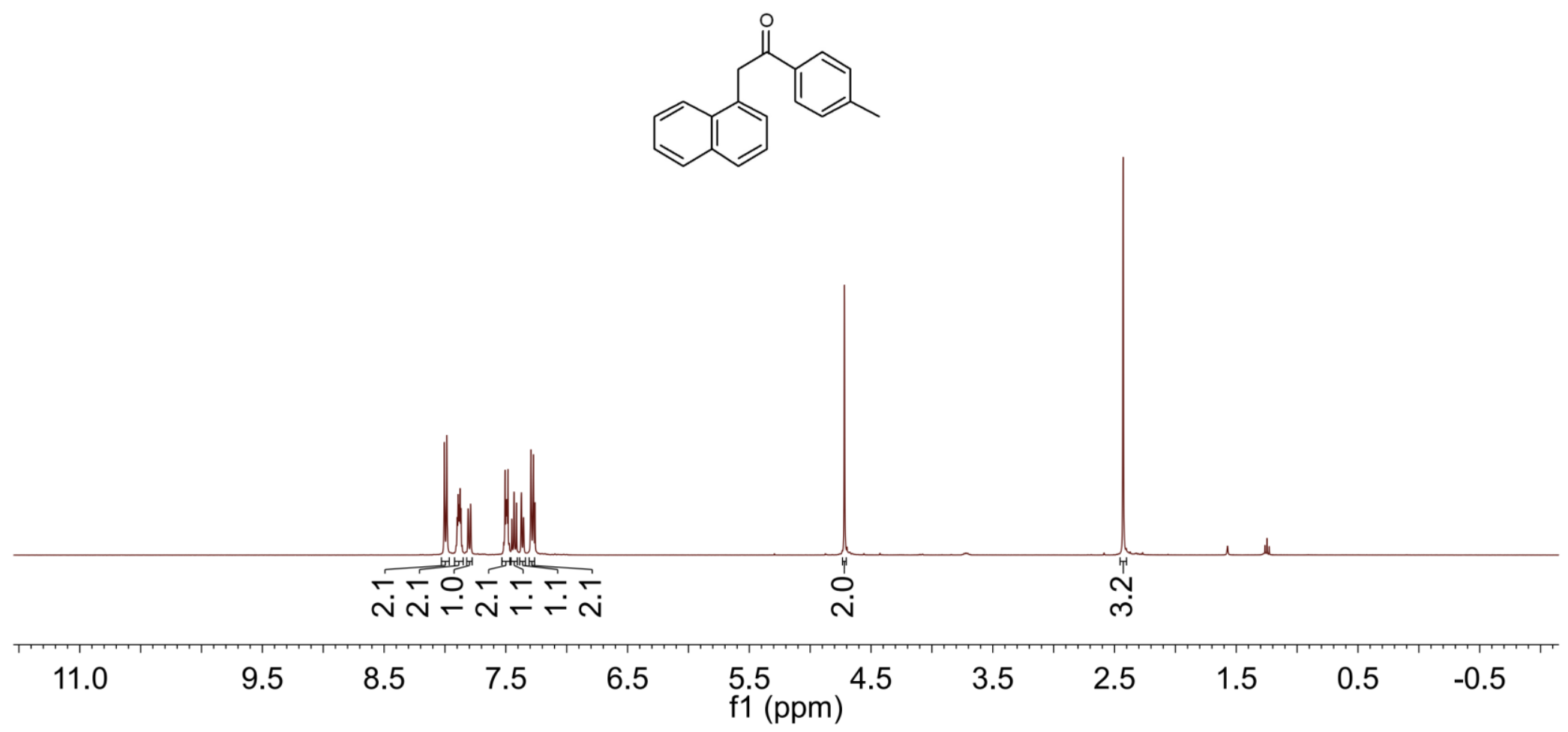

S126 

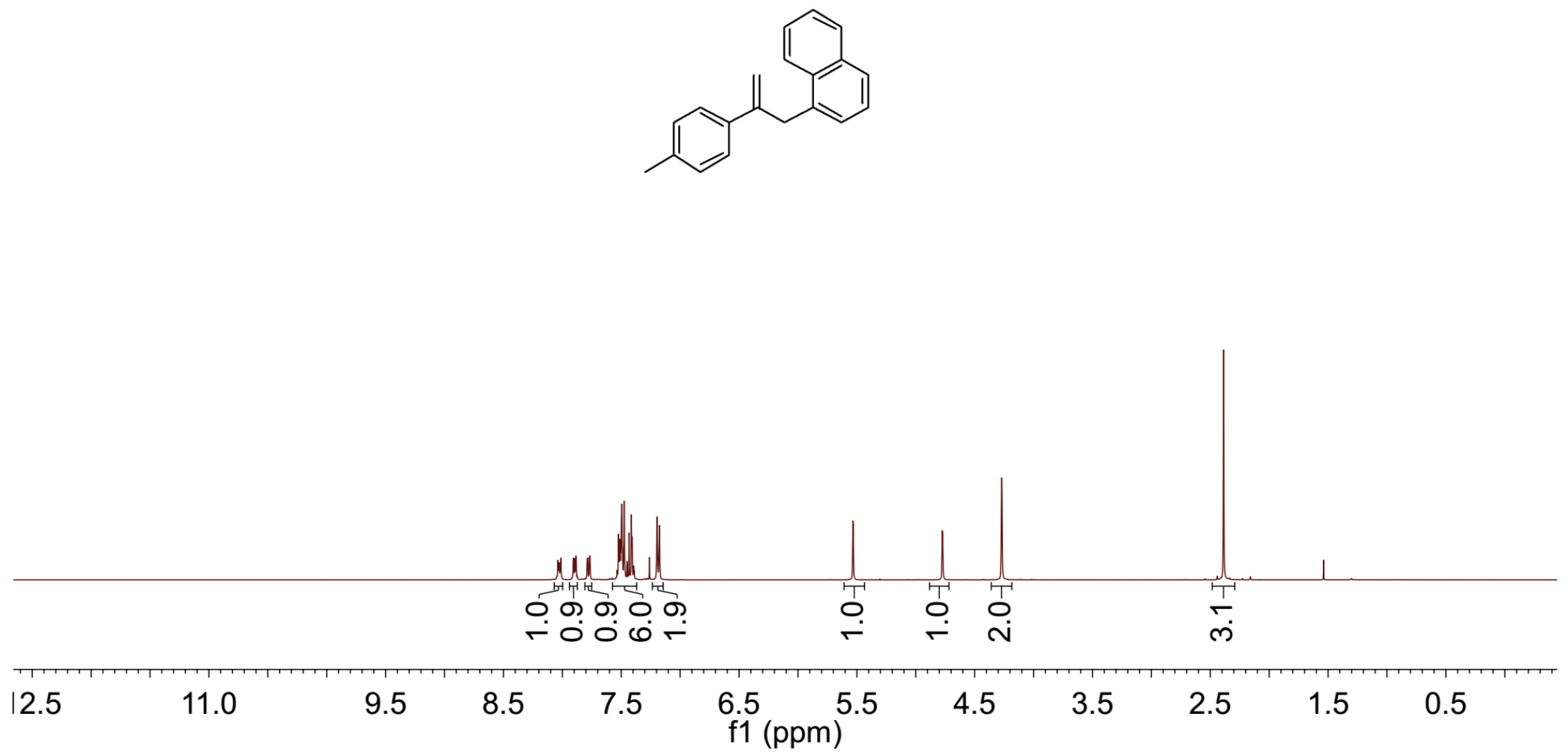
Oae ${ }^{1} \mathrm{H}$ NMR

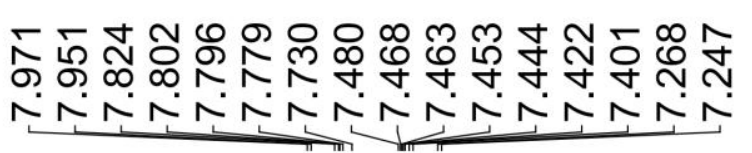

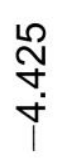

\begin{tabular}{l} 
O \\
\multirow{+}{*}{} \\
I
\end{tabular}
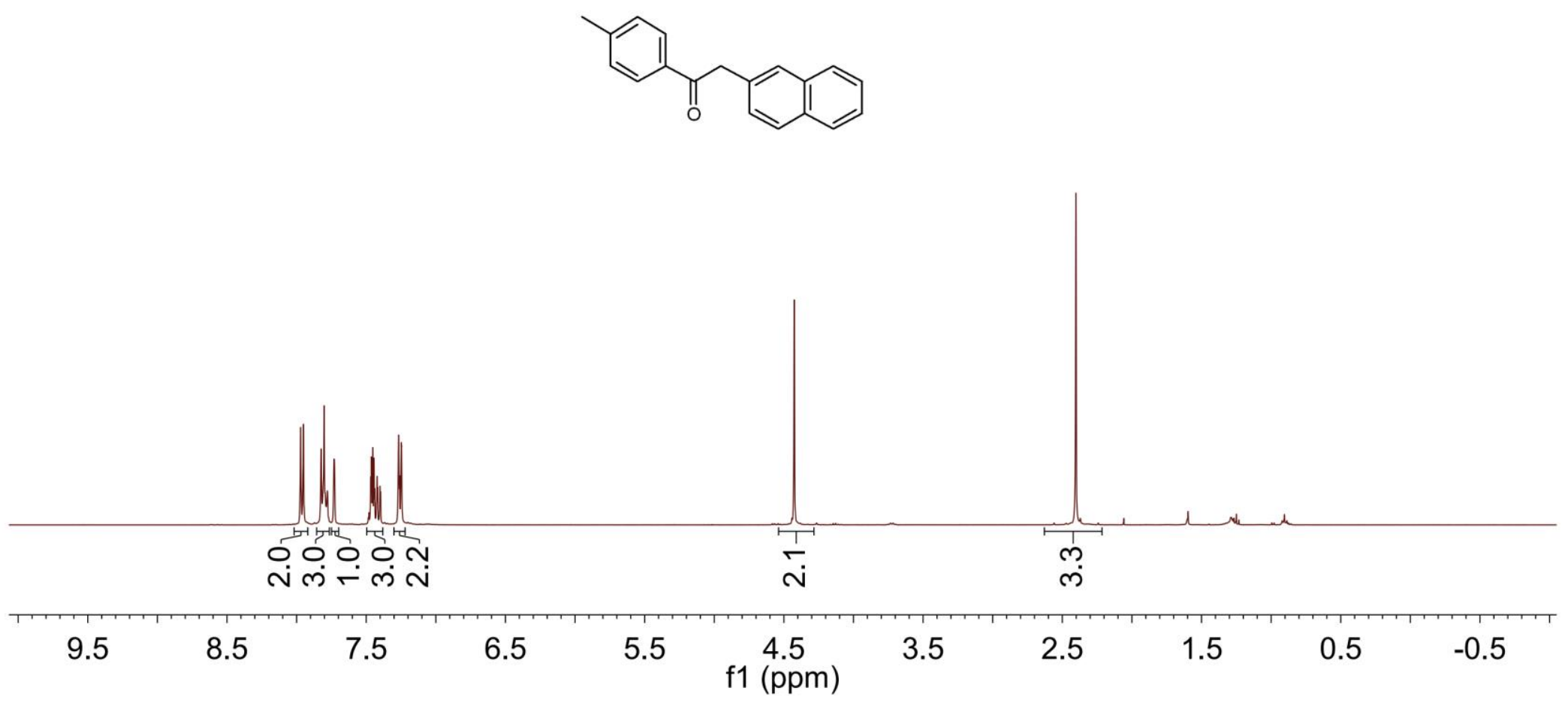

$\mathrm{S} 128$ 
న

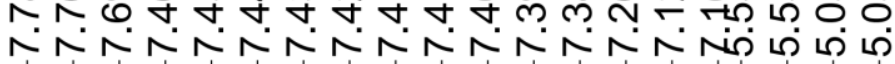

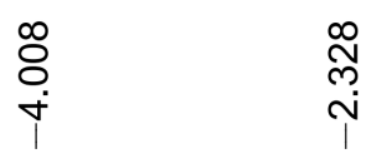

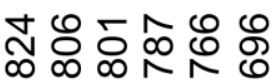

NNNN

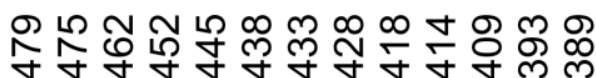

NNNNNNNNNN
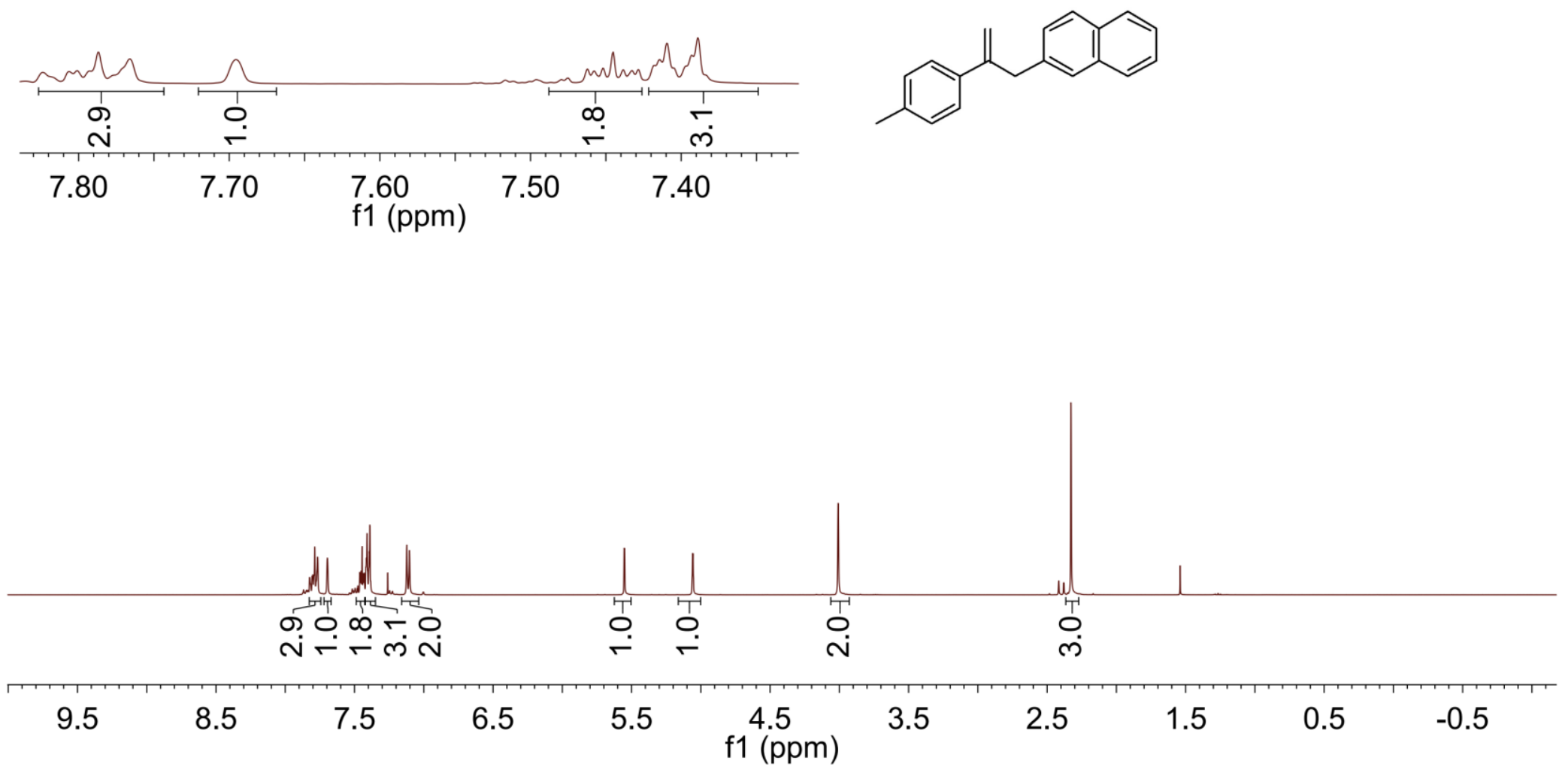

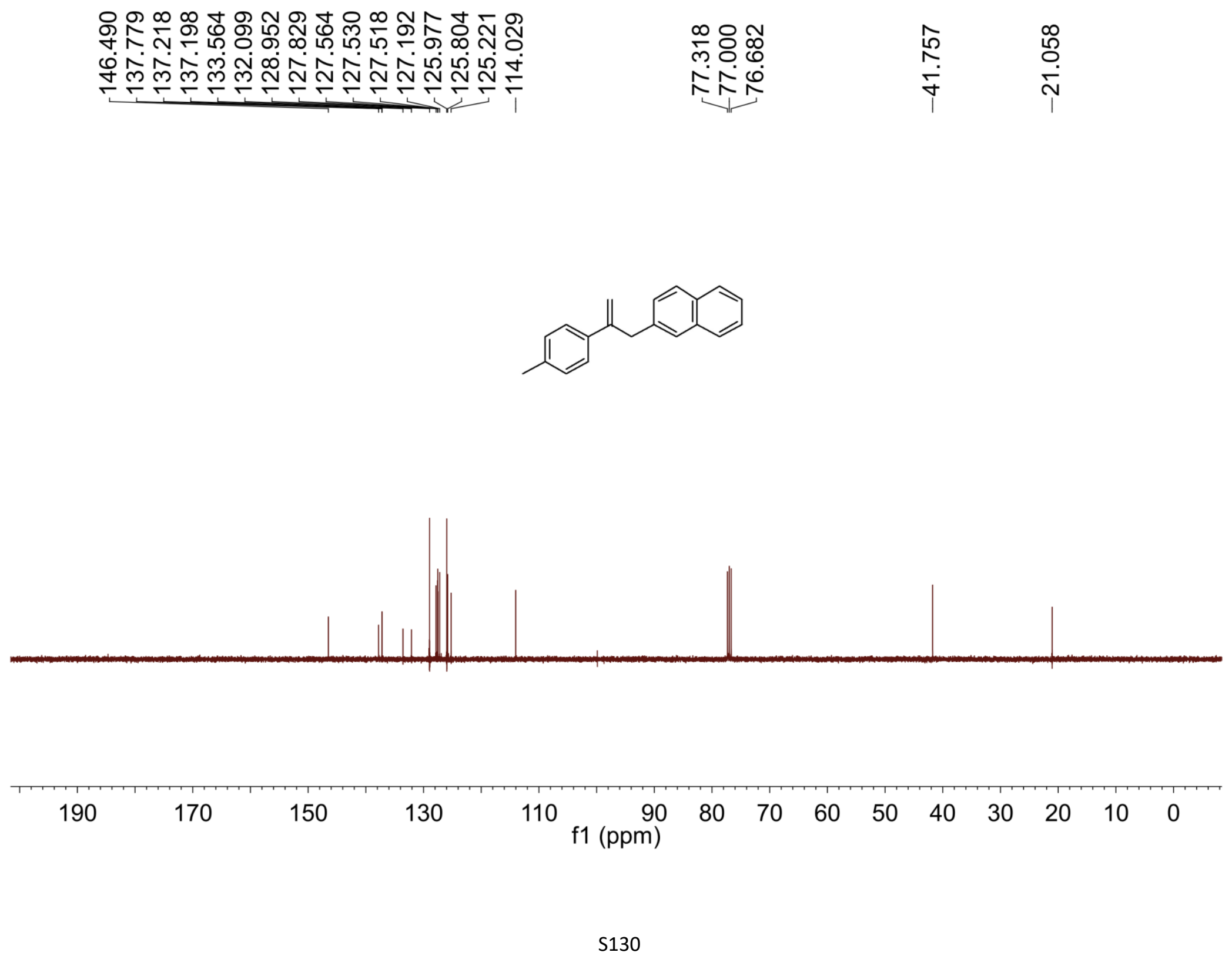


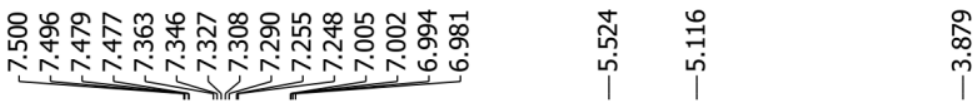

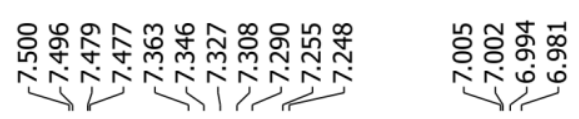
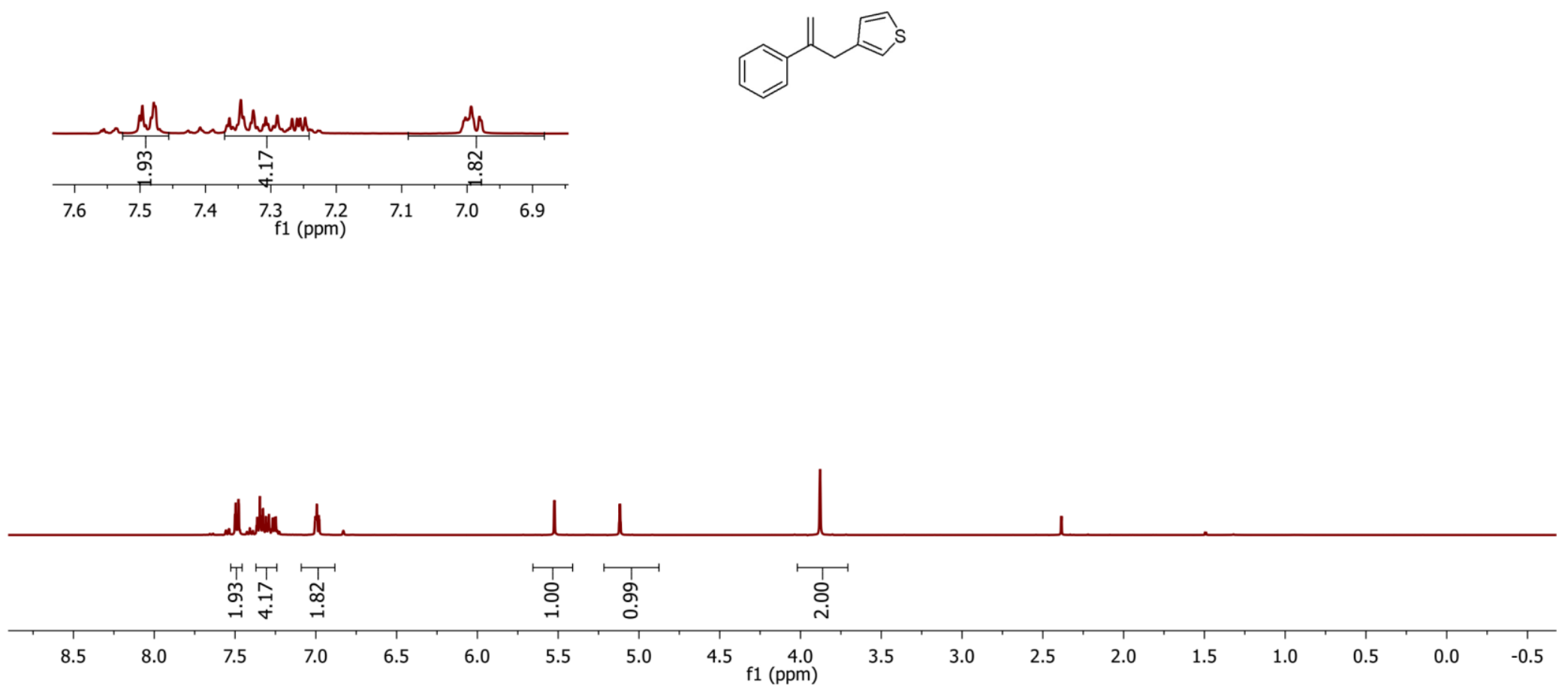
1ag ${ }^{1} \mathrm{H}$ NMR

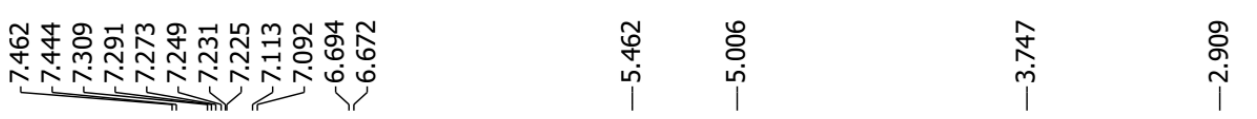

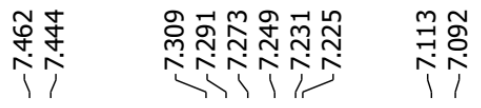
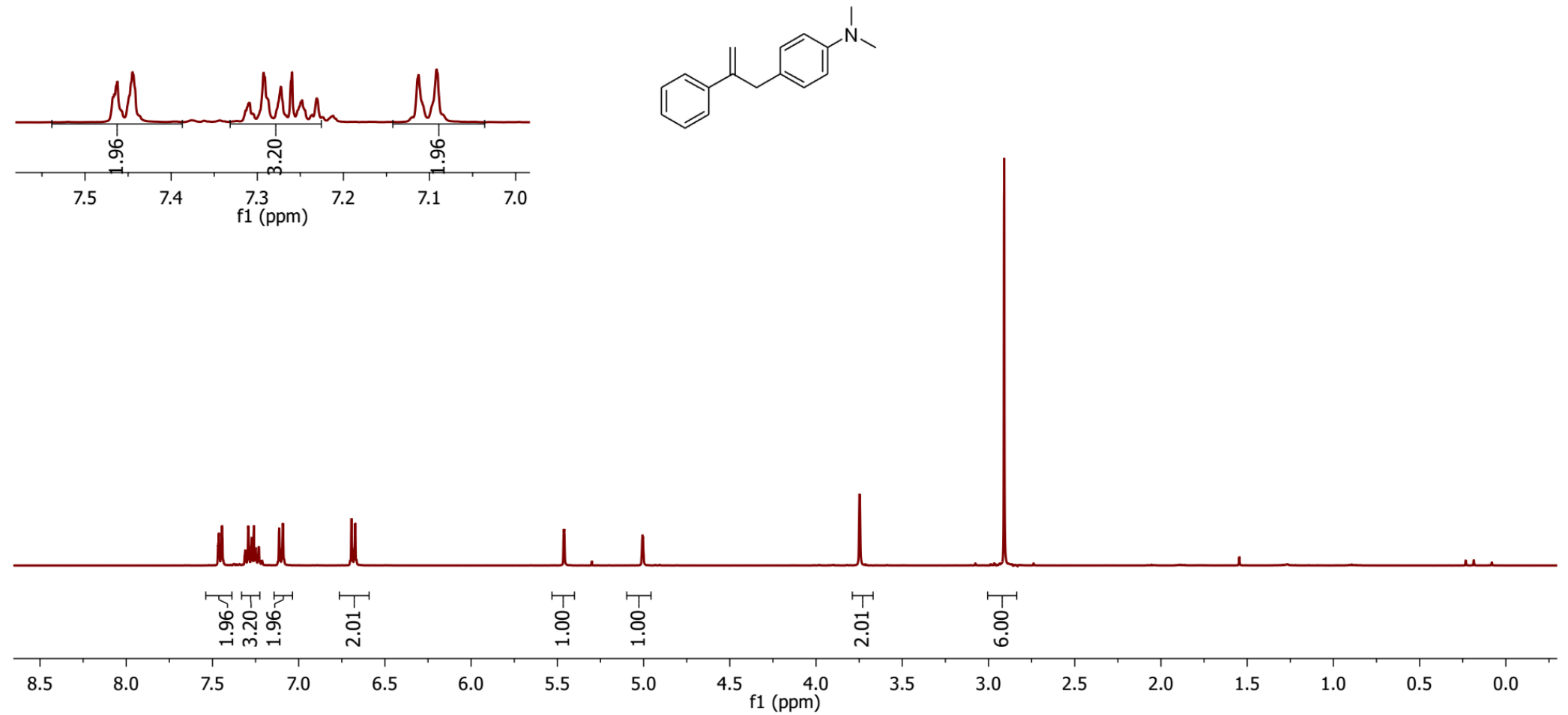
1ah ${ }^{1} \mathrm{H}$ NMR

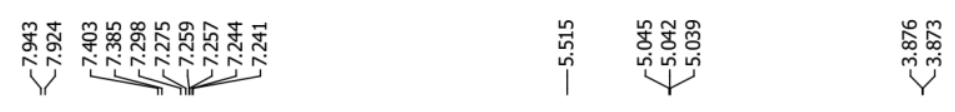
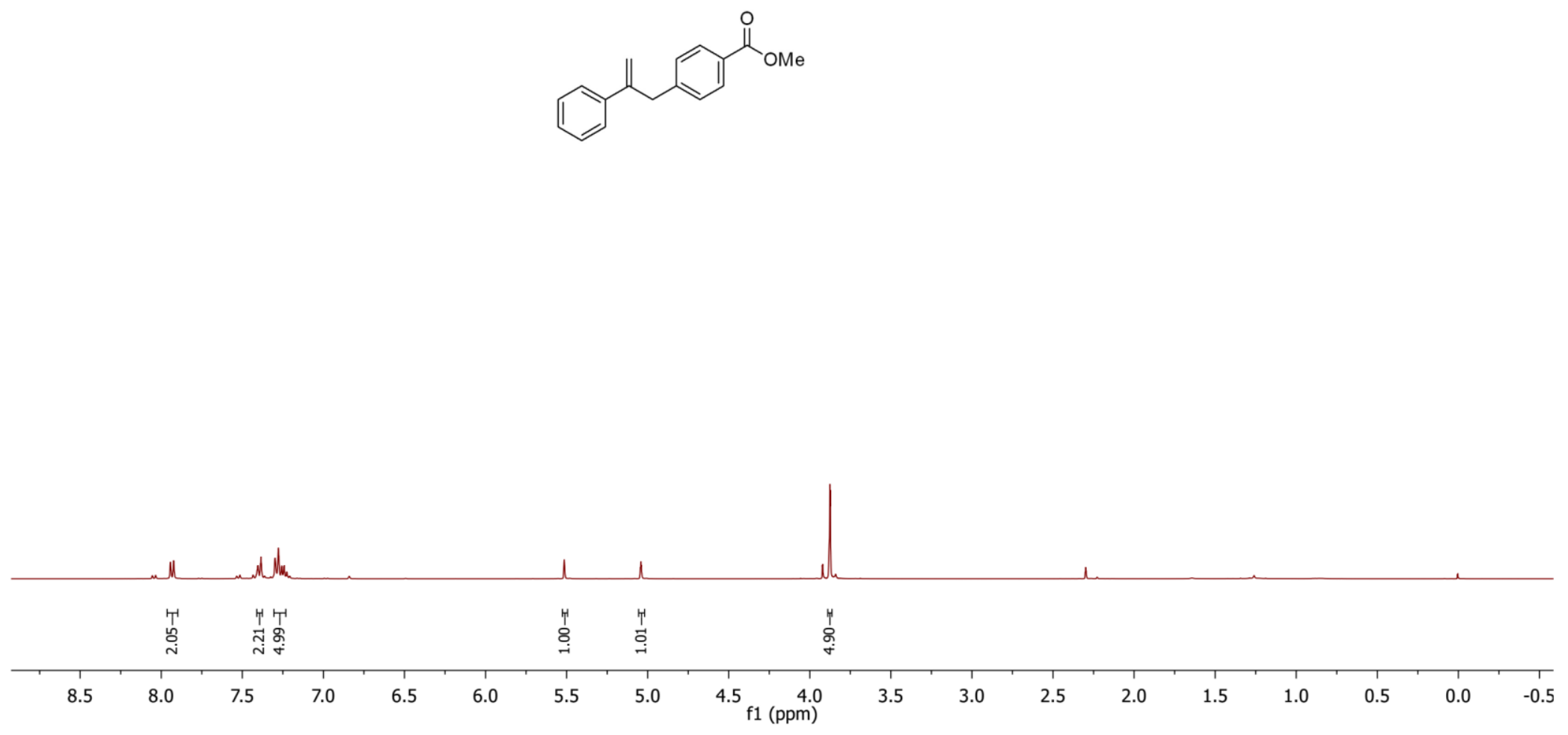


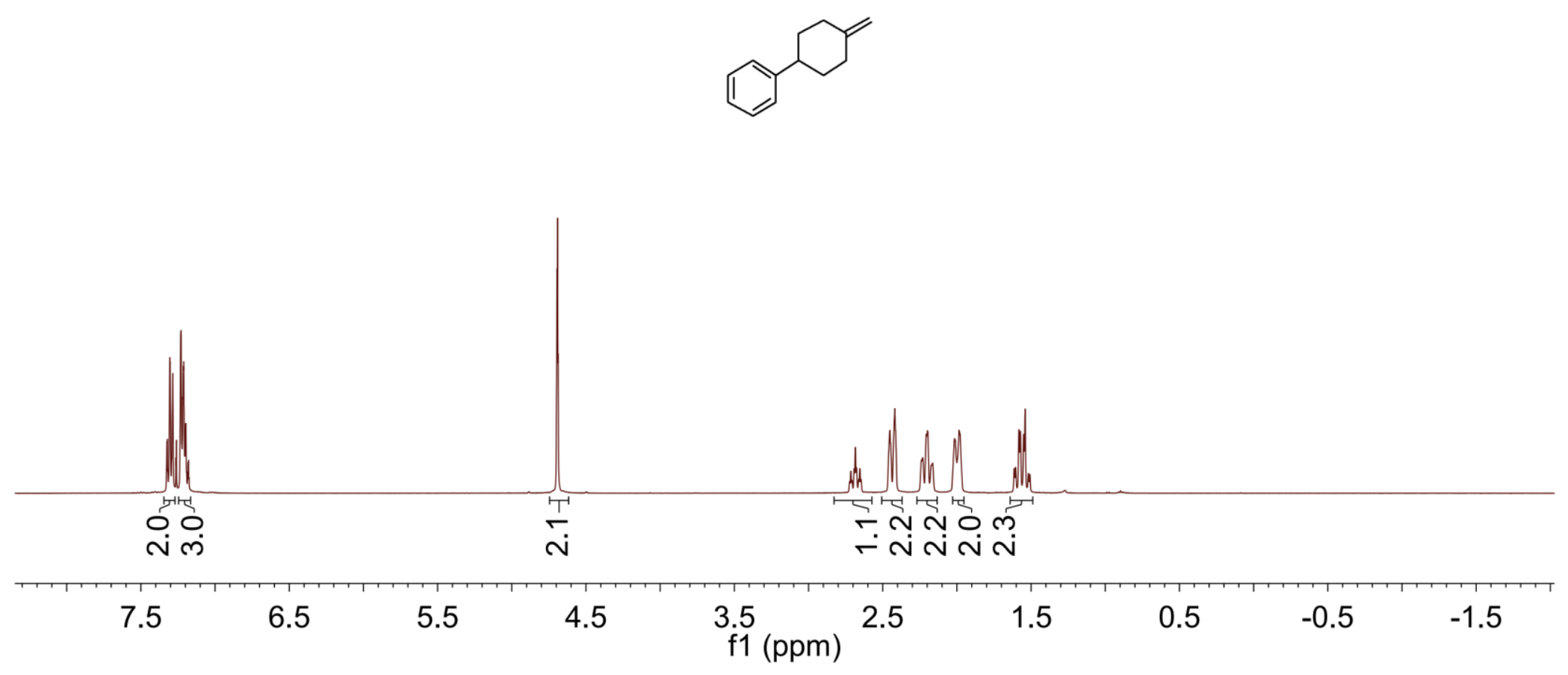


1aj ${ }^{1} \mathrm{H}$ NMR

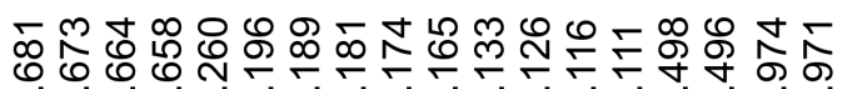

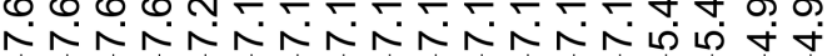

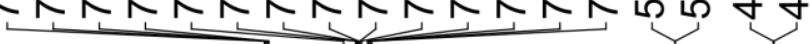

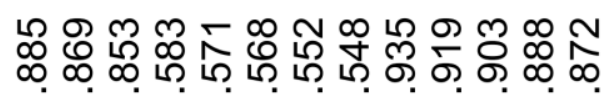

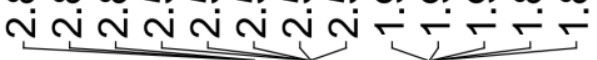
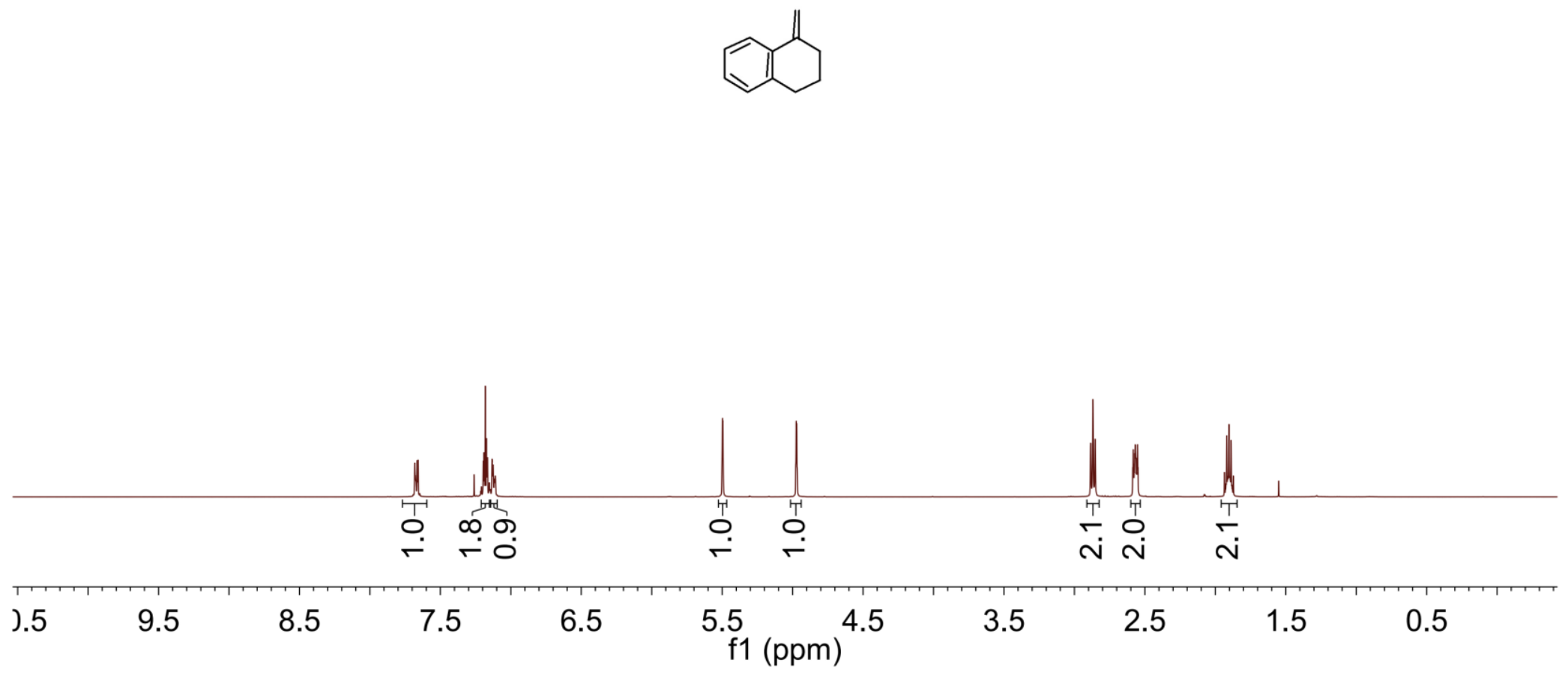


\begin{tabular}{|c|c|c|}
\hline 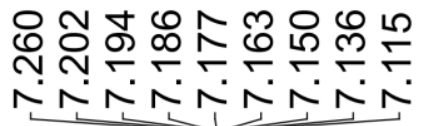 & 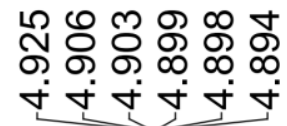 & 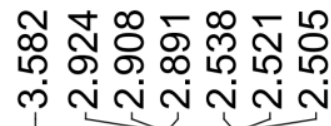 \\
\hline
\end{tabular}
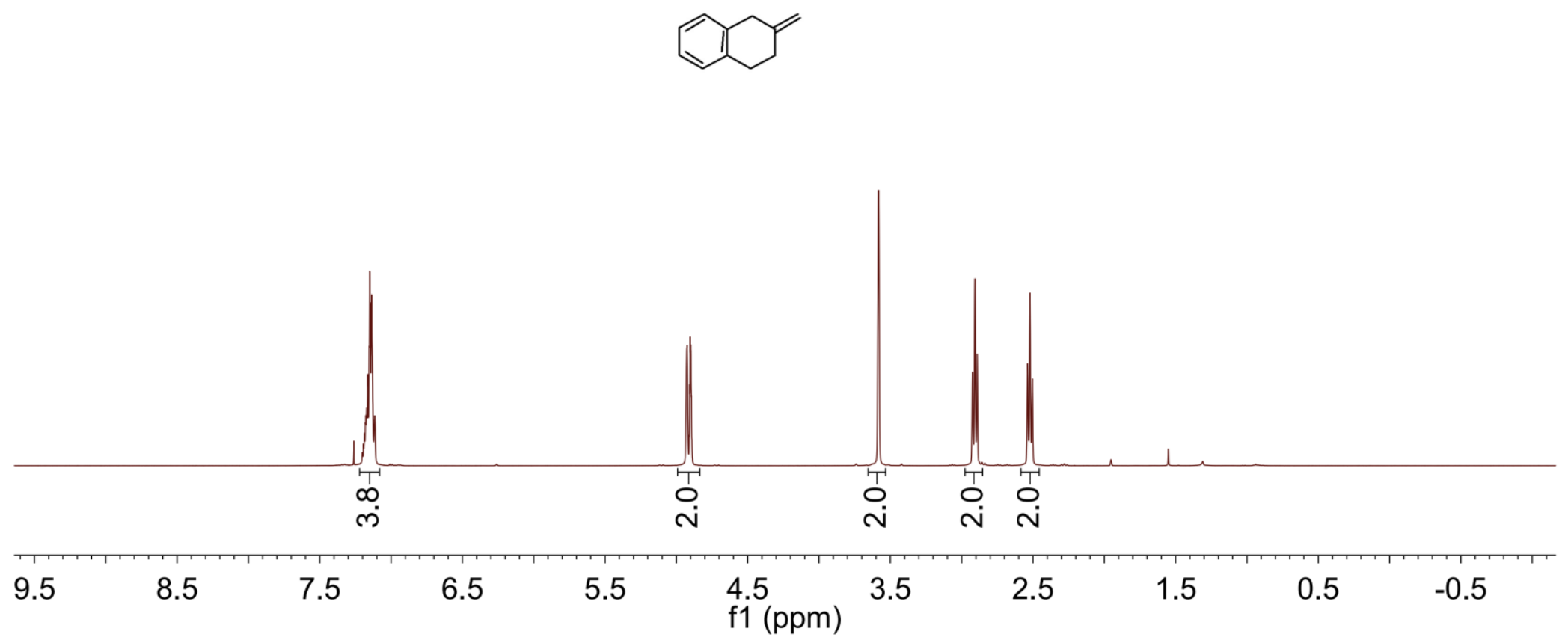


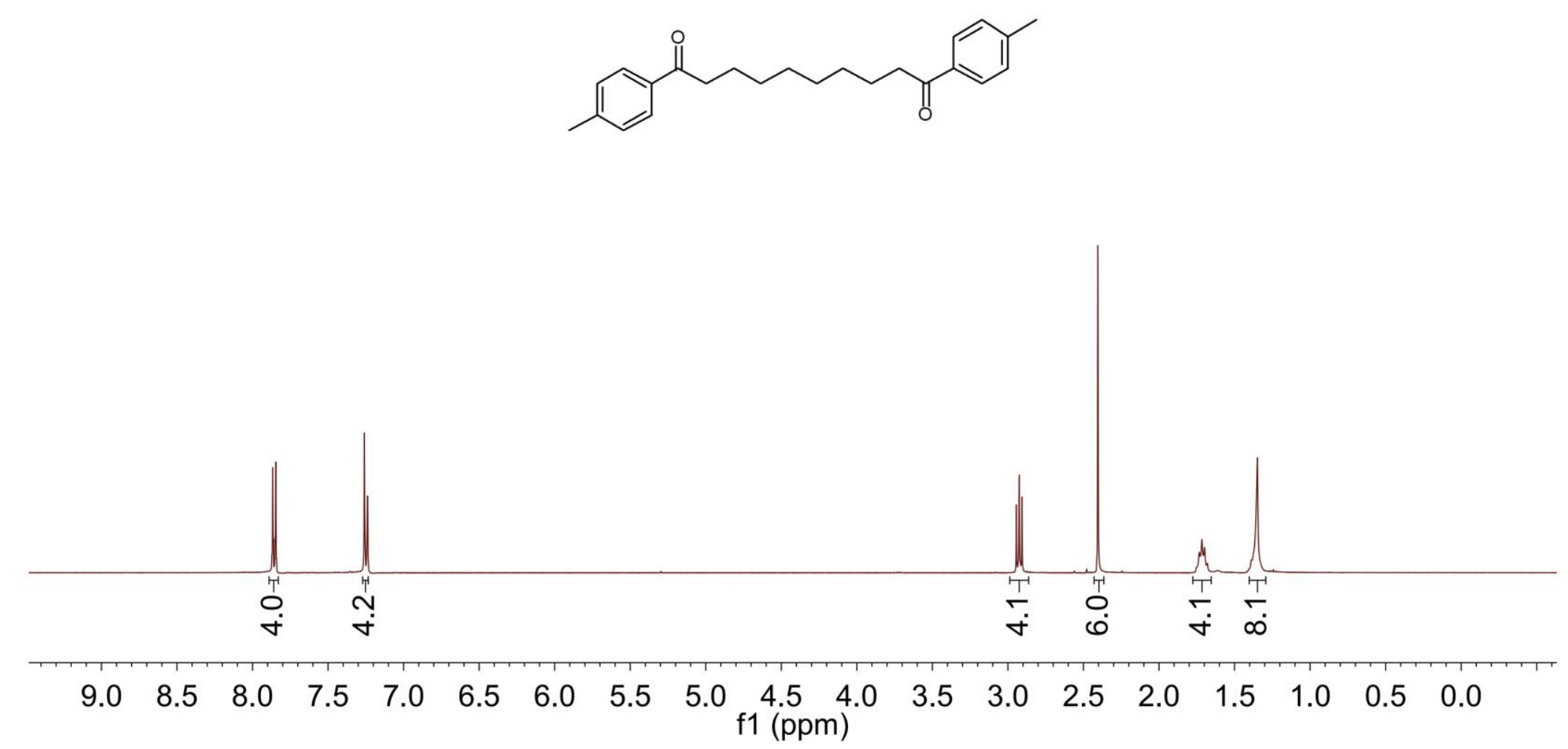




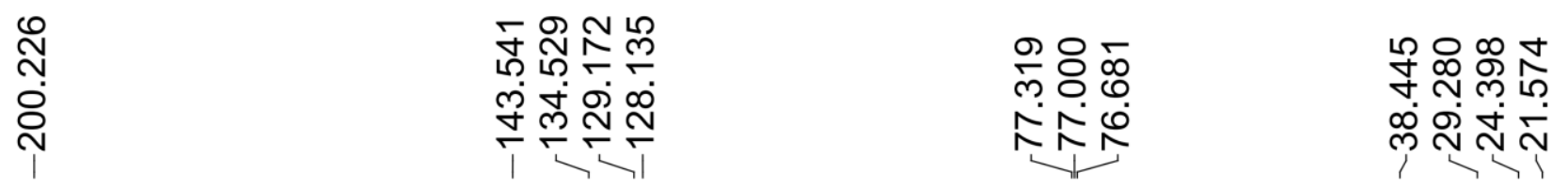

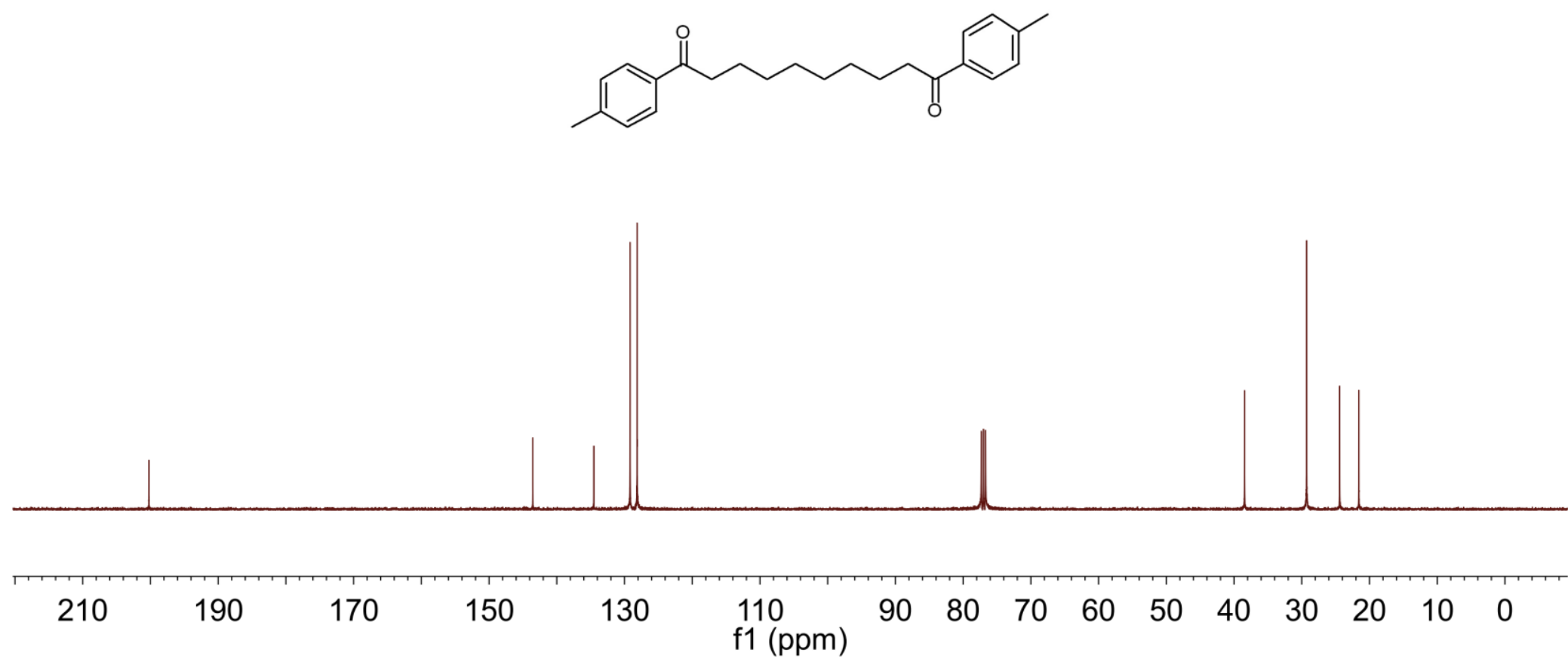




\begin{tabular}{|c|c|c|}
\hline 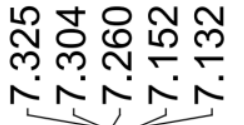 & 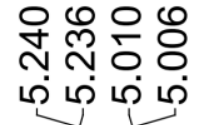 & 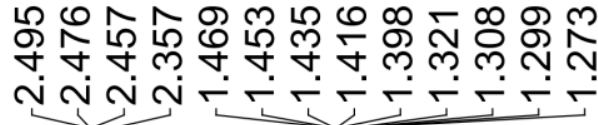 \\
\hline
\end{tabular}

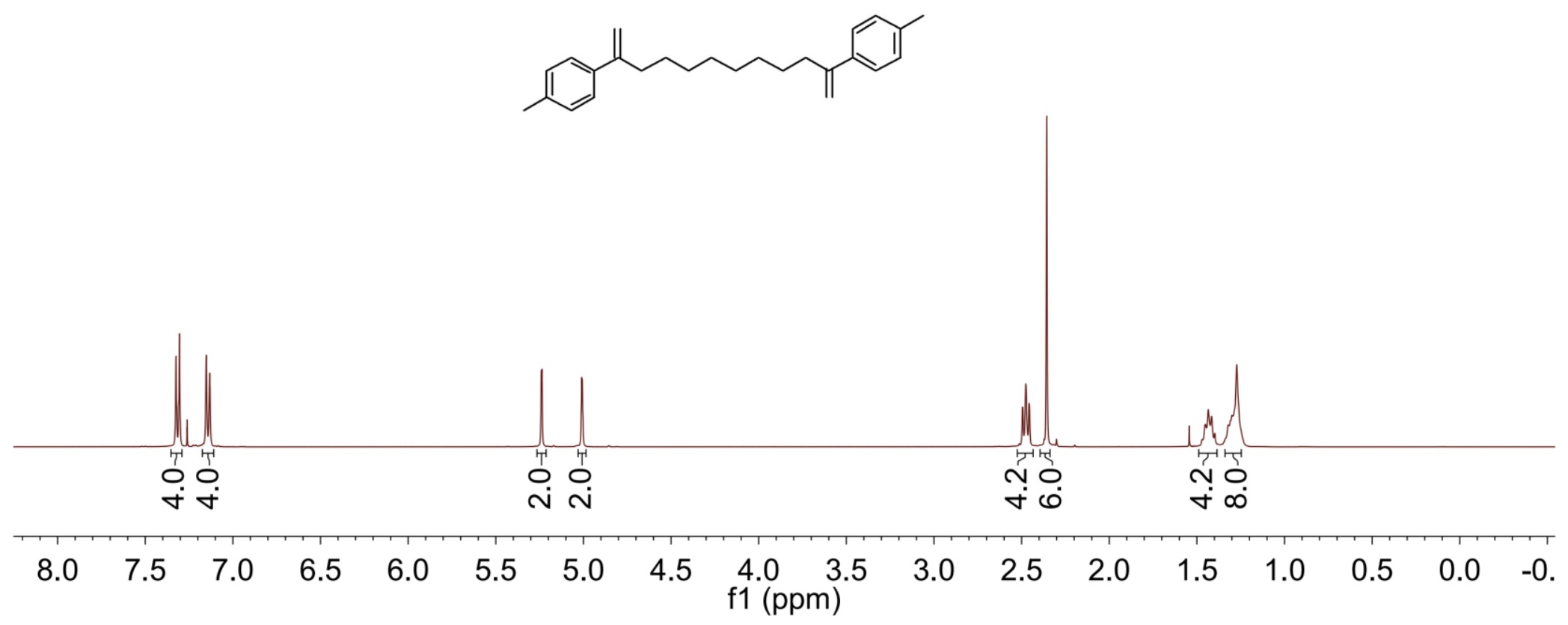




\begin{tabular}{|c|c|c|c|}
\hline 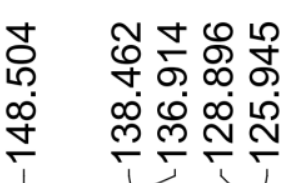 & 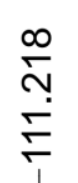 & 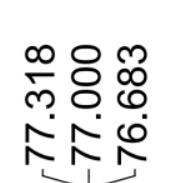 & 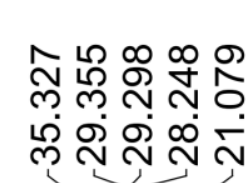 \\
\hline
\end{tabular}

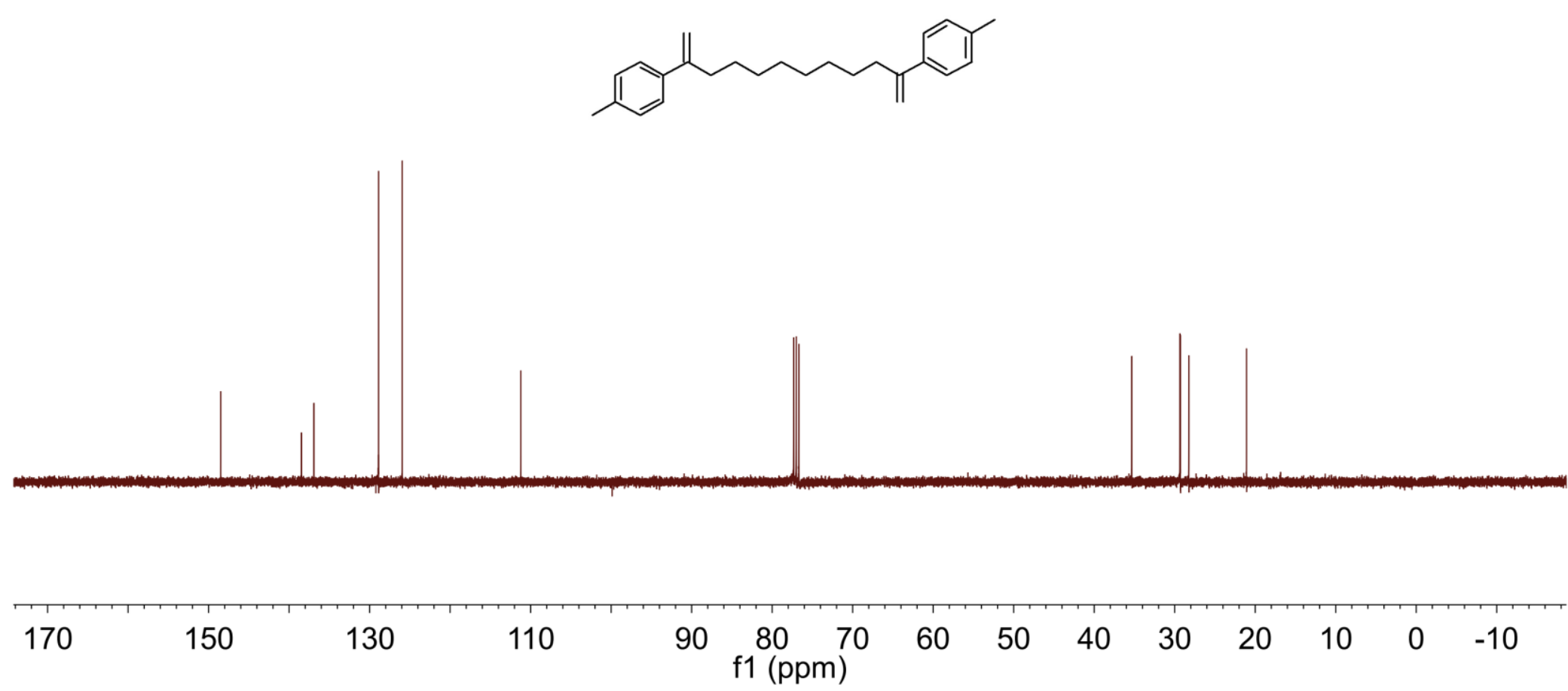


0am ${ }^{1} \mathrm{H}$ NMR

幽

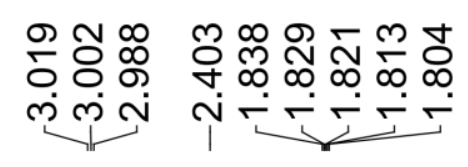

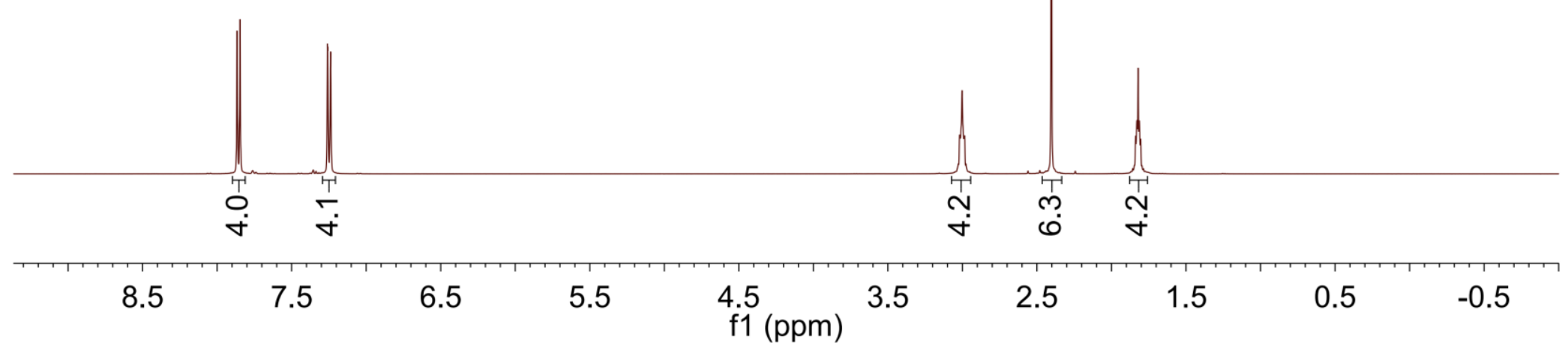

S141 


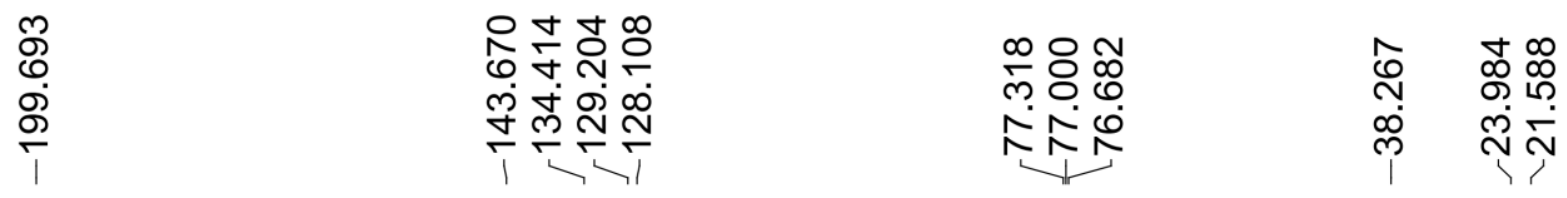
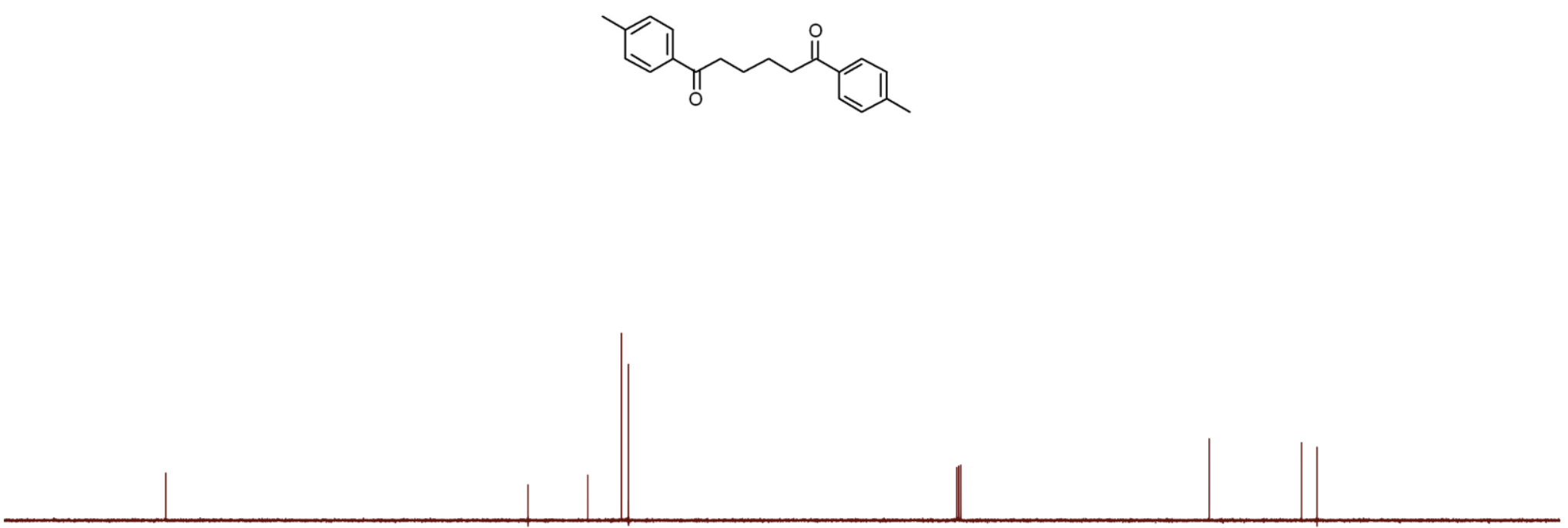

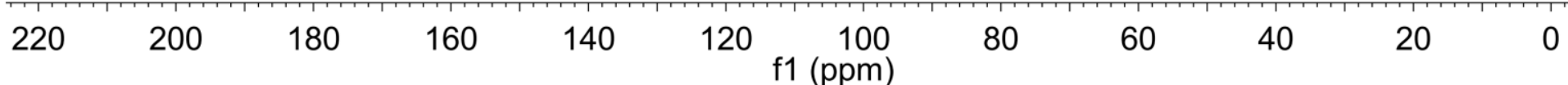


$1 a m{ }^{1} \mathrm{H}$ NMR

\begin{tabular}{|c|c|c|}
\hline 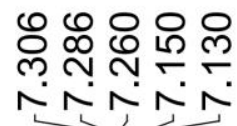 & 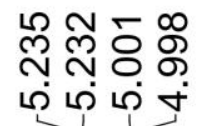 & 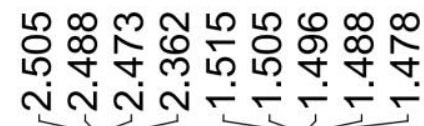 \\
\hline
\end{tabular}

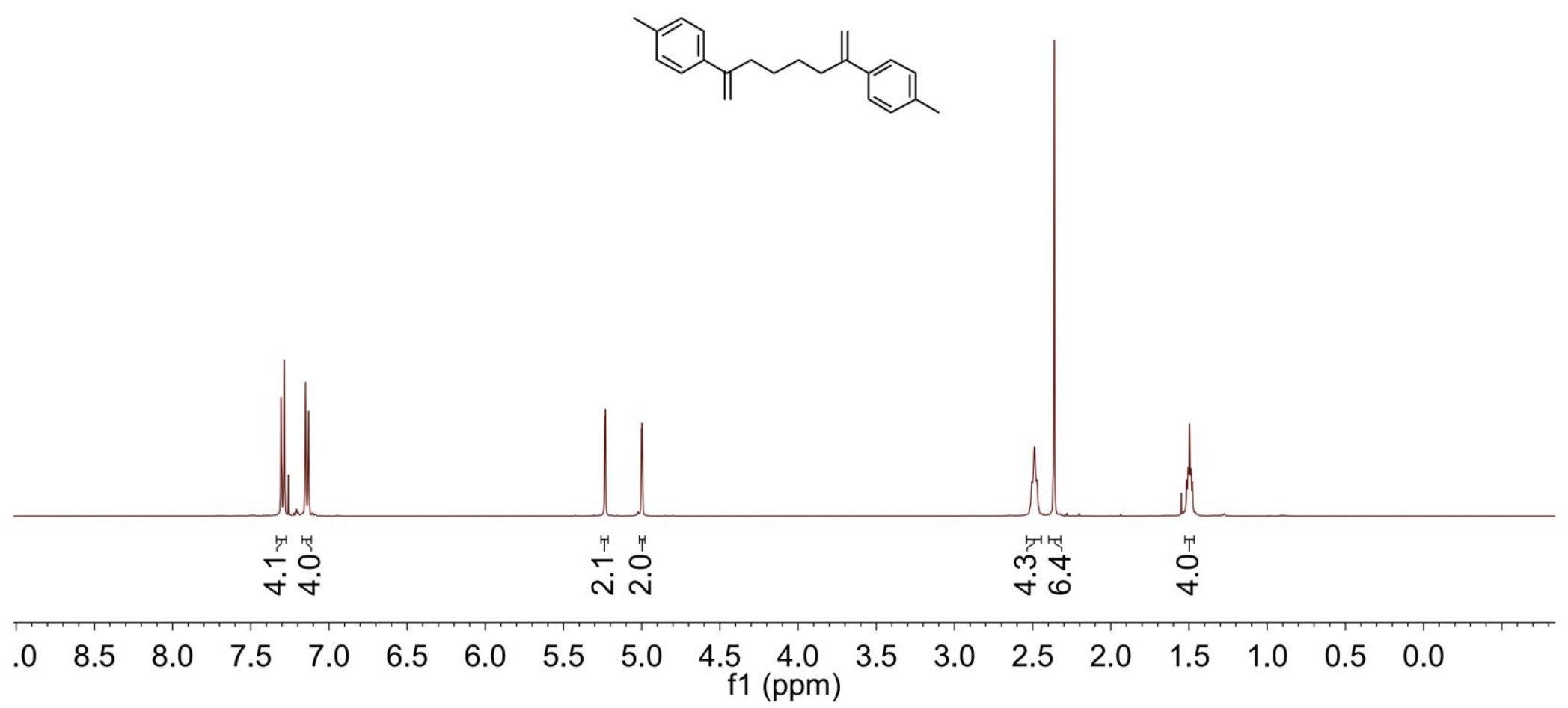




\begin{tabular}{|c|c|c|c|}
\hline 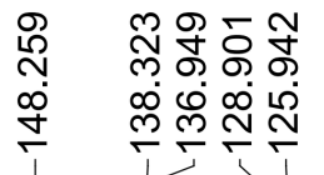 & $\begin{array}{l}\infty \\
\infty \\
\stackrel{\Gamma}{\mp} \\
F\end{array}$ & 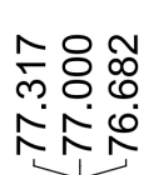 & 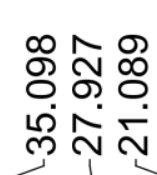 \\
\hline
\end{tabular}
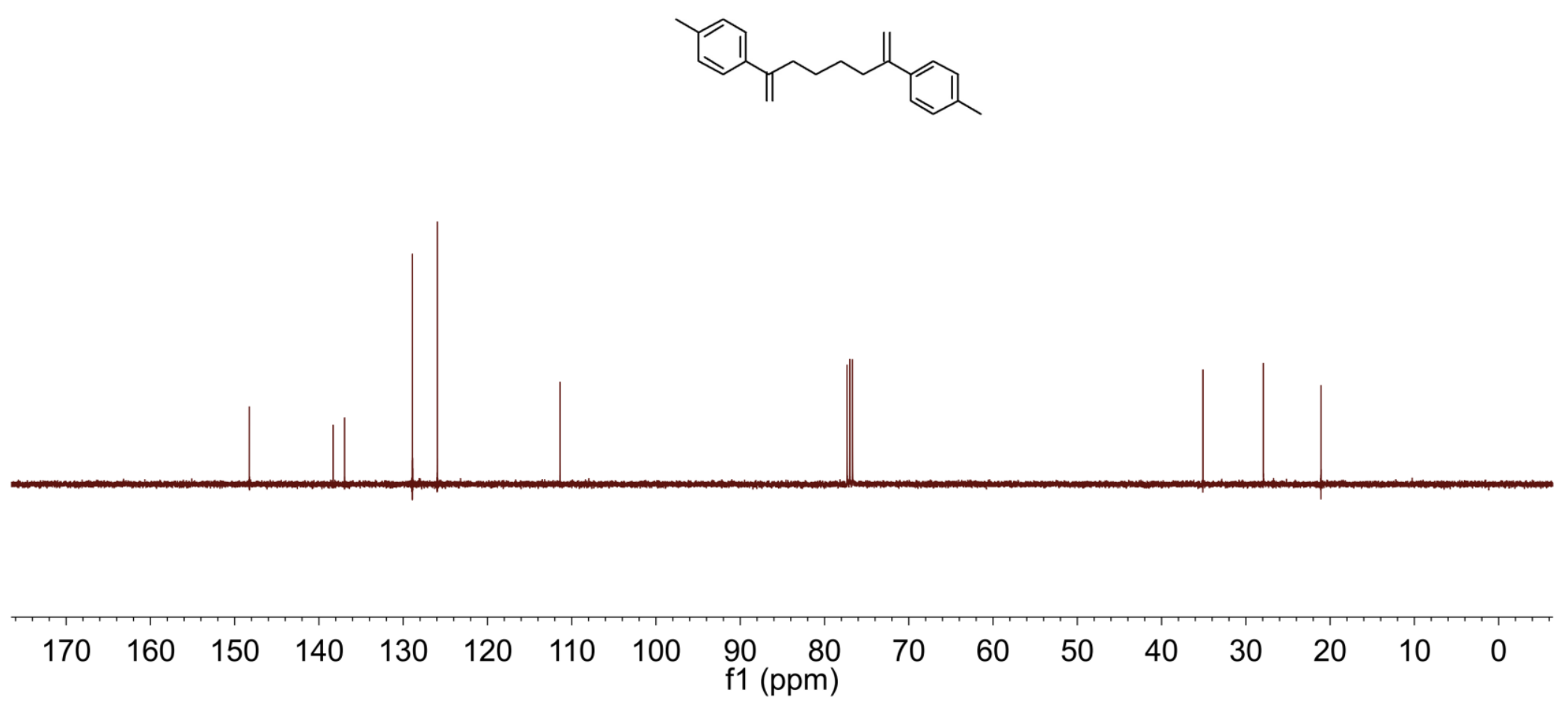
Oan ${ }^{1} \mathrm{H}$ NMR

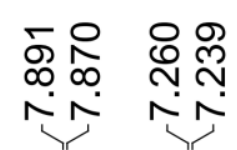

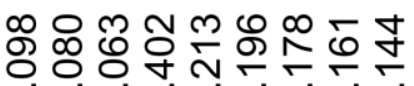

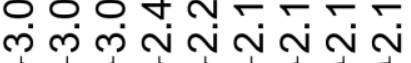
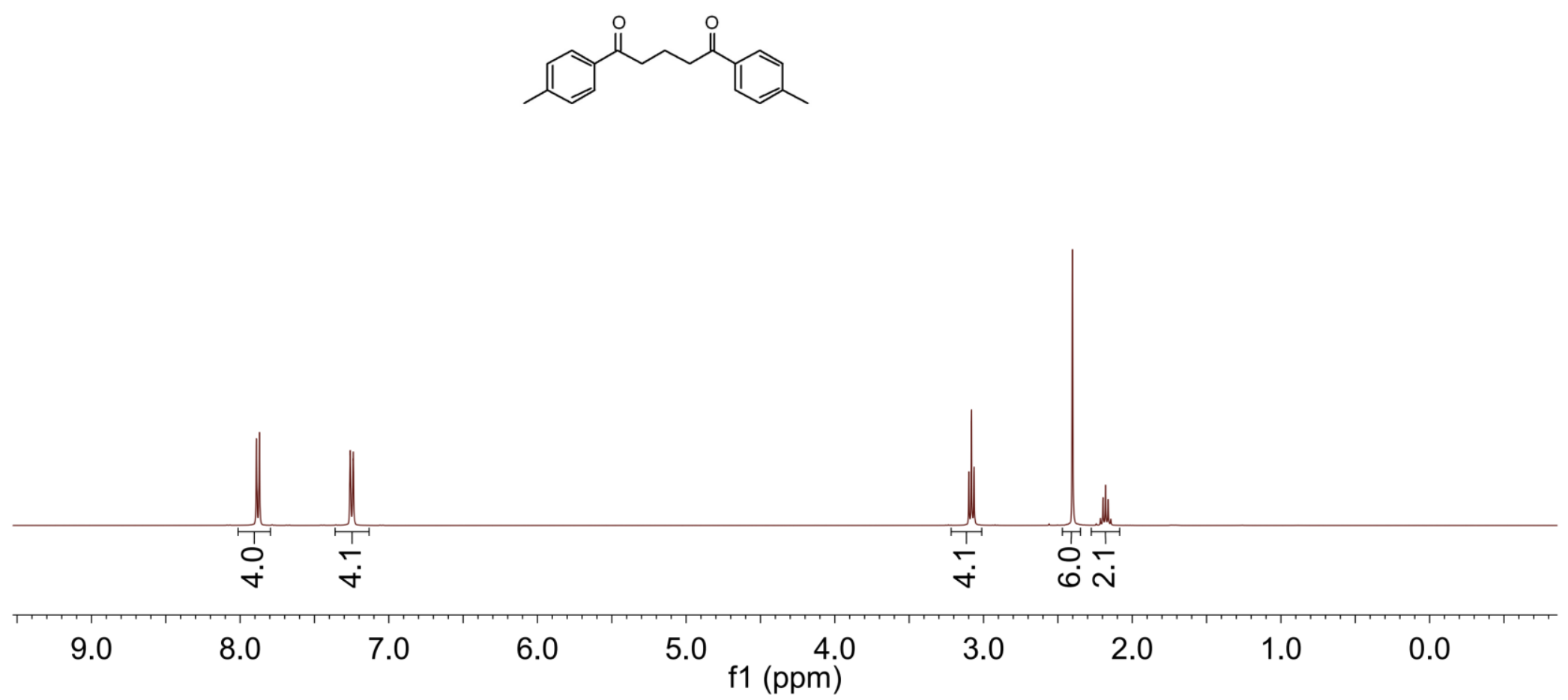
Oan ${ }^{13} \mathrm{C}$ NMR

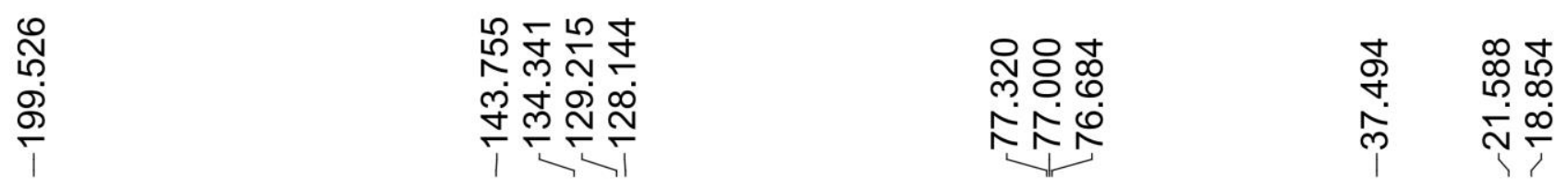

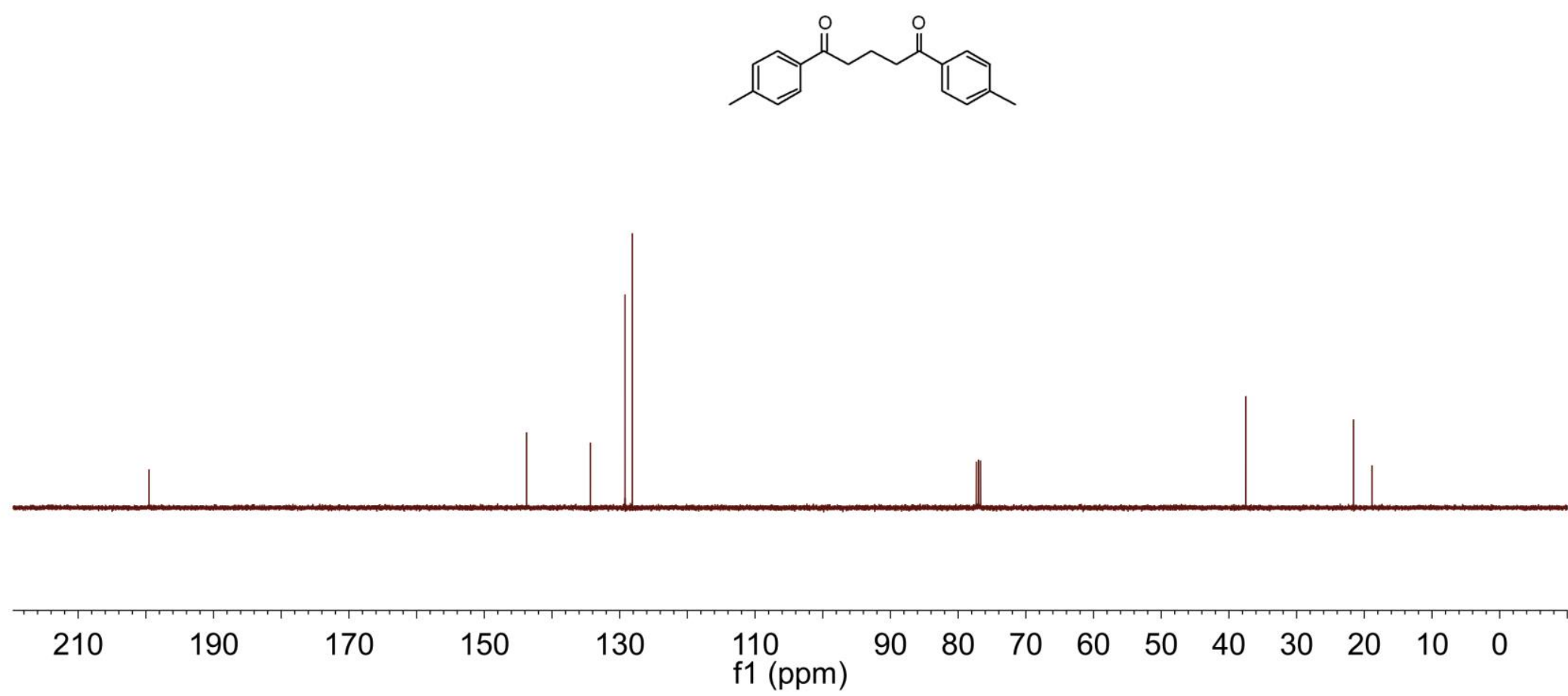




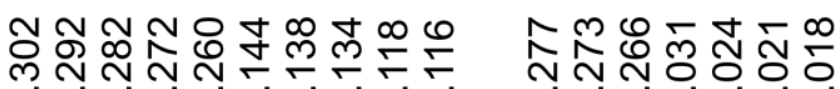

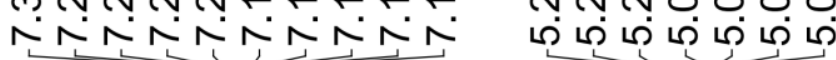

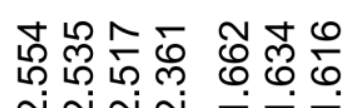

กั่ง

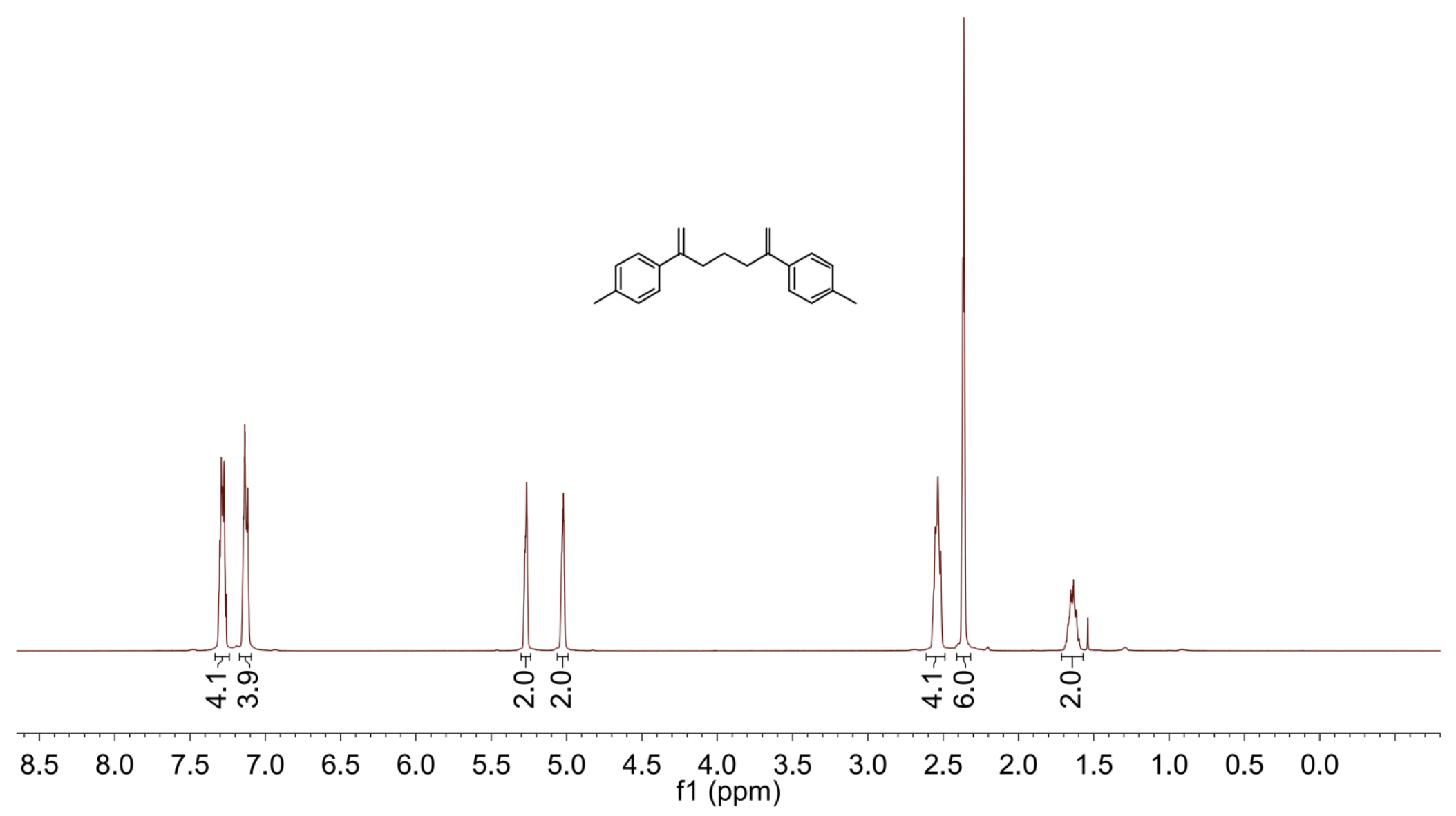


1an ${ }^{13} \mathrm{C}$ NMR

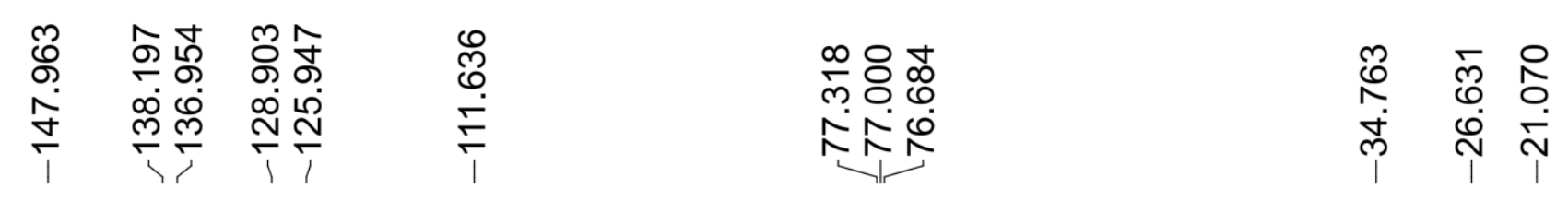
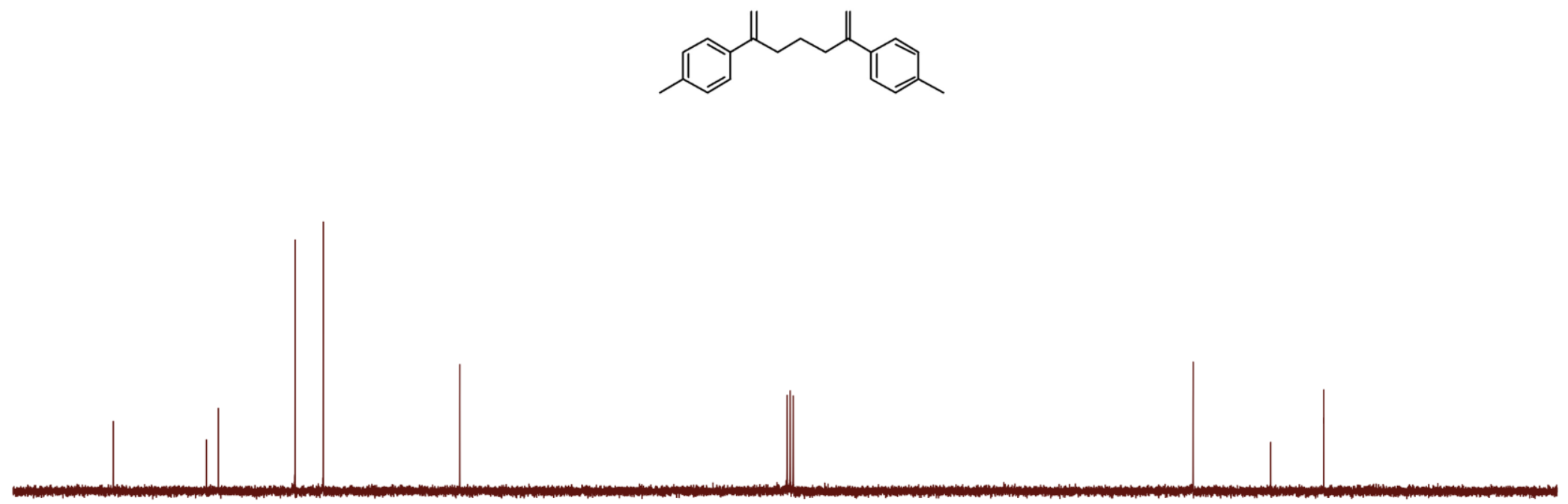

$150 \quad 140 \quad 130 \quad 120$

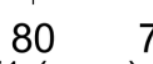

f1 (ppm)

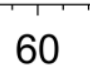

$50 \quad 40$
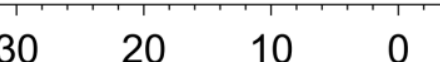
Oao ${ }^{1} \mathrm{H}$ NMR

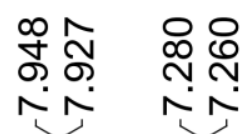

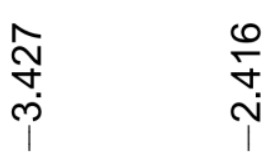
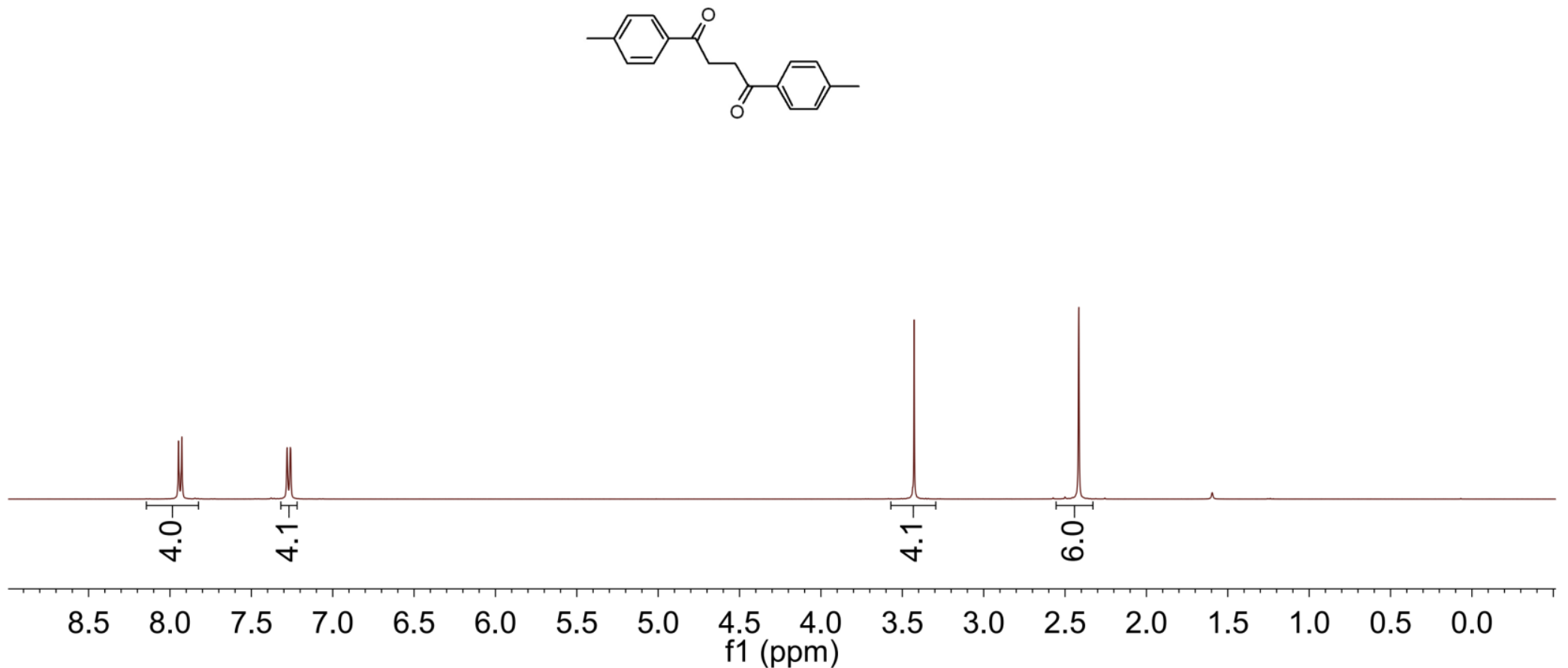
1 ao ${ }^{1} \mathrm{H}$ NMR

\begin{tabular}{|c|c|}
\hline 柋 & 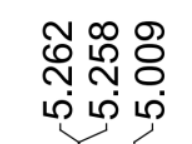 \\
\hline
\end{tabular}
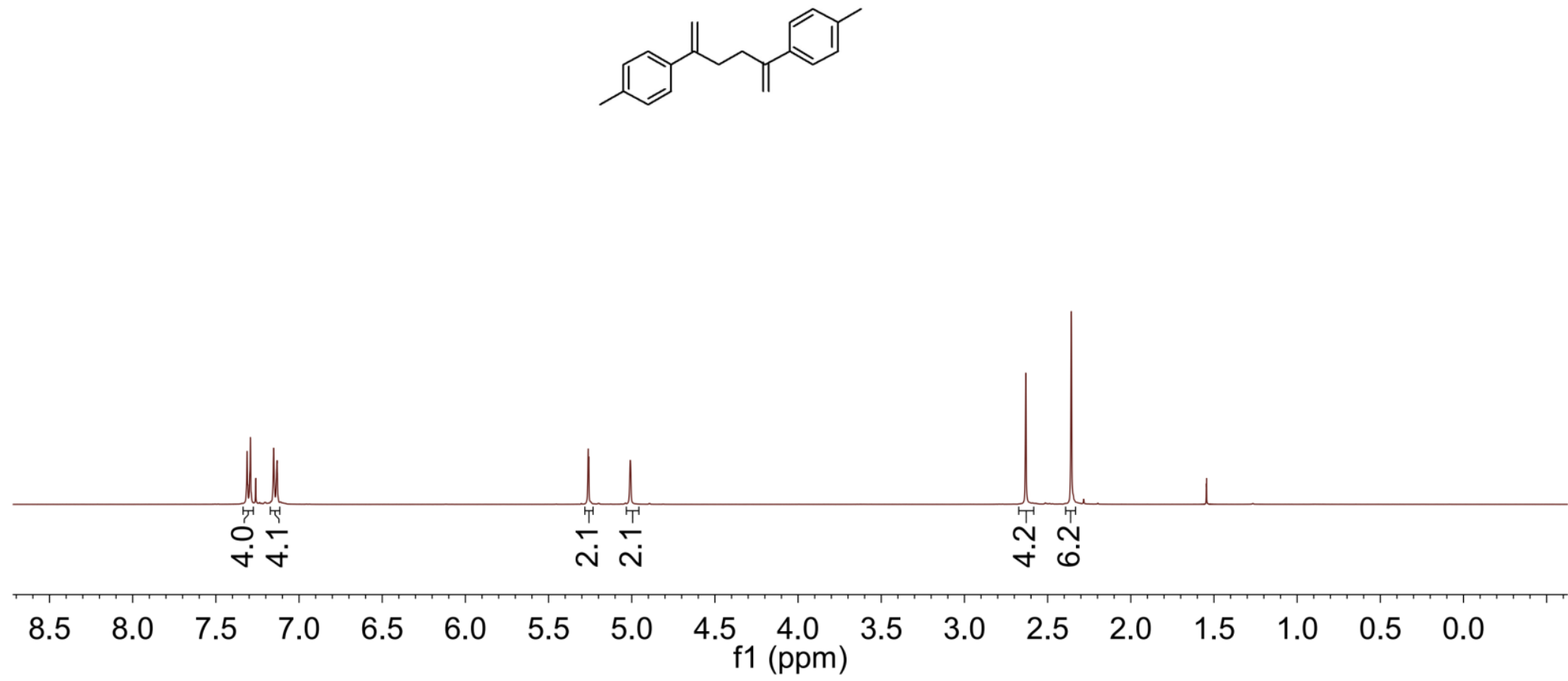
Oap ${ }^{1} \mathrm{H} N M R$

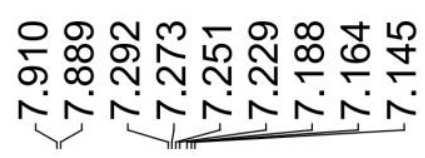

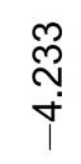

ণ্+ু
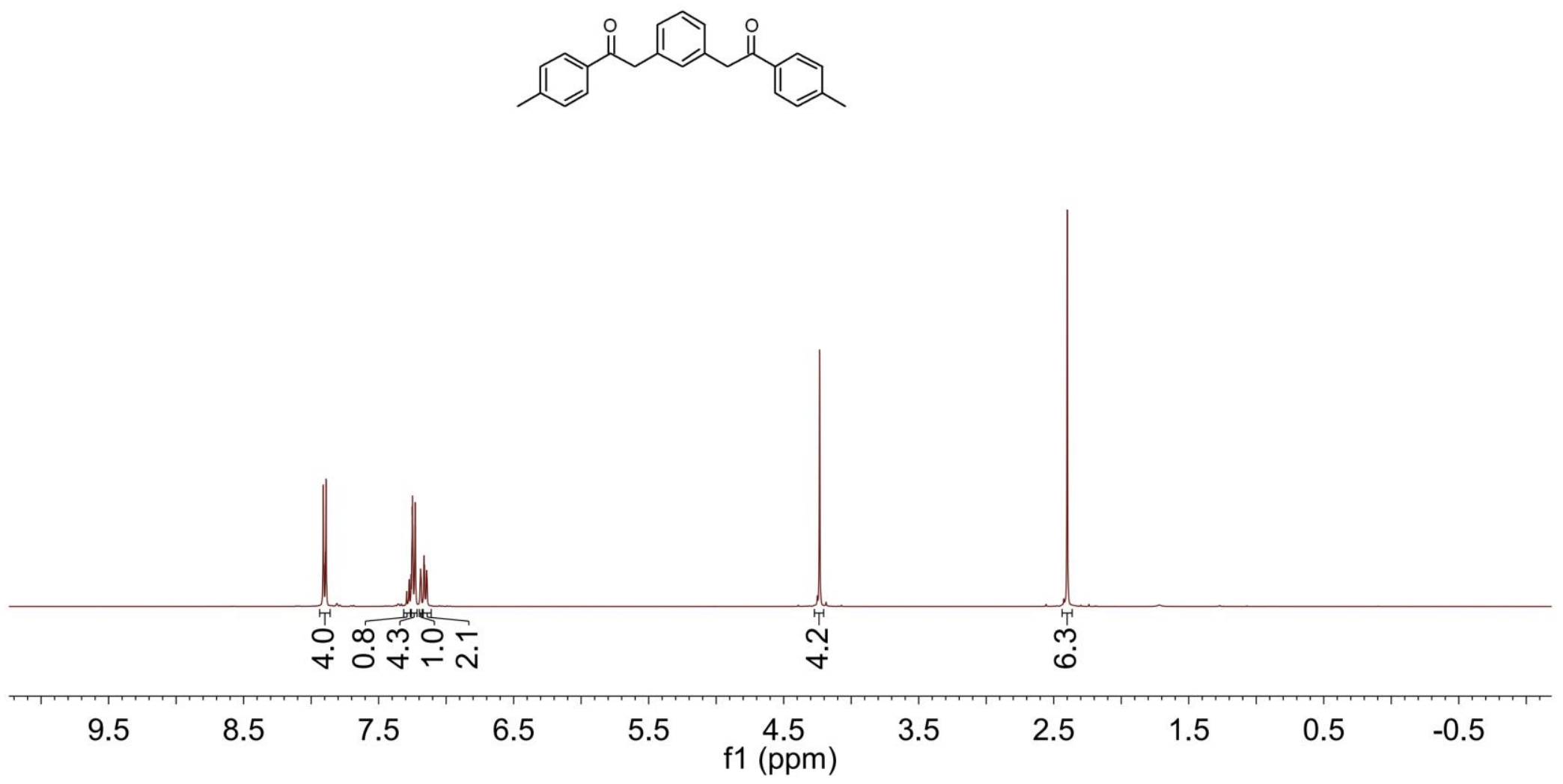

S151 
Oap ${ }^{13} \mathrm{C}$ NMR
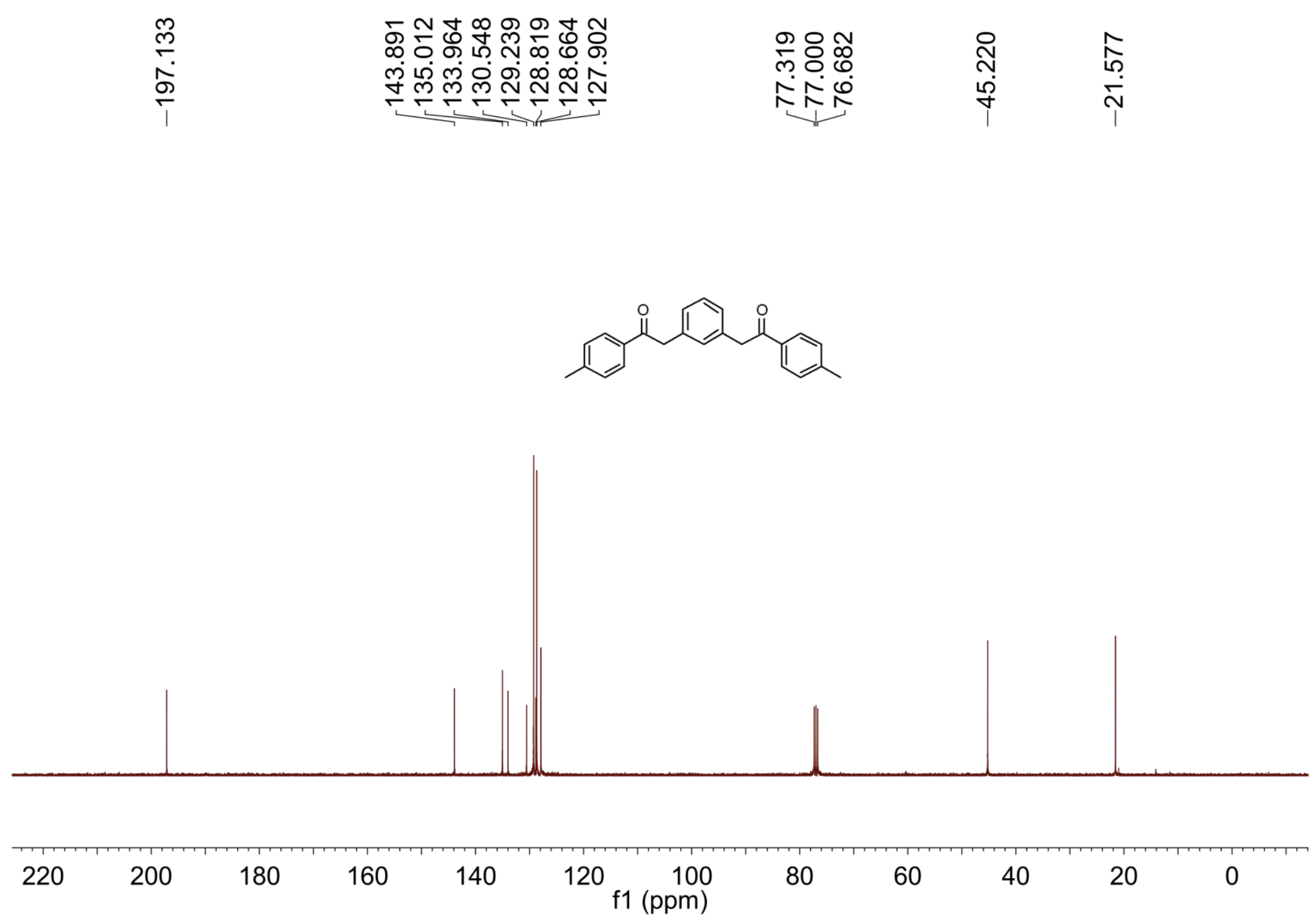

S152 
1ap ${ }^{1} \mathrm{H}$ NMR

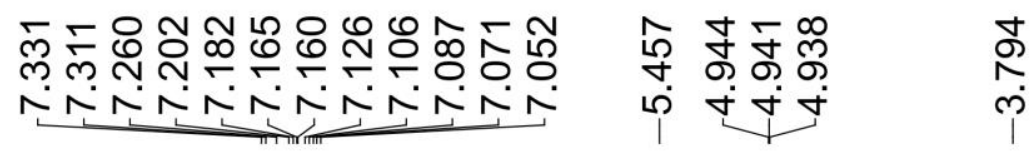

$\stackrel{\text { Fे }}{\text { N }}$
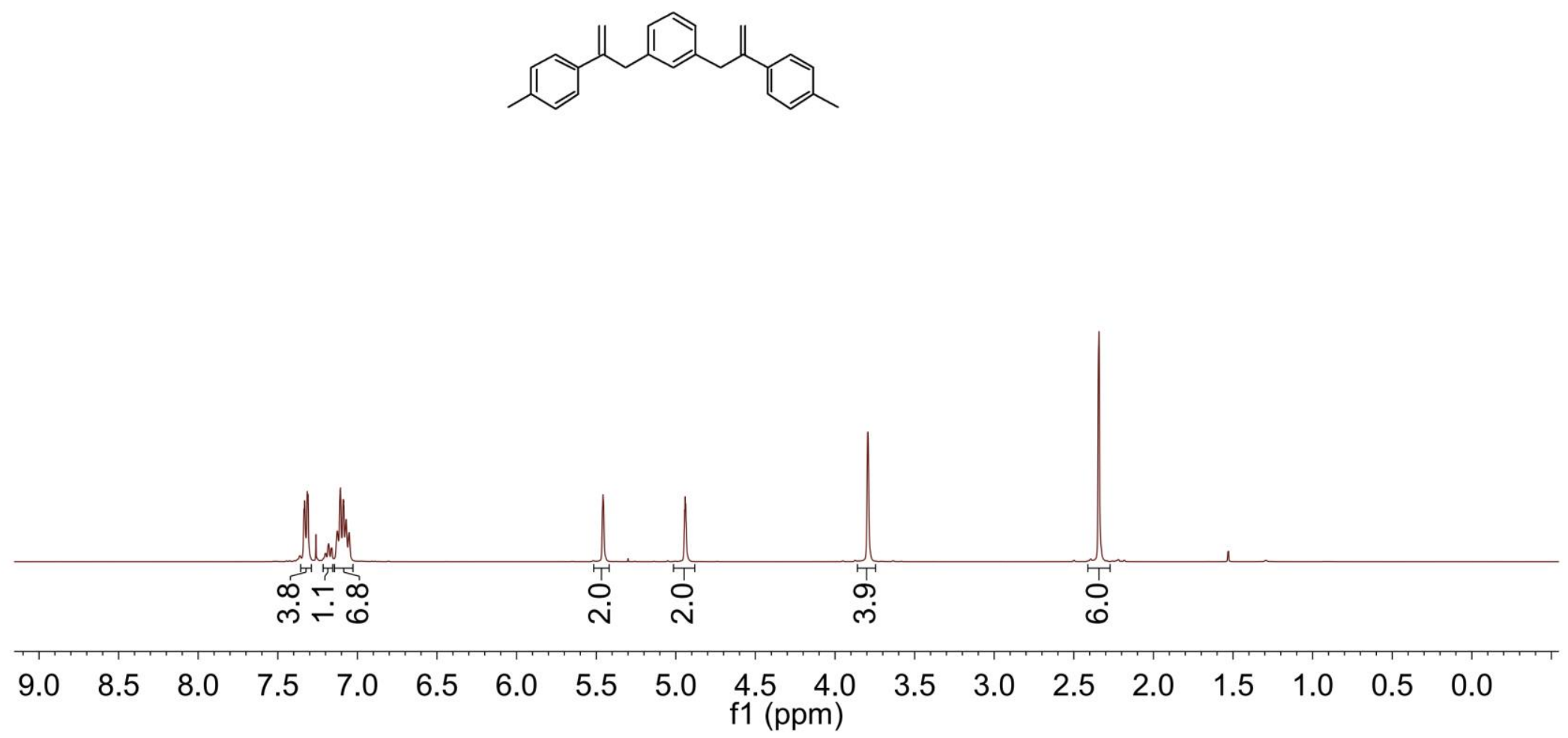

S153 

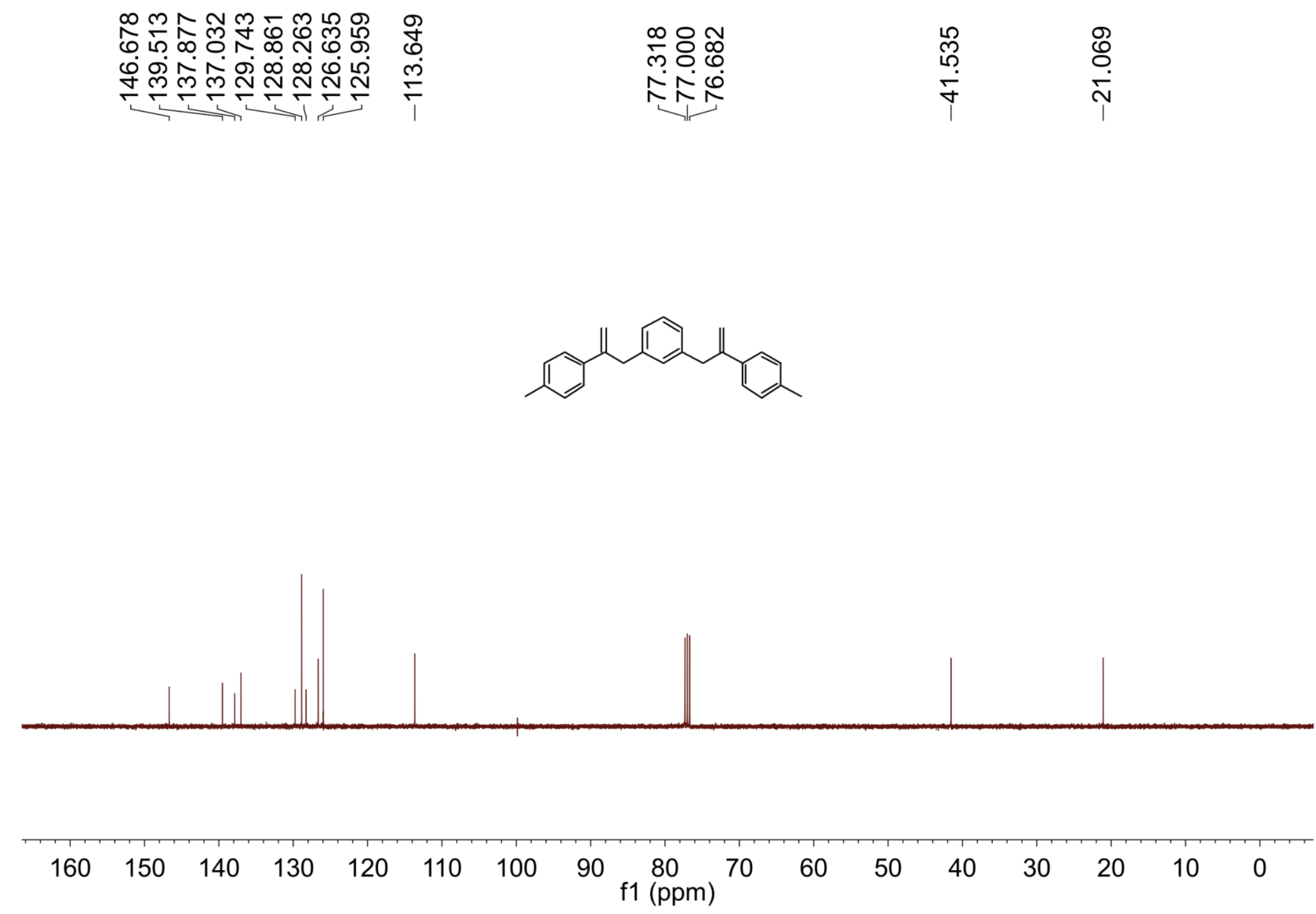


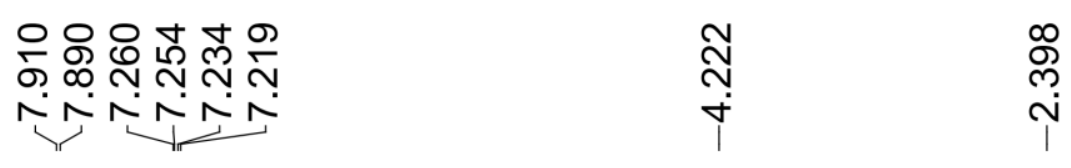

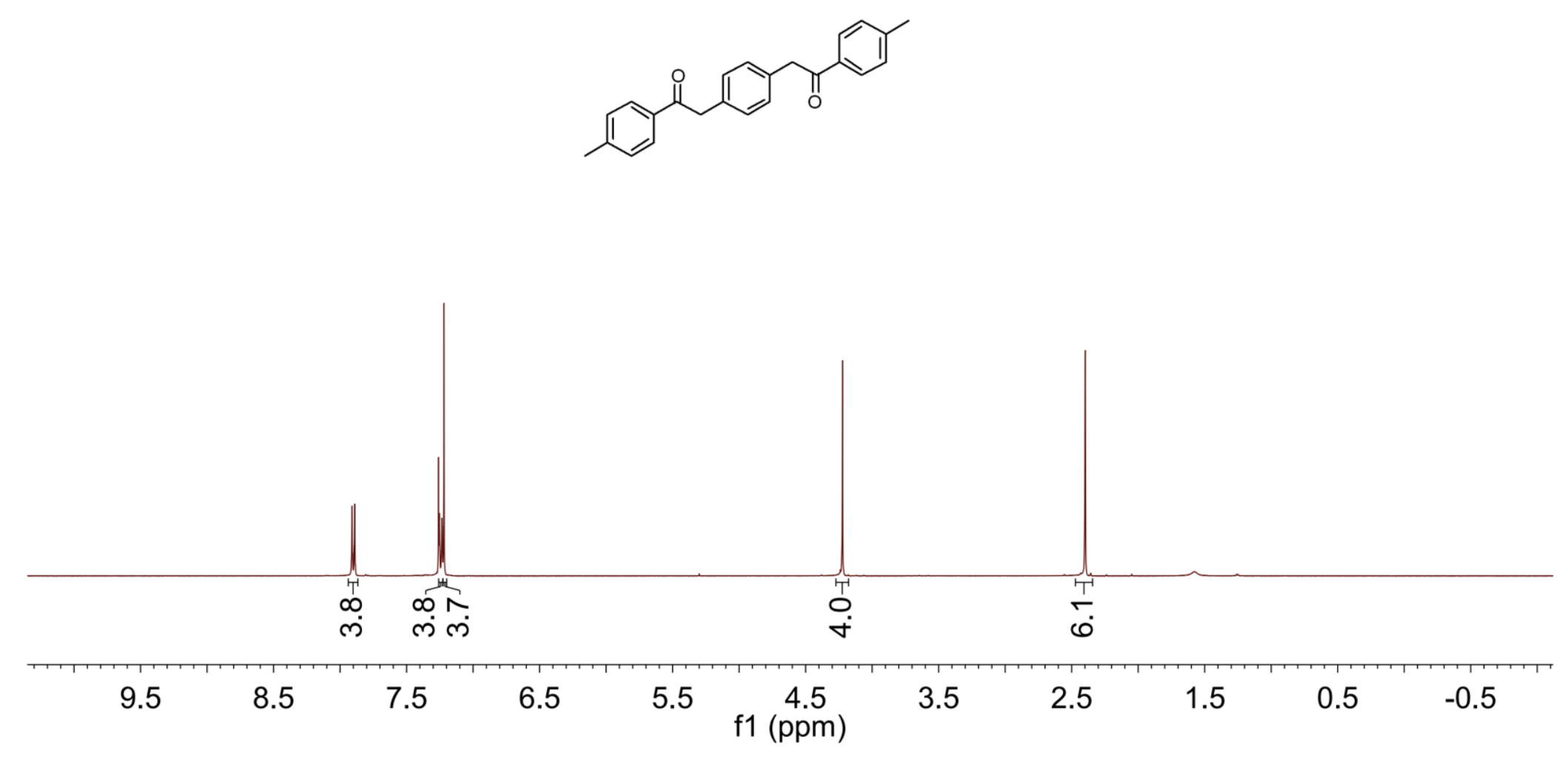


Oaq ${ }^{13}$ C NMR

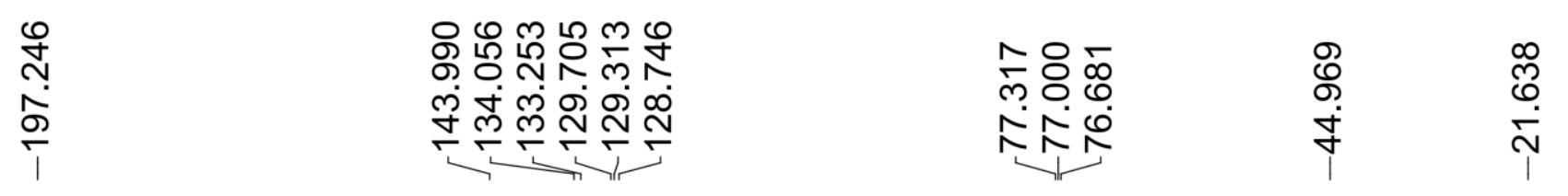

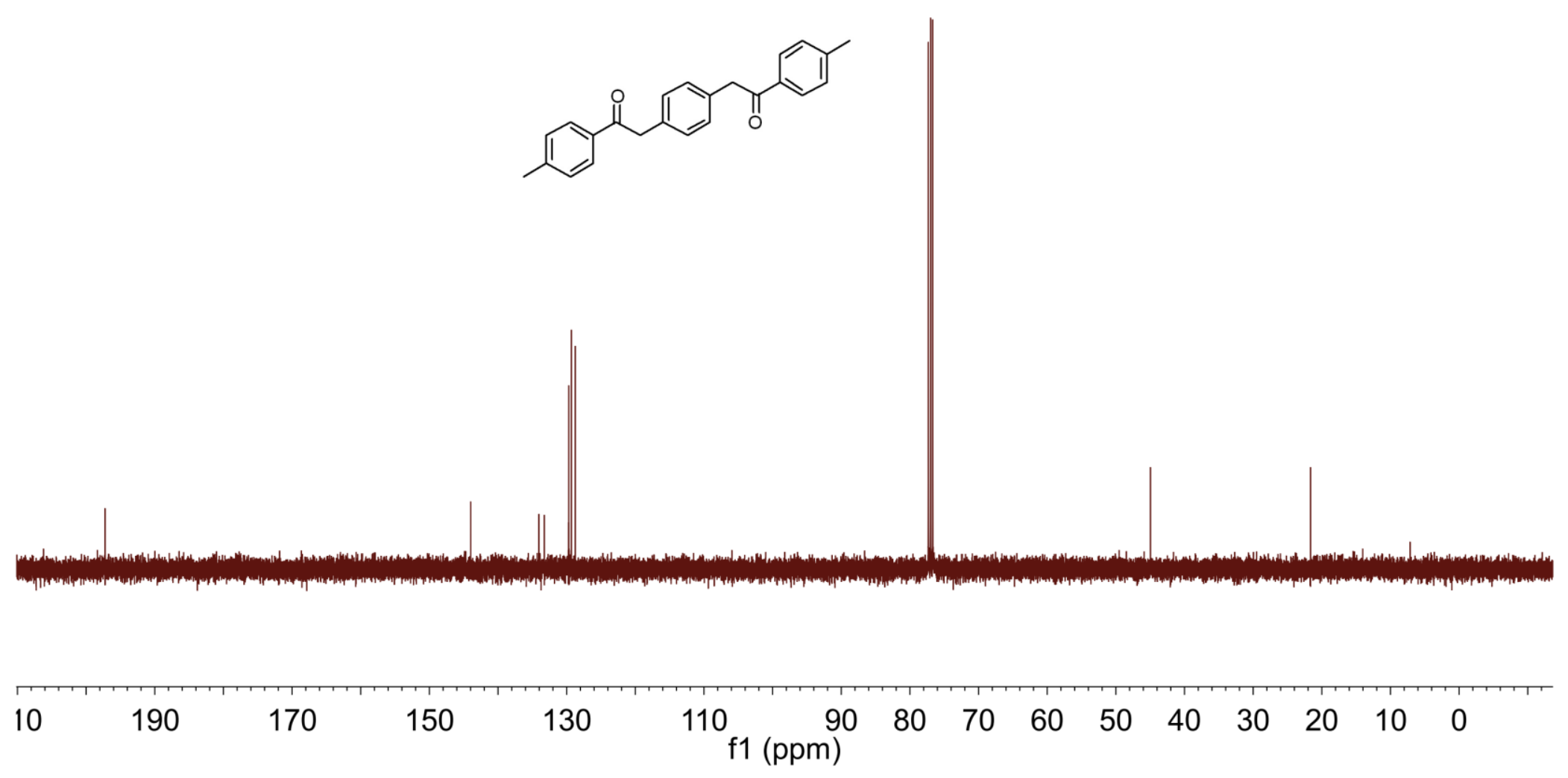




\begin{tabular}{|c|c|c|}
\hline 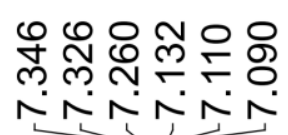 & 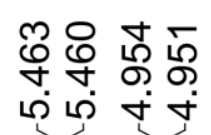 & $\begin{array}{l}\infty \\
\stackrel{\infty}{\infty} \\
\dot{\varphi}\end{array}$ \\
\hline
\end{tabular}

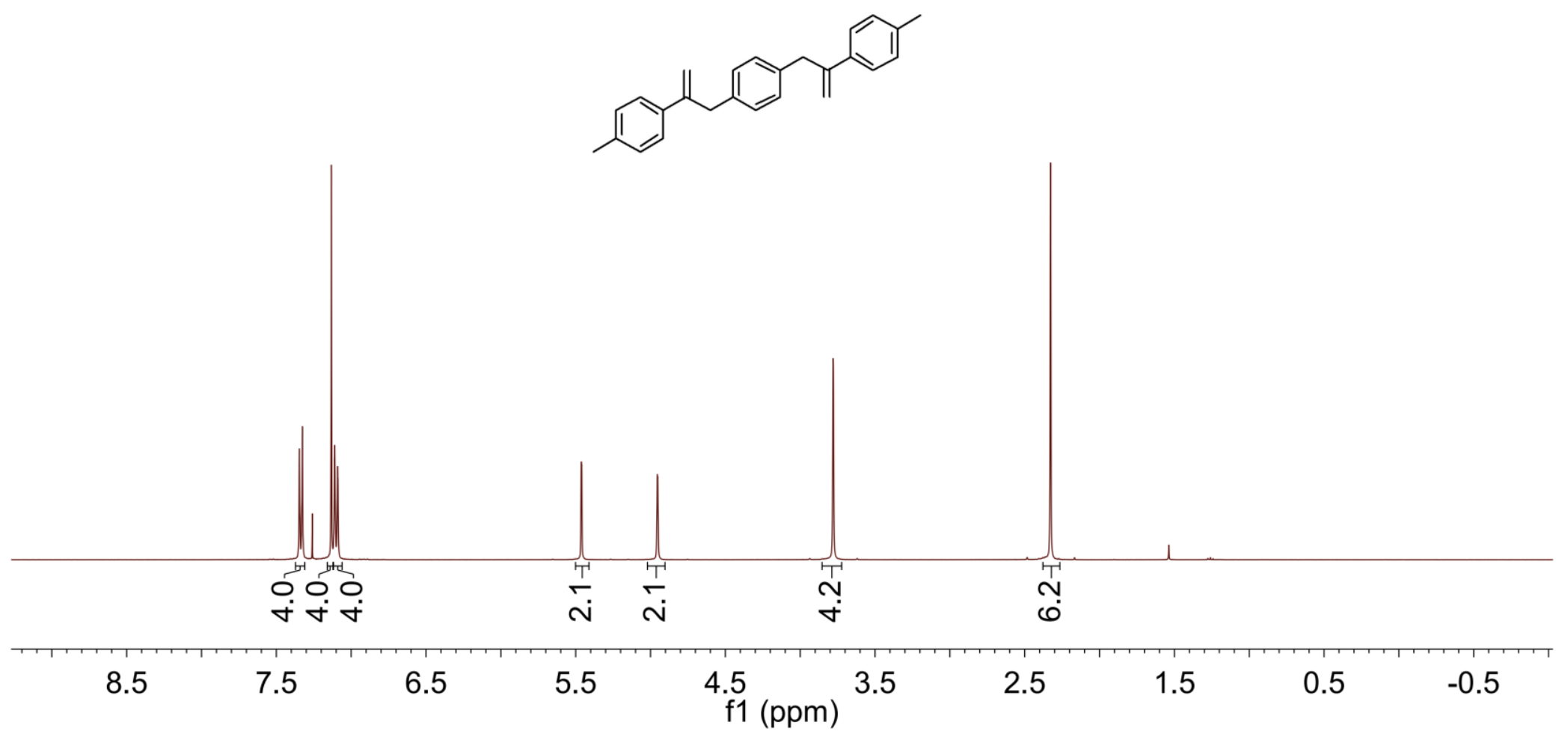


$1 \mathrm{aq}{ }^{13} \mathrm{C}$ NMR

\begin{tabular}{|c|c|c|}
\hline & 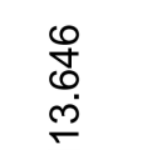 & مِ \\
\hline
\end{tabular}

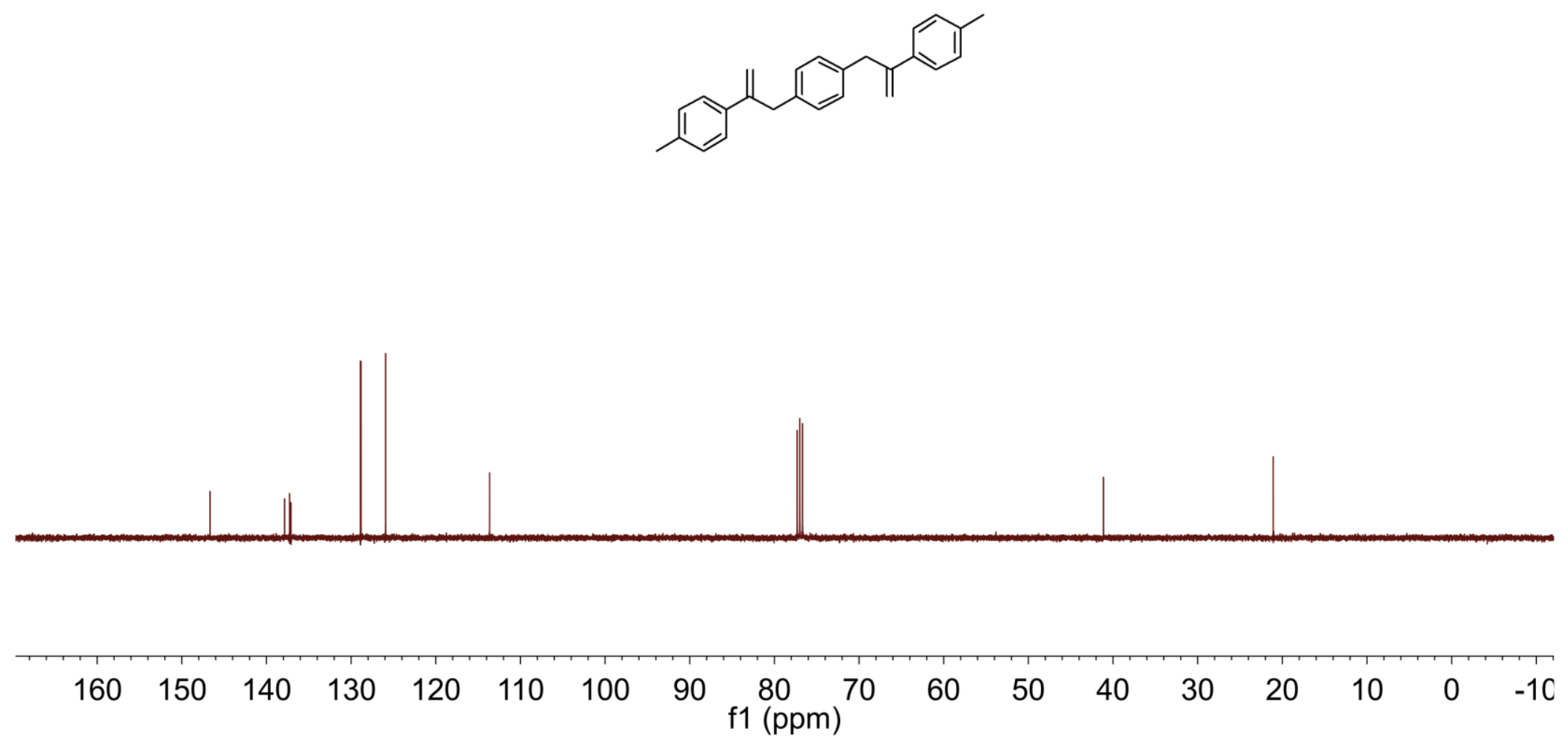




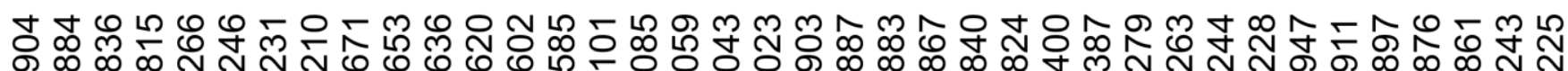

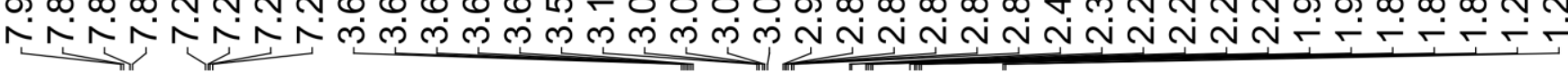
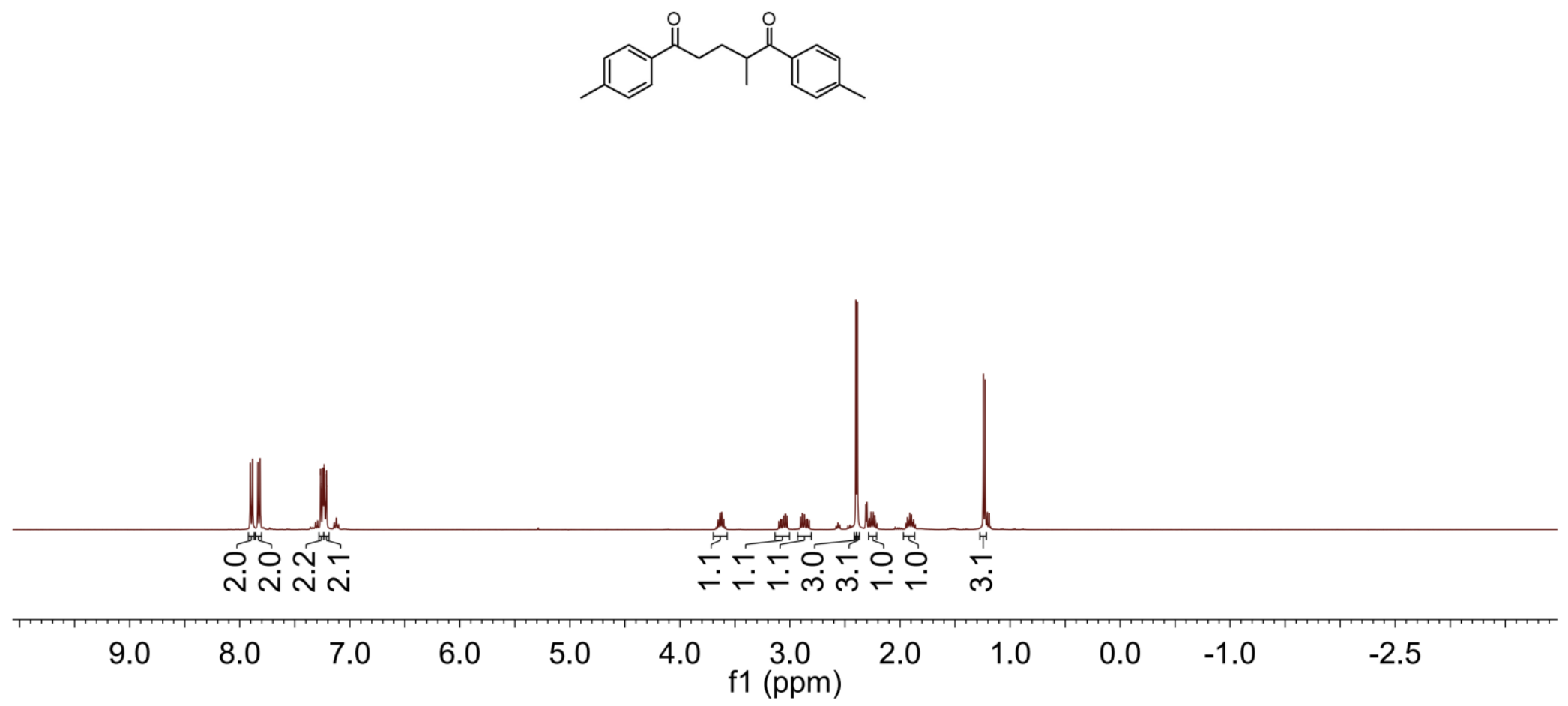
$\operatorname{1ar}{ }^{1} \mathrm{H}$ NMR

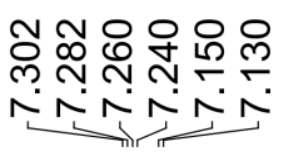

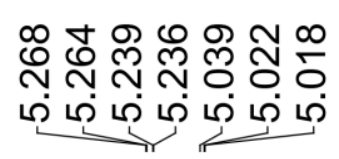

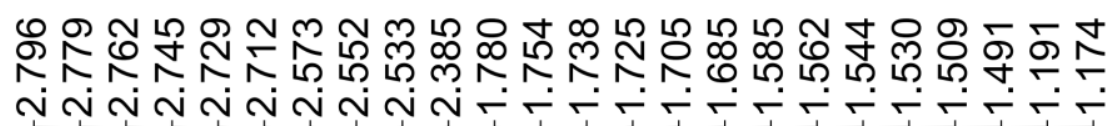

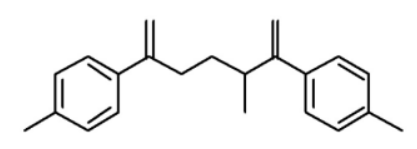

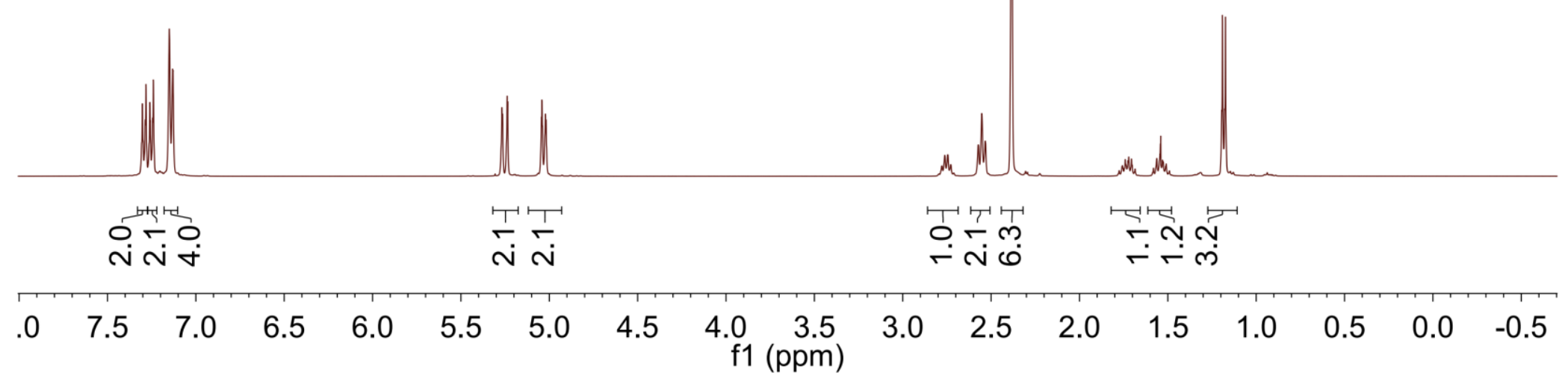

S160 


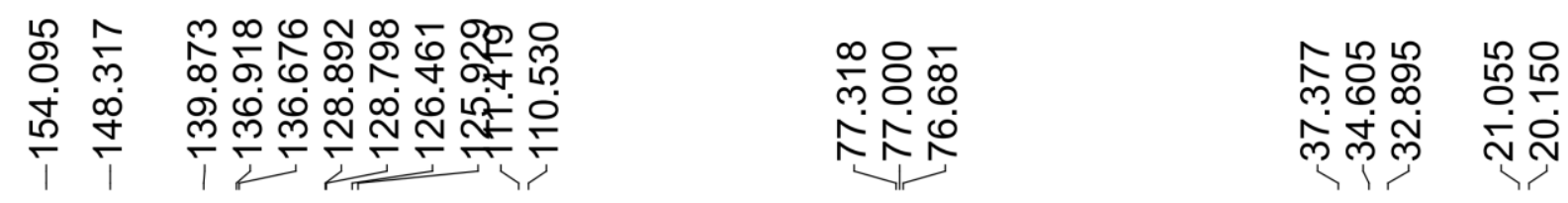

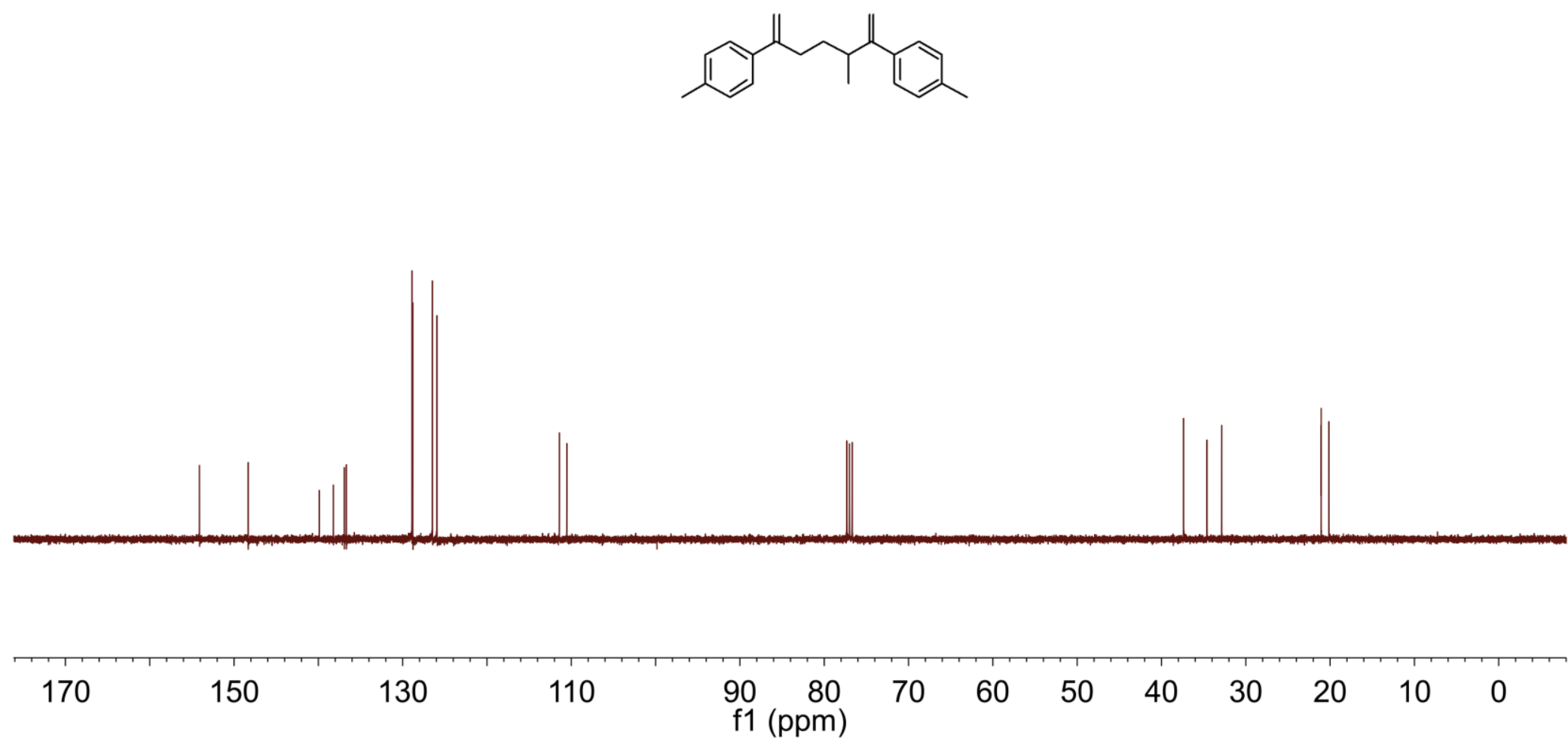




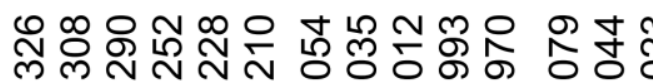

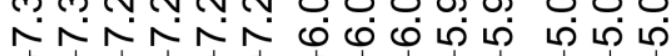

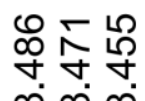

लm

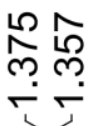
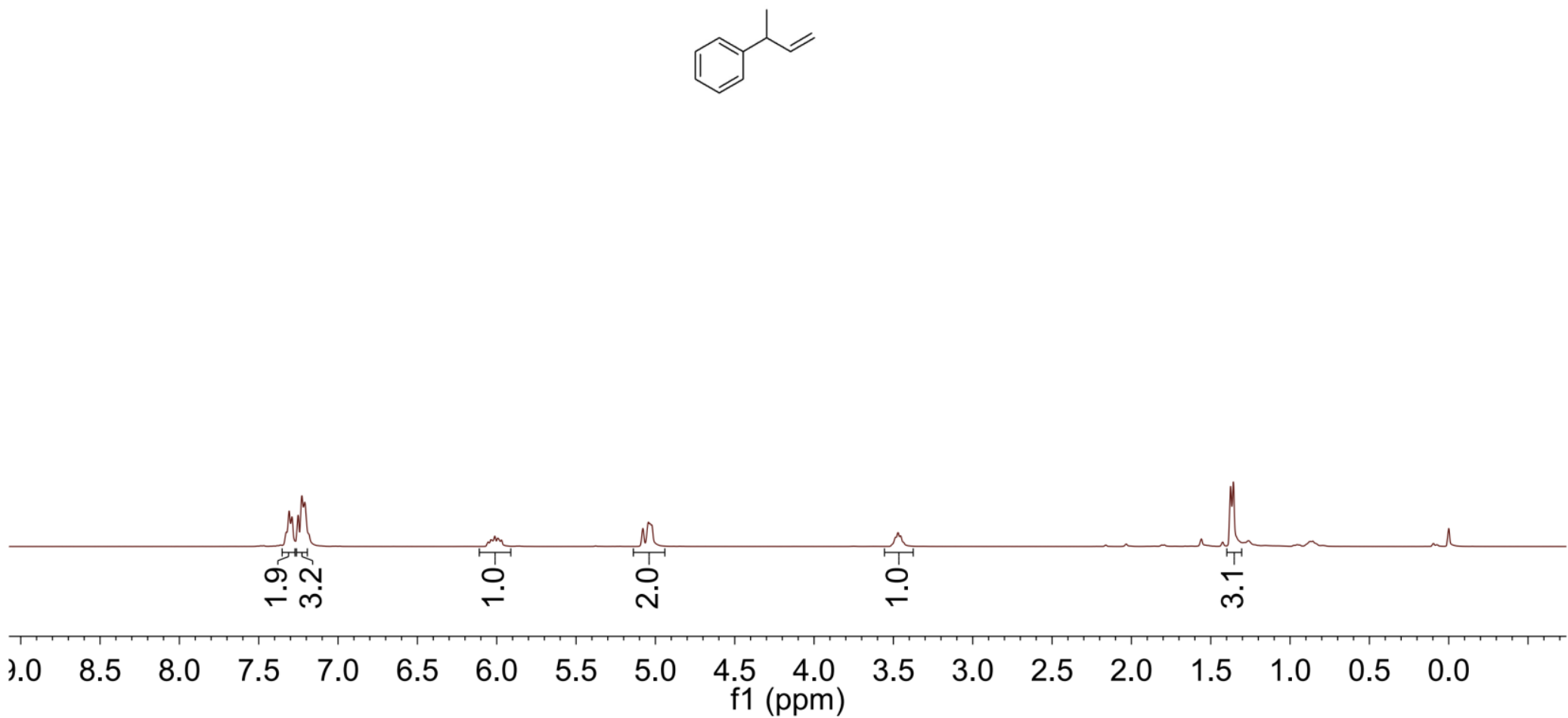


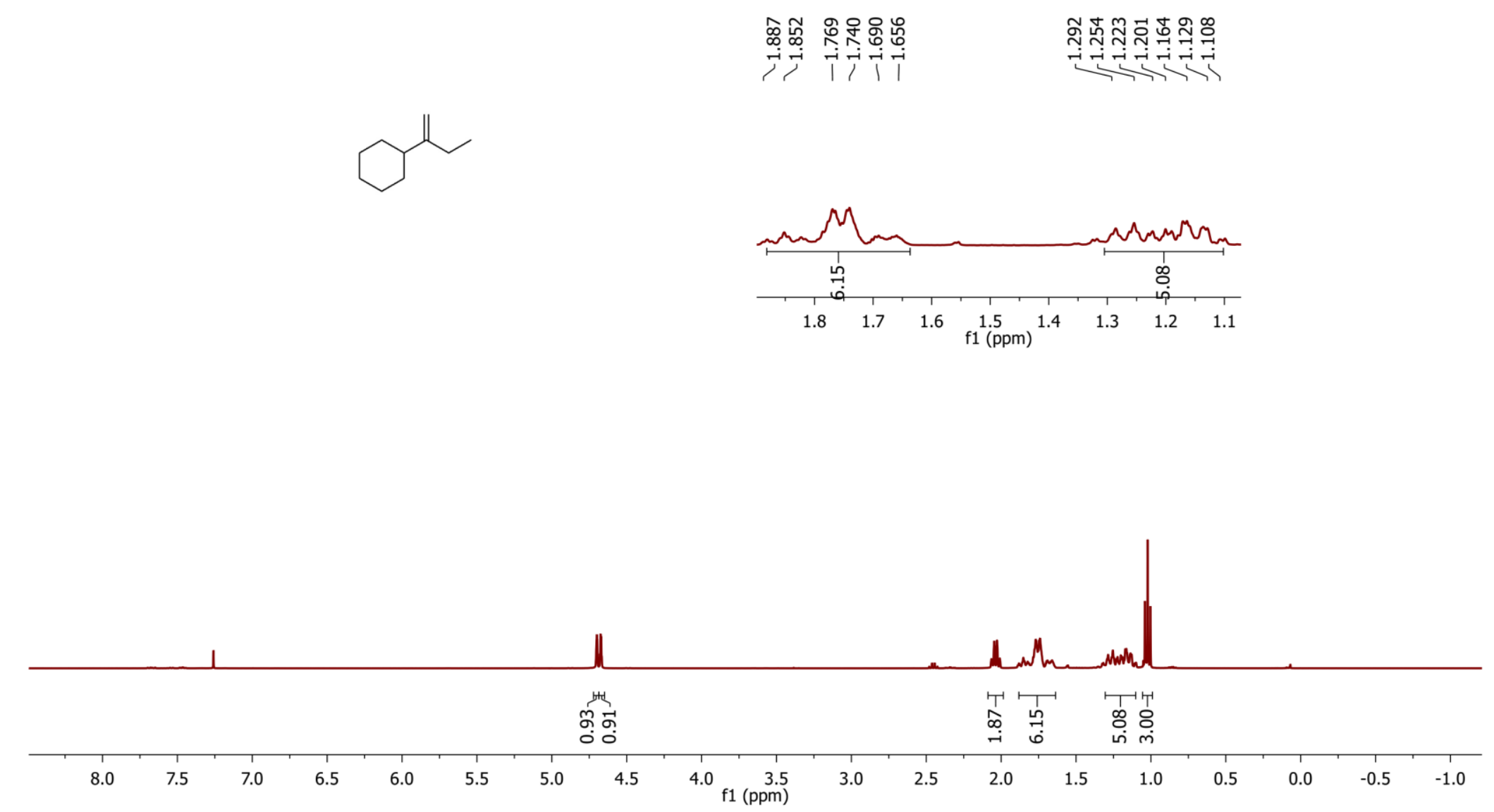



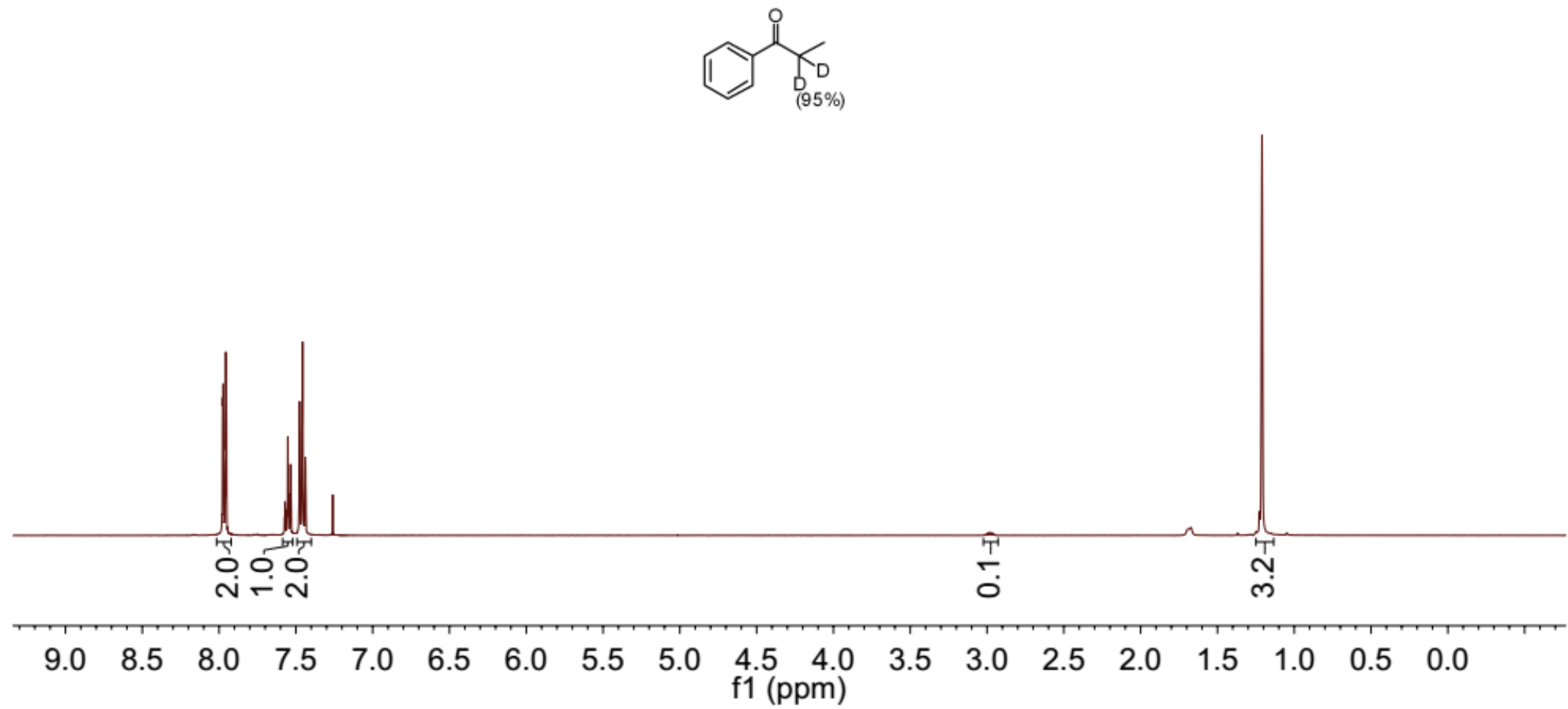
Oa $-D_{2}{ }^{2} \mathrm{H} N M R$ :
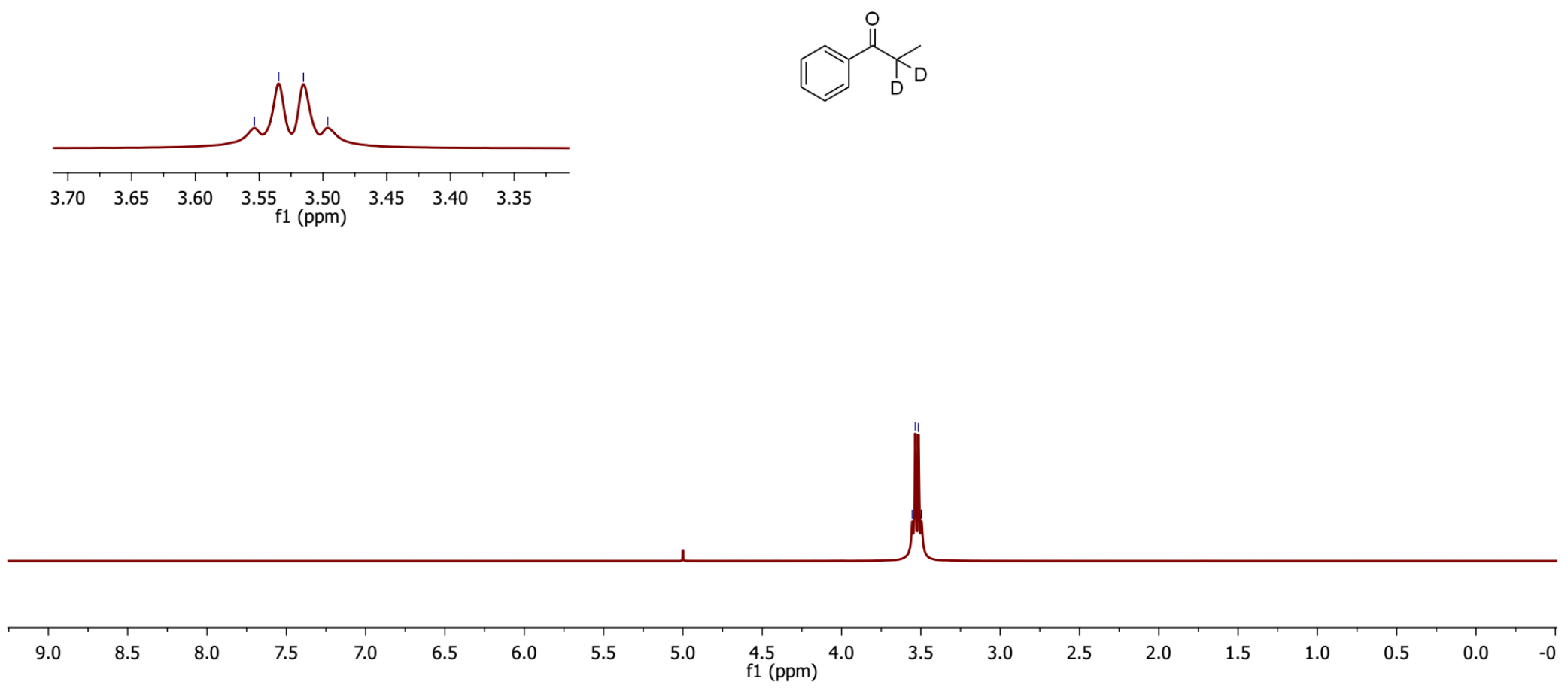
Oa- $D_{2}{ }^{13}$ C NMR:

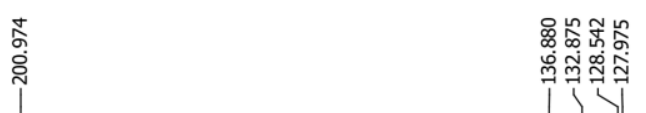

लिलें

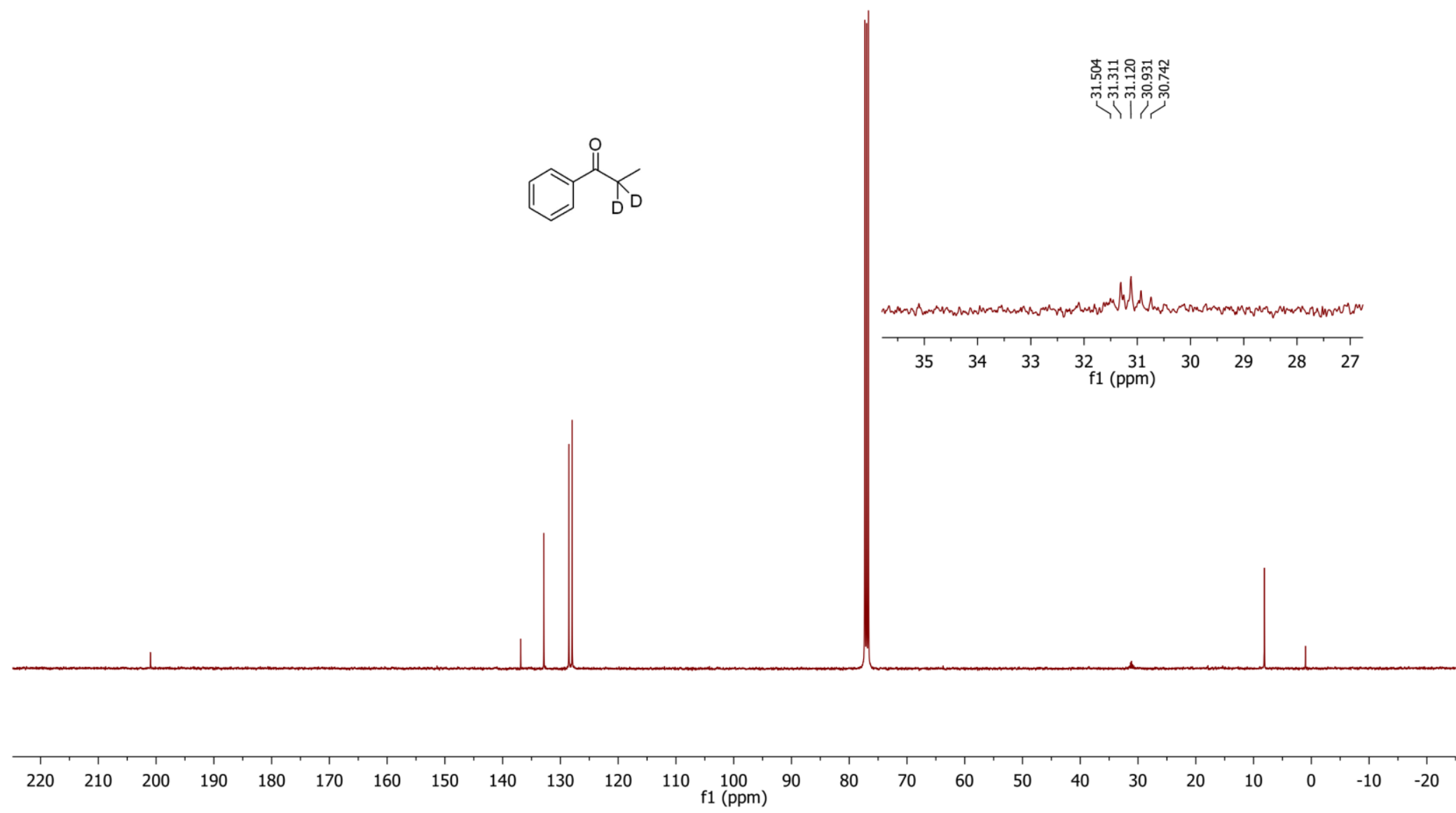




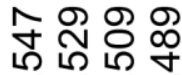

نं نं
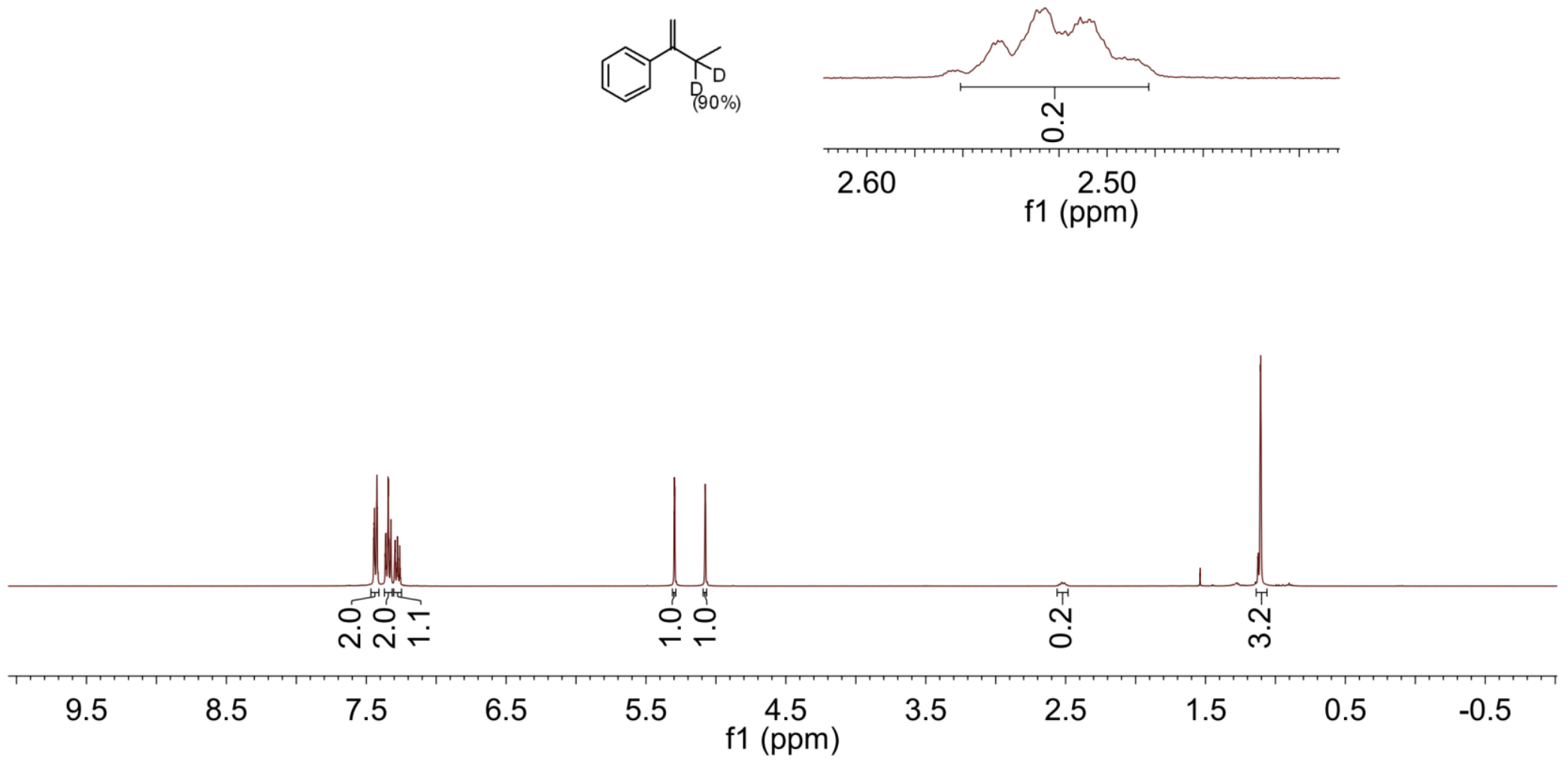


\section{1a-D ${ }^{2} \mathrm{H}$ NMR}
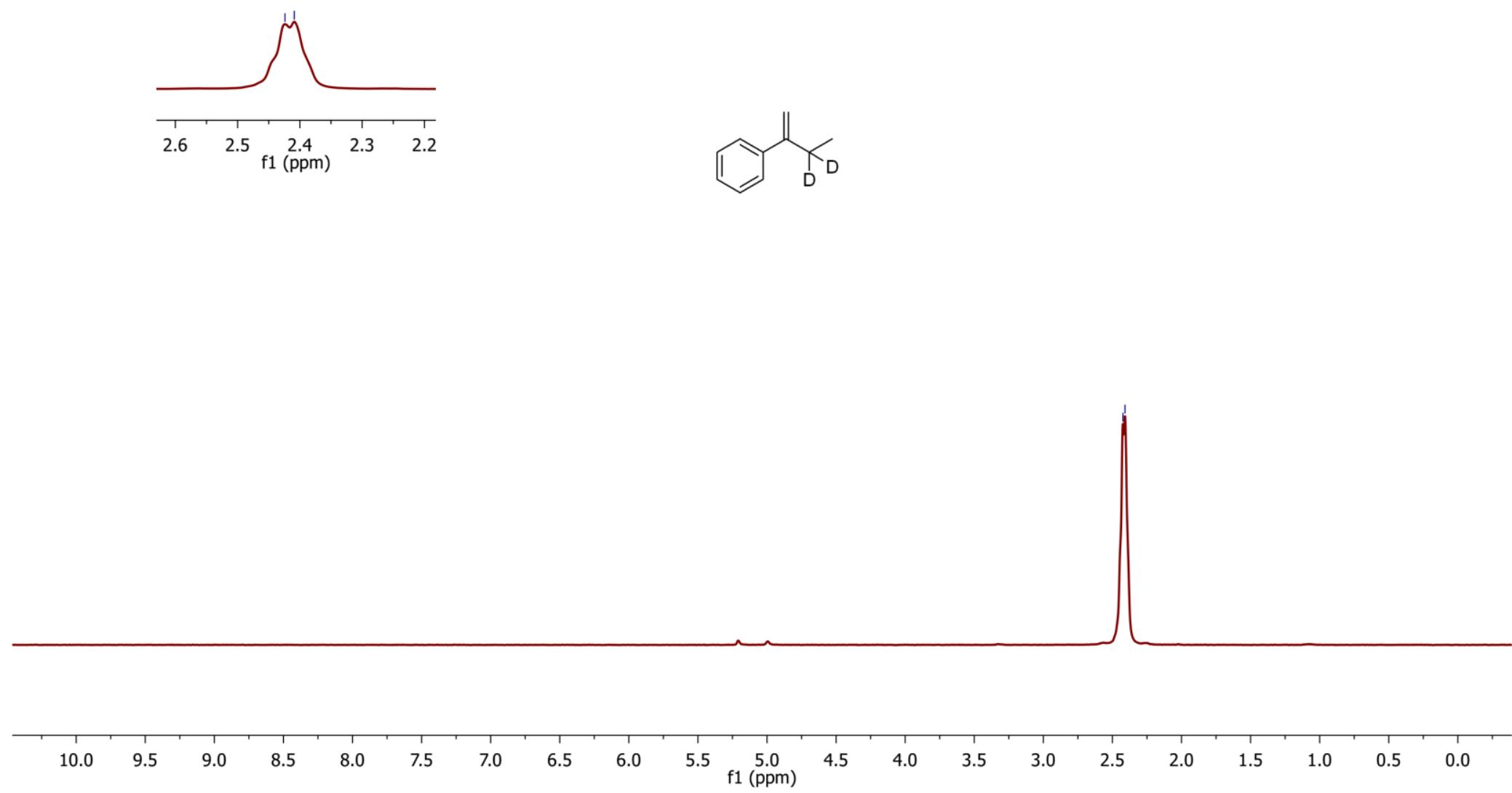
1a- $\mathbf{D}_{\mathbf{2}}{ }^{13} \mathrm{C}$ NMR
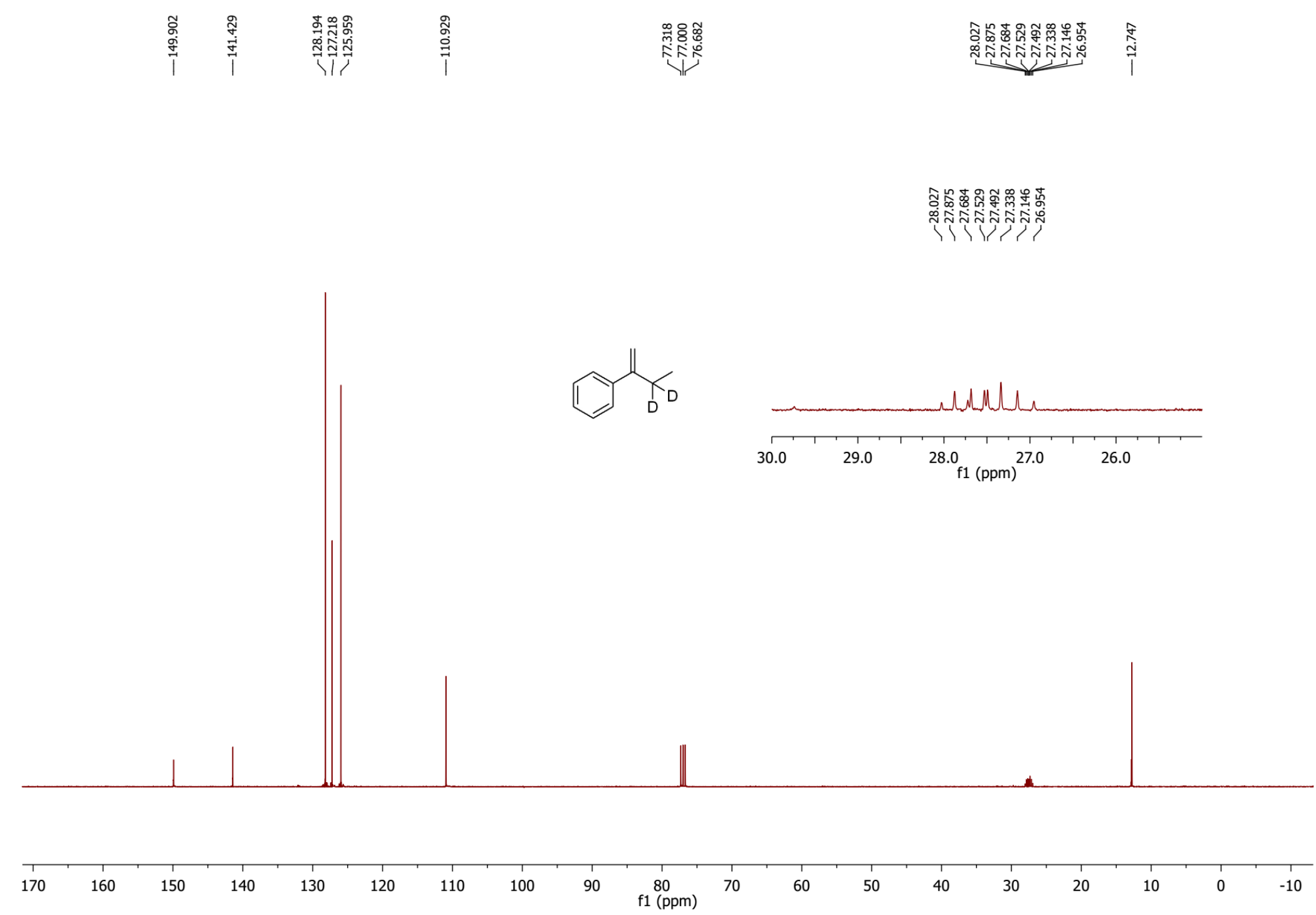


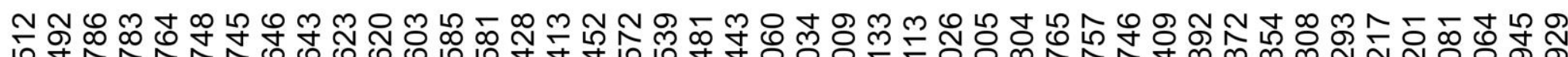

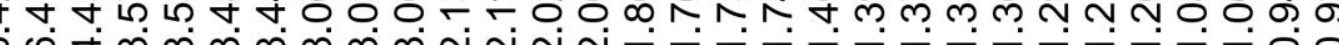
-

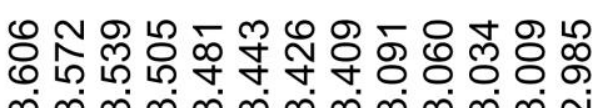
ம்
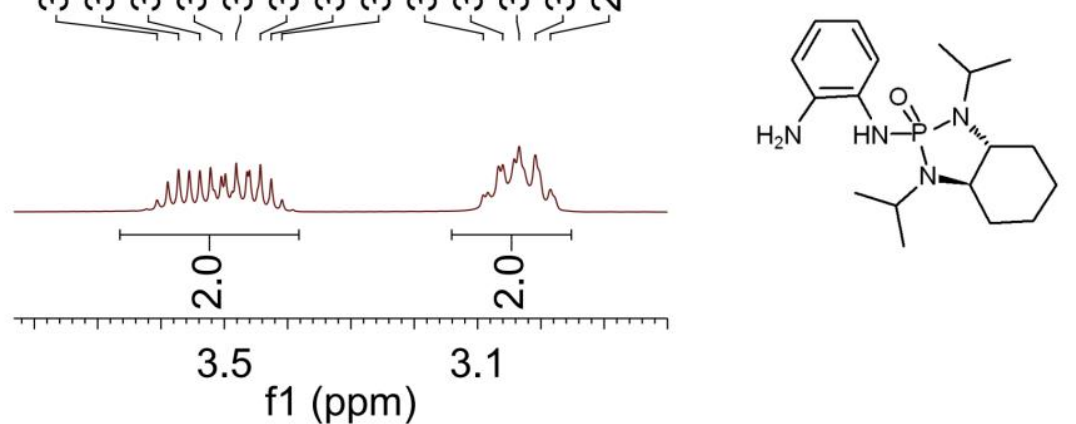

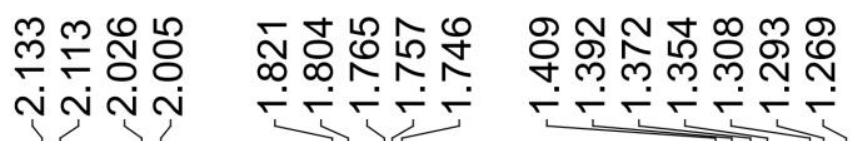
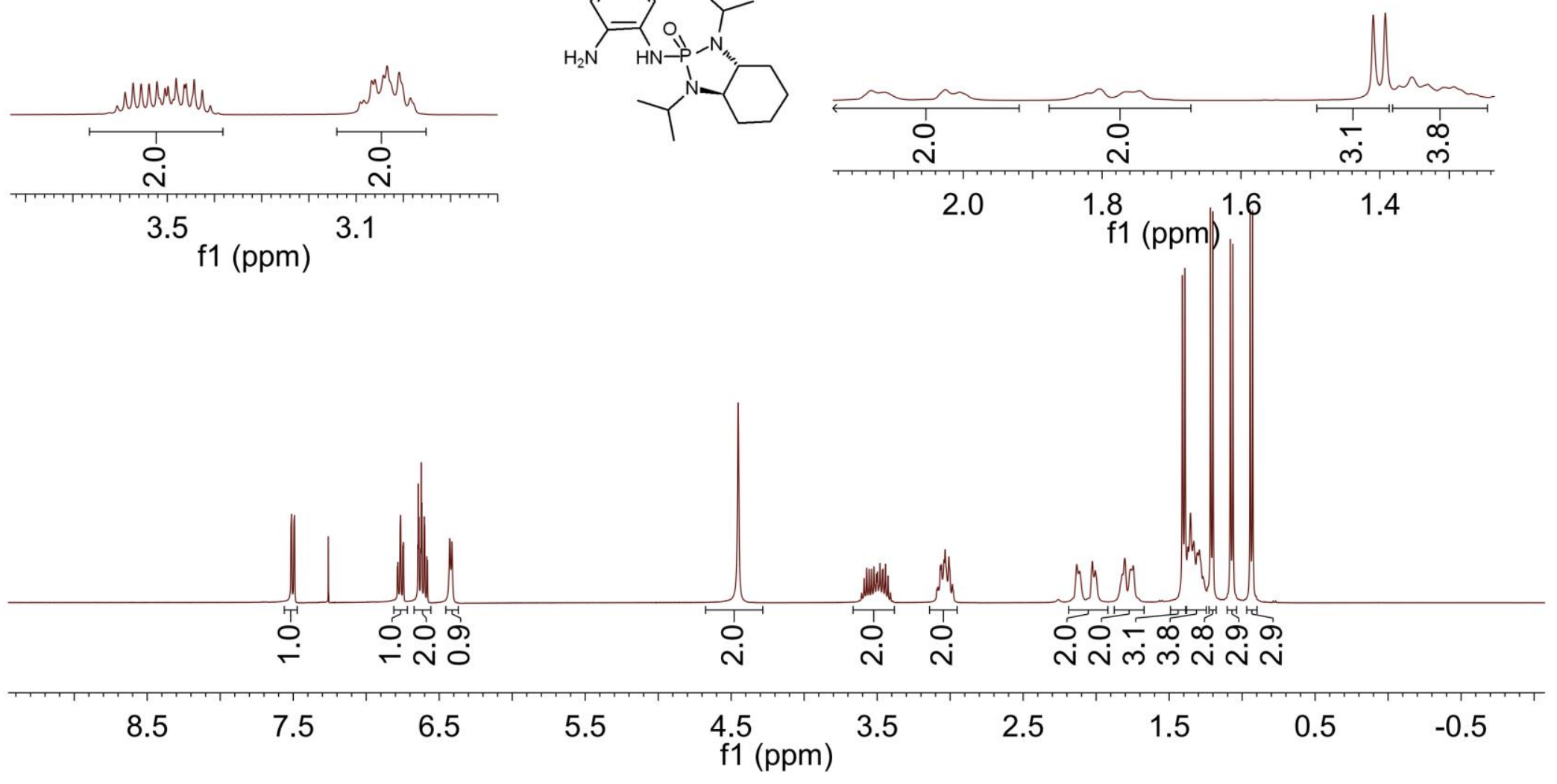


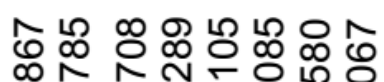

๓

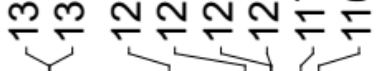
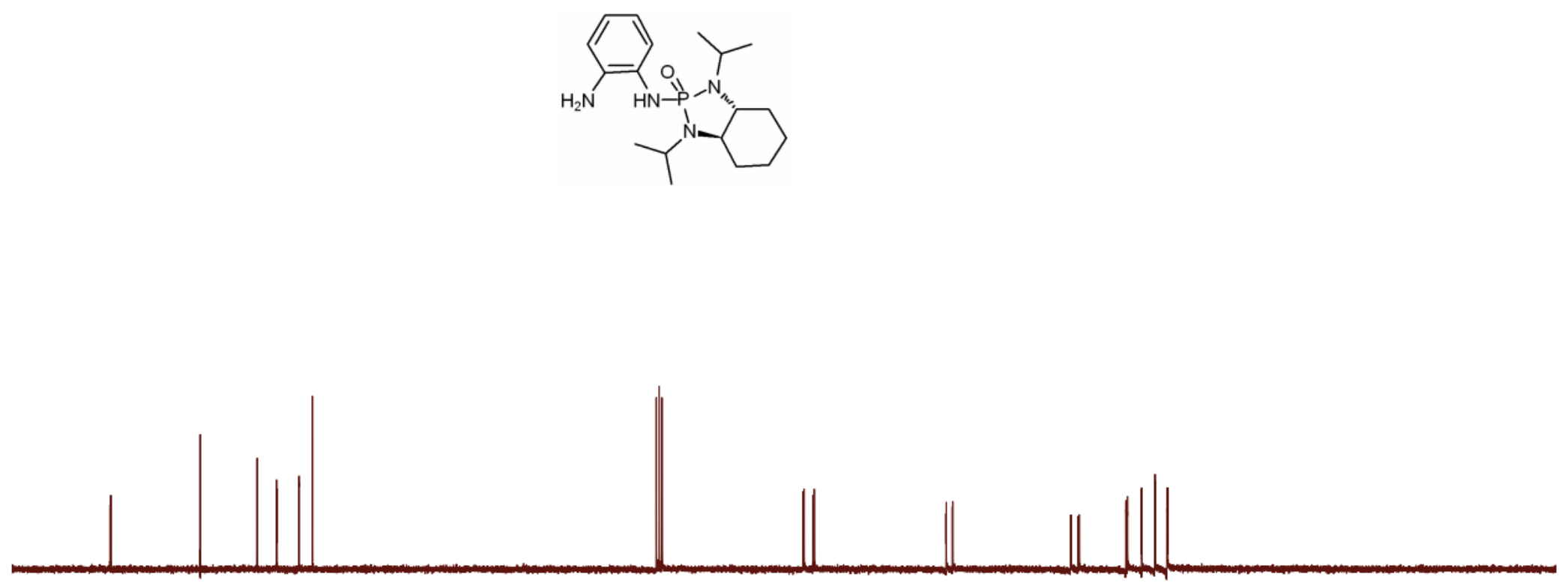

$\begin{array}{lllllllll}140 & 130 & 120 & 110 & 100 & 90 & 80 & 70 & 60\end{array}$

$$
\text { f1 (ppm) }
$$

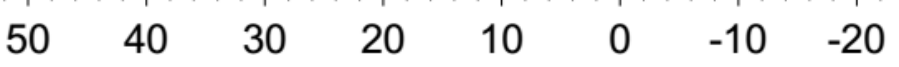



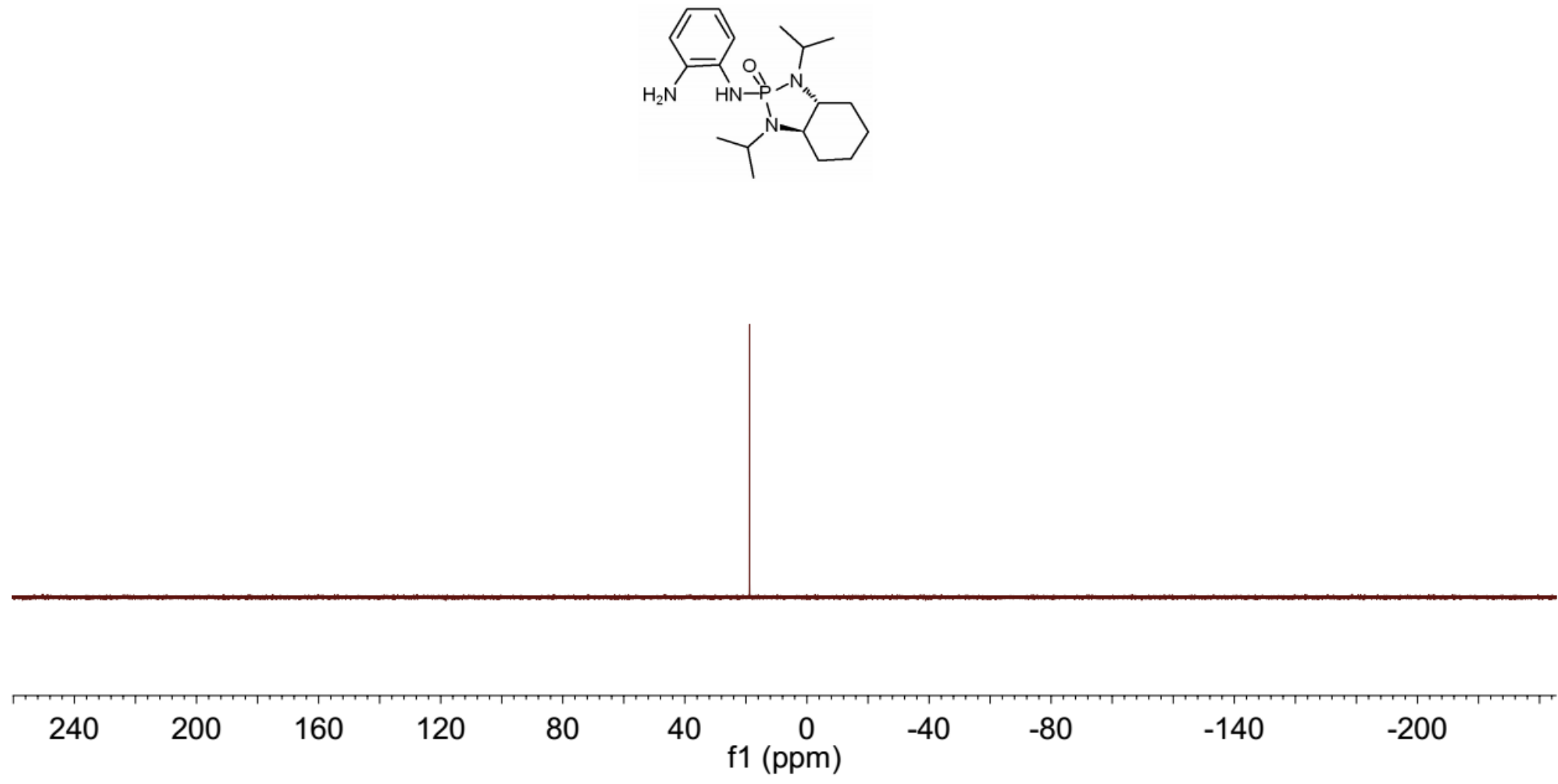


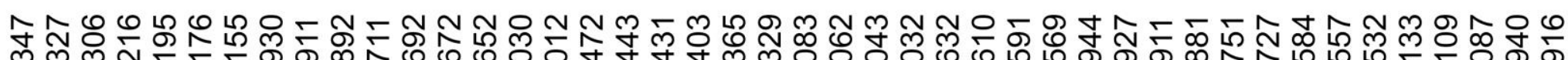
NNN N

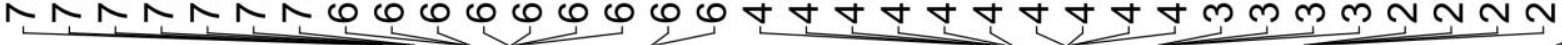
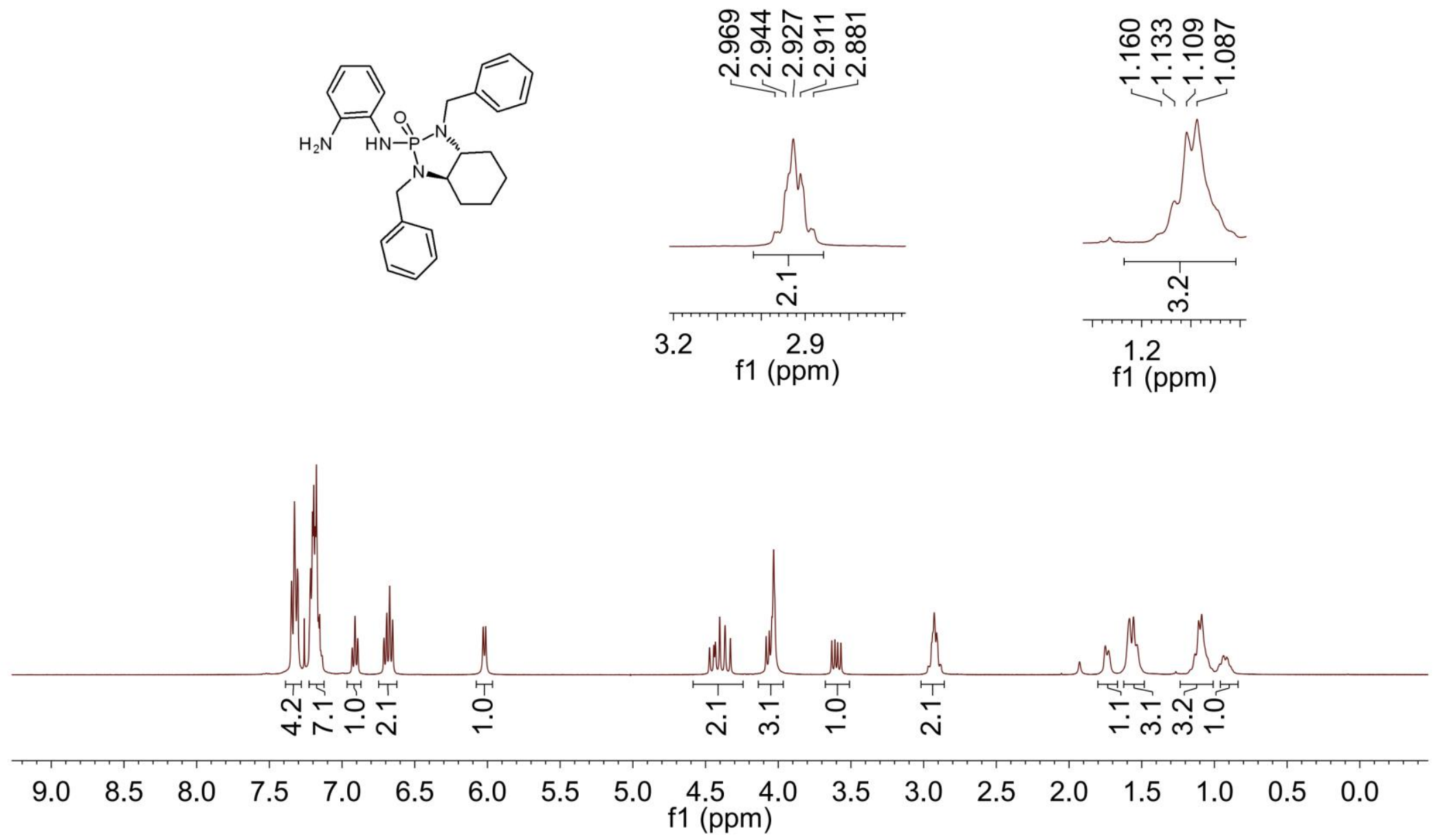


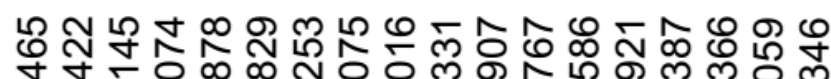
守

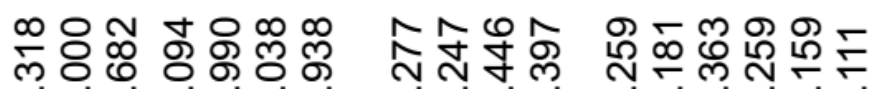

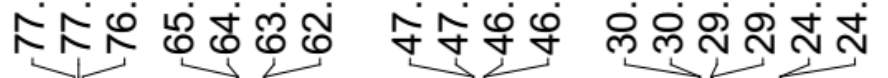

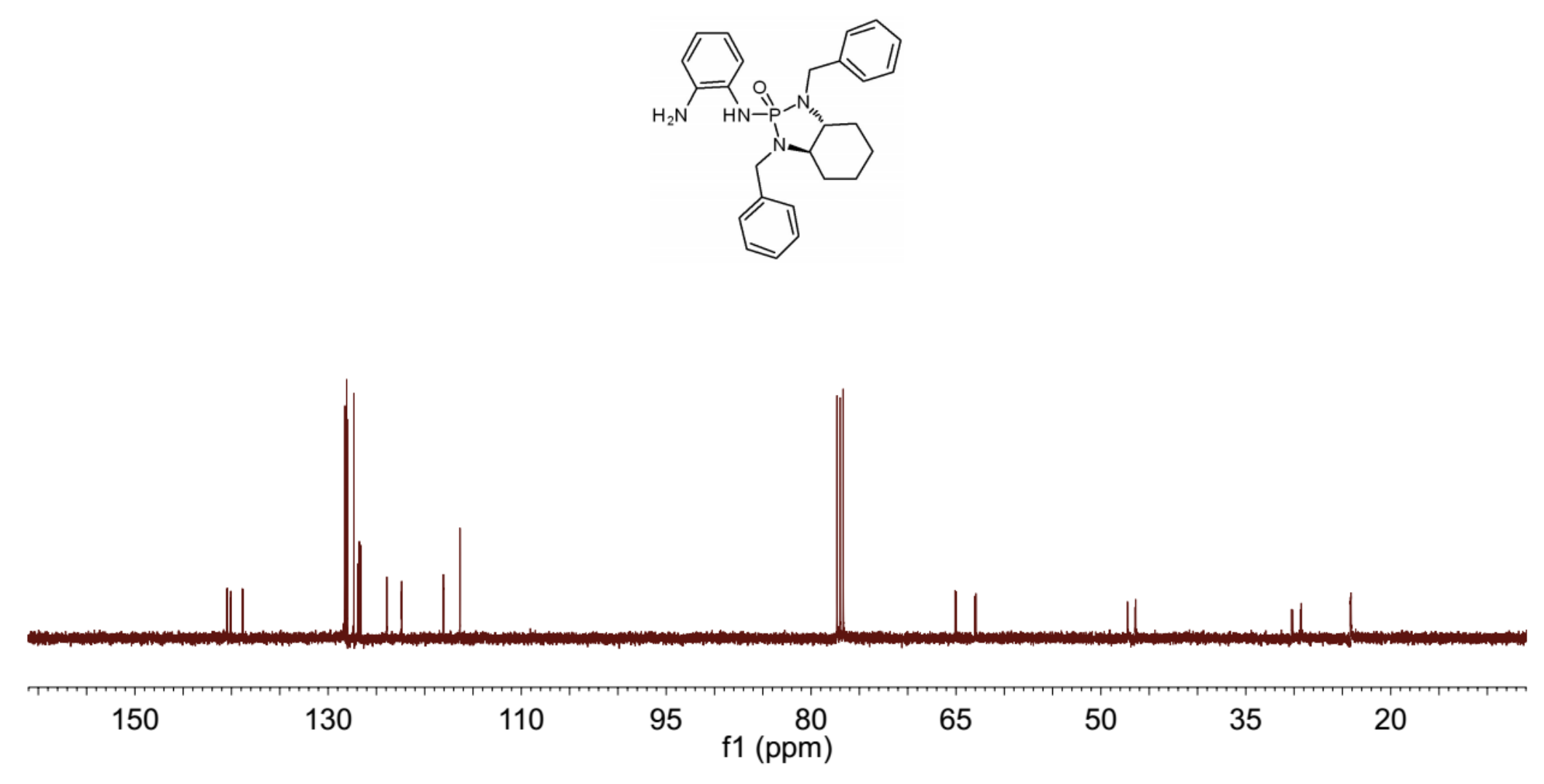



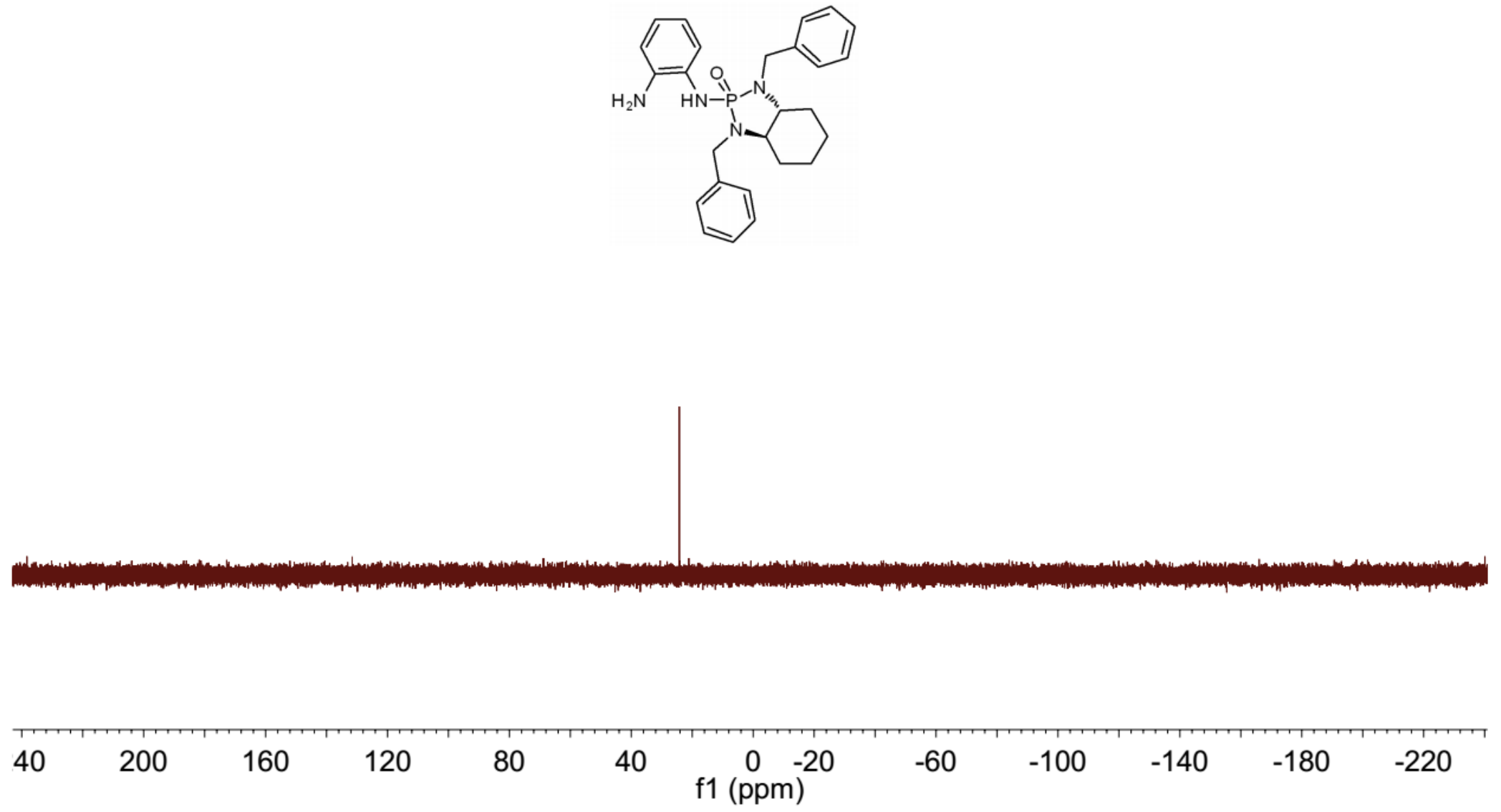


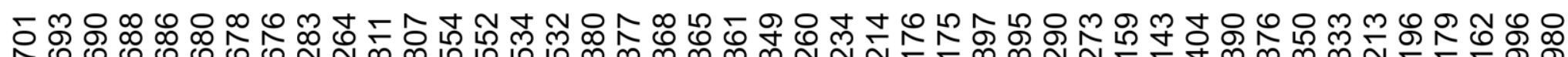

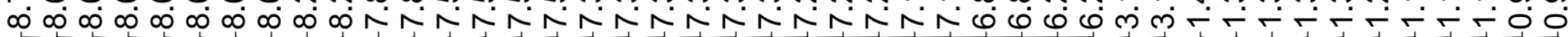

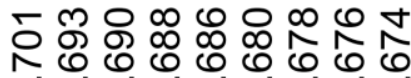
$\infty \infty \infty \infty \infty \infty \infty \infty \infty$

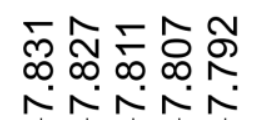

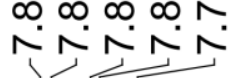

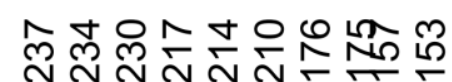

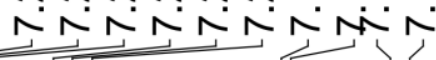
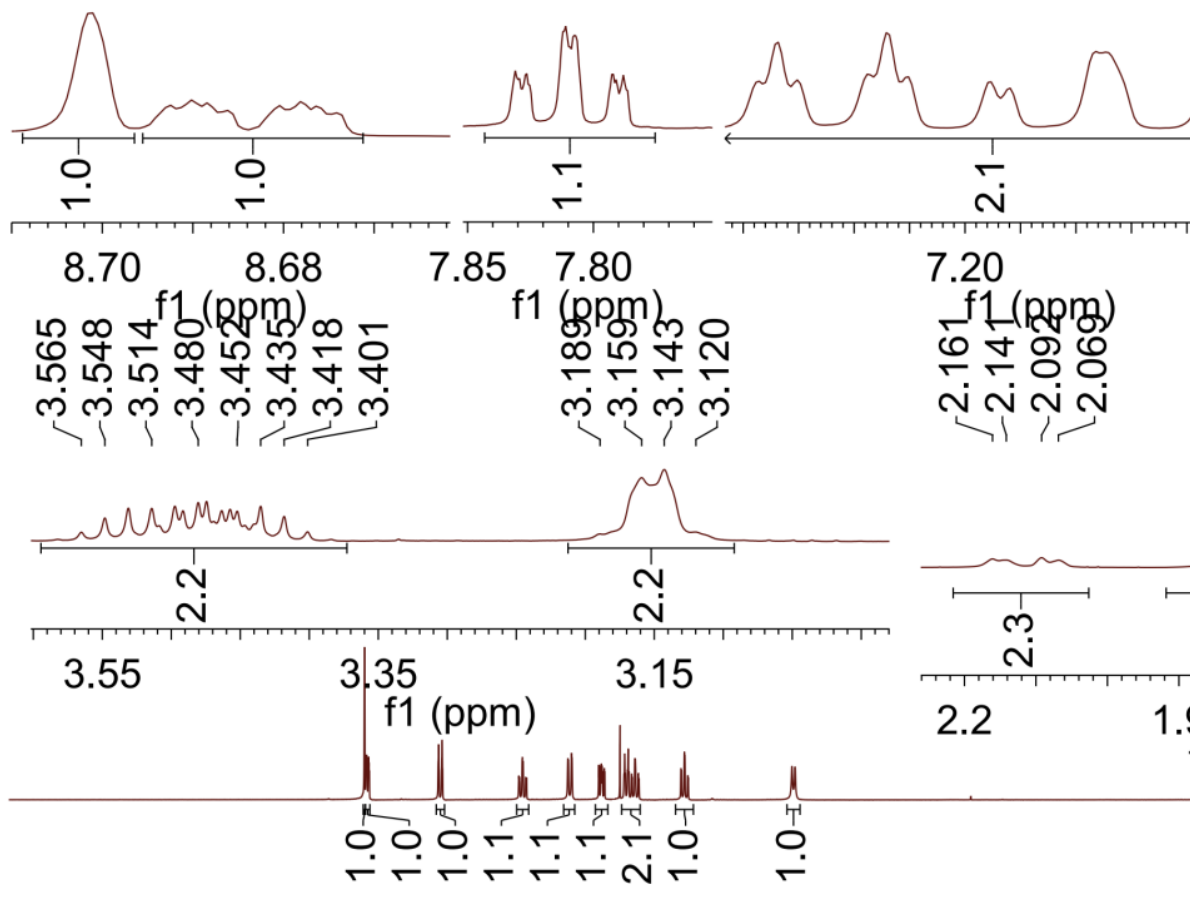

7.20

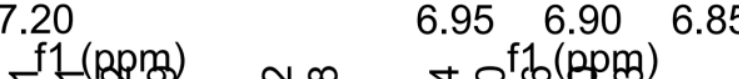

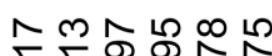
б̆ ம்

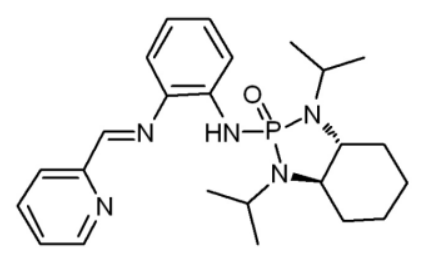

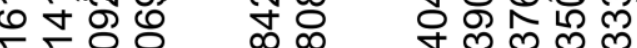

Nin่
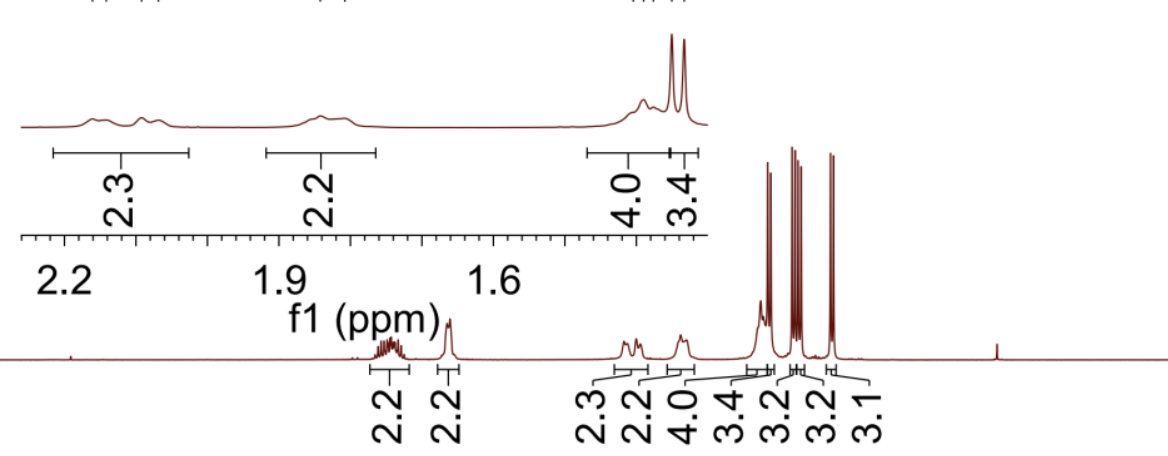
10.0
9.0
8.0
7.0
6.0
5.0
$1(p p m)$
2.0
$\begin{array}{ll}1.0 & 0.0\end{array}$ 


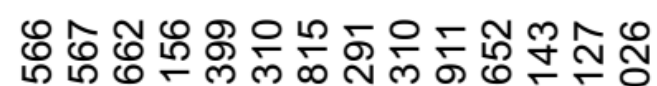
吕

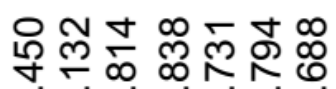
トド்

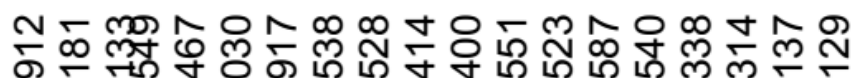
ป๋ 迎
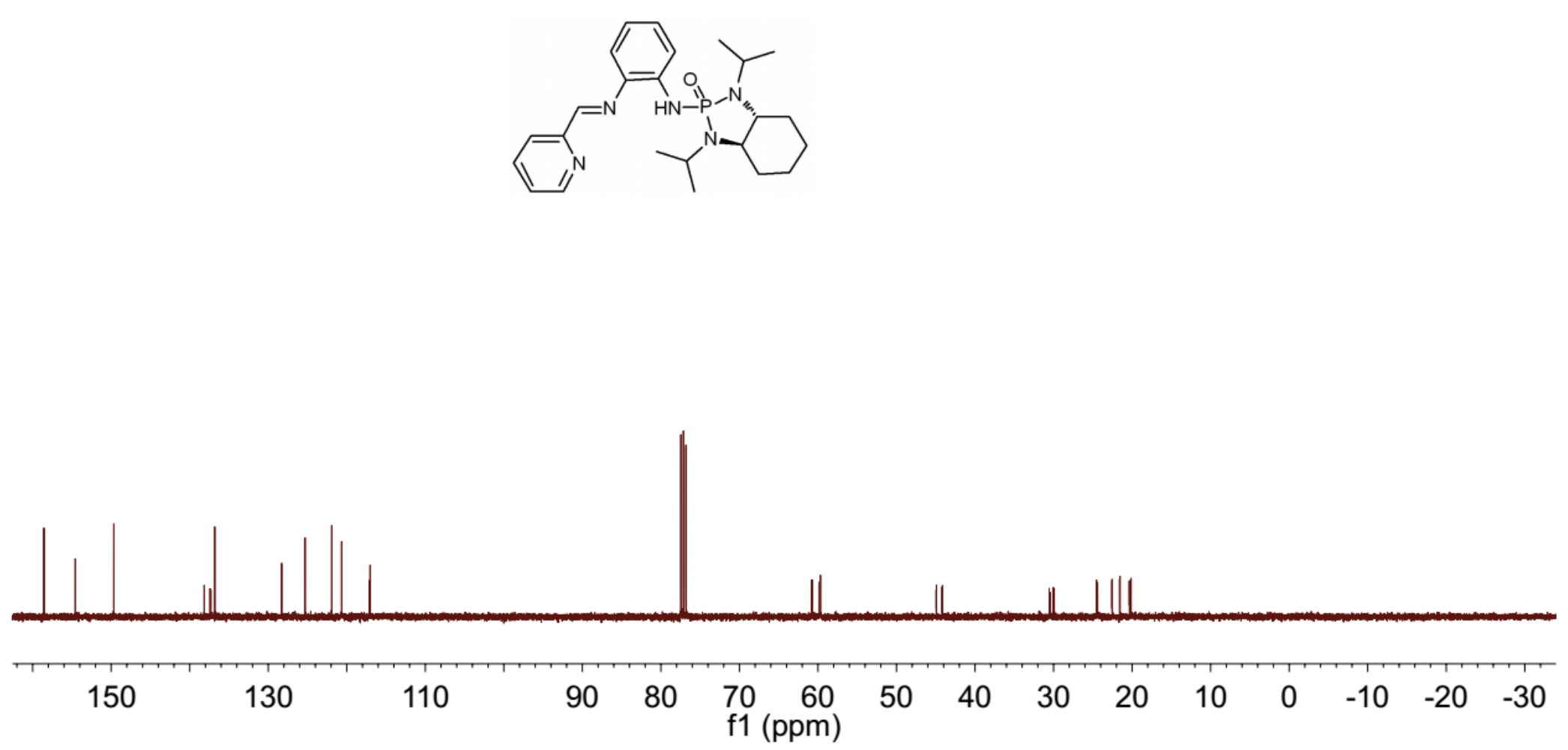

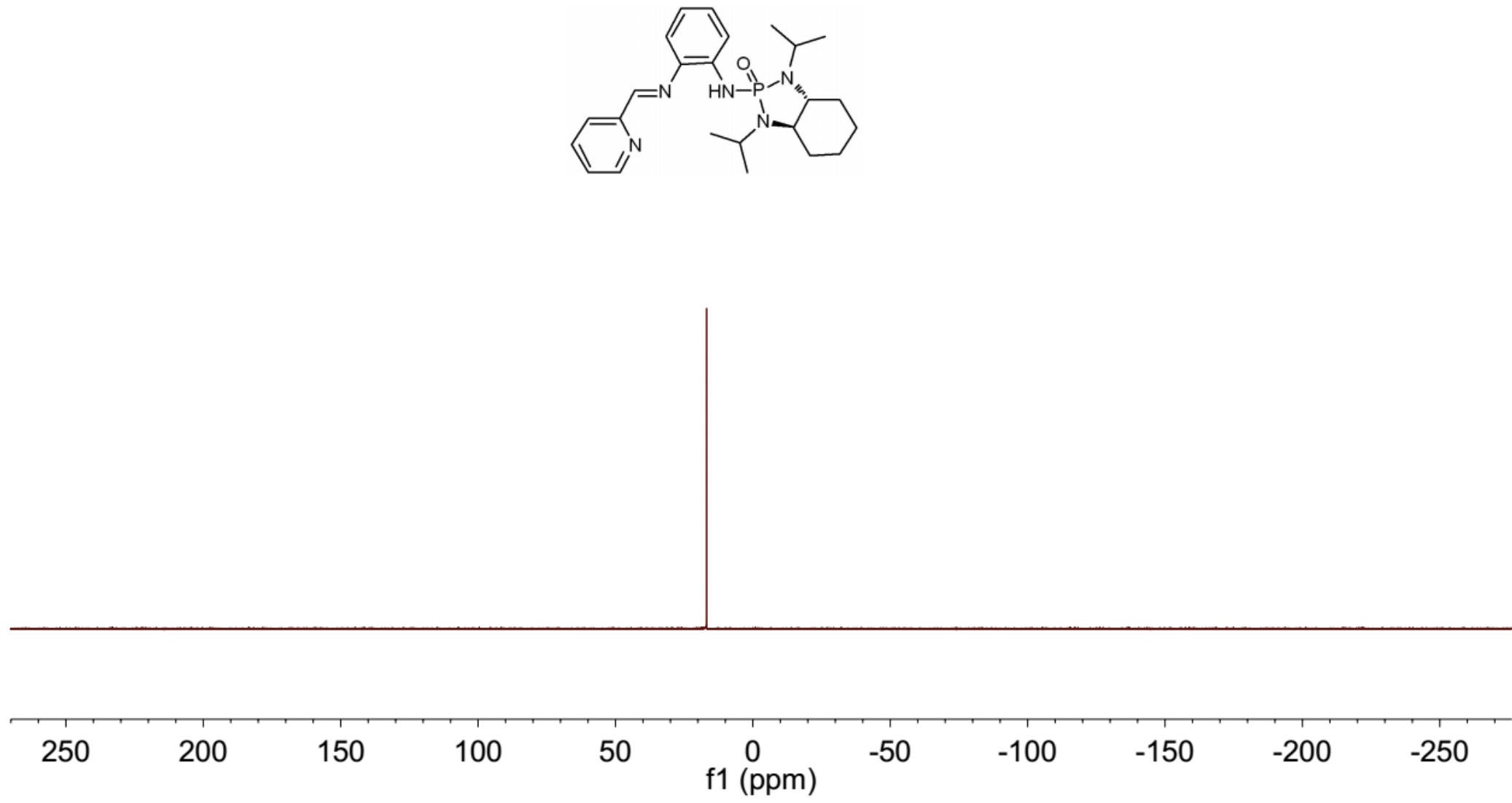


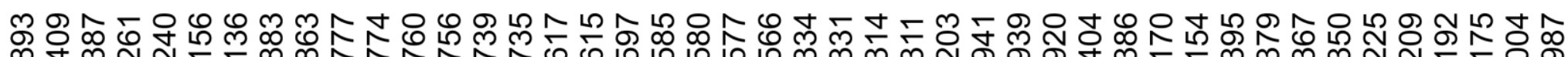

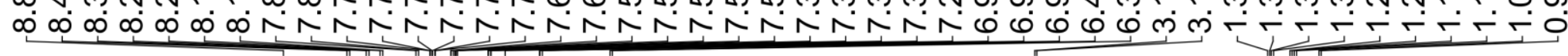

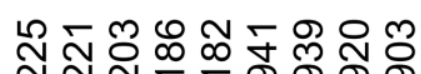

슨유

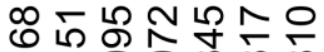

NNNNG

ले लं लं लं लं

テั NNNN
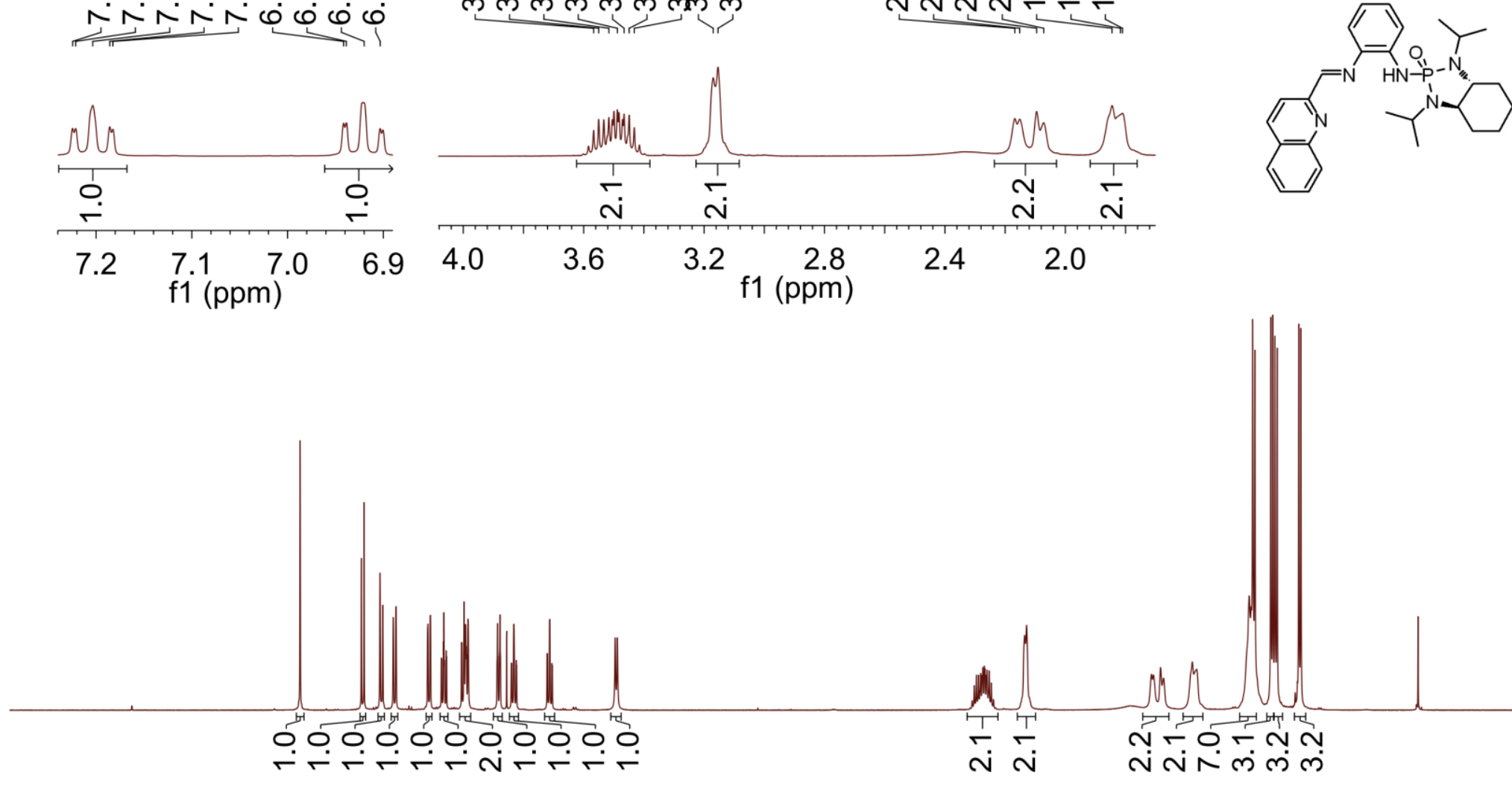

$\begin{array}{lllllll}11.0 & 10.0 & 9.0 & 8.0 & 7.0 & 6.0 & 5.0 \\ \mathrm{f} 1(\mathrm{ppm})\end{array}$
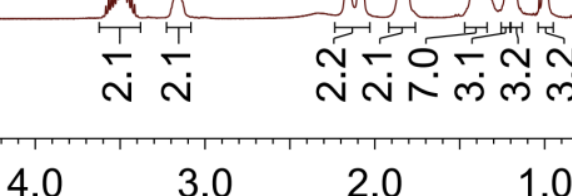

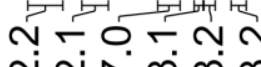
i

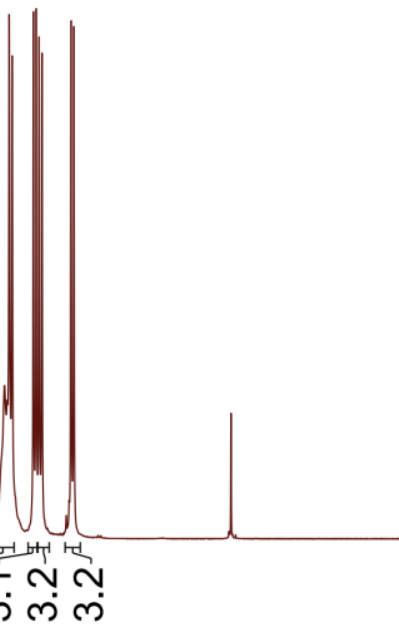




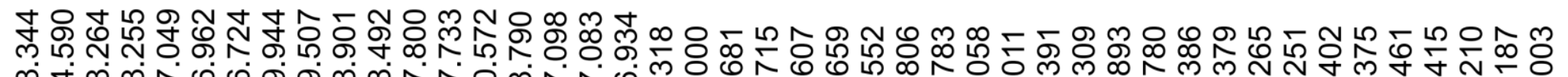

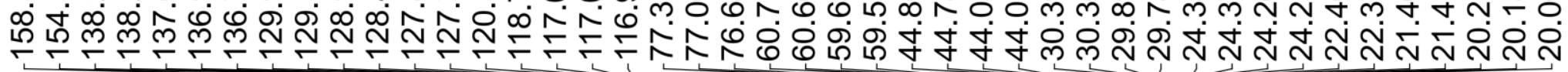
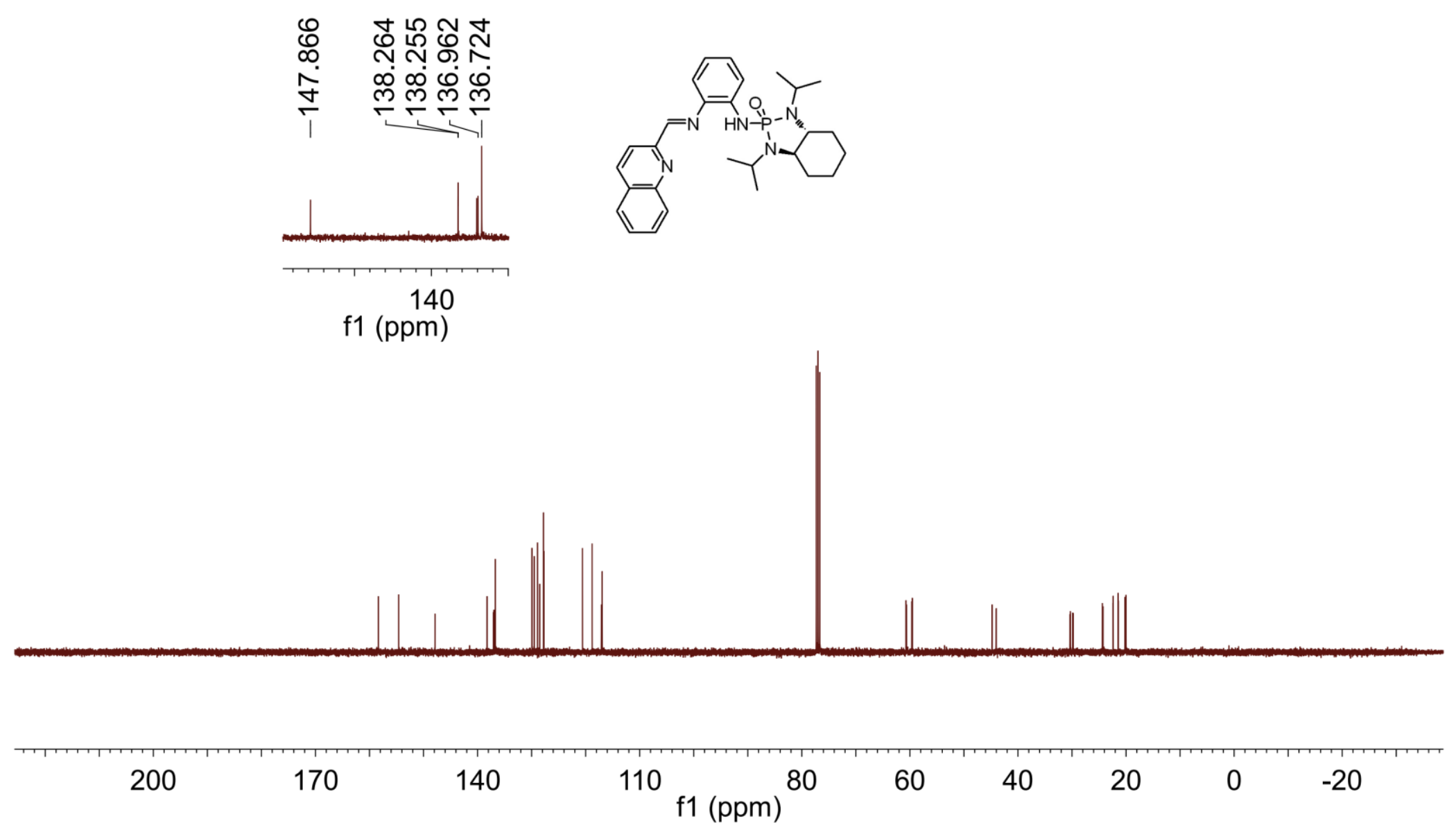
ֻ

เ
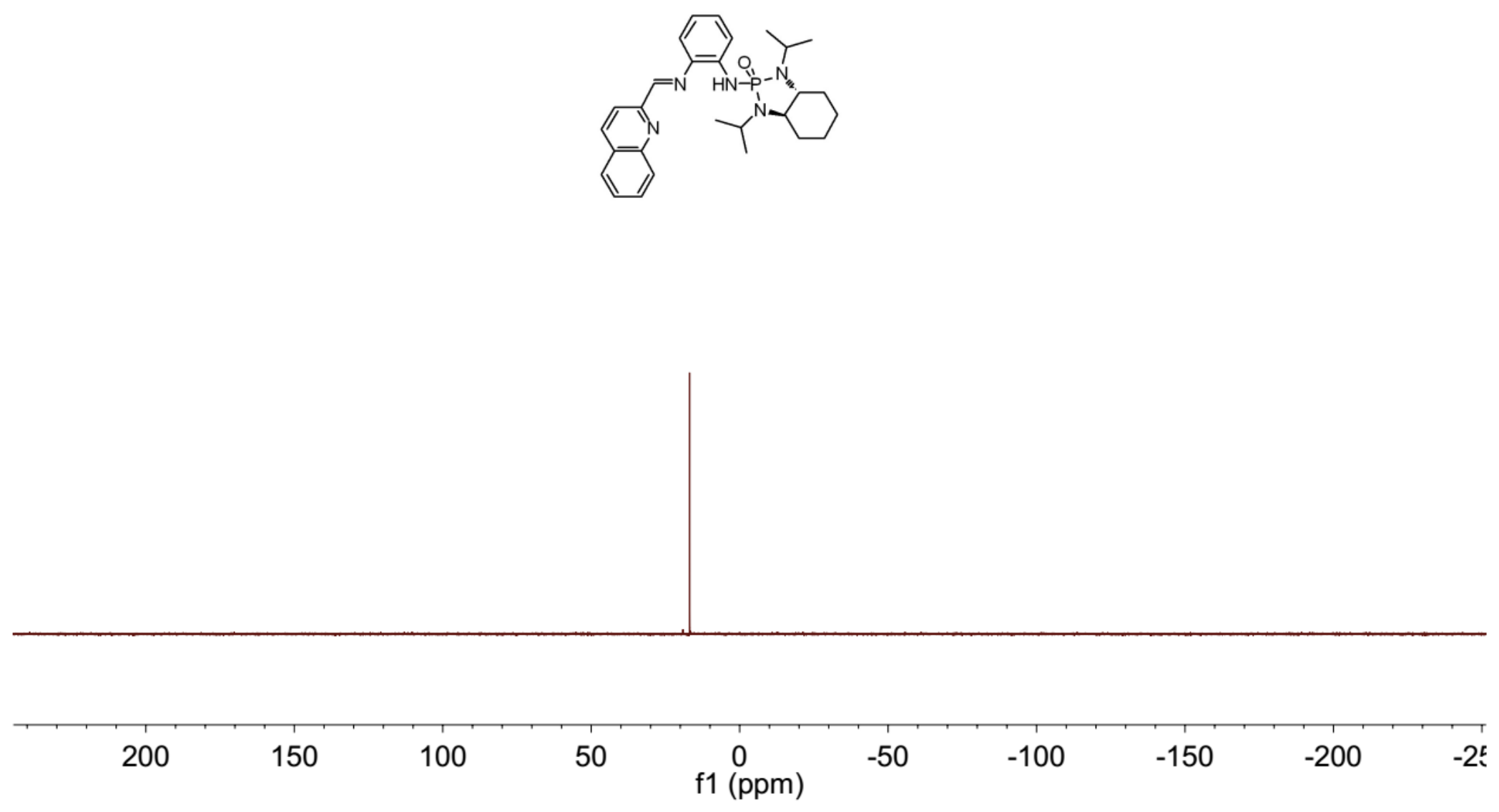


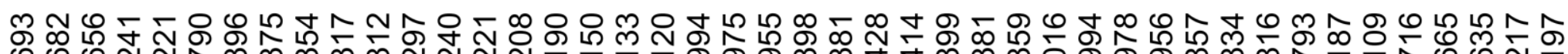

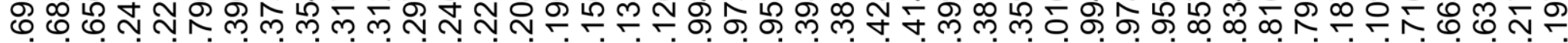
क

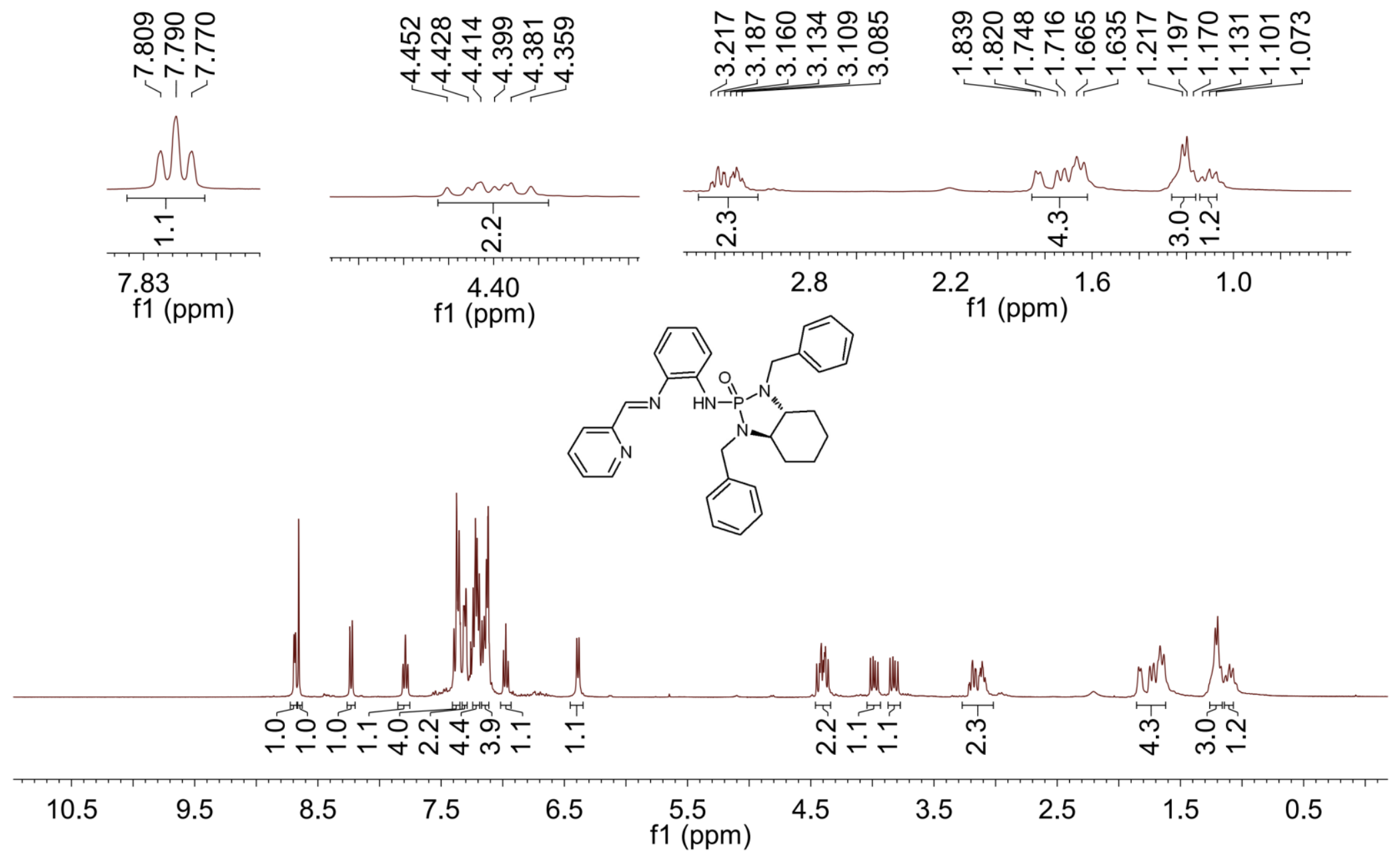




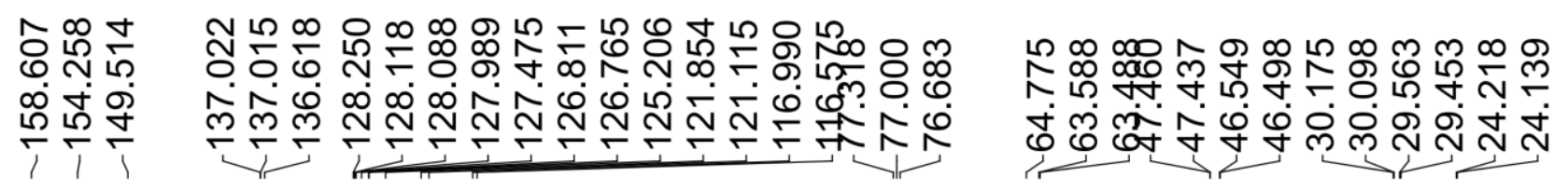

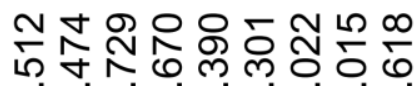

लं
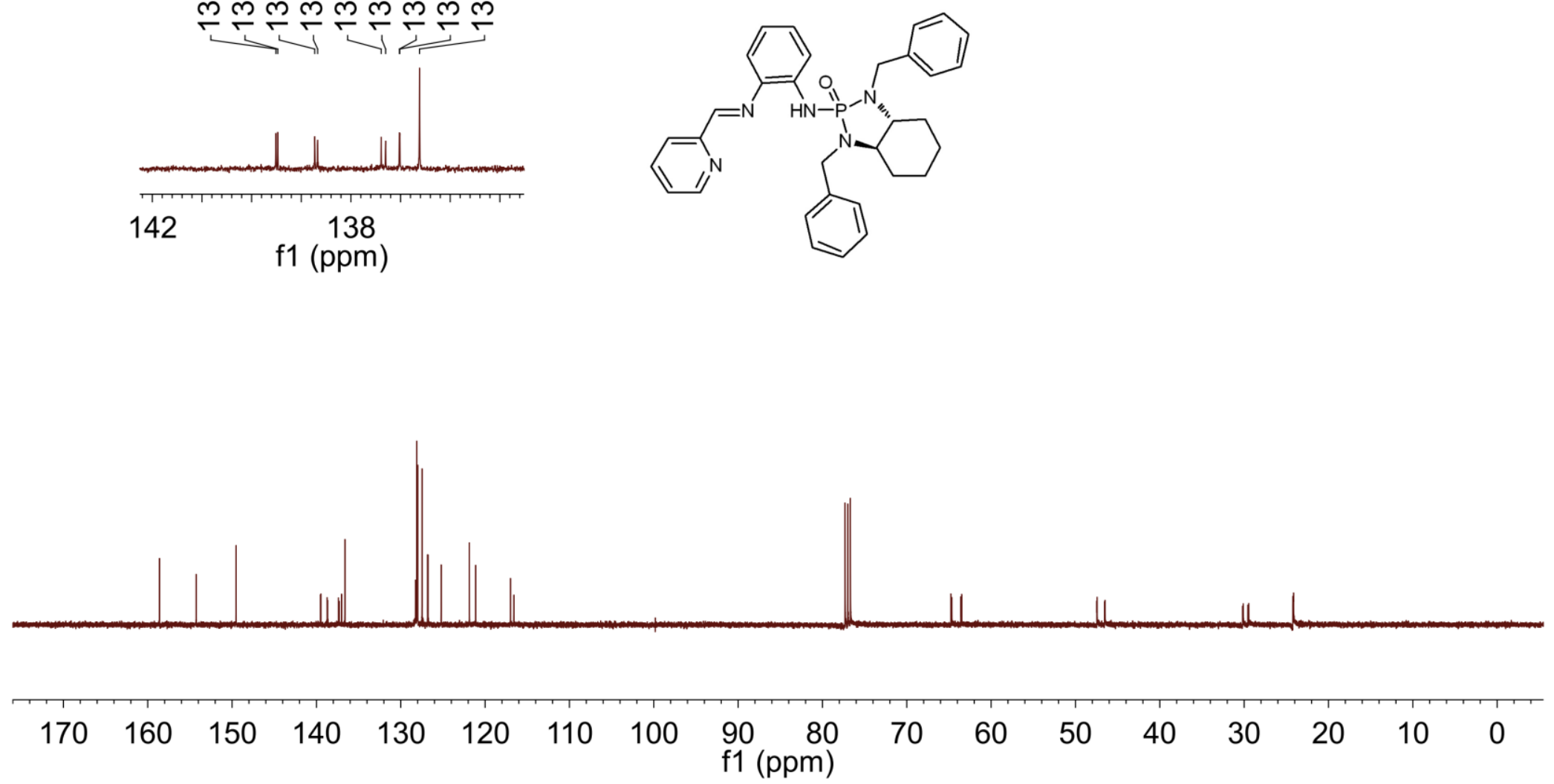
๙ิ
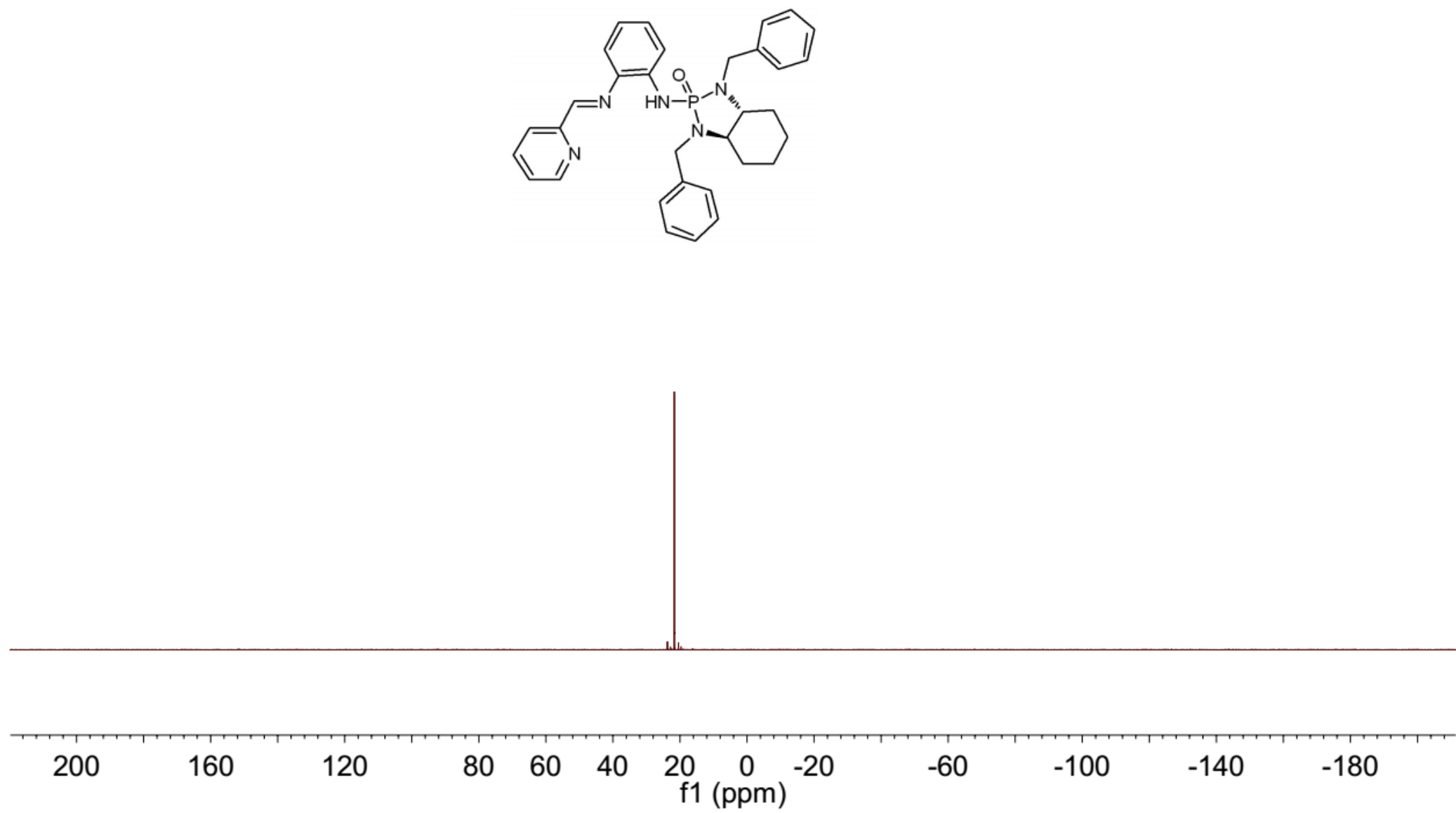


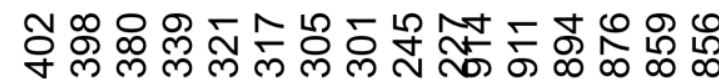

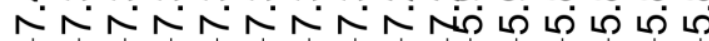

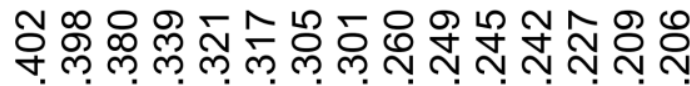

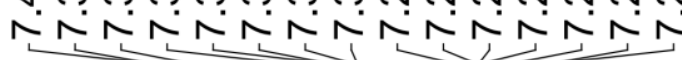
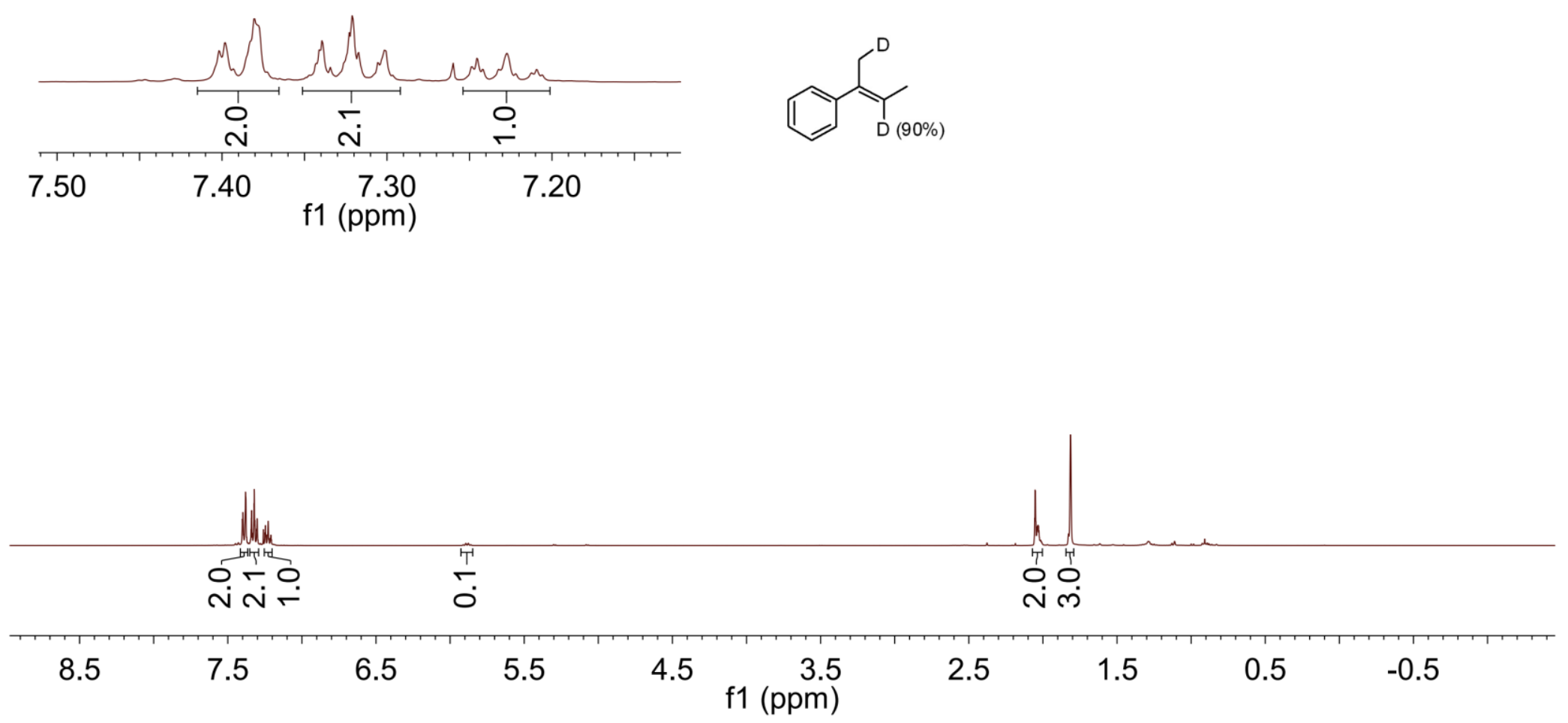

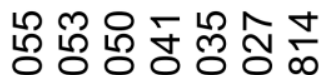


2a- $\mathbf{D}_{\mathbf{2}}{ }^{2} \mathrm{H} N M R$

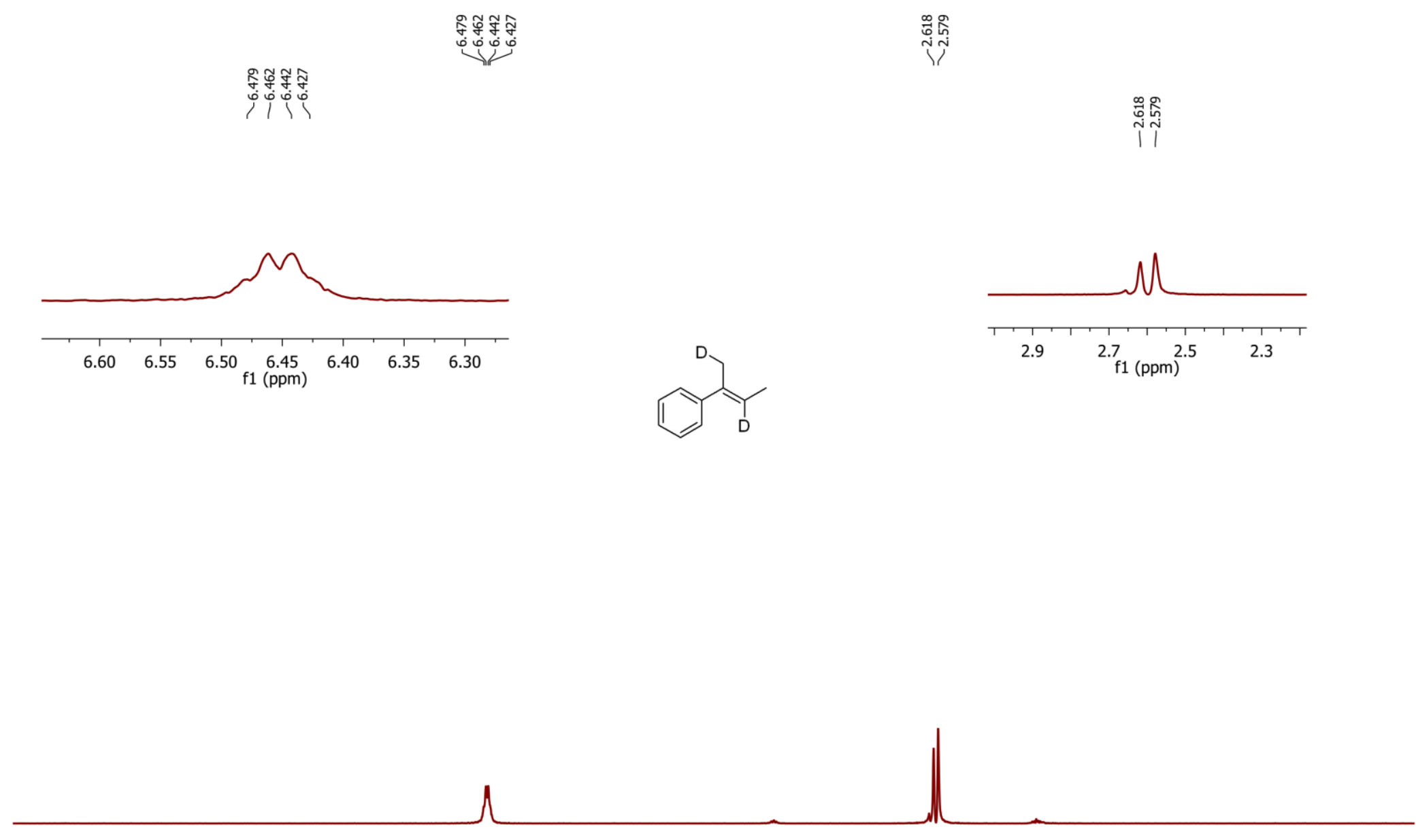

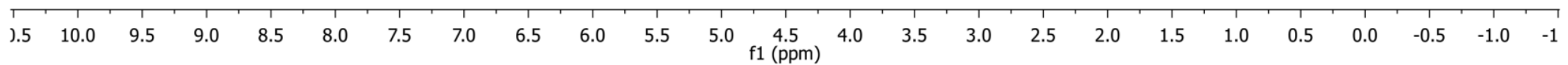


2a- $\mathbf{D}_{\mathbf{2}}{ }^{13} \mathrm{C}$ NMR
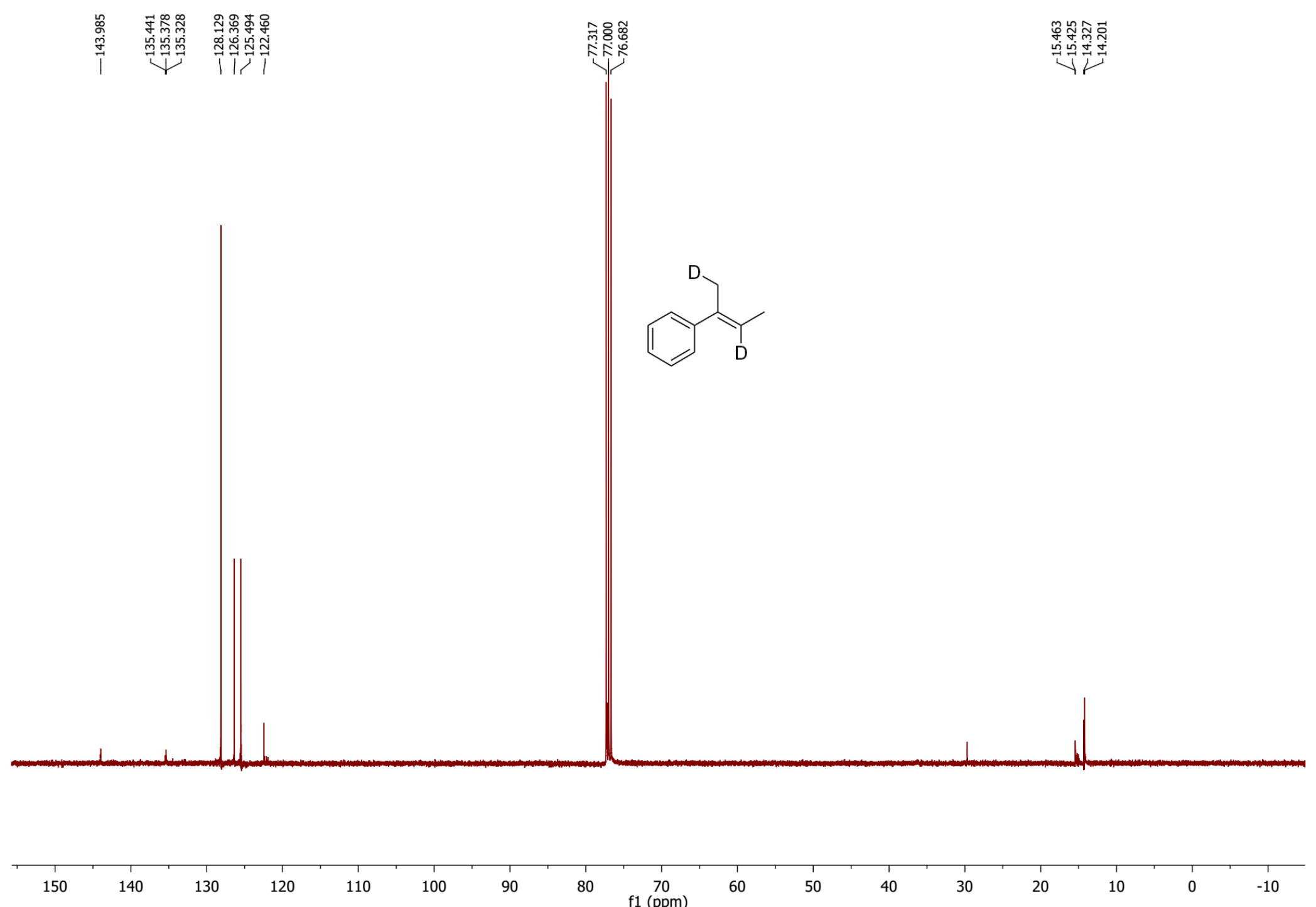
స్

ง N

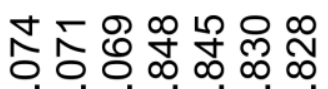

ก ก่ ก
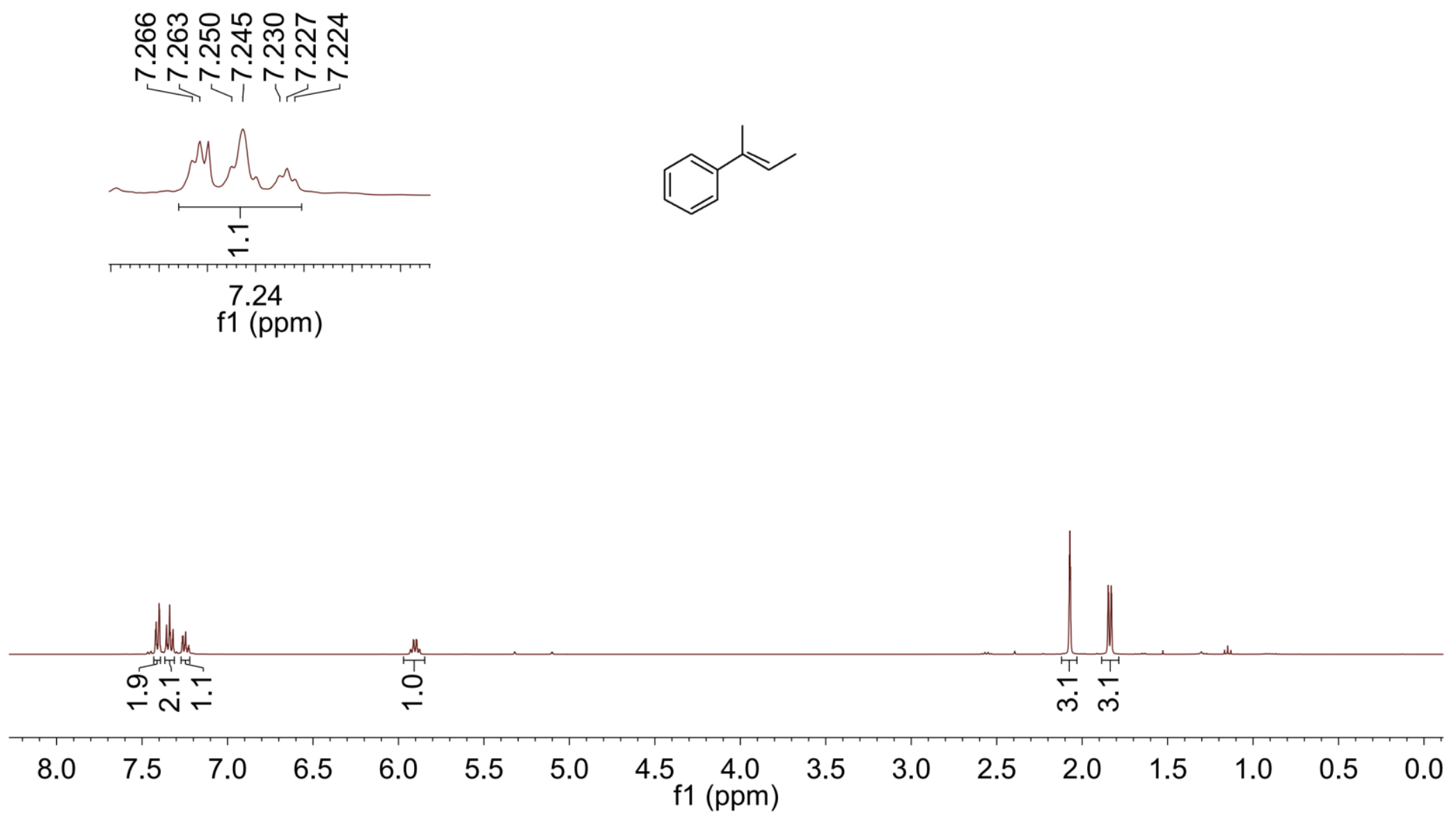
유꿍유요

- $\alpha$.

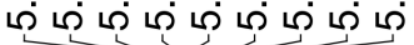

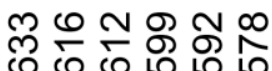 \\ من}

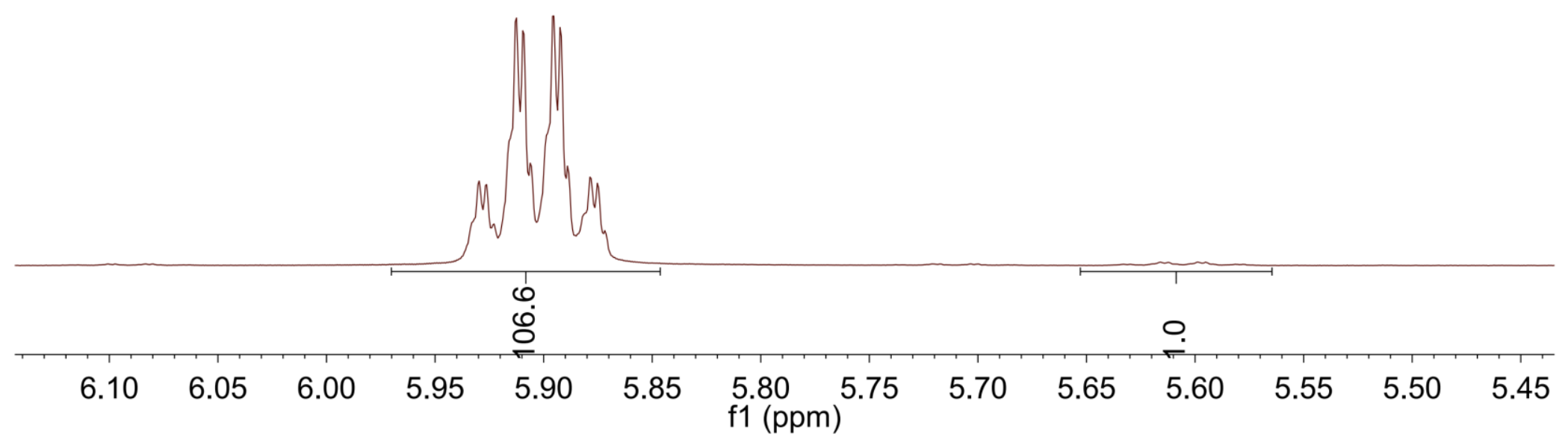




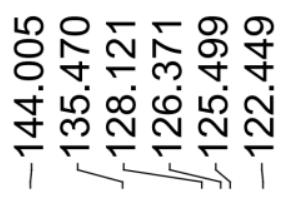

m.

송

莳

เं

난
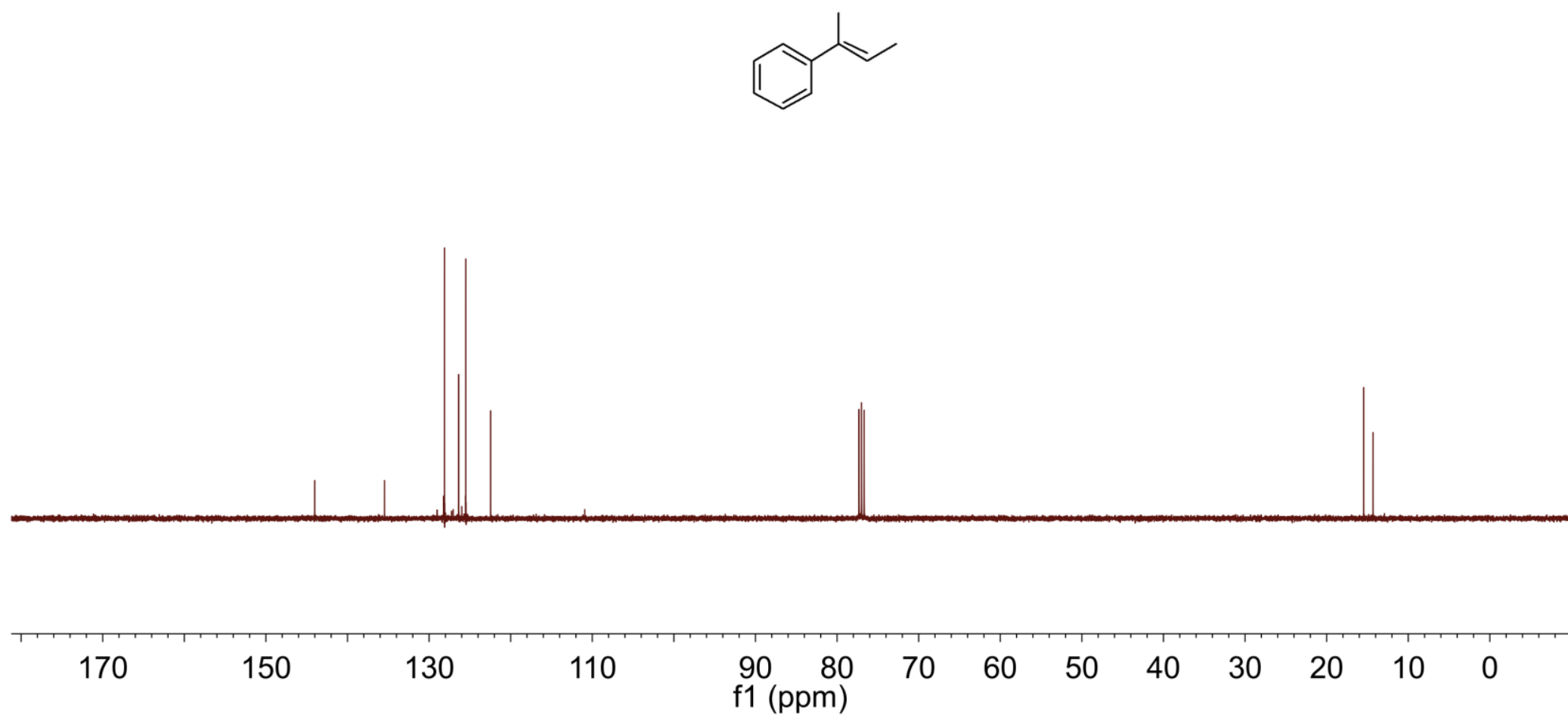

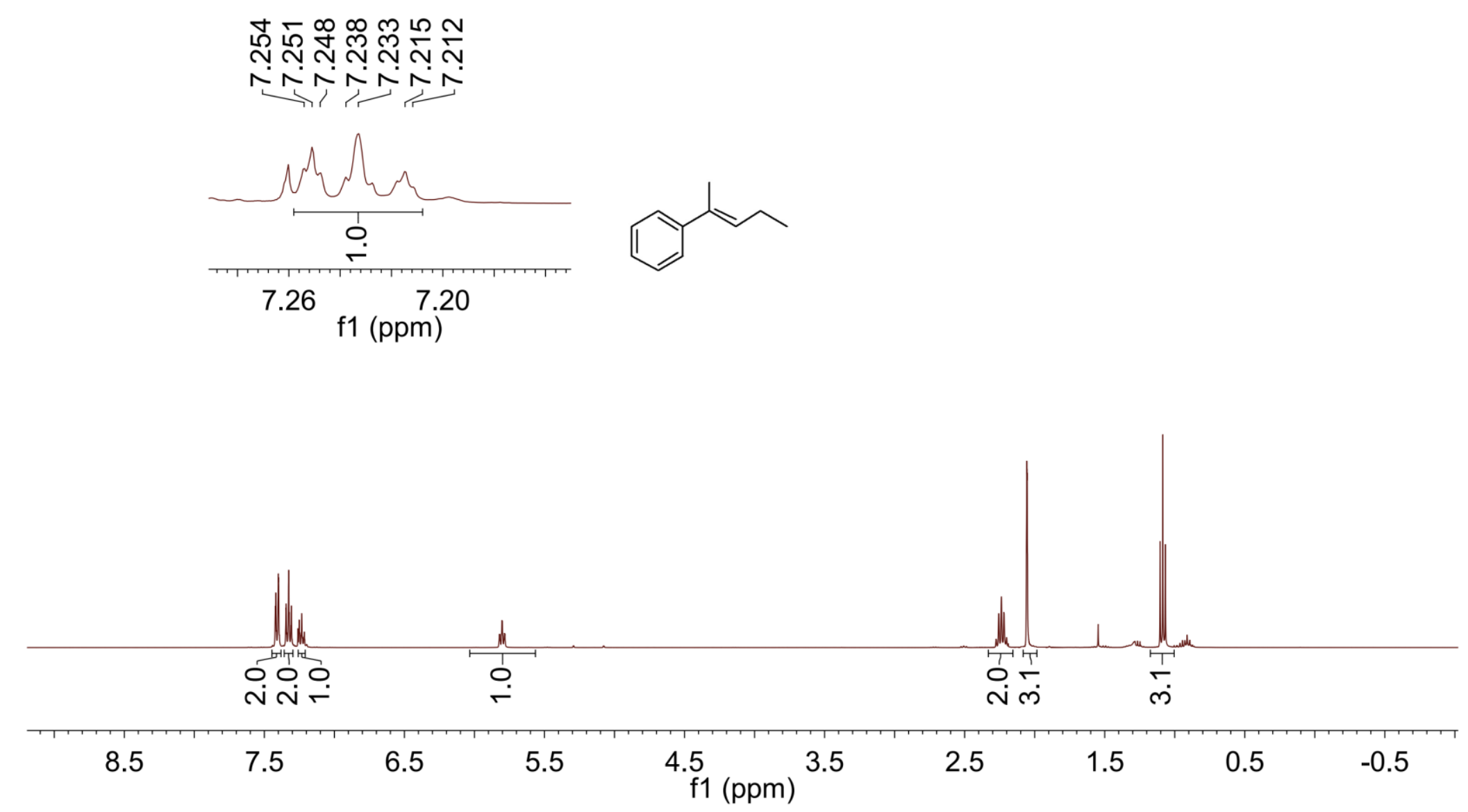


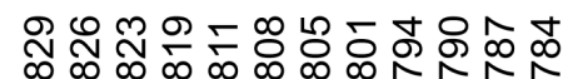

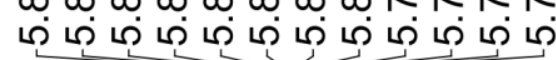

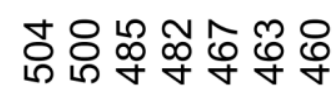

ما

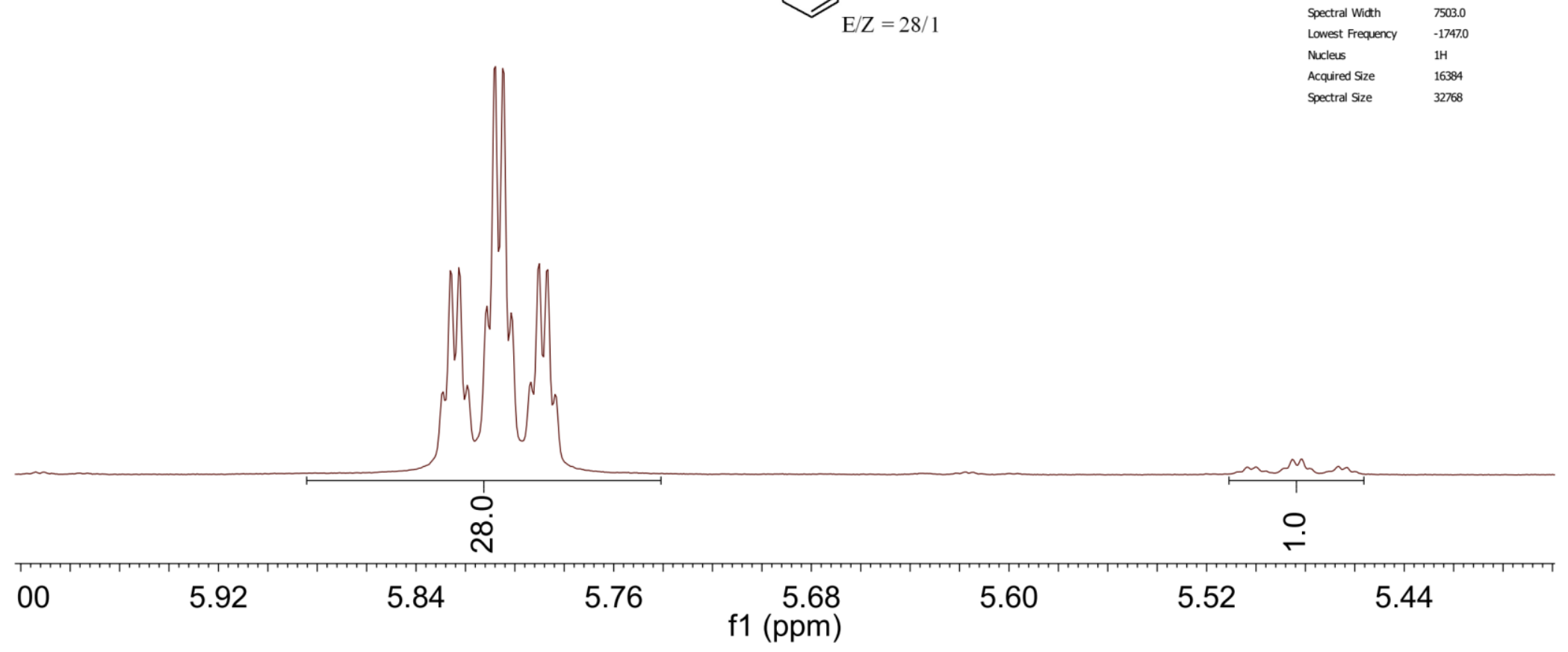




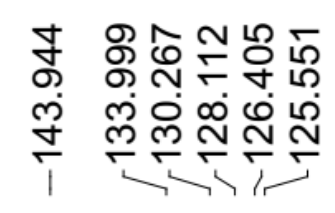

승요

社业

웅

สู่

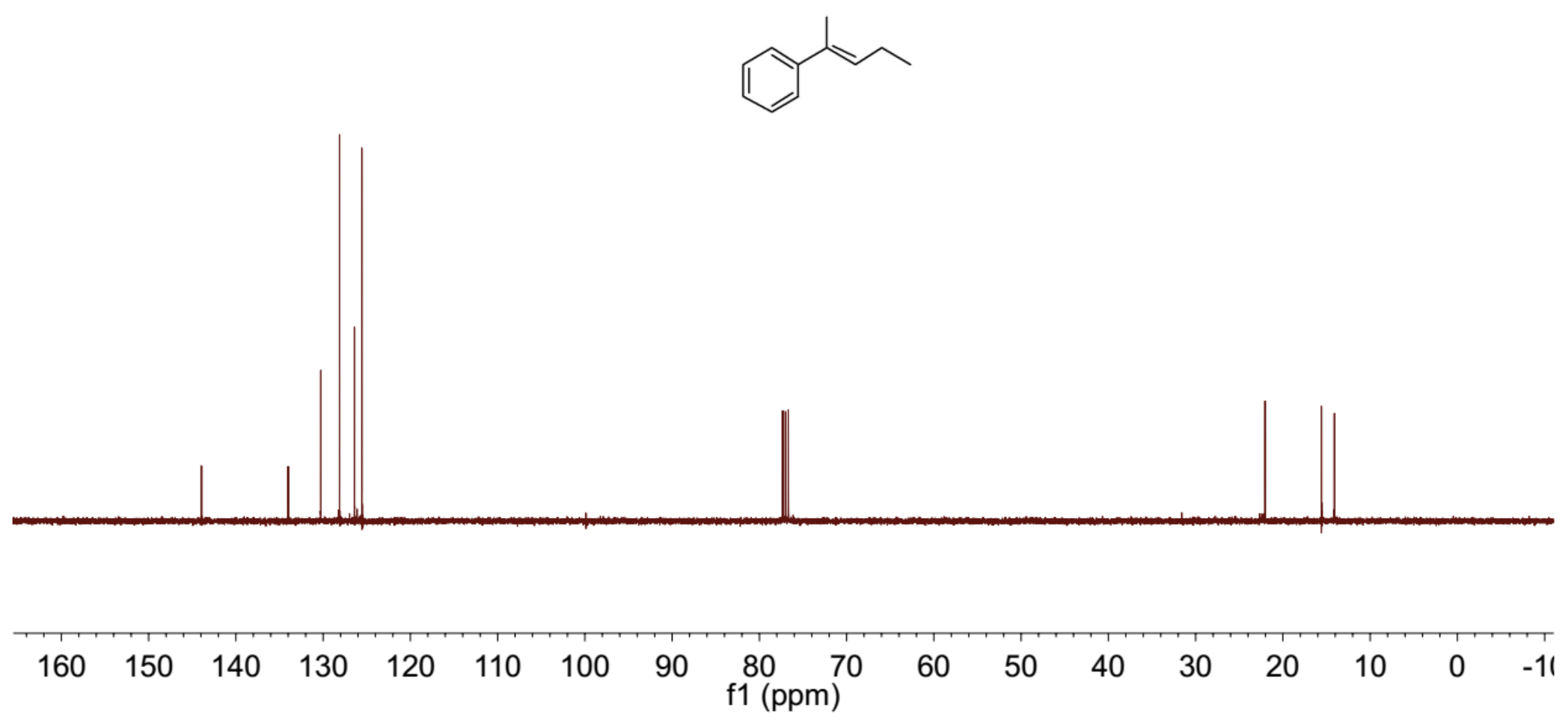




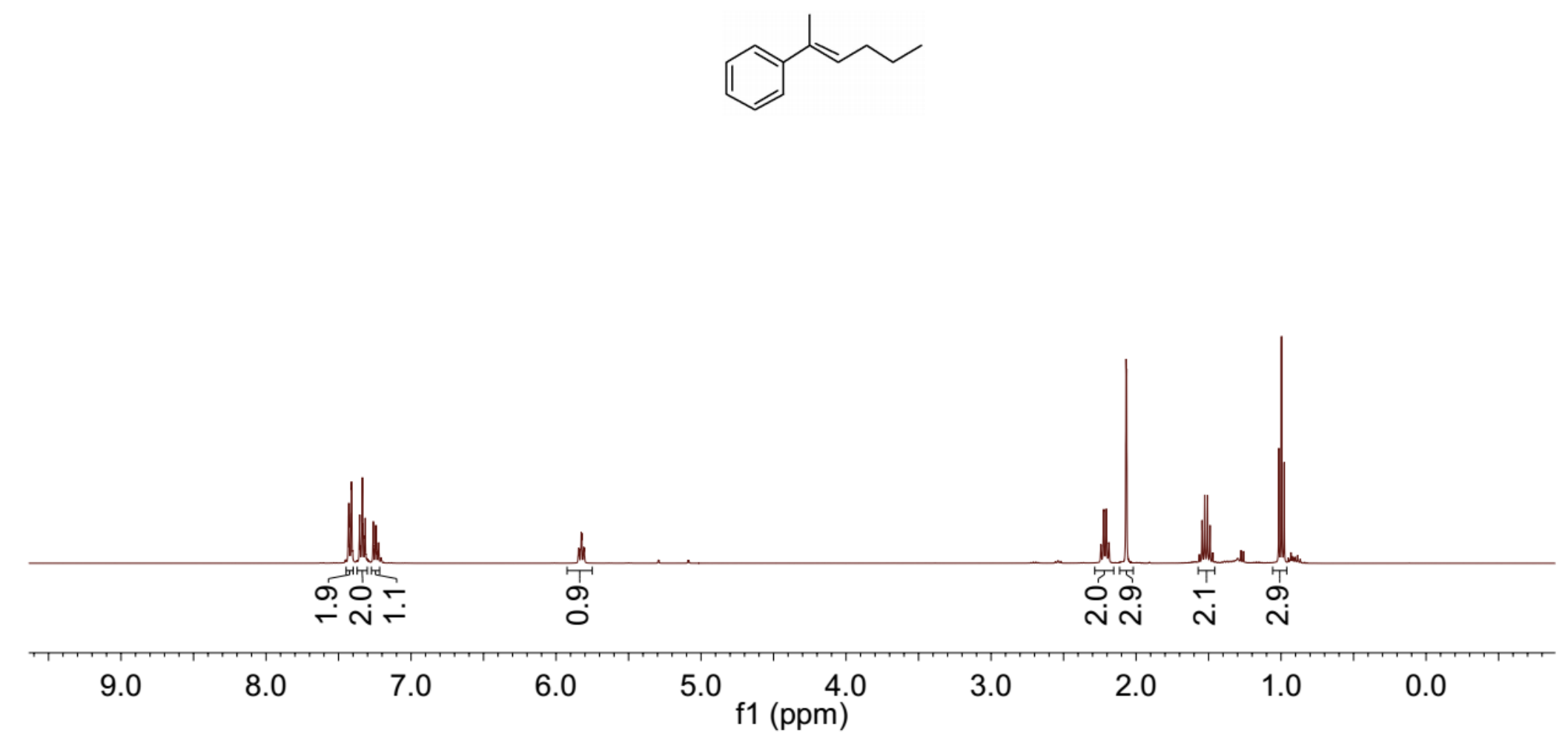




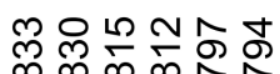

ما ما ما ما ما

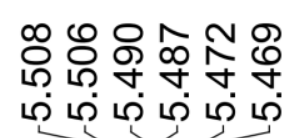

Title $\begin{array}{r}\text { Parameter Valve } \\ \mathrm{S} \# 634795\end{array}$

$\begin{array}{lll}\text { Origin } & \text { JEOL } \\ \text { Spectrometer } & & \end{array}$

Solvent $\quad$ CHOROFORM

Temperature 21.9

Pulse Sequence single pulse.ex

Experiment 1D

$\begin{array}{ll}\text { Number of Scans } & 8 \\ \text { Receiver Gin } & 26\end{array}$

Relaxation Delay 50000

Pulse Woth $\quad 6.4850$

Acquisition Time 2.1837

Spectrometer Frequency 399.78

Spectral Width $\quad 7503.0$

Lowest Frequency -1746.6

Acquired Size

16384

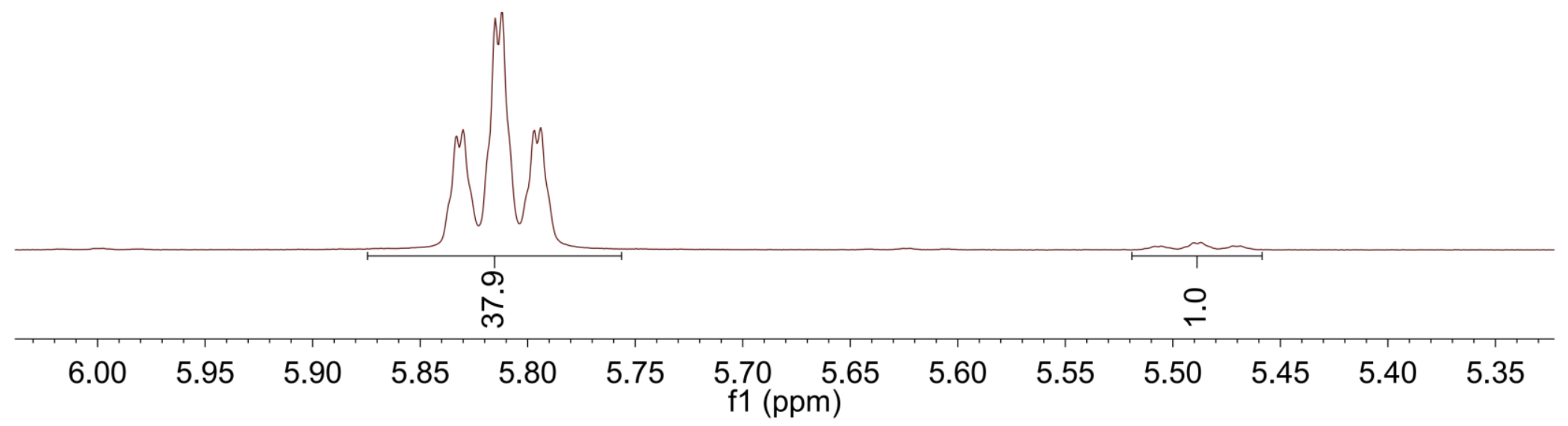


$2{ }^{13}$ C NMR

\begin{tabular}{|c|c|c|}
\hline 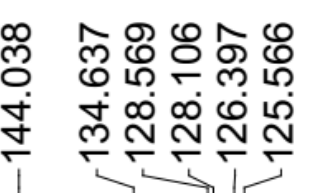 & 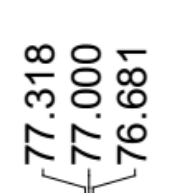 & $\begin{array}{l}\text { o } \\
0 \\
\text { के }\end{array}$ \\
\hline
\end{tabular}
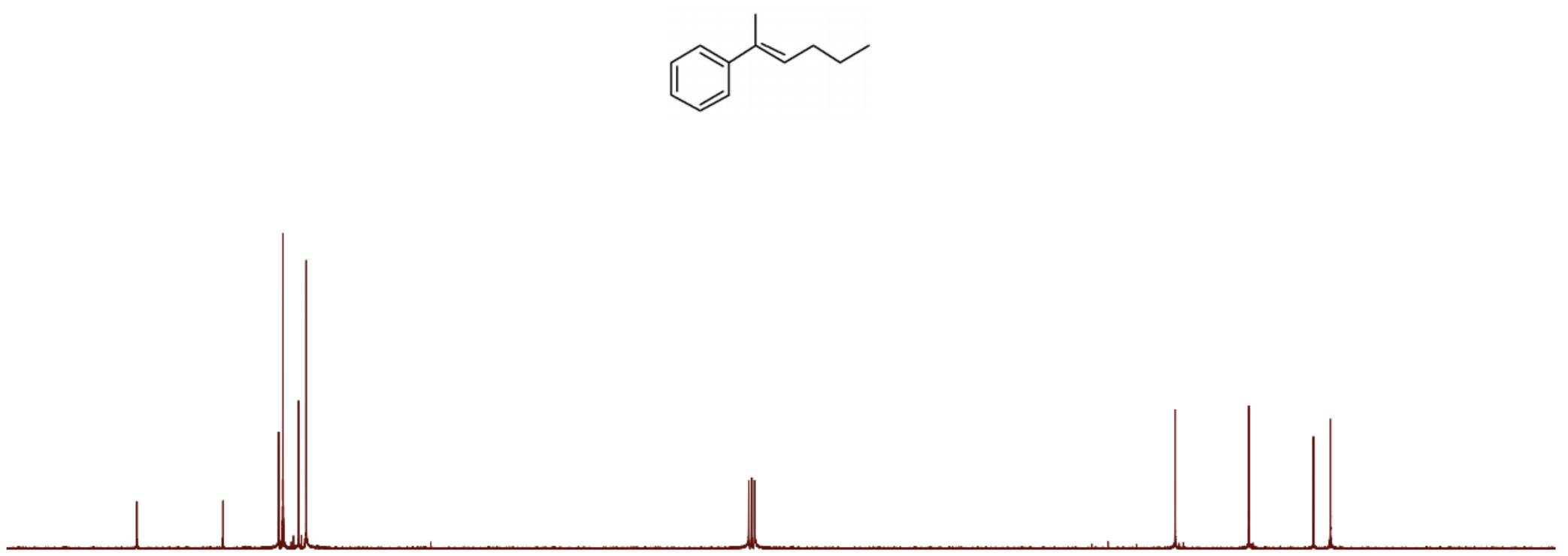

$150 \quad 140 \quad 130 \quad 120$

$80 \quad 70$

60

50

$40 \quad 30$

$\begin{array}{llll}20 & 10 & 0 & -1\end{array}$ 


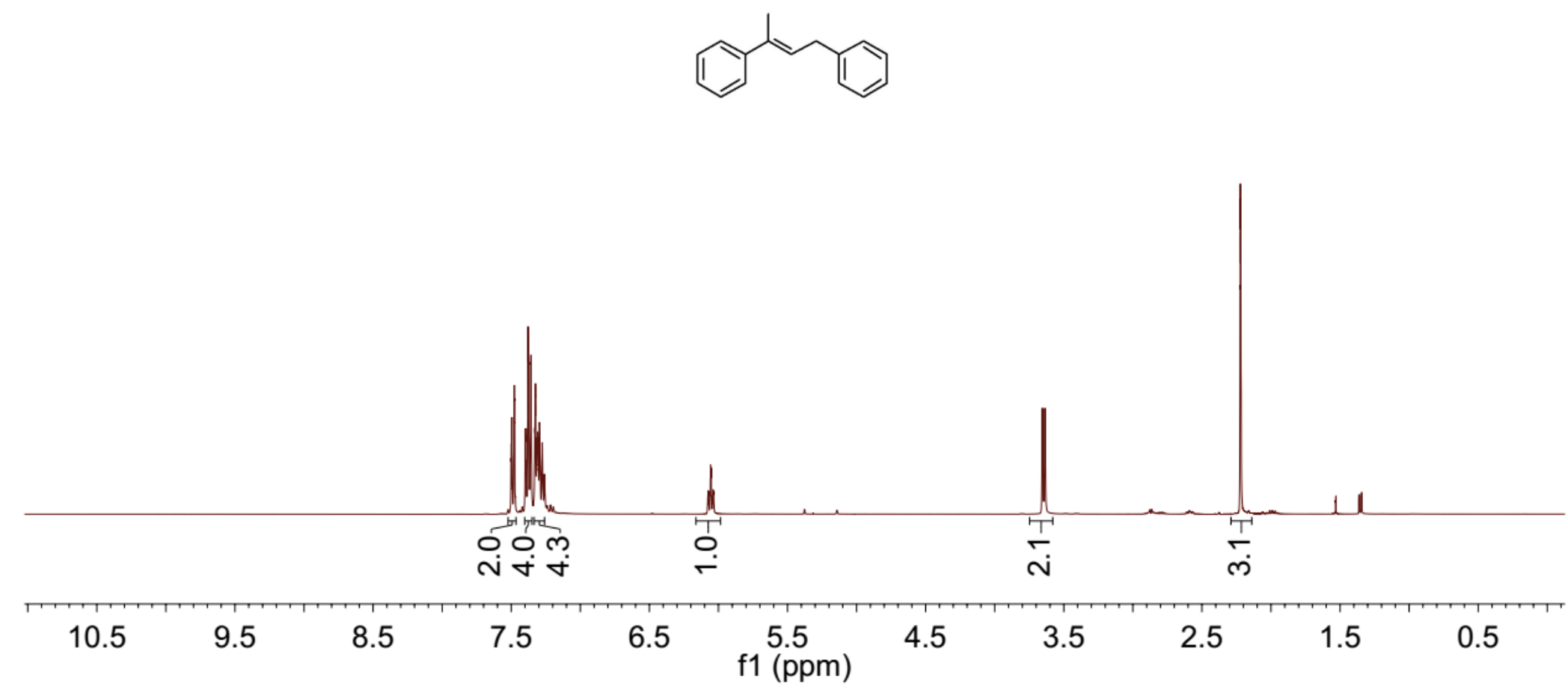


청정

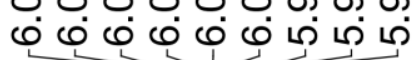

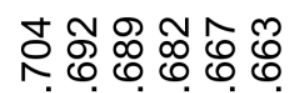

ما
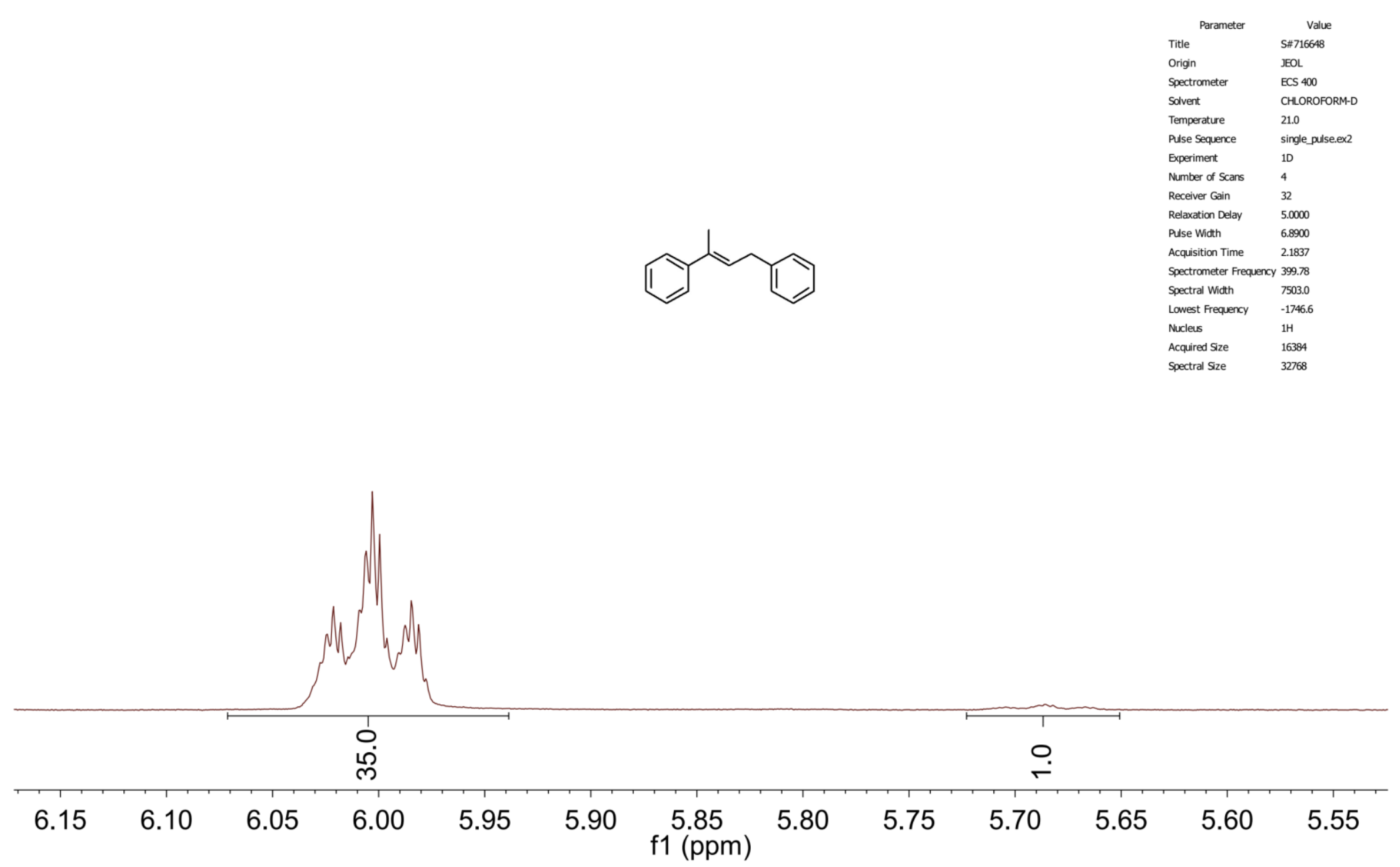


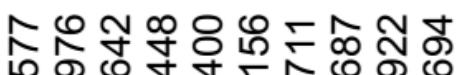
(1)

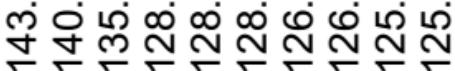
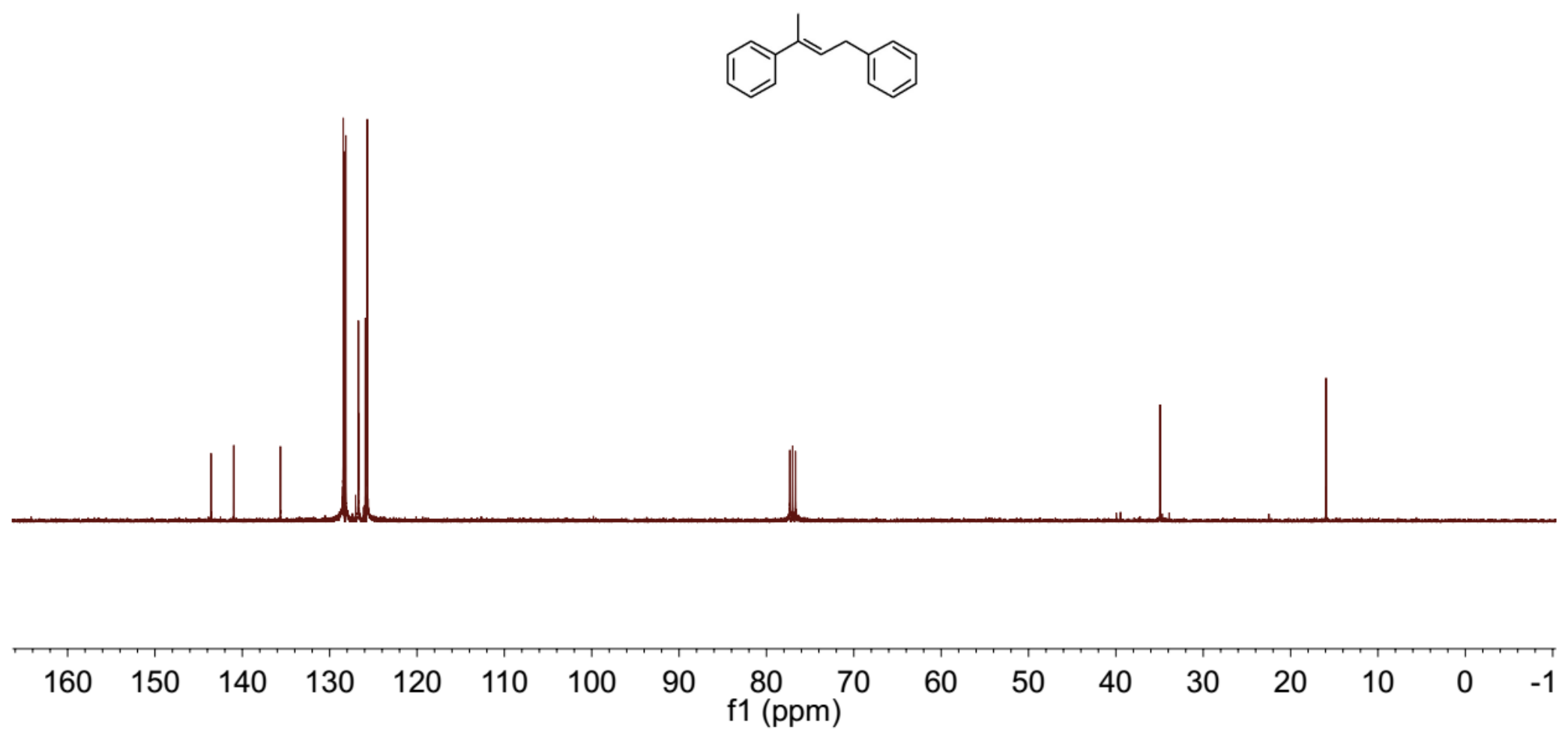


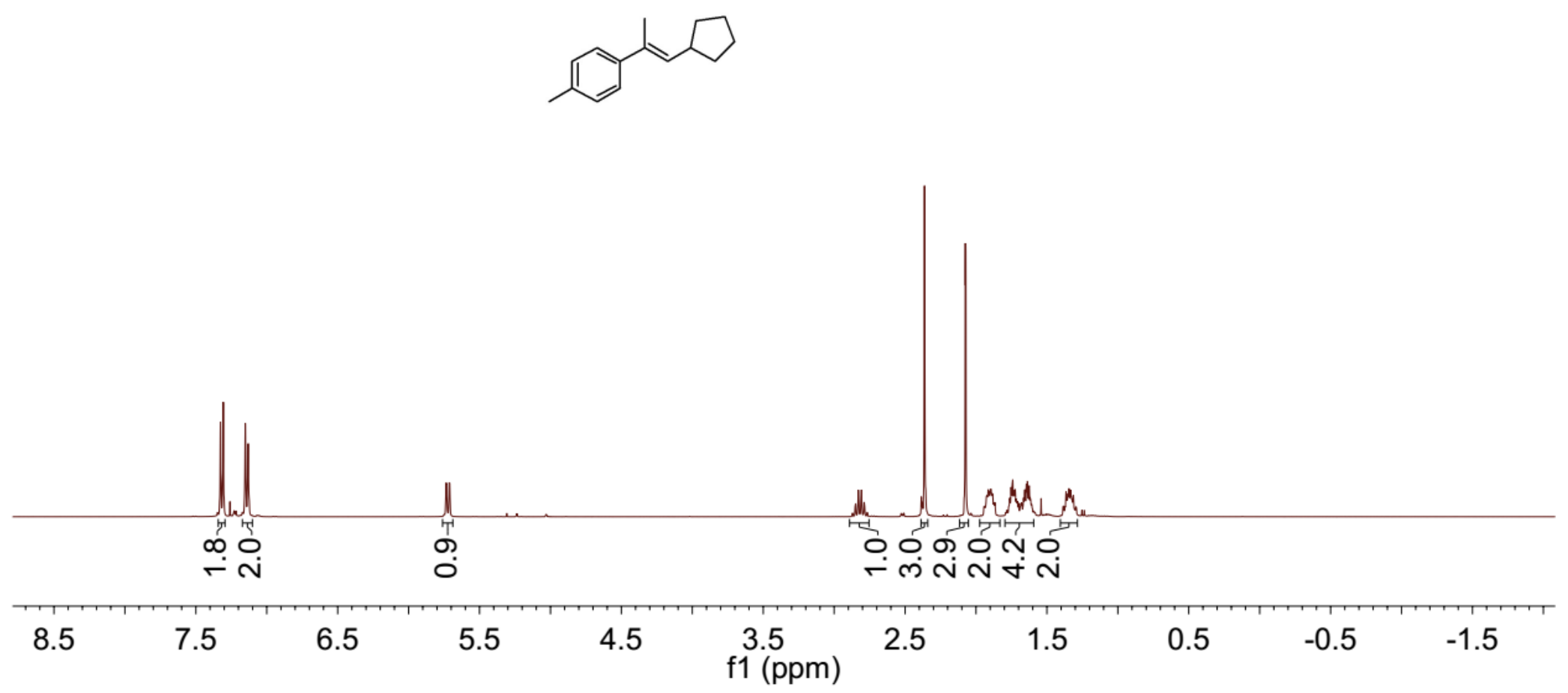


2e $E / Z$

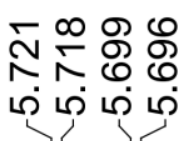

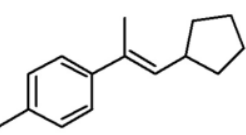

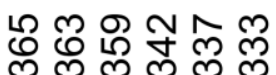

هن

ما

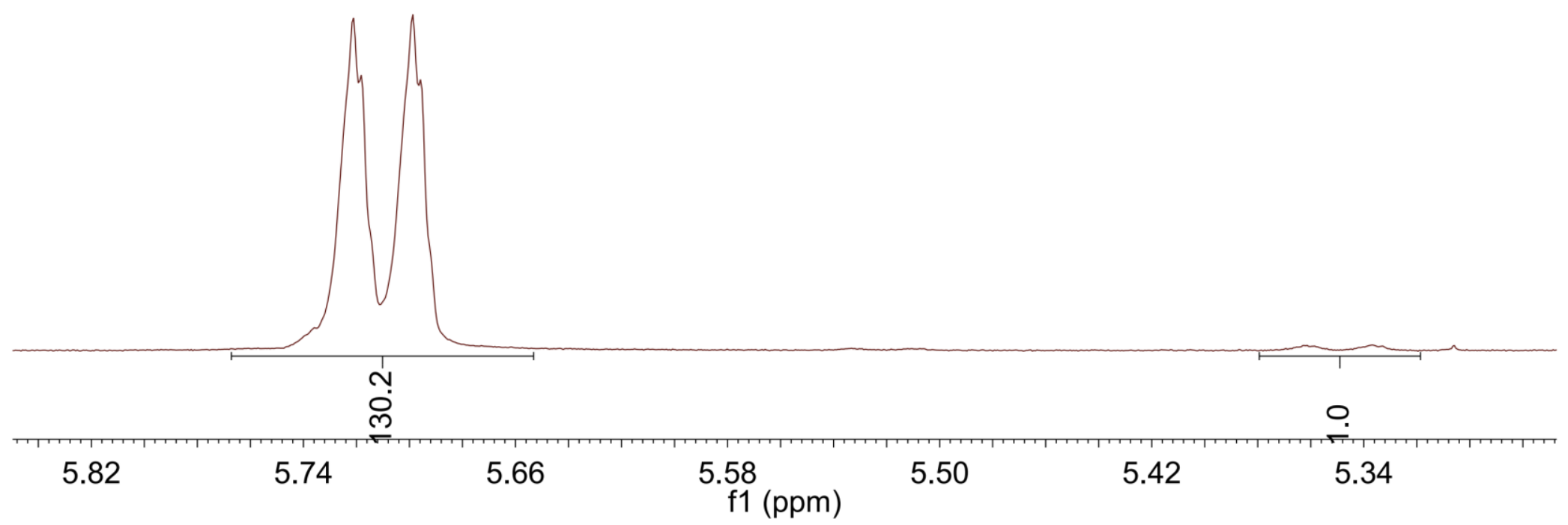




\begin{tabular}{|c|c|c|}
\hline 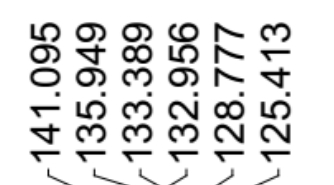 & 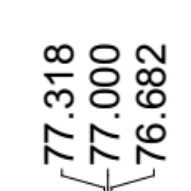 & 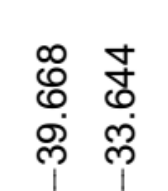 \\
\hline
\end{tabular}

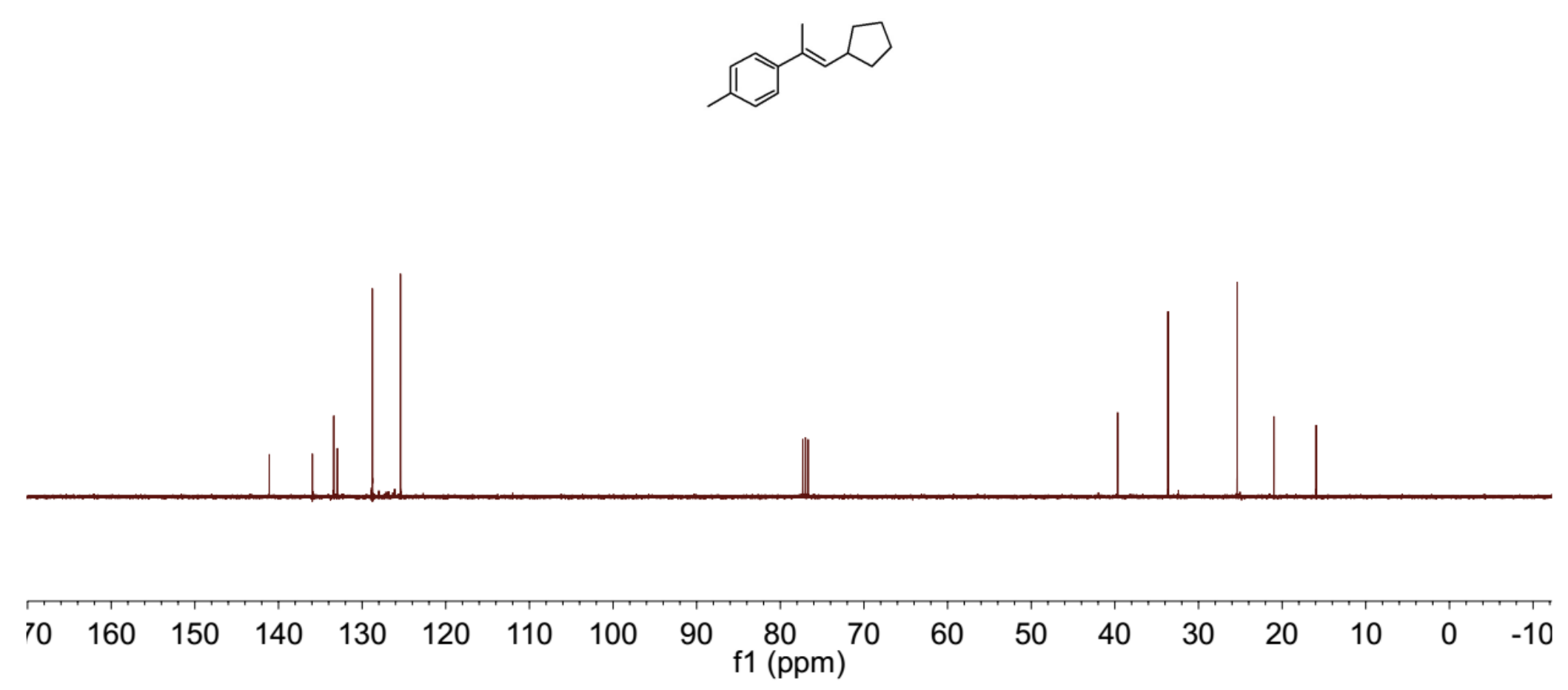




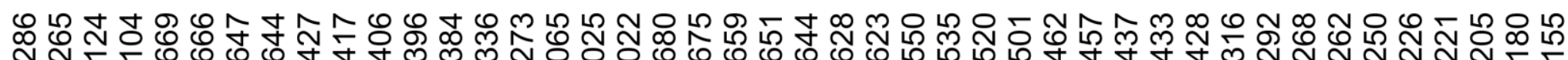
N s

ঙิণ

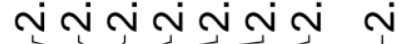
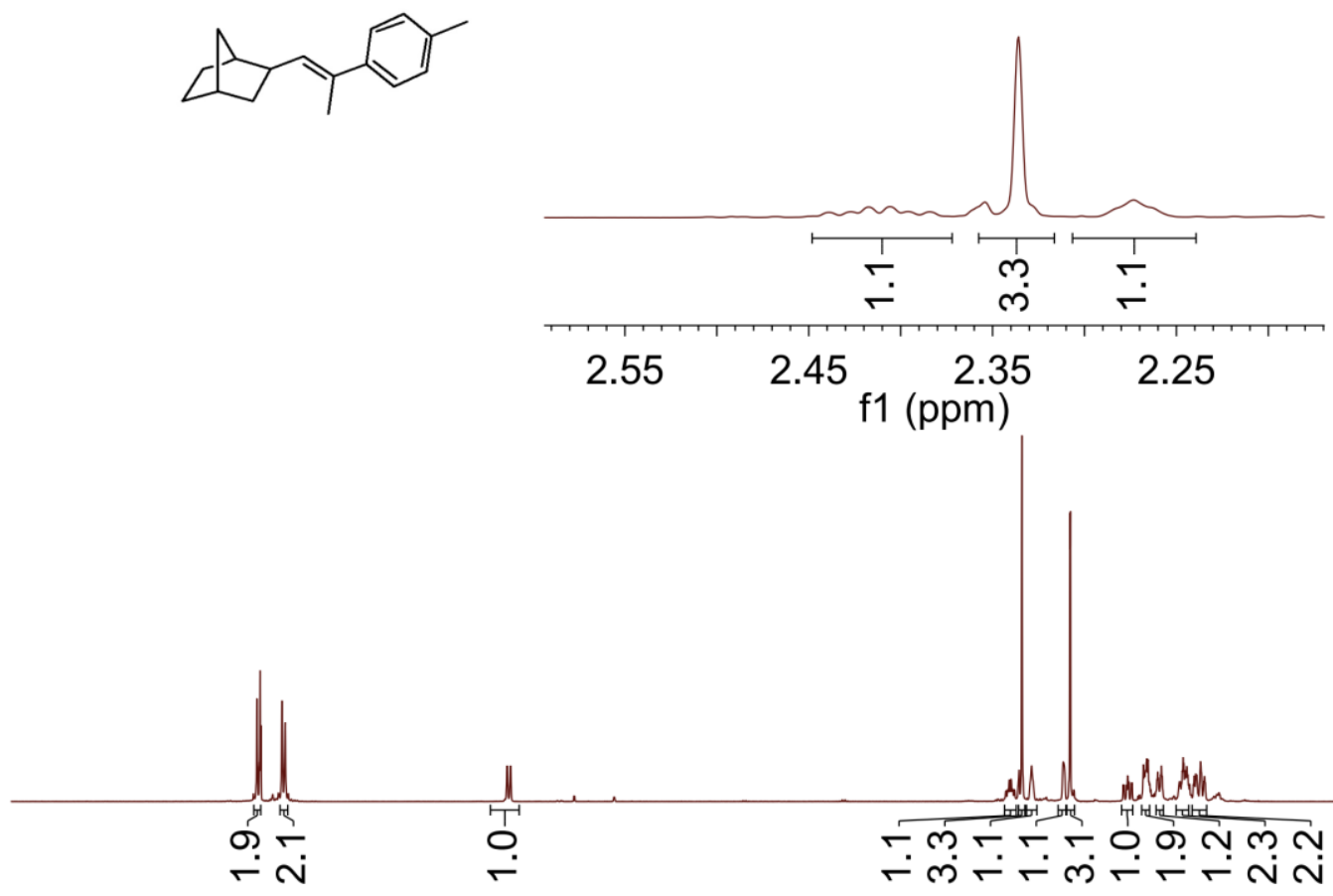

\begin{tabular}{|c|c|c|c|c|c|c|c|c|c|c|}
\hline 8.0 & 7.0 & 6.0 & 5.0 & 4.0 & 3.0 & 2.0 & 1.0 & 0.0 & -1.5 & -3.0 \\
\hline
\end{tabular}




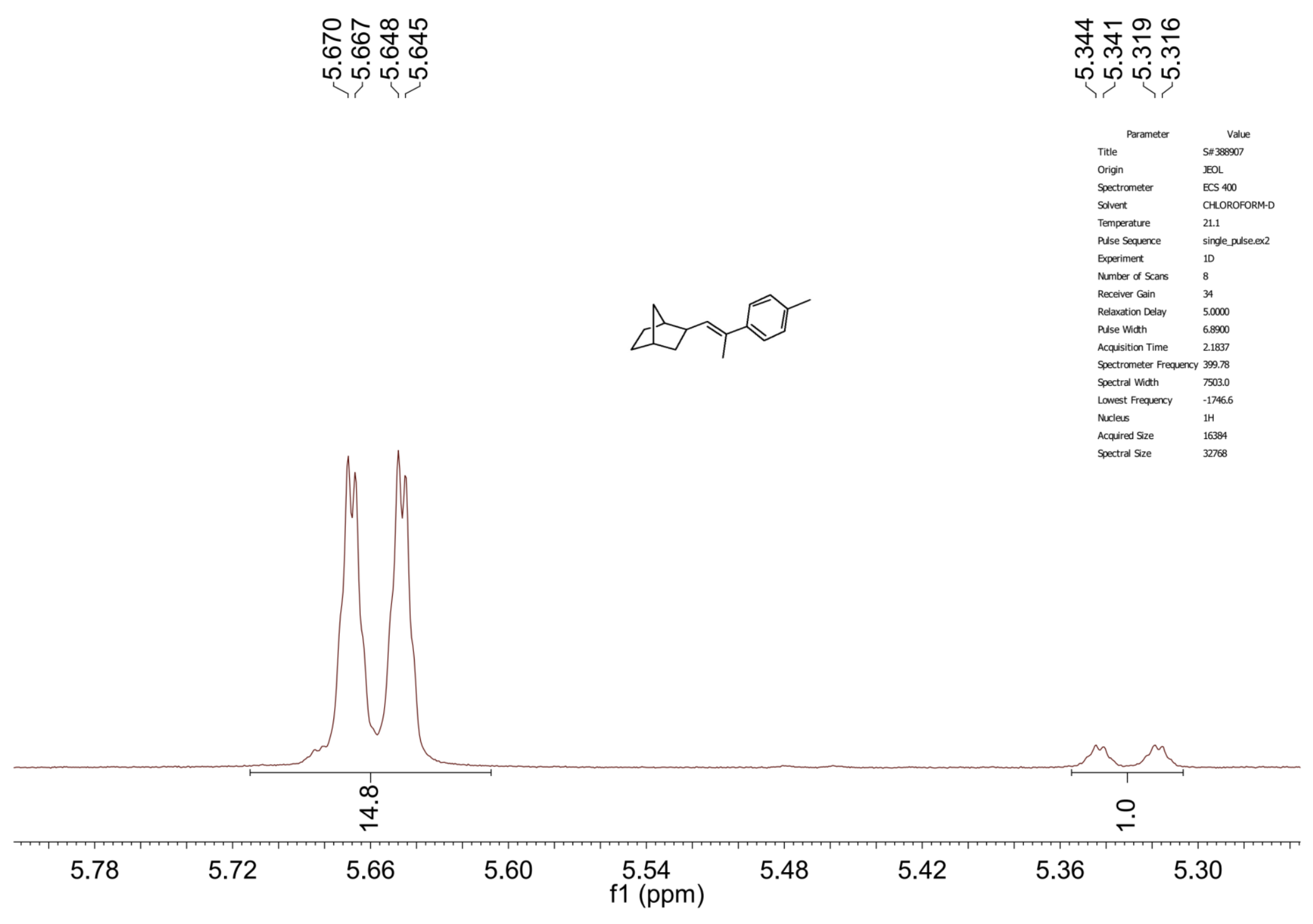


$\infty$

ले 8

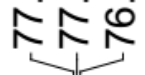

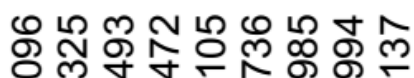

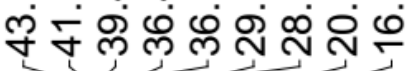

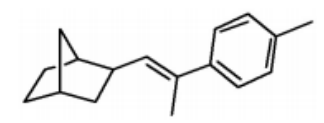

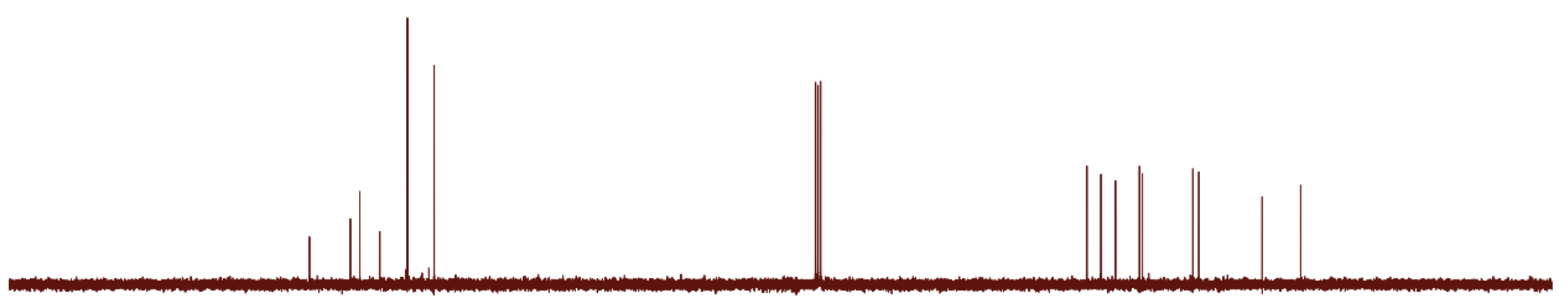

\begin{tabular}{lllllllllllllll}
\hline 170 & 150 & 130 & 110 & 90 & $\mathrm{f} 1(\mathrm{ppm})$ & 70 & 60 & 50 & 40 & 30 & 20 & 10 & 0 & -10
\end{tabular}




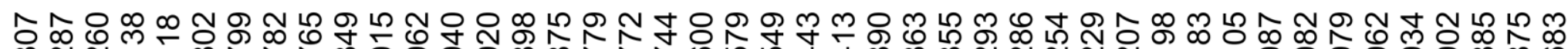

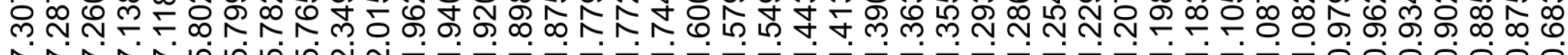
ЛNNN
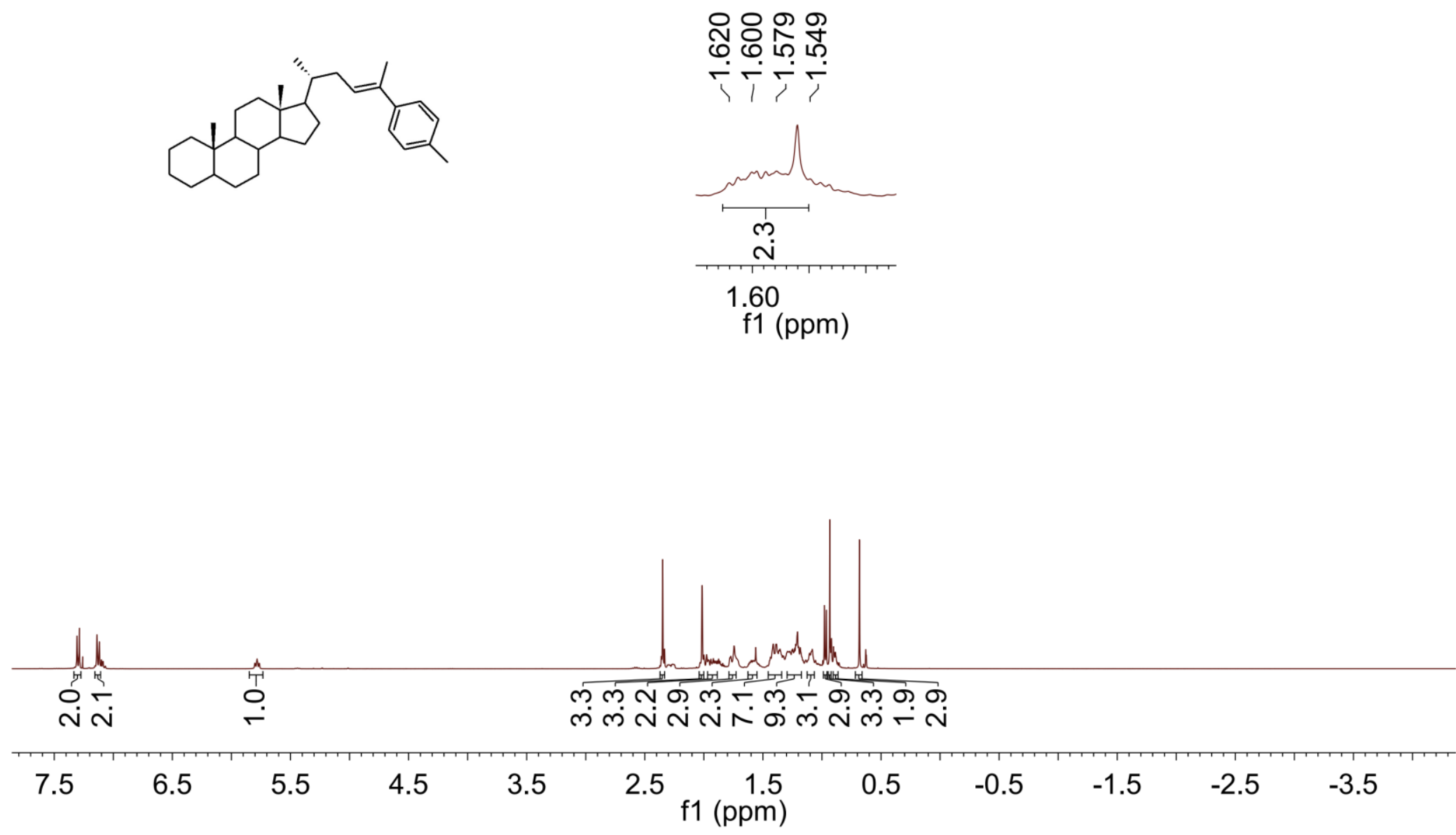


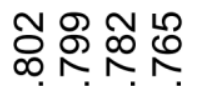 \\ ما}

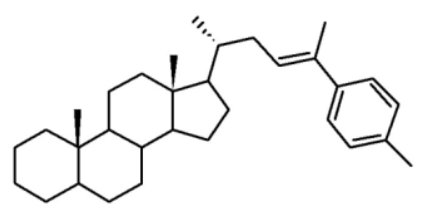

مَ

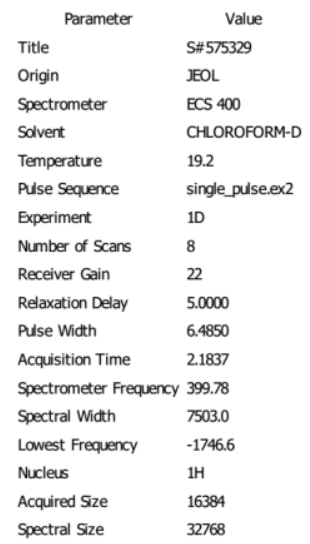

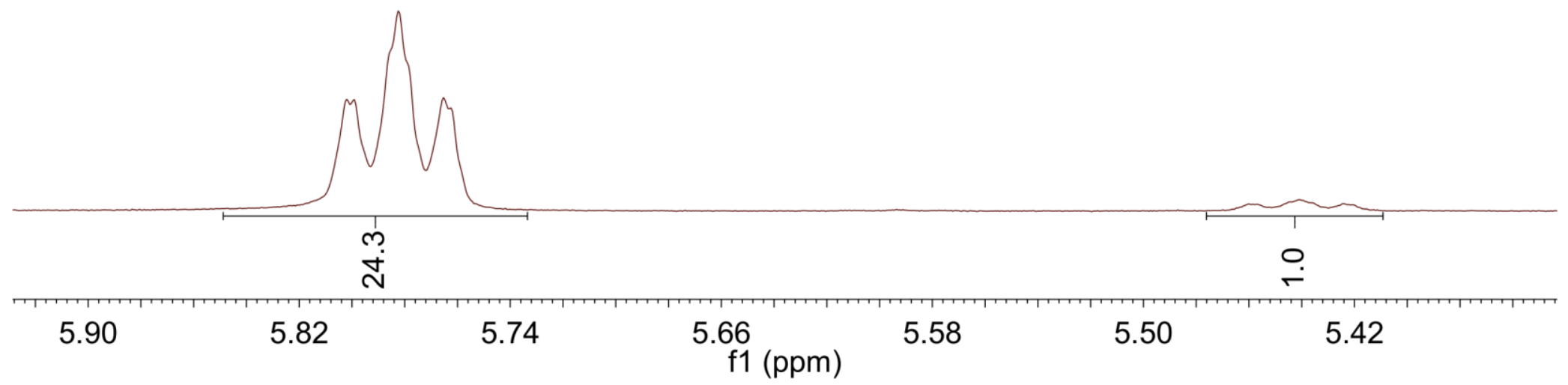




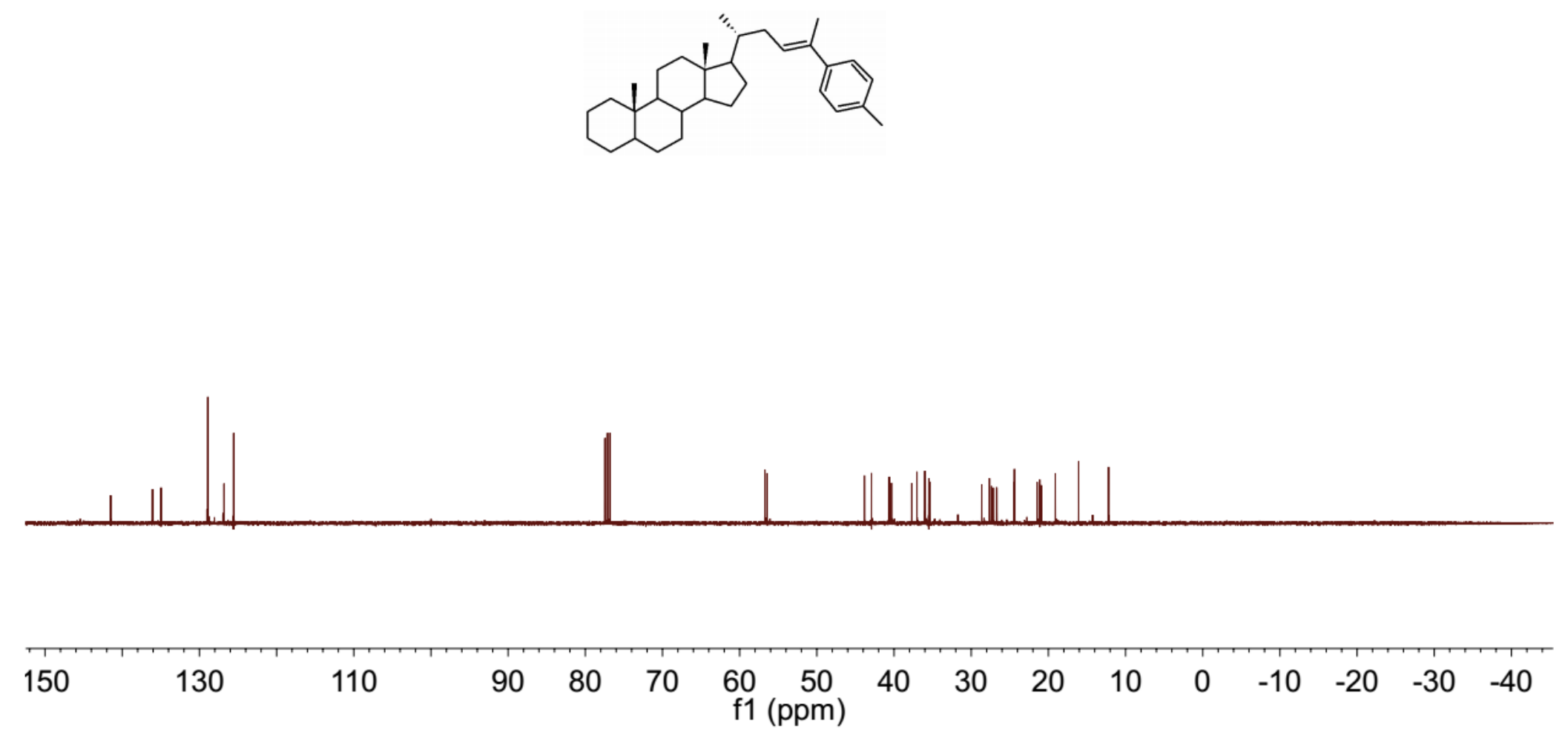



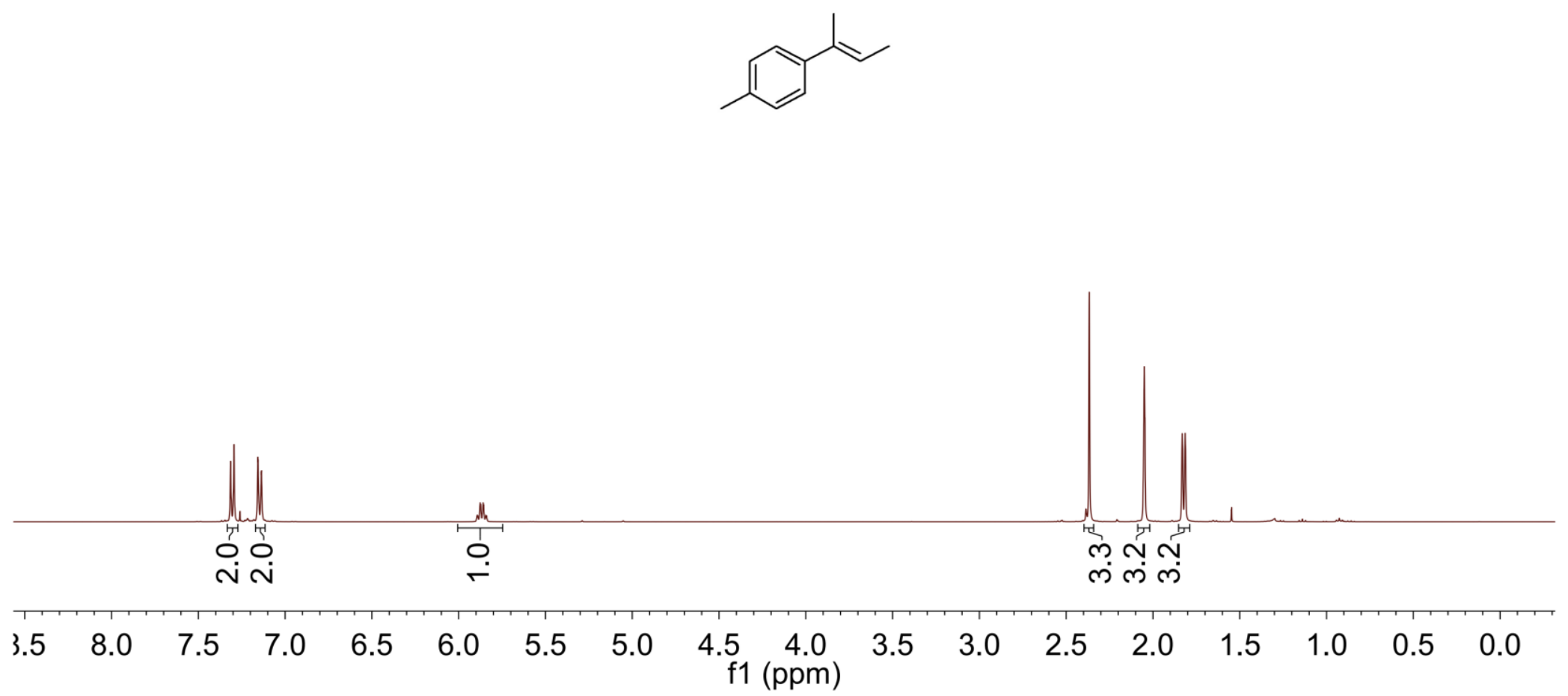
2h E/Z

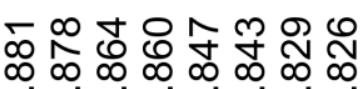

"ما ما ما ما

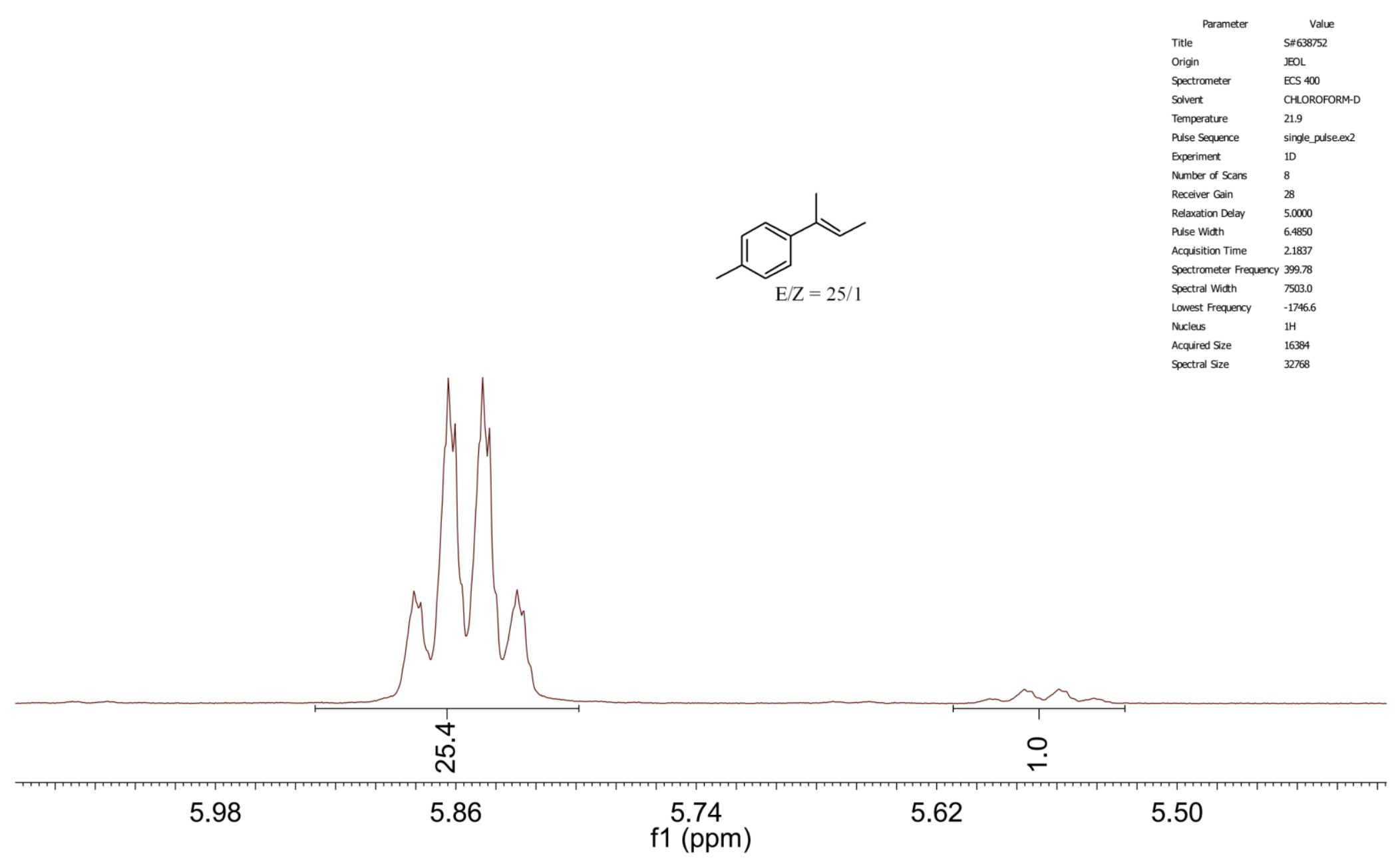

ำ

ए?

ம 


\begin{tabular}{|c|c|}
\hline 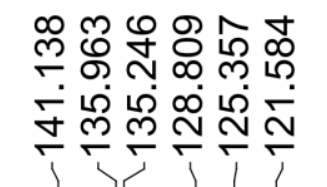 & 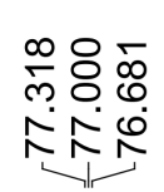 \\
\hline
\end{tabular}

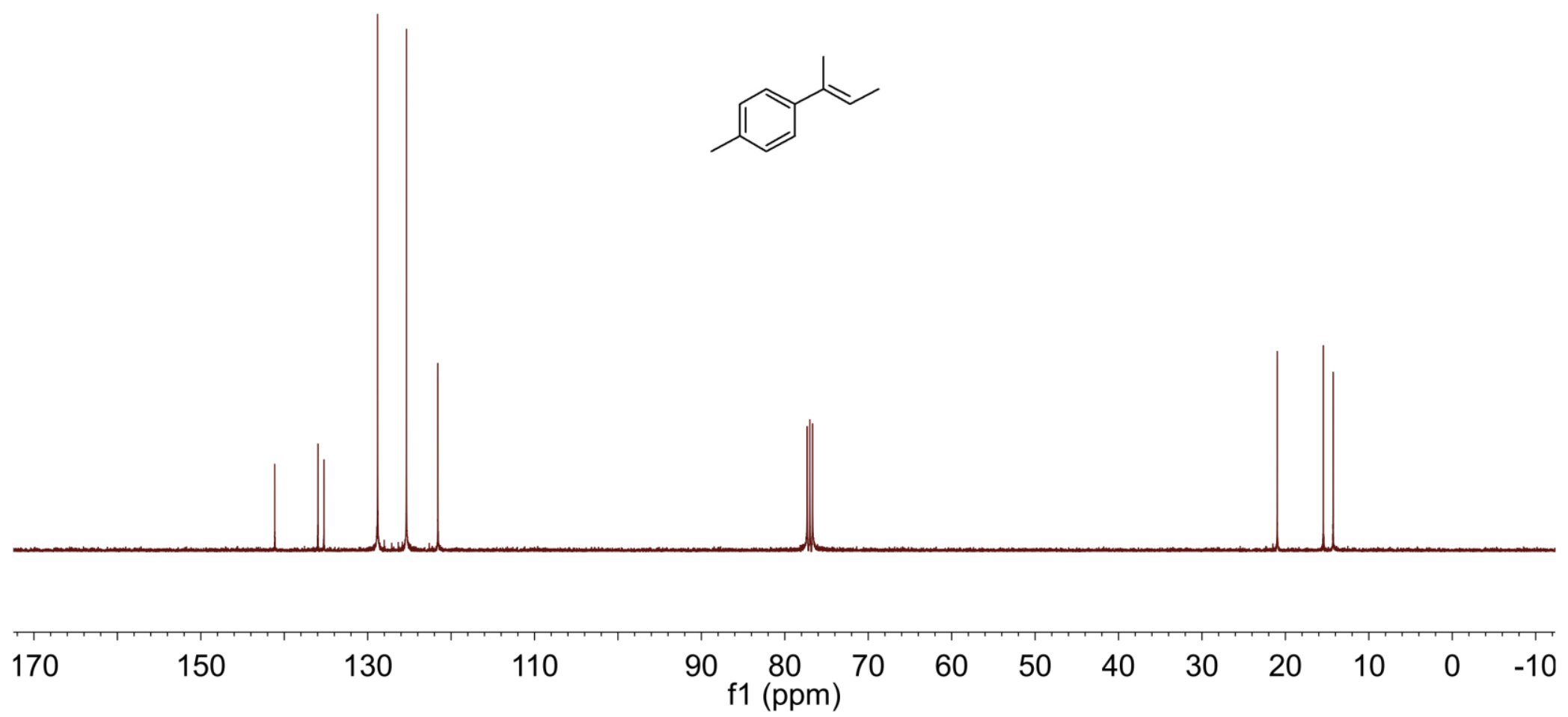




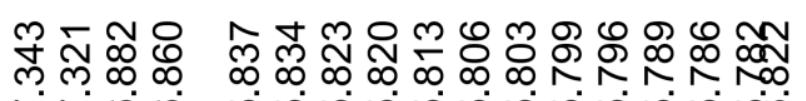

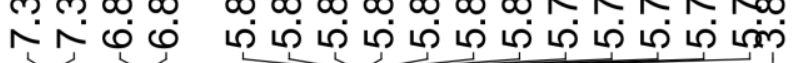

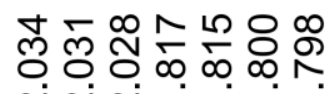

กั่ กั่

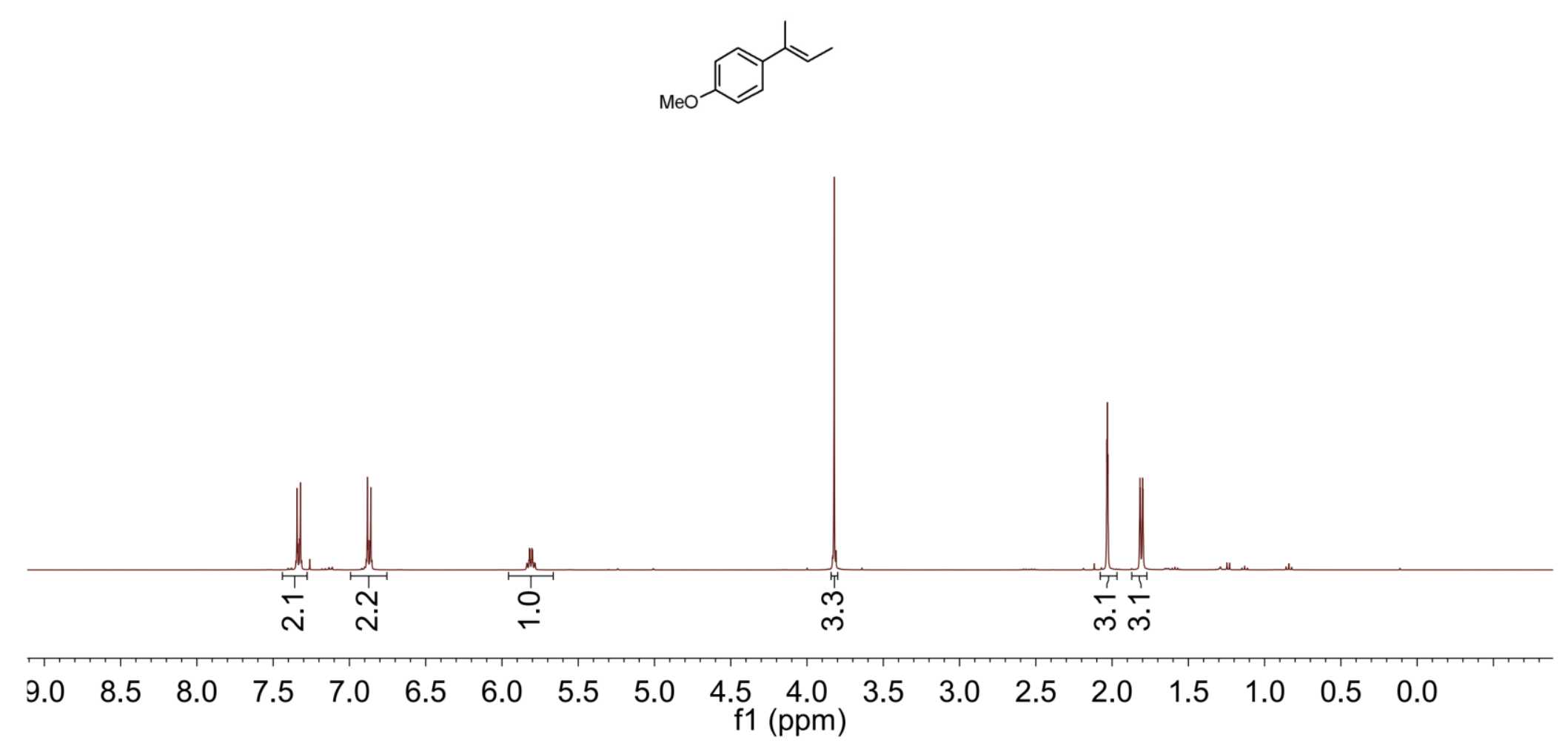




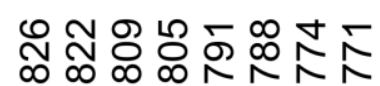

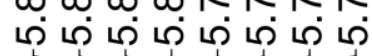

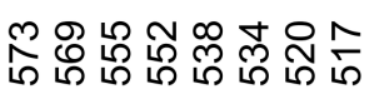

ما

$$
\begin{aligned}
& \text { Title } \begin{array}{c}
\text { Parameter Value } \\
\mathrm{S} \# 496230
\end{array} \\
& \begin{array}{ll}
\text { Origin } & \text { JEOL } \\
\text { Spectrometer } & \text { ECS }
\end{array} \\
& \text { Spectrometer ECS } 400 \\
& \text { Temperature } 20.8 \\
& \text { Puse Sequence single.pulse.ex2 } \\
& \text { Experimert } 10 \\
& \text { Number of Scans } 4 \\
& \text { Relaxation Delay } 50000 \\
& \begin{array}{ll} 
& \text { Relaxation Detay } \quad 5.0000 \\
\text { Puse With } & 68900
\end{array} \\
& \text { Acquistion Time 2.1837 } \\
& \text { Spectrometer Frequency } 399.78 \\
& \text { Spectral With } \quad 7503.0 \\
& \text { Lowest Frequency }-1746 \\
& \text { Nucleus } \\
& \text { Acquired Sze } \\
& 16384
\end{aligned}
$$
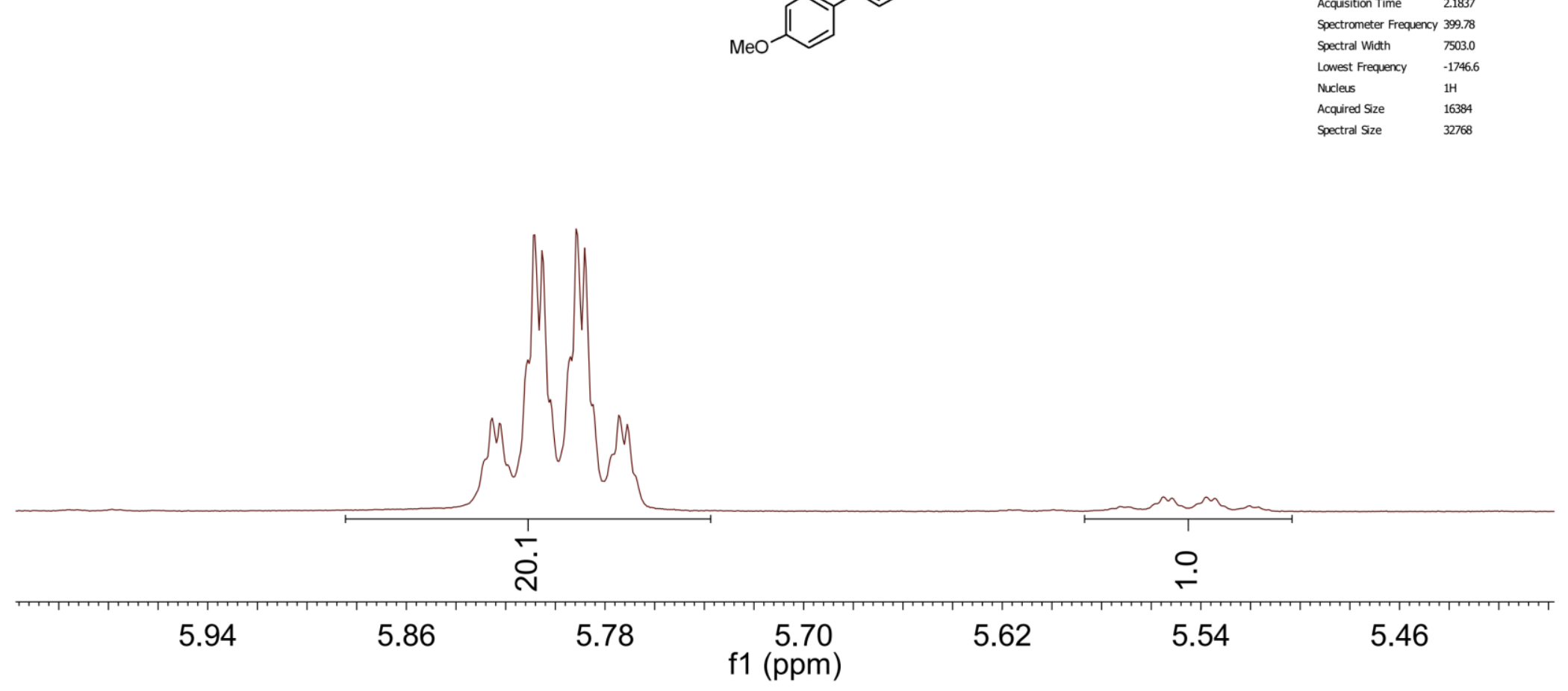
$\mathbf{2 i}^{13}$ C NMR

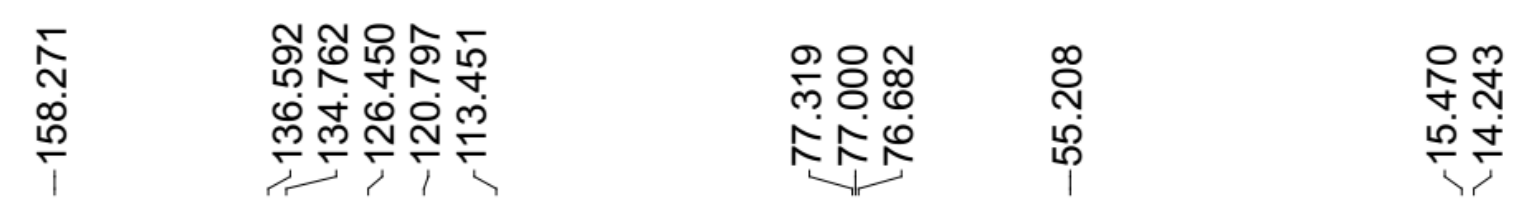
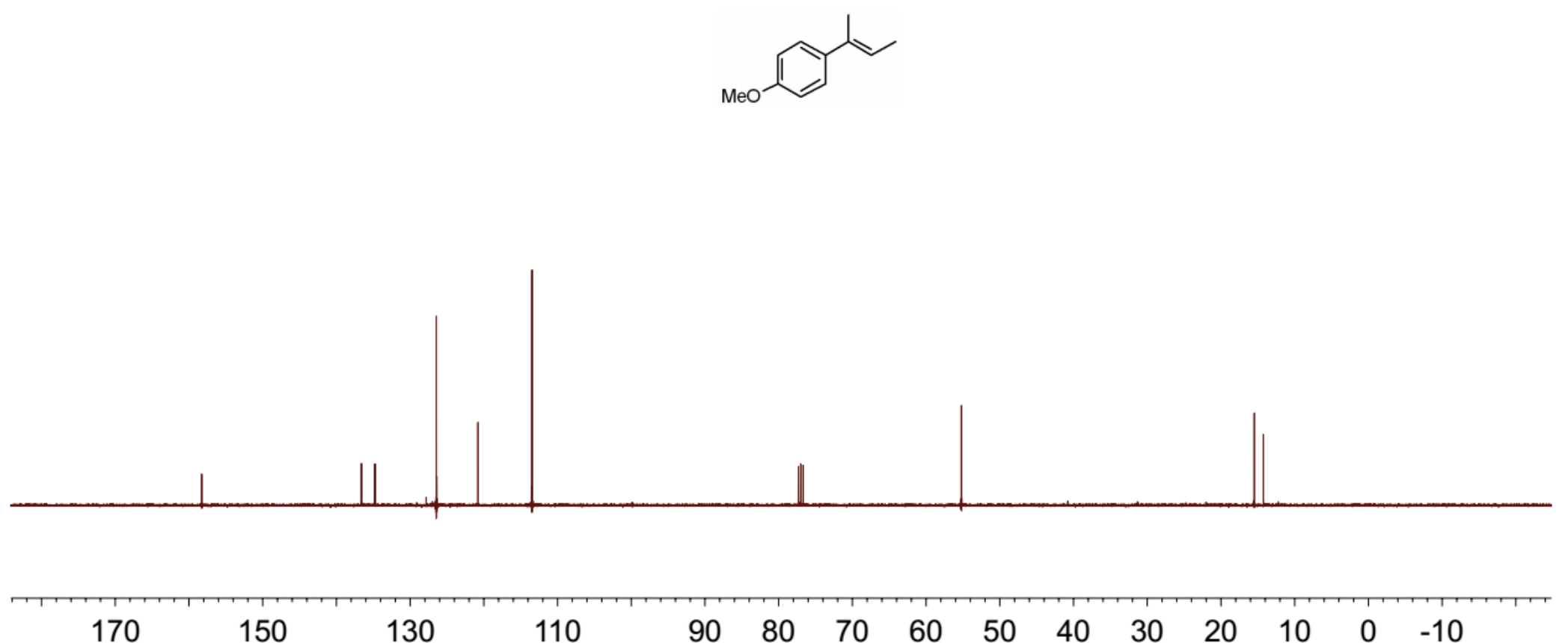

130

110

90

1 (ppm) 


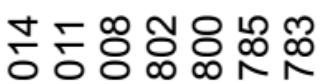

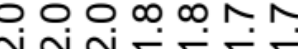

N N

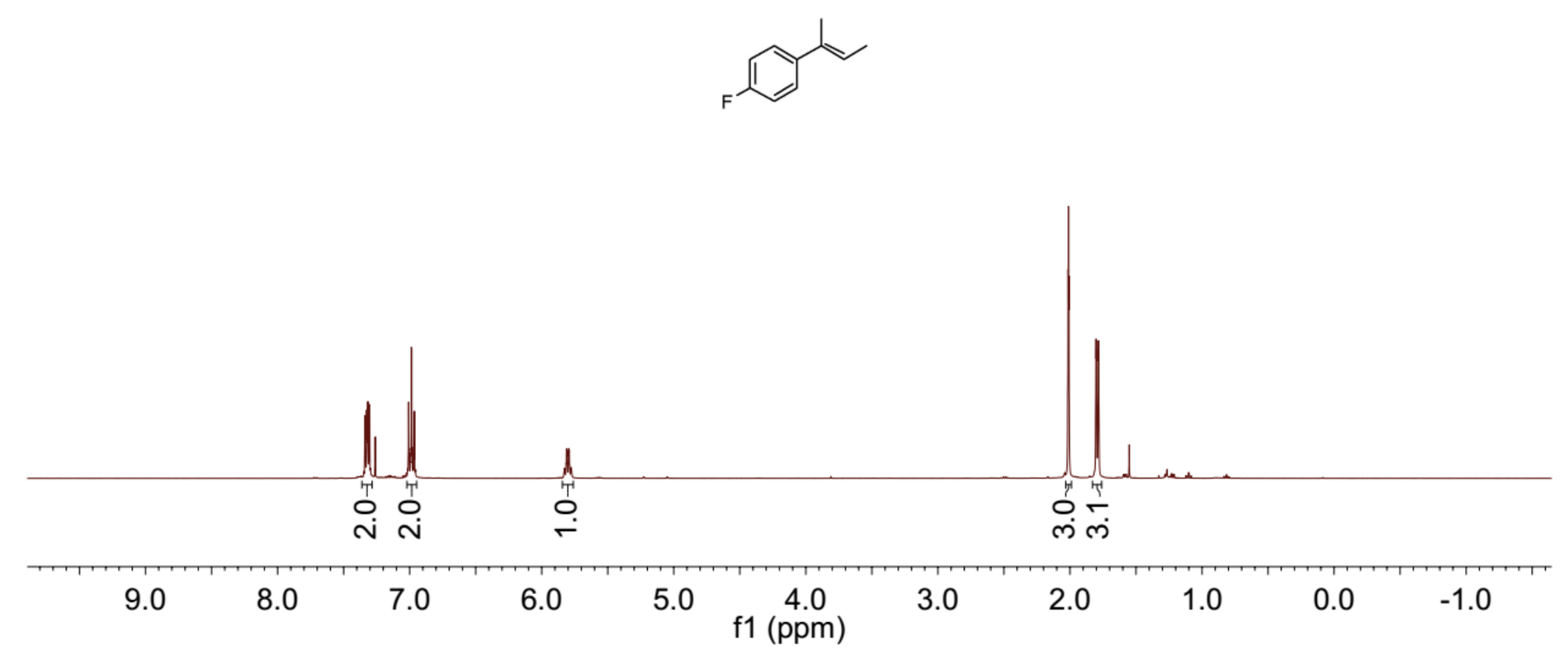


స్లె స్రం

ن

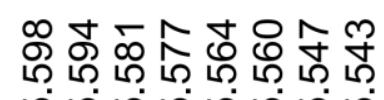

ம
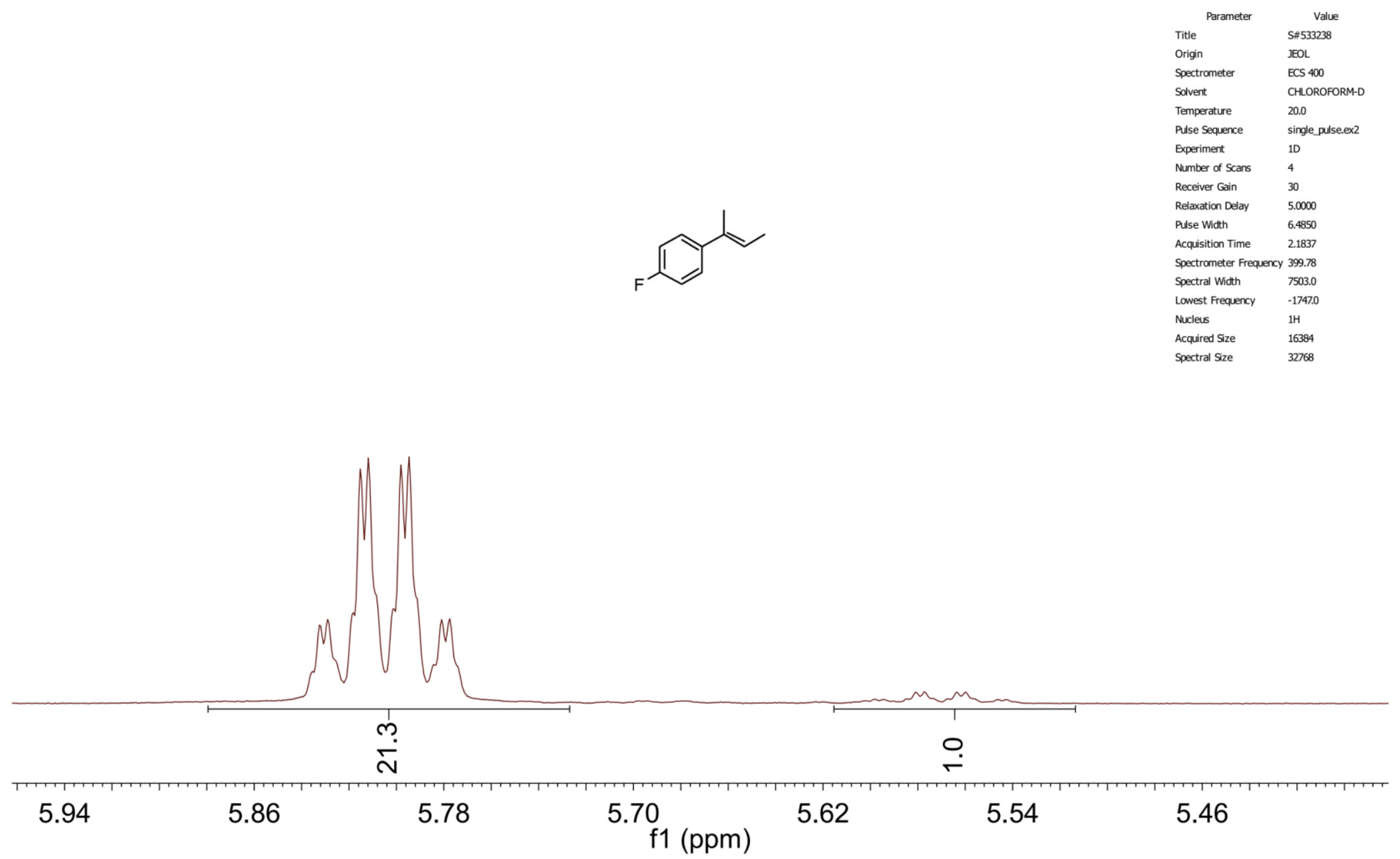
$2{ }^{2}{ }^{13} \mathrm{C}$ NMR

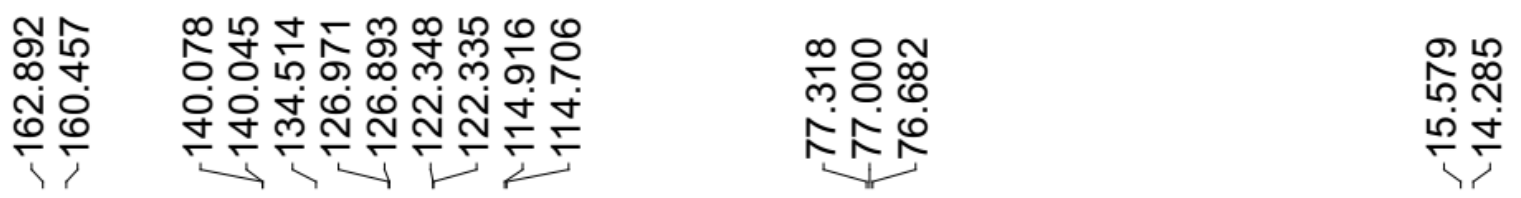

皮

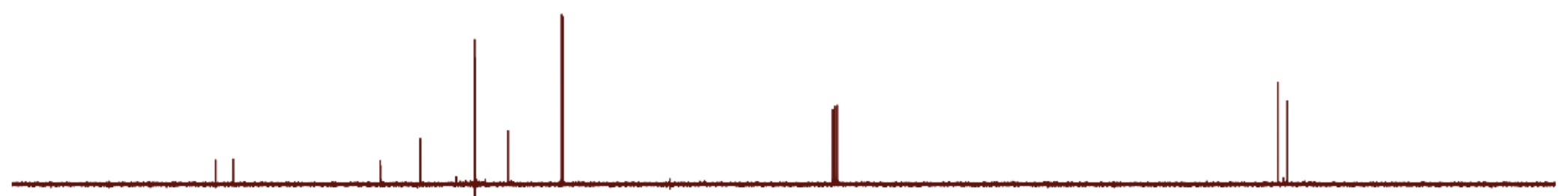

90

$170 \quad 150$

130

110

$90 \quad 80$

f1 (ppm)

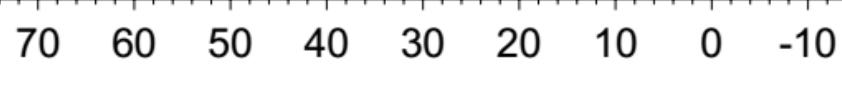



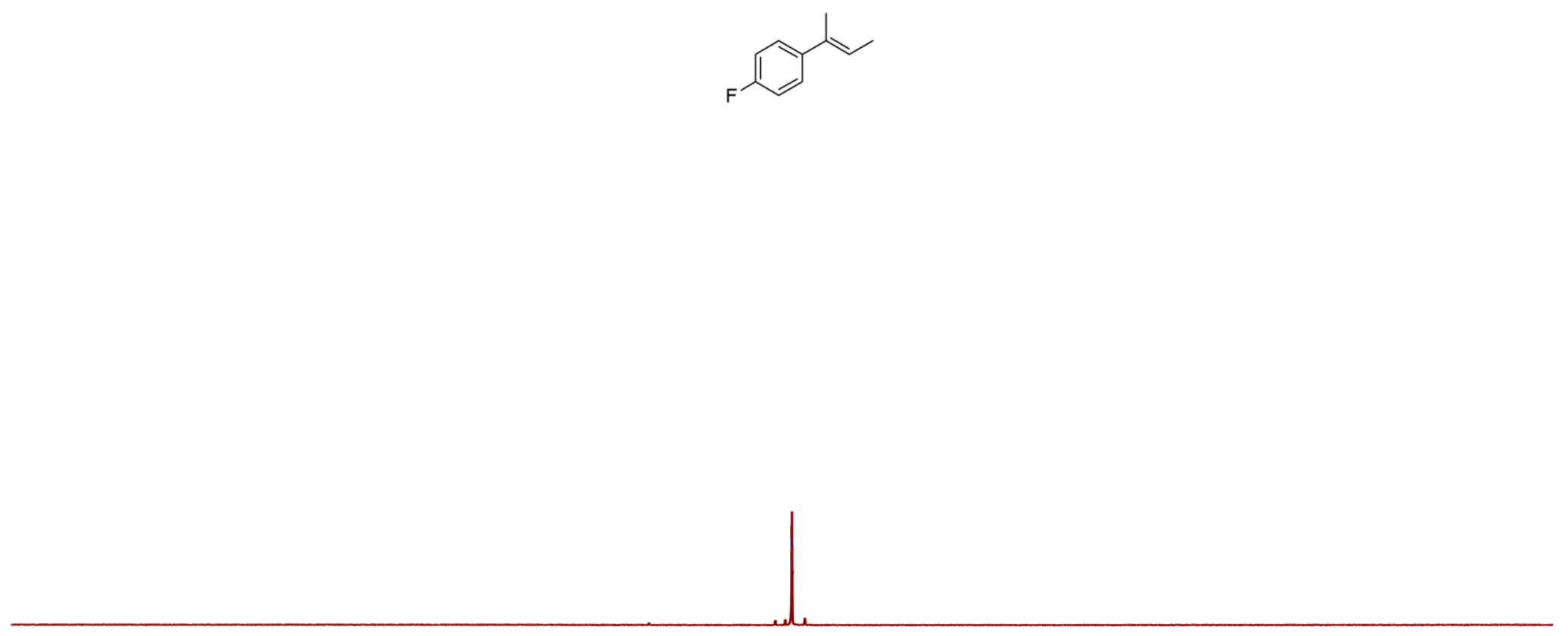

\begin{tabular}{llllllllllllllllllllllllllll}
\hline & -1 & -1 & -60 & -65 & -70 & -75 & -80 & -85 & -90 & -95 & -100 & -105 & -110 & -115 & -120 & -125 & -130 & -135 & -140 & -145 & -150 & -155 & -160 & -165 & -170 & -175 & 1
\end{tabular} 


\section{2j $E / Z{ }^{19} \mathrm{~F}$ NMR}
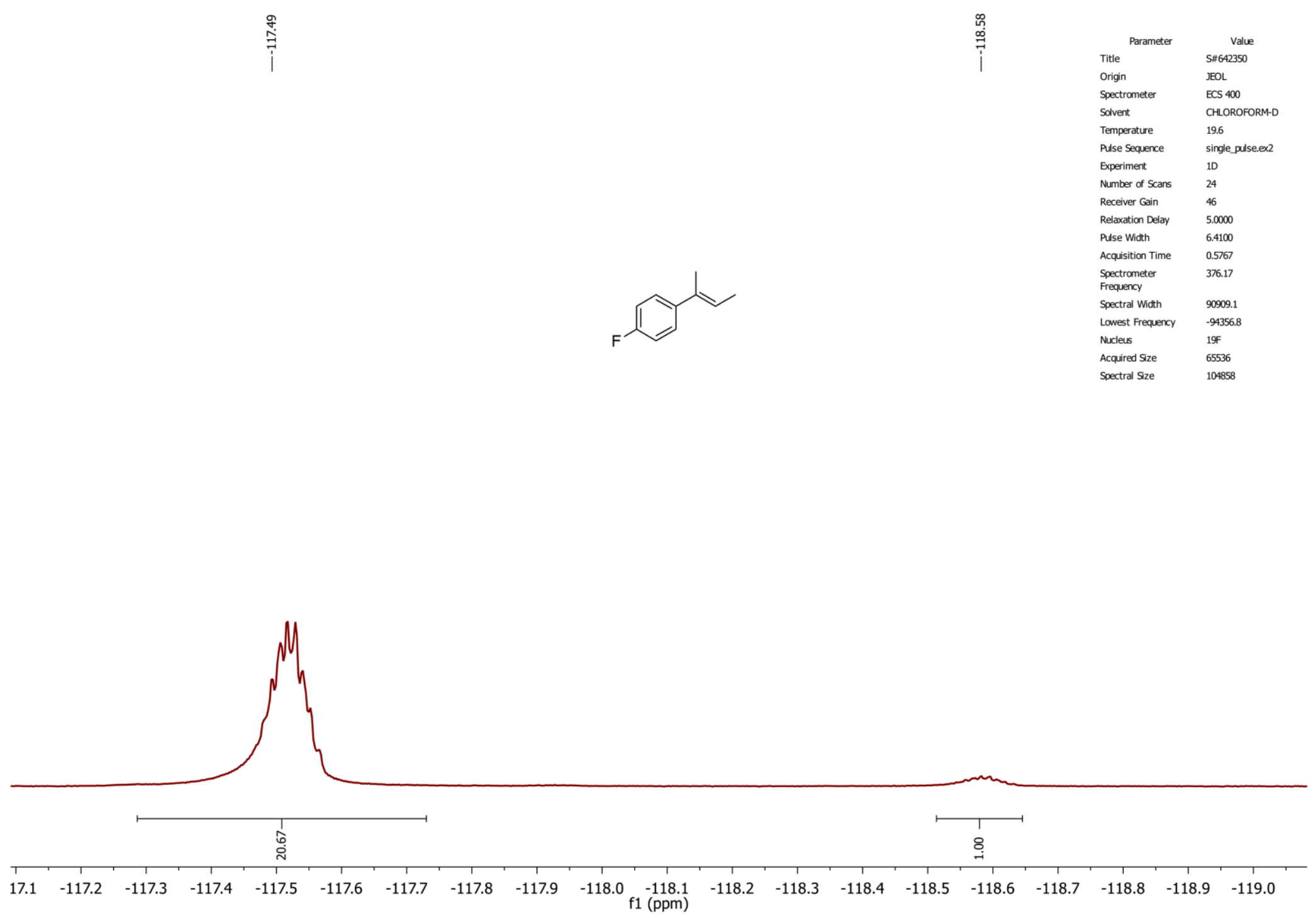


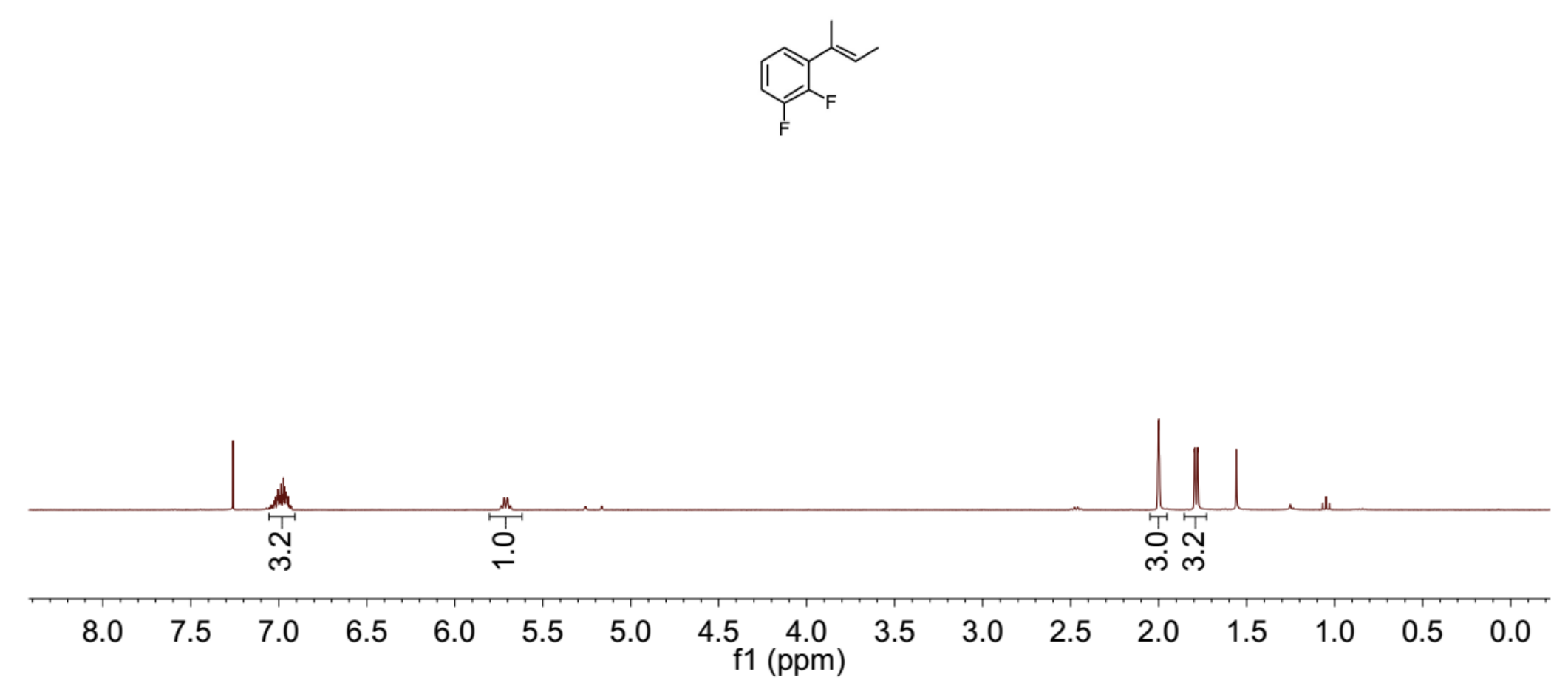


2k E/Z

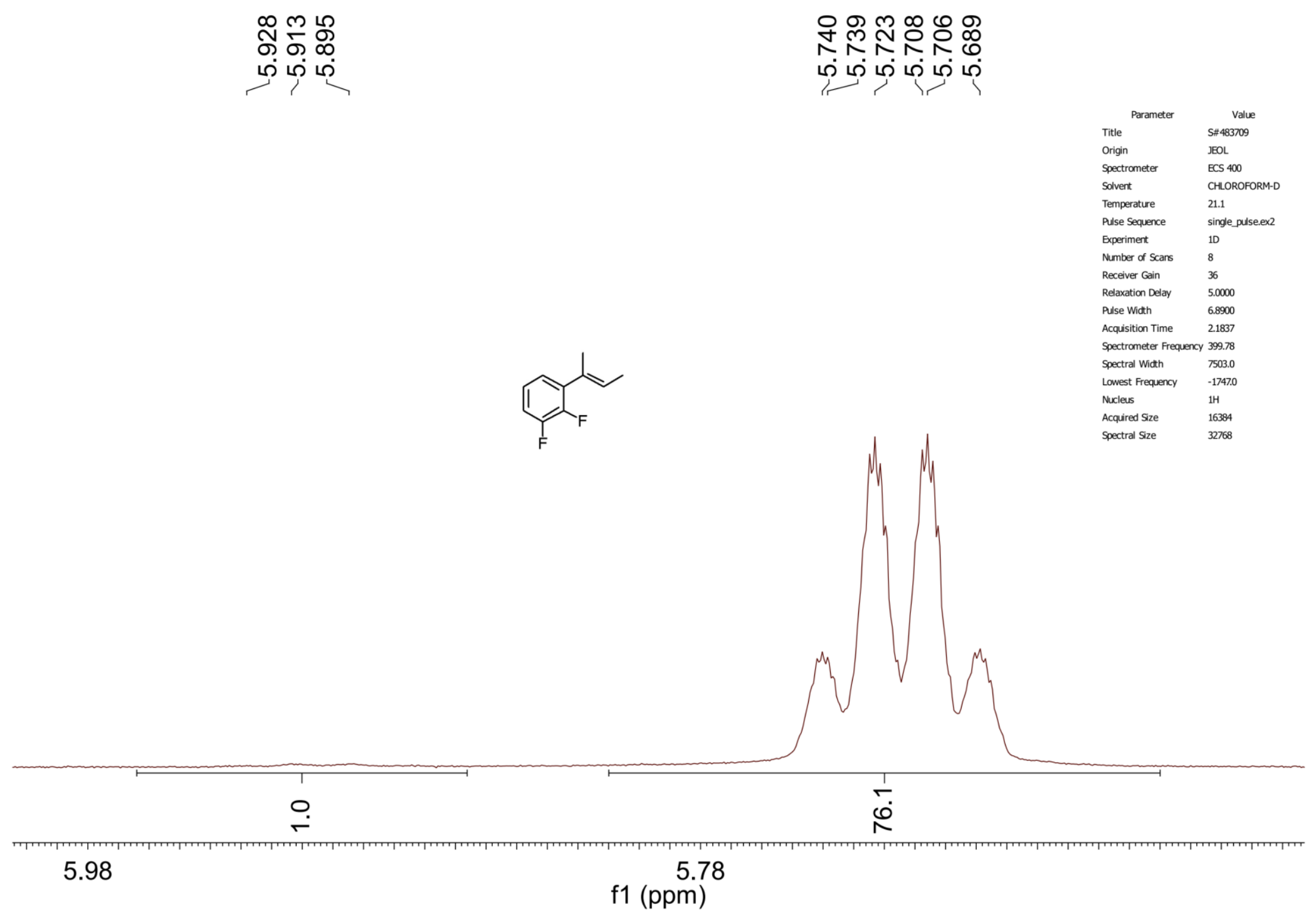




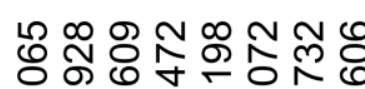

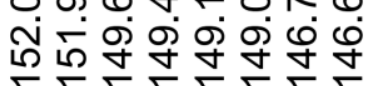

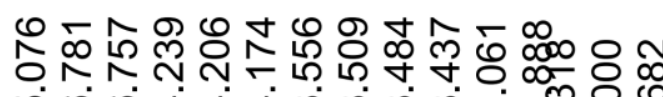

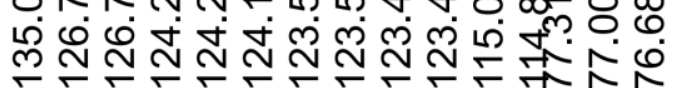

$\because-T-T-T=F$
๒ับำ

ஸ்

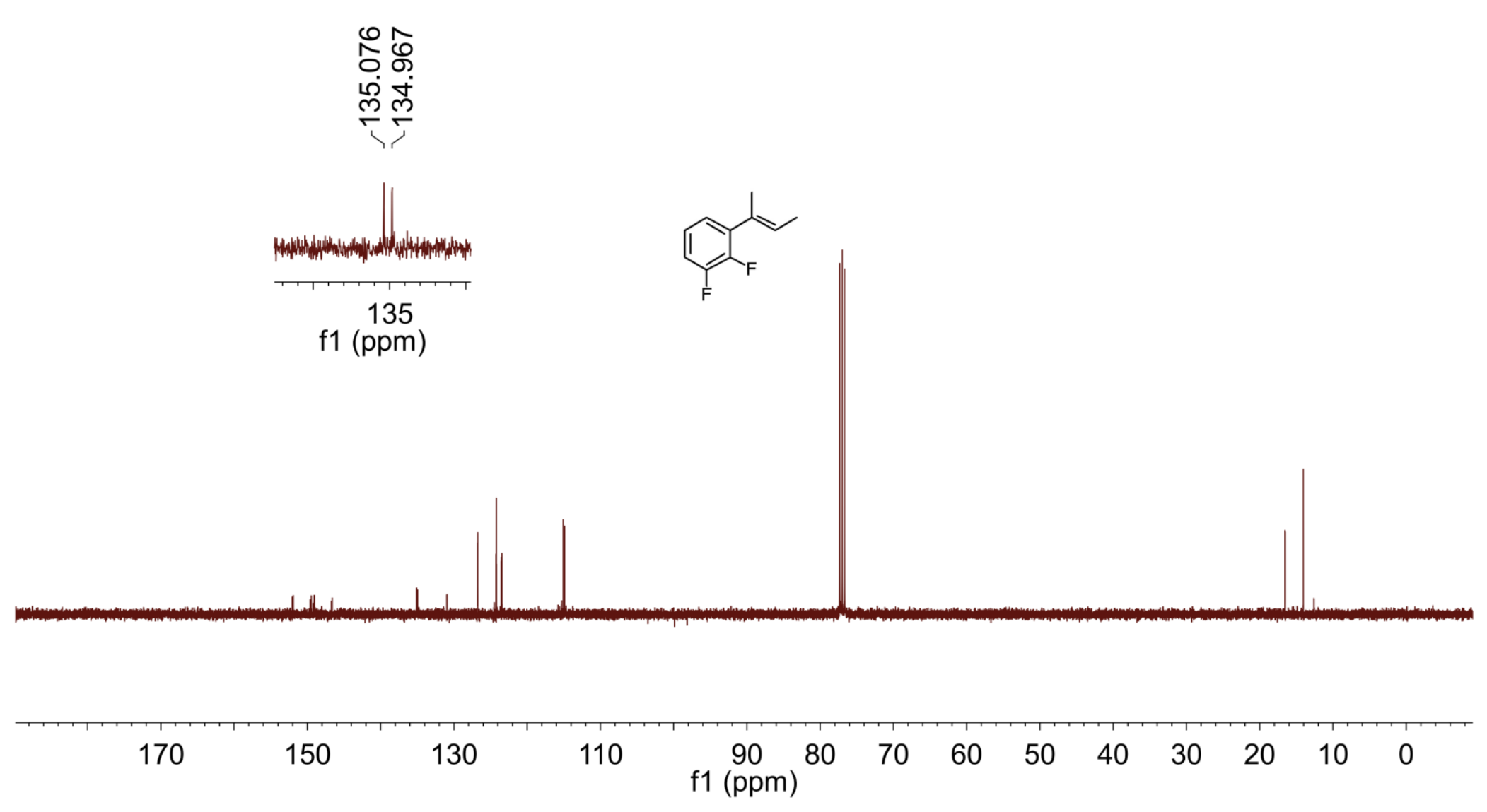


$2 \mathbf{k}^{19}$ F NMR

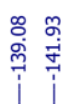
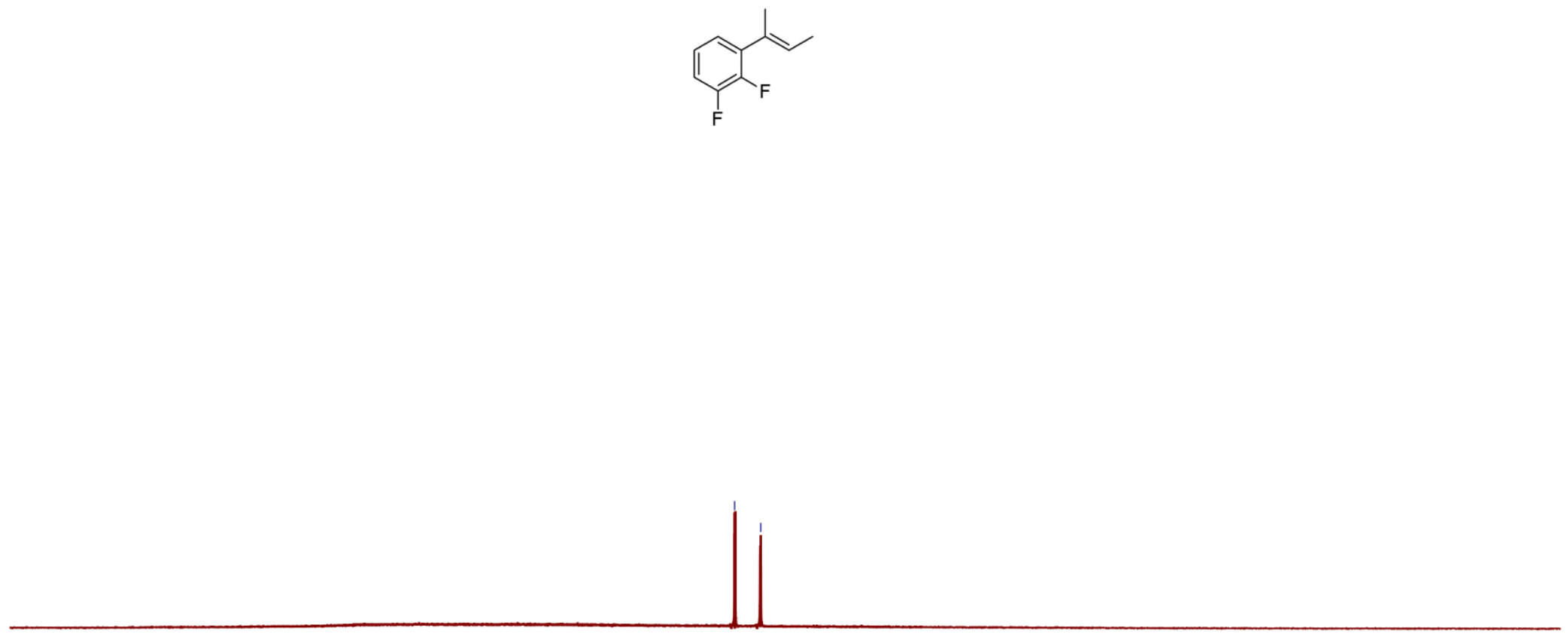

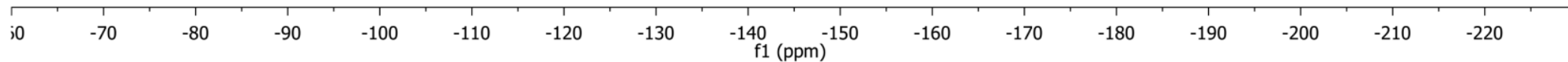




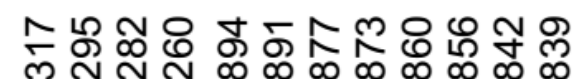

ن

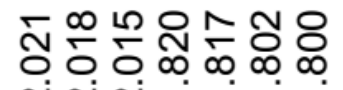

ก่ ก ก

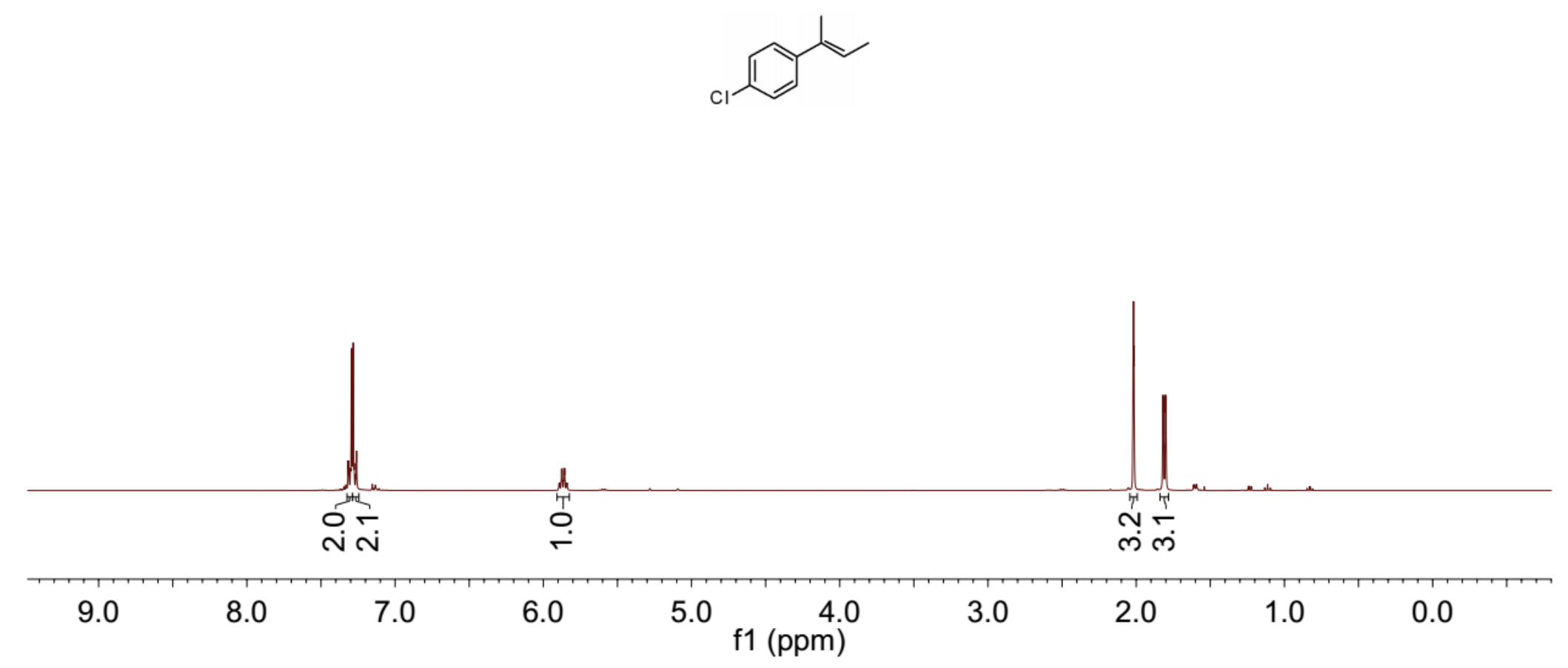


অ্口.

ما

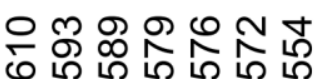

ம0

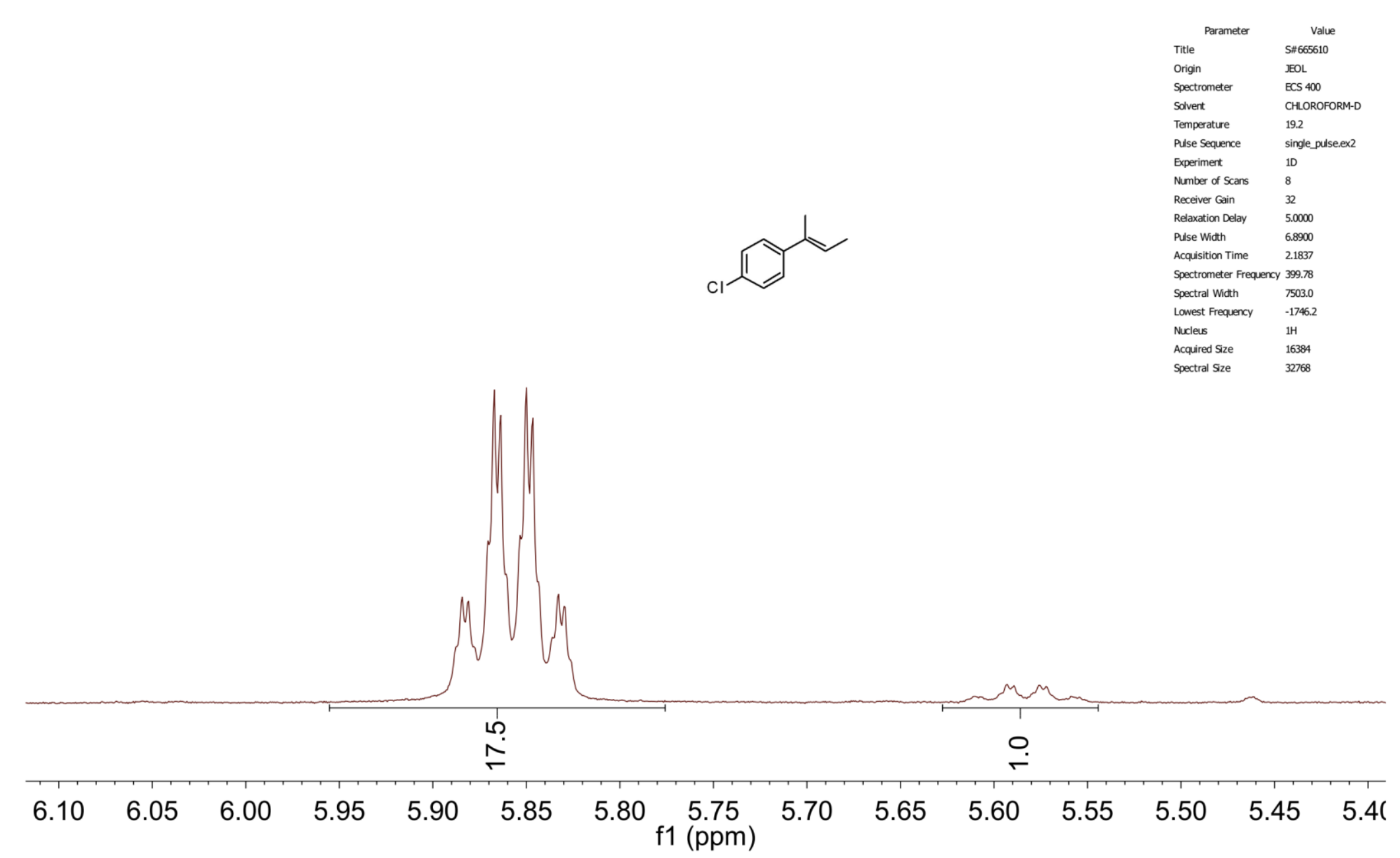


2I ${ }^{13} \mathrm{C} N M R$

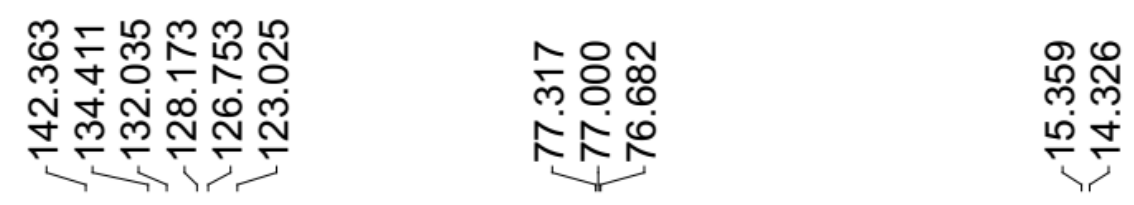

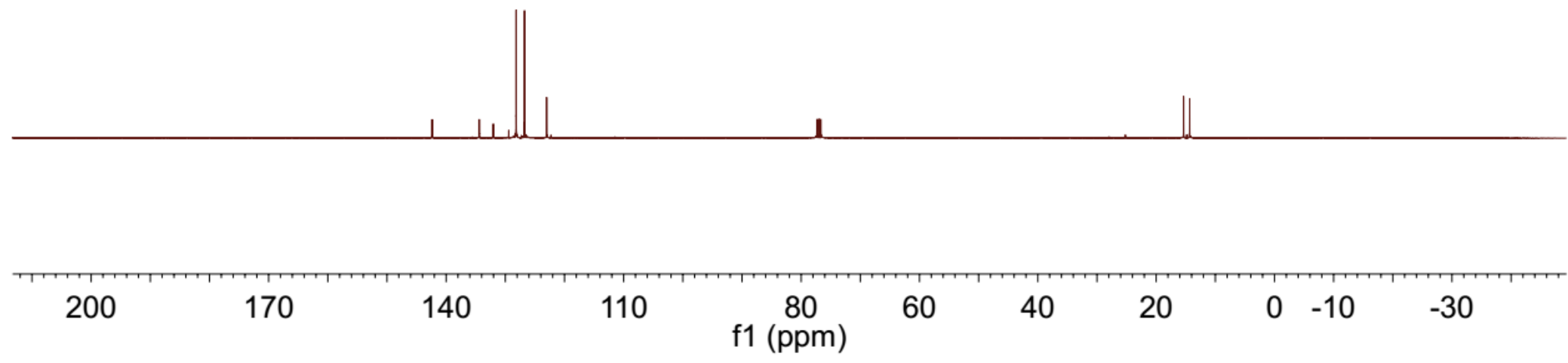




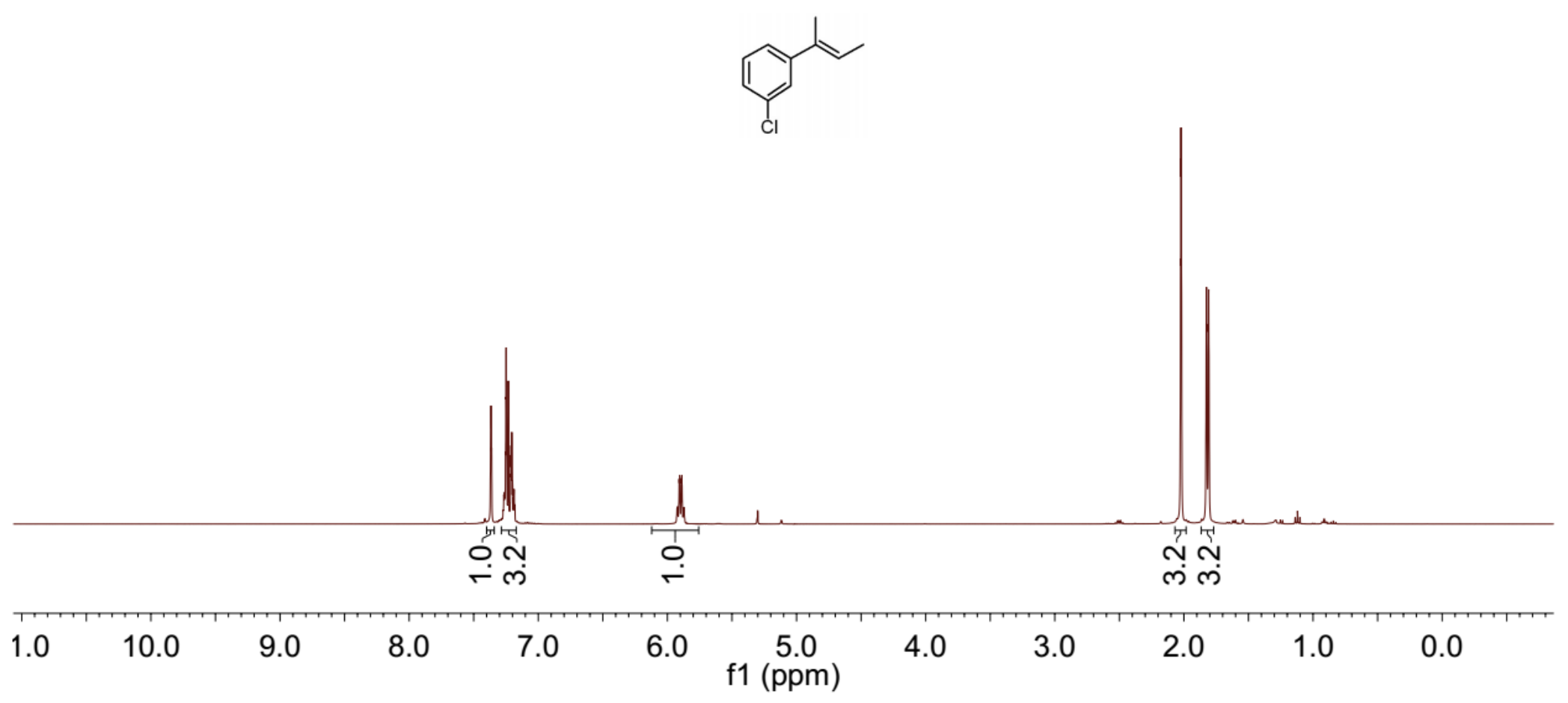


$2 \mathrm{~m} \mathrm{E} / \mathrm{Z}$

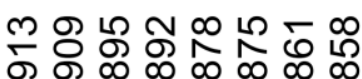

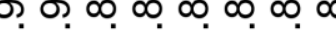

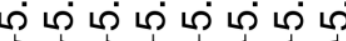

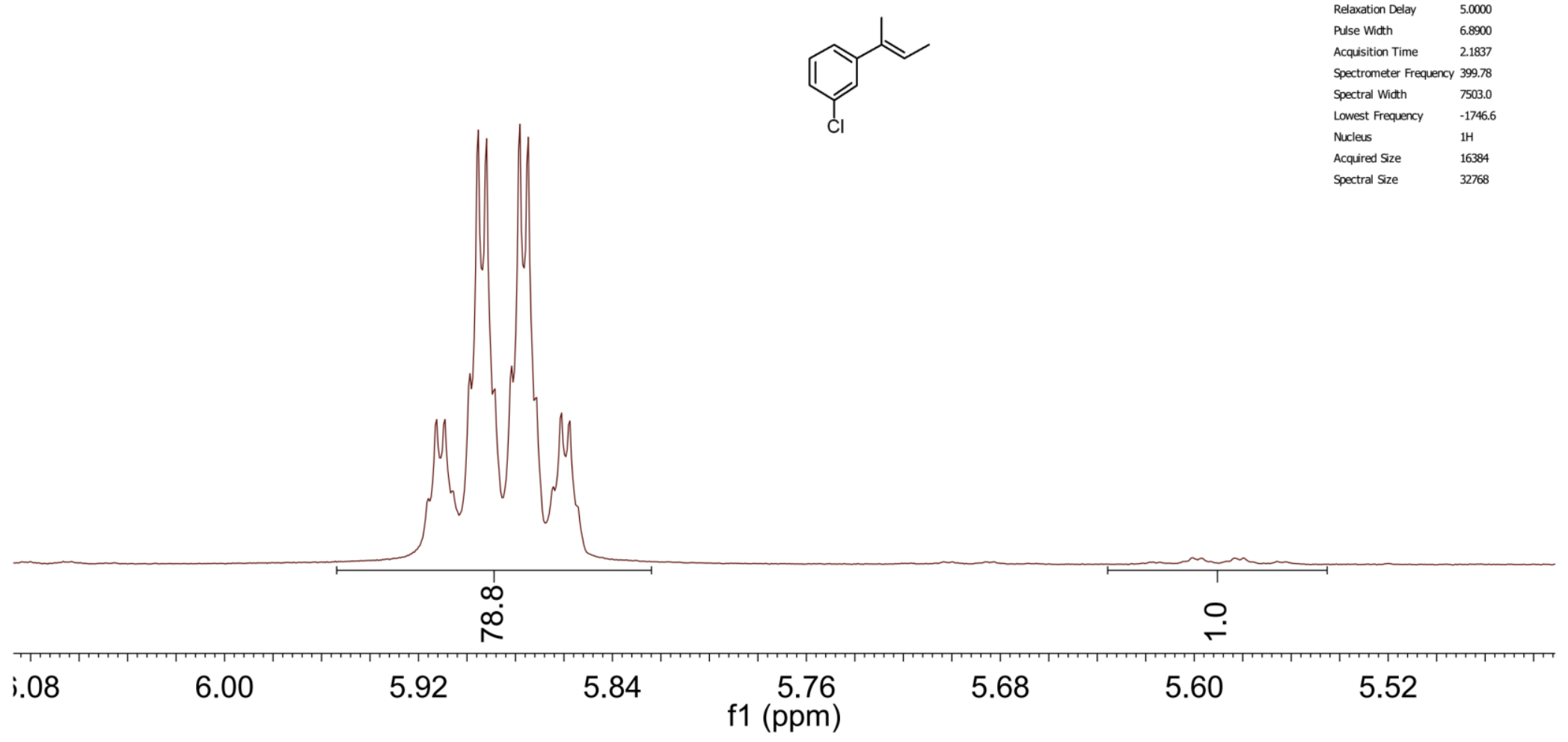

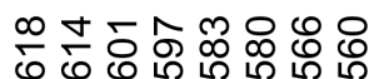

ம் ம் ம் ம் ம் ம்

$$
\begin{aligned}
& \text { Title Parameter } \begin{array}{c}
\text { Value } \\
\mathrm{S} \# 607303
\end{array} \\
& \begin{array}{lll}
\text { Origin } & \text { JEOL } \\
\text { spectrometer } & &
\end{array} \\
& \begin{array}{ll}
\text { Spectrometer } & \text { ECS } 400 \\
\text { Solvent } & \text { CHLOROFORM-D }
\end{array} \\
& \text { Temperature } 21.0 \\
& \text { Puise Sequence single.pusse.ex } \\
& \text { Experiment } 10 \\
& \text { Number of Scans } 8 \\
& \text { Relaxation Delay } \quad 5.0000 \\
& \text { Acquisition Time } \quad 21837 \\
& \text { Spectrometer Frequency } 399.78
\end{aligned}
$$




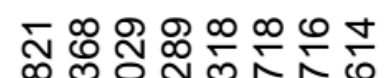

(1)

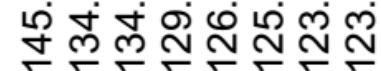

mon

Nरํ

సิำ ్ㅠ

官

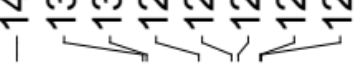

怩

i?
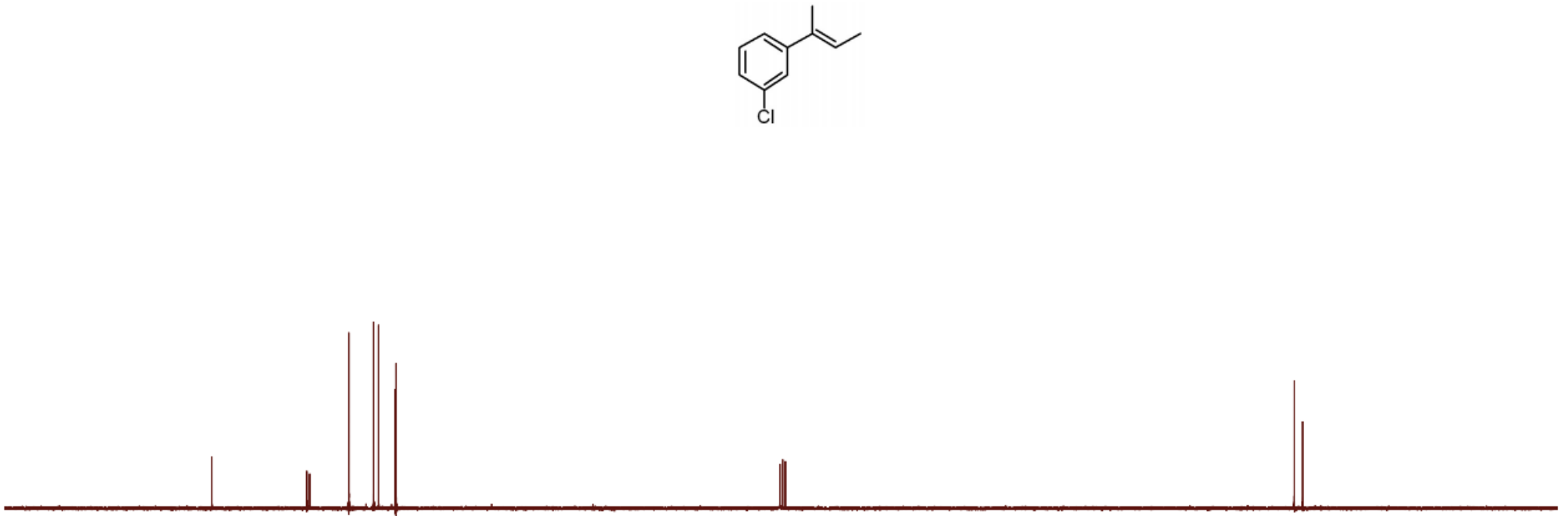

$\begin{array}{llllllllllllll}80 & 150 & 130 & 110 & 90 & \begin{array}{c}80 \\ \mathrm{f} 1(\mathrm{ppm})\end{array} & 60 & 50 & 40 & 30 & 20 & 10 & 0 & -10\end{array}$




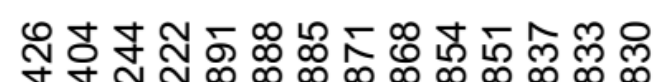

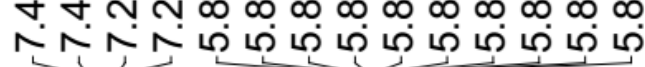

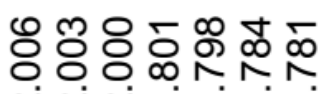 \\ ninirivi}

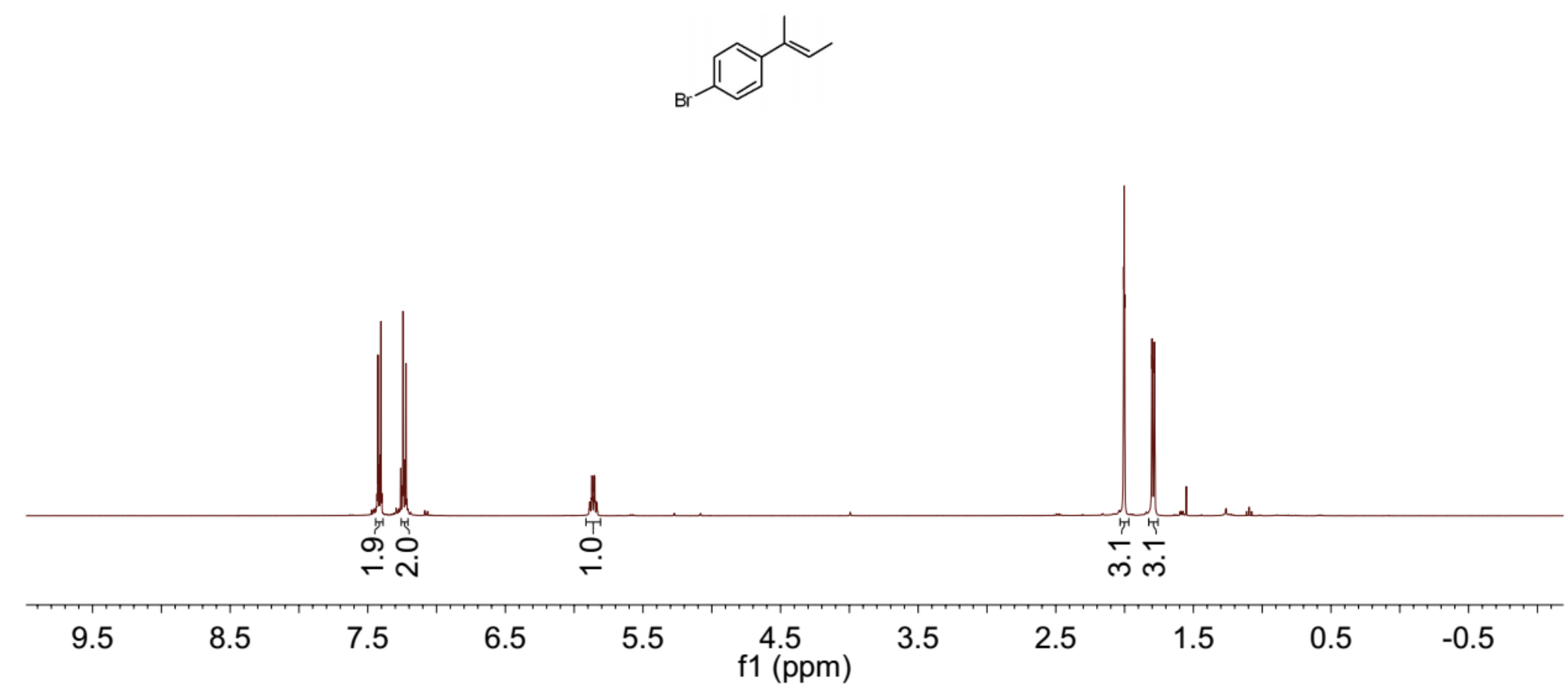




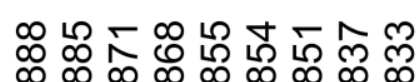

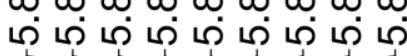

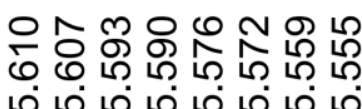

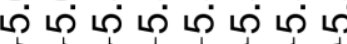

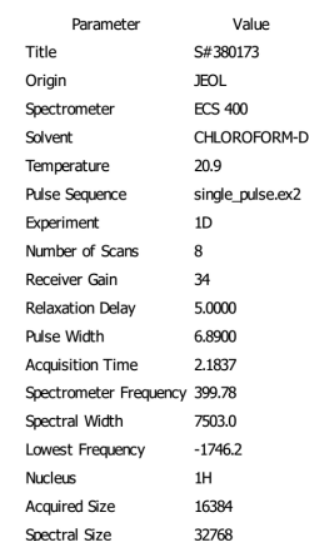

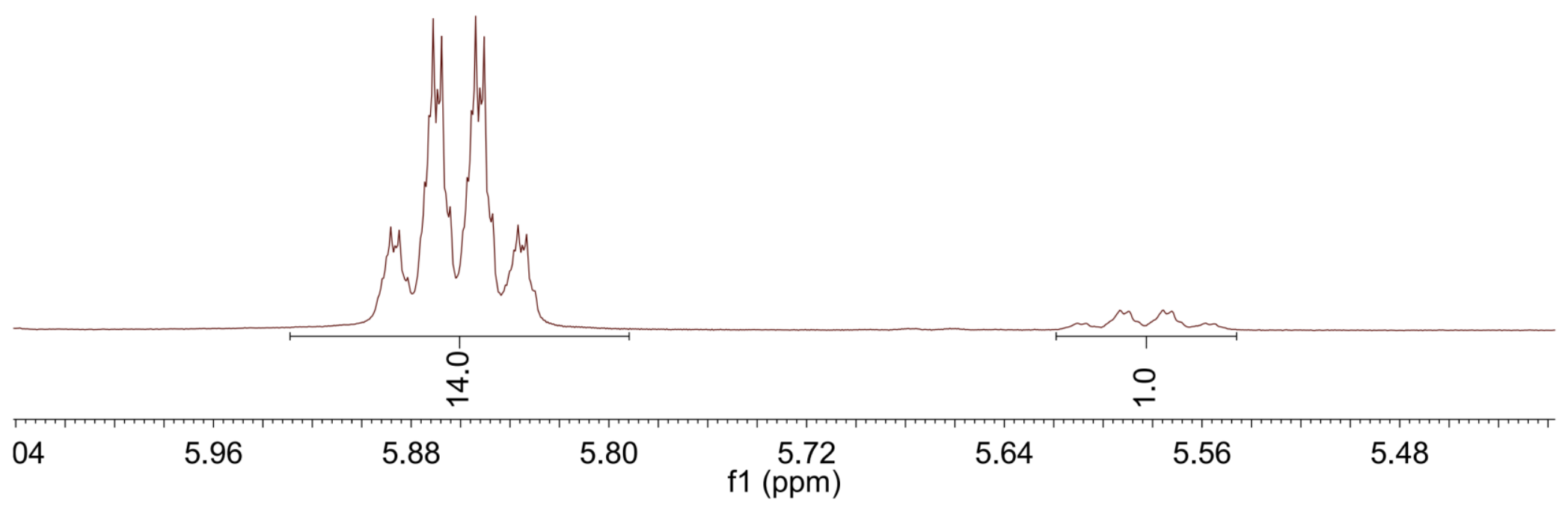




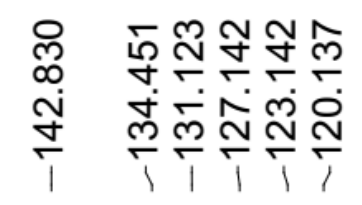

ल요

송

율

மำ
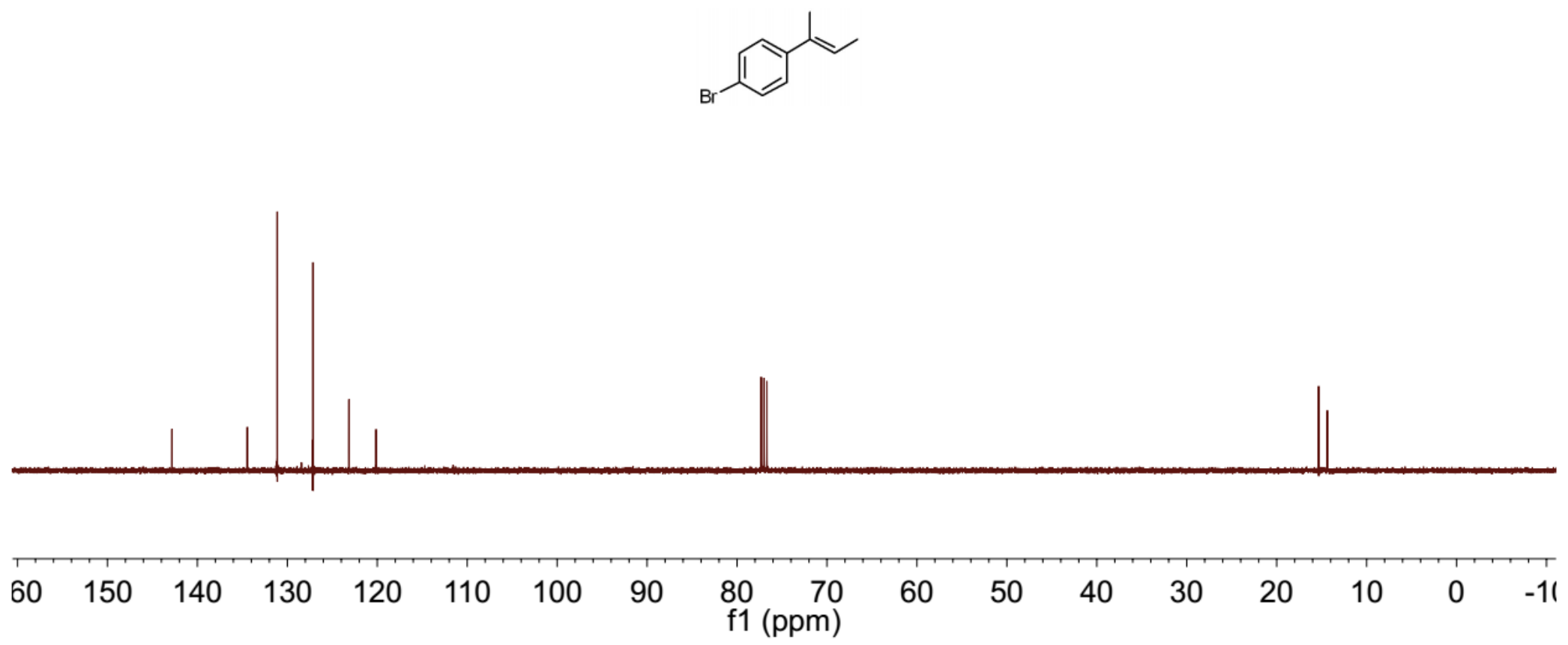


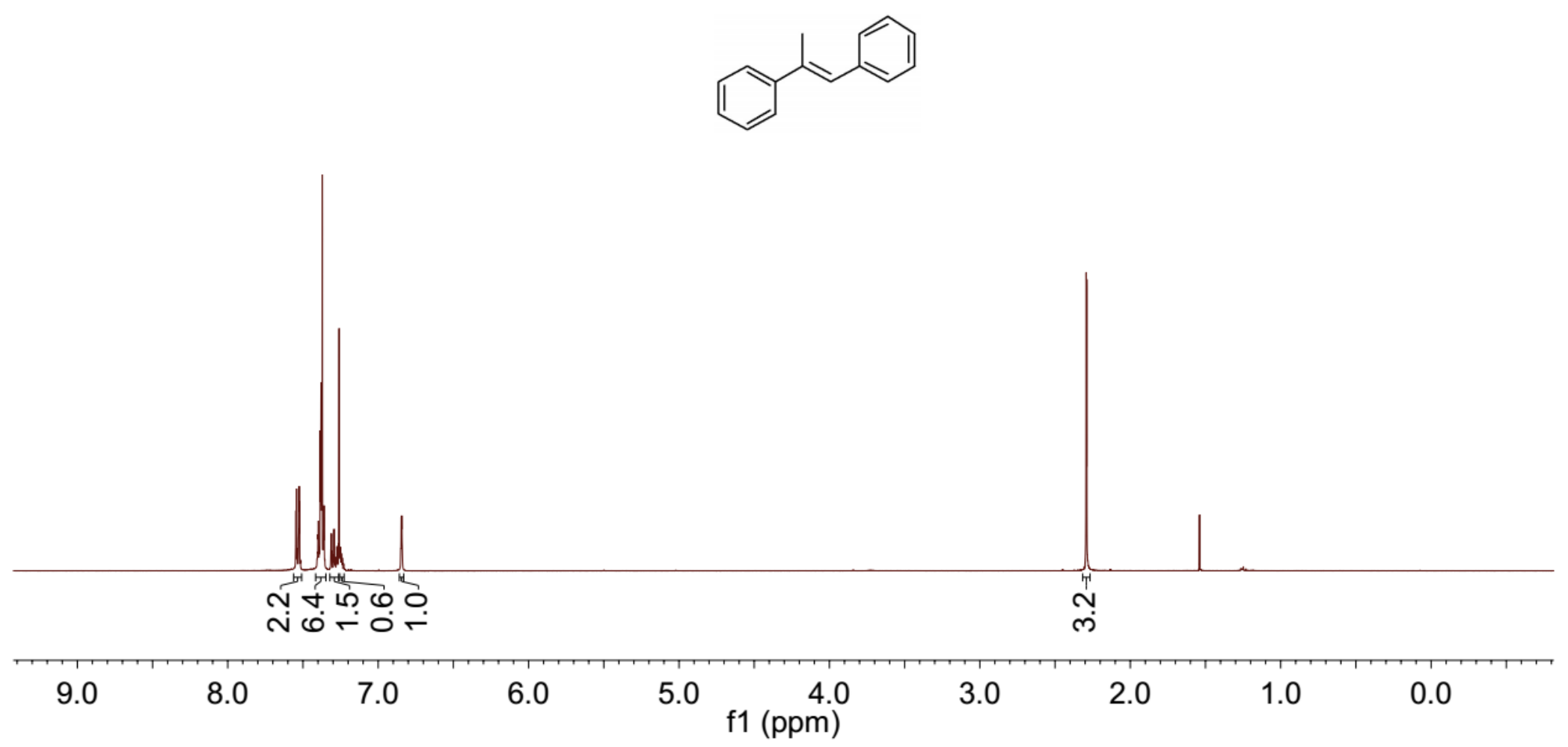


2o $E / Z$

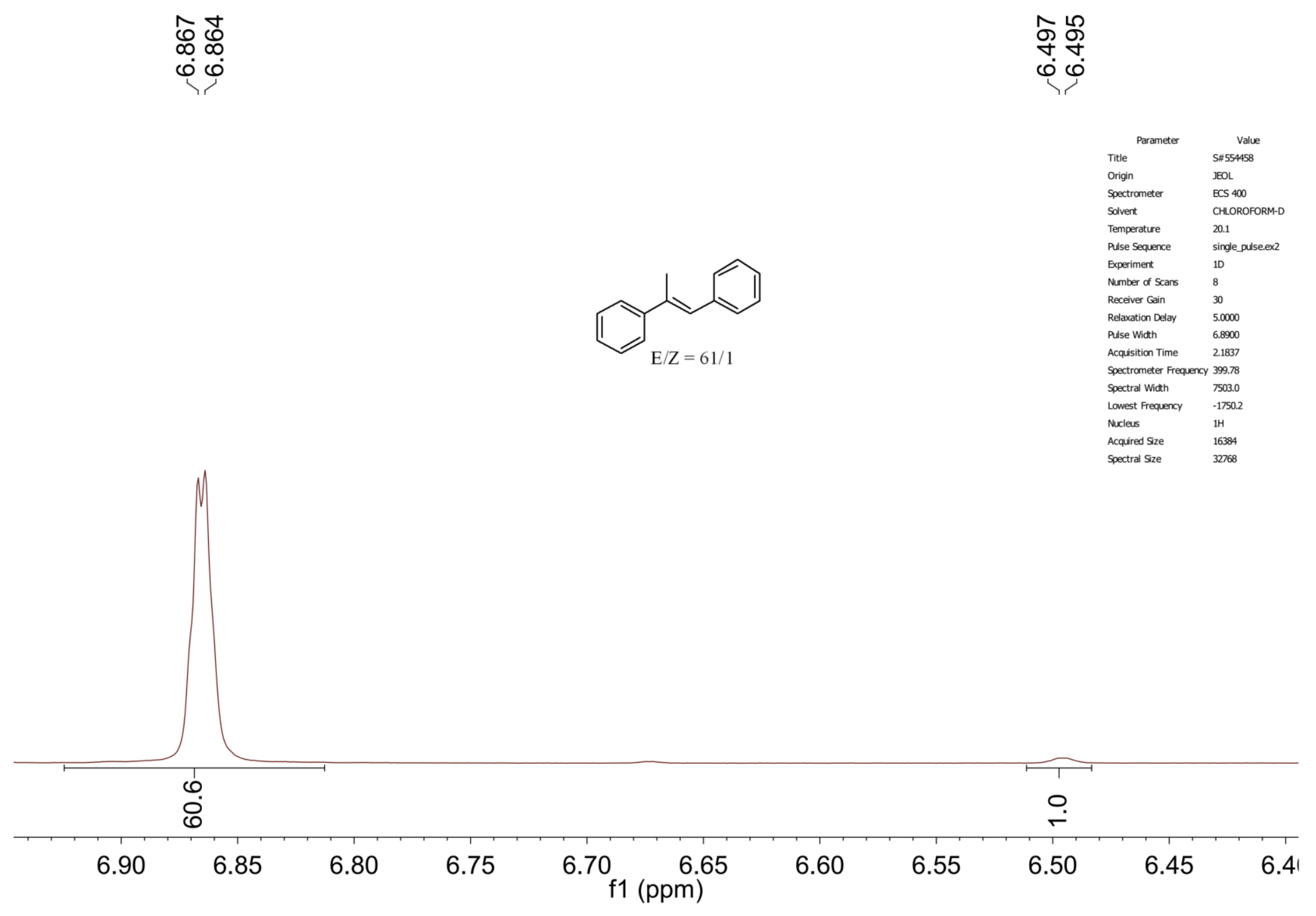



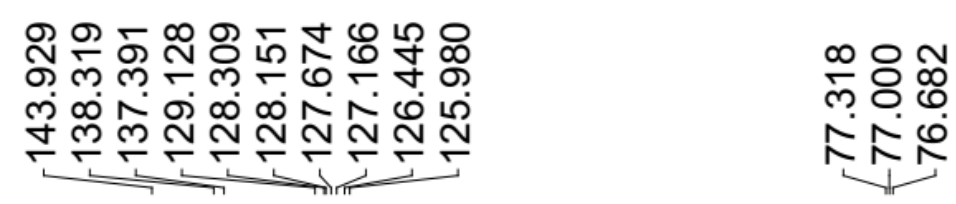

$\frac{\text { 웅 }}{\text { ำ }}$

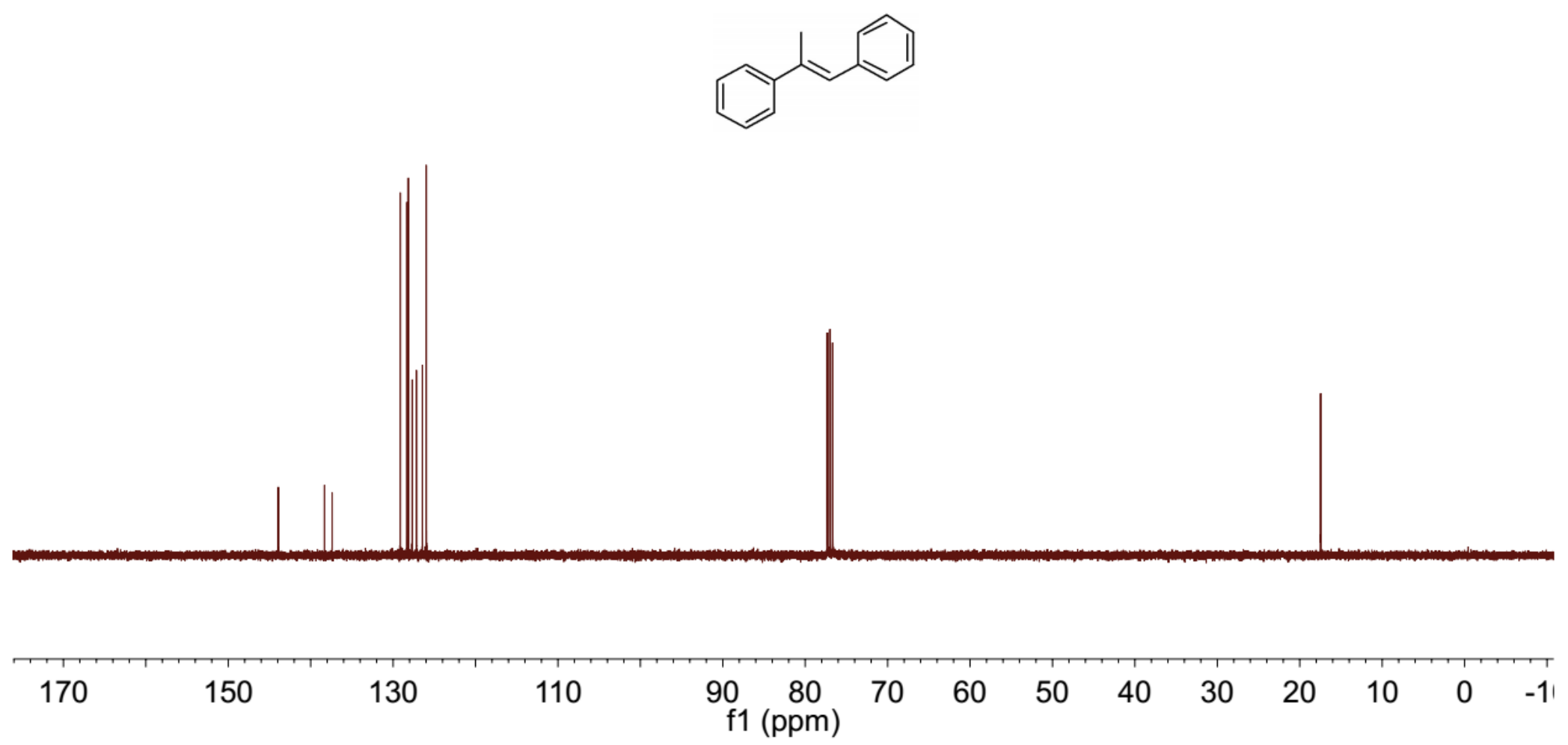



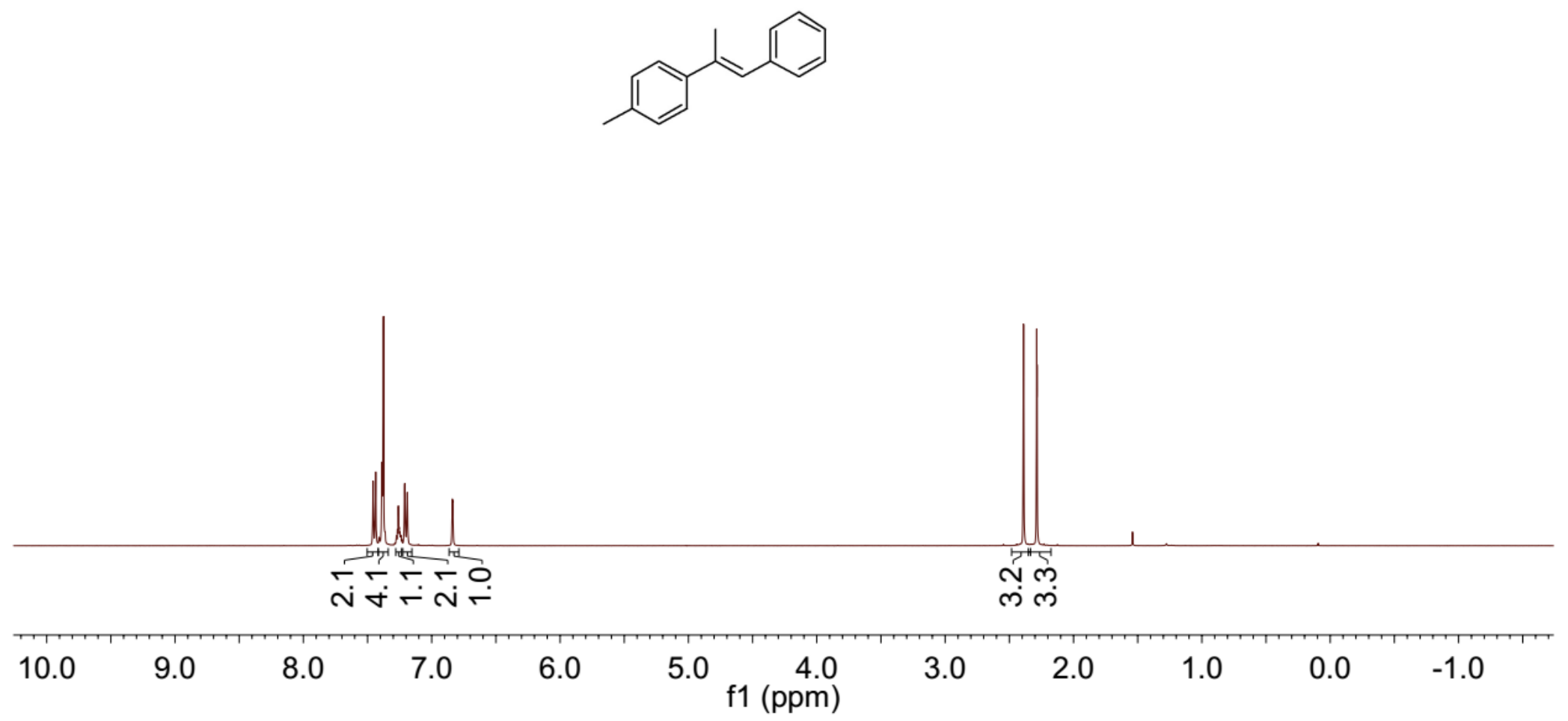
$2 p E / Z$

₹

$\varphi$

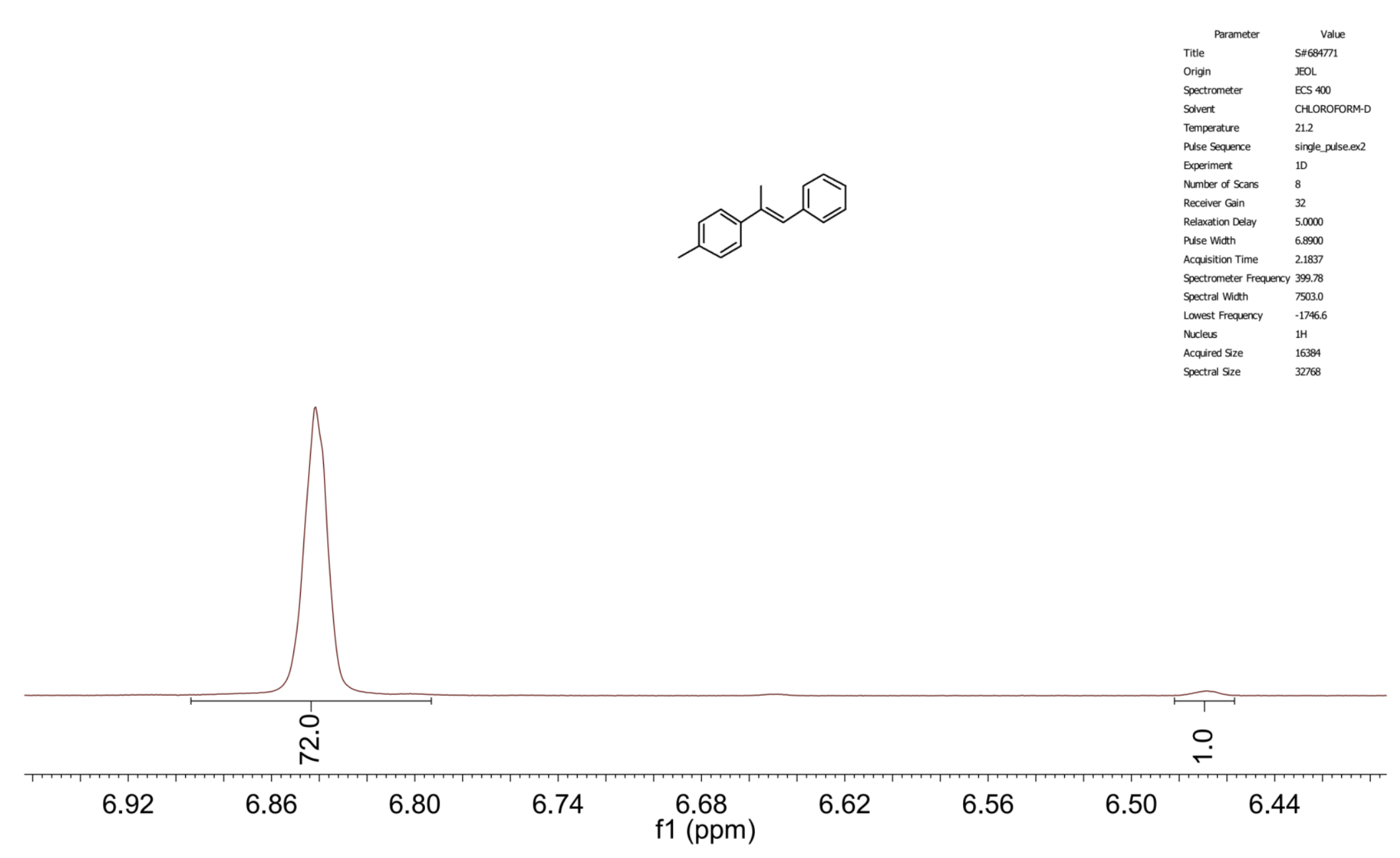




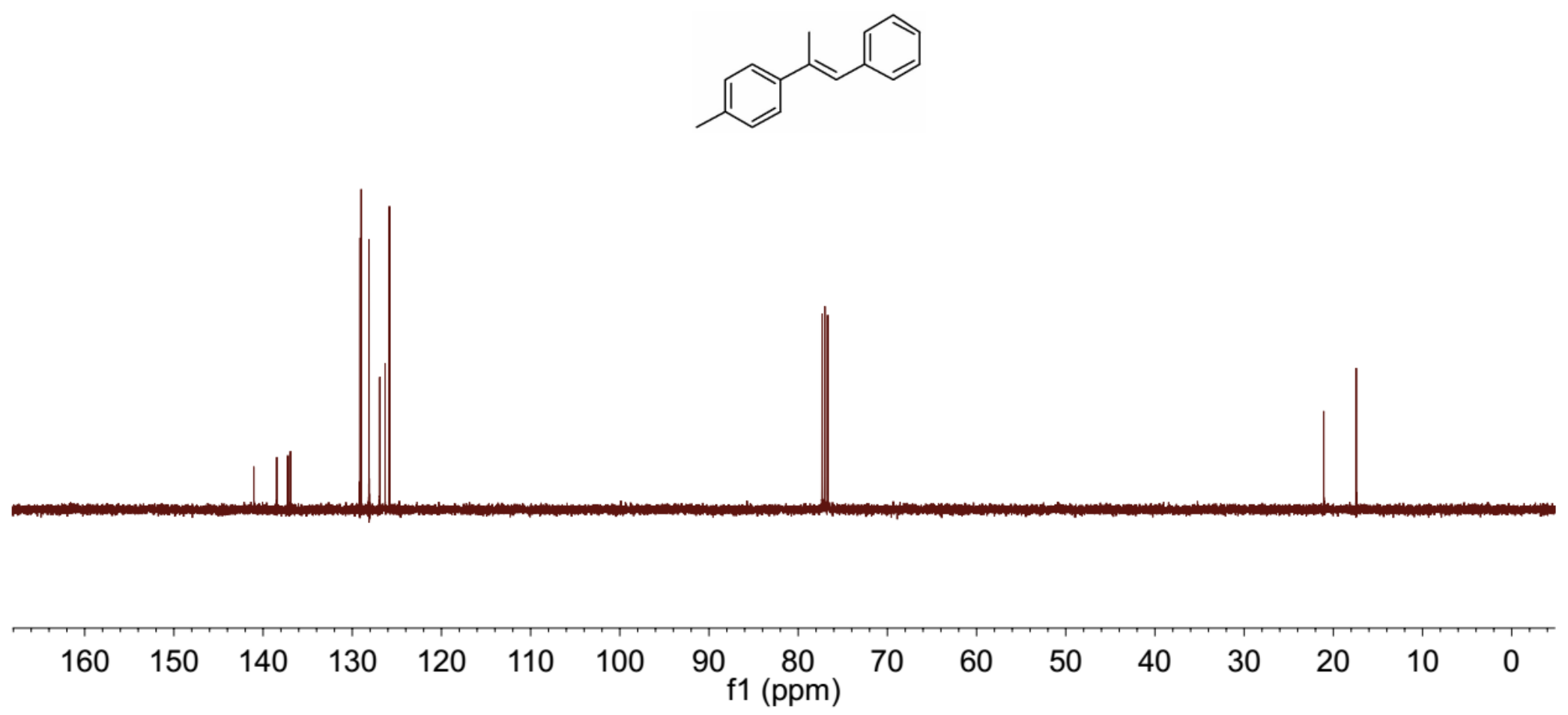


$2 q^{1} H_{N M R}$

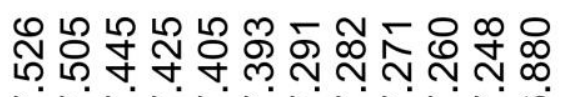

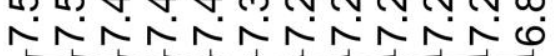

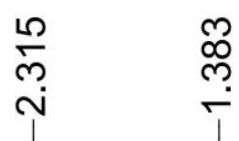
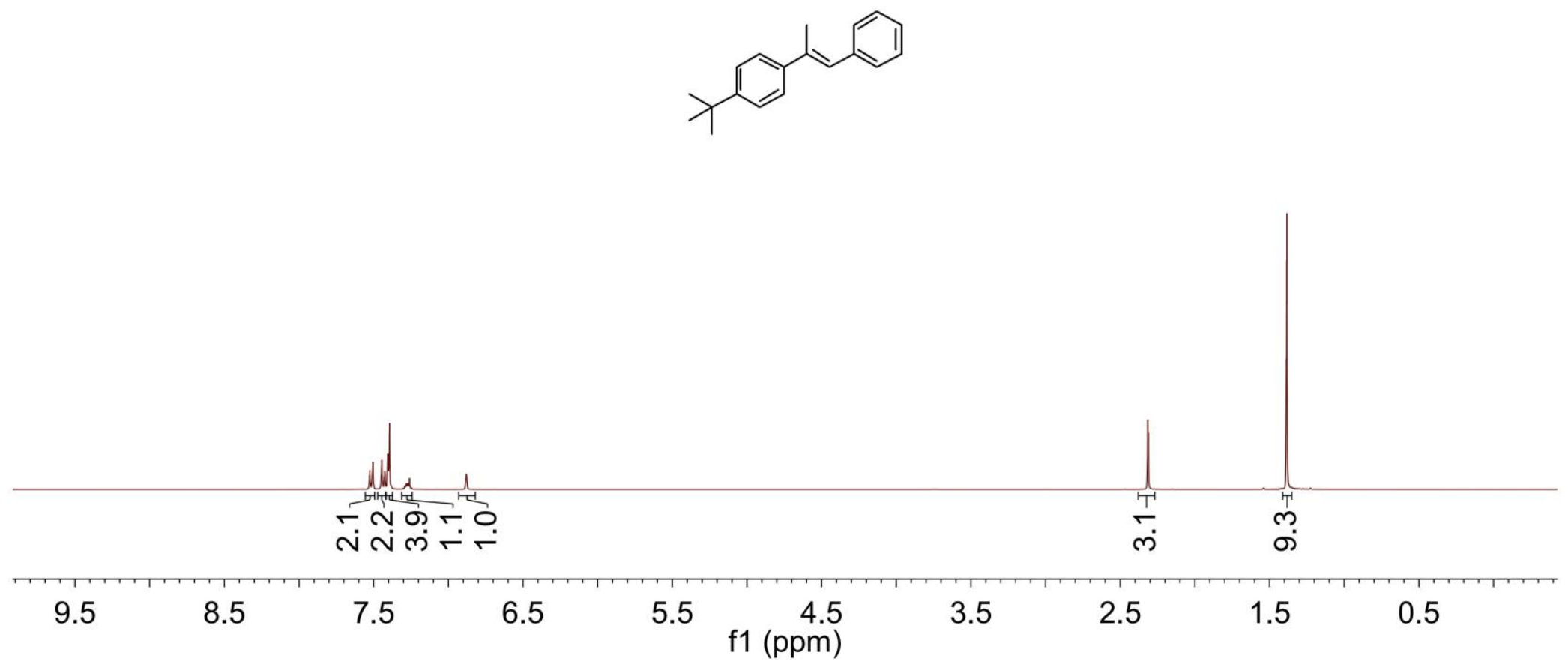
$2 q E / Z$

年㠻

फं

苞

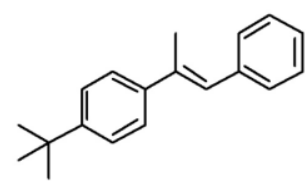

Title $\begin{array}{r}\text { Parameter Value } \\ \text { S\#685607 }\end{array}$

Origin JEOL

ECS 400

Solvert

Puse Sequence single puse

$\begin{array}{ll}\text { Experiment } & 10 \\ \text { Number of Scans } & 8\end{array}$

Receiver Gain 38

Relaxation Delay 5.0000

$\begin{array}{ll} & 6.4550 \\ \text { Pulse Woth } & 21837\end{array}$

spectrometer Frequency 399.78

spectral Width 7503.0

Lowest Frequency $\quad-1747.0$

Acquired Size $\quad{ }_{16384}$

Spectral Size $\quad 32768$

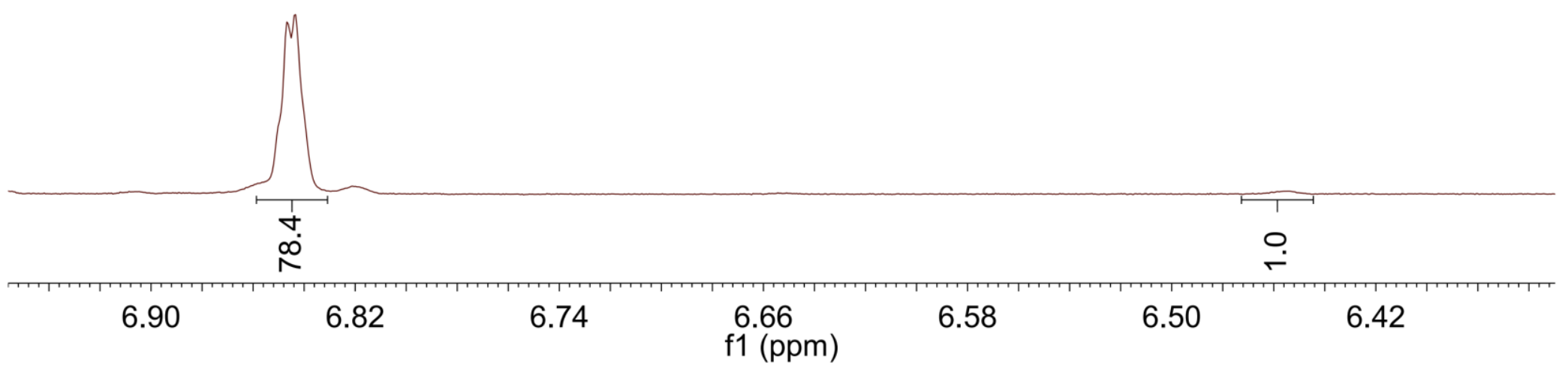




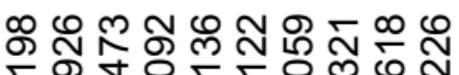

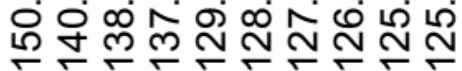

ㄷํ유.

㪘

m్ֻలి స్ల్ల

में न

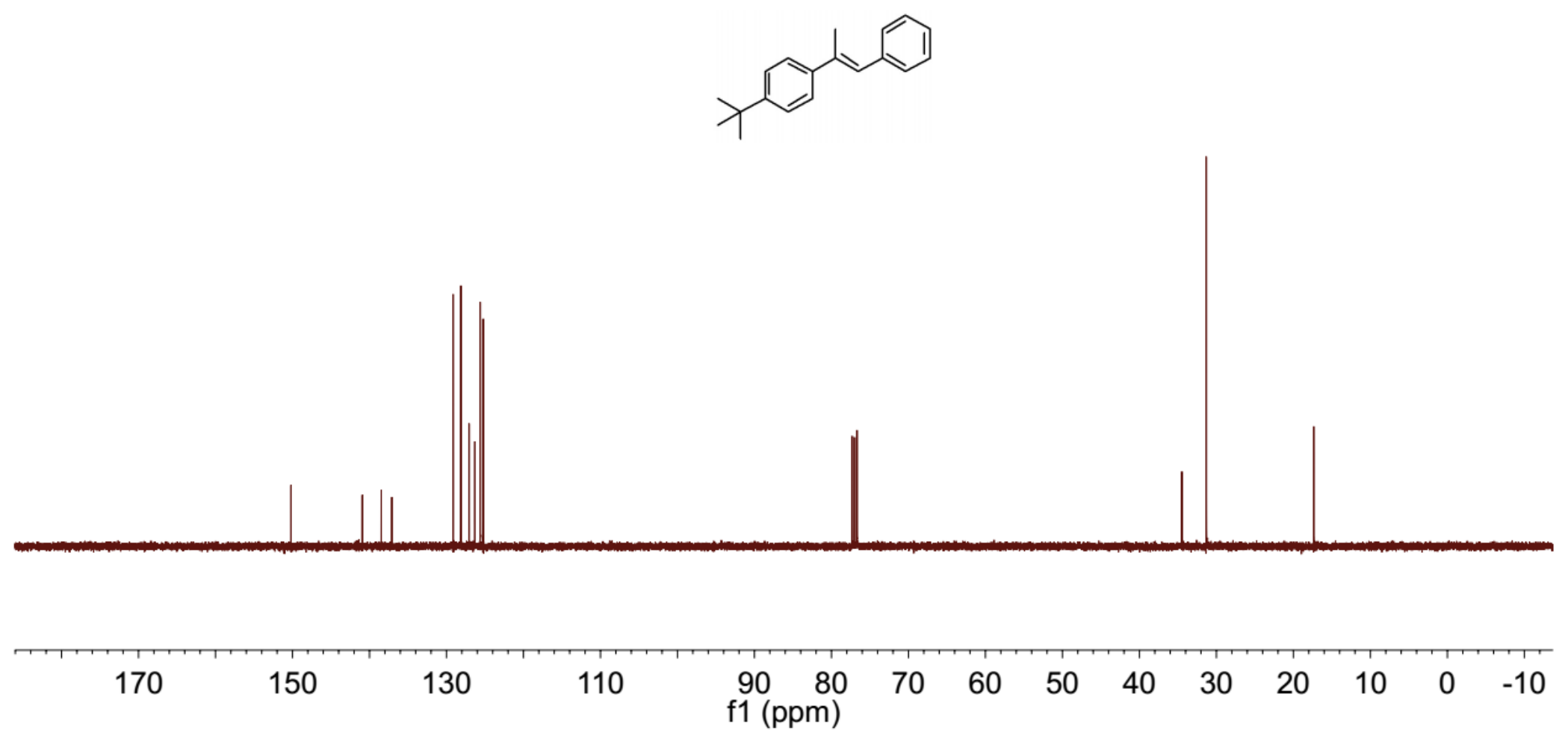




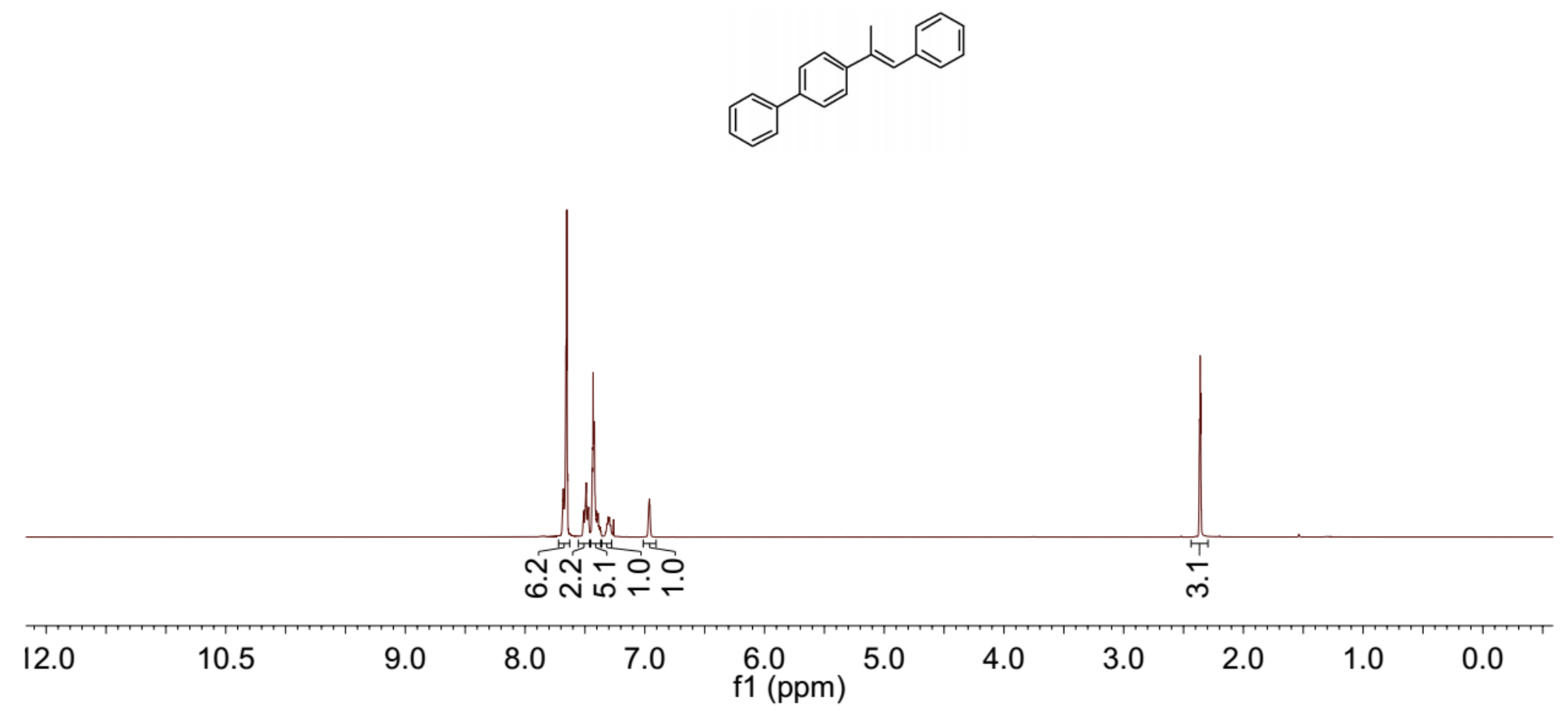


2r E/Z

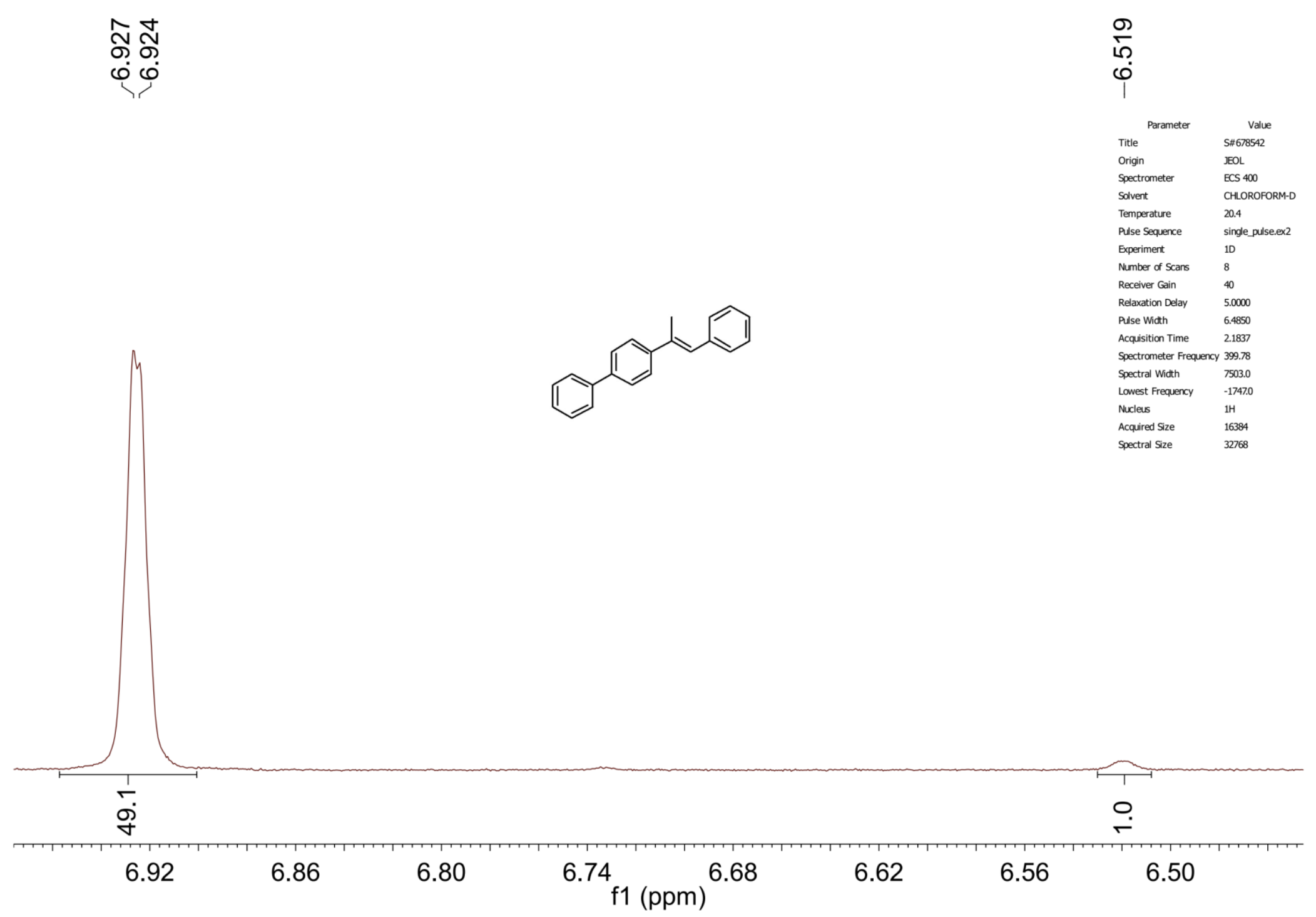



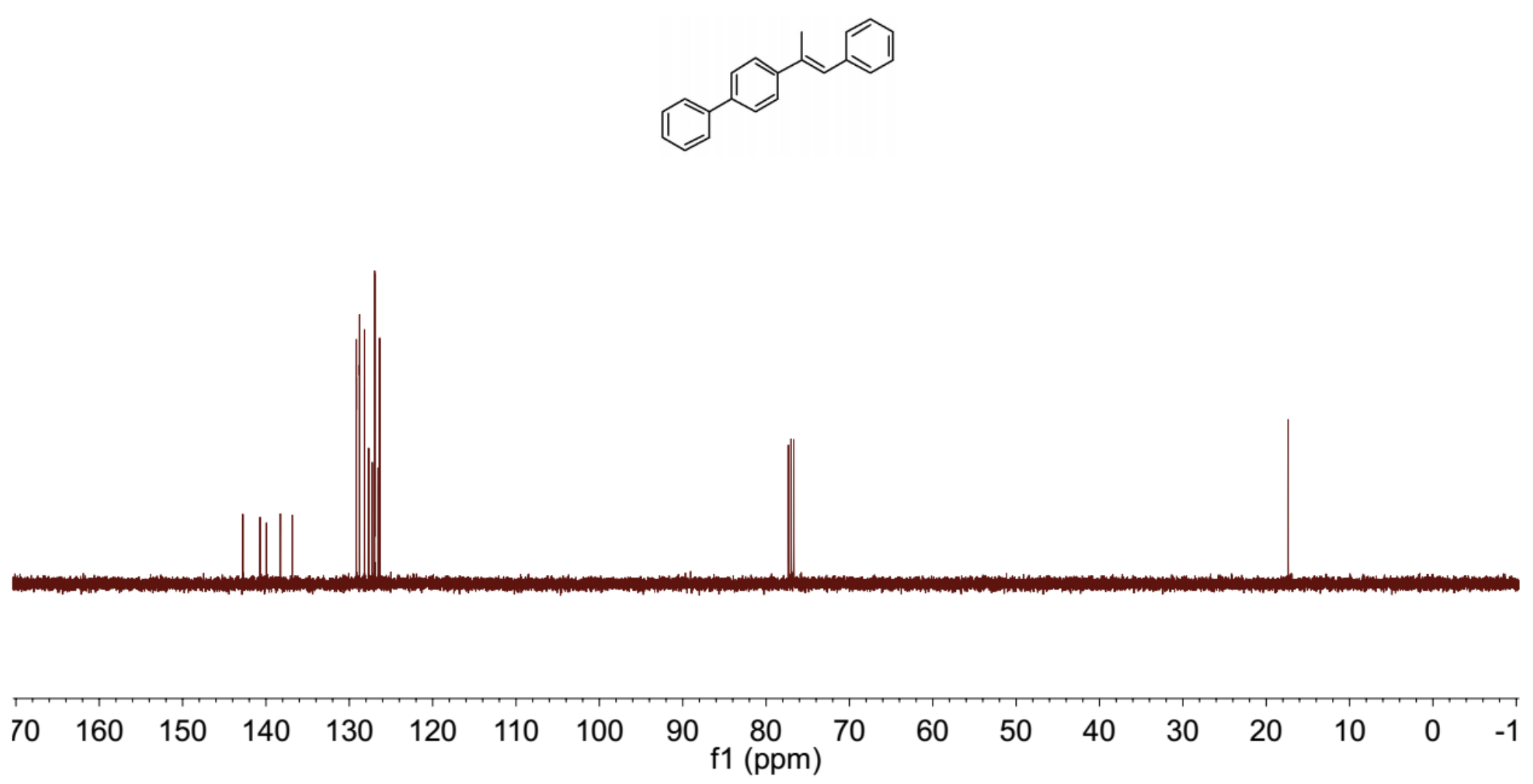


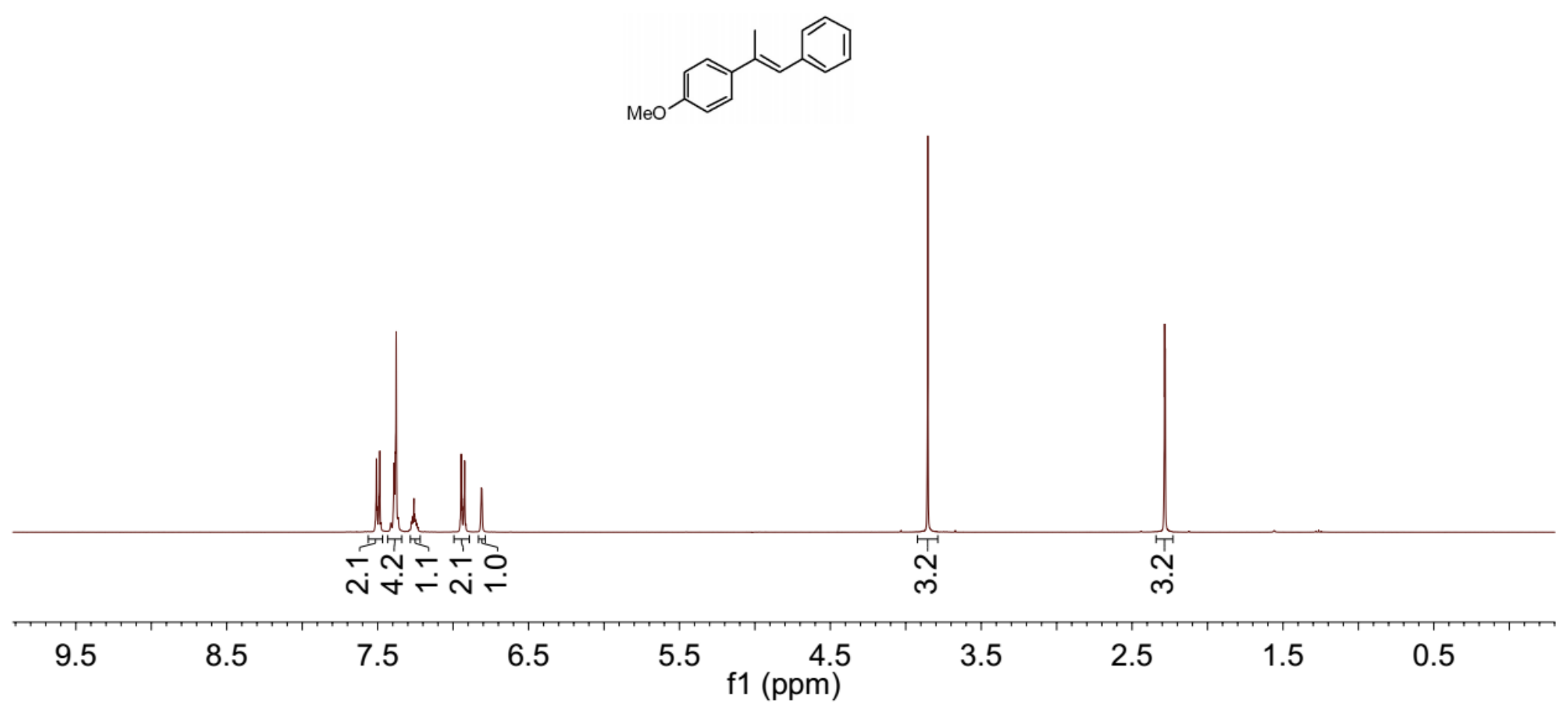


2s E/Z

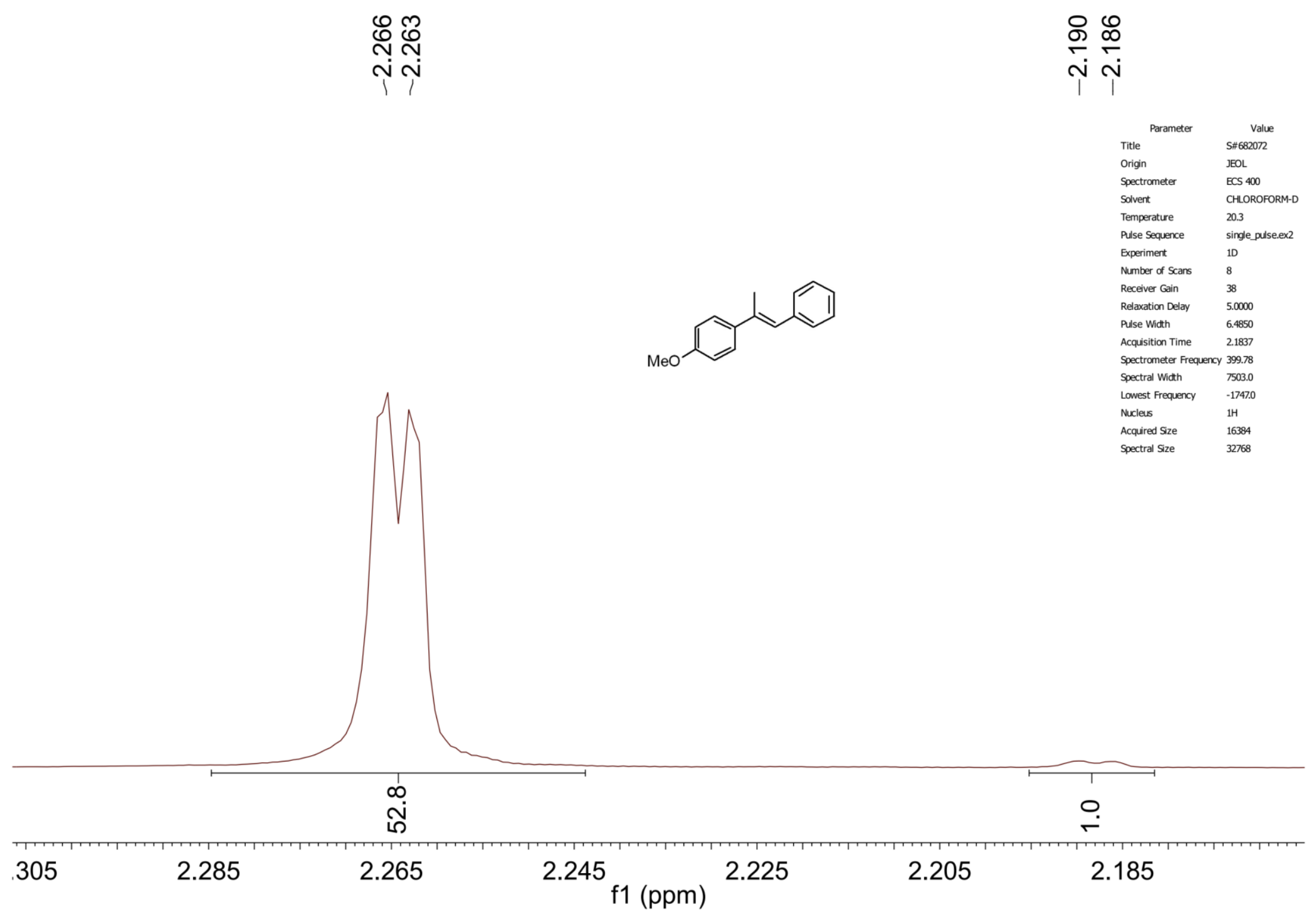



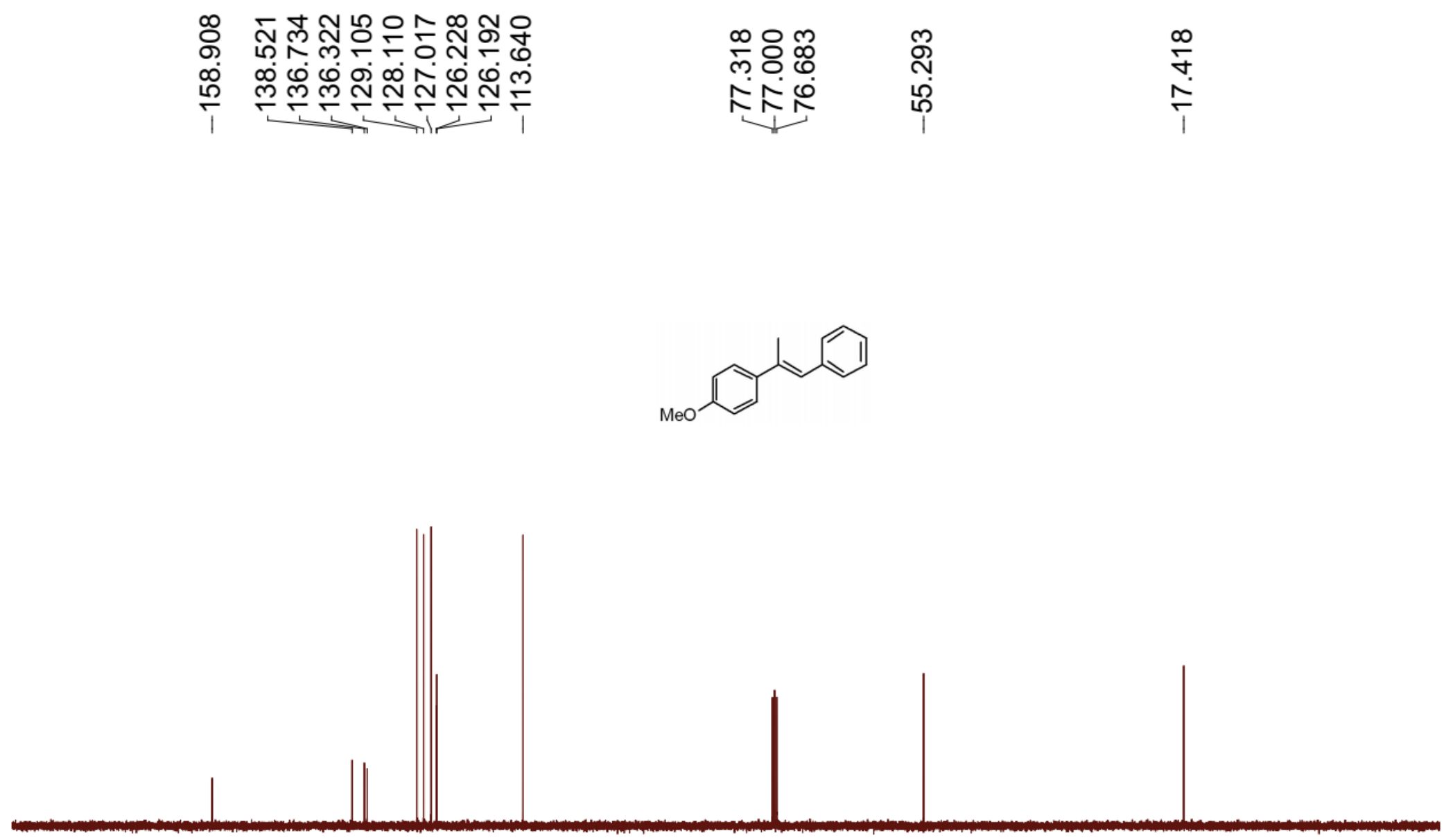


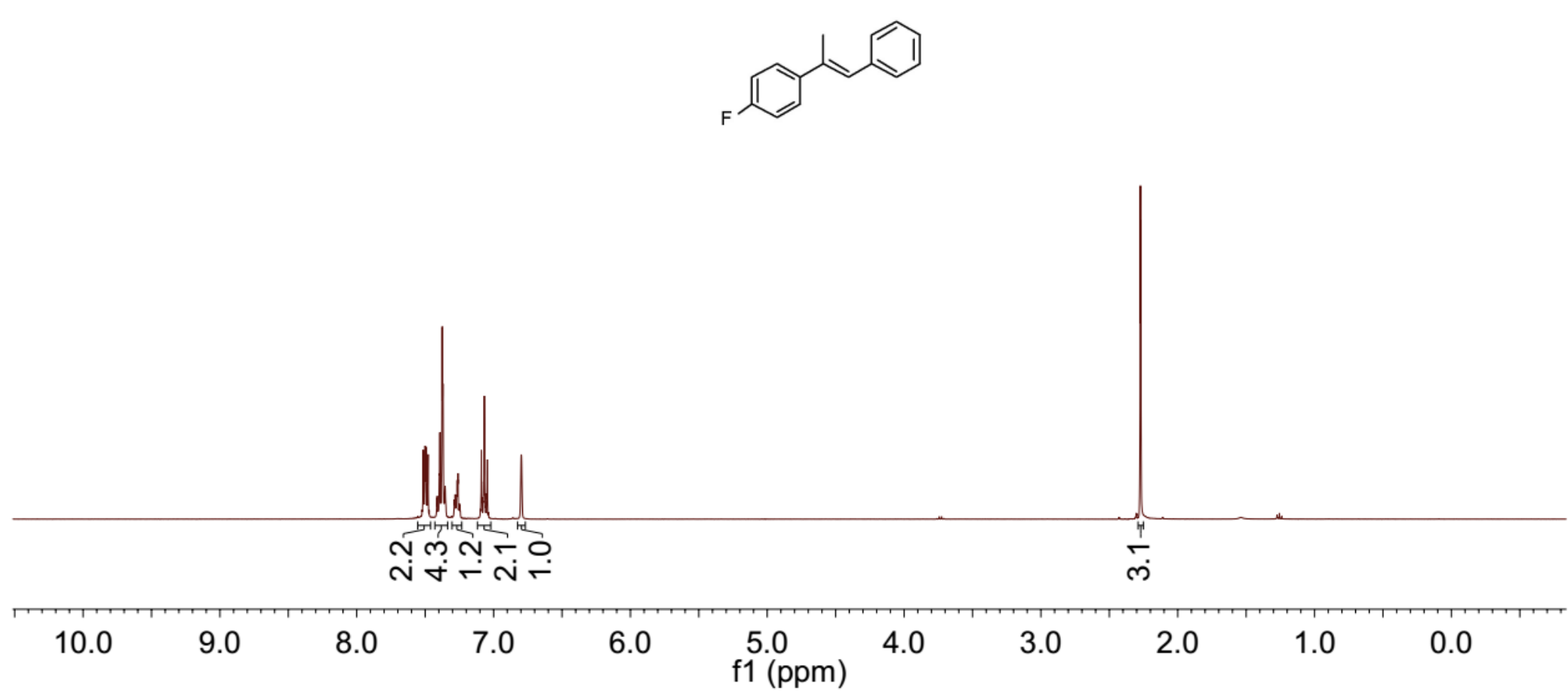


2t E/Z

0
$\stackrel{0}{0}$
$\dot{0}$

$\infty$
0
0
0

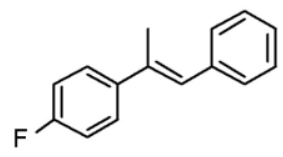

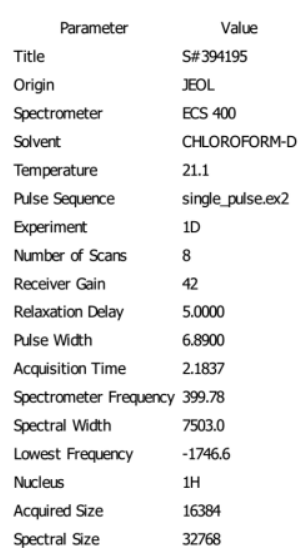
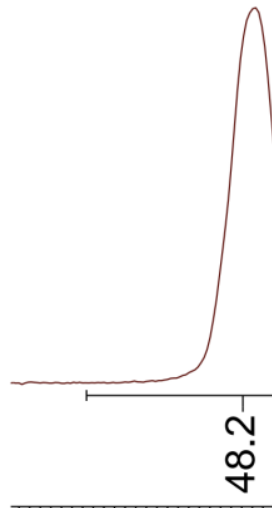

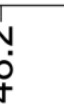

ㅇ.

$\begin{array}{lll}6.74 & 6.64 & 6.54 \\ & \mathrm{f1}(\mathrm{ppm}) & \end{array}$




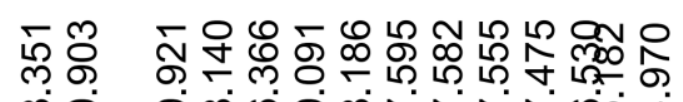

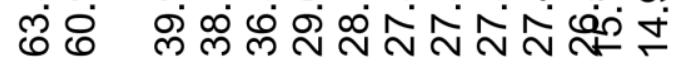

mon

रิ์ị

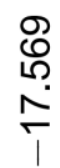
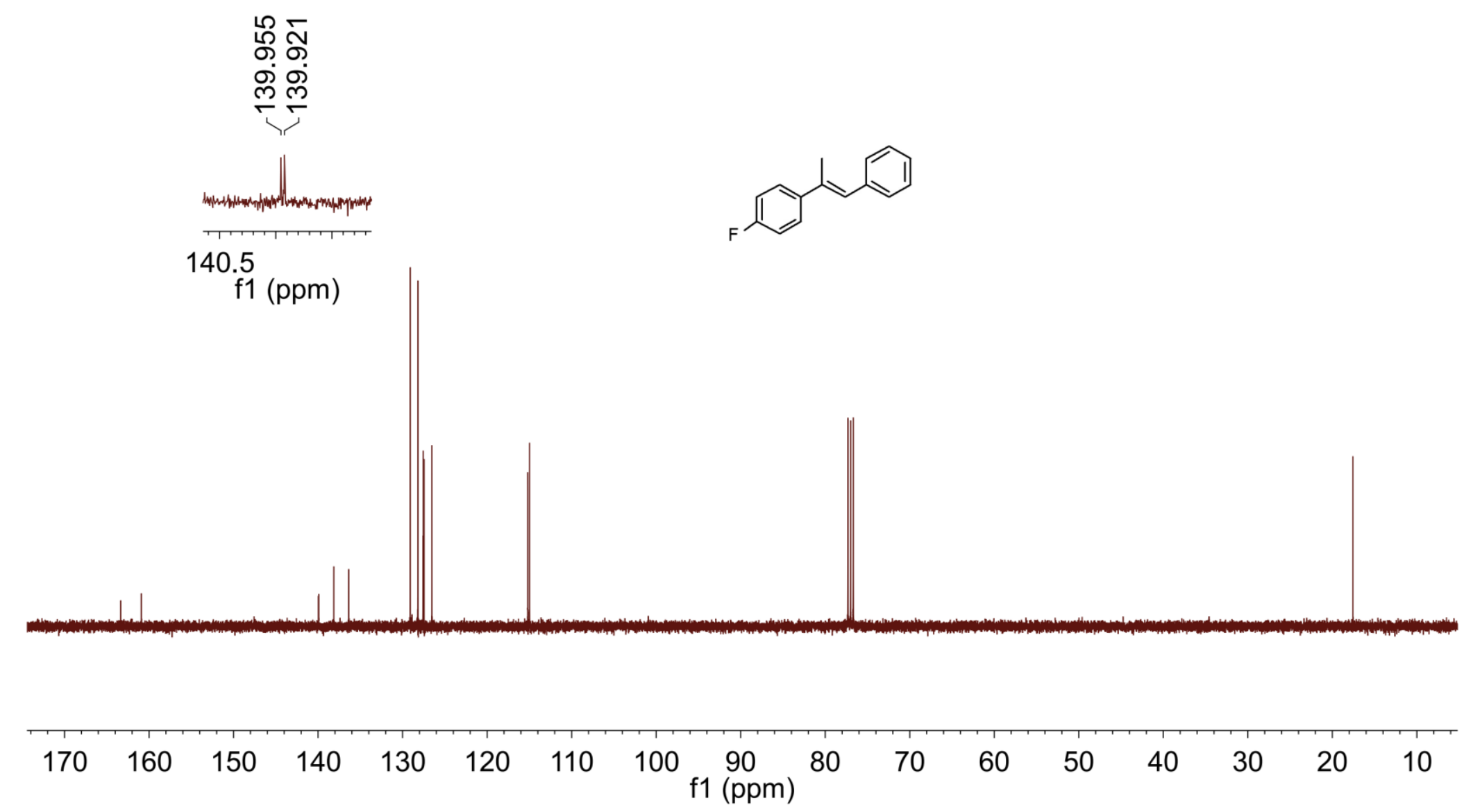
$2 t^{19}$ F NMR

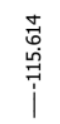
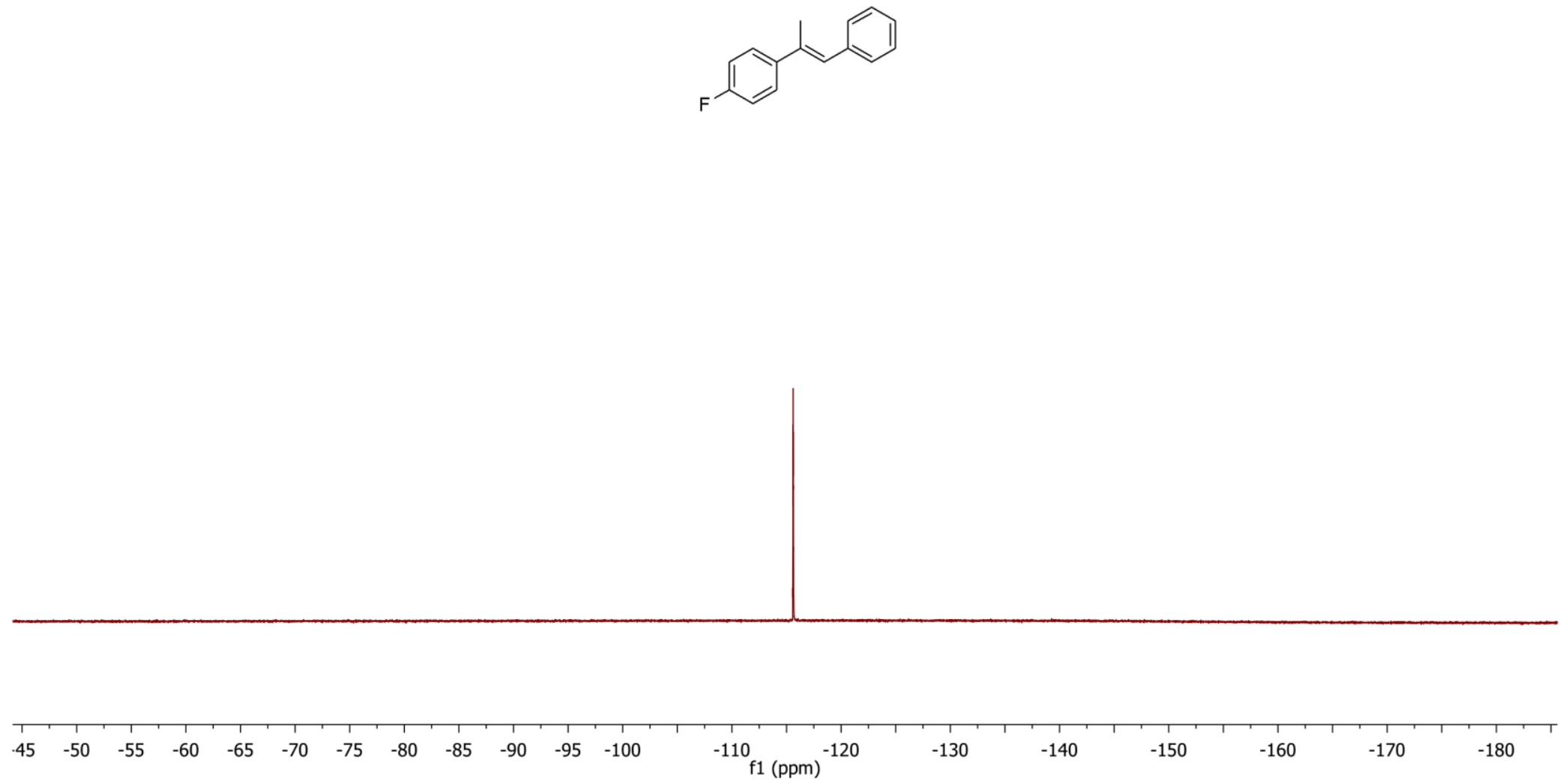


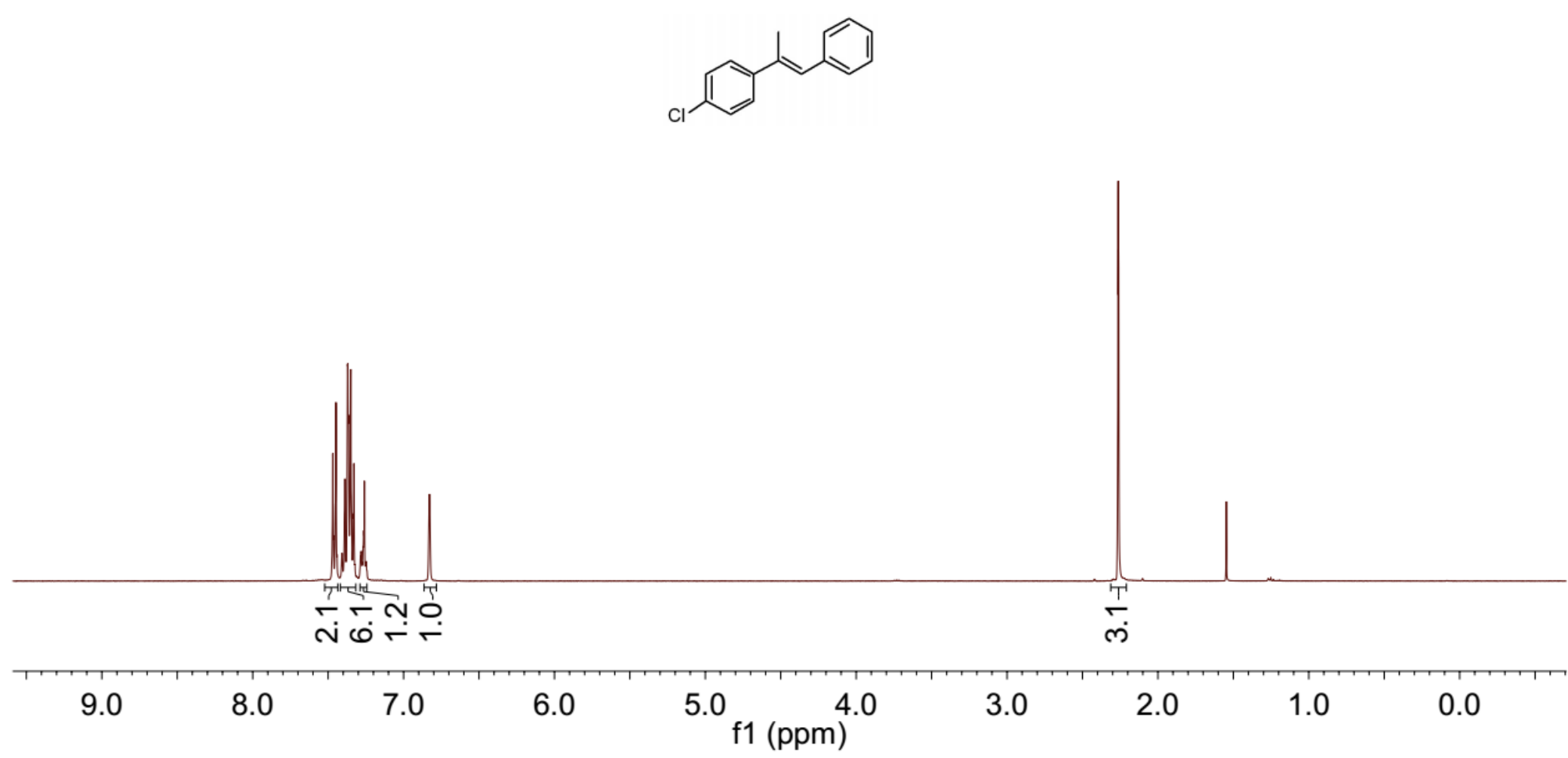


$2 u E / Z$

م્

จิ

0

ต
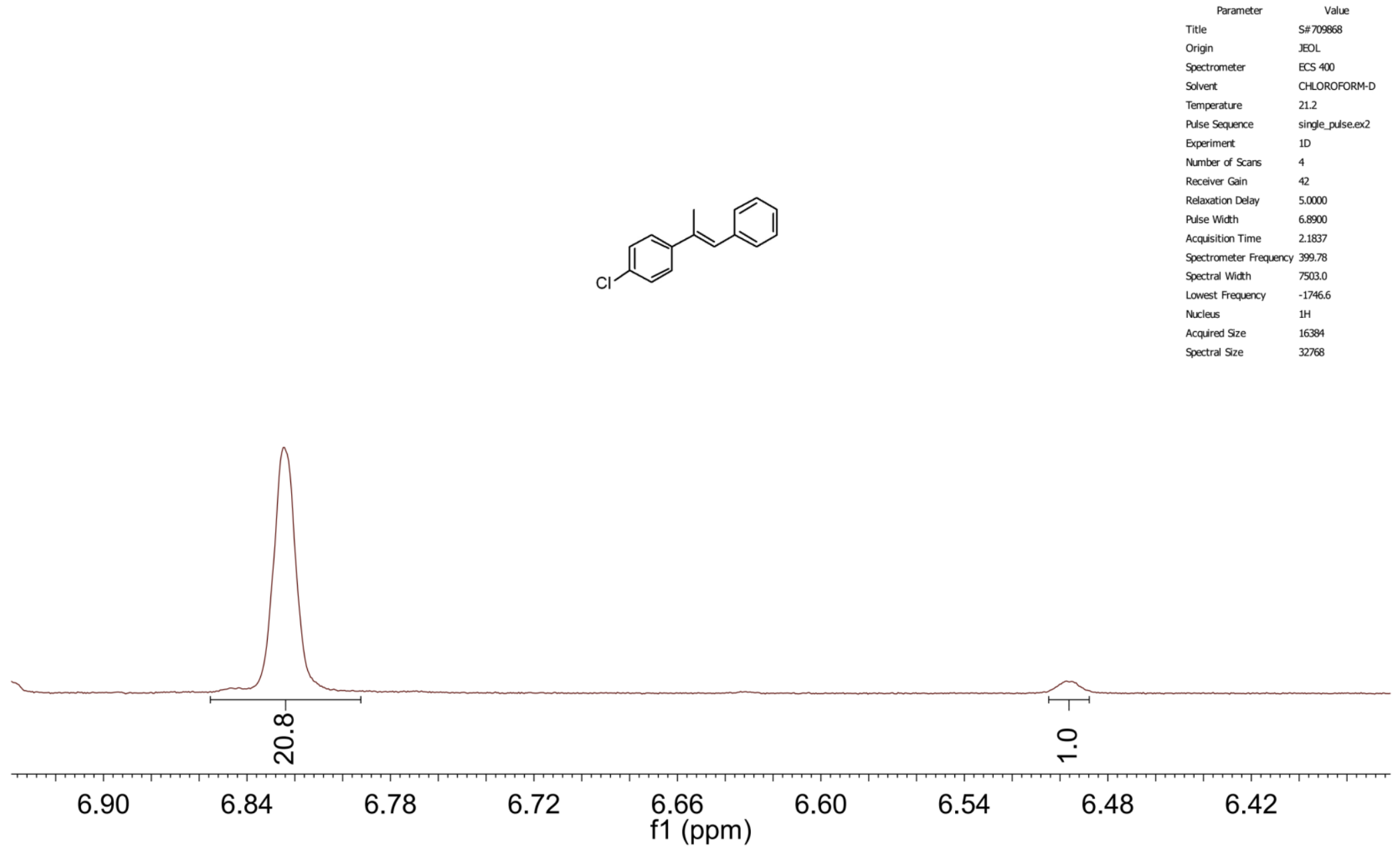


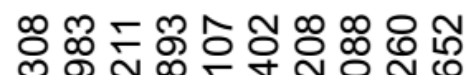

守宁宁

m.

소요

ळ్

$\stackrel{\text { r }}{\stackrel{\infty}{\prime}}$
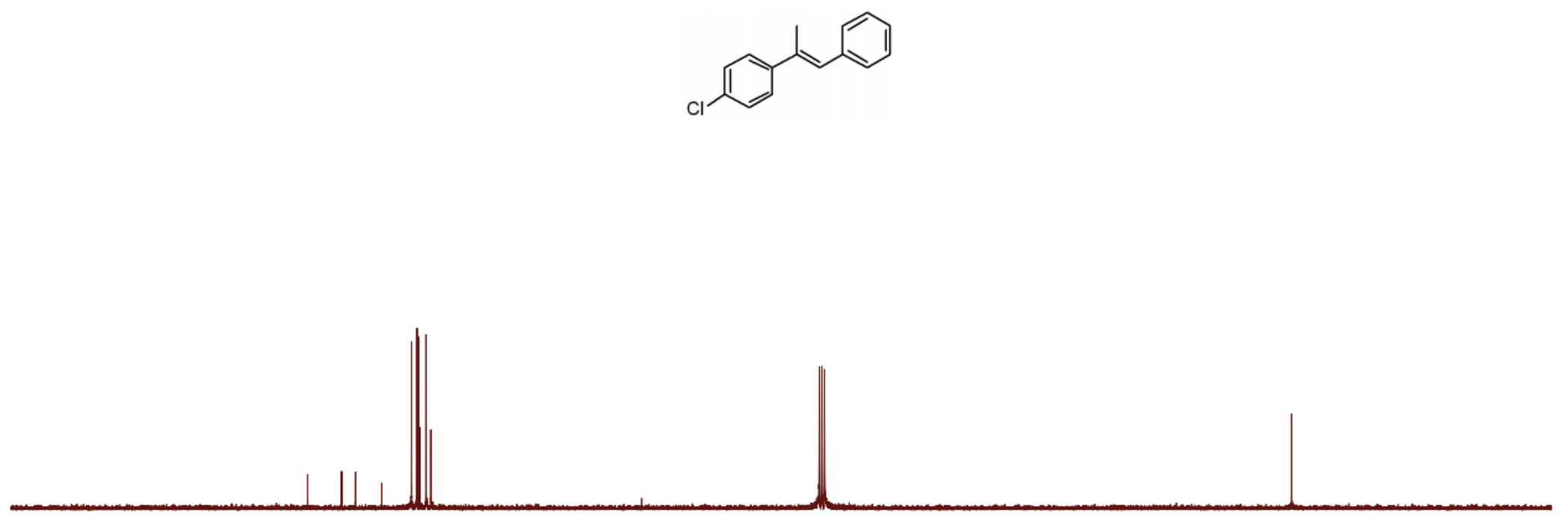

\begin{tabular}{lllllllllllllll}
\hline 170 & 150 & 130 & 110 & $90 \underset{\mathrm{f} 1(\mathrm{ppm})}{80}$ & 70 & 60 & 50 & 40 & 30 & 20 & 10 & 0 & -10
\end{tabular}




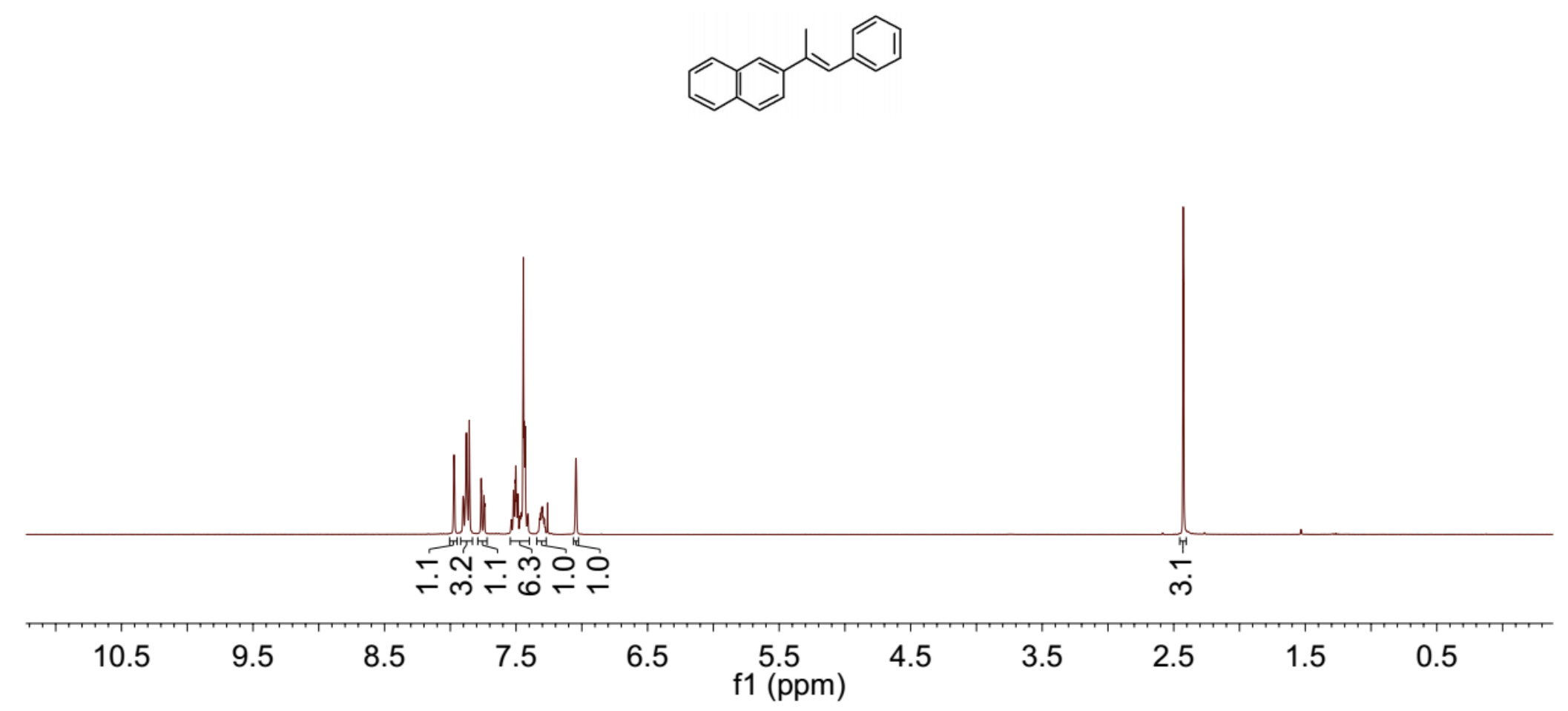


2v E/Z

$\frac{9}{5}$

$\stackrel{\circ}{\circ}$

i

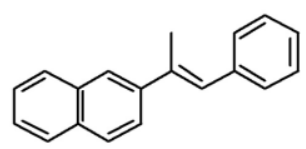

Parameter Valie
5.469395

Origin $\quad \mathrm{EOOL}$

Spectrometer ECS 400

$\begin{array}{ll}\text { Solvent } & \text { CHLOO } \\ \text { Temperature } & 20.3\end{array}$

Puise Sequence single puise.ex2

Experimert 10

Number of Scans 8

Receiver Gain 40

$\begin{array}{ll}\text { Relaxation Delay } & 5.0000 \\ \text { Pulse Widh } & 64850\end{array}$

$\begin{array}{ll}\text { Pulse With } & 6.4550 \\ \text { Accuistion Time } & 2.1837\end{array}$

$\begin{array}{ll}\text { Acquisition Time } & 2.1837 \\ \text { Spectrometer Frequency } 399.78\end{array}$

$\begin{array}{ll}\text { Spectrometer Frequency } & 399.78 \\ \text { Spectral Width } \quad 7503.0\end{array}$

Lowest Frequency $\quad-1746.6$

Nucleus

$1 \mathrm{H}$
16384

spectral Size $\quad 32768$

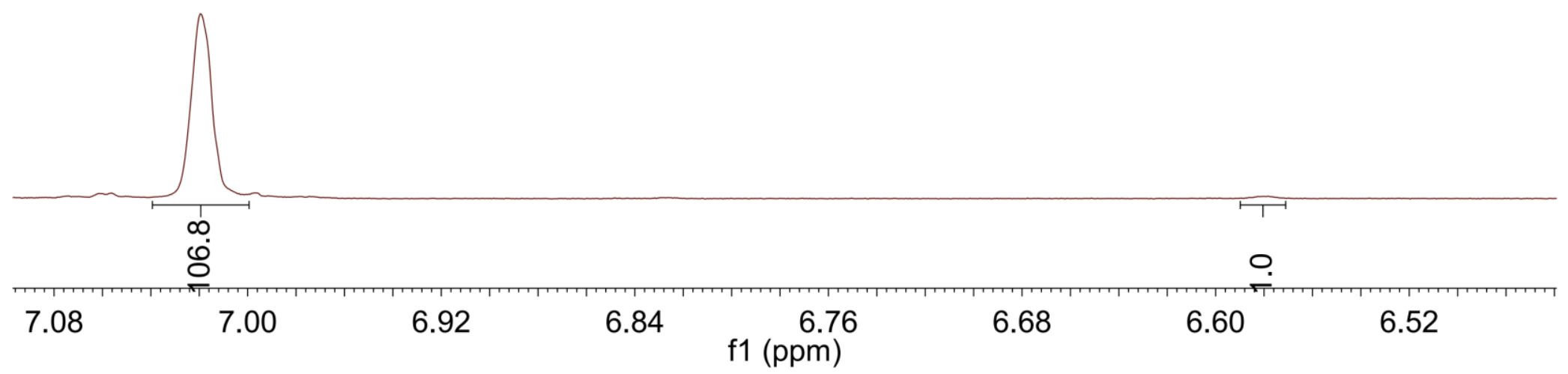




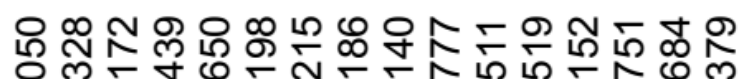

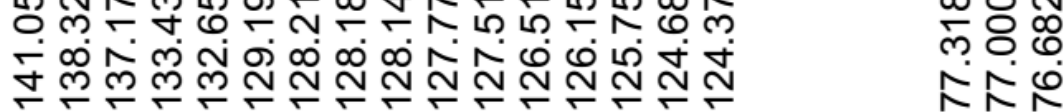
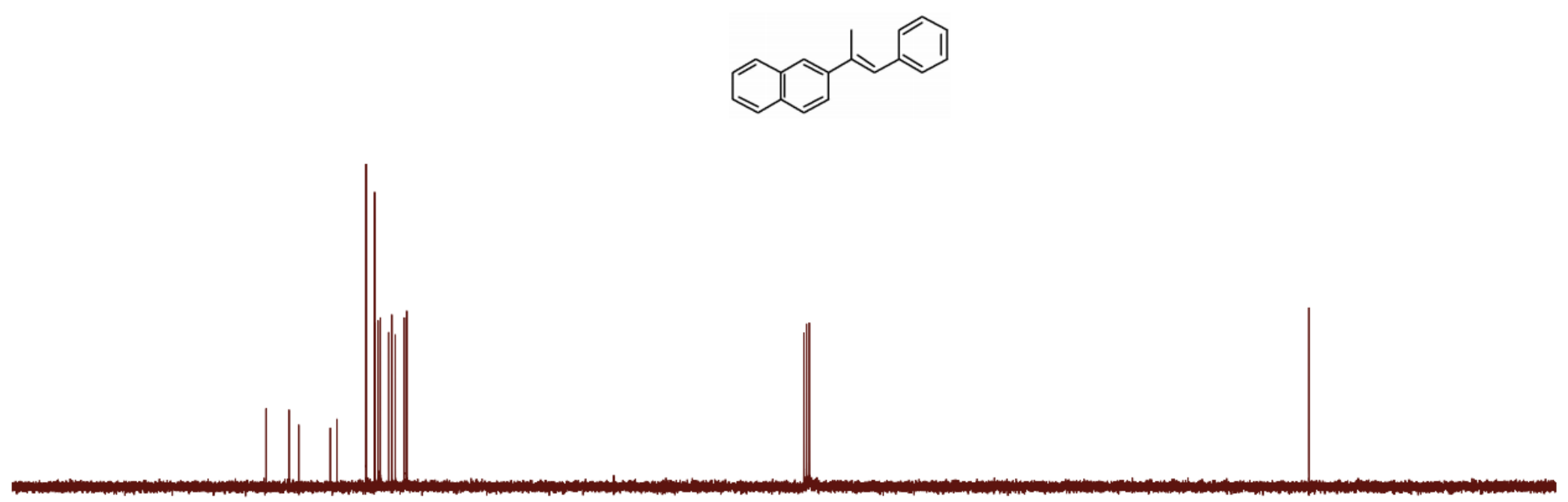

$\begin{array}{lllllllllllllllllll}70 & 160 & 150 & 140 & 130 & 120 & 110 & 100 & 90 & \underset{\mathrm{f} 1}{80}(\mathrm{ppm}) & 70 & 60 & 50 & 40 & 30 & 20 & 10 & 0 & -1 \mathrm{C}\end{array}$ 


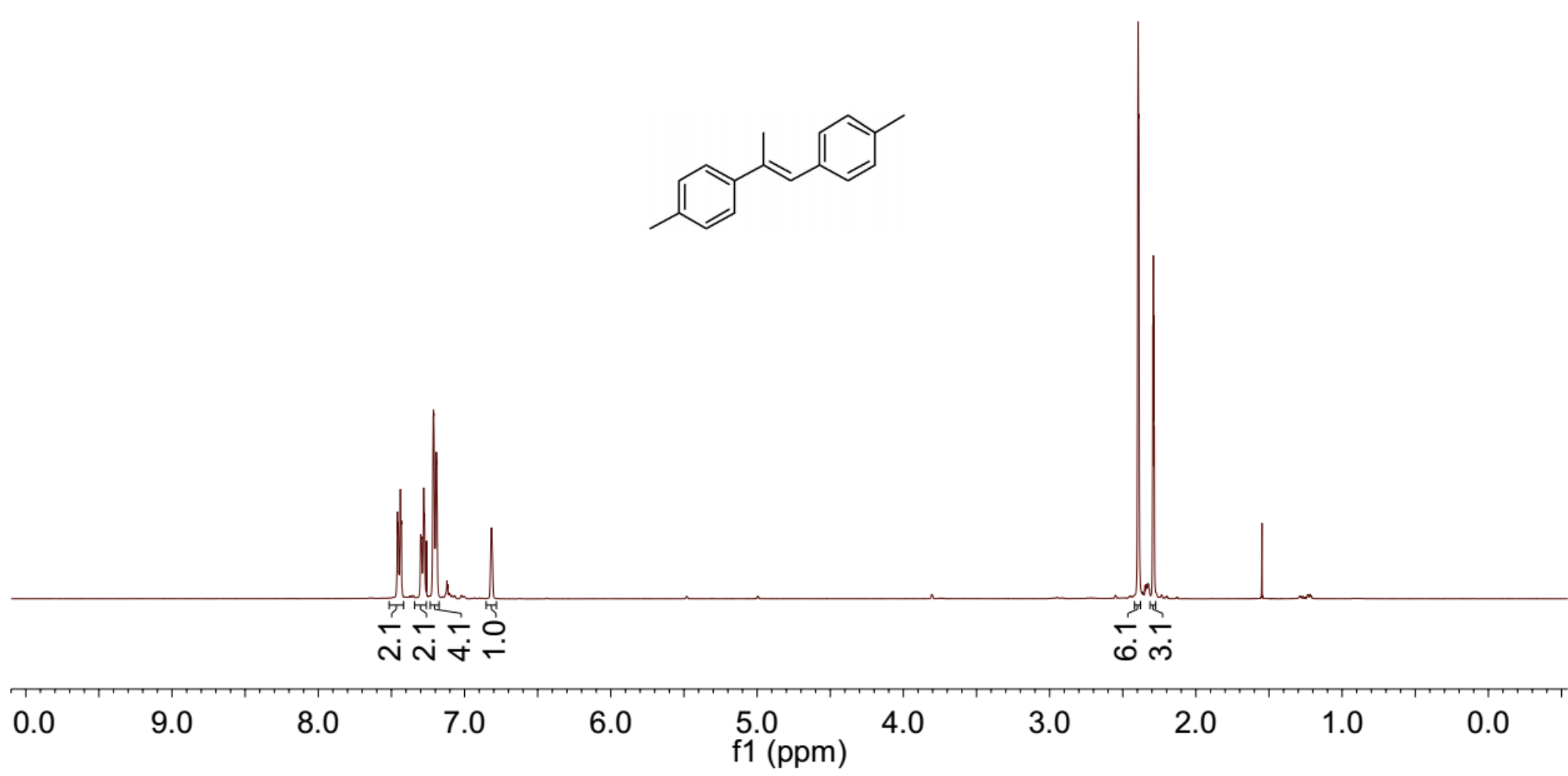


2w E/Z

مิ

\ั7
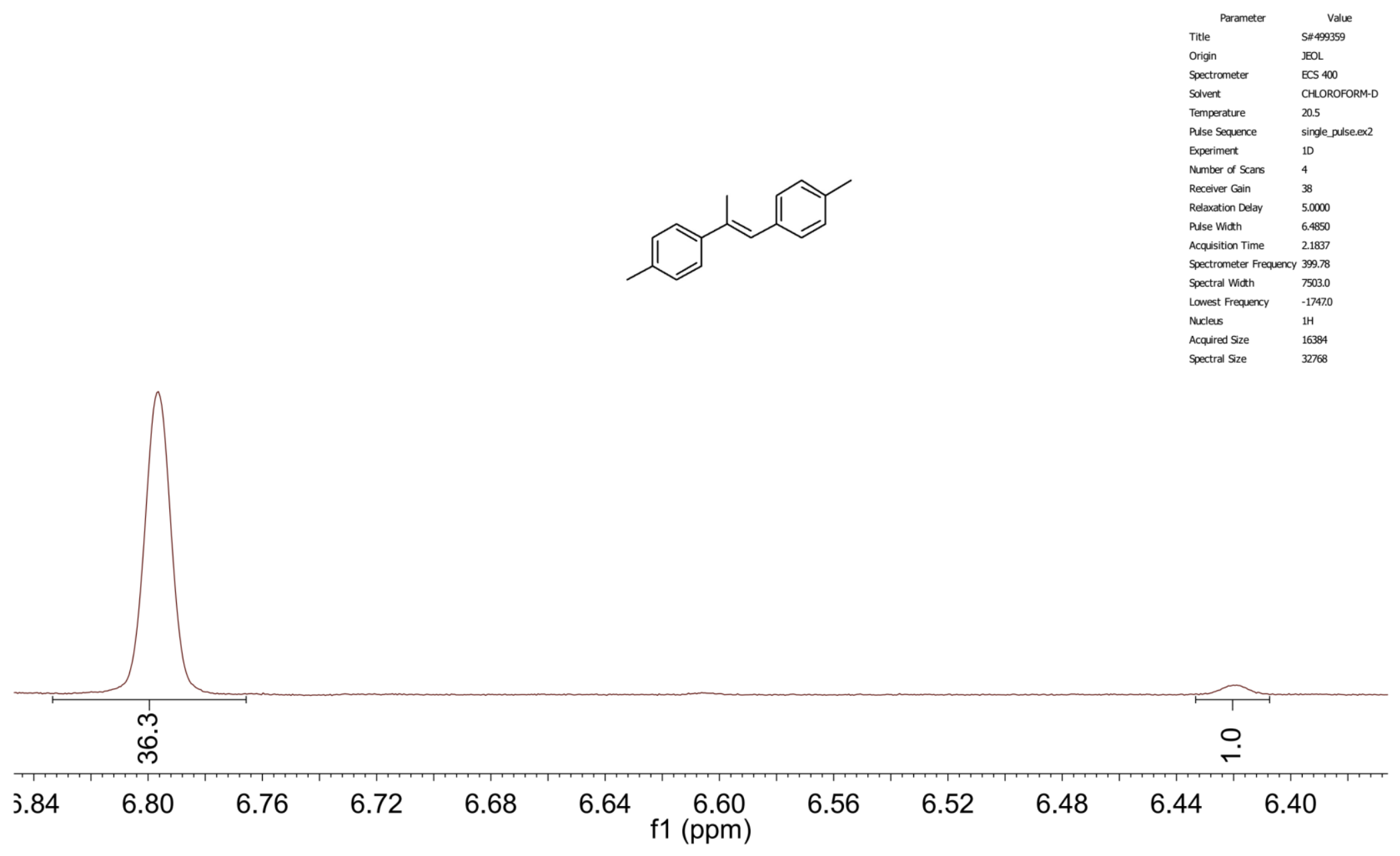


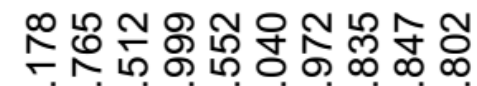

சणं

ㄷํㅇㅇㅛ

소요

๓

可

ล்ํํㄴ

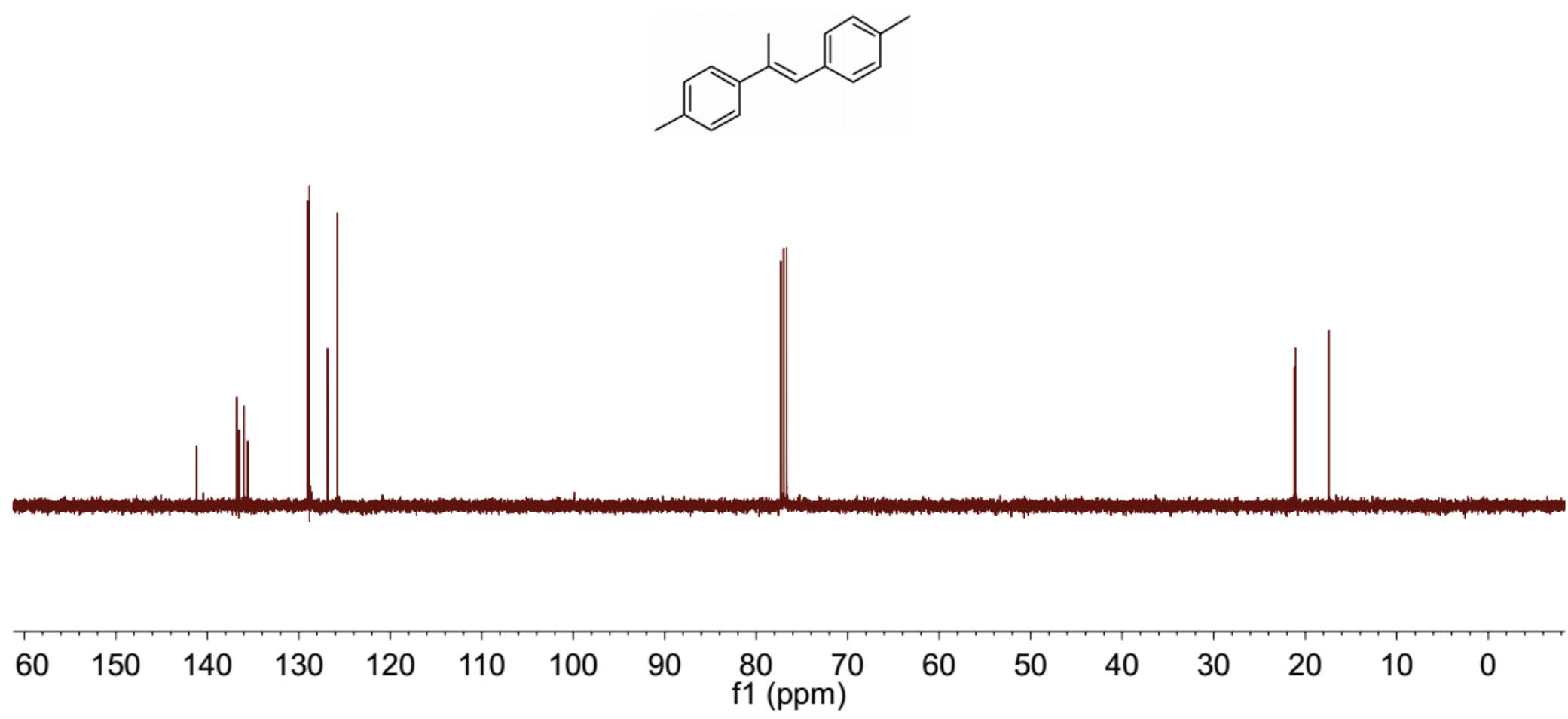



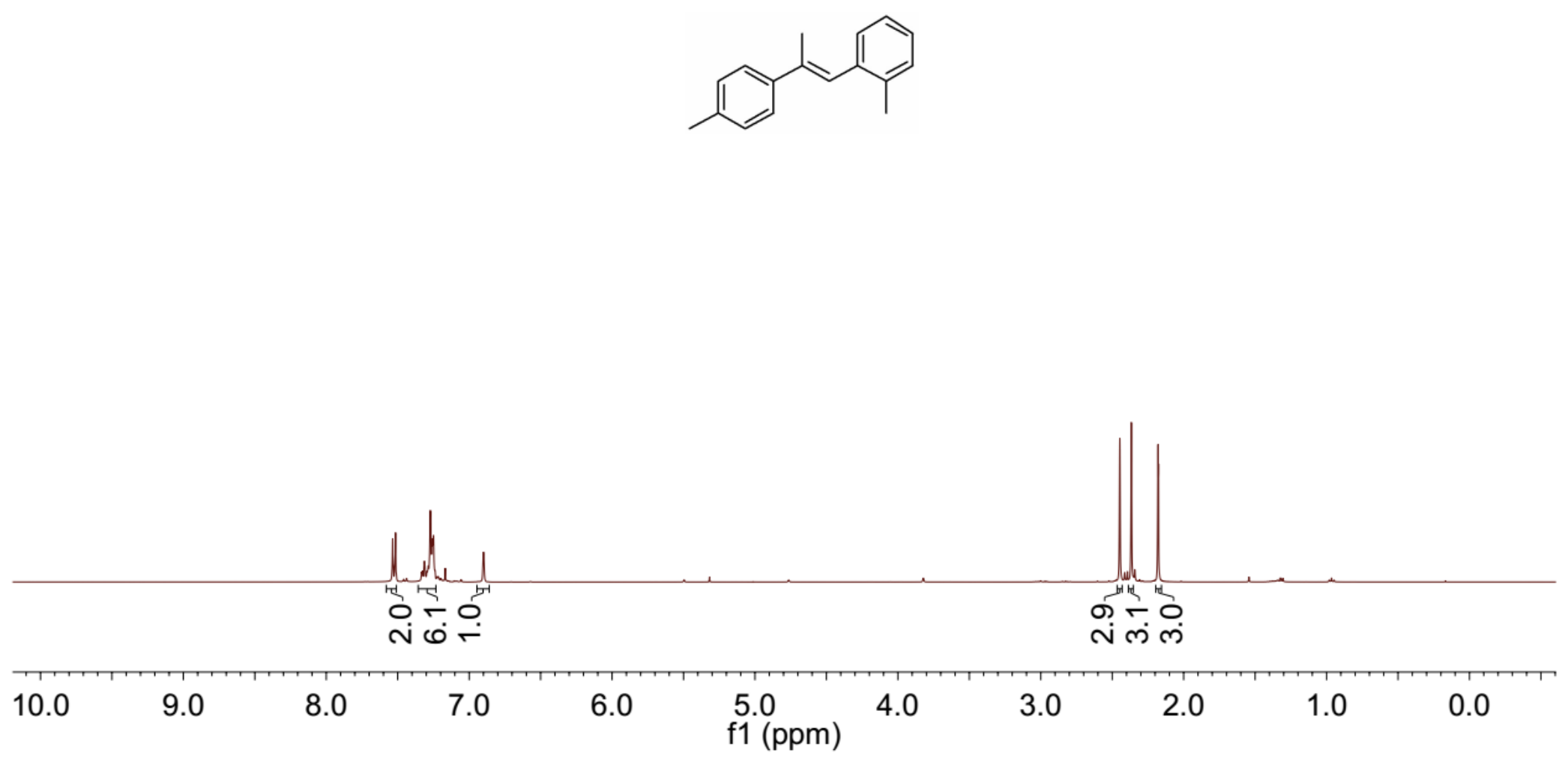


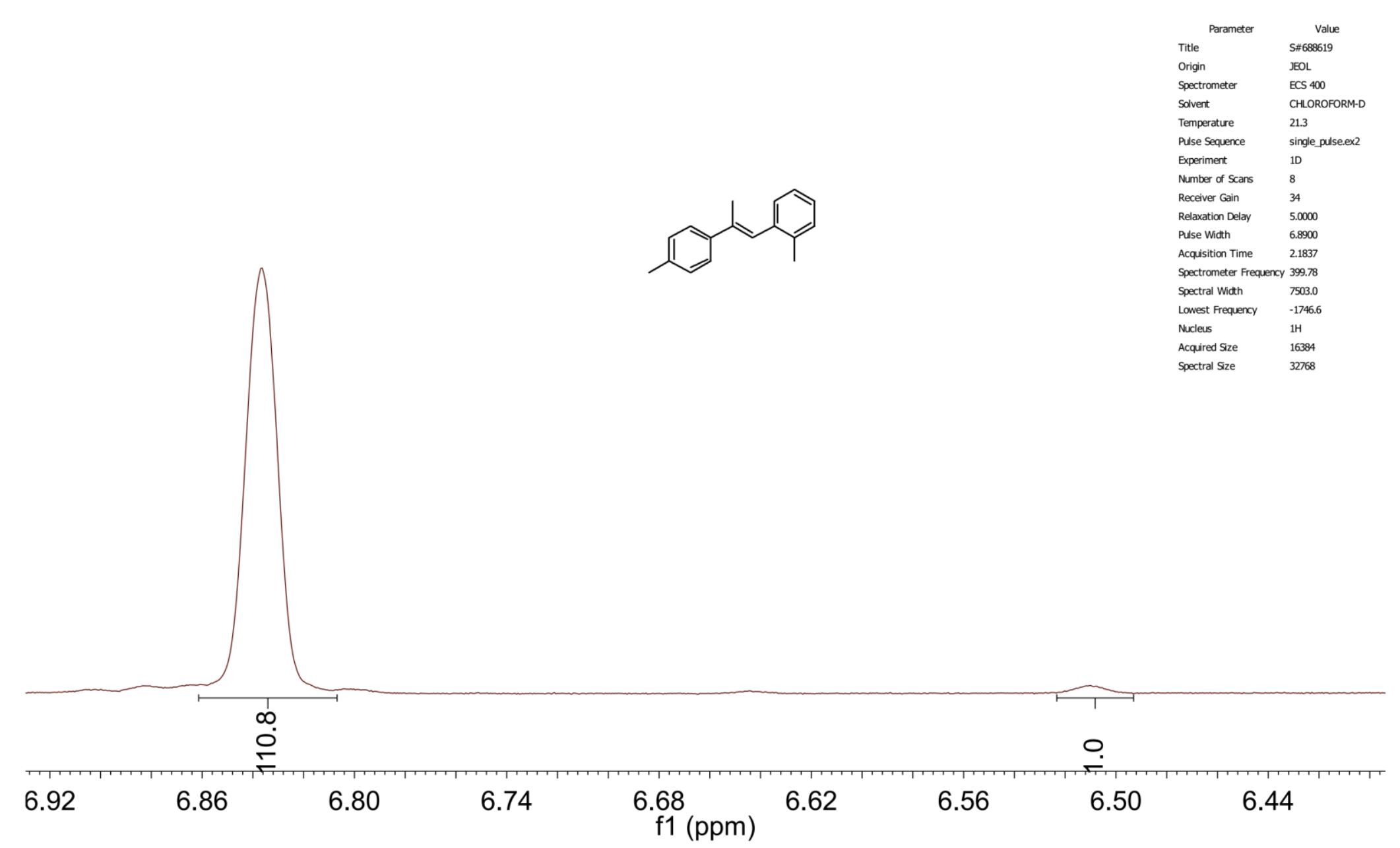




\begin{tabular}{|c|c|c|}
\hline 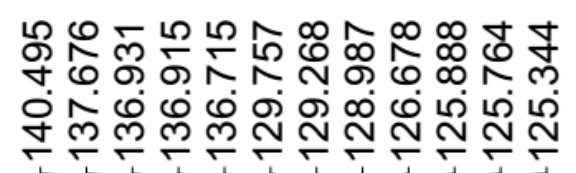 & 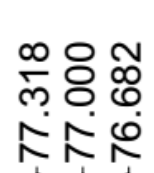 & $\begin{array}{l}\text { 용ㅇㅇ } \\
\text { 휴유 }\end{array}$ \\
\hline
\end{tabular}

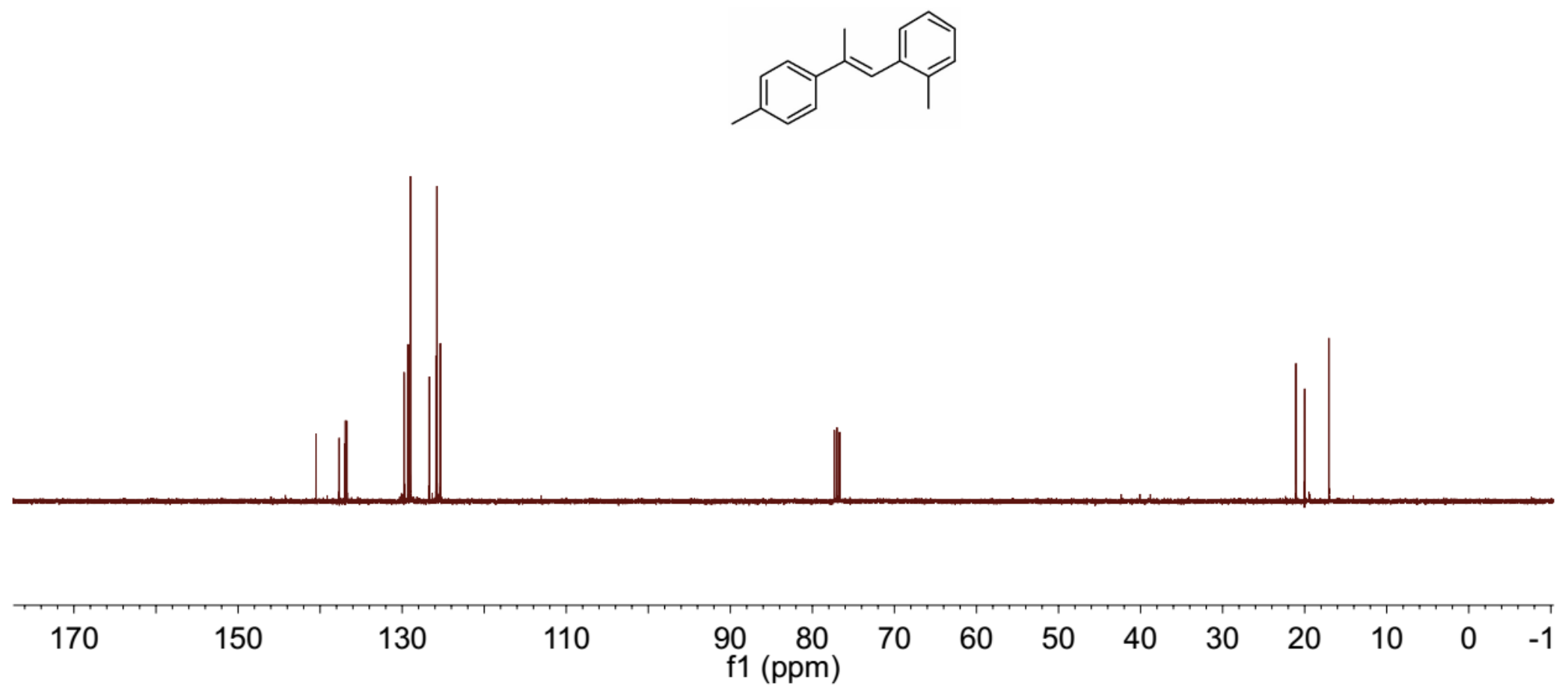




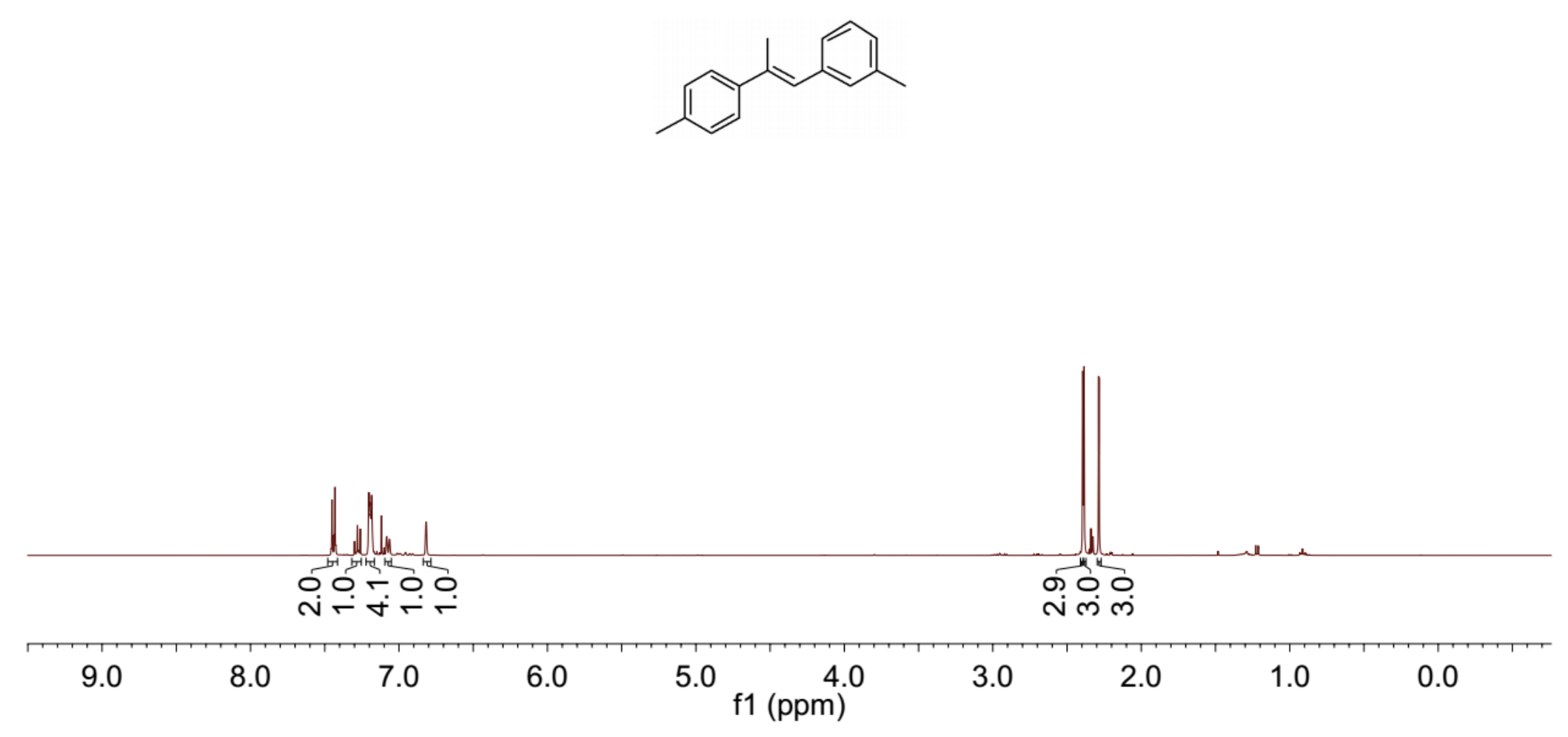


Parameter Value
Title

Origin JEOL

Spectrometer ECS 400

Solvent CHLROFO

$\begin{array}{ll}\text { Temperature } & 23.9 \\ \text { Pusse sequence } & \text { s.9 }\end{array}$

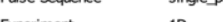

Experiment 10

Receiver Gain $\quad 30$

Relaxation Delay $\quad 5.0000$

Pulse With $\quad 6.4850$

Acquistion Time $\quad 2.1837$

Spectrometer Frequency 399.78

$E / Z=112 / 1$

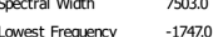

Nucleus $1 \mathrm{H}$

$\begin{array}{ll}\text { Acquired Sze } & 16394 \\ \text { Spectral Sze } & 32768\end{array}$

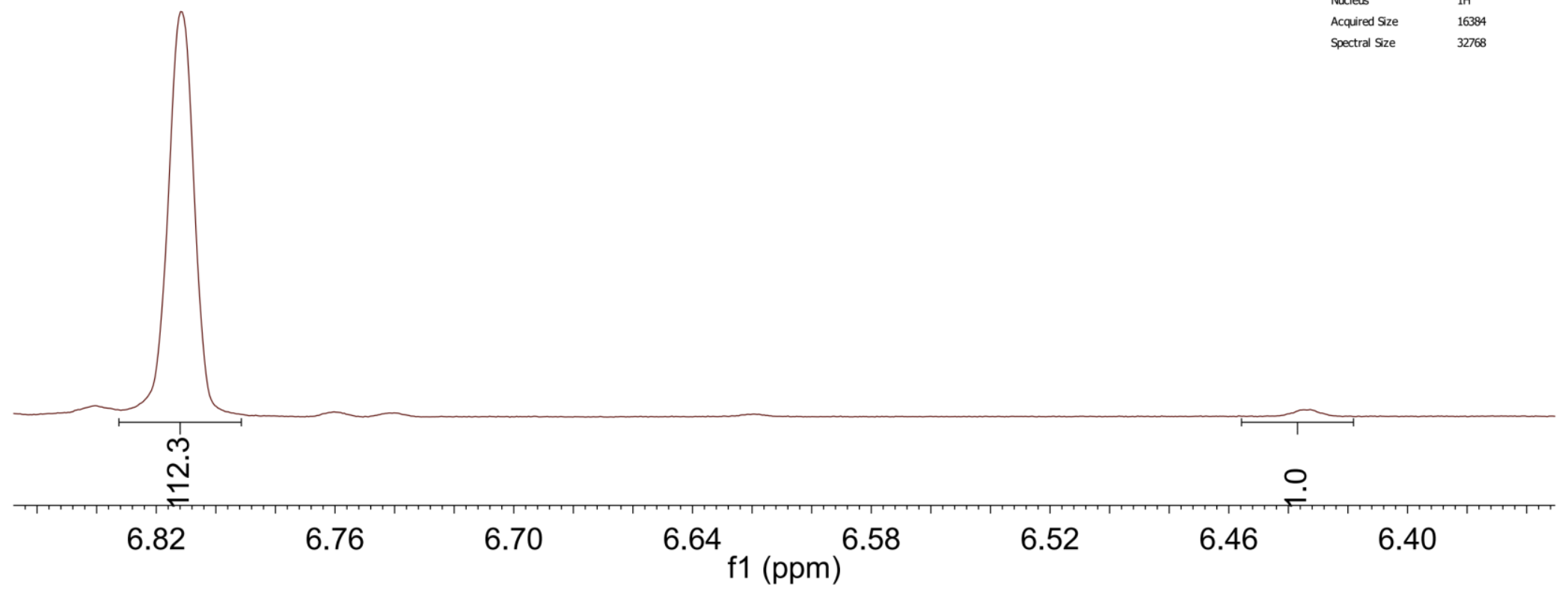




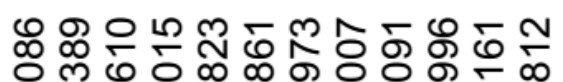

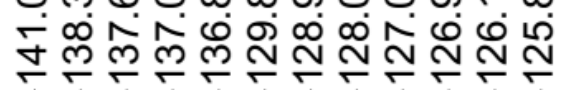

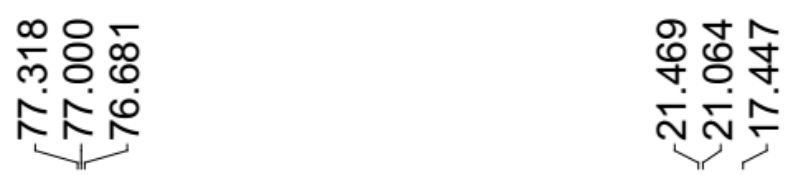

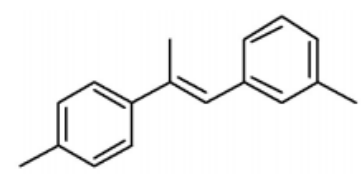

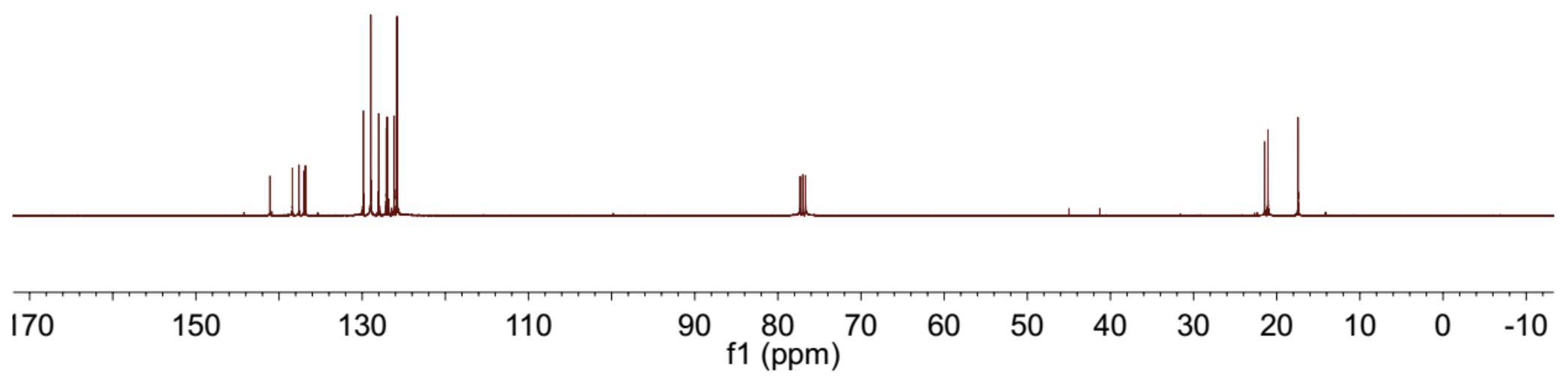




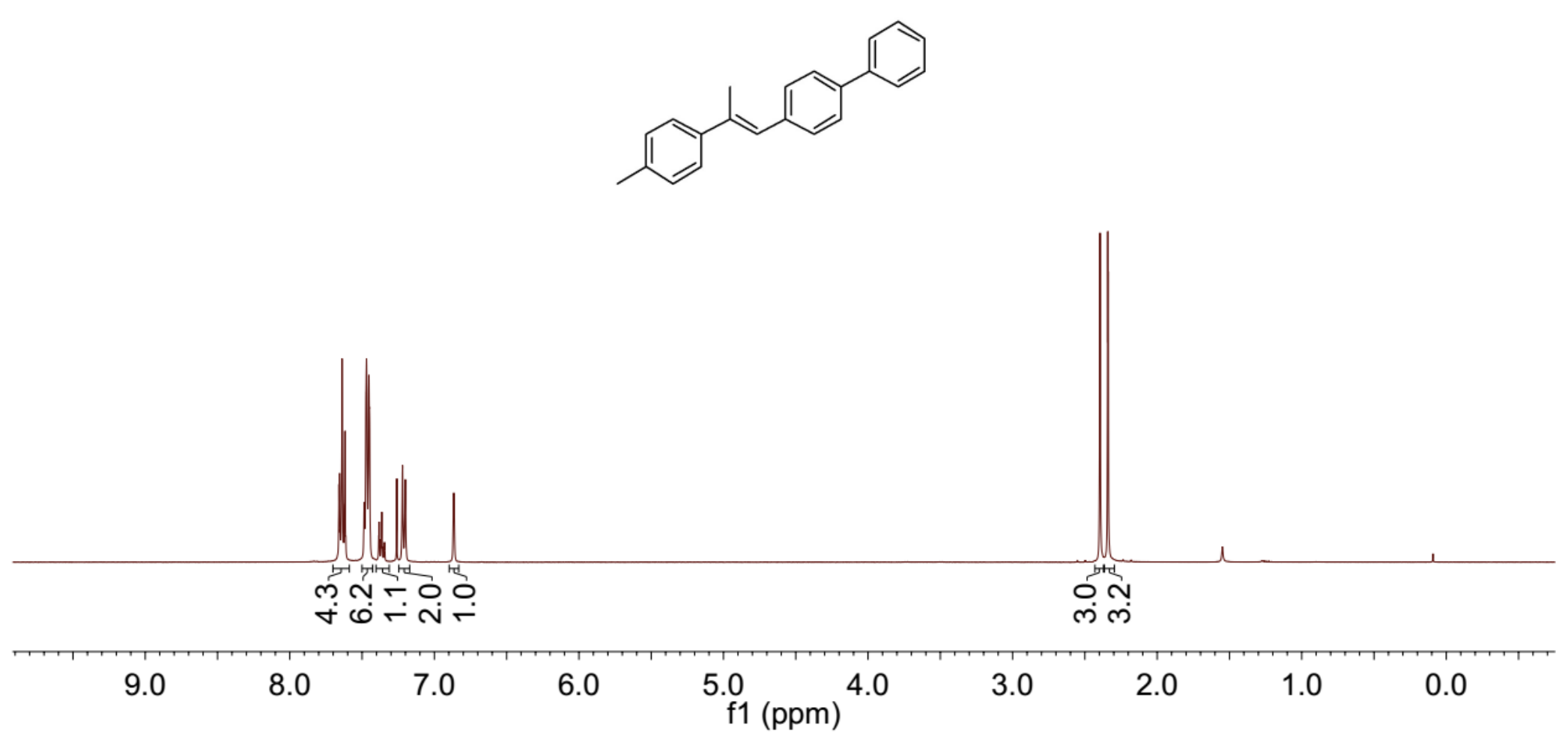




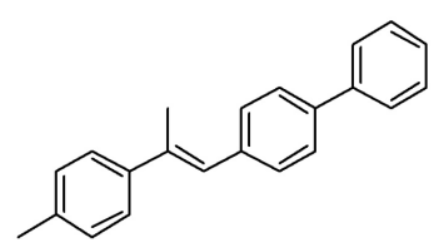

$$
\begin{aligned}
& \begin{array}{c}
\text { Parameter Value } \\
54502330
\end{array} \\
& \text { Title } \quad \text { S\#50350 } \\
& \begin{array}{lll}
\text { Origin JEOL } \\
\text { Spectrometer }
\end{array} \\
& \text { Solvert } \quad \text { CCS } 400 \\
& \text { Temperature } 205 \\
& \text { Puse Sequence } \quad 20.5 \\
& \text { Experiment } 10 \\
& \text { Number of Scans } 4 \\
& \text { Recelver Gain } 40
\end{aligned}
$$

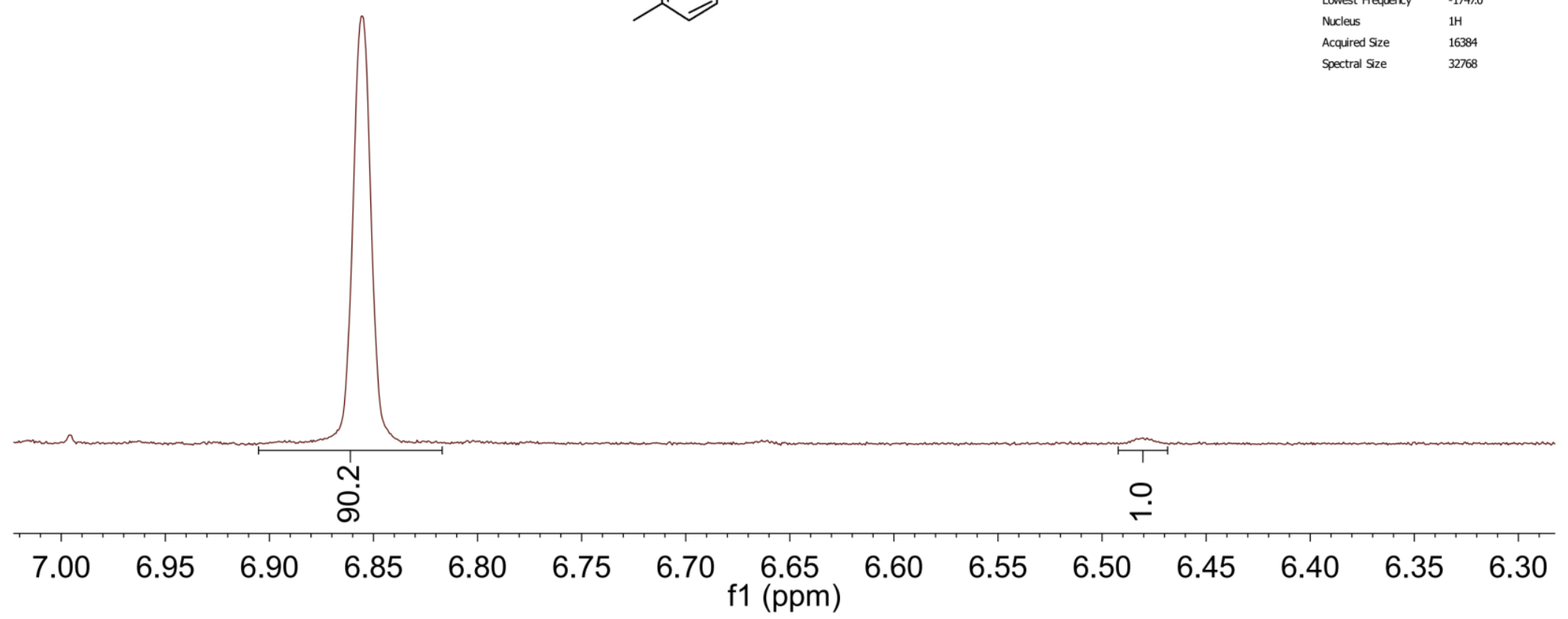




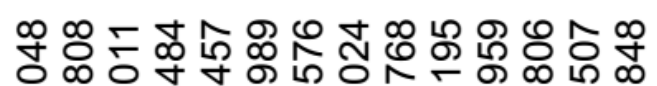

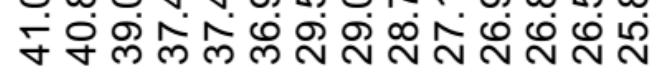

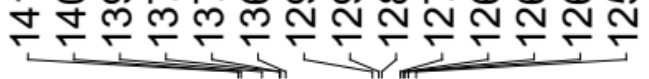

\section{ल유: \\ 슈}

융

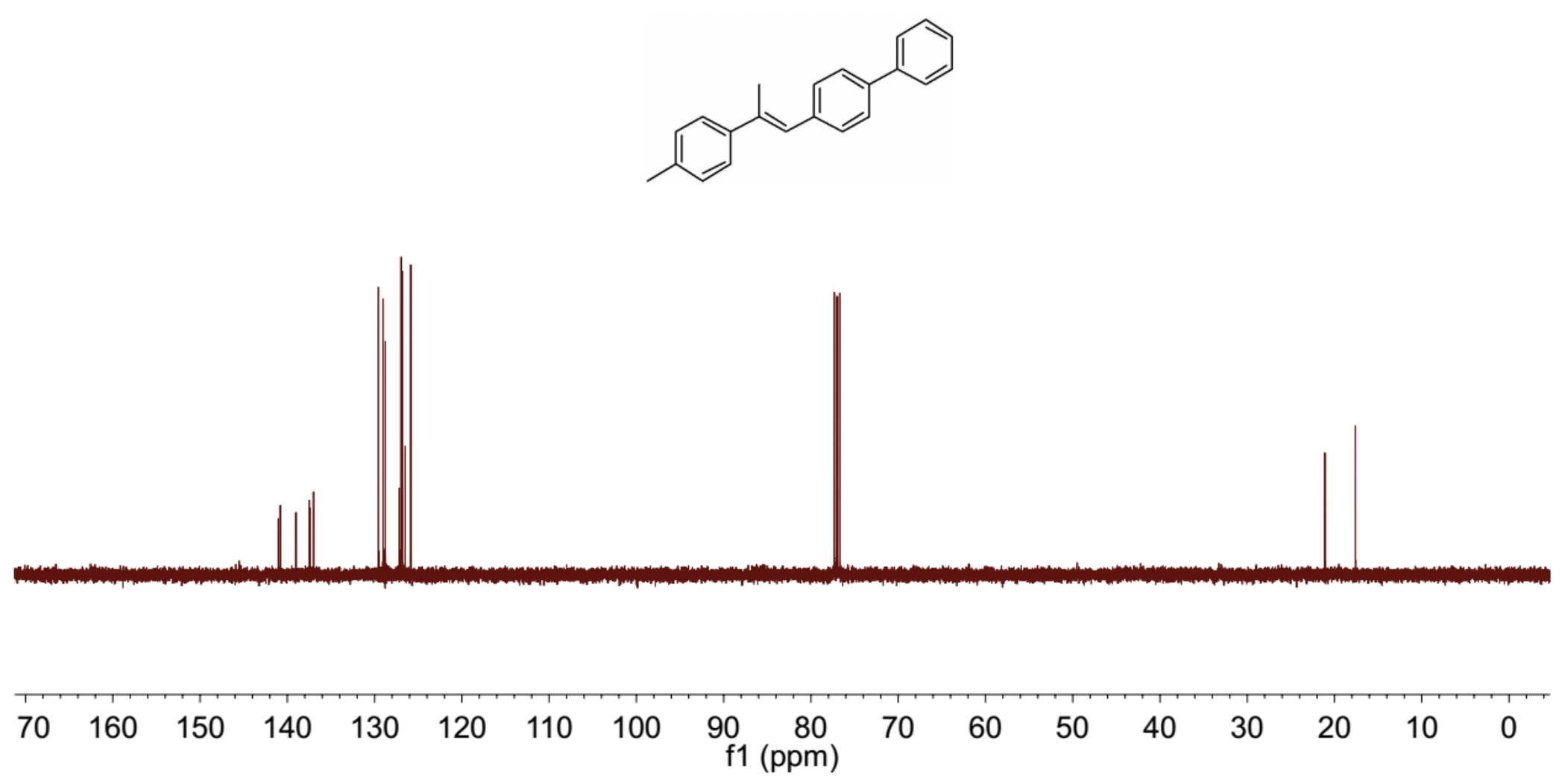




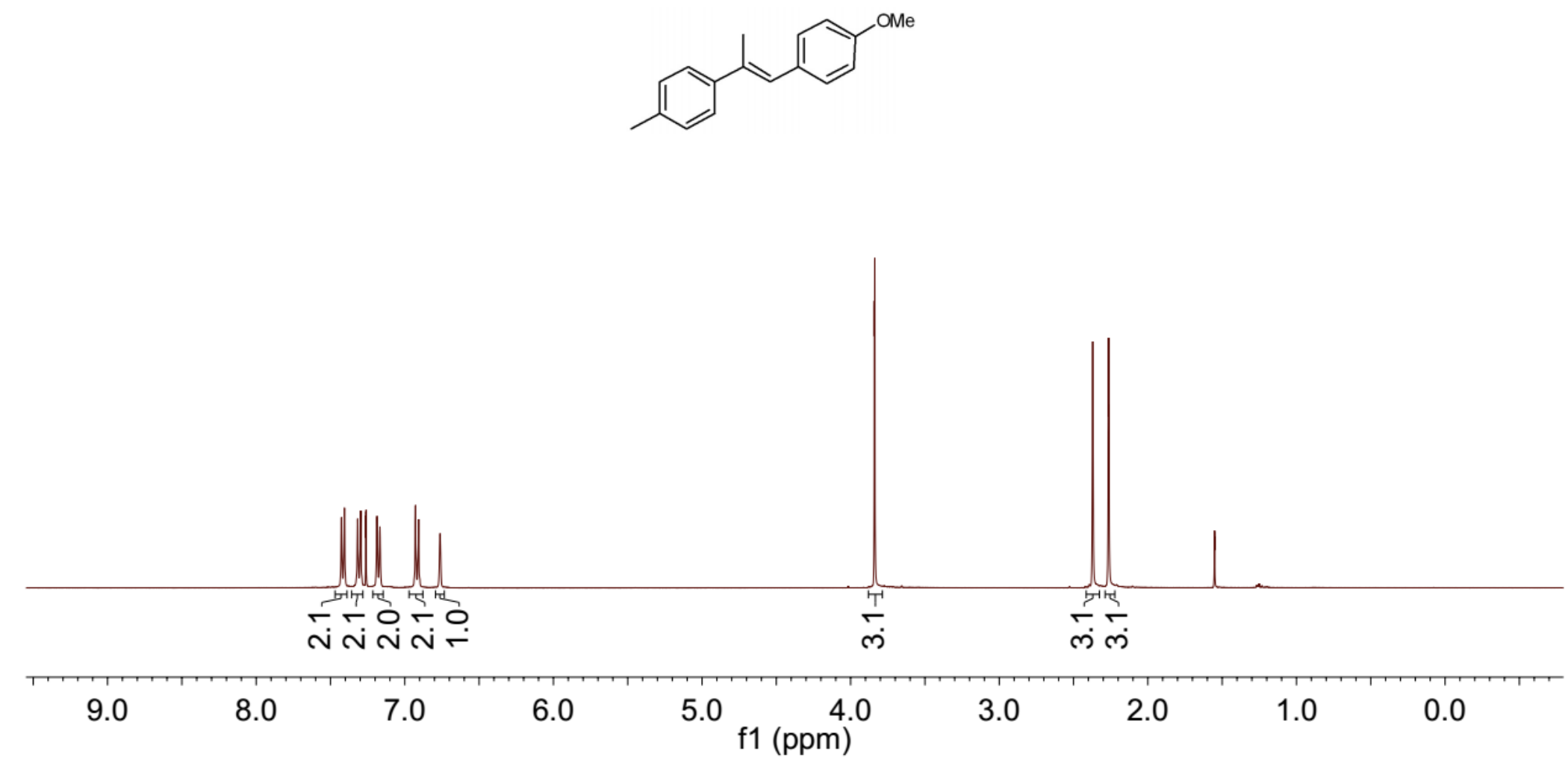


2aa $E / Z$

8
0
0
0

훙ำ

ตै

Parameter Valve
Tithe

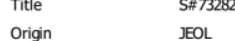

Spectrometer ECS 400

Solvent CHLROFORM-

Temperature $\quad 20.1$

Pulse Sequence single.pulse.er

Experiment 10

$\begin{array}{ll}\text { Number of Scans } & 8 \\ \text { Receiver Gain } & 32\end{array}$

Relaxation Delay $\quad 5.0000$

Pulse Wath $\quad 6.9900$

Acquistion Time $\quad 2.1837$

$\mathrm{E} / \mathrm{Z}=71 / 1$

Spectrometer Frequency 399.78

7503.0

Nucleus

Acquired Size $\quad 16384$

Spectral Size

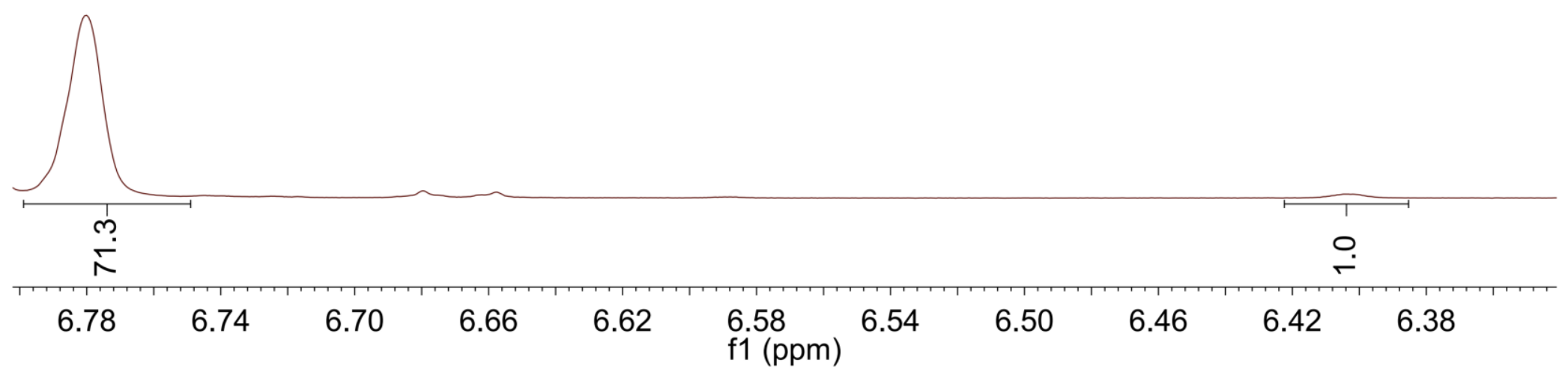




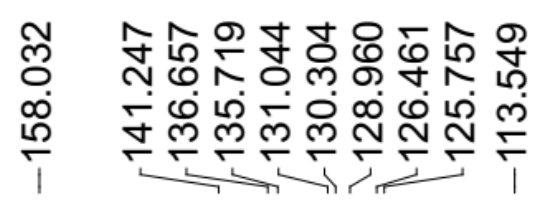

ㄷํㅇ $\frac{2}{8}$

춘

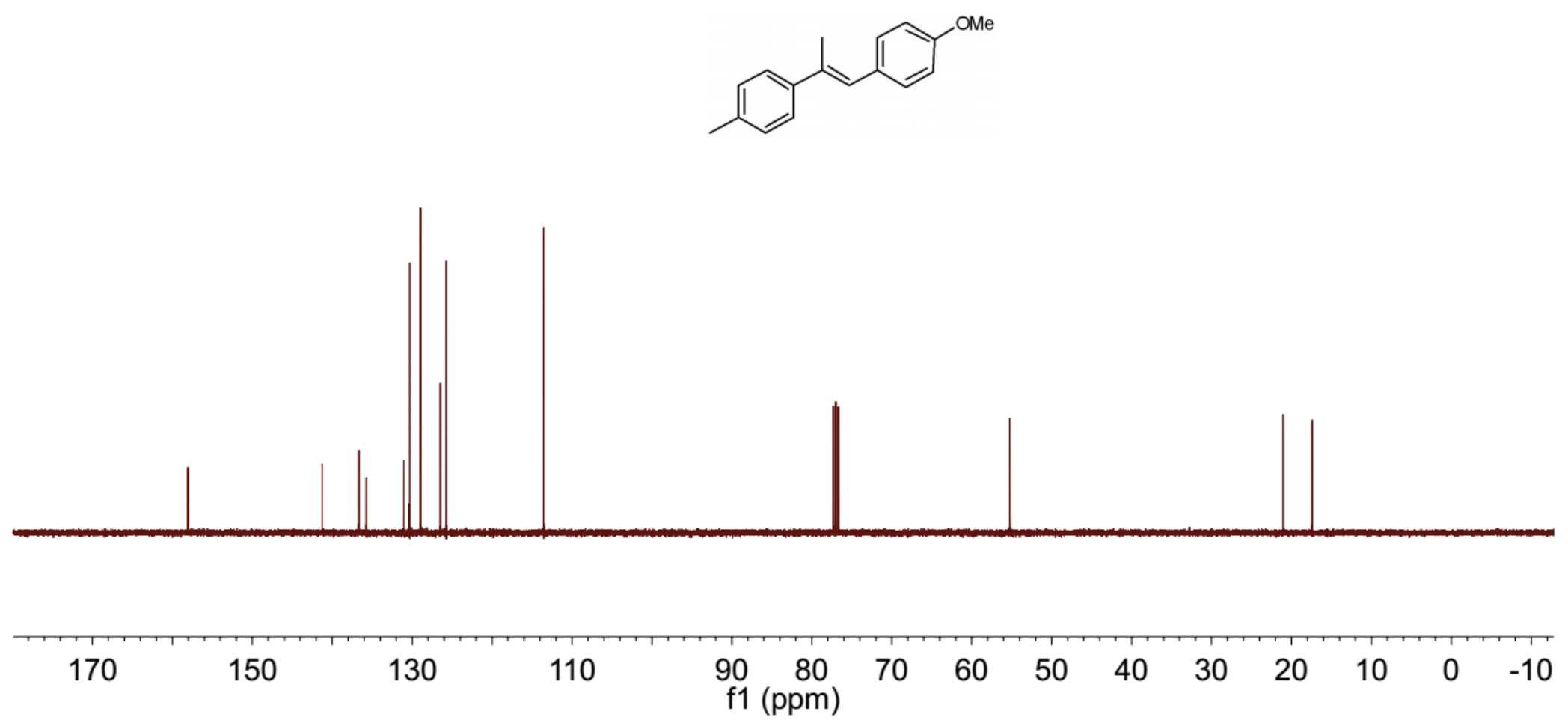




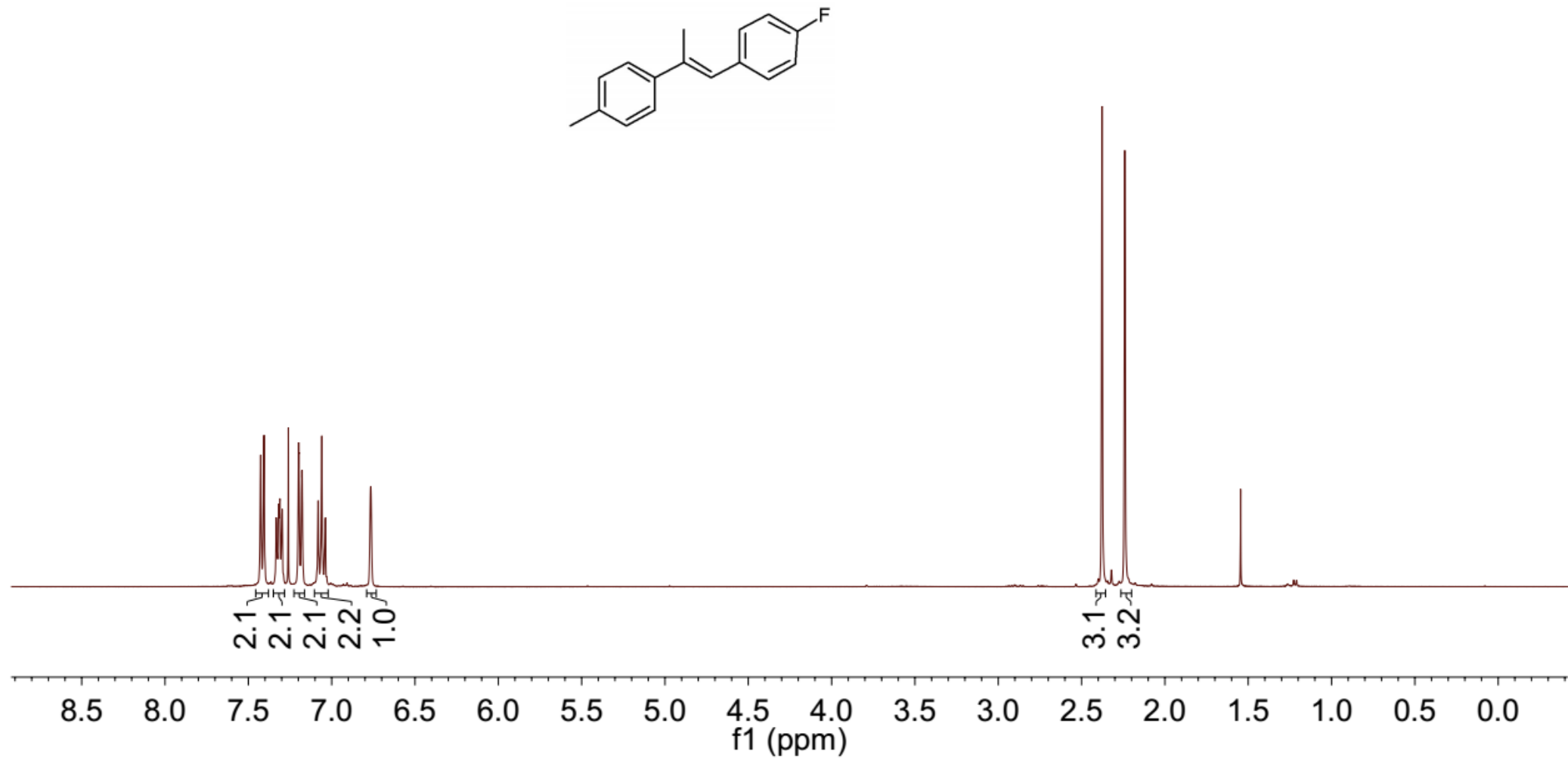


$2 a b$ E/Z

ก

웅ำ

ตं

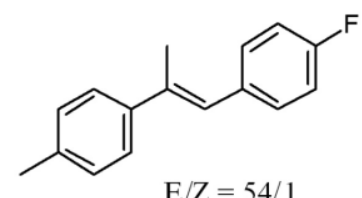

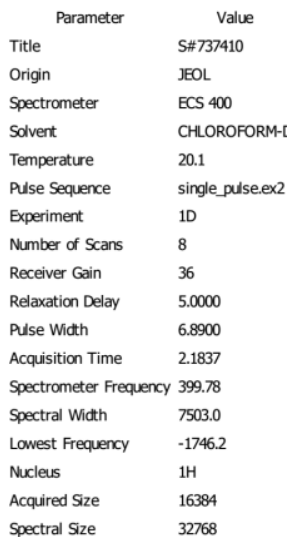

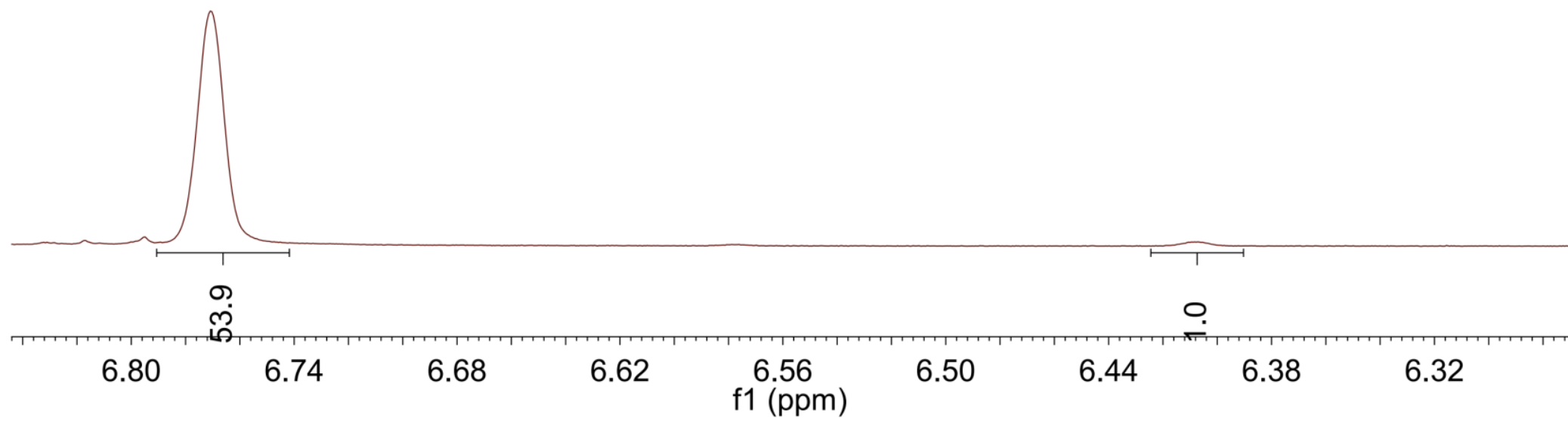




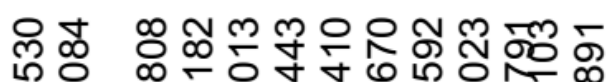

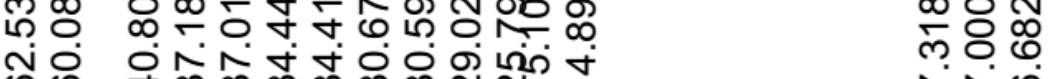

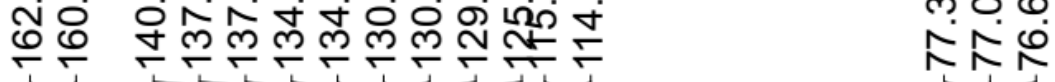

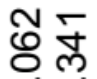

ஸे

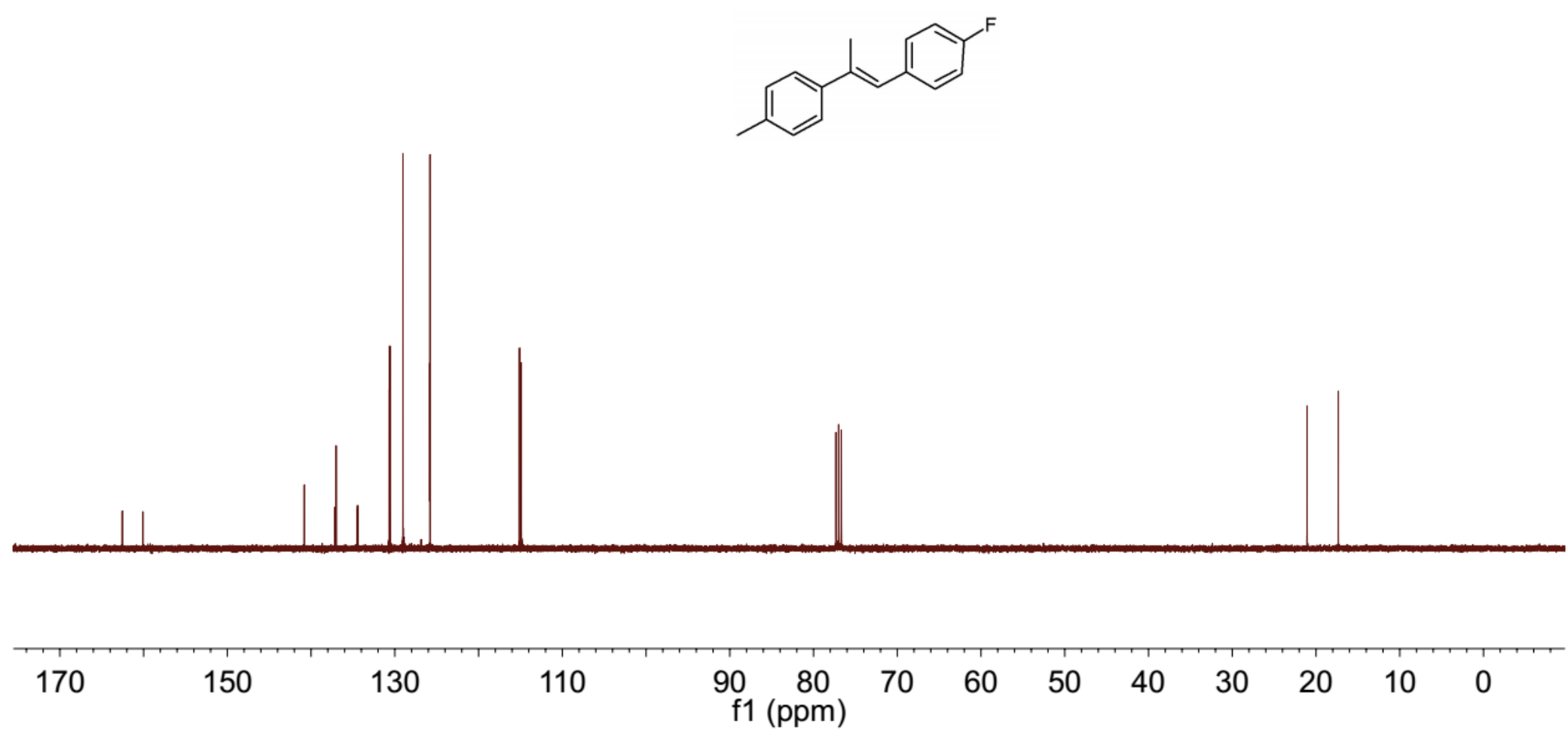



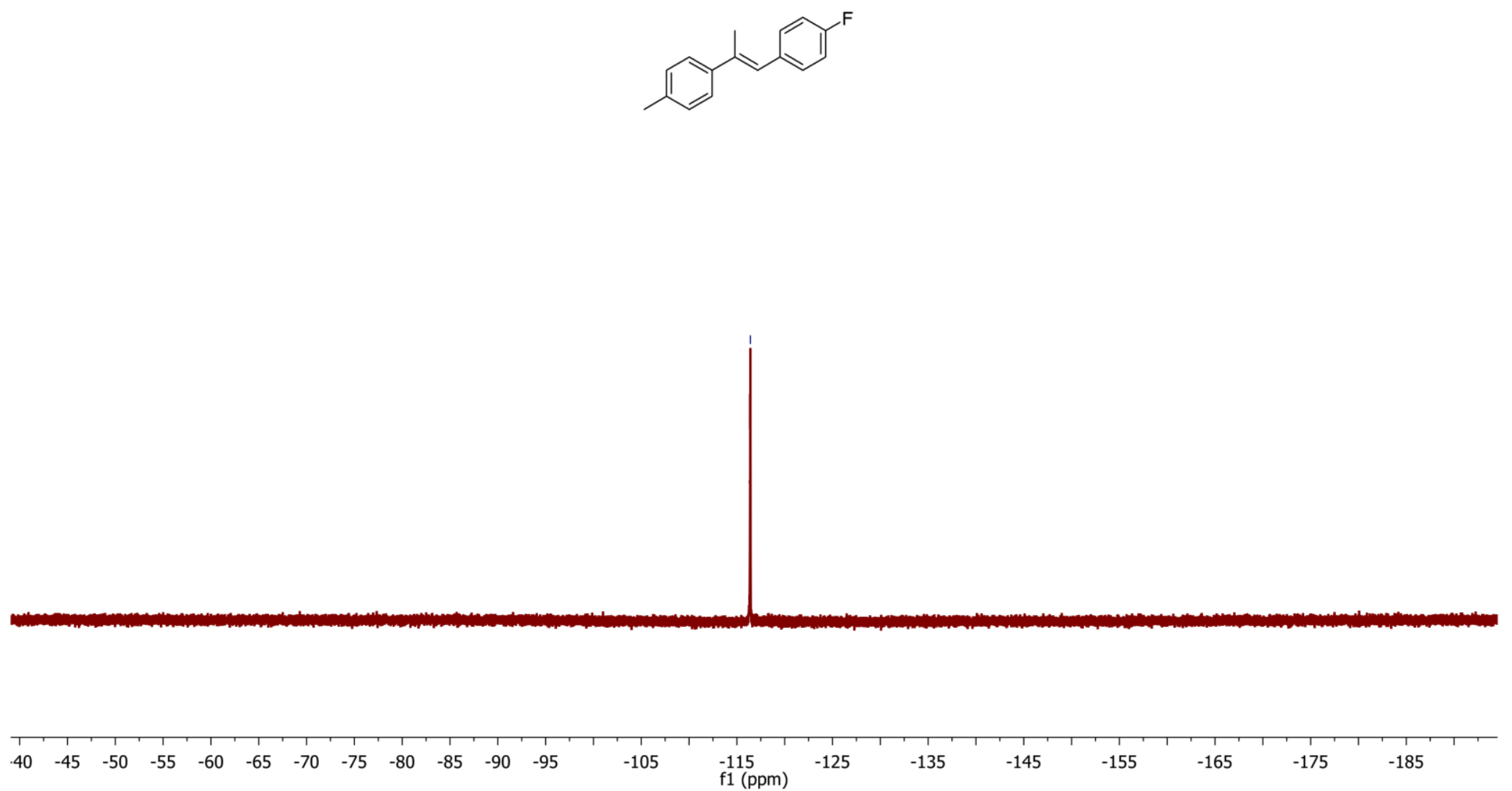


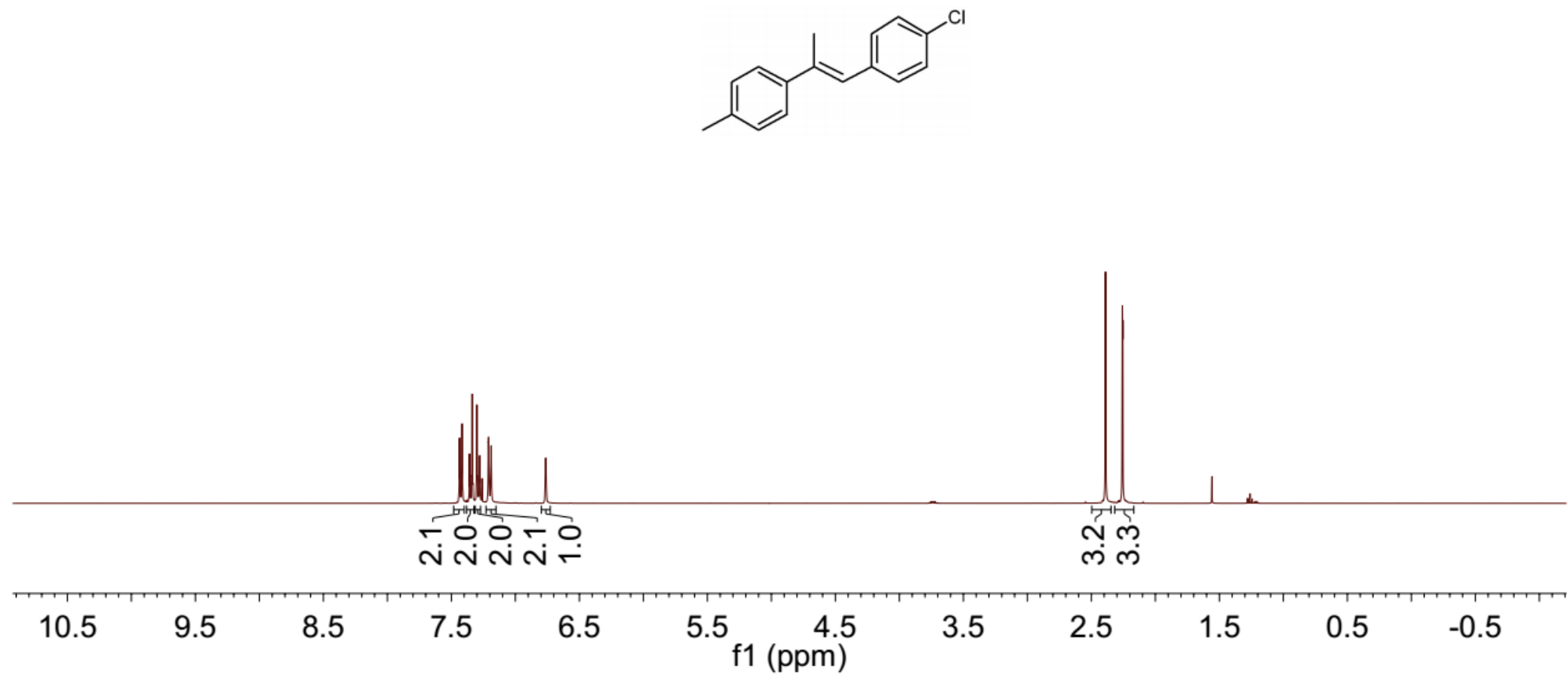


2ac E/Z

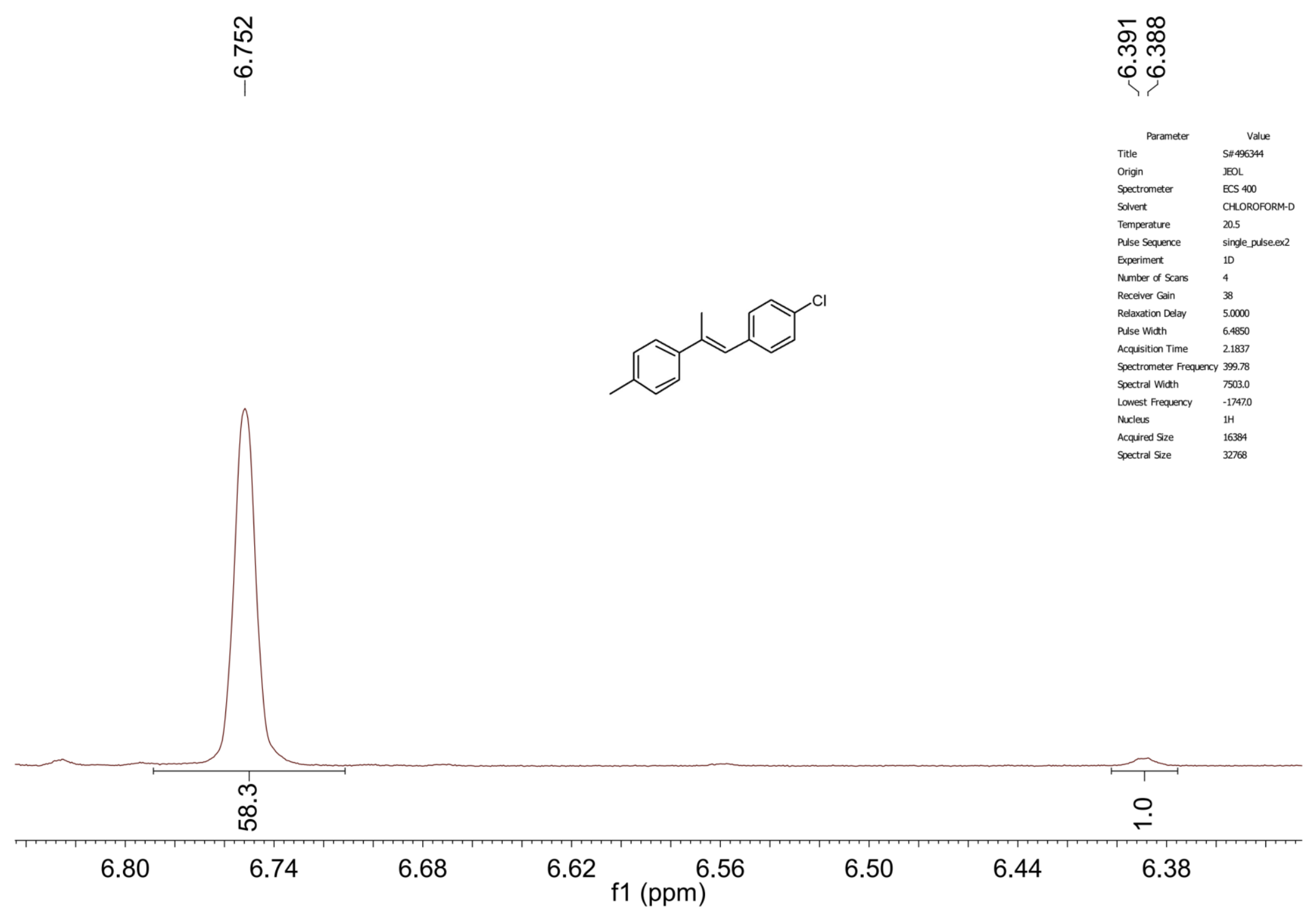




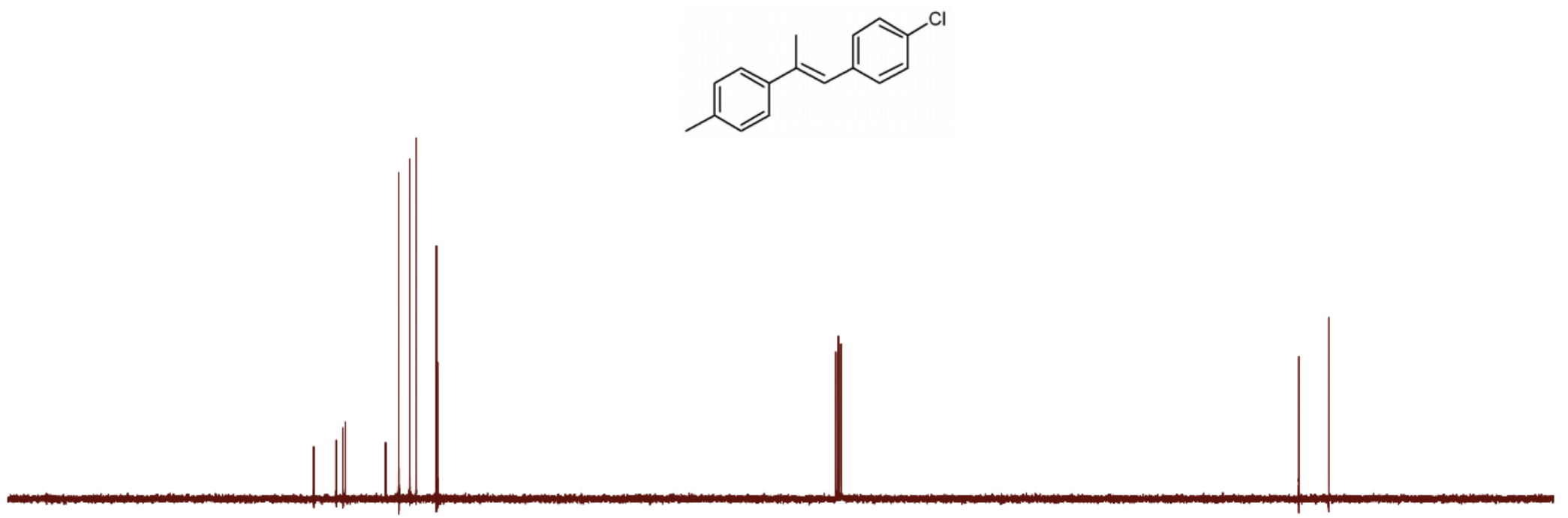

$\begin{array}{lllllllllllll}170 & 150 & 130 & 110 & \begin{array}{r}90 \\ \mathrm{f} 1(\mathrm{ppm})\end{array} & 70 & 60 & 50 & 40 & 30 & 20 & 10 & 0\end{array}$




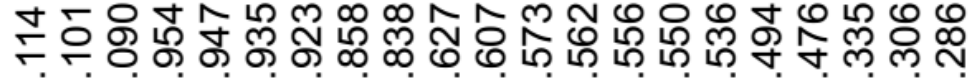

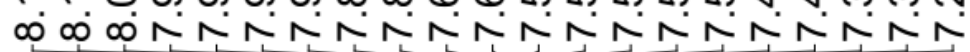

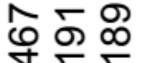

ขู่

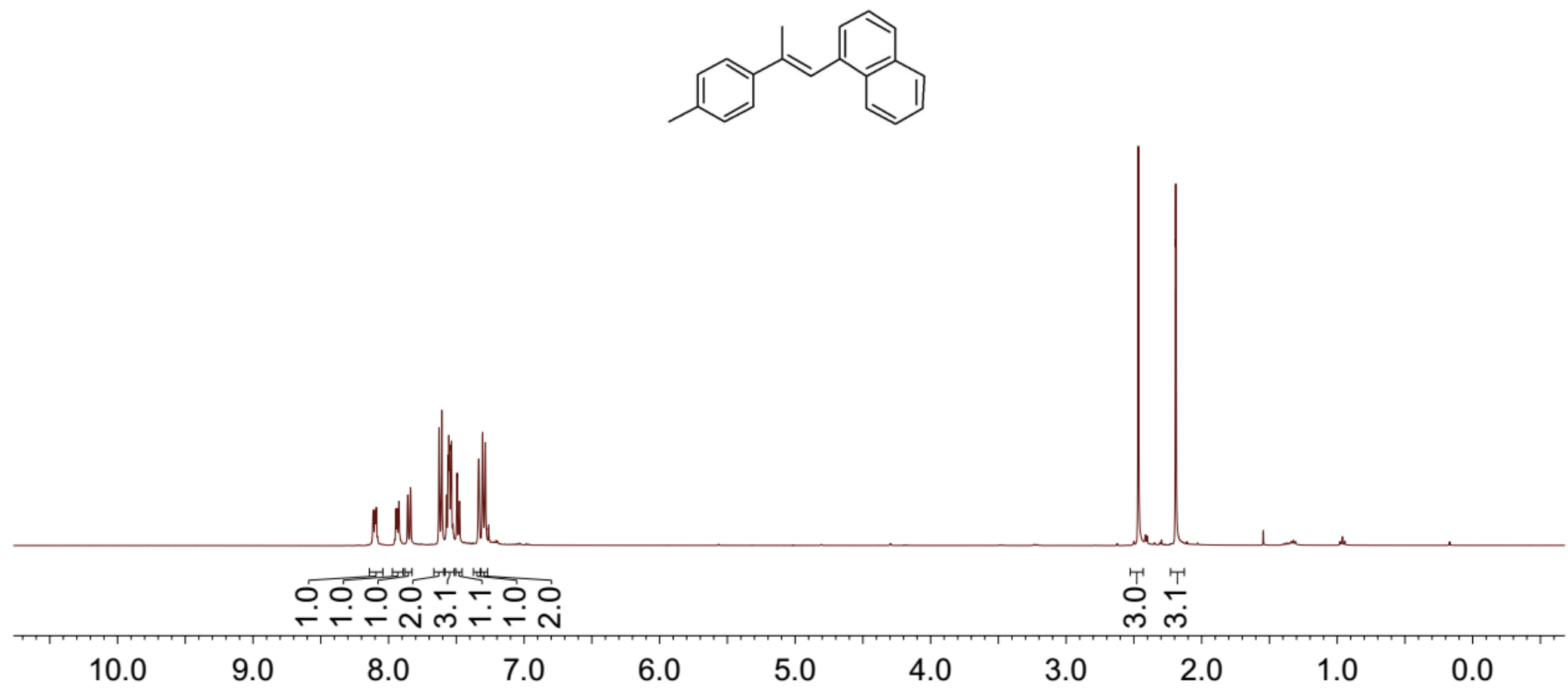


$\operatorname{2ad} E / Z$

$$
\text { ํㅠㄹㅠ }
$$

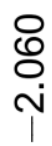
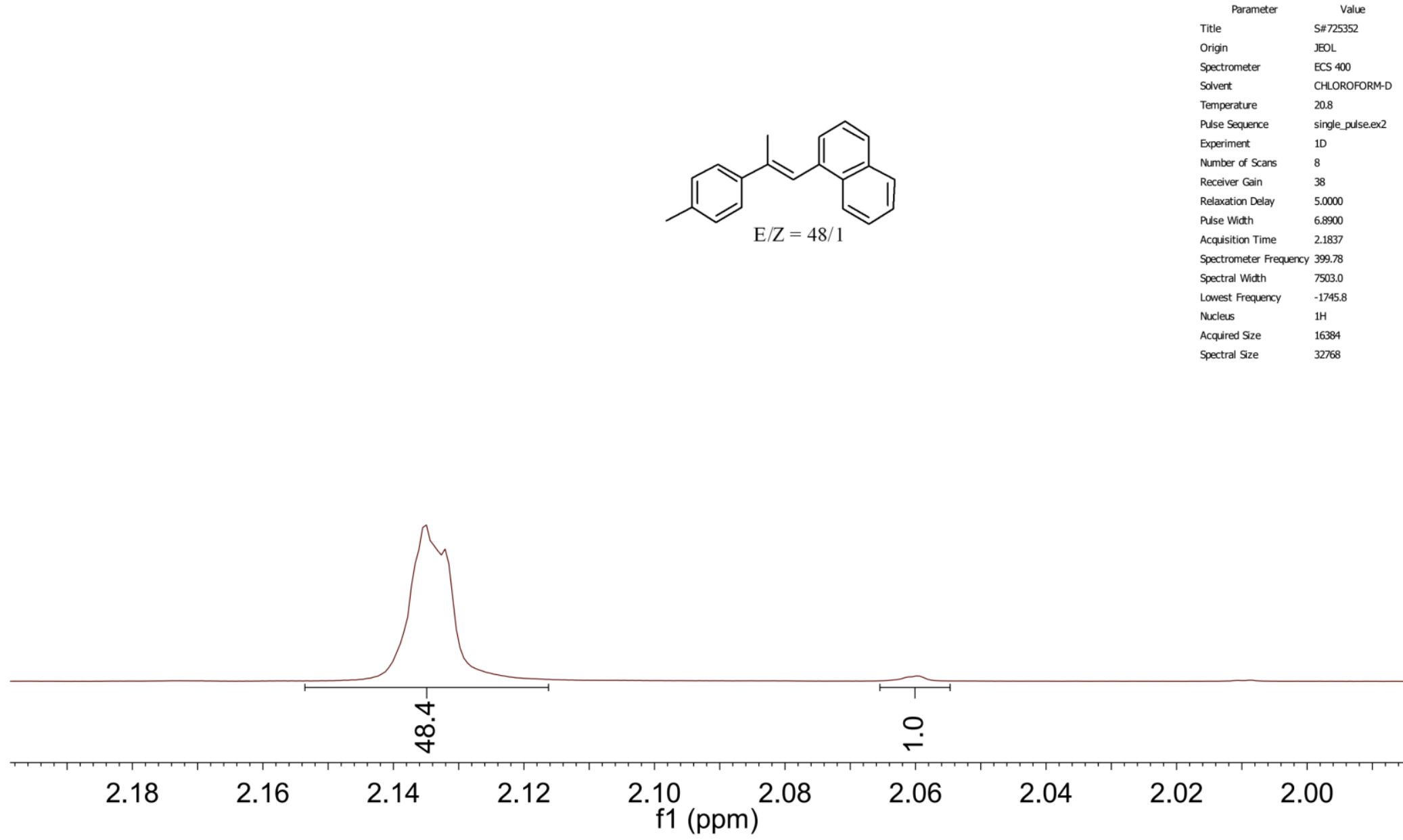

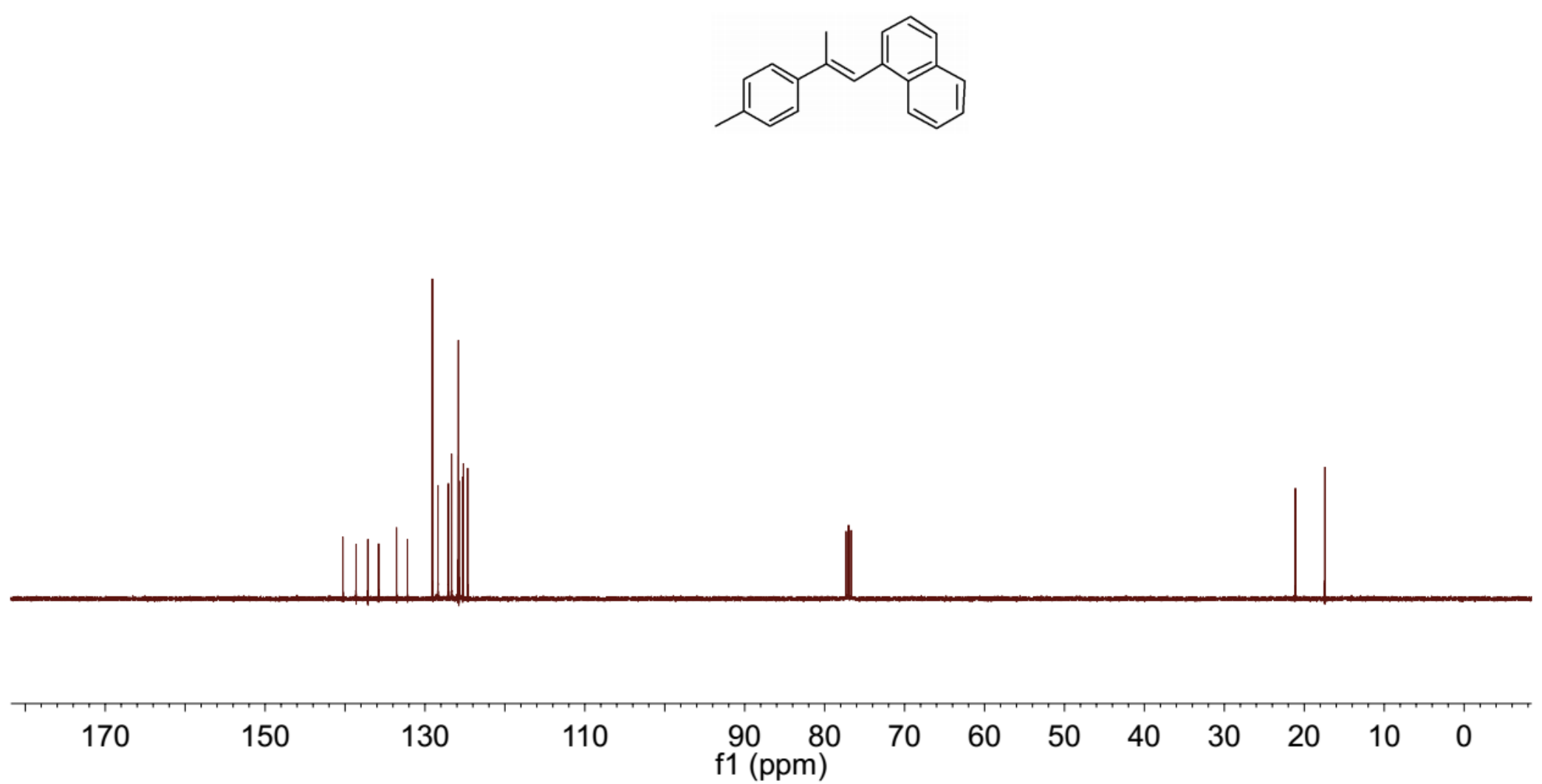

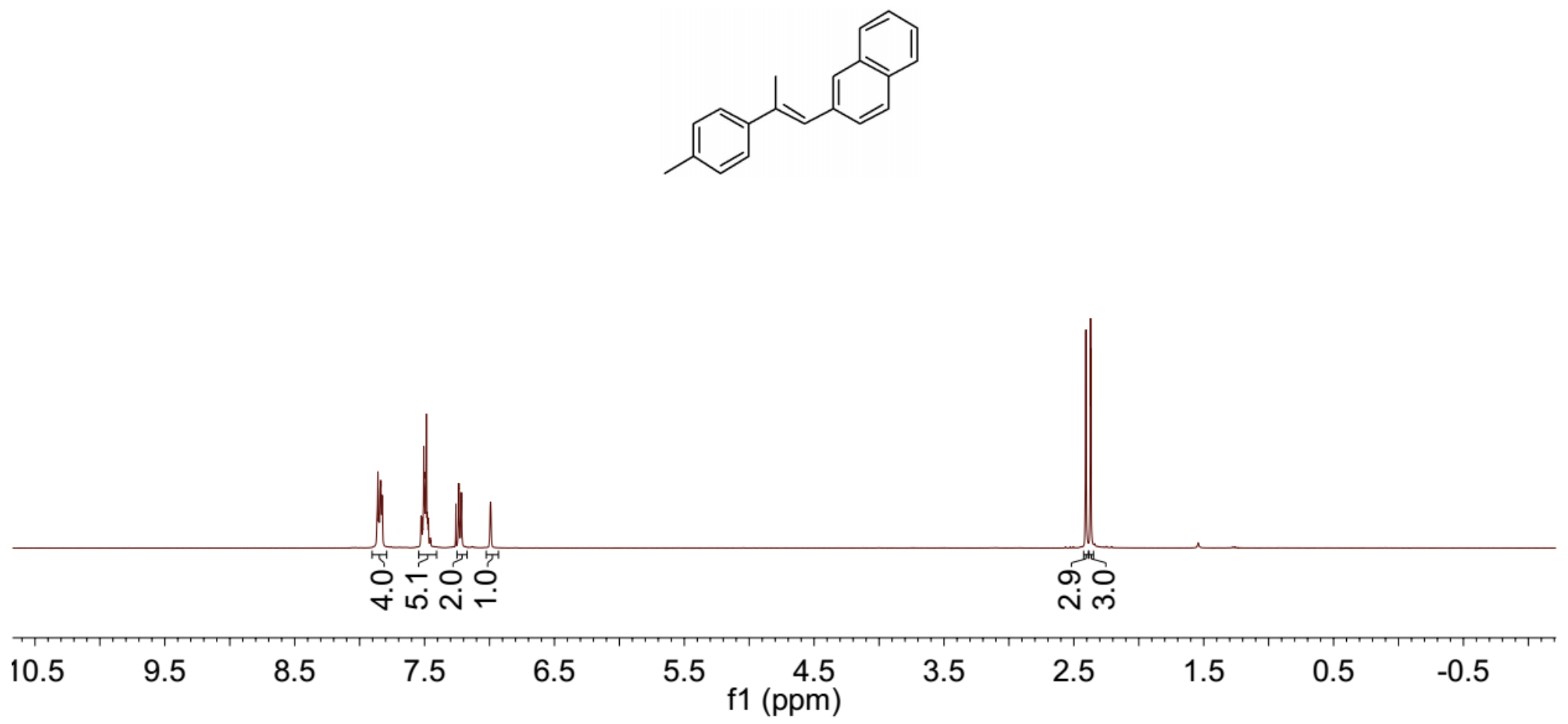
2ae E/Z

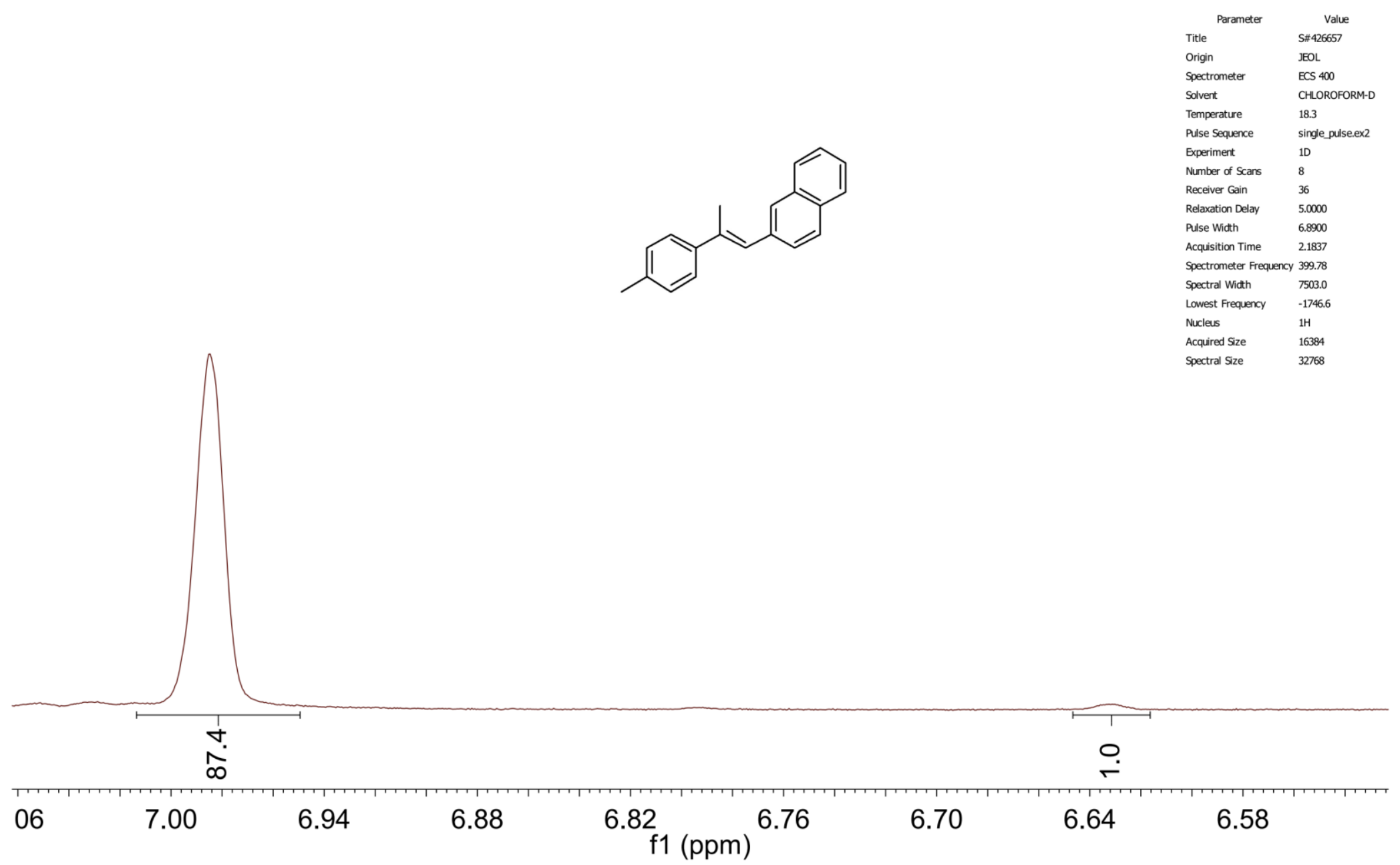




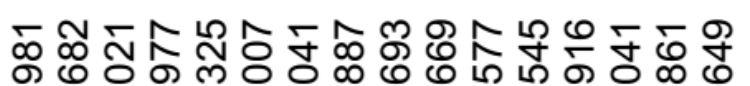

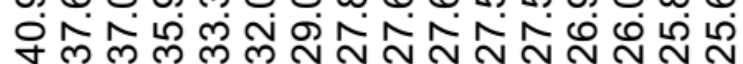

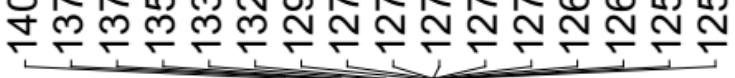

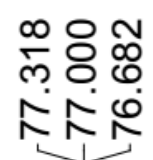

중ำ

ণิ
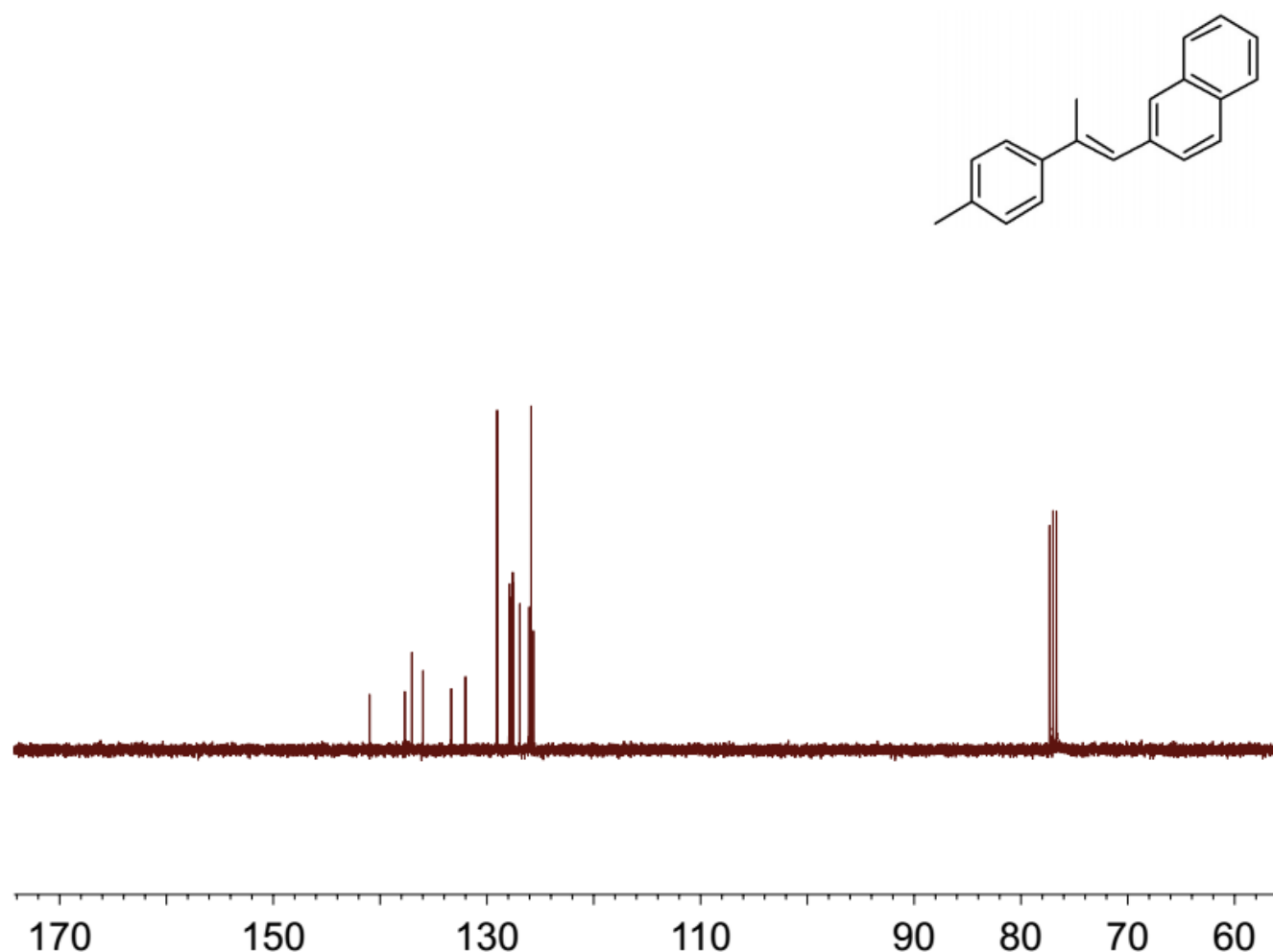

130

110

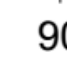

9080

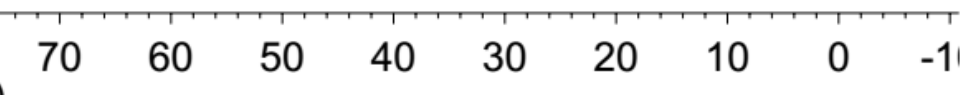


2af ${ }^{1} \mathrm{H}$ NMR

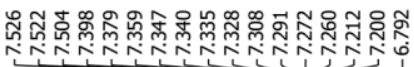

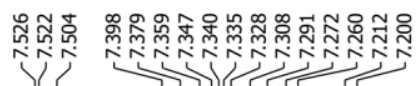
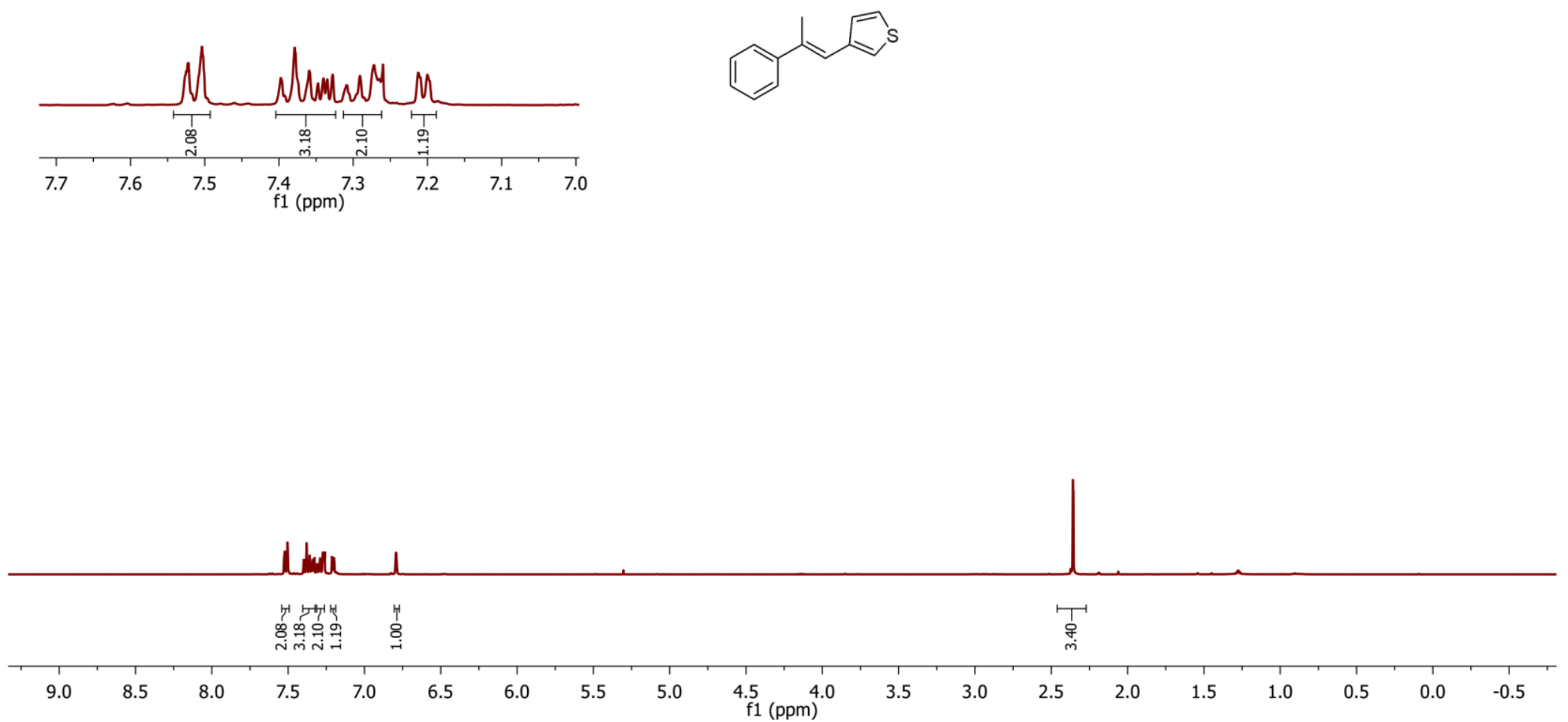
2af $E / Z$

$$
\begin{aligned}
& \text { ริ } \\
& \text { 1 }
\end{aligned}
$$

i

\begin{tabular}{|c|c|}
\hline Parameter & value \\
\hline Title & 1H_DB2238_2 \\
\hline Origin & JEOL \\
\hline spectrometer & ECS 400 \\
\hline Solvent & CHLOROFORM-D \\
\hline Temperature & 21.1 \\
\hline Pulse Sequence & single pulse.ex2 \\
\hline Experiment & 1D \\
\hline Number of Scars & 8 \\
\hline Receiver Gain & 34 \\
\hline Relaxation Delay & 5.0000 \\
\hline Pulse Widh & 6.4850 \\
\hline Acquisition Time & 2.1837 \\
\hline Spectrometer Frequency & 399.78 \\
\hline Spectral With & 6002.4 \\
\hline Lowest Frequency & -996.3 \\
\hline Nucleus & $1 H$ \\
\hline Acquired Size & 16334 \\
\hline & 430 \\
\hline
\end{tabular}

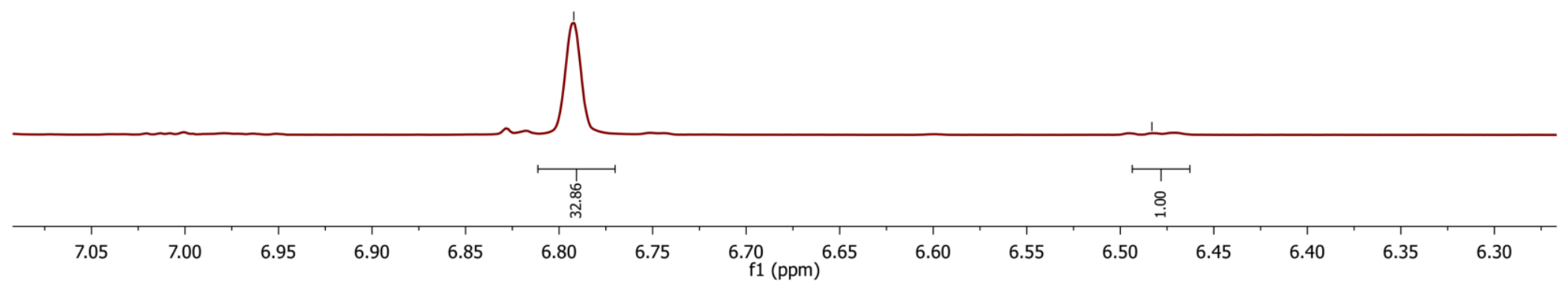


2af ${ }^{13} \mathrm{C}$ NMR

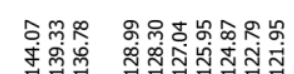

ज小

용

$\stackrel{\infty}{\infty}$

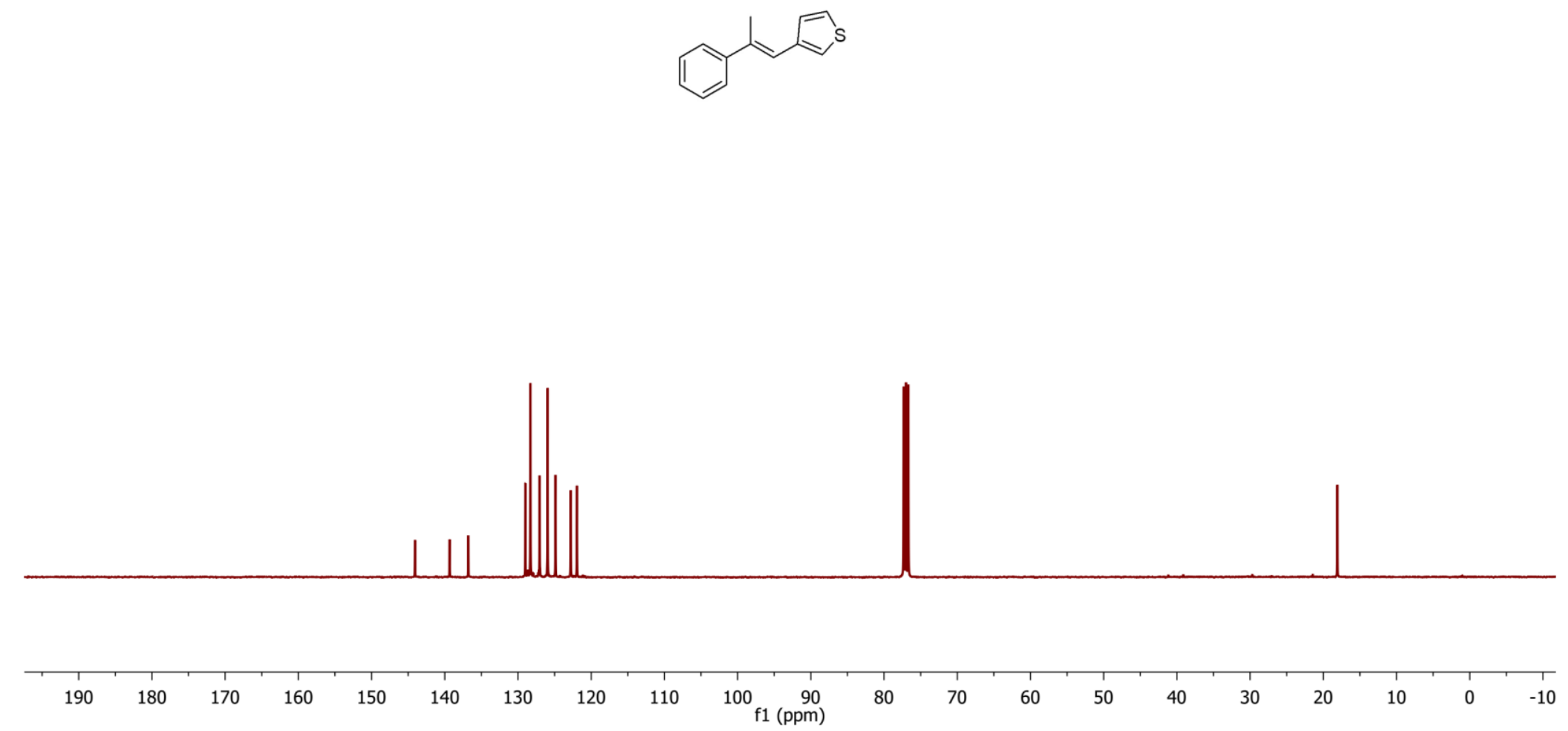


2ag ${ }^{1} \mathrm{H} N M R$

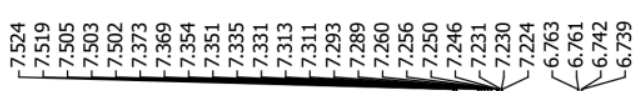

选

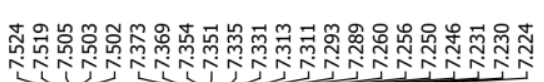
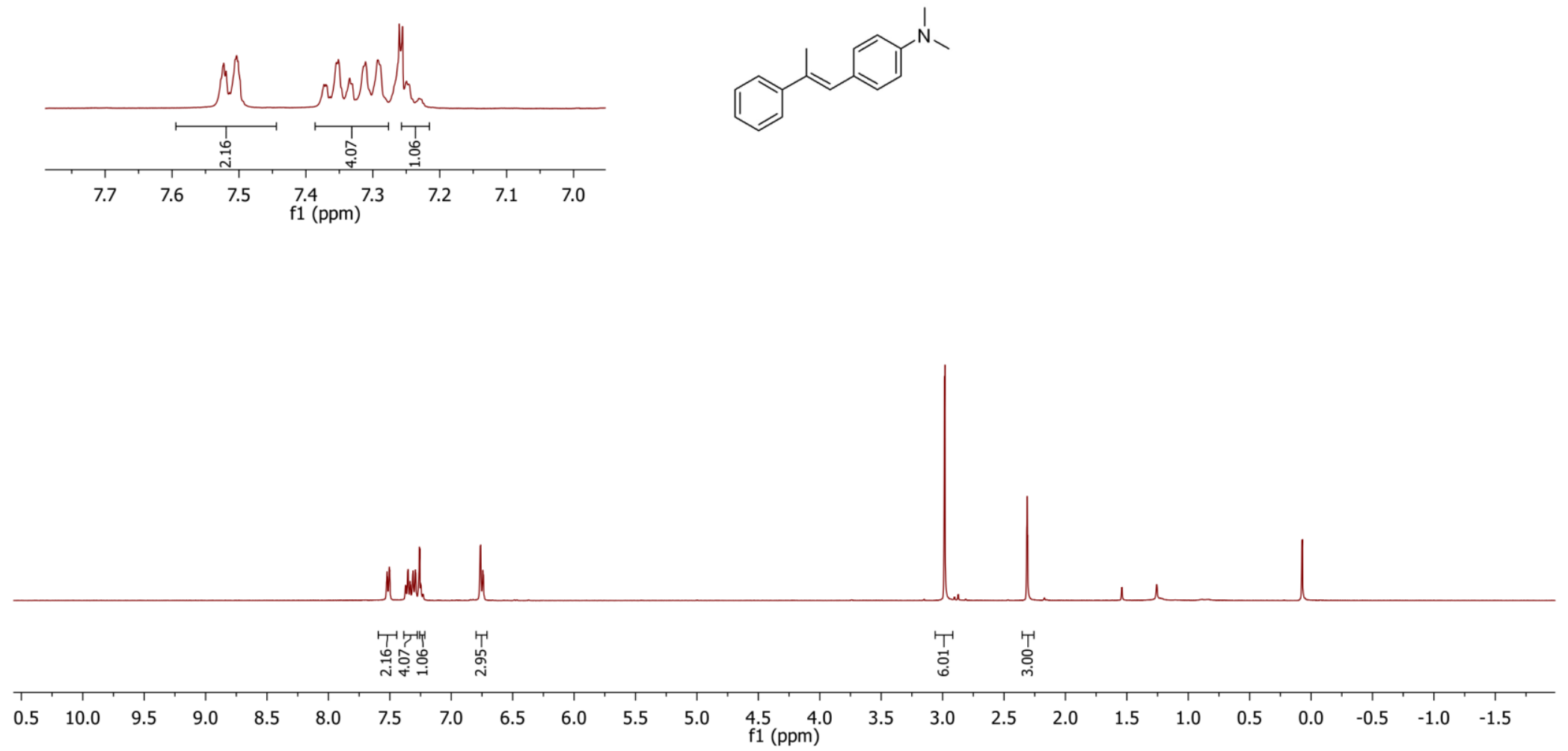

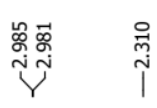

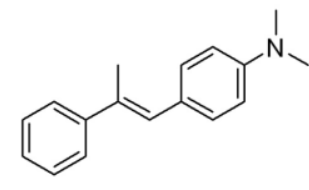


2ag $E / Z$

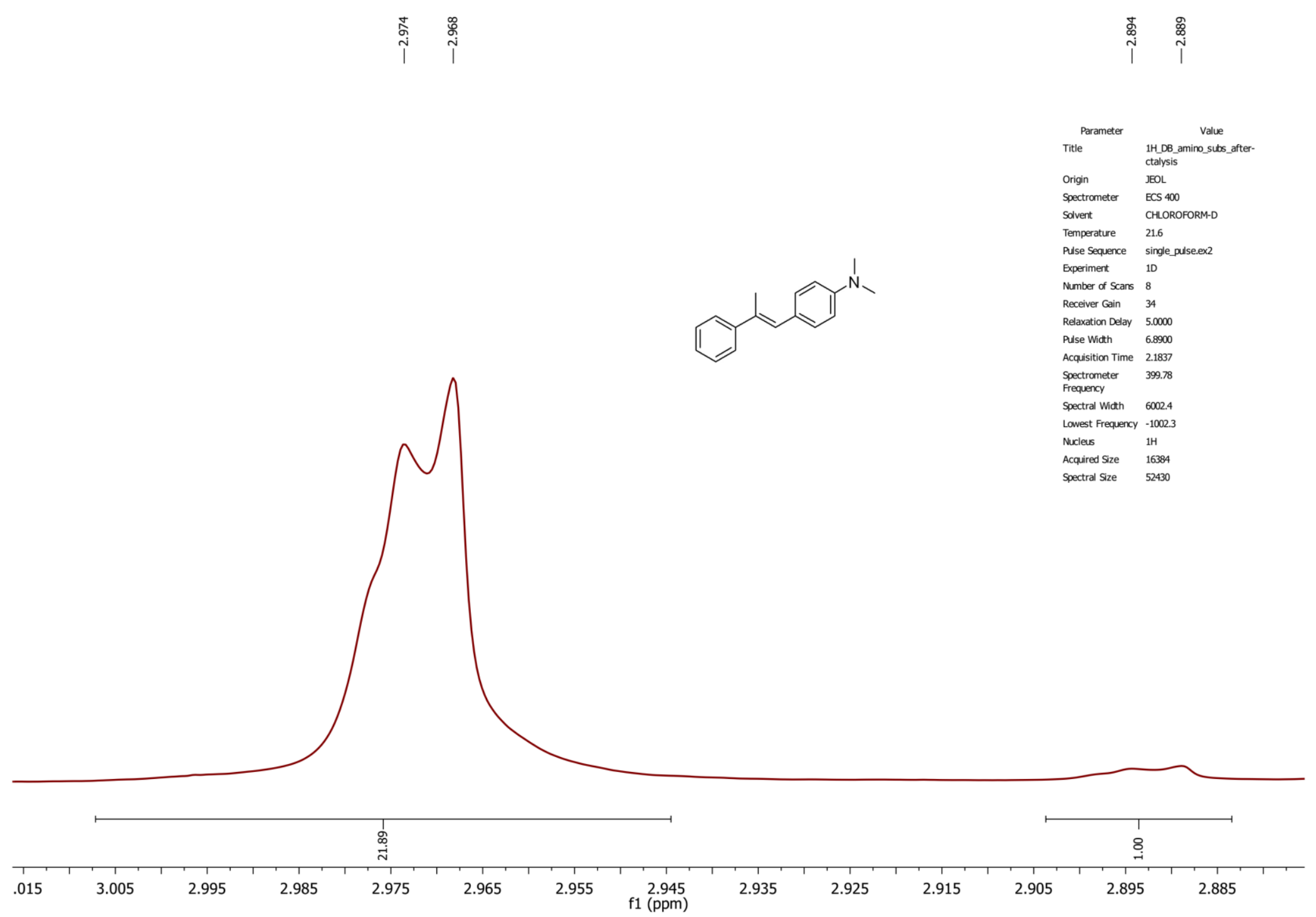


2ag ${ }^{13} \mathrm{C}$ NMR

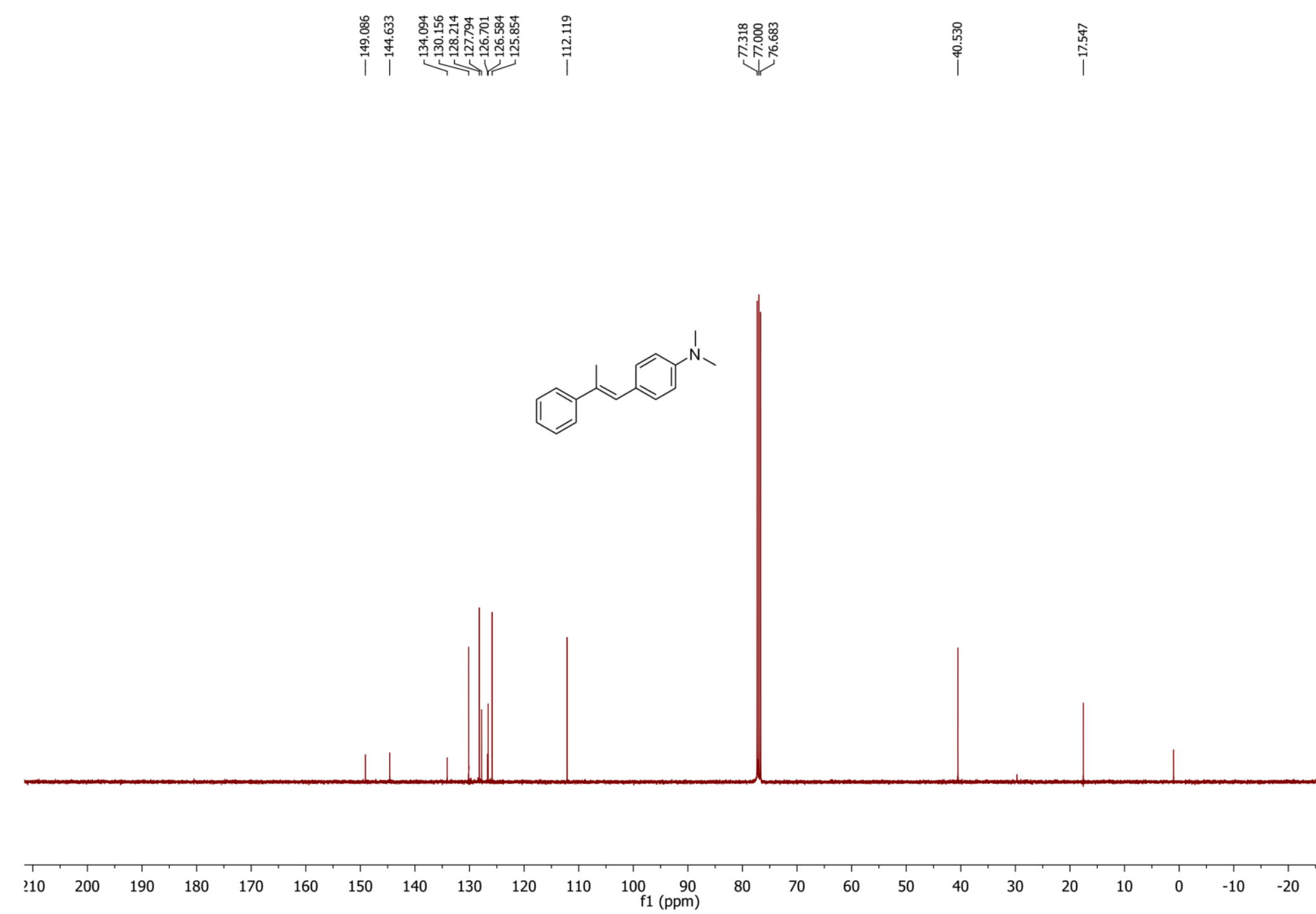


2ah crude product ${ }^{1} \mathrm{H}$ NMR

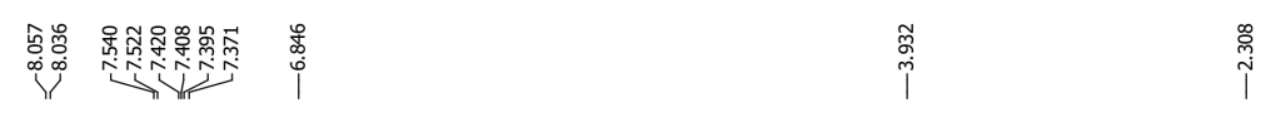
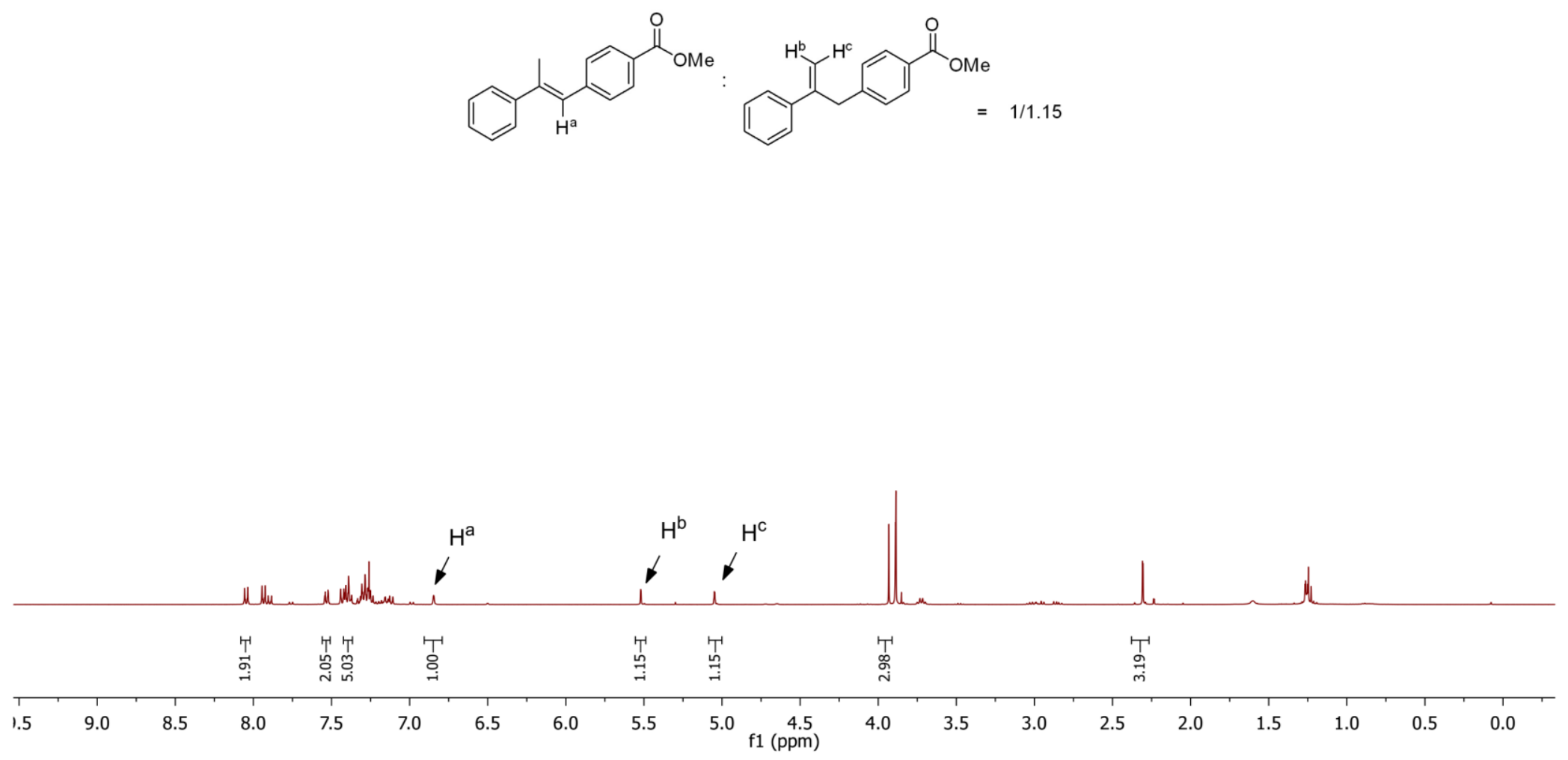
2ah E/Z

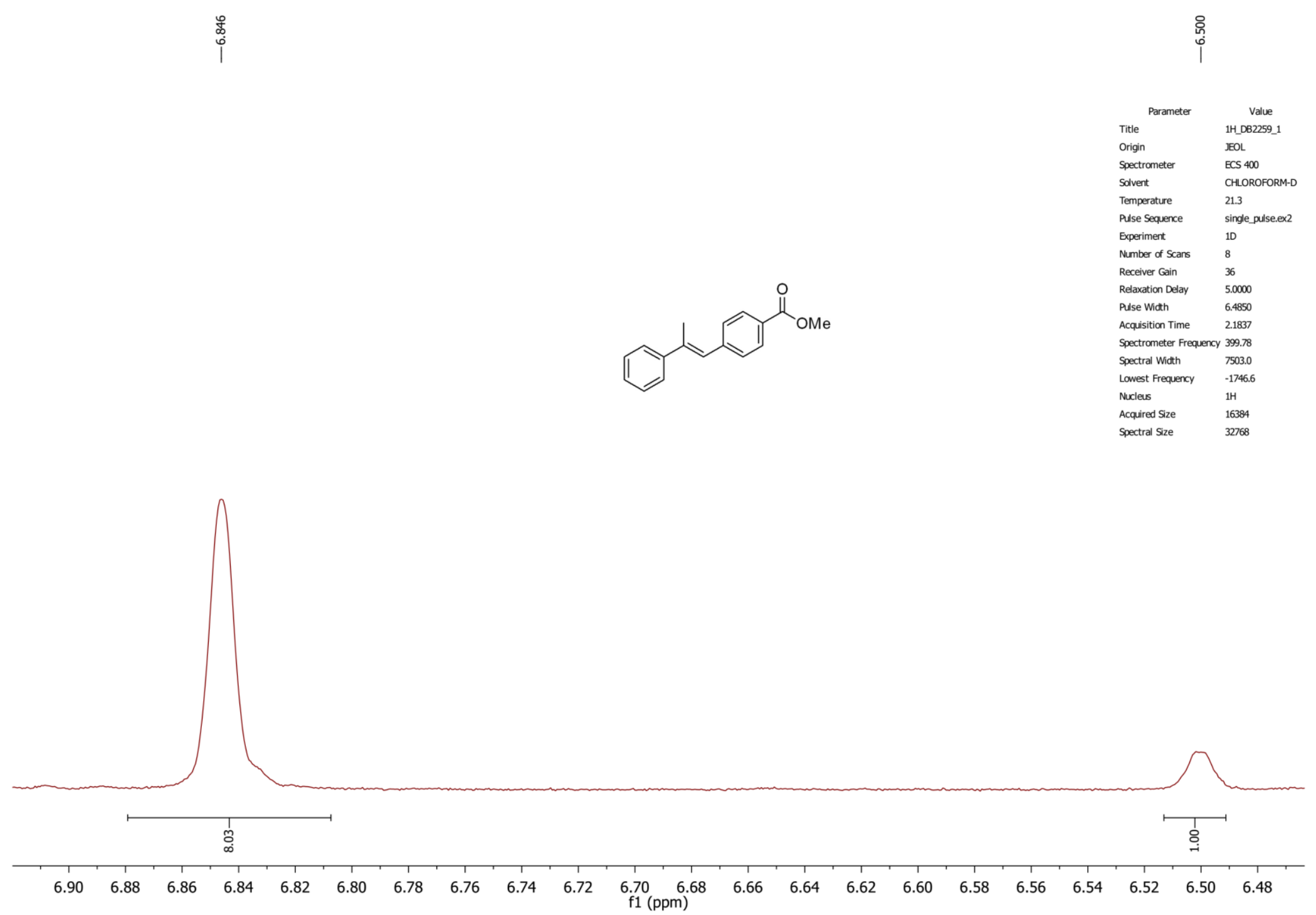




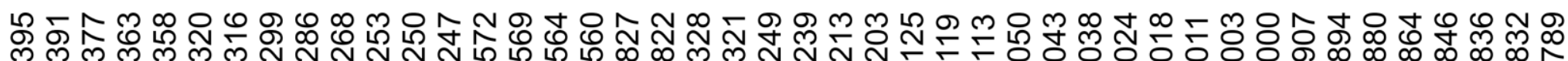

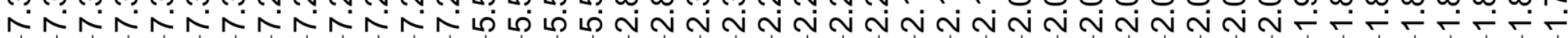

\begin{tabular}{|c|c|c|c|c|}
\hline 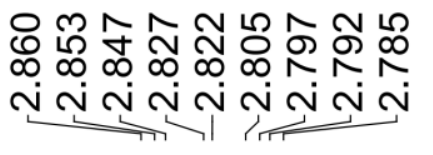 & $\begin{array}{l}\text { ్ְ } \\
\text { ల్ } \\
\text { i }\end{array}$ & $\begin{array}{l}\stackrel{\infty}{N} \underset{N}{m} \\
\underset{y}{\sim}\end{array}$ & 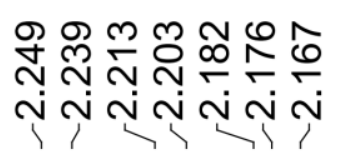 & 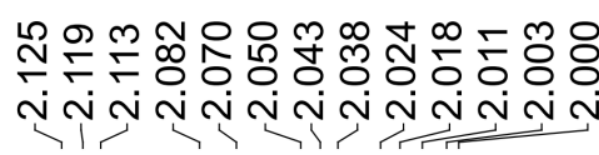 \\
\hline
\end{tabular}
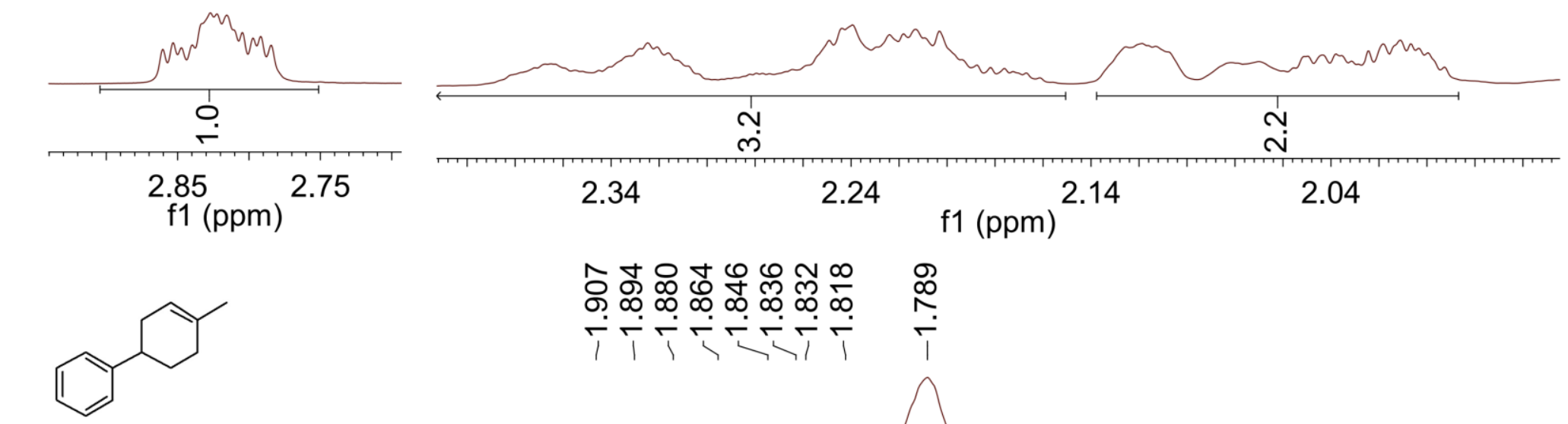

গิ용요
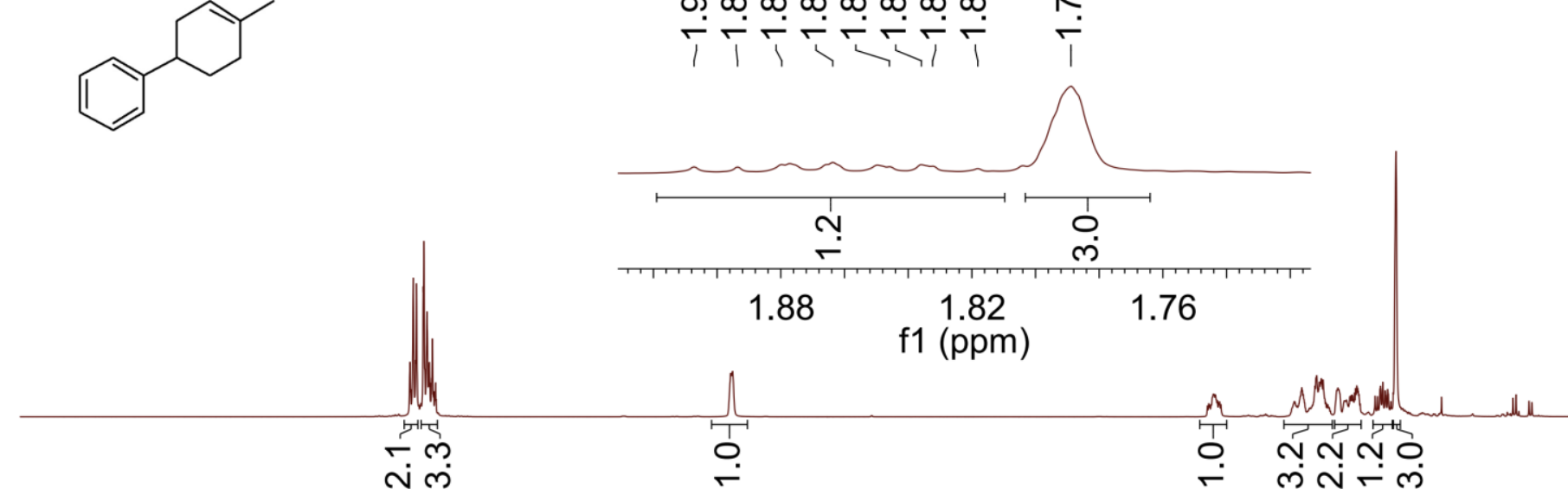

9.5

$\begin{array}{llll}9.0 & 8.5 & 8.0 & 7.5\end{array}$

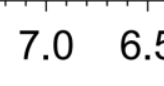

$5.5 \quad 5.0$

$\begin{array}{ll}5.0 & 4.5 \\ \mathrm{f} 1(\mathrm{ppm})\end{array}$ 


\begin{tabular}{|c|c|}
\hline 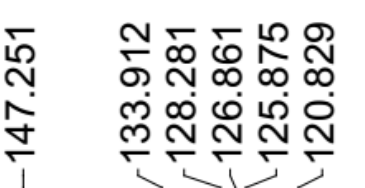 & 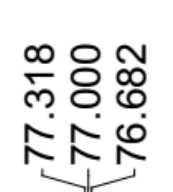 \\
\hline
\end{tabular}

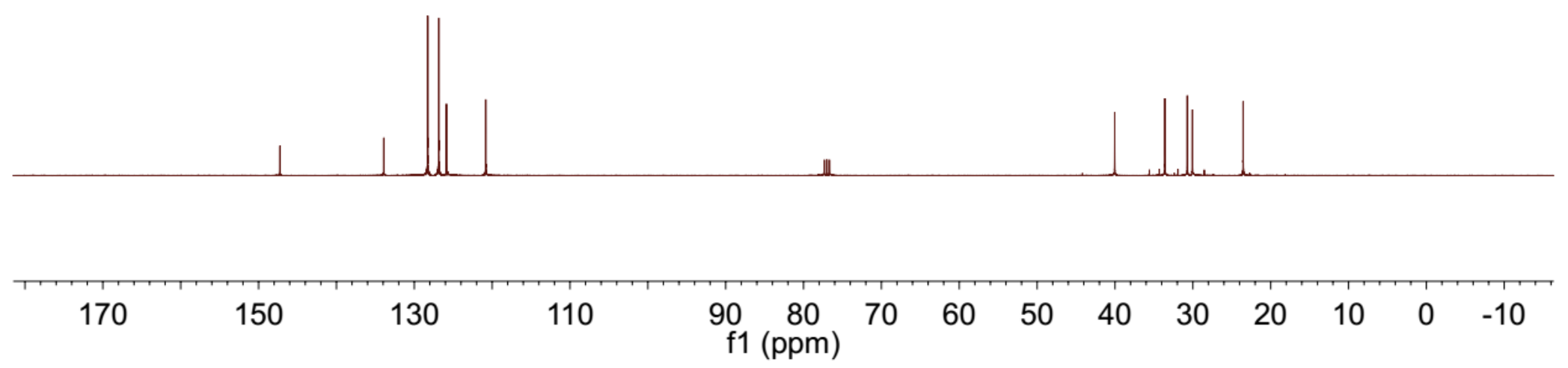




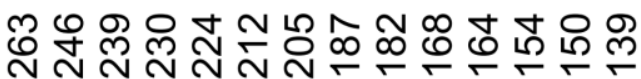

ñNañNañ
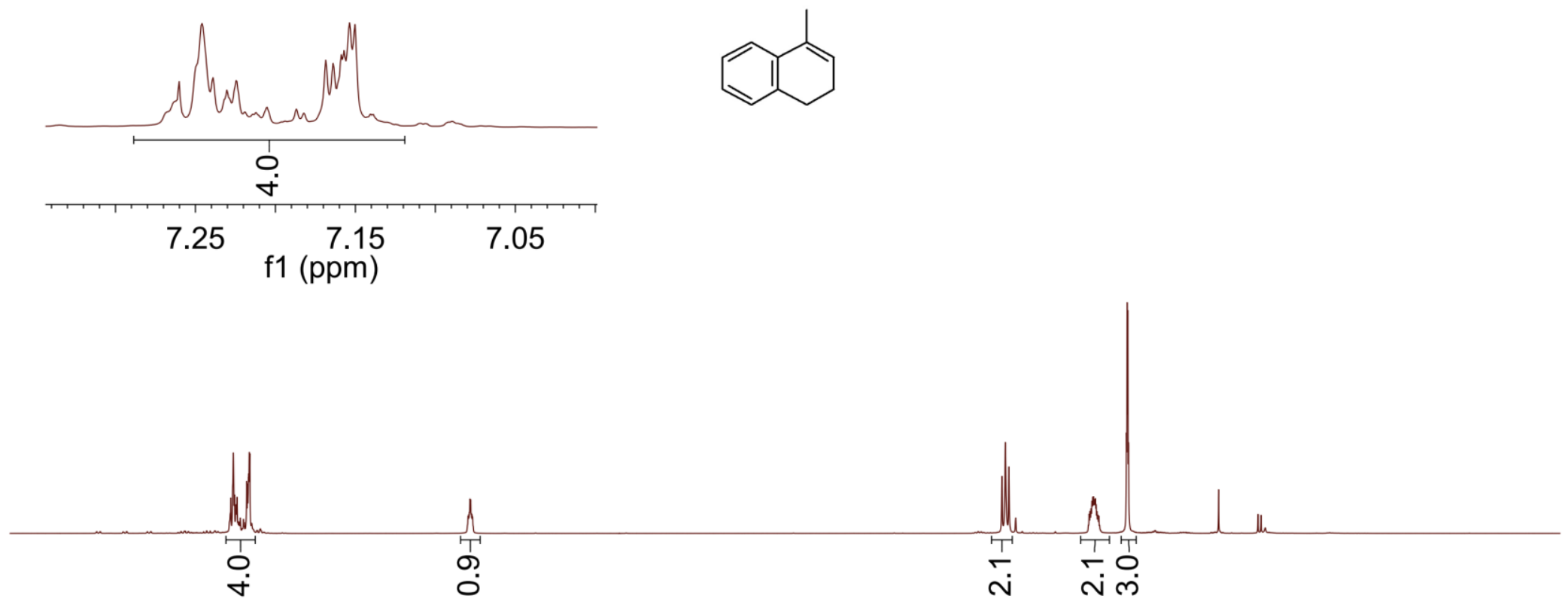

$\begin{array}{llllllllllllllllll}.5 & 8.0 & 7.5 & 7.0 & 6.5 & 6.0 & 5.5 & 5.0 & 4.5 \underset{\mathrm{f} 1(\mathrm{ppm})}{4.0} & 3.5 & 3.0 & 2.5 & 2.0 & 1.5 & 1.0 & 0.5 & 0.0\end{array}$ 
2aj ${ }^{13} \mathrm{C}$ NMR

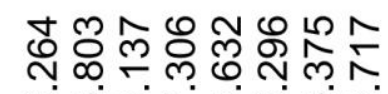

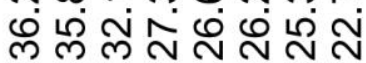

승용

रำ

동 윰용

ஸे ๗ํำ

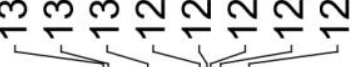

证

TָT
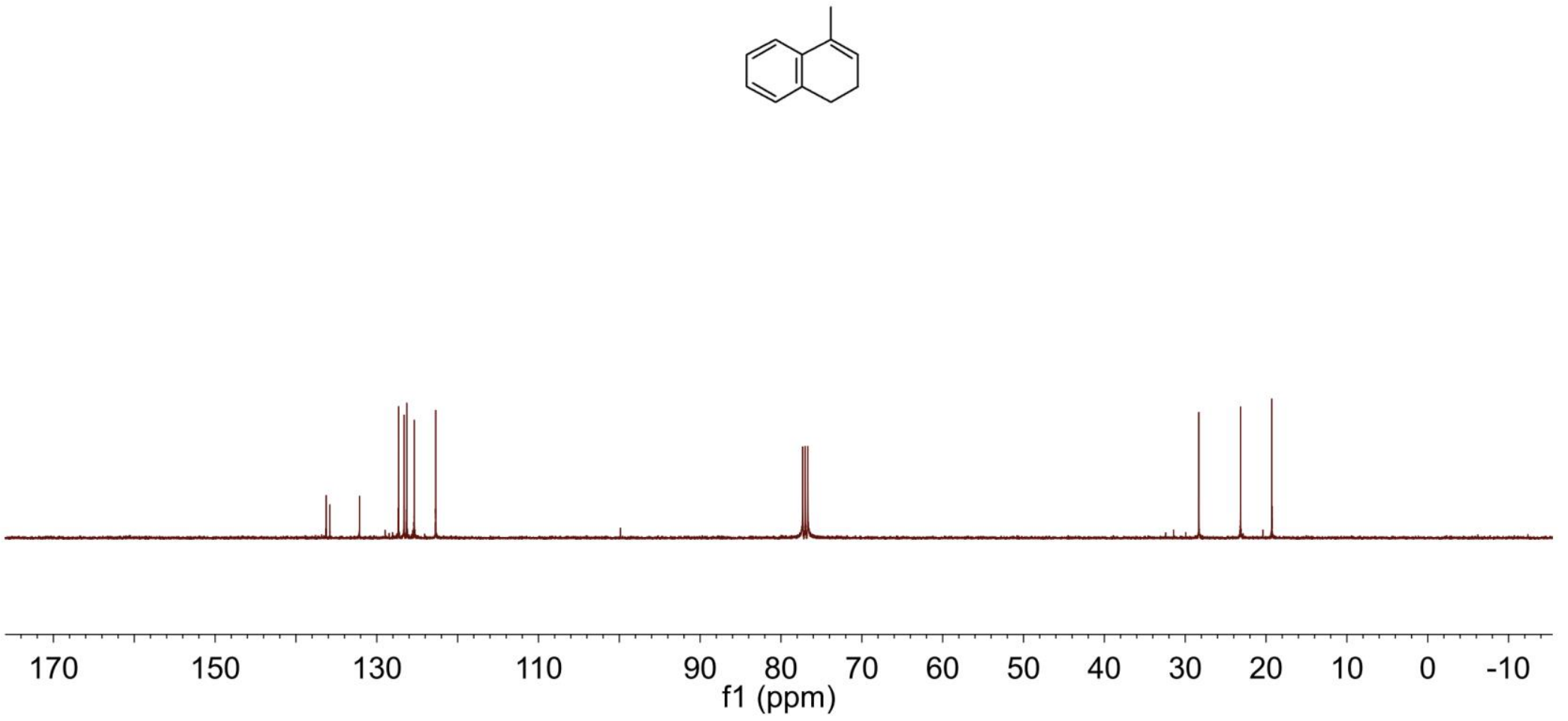

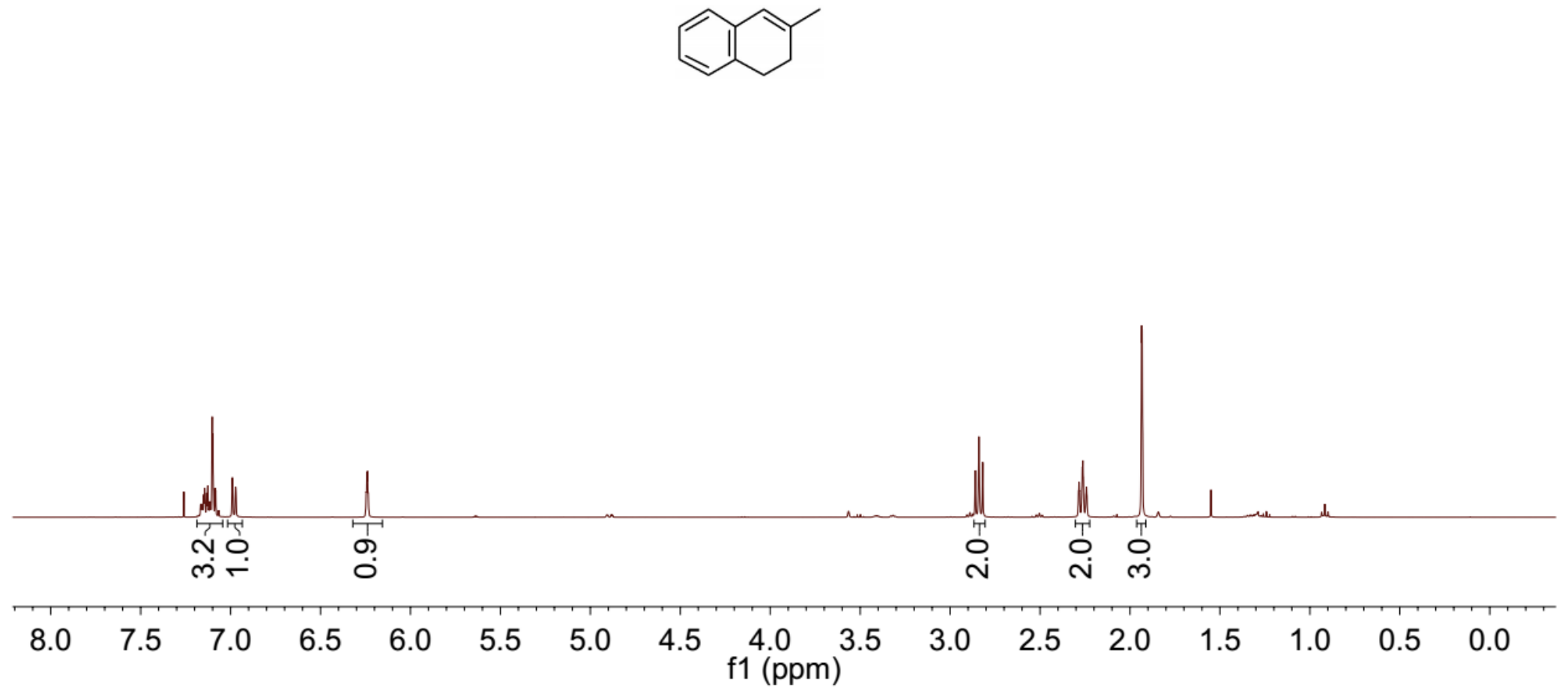
2ak regioselectivity

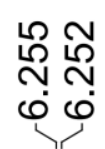

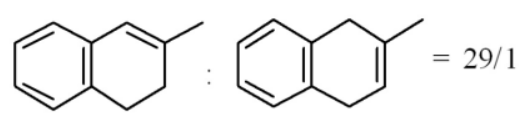

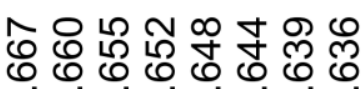

"ما

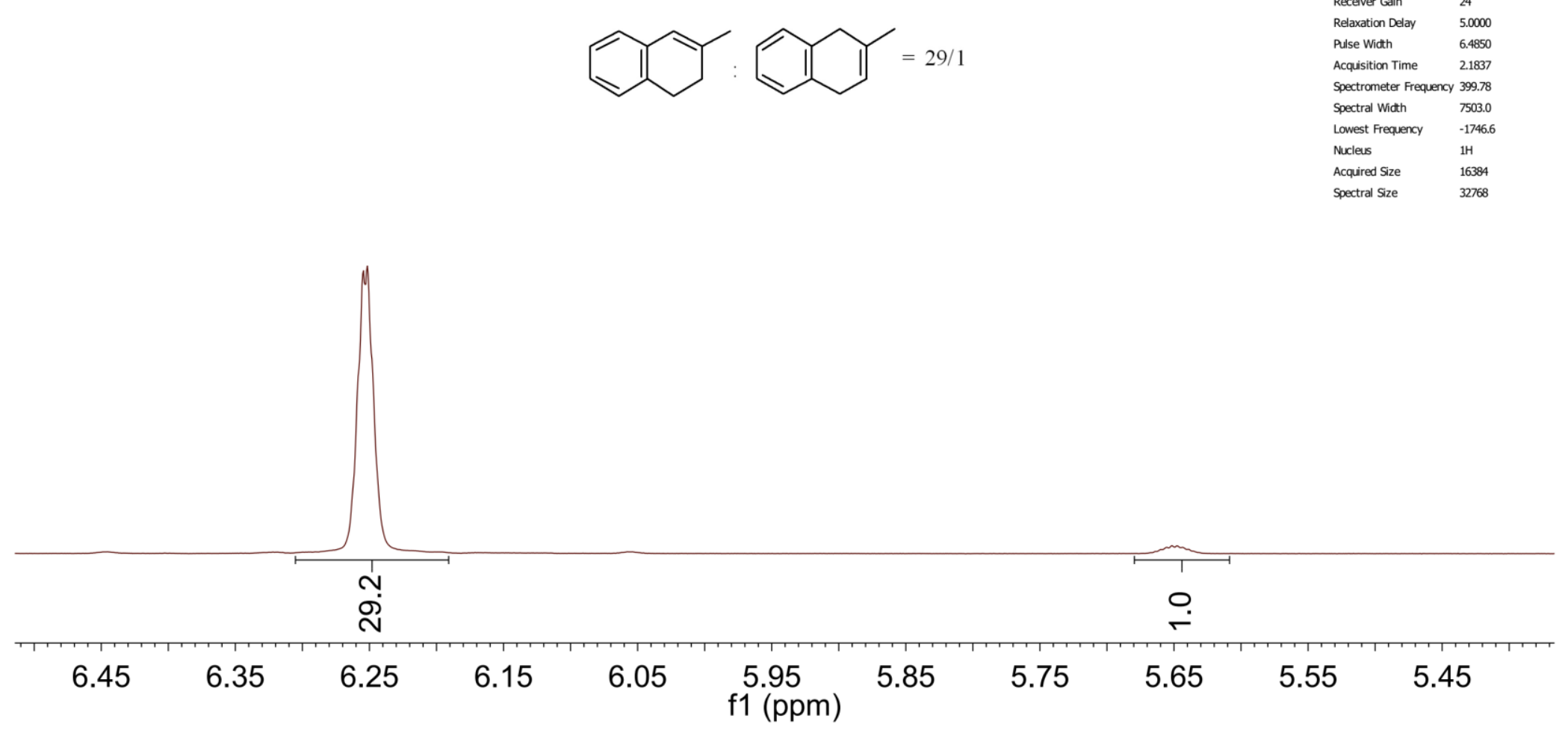

$\begin{array}{cc}\text { Parameter } & \begin{array}{c}\text { Value } \\ \mathrm{S} \# 627636\end{array} \\ \text { Title } & \end{array}$ Origin JEOL $\begin{array}{ll}\text { Spectrometer } & \text { ECS } 400 \\ \text { Solvent } & \text { CHLPOFOPM-D }\end{array}$ Temperature 223 Pulse Sequence single pulse Experiment 10 


\begin{tabular}{|c|c|c|}
\hline 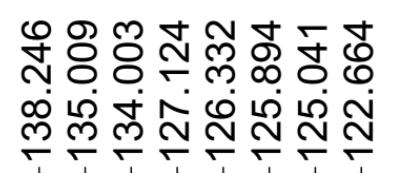 & 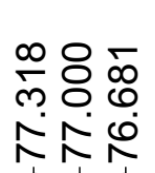 & 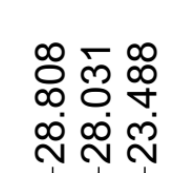 \\
\hline
\end{tabular}
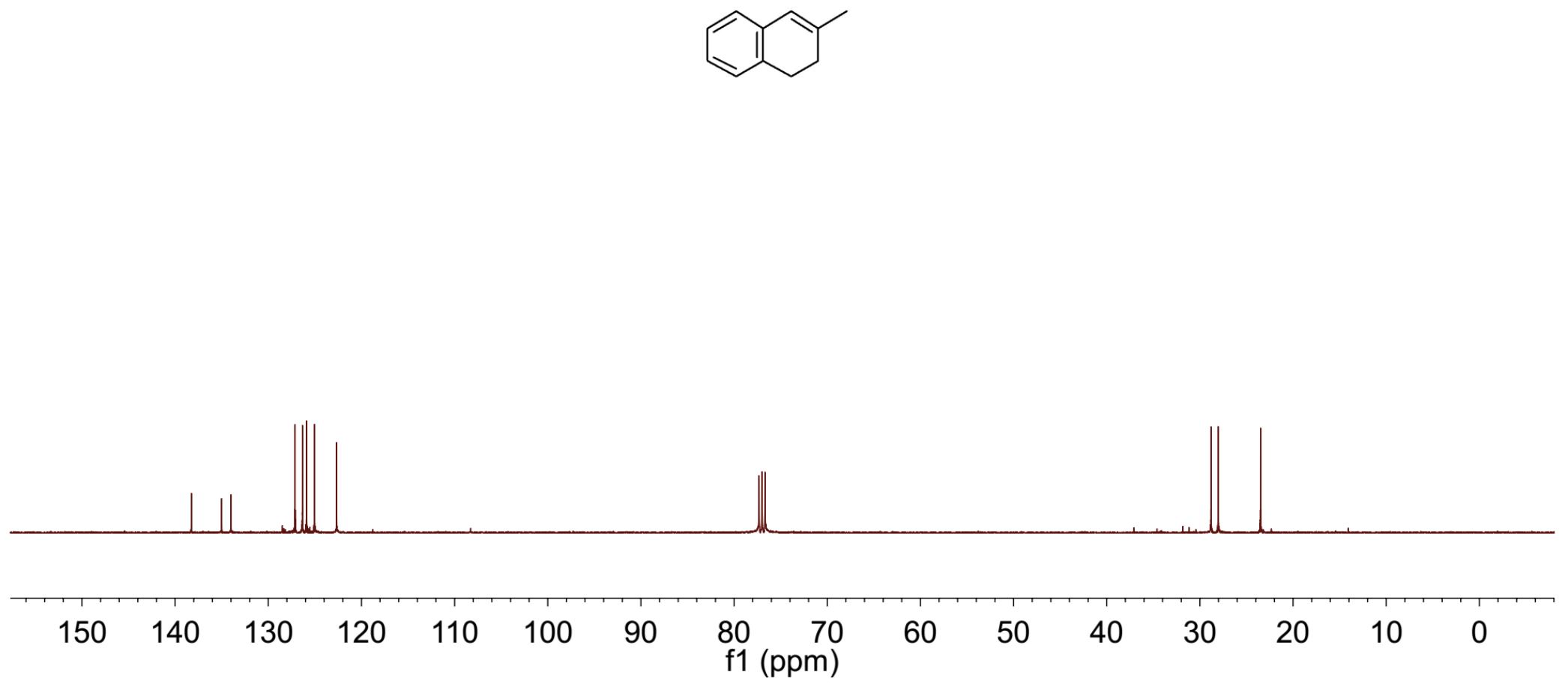
2al ${ }^{1} \mathrm{H}$ NMR

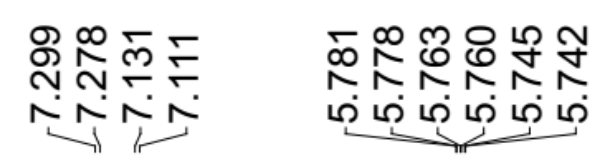

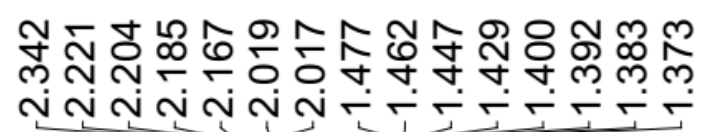

תN
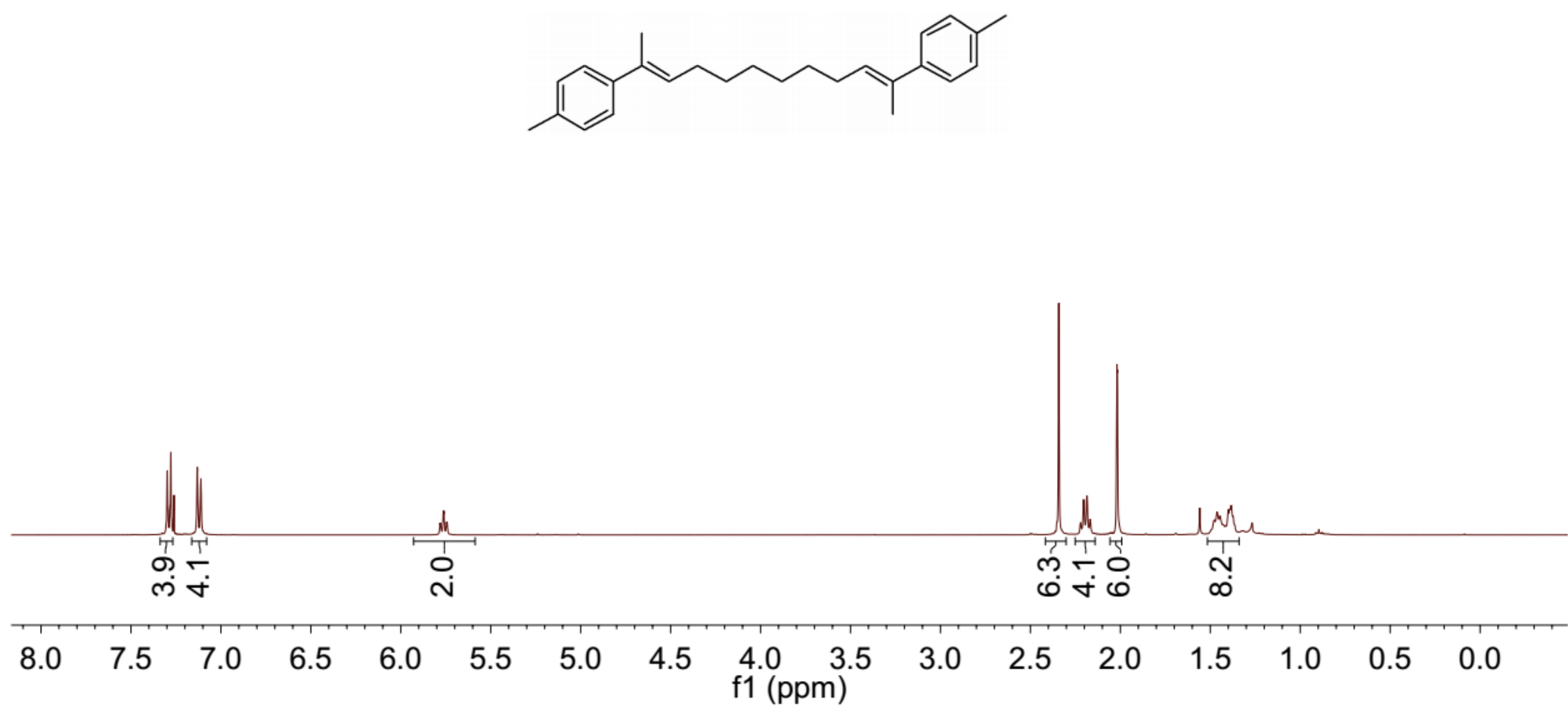
2al E/Z

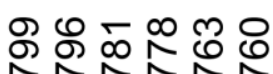

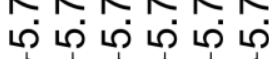 ) ?}

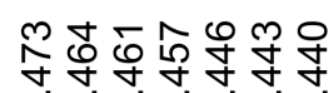

ما

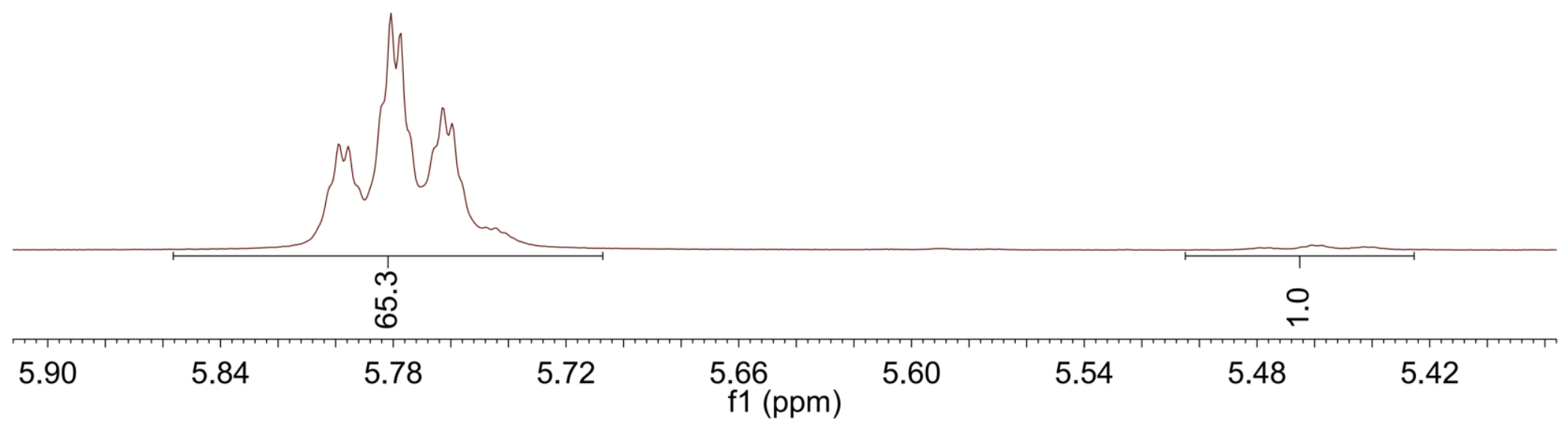


m。

송

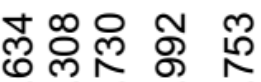

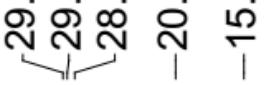
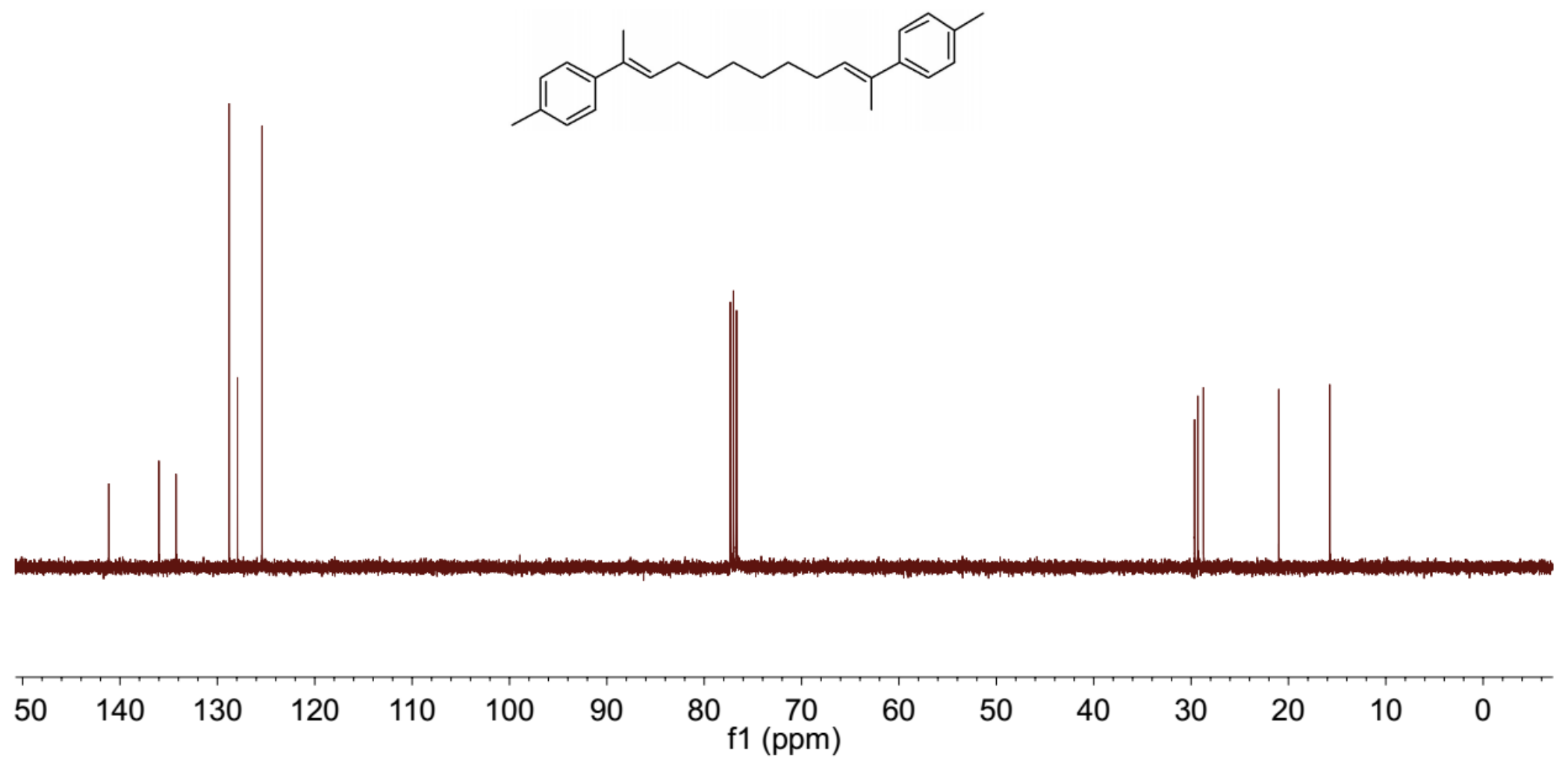


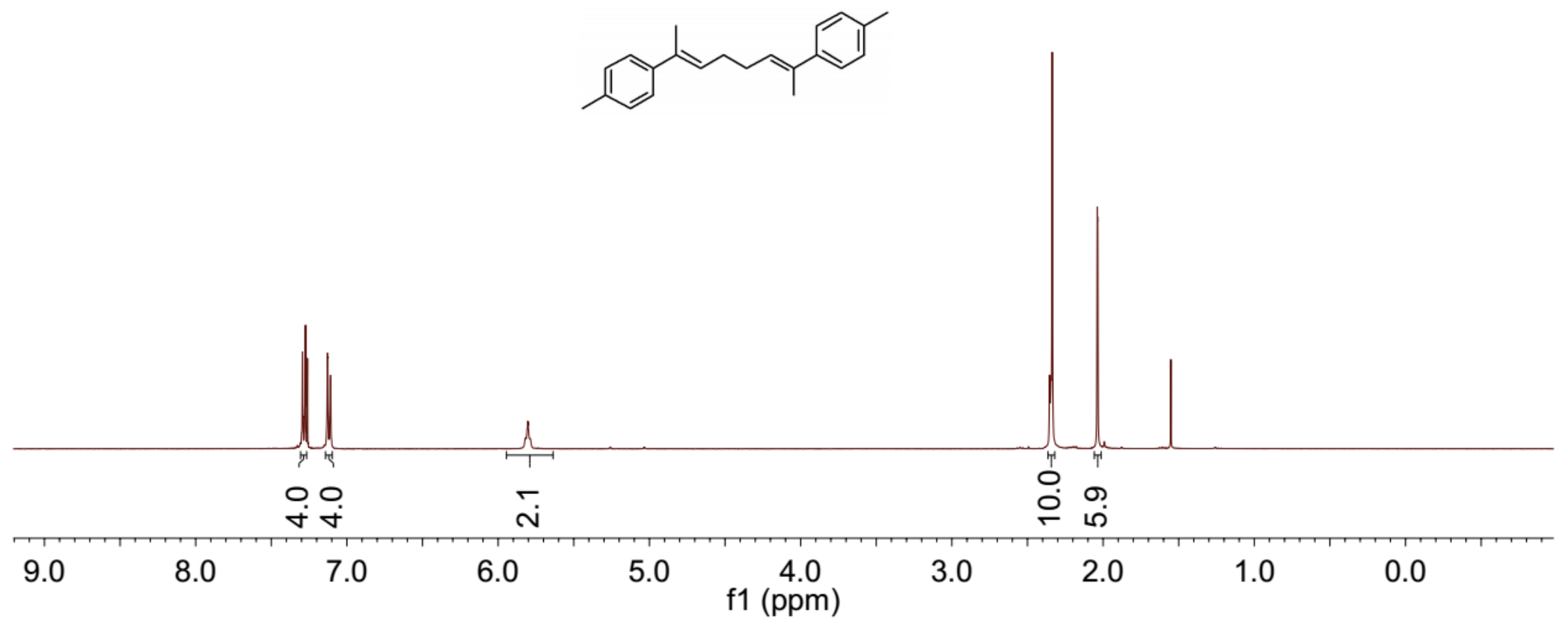


$2 a m E / Z$

등

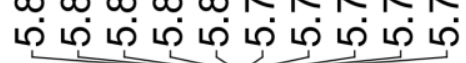

\section{으ำำㄴำ \\ में దि}

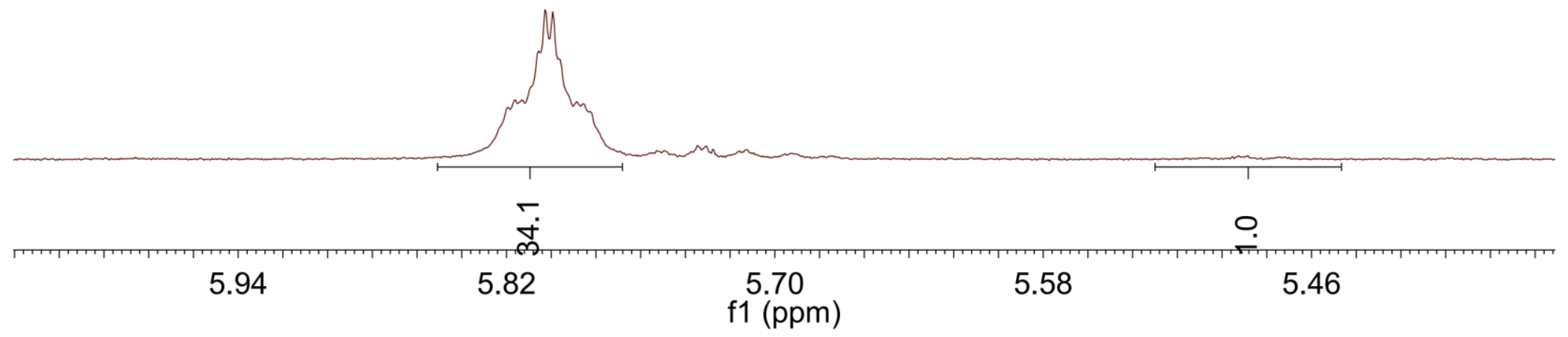




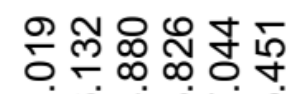

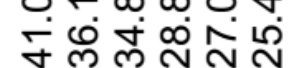

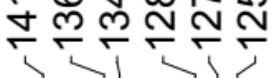

๓ำ

순

œ

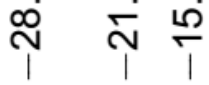

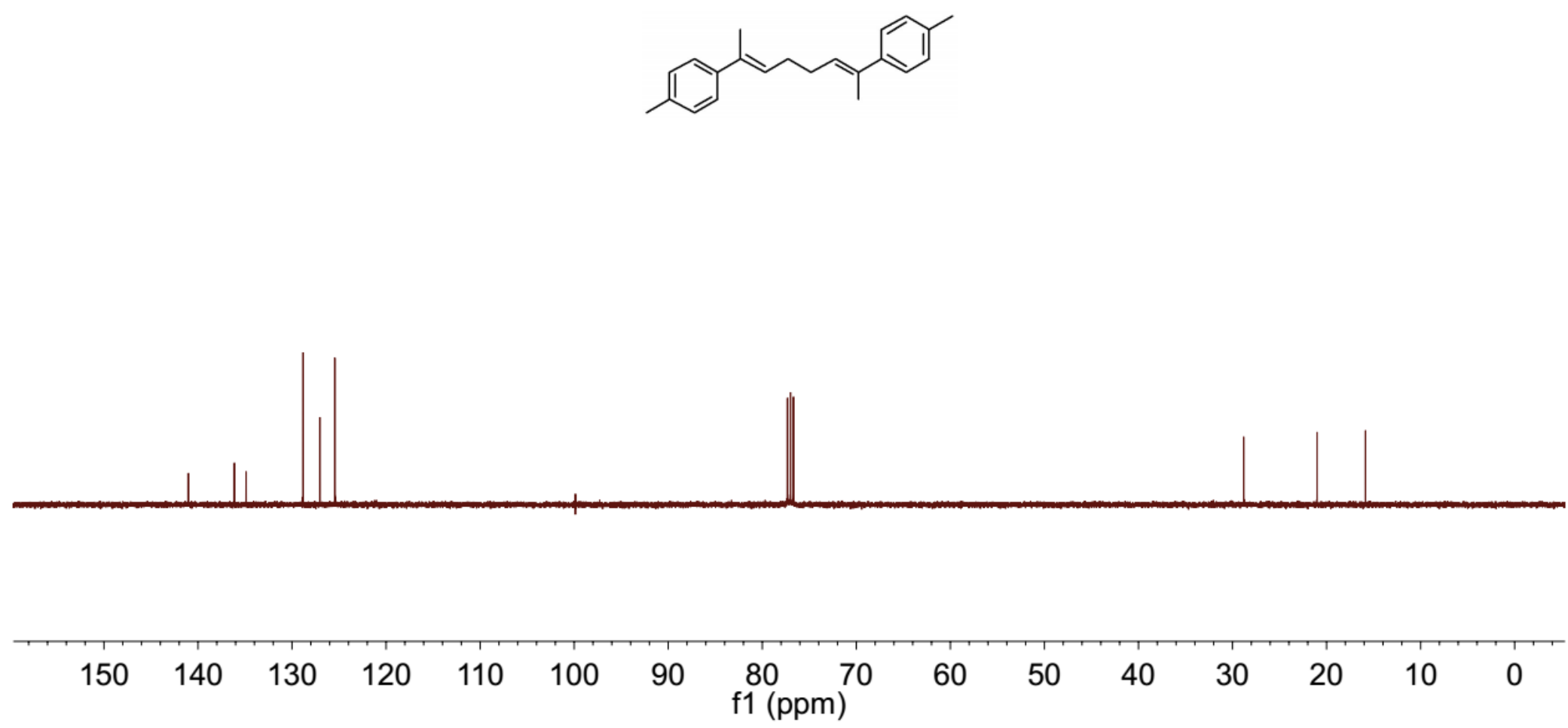


$2 a n{ }^{1} \mathrm{H} N M R$

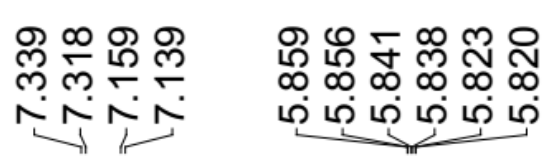

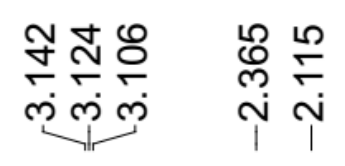
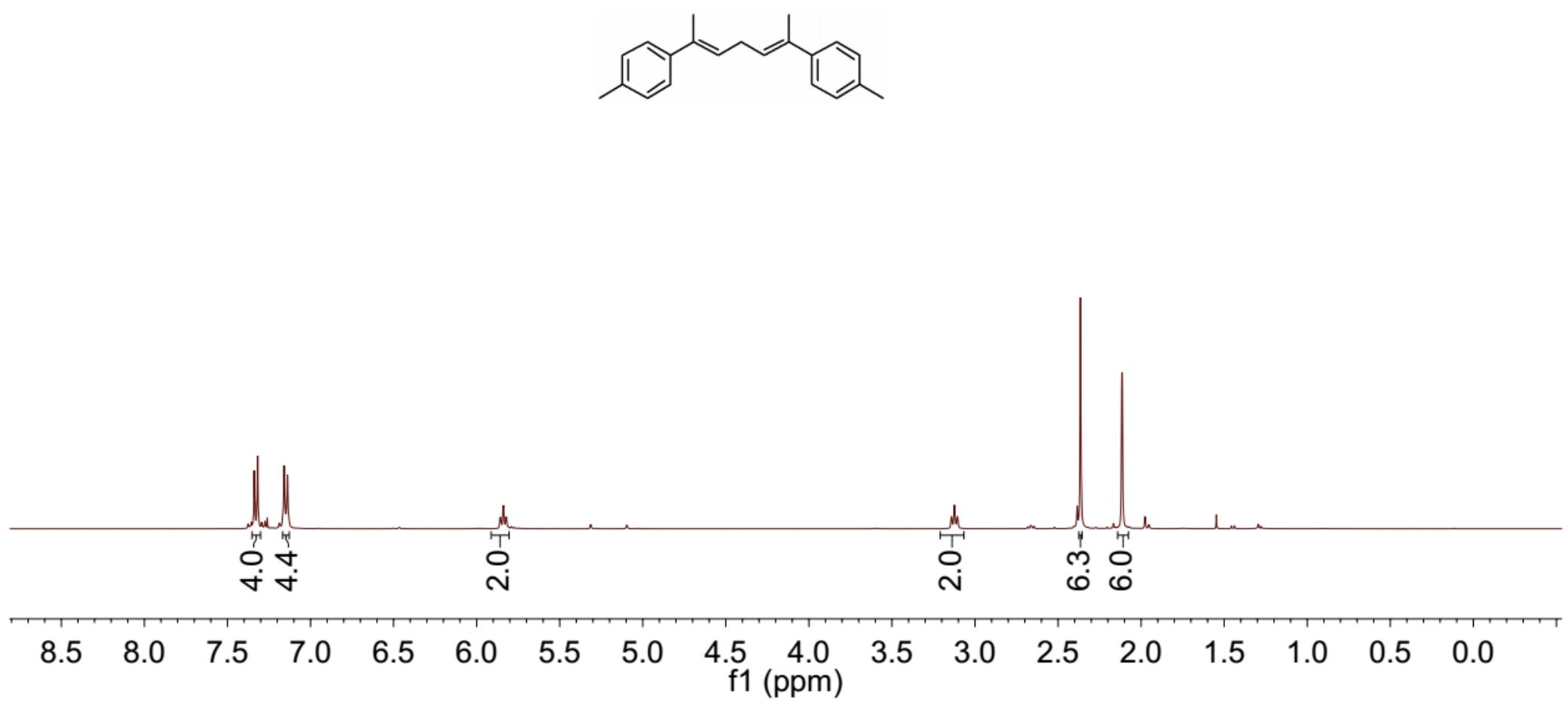

S307 
2an $E / Z$

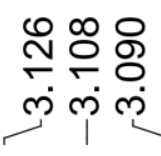

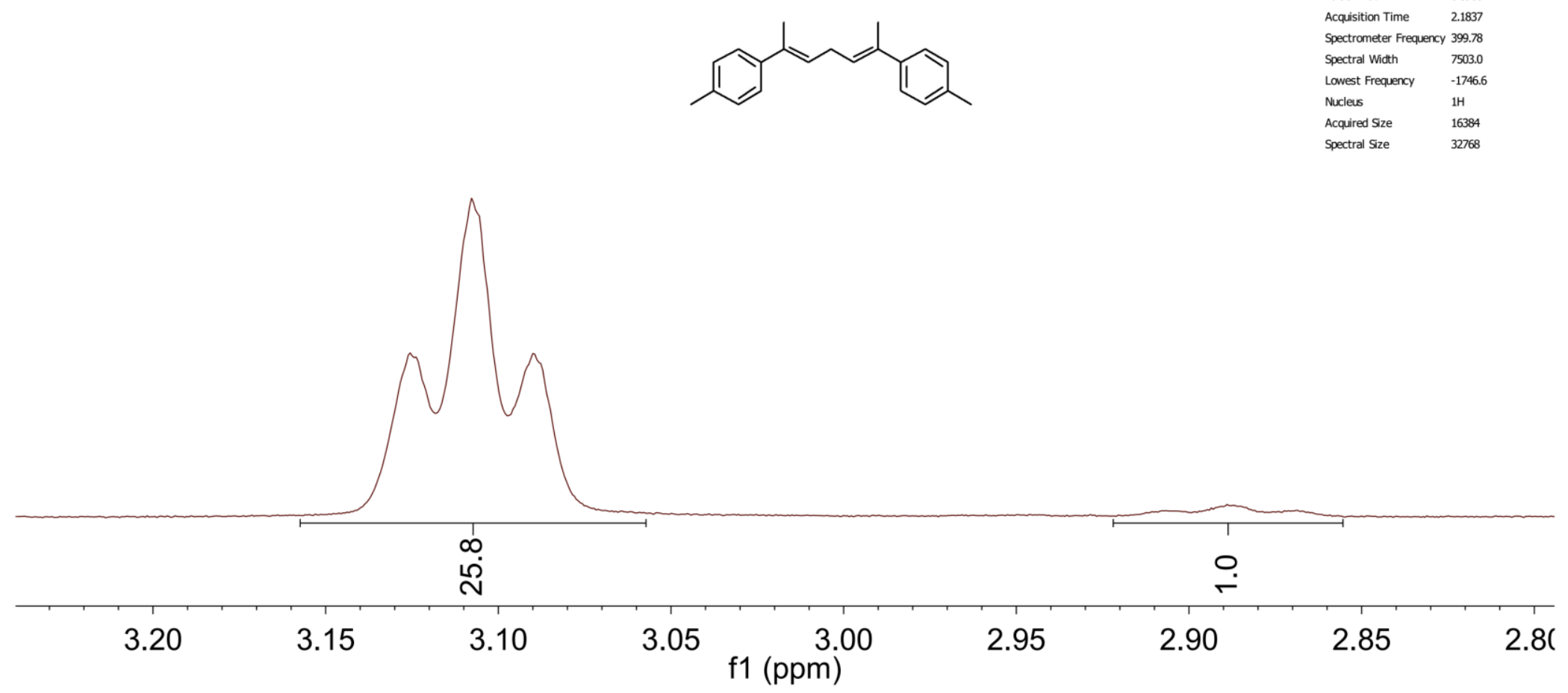

동용

ำ 
$2 a n^{13}$ C NMR

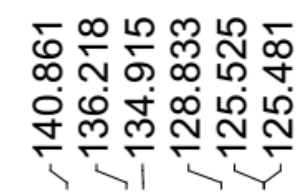

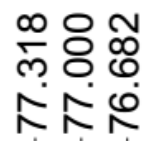

형 命

ণ
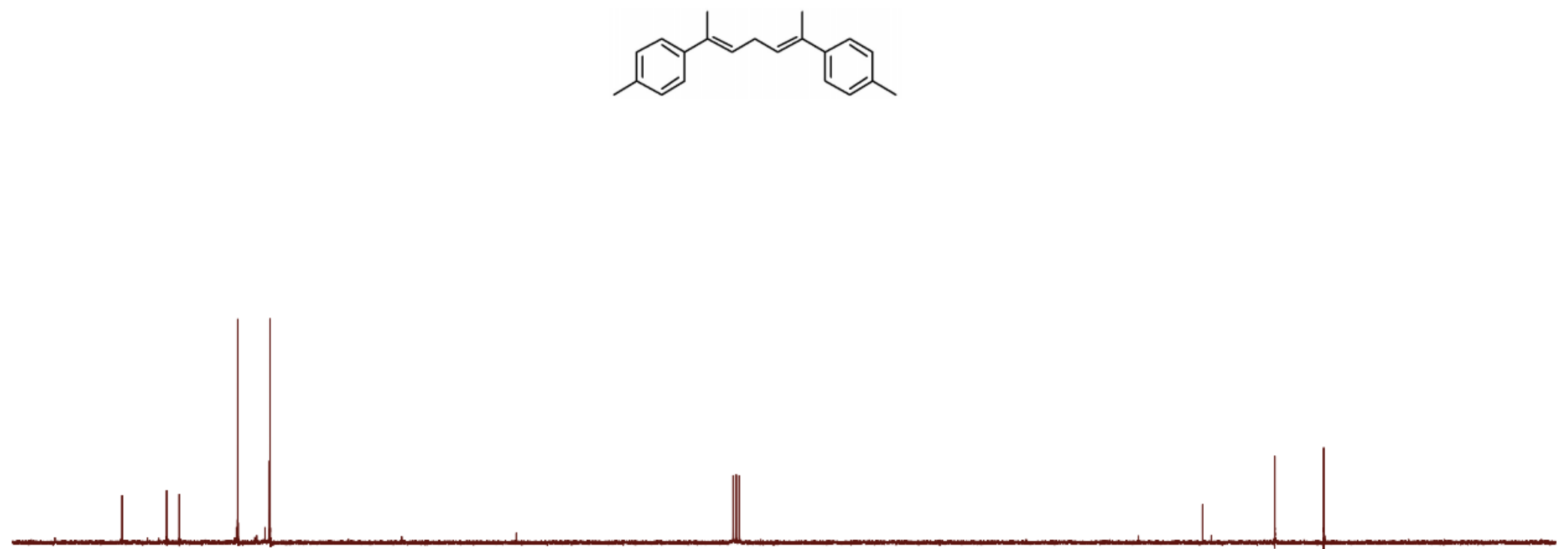

$150 \quad 140 \quad 130$

120

$110 \quad 100$

90

8070

$70 \quad 60$

$\begin{array}{llll}50 & 40 & 30 & 20\end{array}$

100 

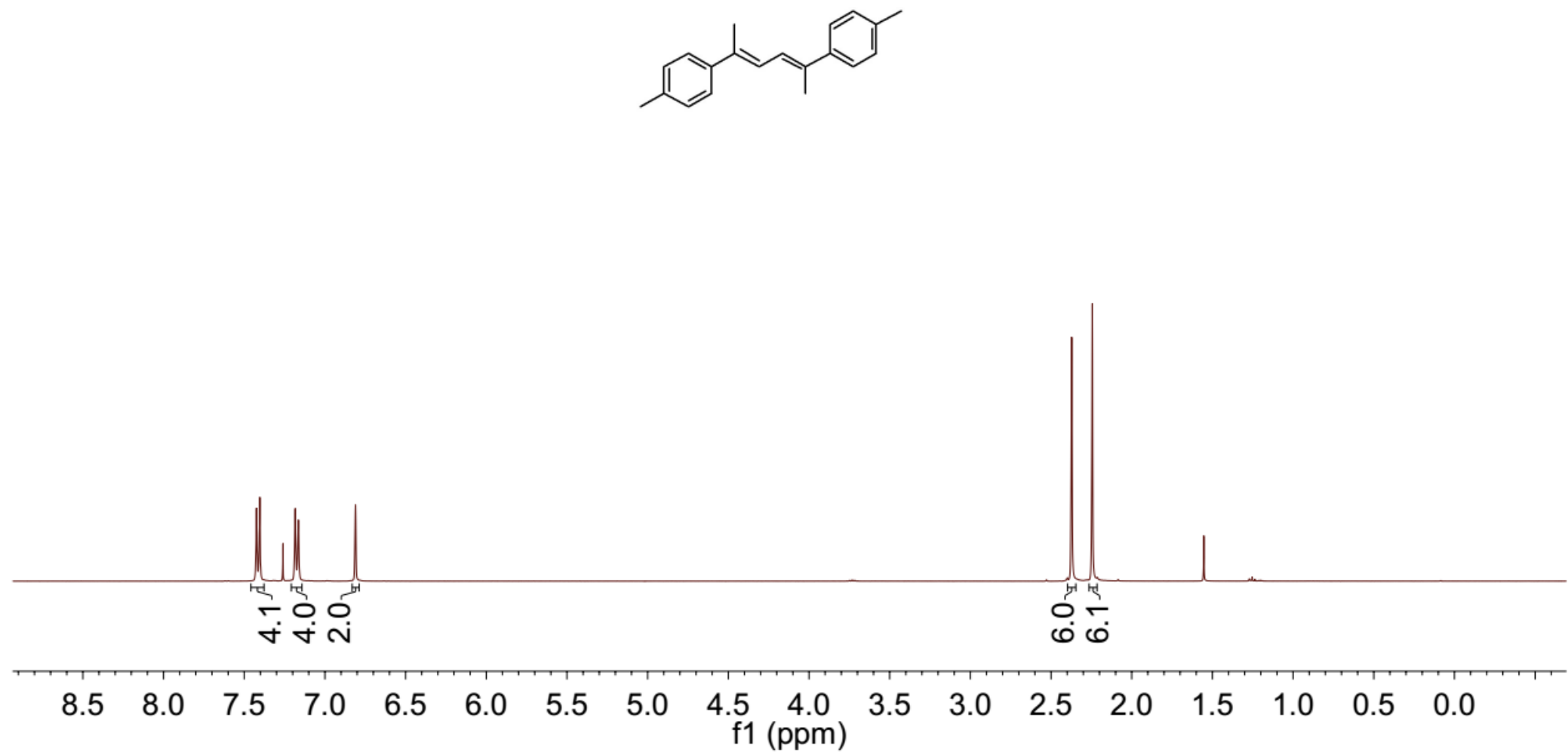
2ao $E / Z$

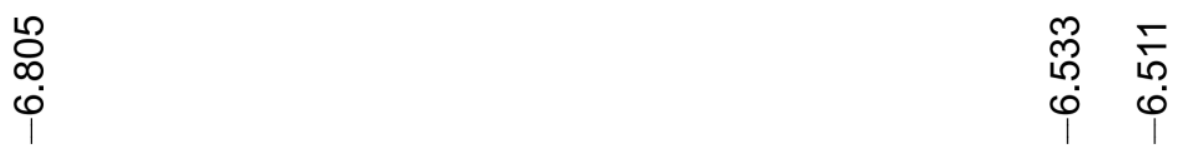

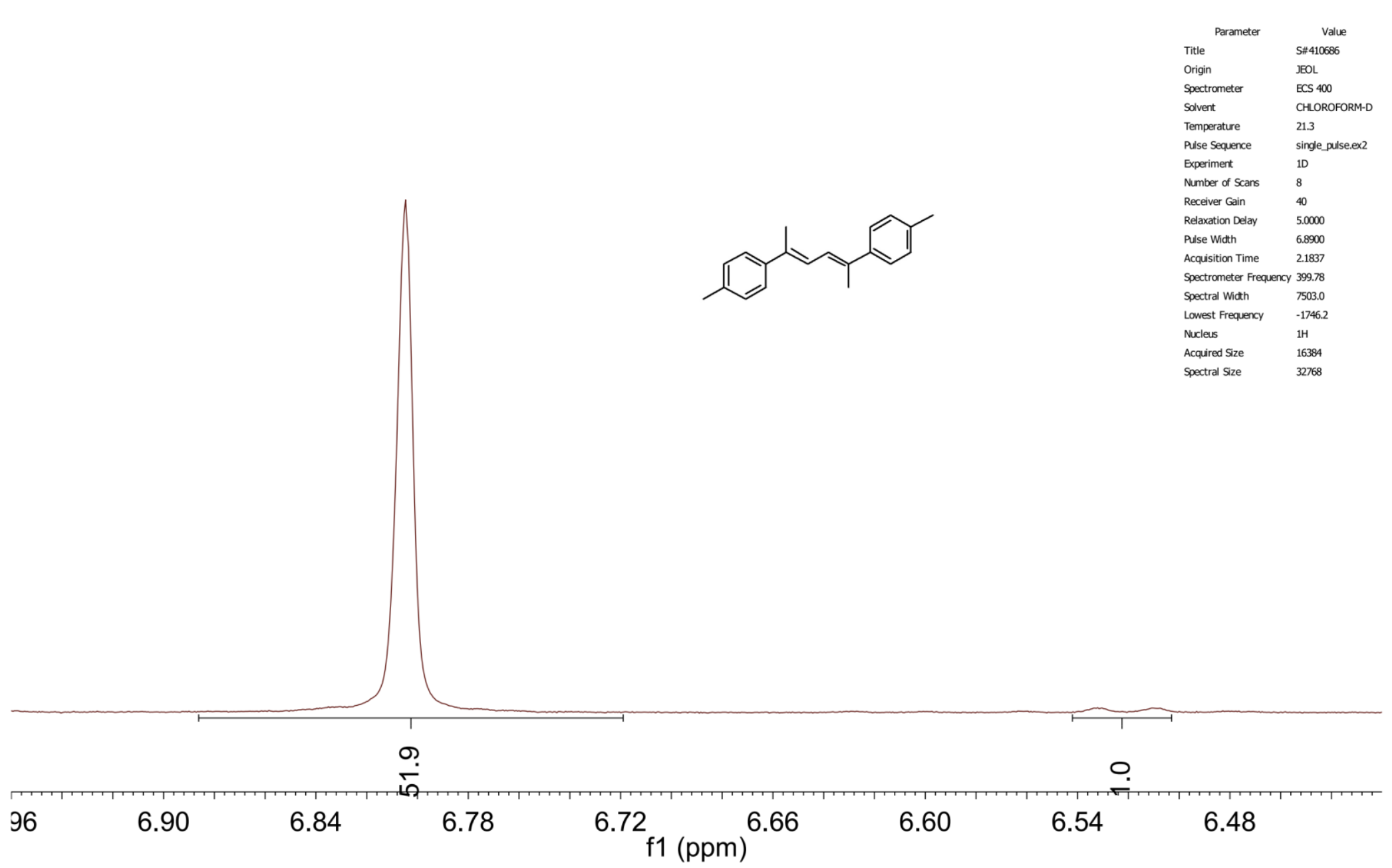


$2 a o{ }^{13}$ C NMR

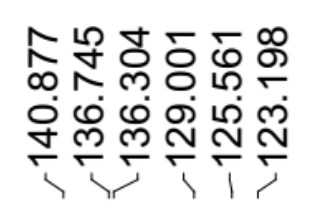

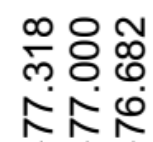

离

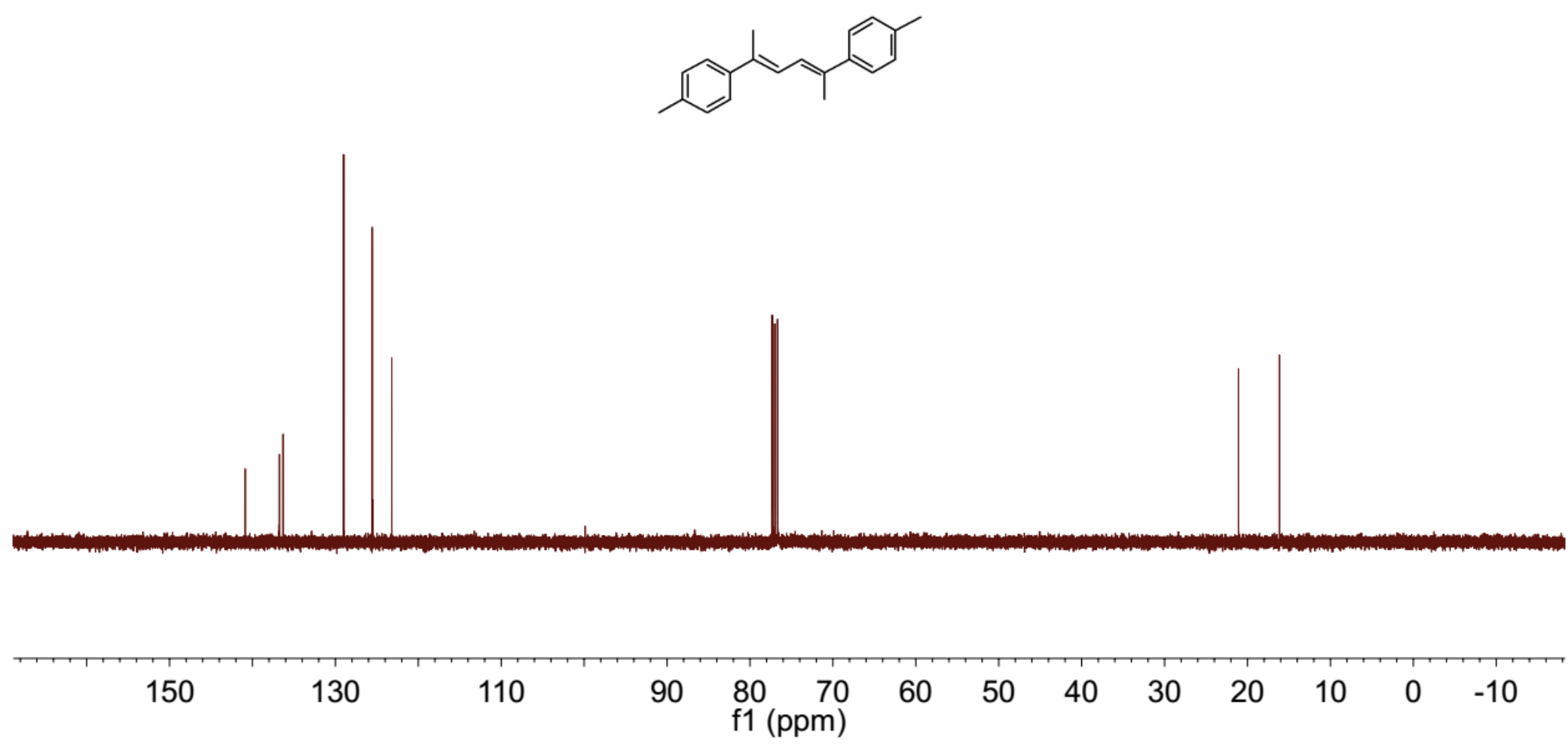



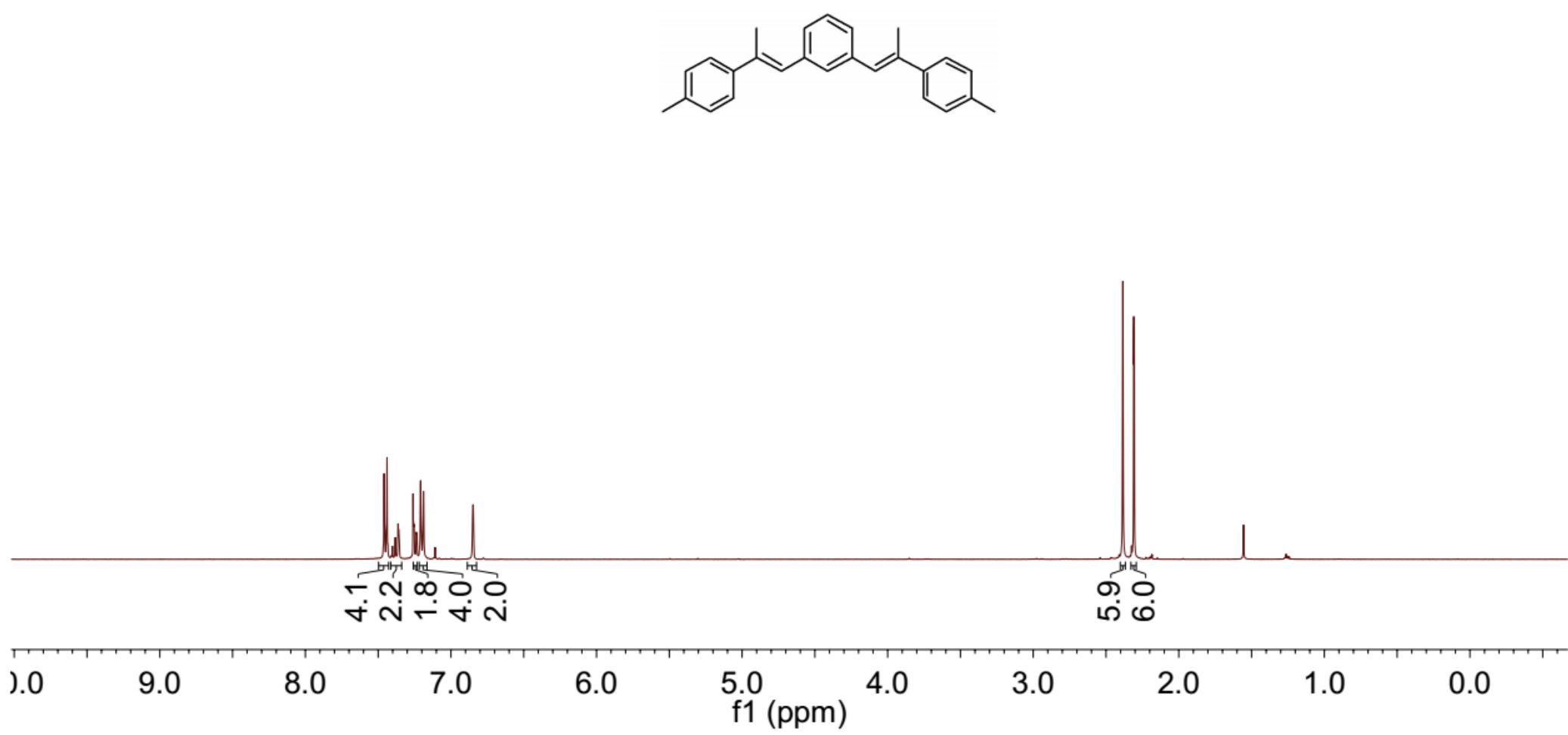
$\operatorname{2ap} E / Z$

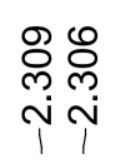

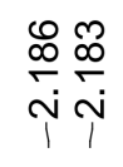

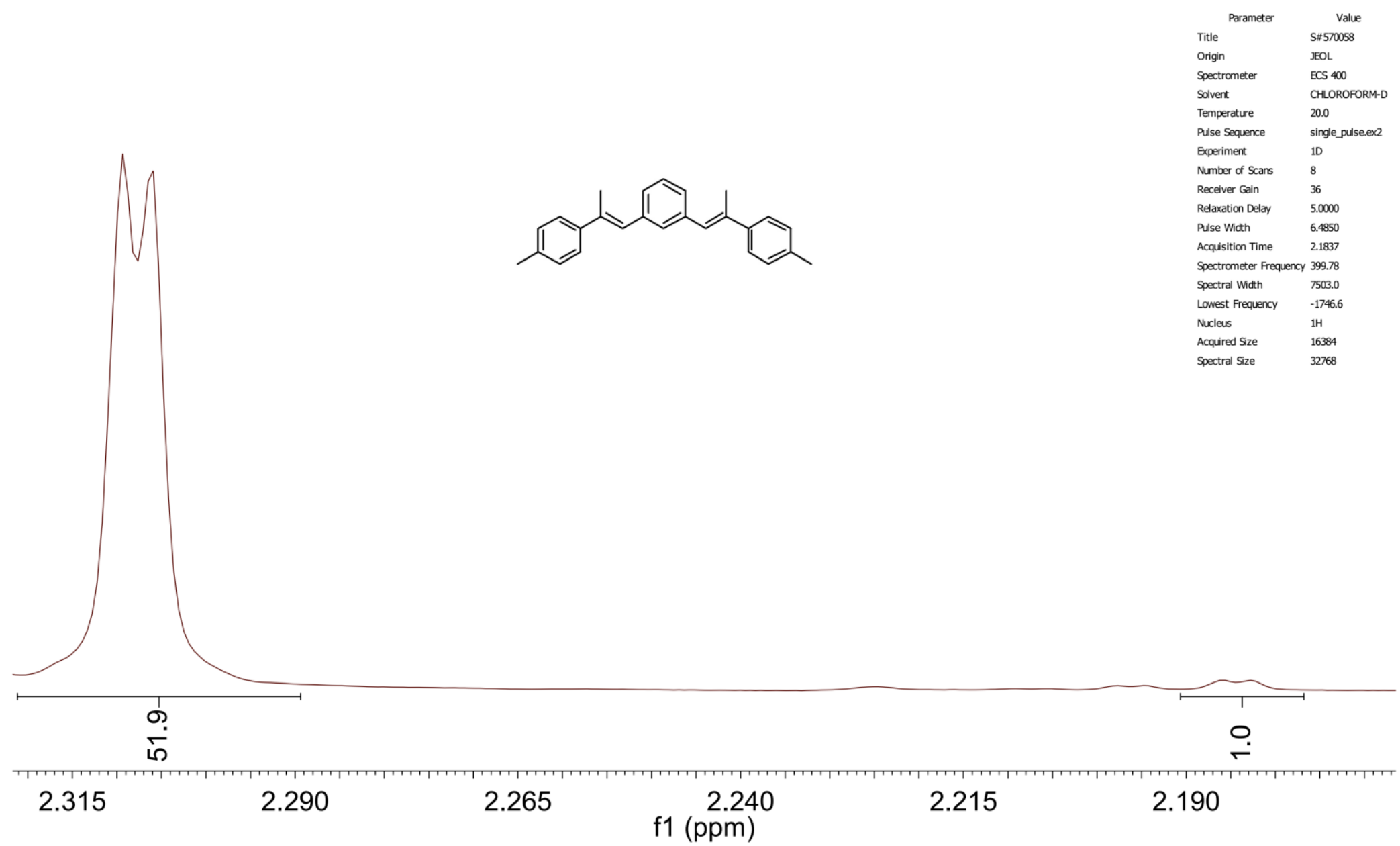

S314 

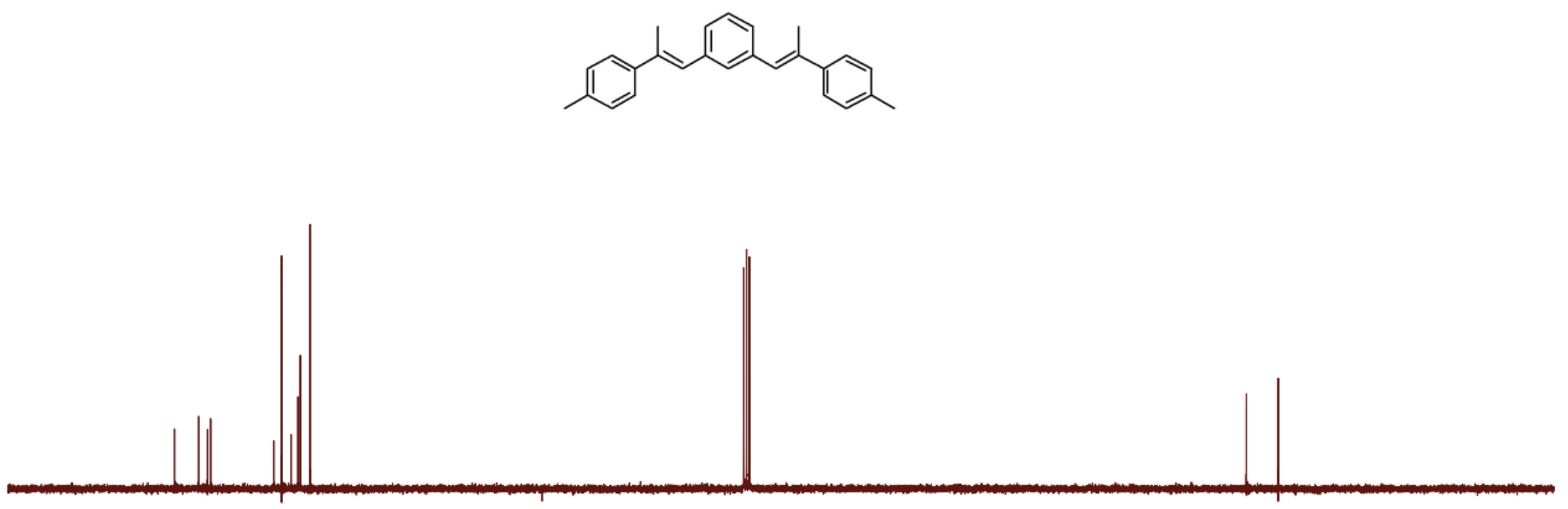

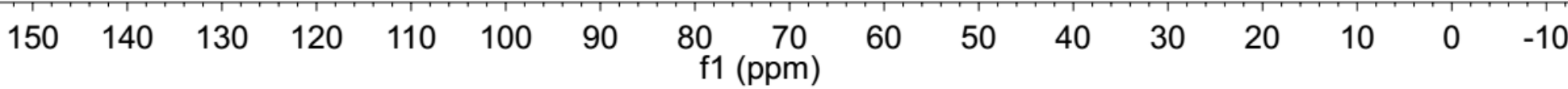



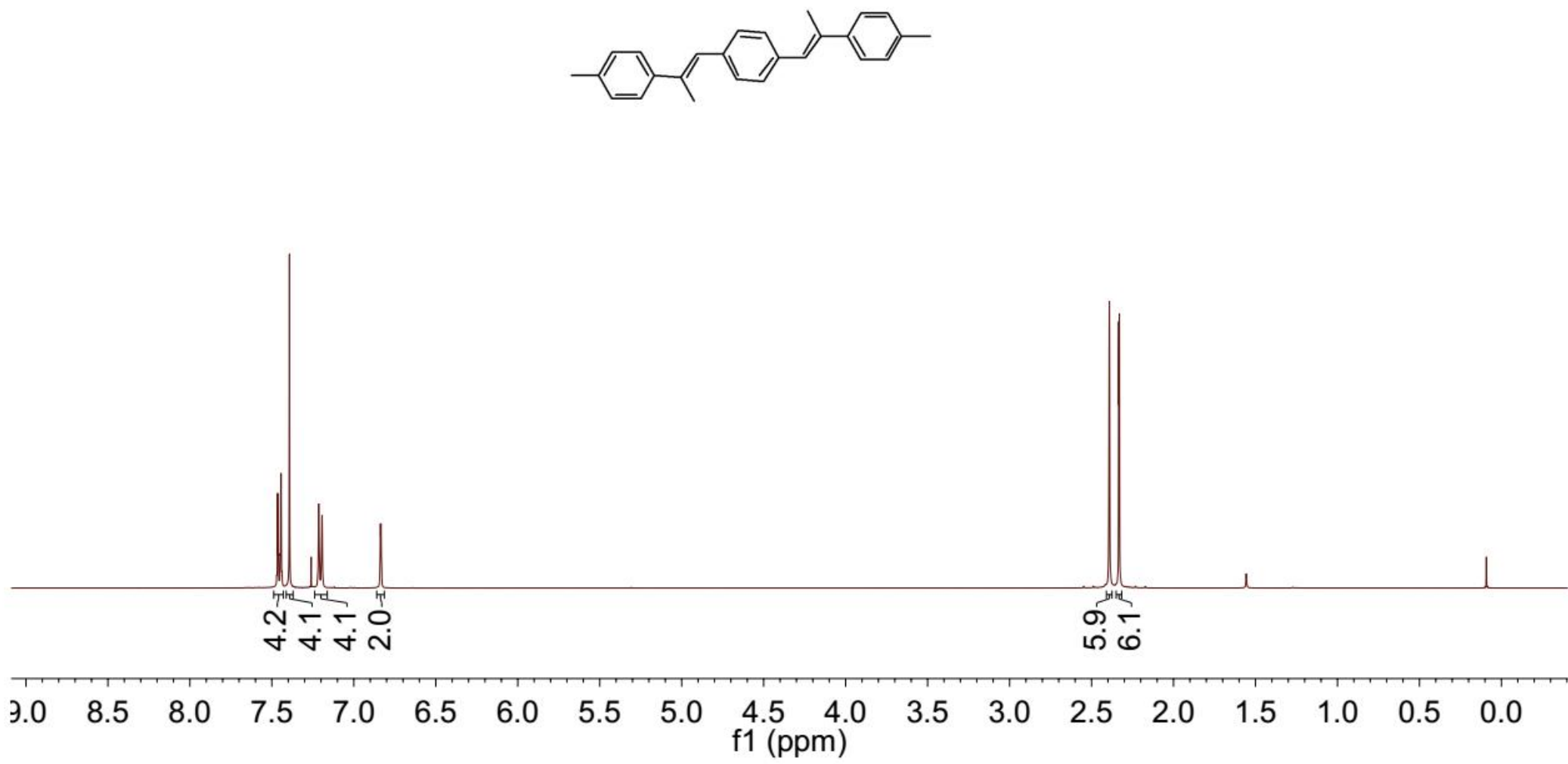
$2 a q E / Z$

$$
\text { }
$$

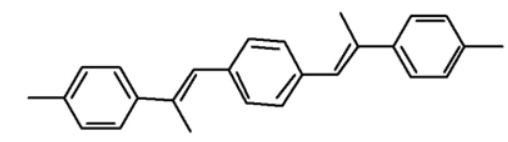

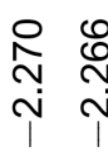
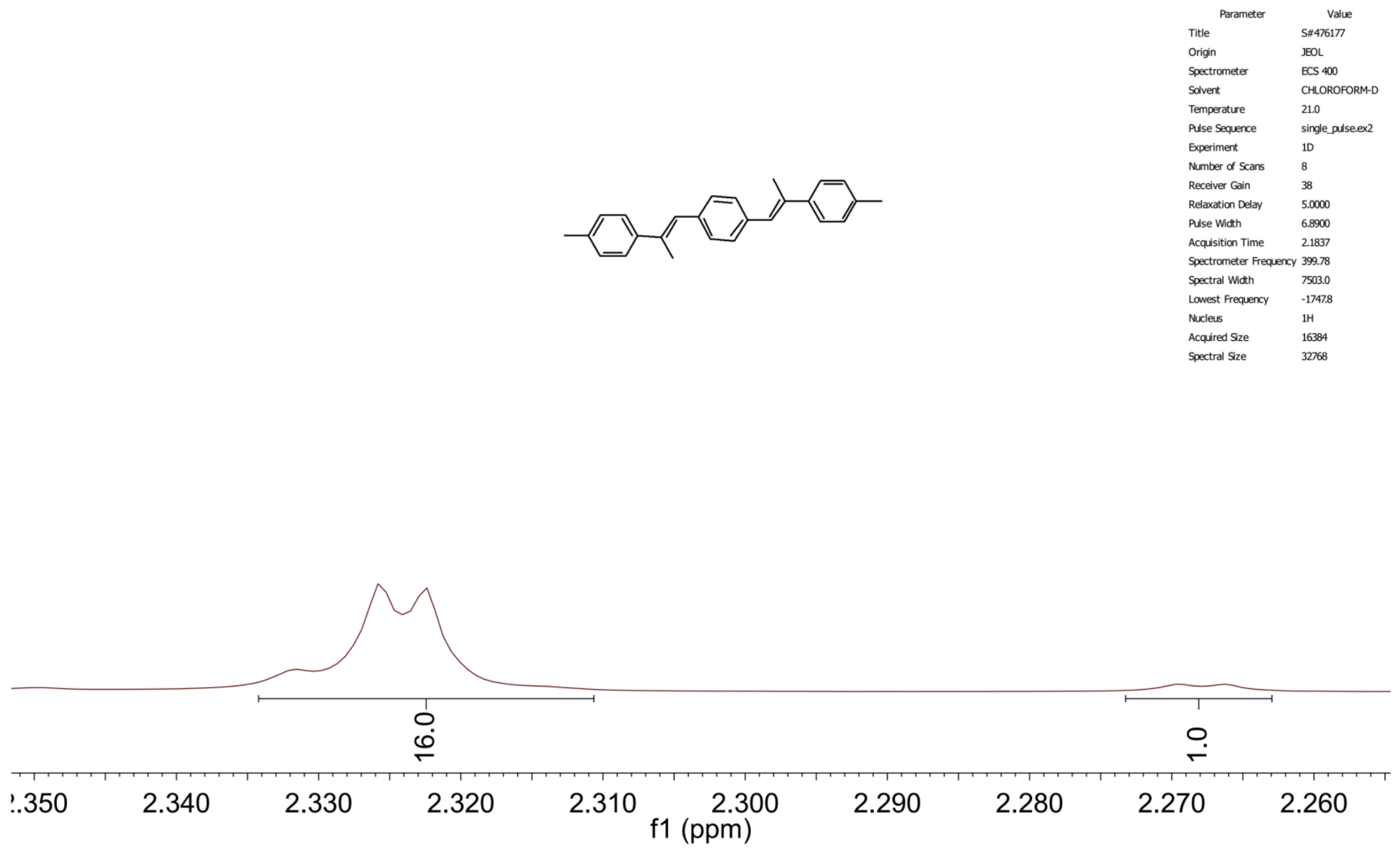


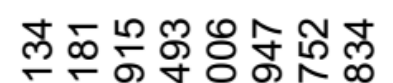 نे

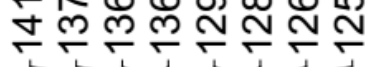

ำㅇํㅇ

र下只

œ్ָ

तิT
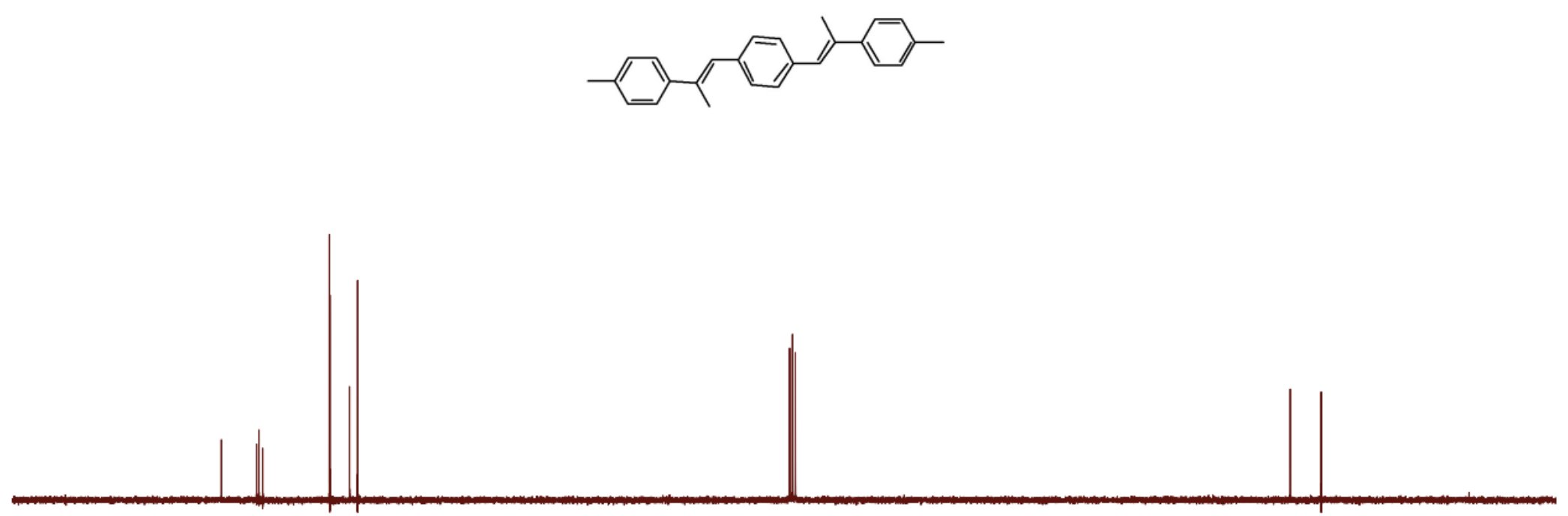

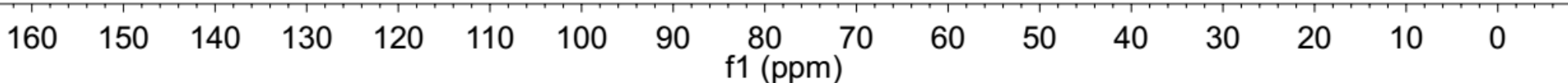




\section{mூ

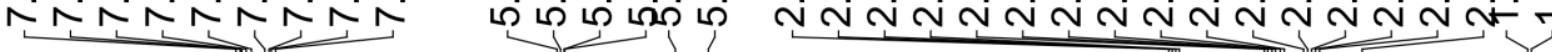
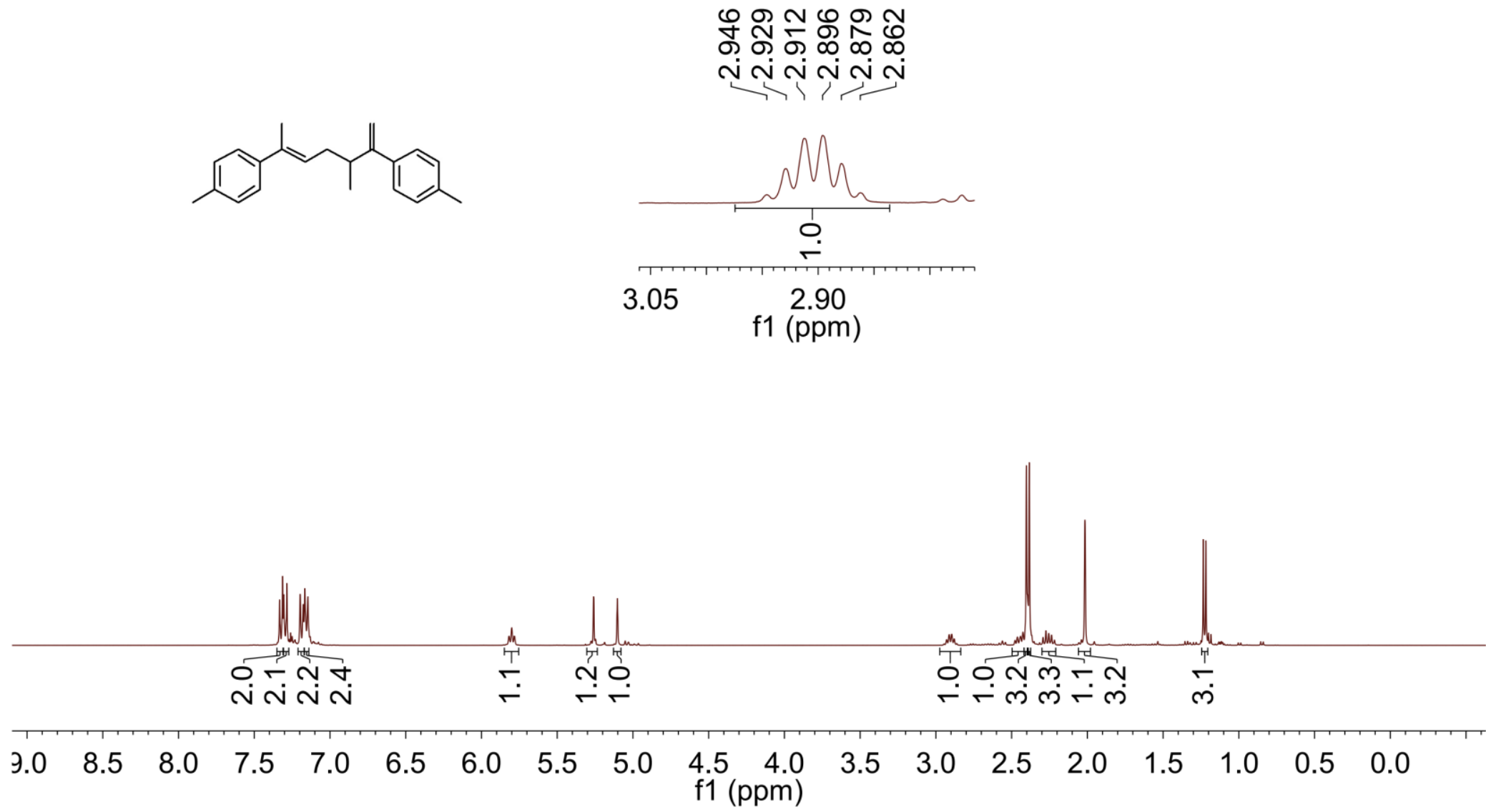


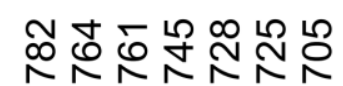

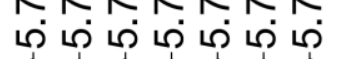

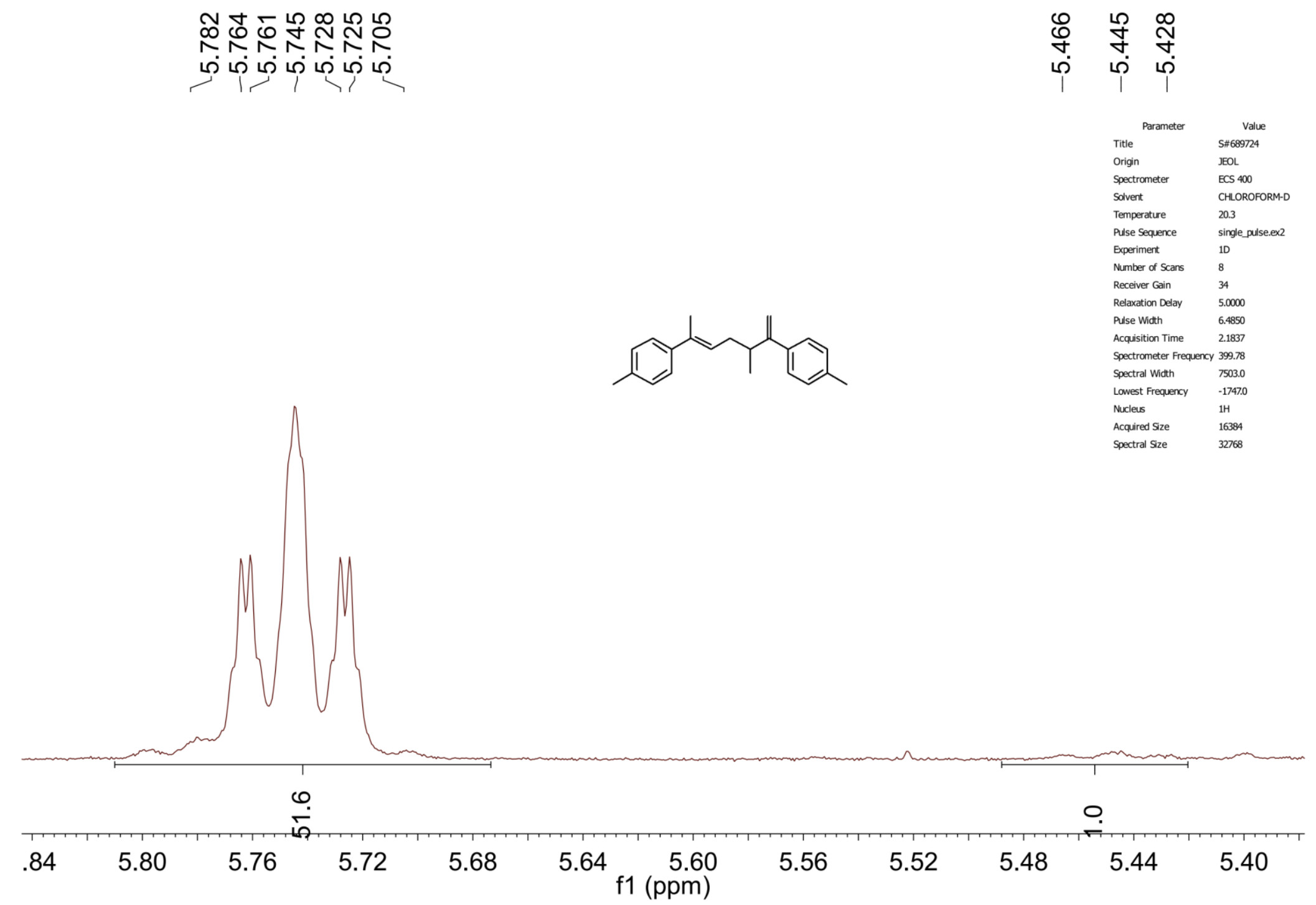




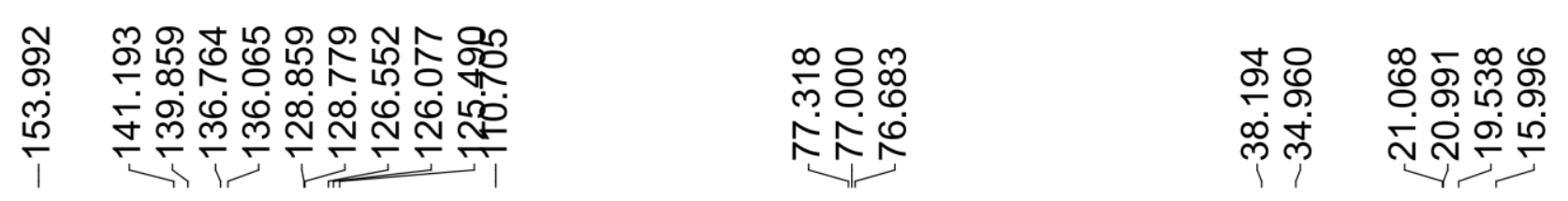

\section{항용요}

कें

mํํㄴ
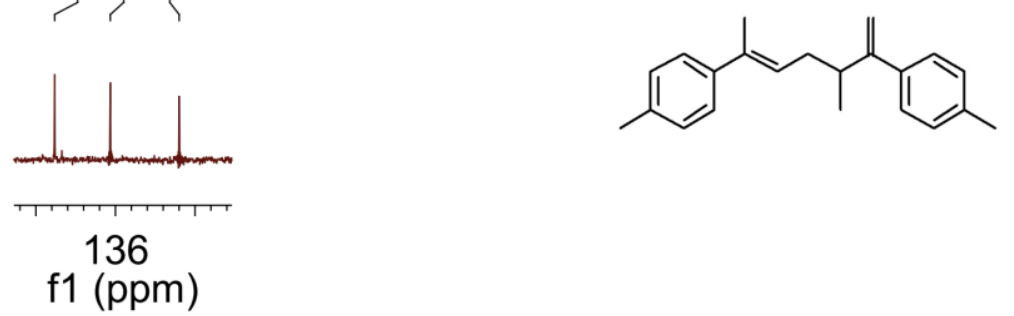

f1 136 (ppm)

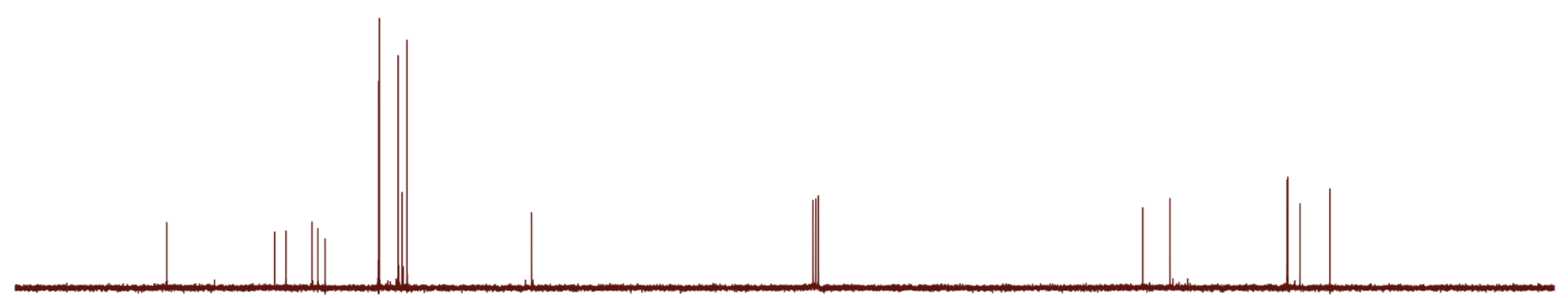

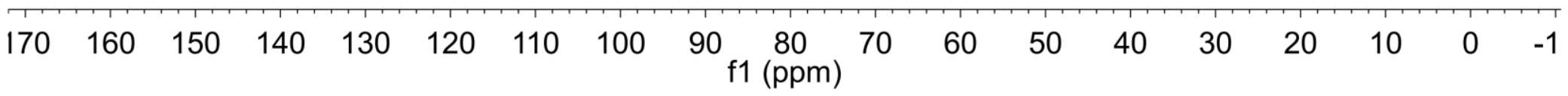

